

\title{
Augmentation of ENDF/B Fission Product Gamma-Ray Spectra by Calculated Spectra
}

\section{Recedvet by 0 OST}

NOV 811991

\section{LOS AIDMOS}

Los Alamos National Laboratory is operated by the University of California for the United States Department of Energy under contract W-7405-ENG-36. 
This work was supported by the US Department of Energy, Office of High Energy and Nuclear Physics, Division of Nuclear Physics.

An Affirmative Action/Equal Opportunity Employer

This report was prepared as an account of work sponsored by an agency of the

United States Government. Neither The Regents of the University of California, the

United States Government nor any agency thereof, nor any of their employees, makes $a .$.

warranty, express or implied, or assumes any legal liability or responsibility for the accuracy. completeness, or usefulness of any information, apparatus, product, or process disclosed, or represents that its use would not infringe privately owned rights. Reference herein to any specific commercial product, process, or service by trade name, trademark, manufacturer, or otherwise, does not necessarily constitute or imply its endorsement, recommendation, or fazoring by The Regents of the University of California, the United States Government, or any agency thereof. The views and opinions of authors expressed herein do not necessarily state or reflect those of The Regents of the University of California, the United States Government, or any agency thereof. 
Augmentation of ENDF/B Fission Product Gamma-Ray Spectra by Calculated Spectra

j. Katakura*

T. R. England 


\section{CONTENTS}

ABSTKACT 1

I. INTRODUCTION 1

II. CALCULATION OF GAMMA-RAY SPECTRA 3

III. AUGMENTED SPECTRA WITH THE CALCULATED ONES _ 5

IV. CALCULATION OF AGGREGATE SPECTRUM 7

A. Comparison with Oak Ridge National Laboratory Measurements 8

B . Comparison with University of Tokyo Measurements (YAYOI Facility) 8

C. Comparison with Los Alamos National Laboratory Measurements 9

V. SUMMARY 10

ACKNOWLEDGMENTS 10

Figure 1--Figure 411 $11-117$

REFERENCES 118

APPENDIX A--COMPARISONS USING ENDF/B-VI PRELIMINARY DATA 120

Figure A-1--Figure A-6 $121-123$

APPENDIX B--CALCULATION OF BETA-RAY SPECTRUM 124

Figure B-1--Figure B-2 126

APPENDIX C--FISSION PRODUCTS: PRELIMINARY DECAY ENERGIES, HALF-LIVES, AND BRANCHINGS FOR ENDF/B-VI 127

TABLE C-1--PRELIMINARY LIST OF ENDF/B-VI FISSION-PRODUCT PARAMETERS

ENDF/B-IV Decay File: Summary of Modifications, Supplements, and General Sources 155

Figure C-1--Figure C-3 $157-158$

TABLE C-2--FISSION-PRODUCT DECAY FILE: SUPPLEMENTS, ADDITIONS, SOURCES 


\title{
AUGMENTATION OF ENDF/B FISSION PRODUCT GAMMA-RAY SPECTRA BY CALCULATED SPECTRA
}

\author{
by
}

\author{
J. Katakura and T. R. England
}

\begin{abstract}
Gamma-ray spectral data of the ENDF/B-V fission product decay data file have been augmented by calculated spectra. The calculations were performed with a model using beta strength functions and cascade gamma-ray transitions. The calculated spectra were applied to individual fission product nuclides. Comparisons with several hundred measured aggregate gamma spectra after fission were performed to confirm the applicability of the calculated spectra. The augmentation was extended to a preliminary ENDF/B-VI file (Appendix A), and to beta spectra (Appendix B). Appendix $C$ provides information on the total decay energies for individual products and some comparisons of measured and aggregate: values based on the preliminary ENDF/B-VI files.
\end{abstract}

\section{INTRODUCTION}

Aggregate gamma-ray spectrum of fission products (FP) after fission of a fissionable nuclide is a summation of the spectra of individual FP nuclides. In order to understand the behavior of the aggregate gamma-ray spectra, it is necessary to know the spectrum of each individual FP nuclide. However, there are many short-lived FP nuclides whose spectra data have not been measured at all or that have been only partially done. If we apply such spectra to the calculation of the aggregate ones, we will obtain an underestimation of the spectral values.

This situation has been recognized in the work on decay heat after fission ${ }^{1}$ that uses average decay energy values. The gamma-ray component of the decay heat calculated by using the average energy values derived from measured spectra data usually underestimates the measured decay he: it values. To reduce the underestimation, theoretically calculated average energy values are adopted for nuclides with no measured spectra data and those considered to have partially measured ones. The calculated energy values have reproduced the measured decay heat values well; ${ }^{2}$ however, the evaluation work has focussed attention primarily on the average energy values.

Recently an attempt to adopt the theoretical calculation to gamma-ray spectra of FP nuclides was tried. ${ }^{3}$ In this attempt the calculations were performed for 32 "typical" FP nuclides characterized by mass (light and heavy), even-oddness of protons and neutrons and four $Q_{\beta}$ values (4, 7, 9, and $11 \mathrm{MeV}$ ). The "typical" spectra were applied to nuclides with partially measured spectra data, as well as those with none, for calculating the aggregate gamma-ray spectra. The resultant spectra showed fairly good agreement with the measured ones. 3 
The evaluated nuclear data file (ENDF/B-V) has not contained such theoretical spectra data; these are particularly applicable to the prediction of the aggregate gamma-ray spectra at short cooling times after fission ${ }^{4}$ because of the absence of measured spectra data of short-lived nuclides. We tried to apply the calculated spectra to augment the ENDF/B-V line spectral data. It was felt that the set of spectra ralculated for individual nulcides would provide better applicability of the spectra to the aggregate spectrum calculation than would the "typical" ones. We prepared the calculated spectra of the individual nuclides, taking into account the character of each nuclide, e.g., mass, $Q_{\beta}$ values, etc.

The ENDF/B-V file contains the FP data for 877 total nuclides. Of these, 750 nuclides are unstable and decay to another nuclide. All of the nuclides have average decay energy values for decay heat application but only 248 nuclides have gamma-ray spectral data. It is understood, however, that some of them may suffer from a problem of missing gamma rays. Therefore, we calculated the spectra of all $\beta$ - decaying nuclides in the ENDF/B-V file. (Electron capturing and/or $\beta^{+}$ decaying nuclides are considered unimportant for the calculation of the aggregate spectra due to their low fission yields.) The calculated spectra amounted to 633; they were used to augment the line spectra contained in the ENDF/B-V file. In the augmentation, the average decay energy values, which give a good prediction of the decay heat, were taken intn account so that the augmented spectra reproduce the average when the spectrum integration is performed.

Calculations of the aggregate spectra using the augmented data were completed and compared with the data measured at the Oak Ridge National Laboratory, 5 the University of Tokyo, ${ }^{6}$ and Los Alamos National Laboratory ${ }^{7}$ to confirm their applicability.

In Sec. II the calculation of he gamma-ray spectra is presented; Section III discusses the augmentation by the calculated spectra, and Sec. IV describes the calculation of the aggregate spectra after fission. The comparisons between measured aggregate gamma spectra and the calculated ones are described in Sec. V, followed by a summary of the entire project in Sec. VI.

In addition, we, have augmented a preliminary (September 1989) ENDF/B-VI file, as summarized in Appendix A. Finally, the beta spectrum also has been augmented in ENDF/B-V, as seen in Appendix B, and the preliminary ENDF/B-VI are also augmented in the same way as discussed there. Appendix $C$ provides some comparisons of aggregate calculations and other information on the preliminary ENDF/B-VI file.

This effort was initiated in order to provide essentially complete fission product spectra for ENDF/B-V and -VI. The files will delineate cases in which theoretical data are partially or entirely in use. 


\section{CALCULATION OF GAMMA-RAY SPECTRA}

We used the Gross Theory $^{8}$ of beta decay and a cascade gamma transition model ${ }^{3}$ for the spectrum calculation. The Gross Theory was employed to calculate the initial level population of a daughter nucleus fed by beta decay. In general, the calculation of the beta strength function of a nuclide needs a detailed knowledge of nuclear structure. Nuclear structure information, however, has not been satisfactory for predicting the strength function of all nuclides because nuclear properties are often experimentally incomplete. The Gross Theory, on the other hand, deals with certain average properties of betadecay and does not require the detailed knowledge of an individual nuclear state. A brief summary of the theory (based on papers by Takahashi, Yamada, and $\mathrm{Kondoh}^{8}$ ) follows.

In the Gross Theory, the summation over final nuclear states appearing in beta decay equations is replaced by an integration, and nuclear matrix elements specifying the transitions are expressed by some simple functional forms whose parameters are evaluated by sum rules and the average properties of beta decay. For example, the total decay constant of the Fermi transition is expressed as follows:

$$
\lambda=\left(\frac{1}{2 \pi^{3}}\right) \int_{Q}^{0} \sum_{\Omega}\left|g_{\Omega}\right|^{2} \cdot\left|M_{\Omega}\left(E_{g}\right)\right|^{2} \cdot f\left(-E_{g}+1\right) d E_{g},
$$

where the symbol $\Omega$ stands for type of beta decay such as Fermi and Gamow-Teller, $f$ is the integrated Fermi function, and $\mathrm{g}_{\Omega}$ is the coupling constant. In this expression, the summation over the final nuclear states is replaced by the integration with respect to the level energy $E_{\mathfrak{g}}$. The $\mid M_{\Omega}\left(E_{\mathcal{g}}\right)^{2}$ is a strength function and is the average of squared matrix elements multiplied by the final level density. The model of theGross Theory expresses the function as

$$
\left|M_{\Omega}\left(E_{g}\right)\right|^{2}=\int_{\varepsilon_{\min }}^{\varepsilon_{\max }} D_{\Omega}\left(E_{g}, \varepsilon\right) \cdot W\left(E_{g}, \varepsilon\right) \frac{d n}{d \varepsilon} d \varepsilon
$$

where $\varepsilon$ is a single nucleon energy of the decaying nucleon in a daughter nucleus, $\frac{d n}{d \varepsilon}$ is a number density of nucleons, and $W\left(E_{g}, \varepsilon\right)$ is a weight function that reflects the degree of vacancy of the final states. The function $D_{\Omega}\left(E_{g}, \varepsilon\right)$ is a single nucleon contribution to $\left|M_{\Omega}\left(E_{g}\right)\right|^{2}$ in the absence of the Pauli principle.

The properties of $D_{\Omega}\left(E_{\mathcal{g}}, \varepsilon\right)$ were studied by sum rules and trial forms were examined. Based on the examination, 8 a modified Lorentzian shape is used on our calculation. 
For the allowed transitions, Fermi and Gamow-Teller transitions are considered and the strength function of total beta decay summed over them,

$$
S_{\beta}(E)=\sum_{\Omega}\left|g_{\Omega}\right|^{2} \cdot\left|M_{\Omega}(E)\right|^{2}
$$

Once the strength function, $S_{\beta}(E)$, is determined, the level population density by beta feeding, $\mathrm{b}(\mathrm{E})$, is given by

$$
b(E)=S_{\beta}(E) \cdot f\left(Z, Q_{\beta}-E\right) \cdot T_{1 / 2}
$$

where $f$ is the Fermi function and $T_{1 / 2}$ is a half-life of beta decay.

After determining the level population density, gamma transitions are treated as successive de-excitation from higher levels and the level population density is changed to be a summation of that by beta feeding and by the gamma de-excitation.

$$
P(E)=b(E)+\int_{E}^{E_{\max }} P\left(E^{\prime}\right) G\left(E^{\prime}-E\right) \rho\left(E^{\prime}\right) d E^{\prime}
$$

where $G\left(E^{\prime}-E\right)$ is a probability of gamma emission and $\rho\left(E^{\prime}\right)$ is the level density of the daughter nucleus.

The intensity of the gamma rays, then, becomes

$$
I\left(E_{\gamma}\right)=\int_{0}^{E_{\max }} d E^{\prime} \int_{E^{\prime}}^{E_{\max }} d E^{\prime \prime} \delta\left(E^{\prime \prime}-E^{\prime}-E_{\gamma}\right) P\left(E^{\prime \prime}\right) G\left(E^{\prime \prime}-E^{\prime}\right) \rho\left(E^{\prime}\right)
$$

The probability $G(E)$ depends upon the type of the gamma transition, such as $E 1, M 1, E 2$, etc. In our calculations, we take the assumed $\mathrm{E} 1$ transitions. In this case, $\mathrm{G}(\mathrm{E})$ is expressed as

$$
\mathrm{G}(\mathrm{E})=\mathrm{E}^{3} \cdot \mathrm{S}_{\gamma}(\mathrm{E})
$$

where $S_{\gamma}(E)$ is the gamma-ray strength function. We used the strength function proposed by Brink $^{9}$ and by Axel ${ }^{10}$ and the level density based on Gilbert and Cameron. ${ }^{11}$

In the frame of the Gross Theory, a parameter Q00 (introduced to take into account the effect of the selection rules applied to decays to low-lying states) plays an important role in the 
calculation of average decay energy values. ${ }^{12-13}$ In the calculation of the gamma-ray spectrum based on the present model, however, the introduction of a non-zero value to the parameter produces a discontinuity in the energy of the Q00 parameter (see Figs. 1-4). To avoid this discontinuity, we have used a value of zero for the calculation of the spectra.

Using the above procedure, the gamma spectra were calculated for all $\beta$-decaying nuclides in the ENDF/B-V file. The calculated spectra have an energy bin structure of $10 \mathrm{keV}$, and the ener $y$ spectra were normalized to 1.0 (the ENDF/B-V spectra is truncated for some nuclides and may not integrate to 1.0 ).

Examples of the calculated spectra are shown in Figs. 5-8 for nuclides with high $Q_{\beta}$ values. In these figures the "typical" spectra (used in Ref. 3) are also shown. As seen in these figures, the "typical" energy spectra are softer than those currently calculated. It is expected that the enhancement at about $2 \mathrm{MeV}$ (again, in Ref. 3) should be depressed.

The calculations of the aggregate spectra using the present calculated spectra for all $\beta$ - decaying nuclides are shown in Figs. 9-12, together with the calculations for the ENDF/B-V spectra data and the measured aggregate spectra of ${ }^{235} \mathrm{U}$ and ${ }^{239} \mathrm{Pu}$ thermal neutron fission. 5 The calculational method of the aggregate spectra will be described in Sec. IV. The measured data were taken from Dickens et al. ${ }^{5}$ Figures 9 and 10 illustrate ${ }^{235} \mathrm{U}$ fission and Figs. 11 and 12 . the ${ }^{239} \mathrm{Pu}$ fission. Figures 9 and 11 cover the full energy range of the measured spectra, while Figs. 10 and 12 show the low-energy part up to $2 \mathrm{MeV}$. It can be seen from these figures that the calculations with only the ENDF/B-V spectra underestimate the measured values for the full energy range. Alternatively, the calculations using only the present calculated spectra [GT (Gross Theory) spectra only] show overall agreement with the measurements except for the detailed fine structure and the overestimation seen at energies between 2 and $3 \mathrm{MeV}$.

\section{AUGMENTED SPECTRA WTTH THE CALCULATED ONES}

It is important to decide if the spectrum should be augmented or not in the cases in which a nuclide has a measured spectrum. A definitive means for making this decision does not exist. However, the average decay energy set, which gives a good prediction for the many temporal values of decay heat after fission, can be used as an accurate measurement of the defective spectrum. It is known from decay heat studies that some of the average energy values derived from the measured spectra data show an underestimation of gamma-energy values due to missing gamma rays. ${ }^{1}$ This means that the gamma-energy value based on the measured spectral data is smaller than that which is used for the decay heat prediction. In this case, the calculated spectra should be added to the measured ones in order to compensate for the difference between them. That is, if $E_{a}$ is the average energy value for the decay heat calculation and $E_{s}$ is from measured spectral data, $\Delta E=E_{a}$ $E_{S}$ is proportional to the defect of the spectrum. The normalized energy spectrum calculated in 
Sec. $I I$ is multiplied by the $\Delta \mathrm{E}$ value for the augmentation. Thus, the augmented energy spectrum $A\left(E_{\gamma}\right)$ is expressed by a sursmation of the experiment one, $E\left(E_{\gamma}\right)$ and the calculated one, $C\left(E_{\gamma}\right)$, as follows:

$$
A\left(E_{\gamma}\right)=E\left(E_{\gamma}\right)+\Delta E \cdot C\left(E_{\gamma}\right)
$$

By this expression, the energy value averaged with the above spectrum becomes equal to that for the decay heat calculation.

$$
\begin{aligned}
\bar{E} & =\int A\left(E_{\gamma}\right) d E \\
& =E_{s}+\Delta E \\
& =E_{s}+\left(E_{a}-E_{s}\right) \\
& =E_{a}
\end{aligned}
$$

In the present calculation, we used the JNDC V2 library ${ }^{13}$ as an average energy set to partially assist us in the determination of when an augmented spectra appeared to be needed.

Examples of the augmented spectra are seen in Figs. 13 and 14, as well as the measured spectra. In these figures the lower part indicates the measured line spectrum and the upper part, the augmented one. Figure 13 shows the ${ }^{98} \mathrm{Sr}$ decay, the $\mathrm{Q}_{\beta}$ value of which is $5.8 \mathrm{MeV}$. In the ENDF/B-V file, there are 11 gamma rays emitted through ${ }^{98} \mathrm{Sr}$ decay, and the highest energy of the gamma ray is $600 \mathrm{keV}$. The average energy from the spectral data is $0.176 \mathrm{MeV}$; that in the JNDC V2 library, on the other hand, is $1.051 \mathrm{MeV}$. The difference between the two is augmented by the calculated spectra. The calculated spectrum used for the augmentation is a continuous one and smoothly extends to the $Q_{\beta}$ value limit. Another example, shown in Fig. 14, is the case of $97 \mathrm{~m} Y$ decay. The highest energy of the gamma ray in this case is much lower than the $Q_{\beta}$ value. The average energy from the spectral data is $1.82 \mathrm{MeV}$ and that in the JNDC V2 library is $3.34 \mathrm{MeV}$. The calculated spectra of the nuclide is used to augment the difference between them.

This concludes the brief discussion regarding the method of Gross Theory calculations and the augmentation of selected measured spectra. The reason for the augmentation of each spectra was considered in more detail than we have presented. Many nuclides are known to have an incomplete spectra, and there are other parameters, such as Qp, which indicate inadequate spectra when compared with average energies derived from spectra. We acknowledge that our reasons for a few of the augmentations could be in error. The new files will contain sufficient information for users to examine the augmentations, and no evaluated measurements will be missing from the files. 


\section{CALCULATION OF AGGREGATE SPECTRUM}

Aggregate spectrum is a summation of the spectra of FP nuclides produced after nuclear fission.

$$
A\left(E_{\gamma}, t\right)=\sum_{j} \lambda_{j} \cdot N_{j}(t) \cdot a_{j}\left(E_{\gamma}\right)
$$

where $a_{j}\left(E_{\gamma}\right)$ is the spectra of the $j$-th FP nuclide, $\lambda_{j}$ is the decay constant, and $N_{j}(t)$ is the nuclide concentration at time $t$. The nuclide concentrations of FP nuclides at time $t$ were calculated with the CINDER-10 code, ${ }^{14}$ which has been developed at Los Alamos National Laboratory. The library for CINDER-10 calculations contains all nuclear data needed for the calculation of the nuclide concentration. However, we used preliminary fission yield data evaluated by England et al. 15 for the ENDF/B-VI file. After obtaining the nuclide concentrations at various cooling times, the aggregate spectra were calculated by summing up the spectrum of each nuclide at these cooling times.

In the comparison with measurement, however, the measured data are broadened because of the finite energy resolution of the detector. In order to take into account the effect of broadened spectra in the comparison, we used the detector resolution reported with the measired data to broaden each fission-product spectra used in calculations.

Figures 15-18 show the comparisons of the calculated aggregate spectra after the fission event with the same measured data in Figs. 9-12. Three kinds of calculations are illustrated in these figures: the ENDF/B-V line spectra augmented by the present calculated ones (ENDF/B-V + GT spectra); the JNDC line spectra augmented by the present calculated ones [JNDCV2 (line) + GT spectra]; and the JNDC line spectra augmented by the "typical" ones used in Ref. 3 (JNDCV2 only). The first and second ones show nearly the same behavior, the difference between them being due to the line spectra contained in each file. Their agreement with measured data is better than that of the third, which shows the digression at higher energies, especially above $6 \mathrm{MeV}$, and the overestimation at low energies. This behavior reflects the "softness" of the "typical" spectra used in the calculation. As is evident in these figures, the present spectra seem to be applicable to the calculation of the aggregate spectra after fission of a fissionable nuclide. (Only the spectra change in these comparisons, not, e.g., densities.)

\section{COMPARISON WITH MEASURED AGGREGATE SPECTRA}

In confirming the applicability of the calculated spectra of individual FP nuclides to the calculation of the aggregate gamma-ray spectra after a fission event, we performed comparisons with aggregate spectra measured at Oak Ridge National Laboratory, the University of Tokyo, and Los 
Alamos National Laboratory. In the comparisons, the line spectra of gamma rays were broadened according to the detector resolution reported. The calculated GT spectra, due to their smoothness and continuous nature, were not considered in need of broadening. The energy group structure of the calculated aggregate spectra was also matched to that of each measurement. Additionally, the measurements are made over a counting period, but the calculations are carried out for a specific time that corresponds to the average time during the counting period.

All of the measured aggregate gamma spectra are compared with the calculations using the ENDF/B-V spectra data (i.e., caloglations using the ENDF/B-V only) and the augmented one (using ENDF/B-V + GT spectra).

In the following comparisons, the unit of the spectrum is expressed as $\mathrm{MeV} / \mathrm{sec} /$ fission/bin; i.e., the energy release rate per fission divided by the bin width in energy units.

\section{A. Comparison with Oak Ridge National Laboratory Measurements.}

The aggregate gamma-ray spectra from ${ }^{235} \mathrm{U},{ }^{239} \mathrm{Pu}$, and ${ }^{241} \mathrm{Pu}$ thermal neutron fission were measured by Dickens $e t$ al. at Oak Ridge. ${ }^{5}$ The spectra data are reported up to $8 \mathrm{MeV}$. The average cooling times after fission cover from $2.7 \mathrm{~s}$ to $12000 \mathrm{~s}$.

The compared results are shown in Figs. 19-61 for ${ }^{235} \mathrm{U}$, Figs. $62-104$ for ${ }^{239} \mathrm{Pu}$, and Figs. $105-148$ for ${ }^{241} \mathrm{Pu}$. As is evident in these figures, the augmentation by the GT spectra improves the calculation of the aggregate spectra at cooling times shorter than a few hundred seconds. In this cooling-time region, the augmented spectra reproduce the measured ones rather well for these fissionable nuclides, irrespective of their different fission yields. In particular the reproduction is good at energy regions lower than $3 \mathrm{MeV}$. Above $3 \mathrm{MeV}$, the calculated spectra cannot make some peaks, but they seem to show the overall agreement.

After a few hundred seconds, the difference between the calculation with the ENDF/B-V spectral data and the augmented ones is not seen. This indicates that the nuclides with measured, augmented spectra data hardly contribute to the aggregate spectra at long cooling times. However, the discrepancies between the calculation and measurement seen at long cooling time regions seem to show that even the nuclides with long half-lives may have insufficient spectral data for application to the calculation of the aggregate spectrum. Further examination of measured spectra data for these nuclides may well be needed.

\section{B. Comparison with University of Tokye Measurements (YAYOI Facility).}

The aggregate gamma-ray spectra from ${ }^{233} \mathrm{U},{ }^{235} \mathrm{U},{ }^{238} \mathrm{U},{ }^{239} \mathrm{Pu}$, and ${ }^{232} \mathrm{Th}$ fast neutron fission were measured by Akyama et al. at the University of Tokyo. ${ }^{6}$ Measurements up to $5 \mathrm{MeV}$ were taken, with average cooling times after fission covering from $19 \mathrm{~s}$ to $24000 \mathrm{~s}$. 
The compared results are seen in Figs. $149-188$ for ${ }^{233} \mathrm{U}$, in Figs. $189-238$ for ${ }^{235} \mathrm{U}$, in Figs. $239-286$ for ${ }^{238} \mathrm{U}$, in Figs. $287-327$ for ${ }^{239} \mathrm{Pu}$, and in Figs. $328-375$ for ${ }^{232} \mathrm{Th}$. In the cases of $238 \mathrm{U}$ and $232 \mathrm{Th}$, the measured data have the contributions from the products by neutron capture reactions. The chains of the products are as follows:

$$
\begin{aligned}
& { }^{238} \mathrm{U} \rightarrow{ }^{239} \mathrm{U} \rightarrow{ }^{239} \mathrm{~Np} \rightarrow{ }^{239} \mathrm{Pu} \\
& { }^{232} \mathrm{Th} \rightarrow{ }^{233} \mathrm{Th} \rightarrow{ }^{233} \mathrm{~Pa} \rightarrow{ }^{233} \mathrm{U} .
\end{aligned}
$$

The nuclides in the chains of ${ }^{239} \mathrm{Pu}$ and ${ }^{233} \mathrm{U}$ have a sufficiently long half-life $\left(>10^{4} \mathrm{y}\right)$ such that their contributions to the measured spectra are negligible for the cooling time region of the measurements. In our comparisons, the contributions from ${ }^{239} \mathrm{U}$ and ${ }^{239} \mathrm{~Np}$ for ${ }^{238} \mathrm{U}$ fission and those from ${ }^{233} \mathrm{Th}$ and ${ }^{233} \mathrm{~Pa}$ for ${ }^{232} \mathrm{Th}$ fission are taken into consideration.

In the case of ${ }^{238} \mathrm{U}$ neutron capture, the nuclide concentrations of ${ }^{239} \mathrm{U}\left(\mathrm{Nu}^{\mathrm{u}}(\mathrm{t})\right)$ and ${ }^{239} \mathrm{~Np}\left(\mathrm{~N}^{\mathrm{a}}(\mathrm{t})\right)$ are expressed as follows:

$$
\begin{aligned}
& N^{u}(t)=R \cdot e^{-\lambda u t}, \\
& N^{n}(t)=\left[\left(\lambda_{u} R /\left(\lambda_{n}-\lambda_{u}\right)\right] \cdot\left[e^{-\lambda u t}-e^{-\lambda n t}\right],\right.
\end{aligned}
$$

where $R$ is the reaction rate ratio of neutron capture to fission, $\lambda_{u}$ and $\lambda_{n}$ are decay constants of ${ }^{239} \mathrm{U}$ and ${ }^{239} \mathrm{~Np}$, respectively. The spectra from the ${ }^{239} \mathrm{U}$ and ${ }^{239} \mathrm{~Np}$ decay are added to those of fission products in the figures. The reaction rate ratio $R$ is 5.38 for ${ }^{238} \mathrm{U}$ and 23.0 for ${ }^{232} \mathrm{Th}$, respectively. ${ }^{6}$ The spectral data of these nuclides were taken from the ENDF/B-V file.

The calculated aggregate spectra using the augmented nuclides improve the agreement with measured data at cooling times shorter than a few hundred seconds. Even in the fission of ${ }^{238} \mathrm{U}$ and ${ }^{232} \mathrm{Th}$, the agreement was achieved by adding contributions from the products by neutron capture.

\section{Comparison with Los Alamos National Laboratory Measurements.}

The aggregate spectra from ${ }^{233} \mathrm{U},{ }^{235} \mathrm{U}$, and ${ }^{239} \mathrm{Pu}$ thermal neutron fission were measured by Jumey et al. at Los Alamos National Laboratory, ${ }^{7}$ with the energy of range of measurements extending to $7.5 \mathrm{MeV}$. The compared results are shown in Figs $376-387$ for ${ }^{233} \mathrm{U}$, in Figs. 388399 for ${ }^{235} \mathrm{U}$, and in Figs. $400-411$ for ${ }^{239} \mathrm{Pu}$. These comparisons demonstrate no difference between the calculations using the ENDF/B-V spectra data vs the augmented ones. The irradiation time of the measurements is $20000 \mathrm{~s}$, and this is sufficiently long to prohibit the short-lived nuclides, having augmented spectra, from contributing appreciably in such cases. However, the calculated results do show the underestimation at short cooling times for the energy regions above 2 
$\mathrm{MeV}$ (see Figs. 376, 388, and 400). With the exception of the short cooling times, the comparisons show a good agreement.

\section{SUMMARY}

The theoretically calculated gamma spectra of FP nuclides have been used to augment the line spectra in the ENDF/B-V file based on measurements. The augmented spectra have been appliea to the calculation of the aggregate gamma spectra after fission. Calculated results show a good agreement with the measured spectra for various fissionable nuclides. The effect of the augmentation is prominent at short cooling times after fission when the nuclides with partially measured or no measured spectra data contribute to the aggregate spectra. At longer cooling times, the augmentation is not effective because it is considered that almost all nuclides contributing at those cooling times have a well-measured spectra.

From our comparisons, it is concluded that the augmented spectra are applicable to the calculation of aggregate ones at shorter cooling times when the nuclides with incomplete or no measured spectra data contribute. If we use the augmented spectra, we can obtain a spectra consistent with the decay heat calculated by the average decay energy.

\section{ACKNOWLEDGMENTS}

We greatly appreciate the assistance of the Science and Technology Agency (STA) of Japan and the US Department of Energy. One of the authors (J. Katakura) spent one year as a collaborator for the Nuclear Theory and Applications Group at Los Alamos National Laboratory (November 1988-November 1989) under the support of the STA. The work was performed during his stay.

We also wish to acknowledge the productive discussions with Drs. R. E. Schenter, F. M. Mann, J. K. Dickens, C. W. Reich, T. Yoshida, K. Tasaka, and R. Nakasima. All have contributed information and supported the need for a complete data base; they are in no way responsible for any errors we may have made. 






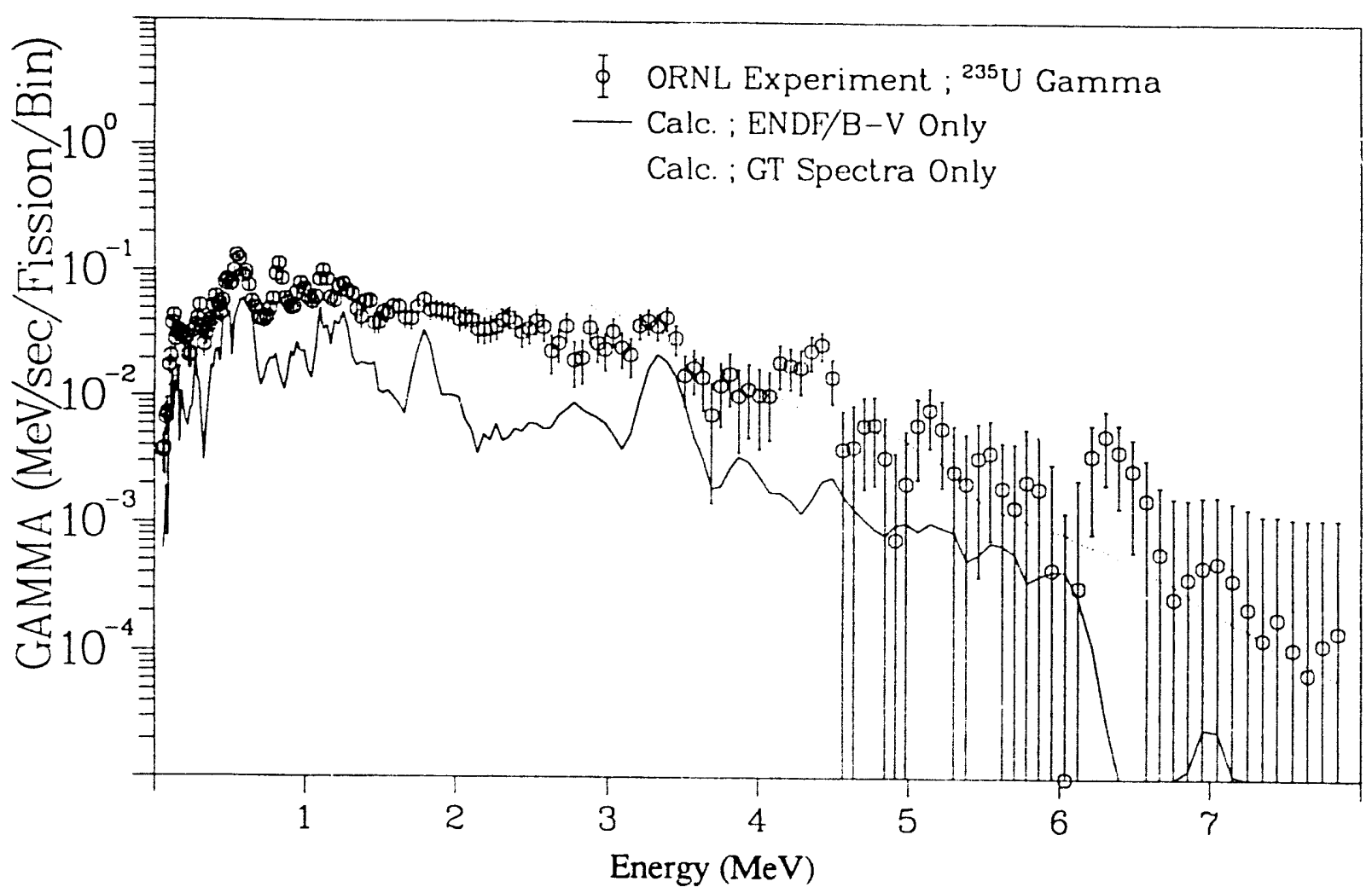

Fig. 9. Gamma spectrum after ${ }^{235} \mathrm{U}$ thermal neutron fission $\left(\mathrm{T}_{\mathrm{irrad}}=1.0 \mathrm{sec}, \mathrm{T}_{\text {cool. }}=2.2 \mathrm{sec}\right)$ (to $8 \mathrm{MeV}$ ).



Fig. 10. Gamma spectrum after ${ }^{235} \mathrm{U}$ thermal neutron fission $\left(\mathrm{T}_{\mathrm{inad} .}=1.0 \mathrm{sec}, \mathrm{T}_{\mathrm{cool}}=2.2 \mathrm{sec}\right)$ (to $2 \mathrm{MeV}$ ). 


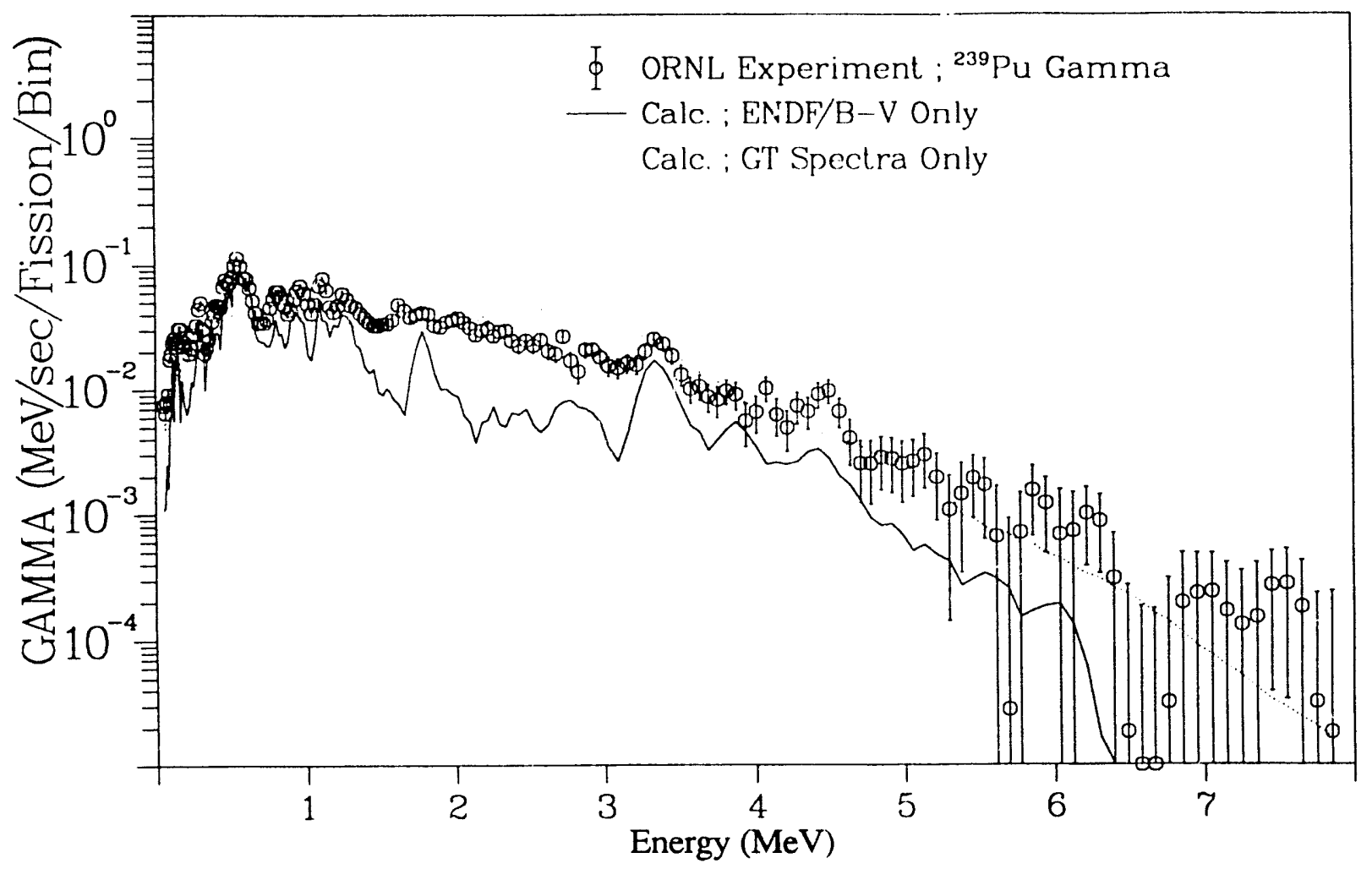

Fig. 11. Gamma spectrum after ${ }^{239} \mathrm{Pu}$ thermal neutron fission $\left(\mathrm{T}_{\mathrm{irrad}}=1.0 \mathrm{sec}, \mathrm{T}_{\text {cool }}=2.2 \mathrm{sec}\right)$ (to $8 \mathrm{MeV}$ ).

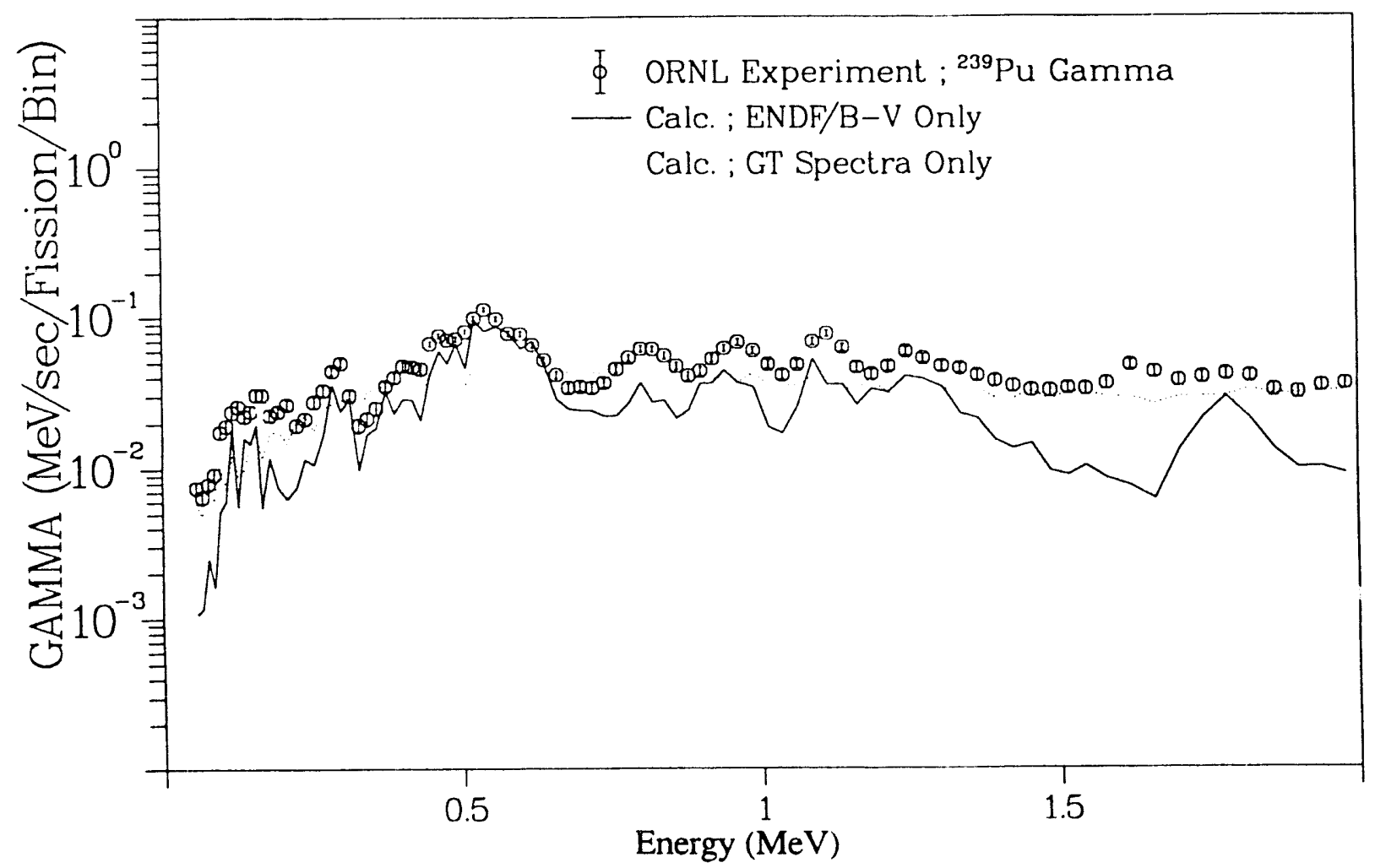

Fig. 12. Gamma spectrum after ${ }^{239} \mathrm{Pu}$ thermal neutron fission $\left(\mathrm{T}_{\mathrm{irrad}}=1.0 \mathrm{sec}, \mathrm{T}_{\text {cool. }}=2.2 \mathrm{sec}\right)$ (to $2 \mathrm{MeV}$ ). 




Fig. 13. Measured and modified energy spectra of ${ }^{98} \mathrm{Sr}$ decay $\left(\mathrm{Q}_{00}=0.00\right)$.



Fig. 14. Measured and modified energy spectra of $97 \mathrm{~m} Y$ decay $\left(Q_{00}=0.00\right)$. 




Fig. 15. Gamma spectrum after $235 \mathrm{U}$ thermal neutron fission $\left(\mathrm{T}_{\text {irrad }}=1.0 \mathrm{sec}, \mathrm{T}_{\text {cool }}=2.2 \mathrm{sec}\right)$ (see Fig. 9).



Fig. 16. Gamm a spectrum after $235 \mathrm{U}$ thermal neutron fission $\left(\mathrm{T}_{\text {irrad. }}=1.0 \mathrm{sec}, \mathrm{T}_{\text {cool. }}=2.2 \mathrm{sec}\right.$ ) (to $2 \mathrm{MleV}$ ). 




Fig. 17. Gamma spectrum after ${ }^{239} \mathrm{Pu}$ thermal neutron fission $\left(\mathrm{T}_{\text {irrad }}=1.0 \mathrm{sec}, \mathrm{T}_{\text {cool. }}=2.2 \mathrm{sec}\right)$ (see Fig. 11).



Fig. 18. Gamma spectrum after $235 \mathrm{U}$ thermal neutron fission $\left(\mathrm{T}_{\text {irrad. }}=1.0 \mathrm{sec}, \mathrm{T}_{\text {cool. }}=2.2 \mathrm{sec}\right)$ (to $2 \mathrm{MeV}$ ). 




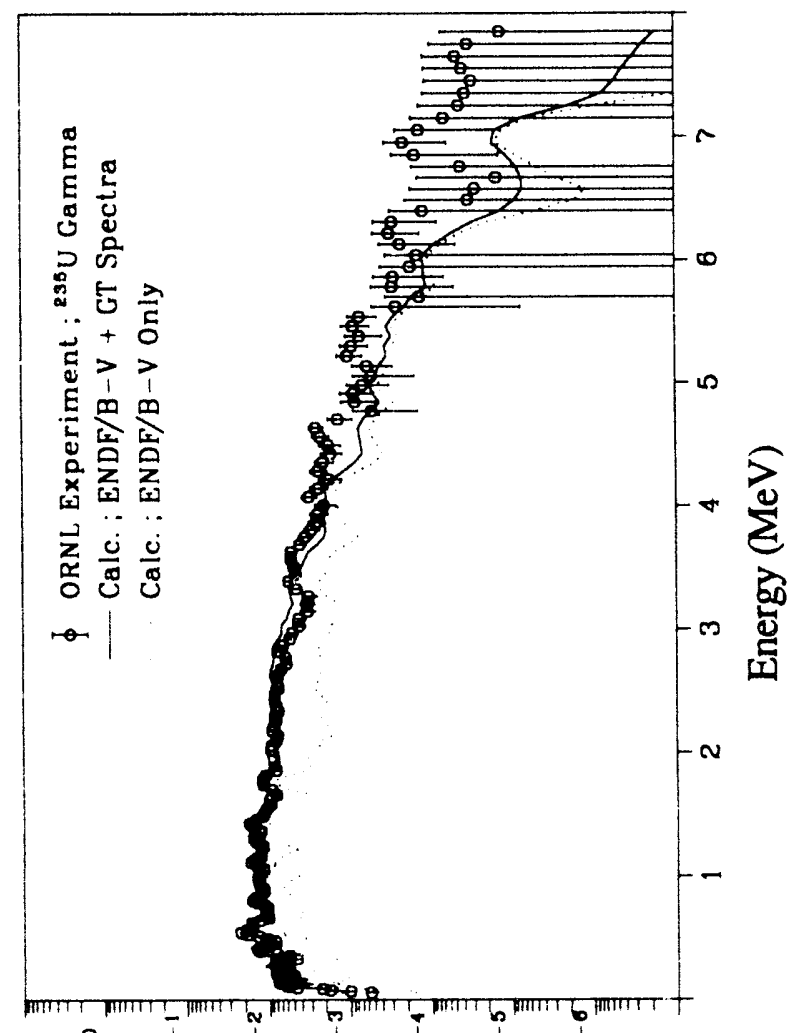

으응으응의



号

然



.



品

$\vec{L}_{0}>0$

E

츨충

xis $\sum_{i=1}$

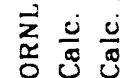

-




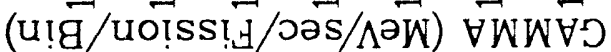






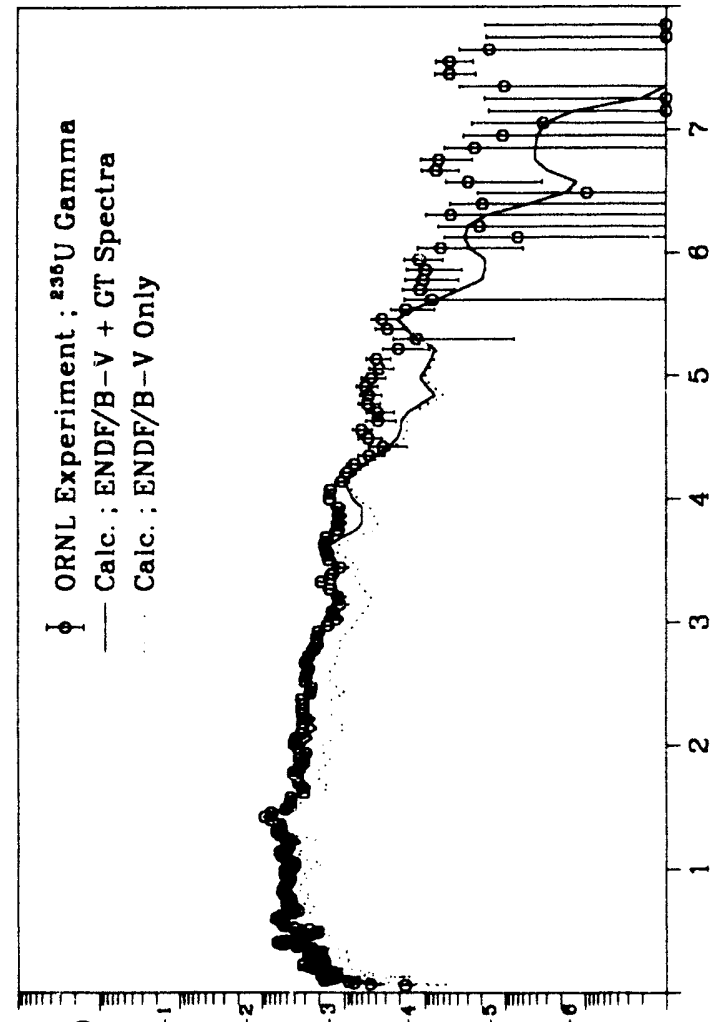

으 으 으 으 으 으은

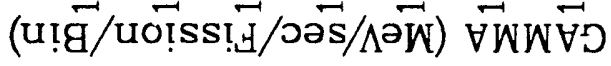

它

is
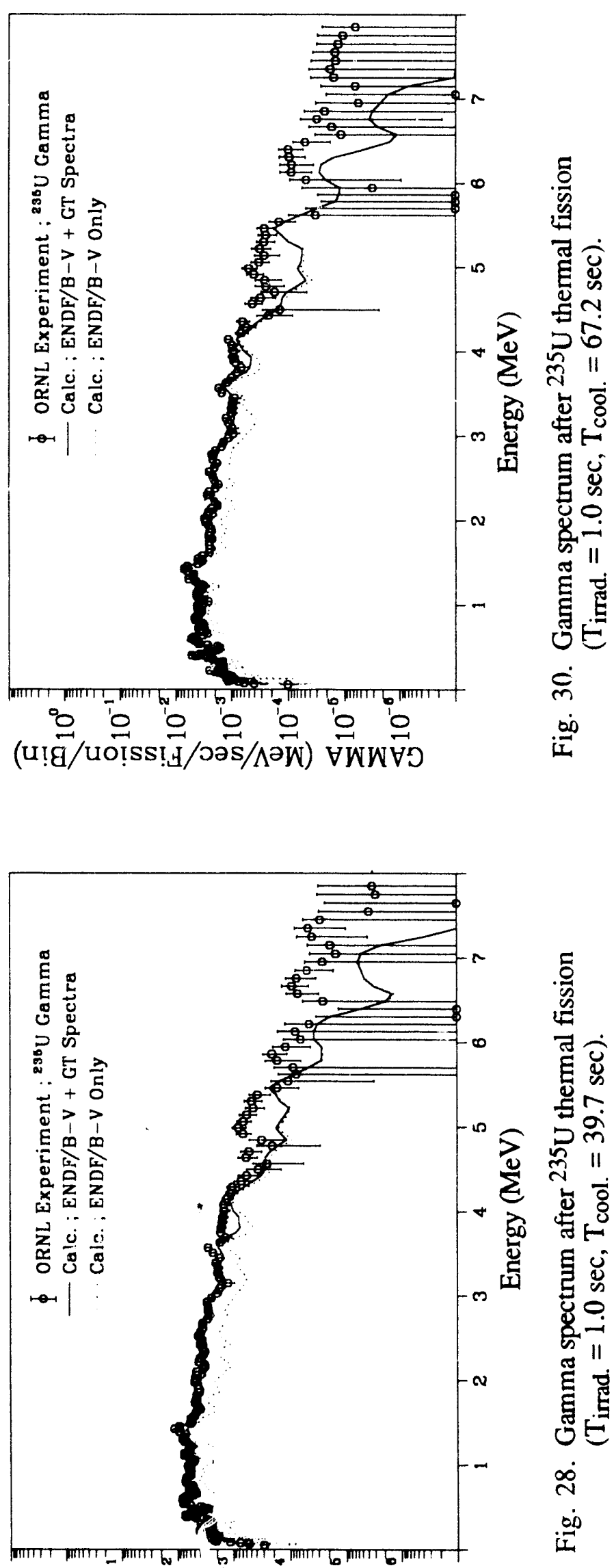

음으을 으 으 으 (u!g/uo!̣s! 




응응ㅇㅇ으의 (u!g/uộss!



$\dot{m}$

$\dot{\infty}$
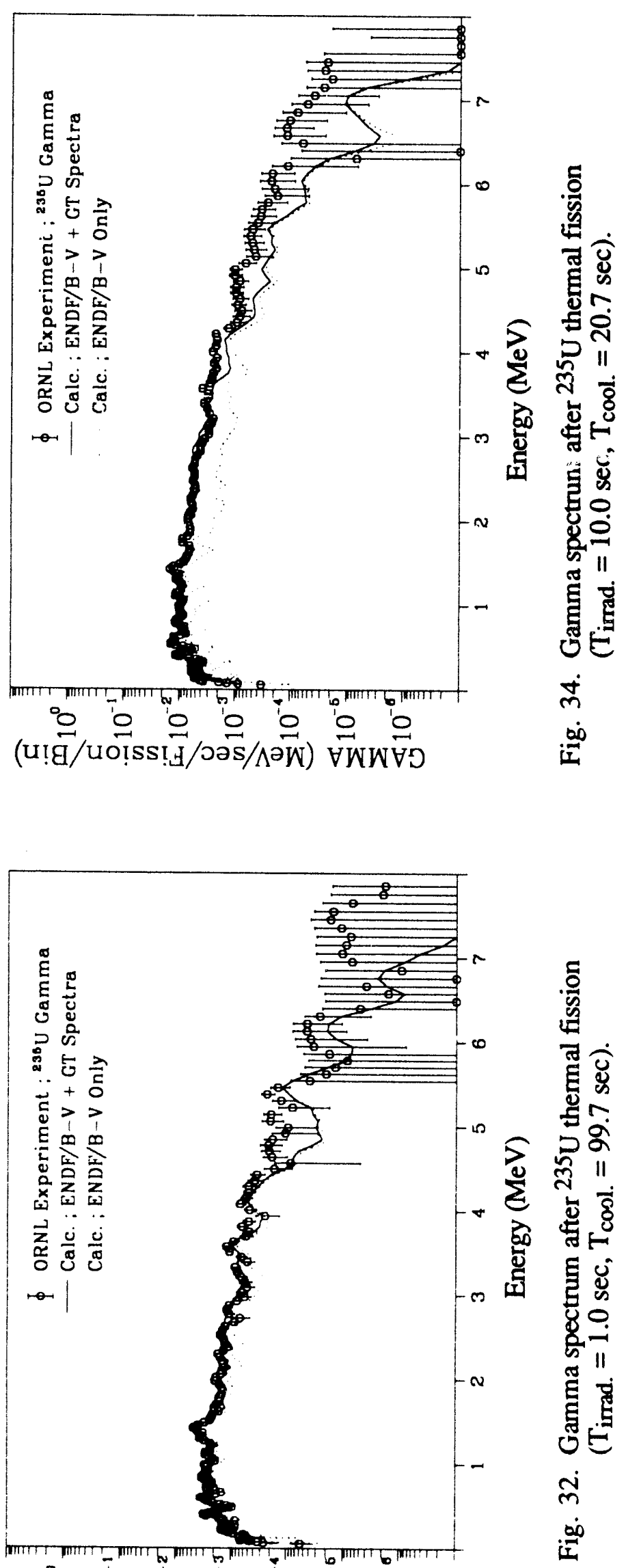













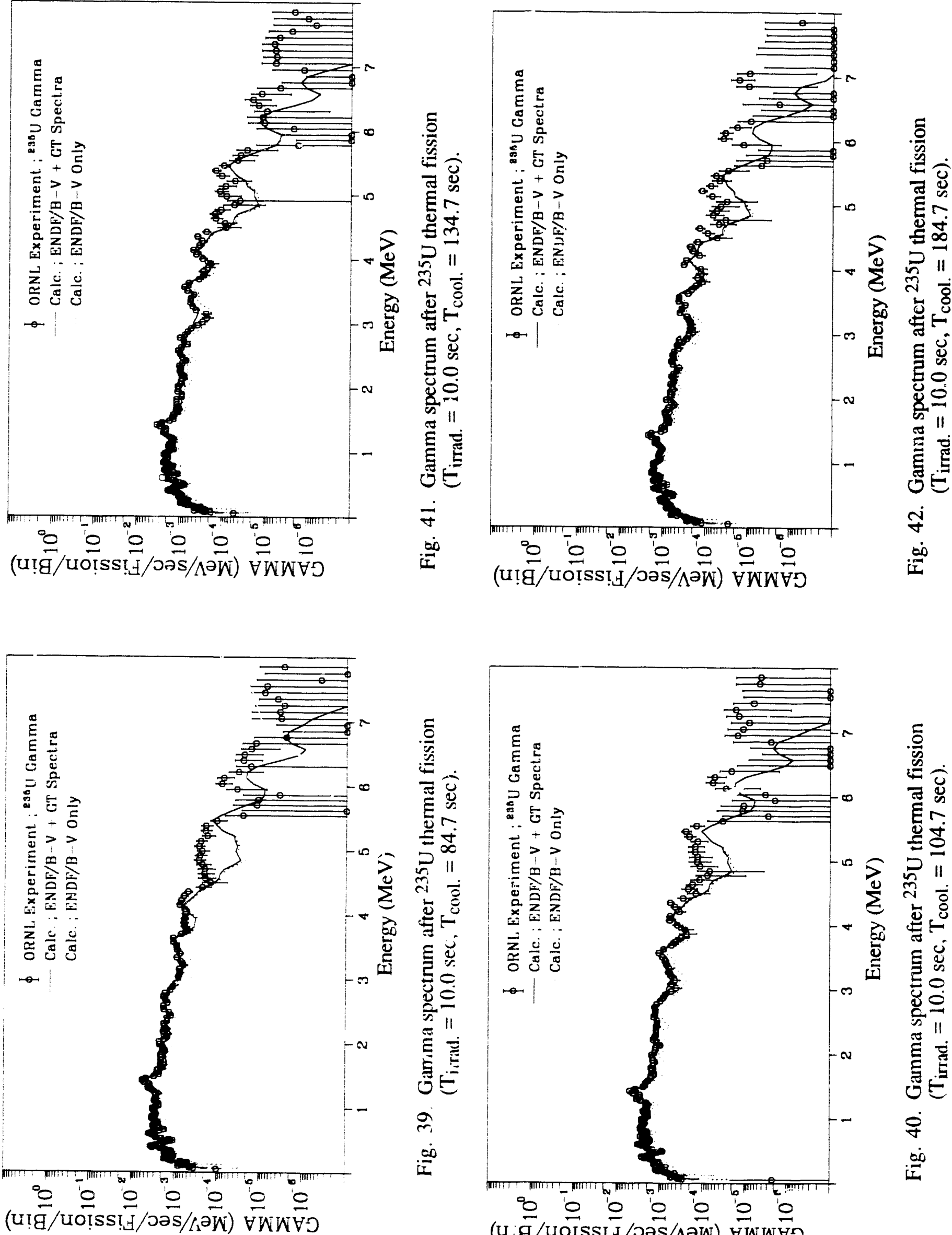







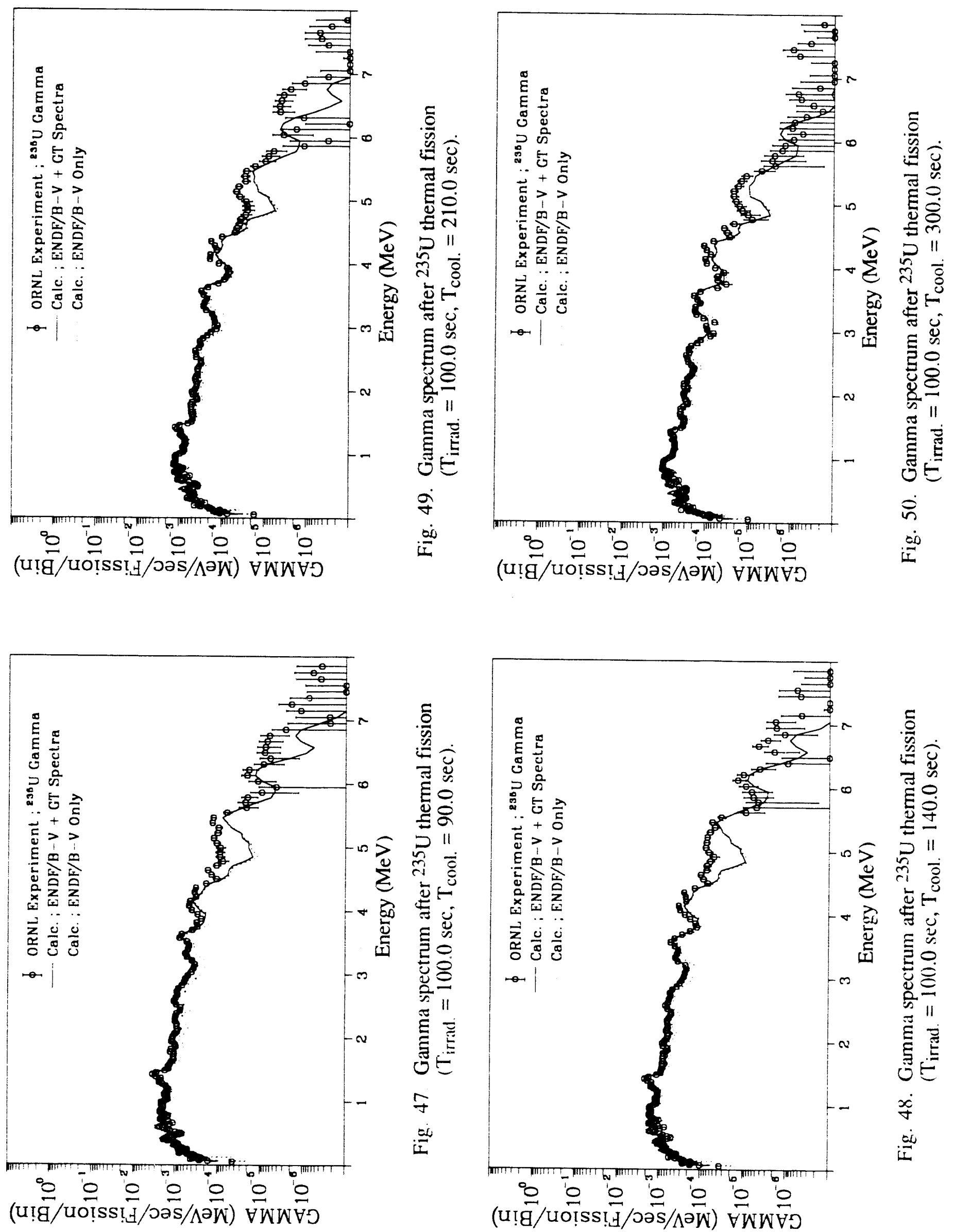

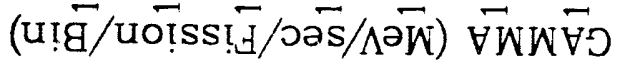







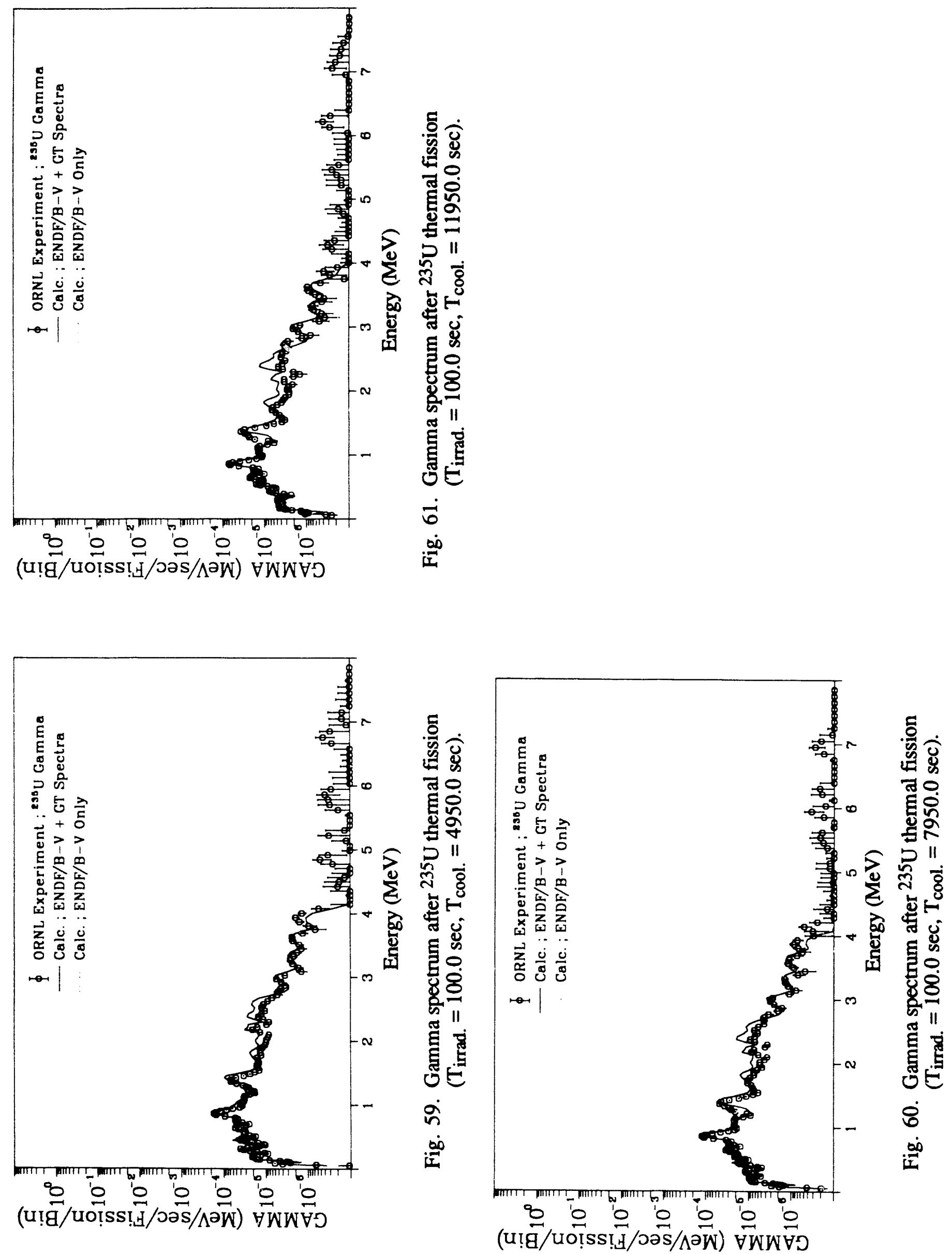

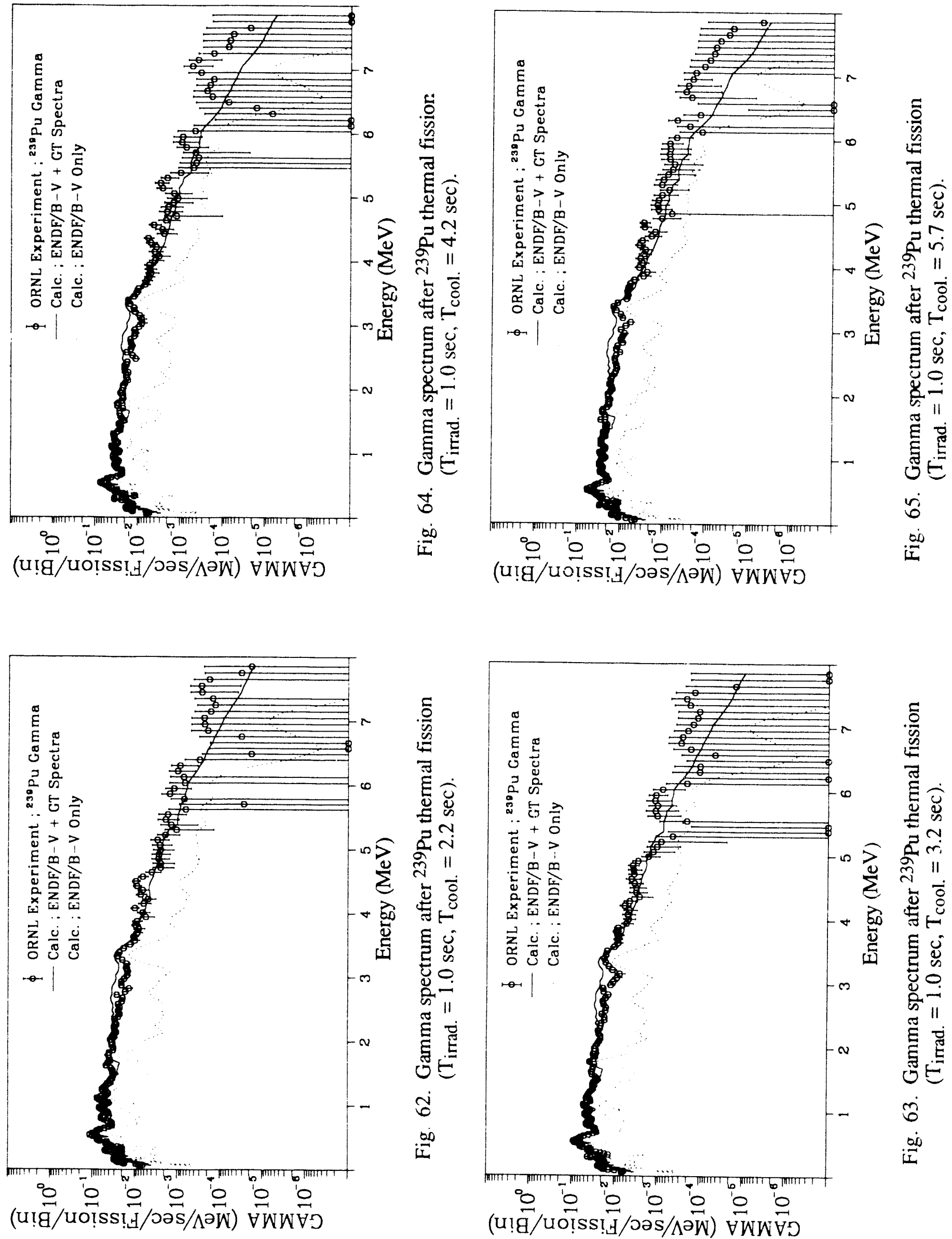

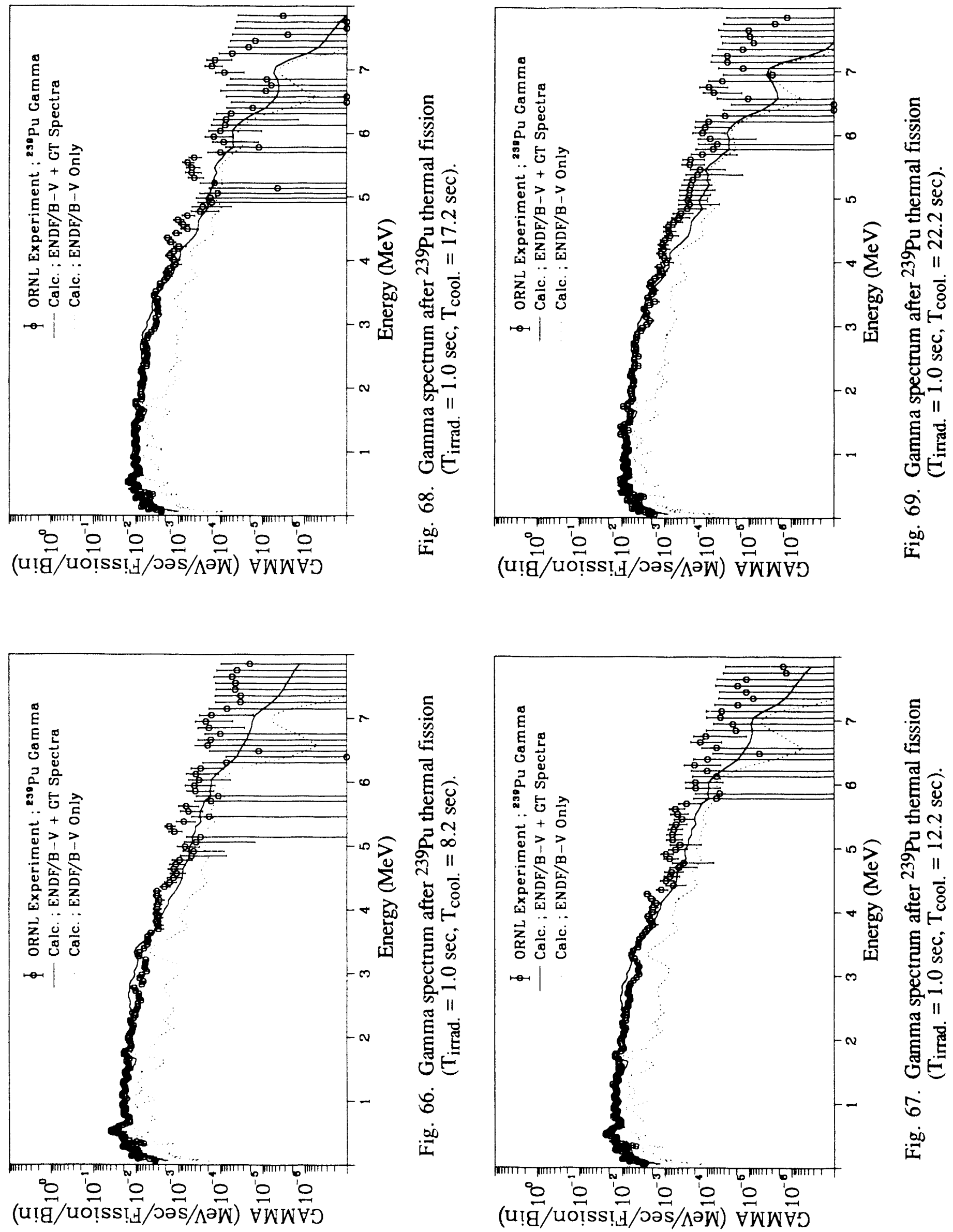

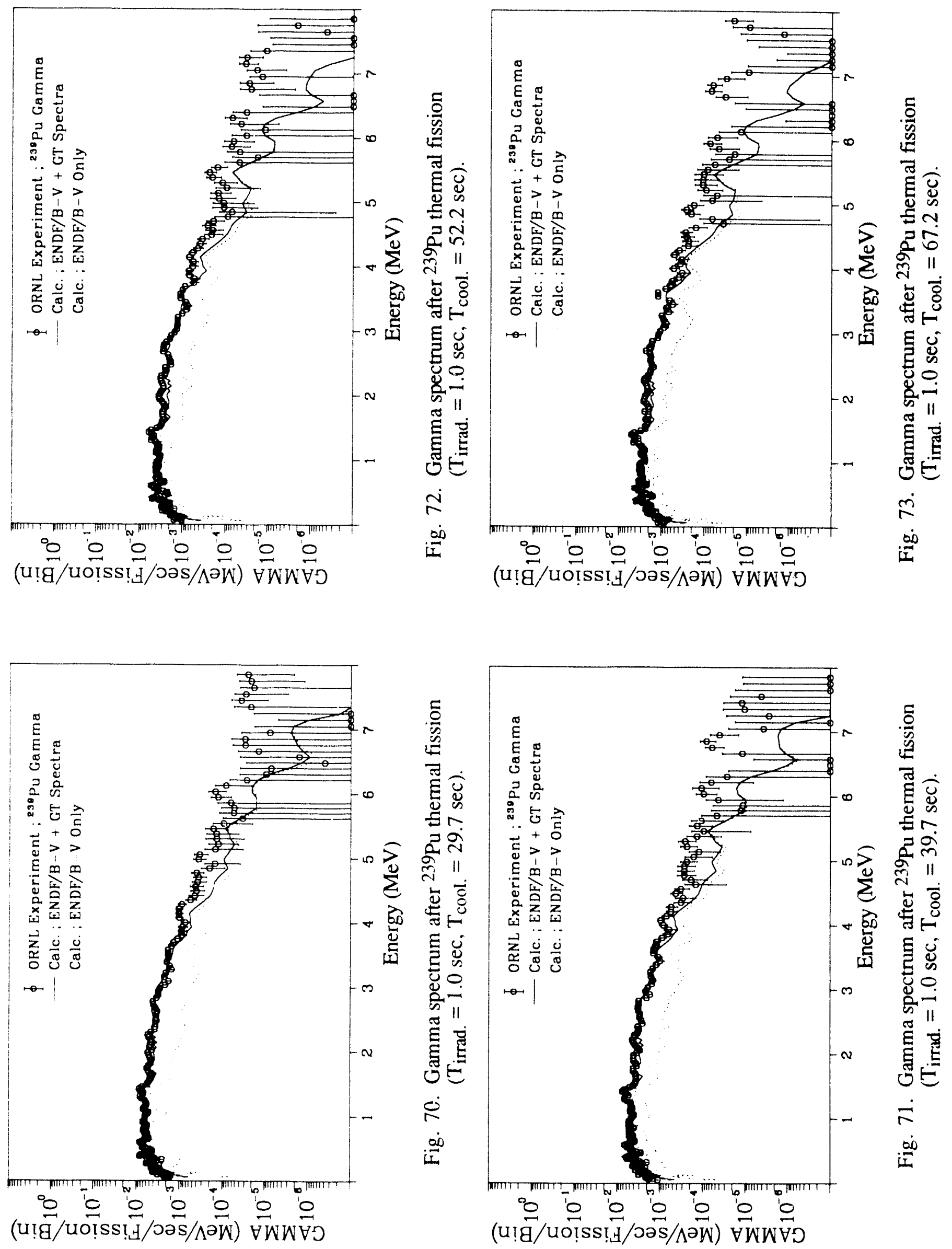


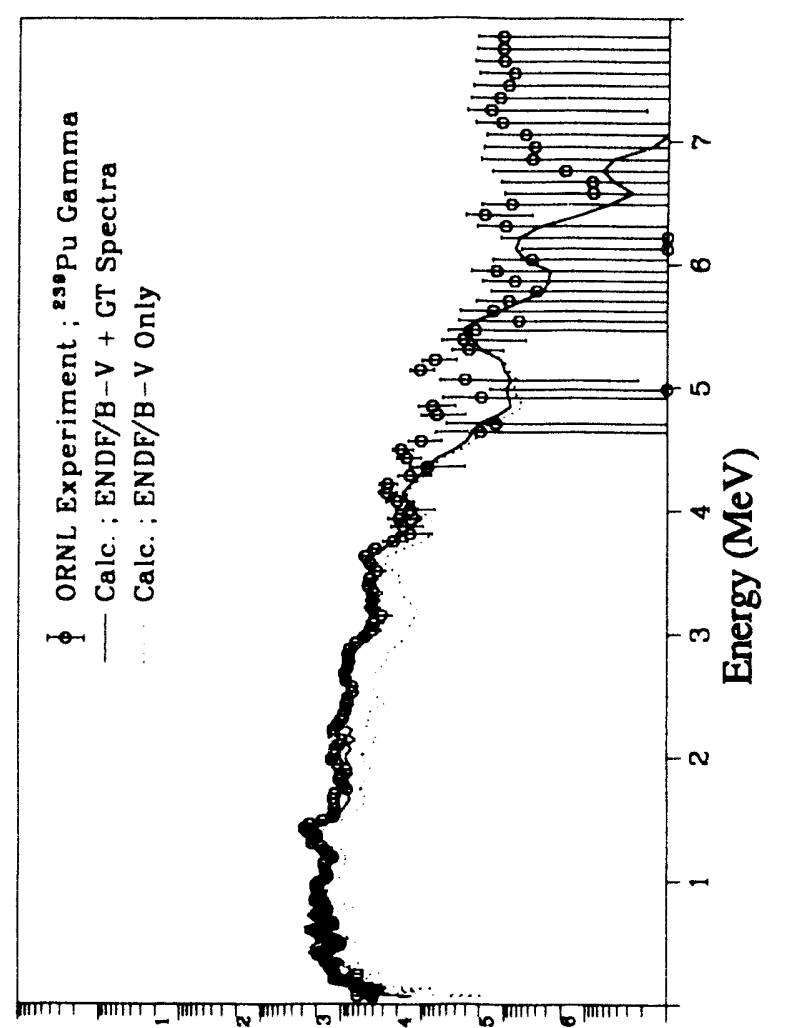

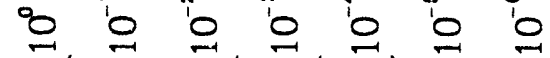

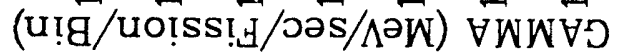

贯
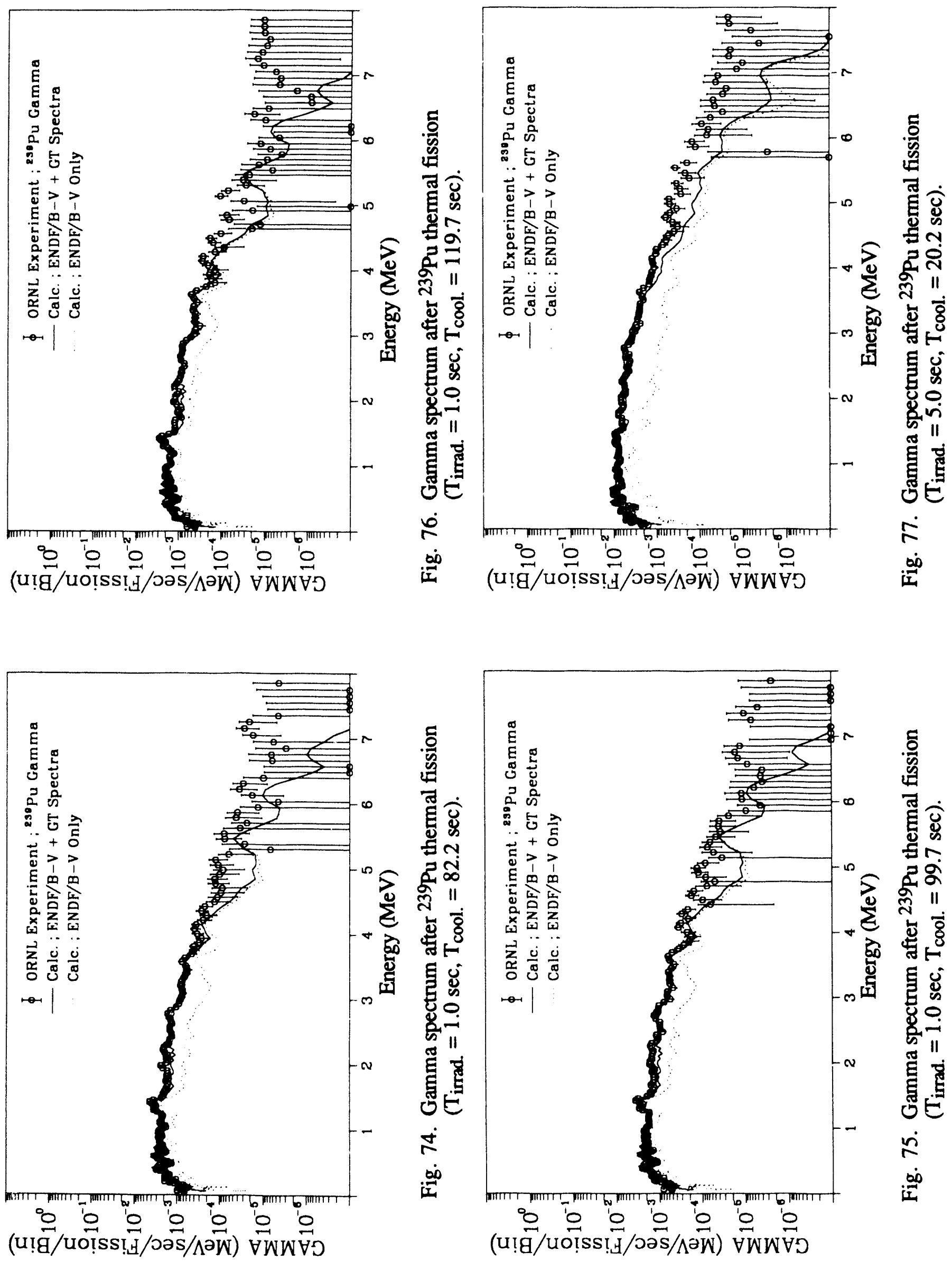

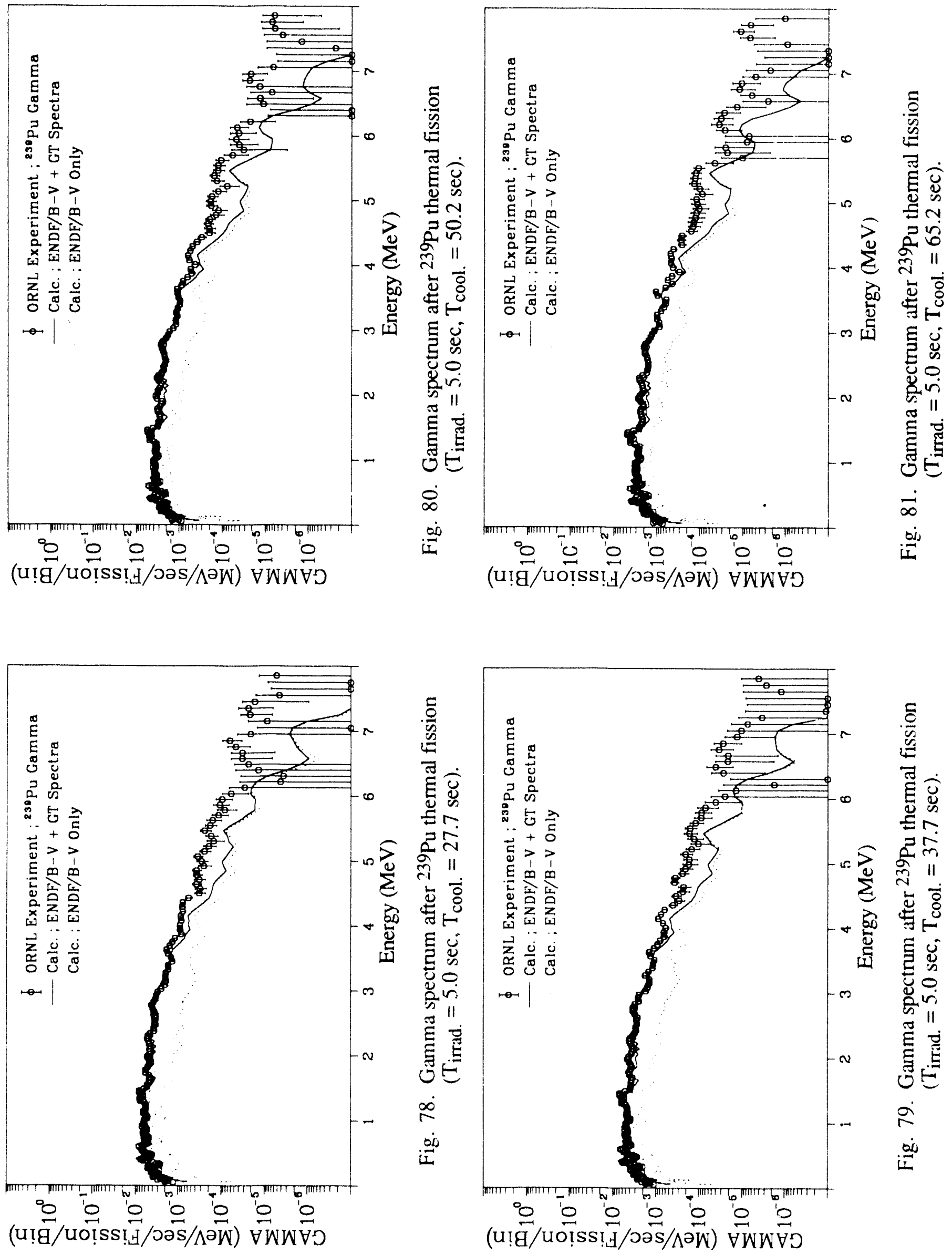

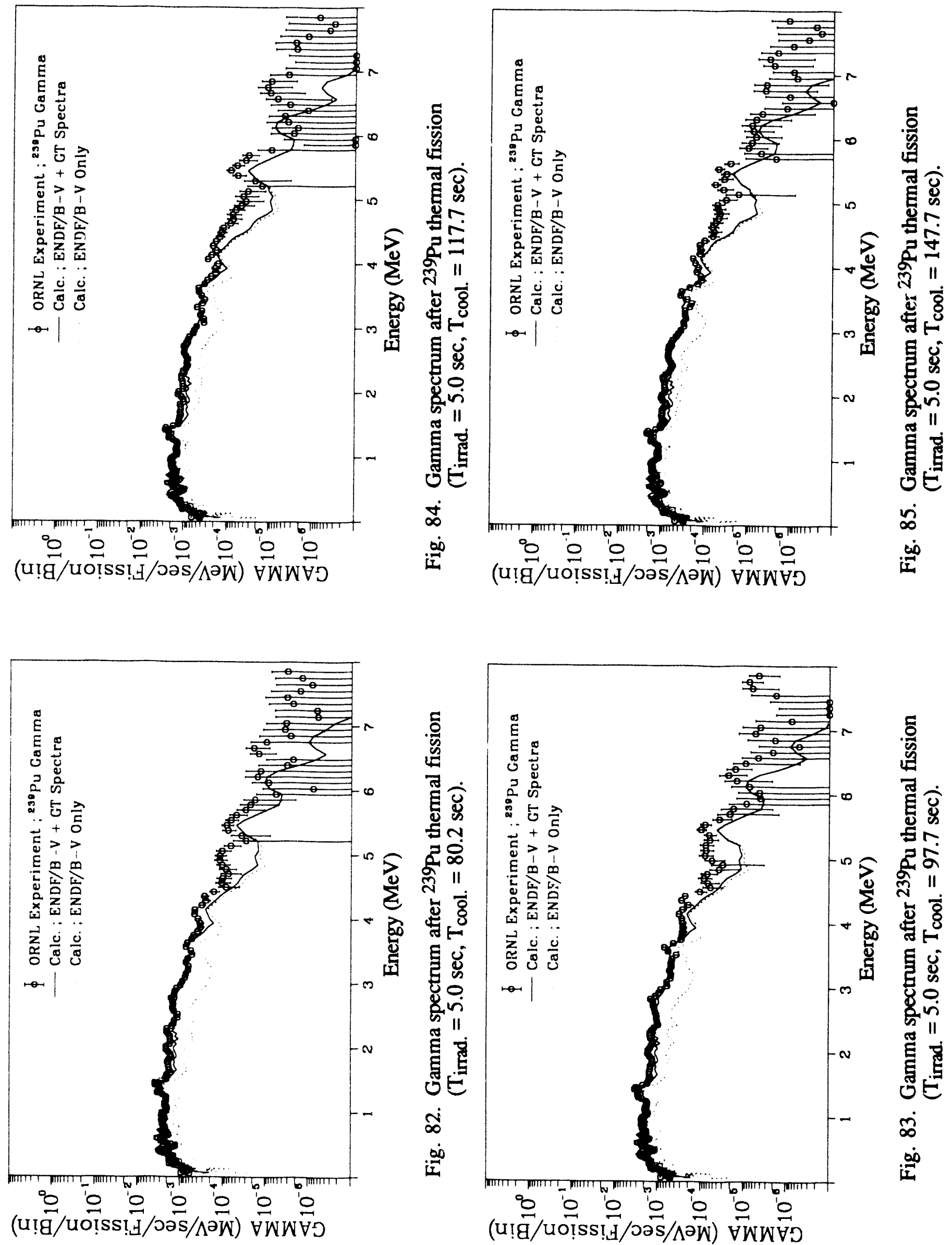

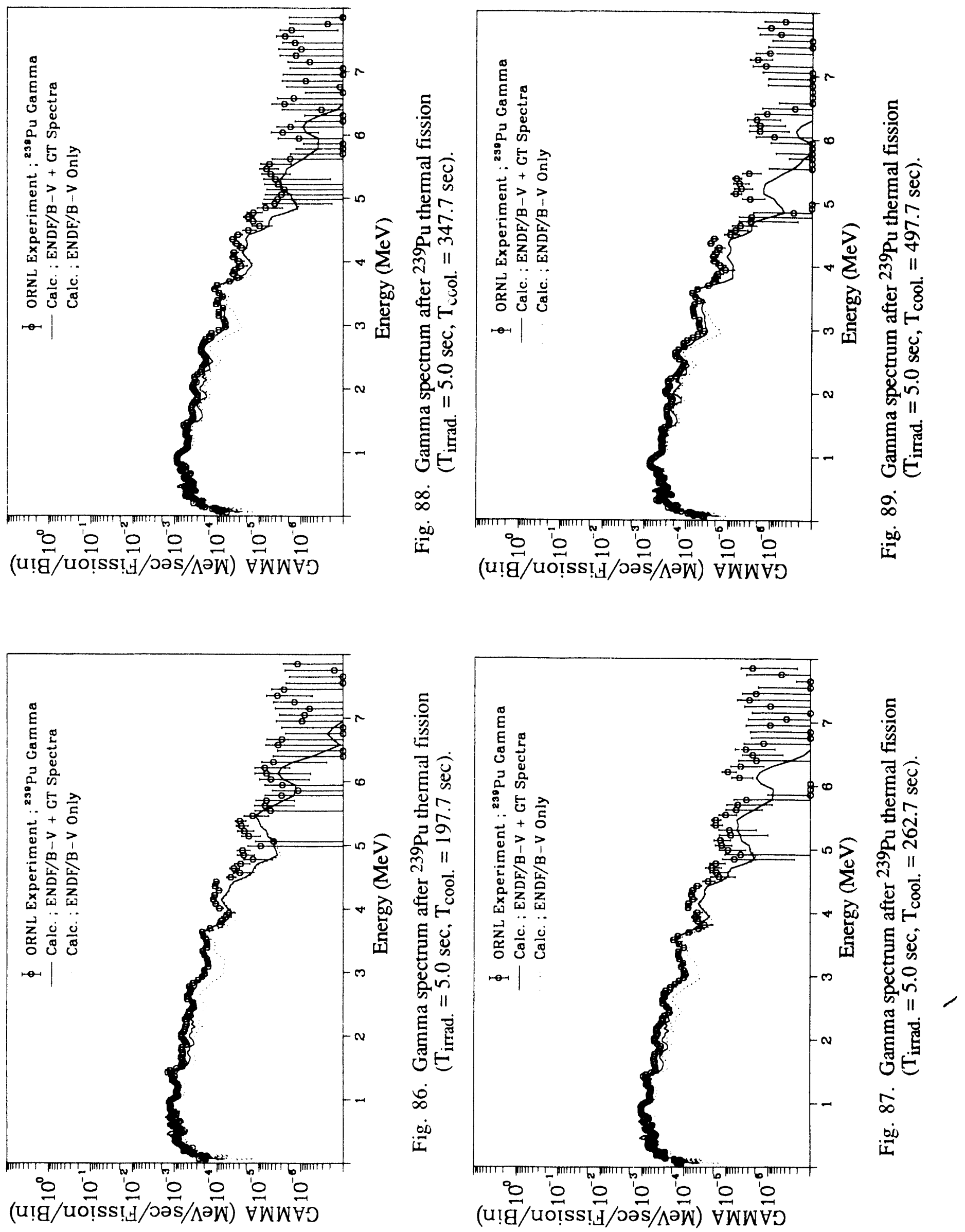

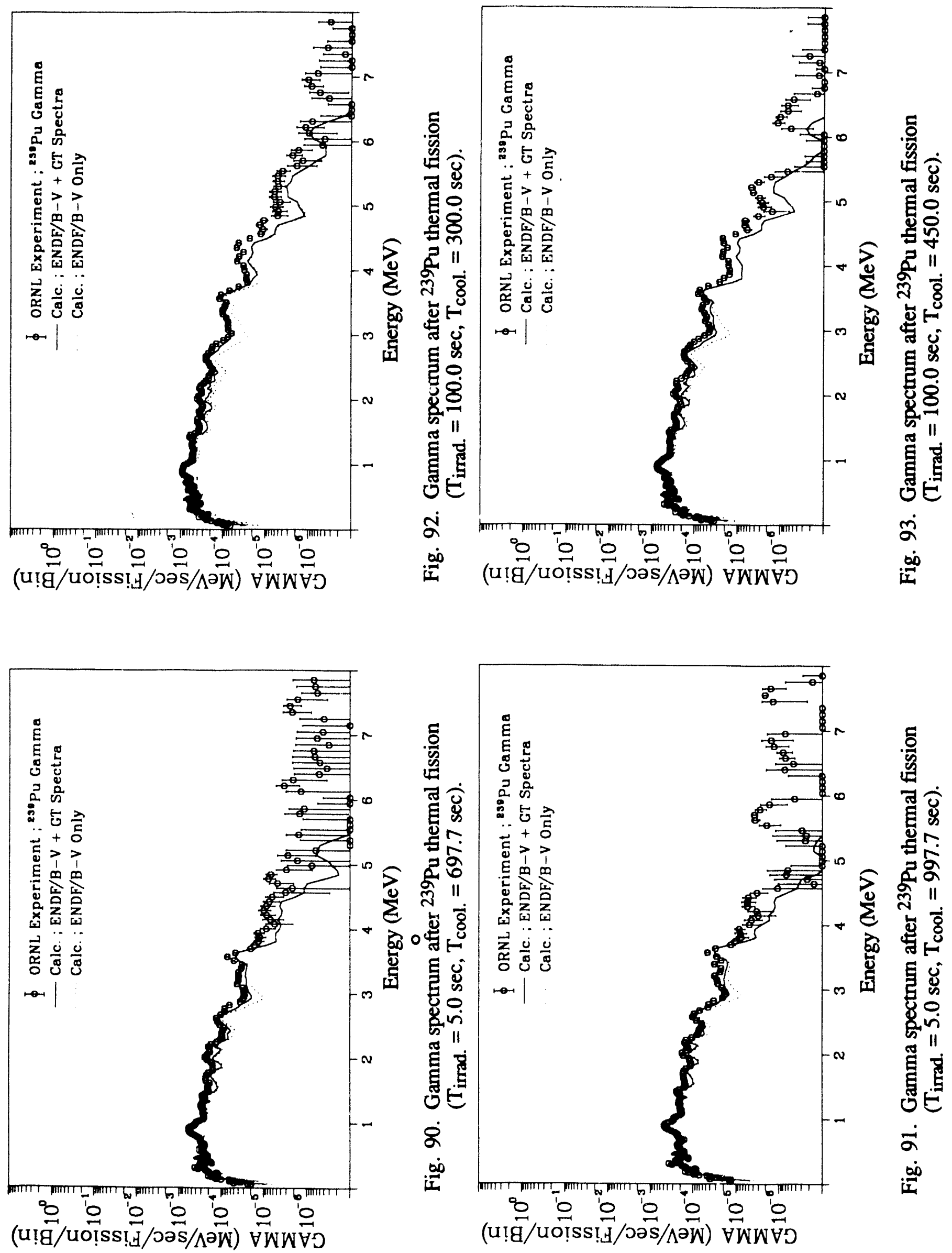

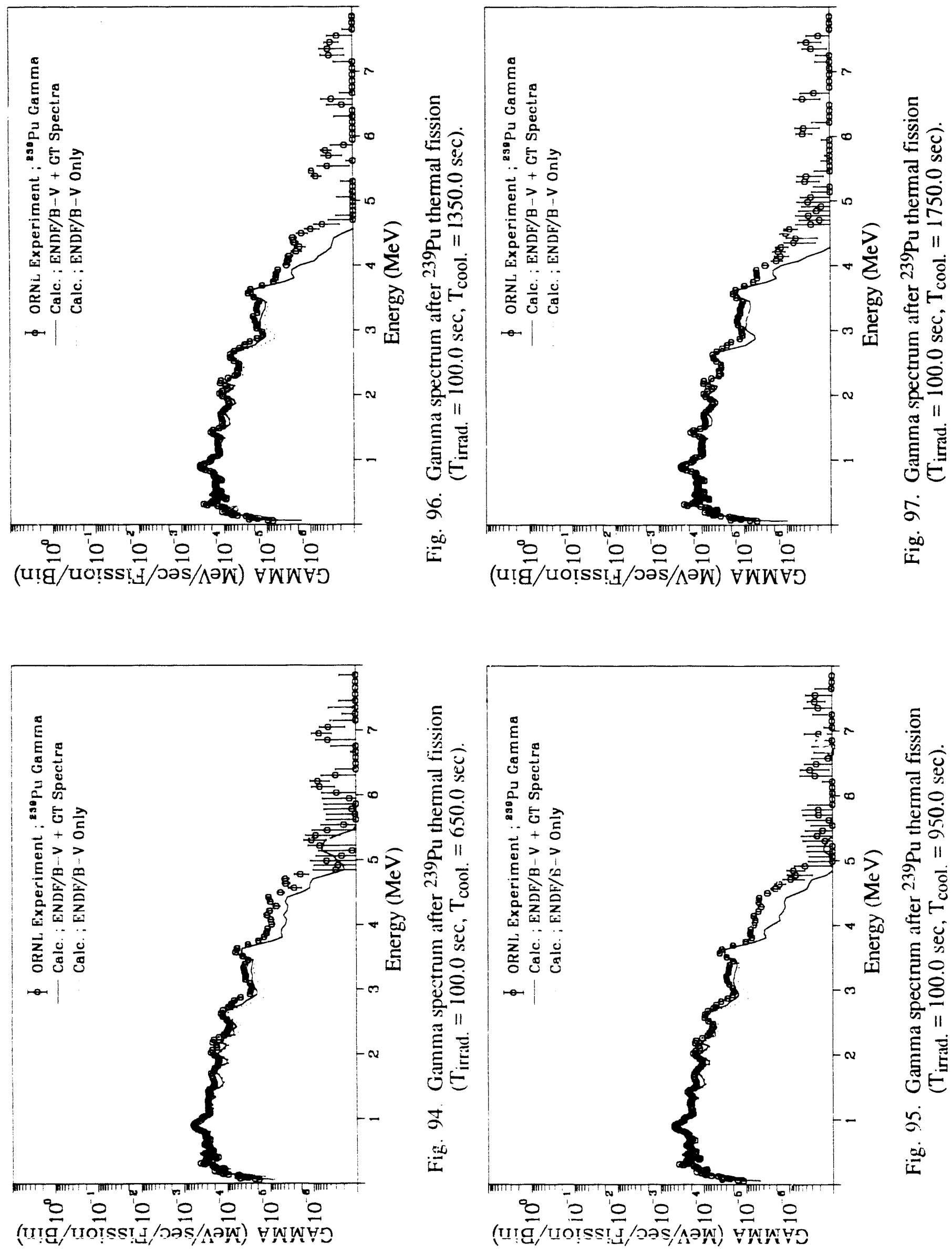


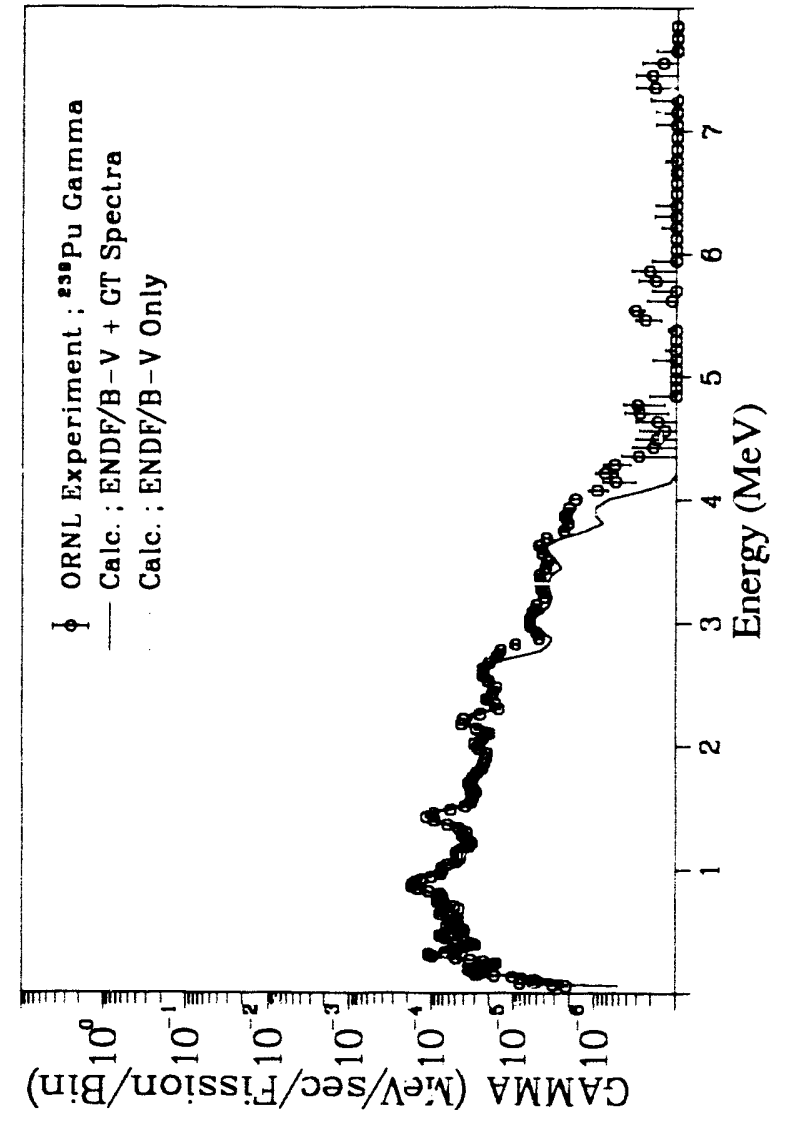

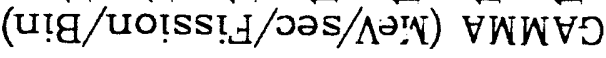

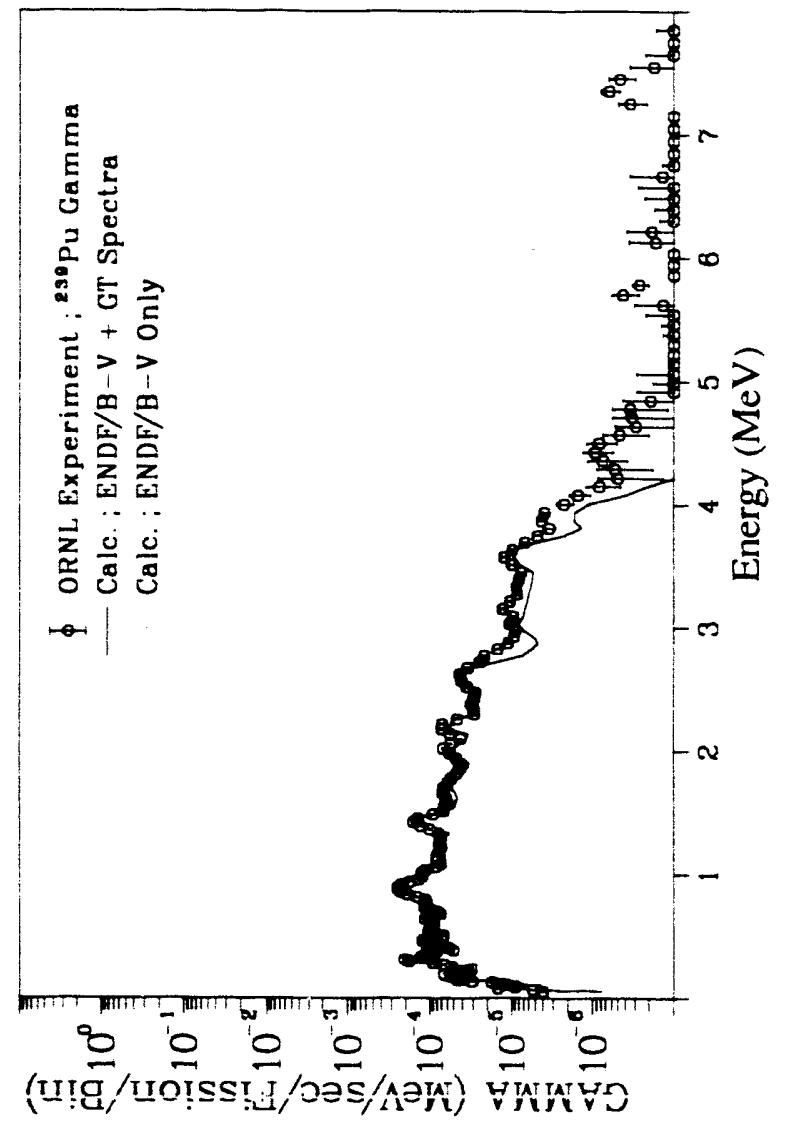

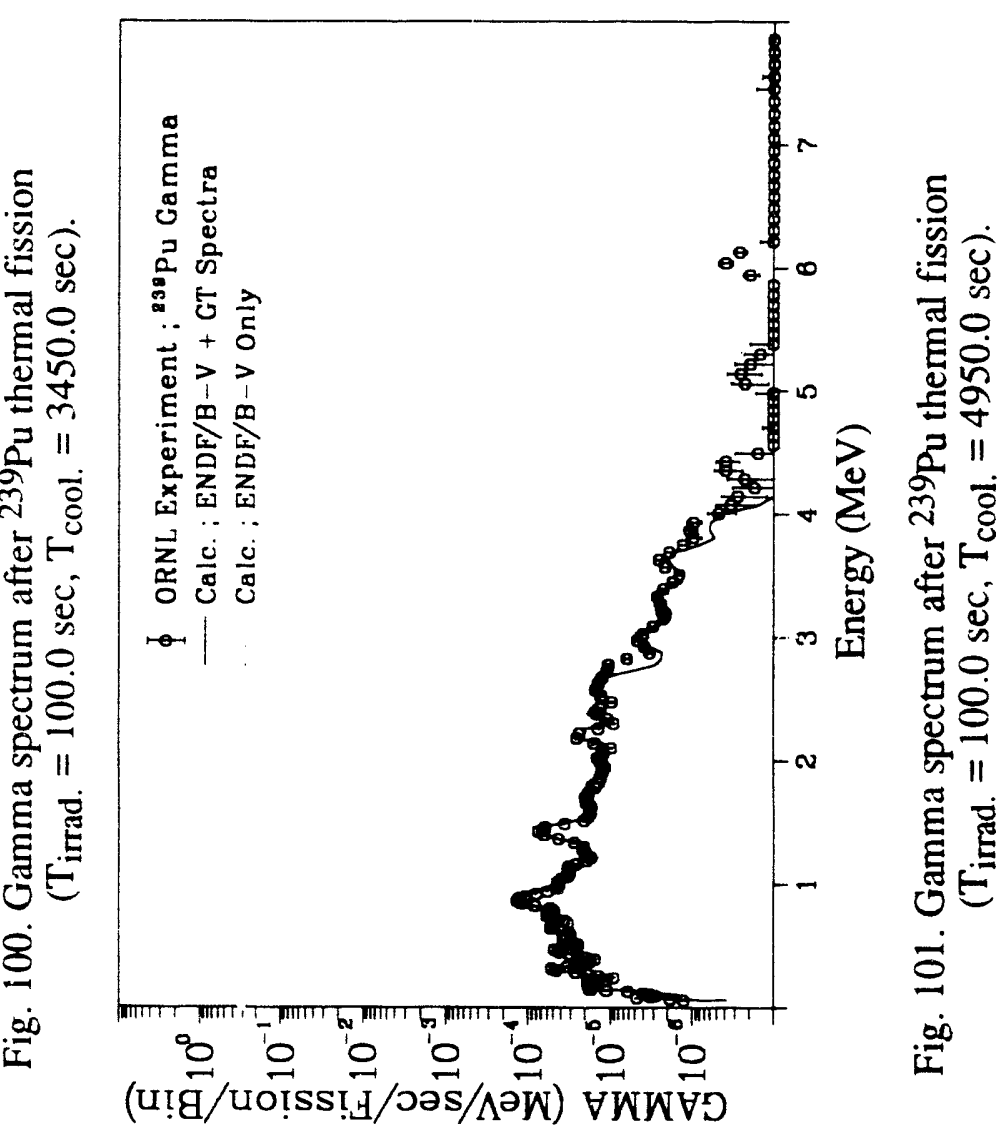

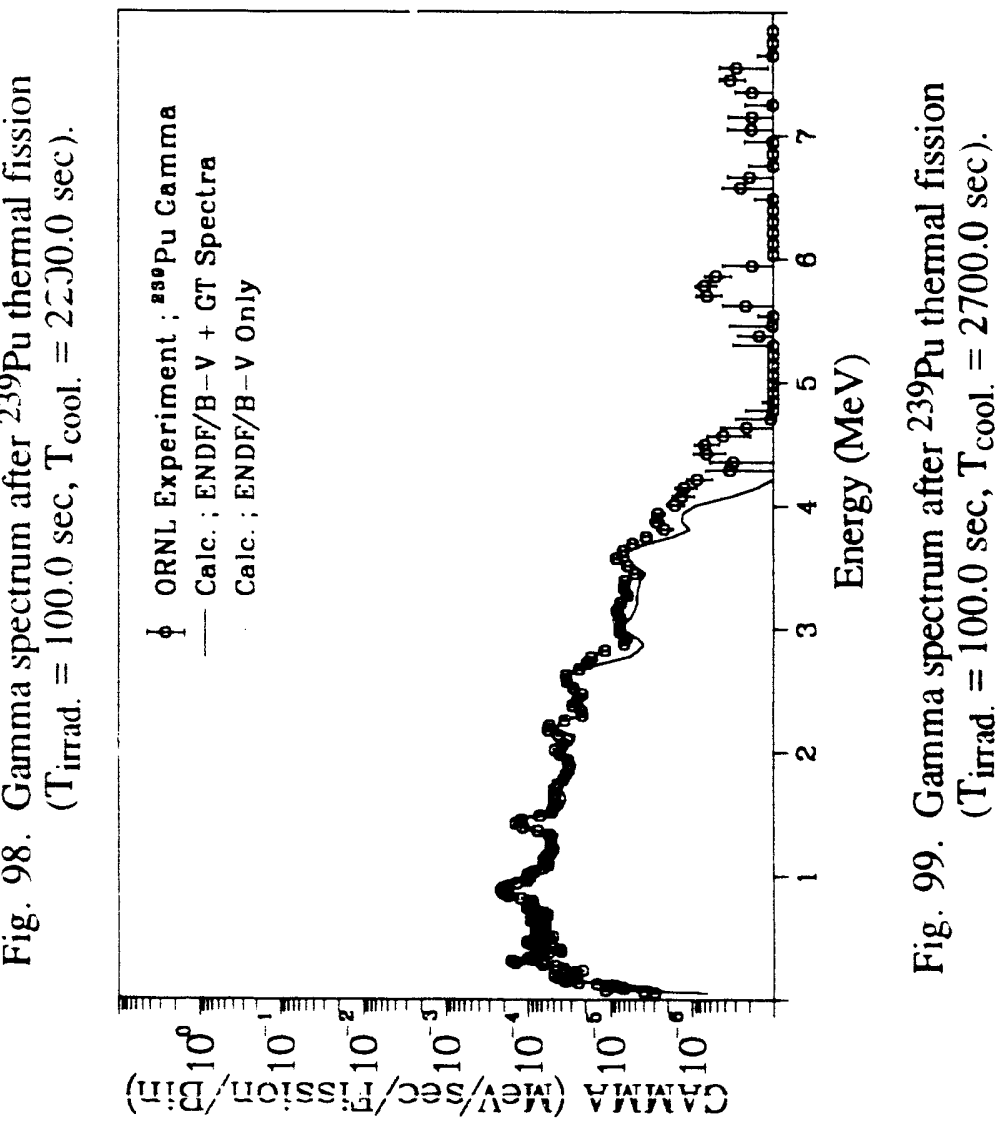



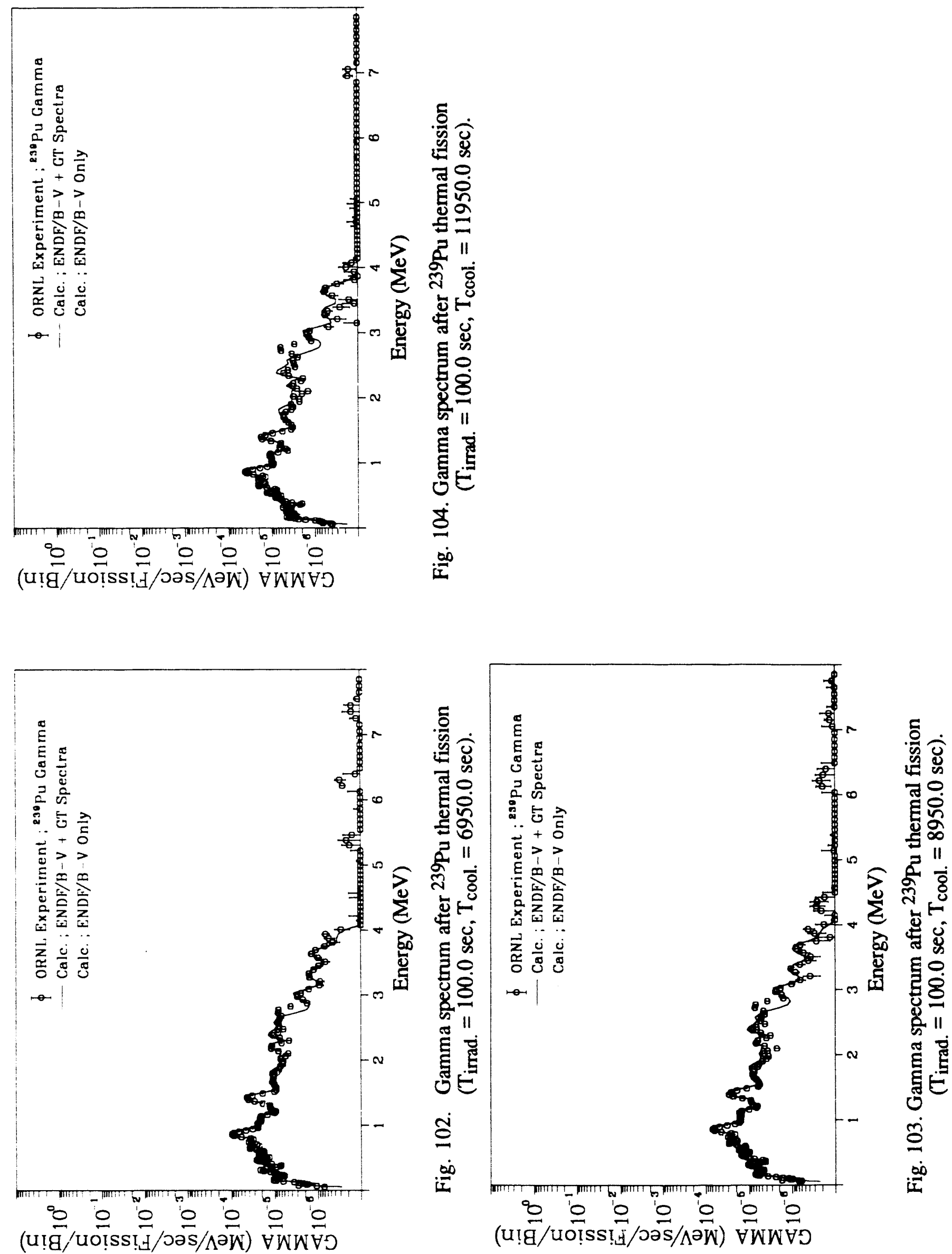

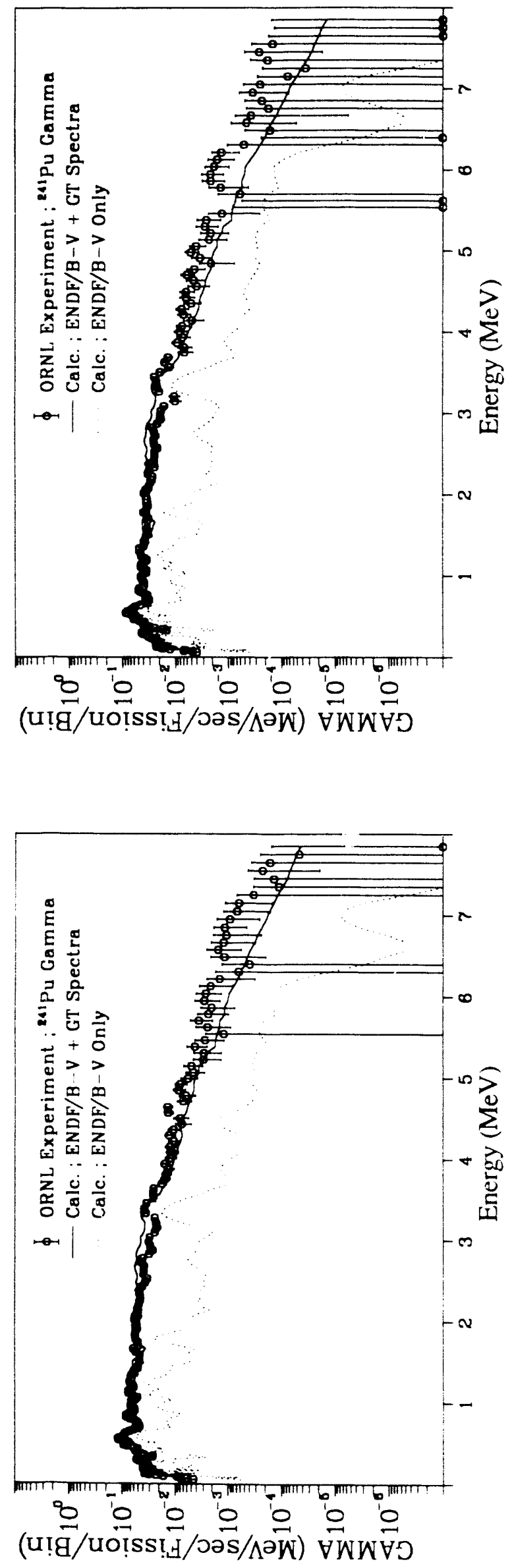
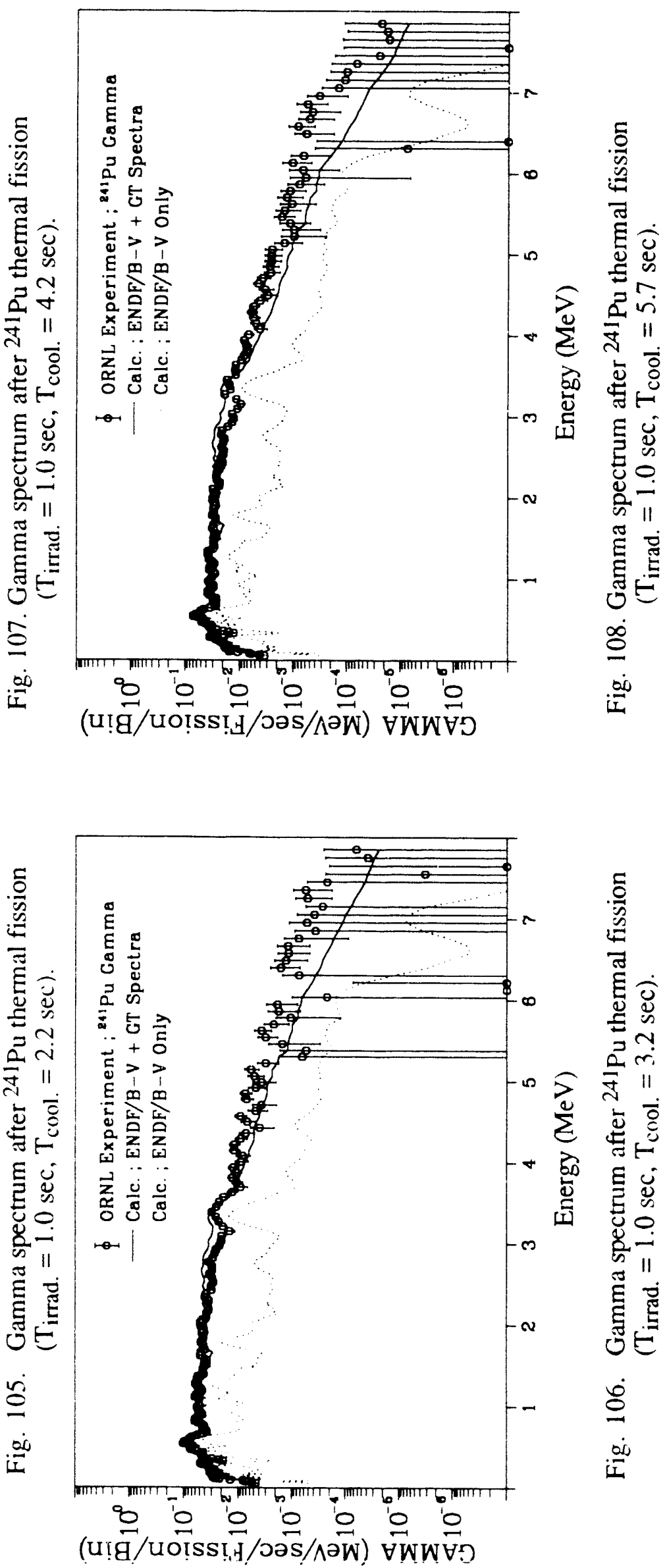





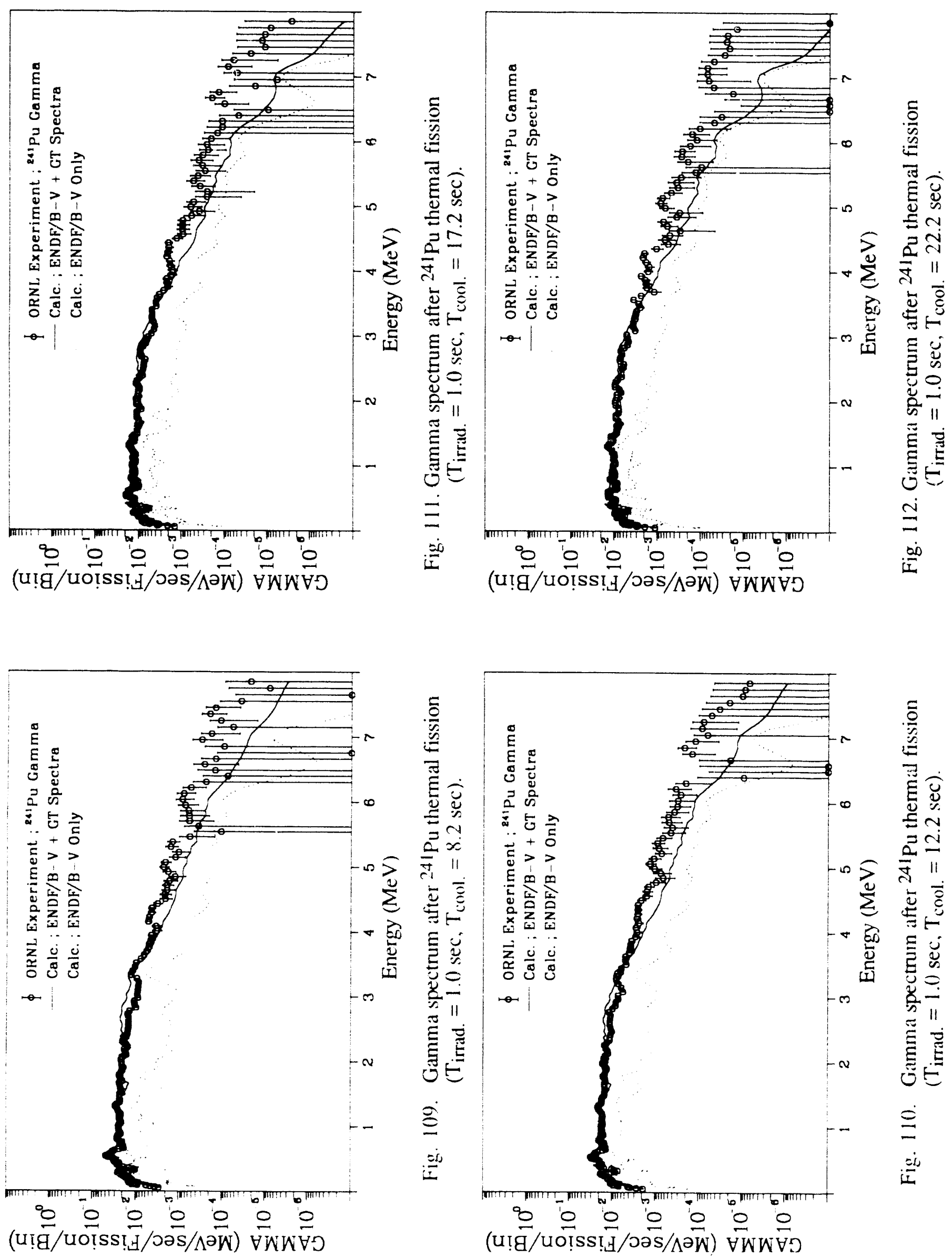




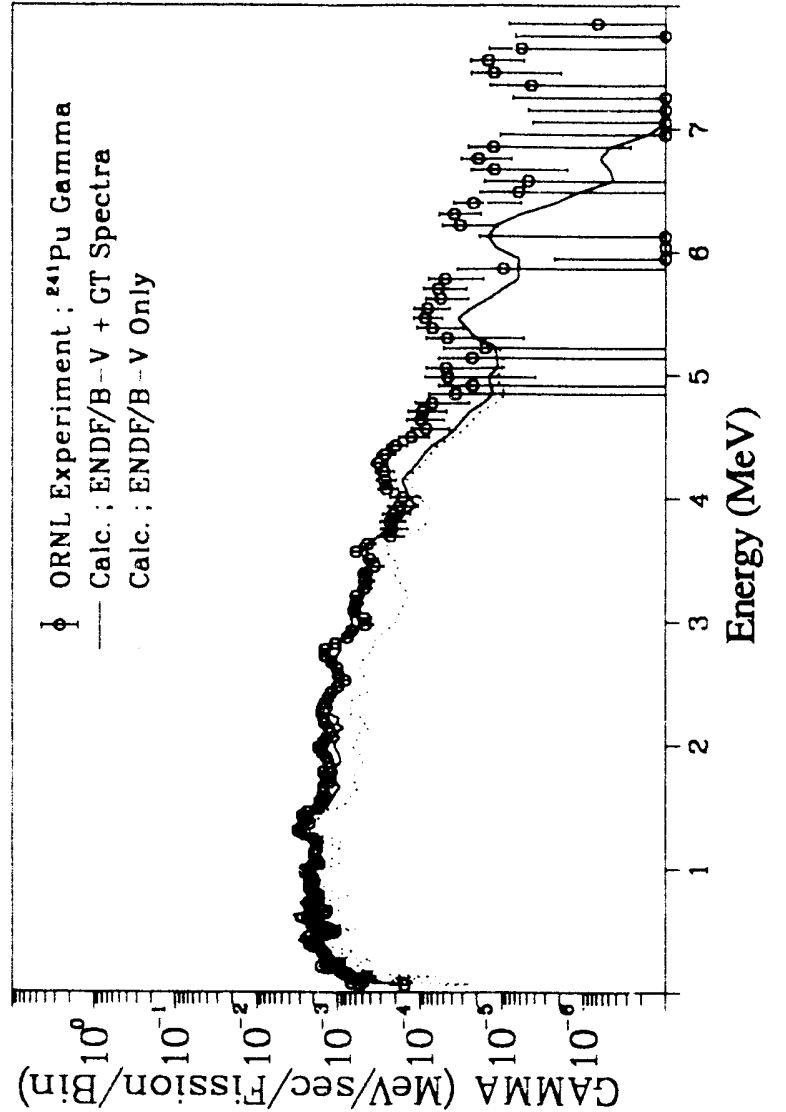

(u!g/uotssî.
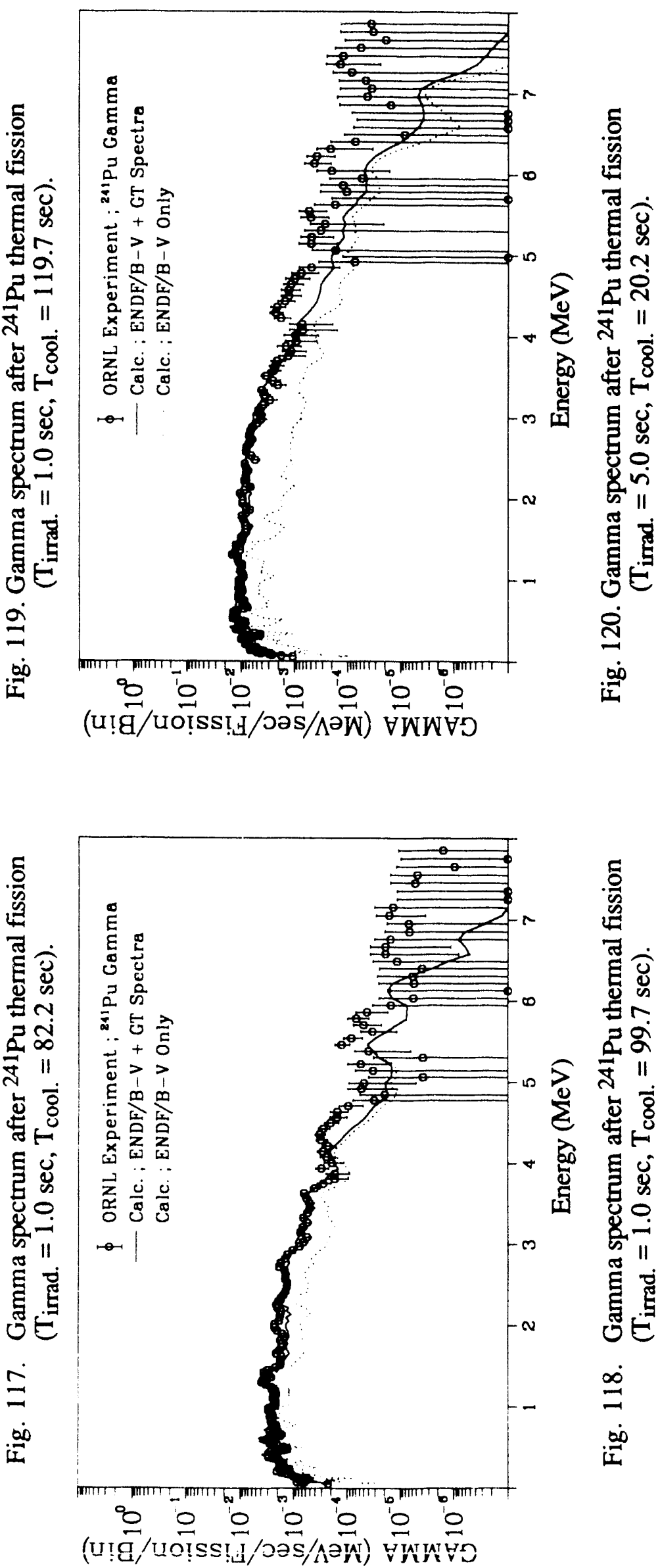

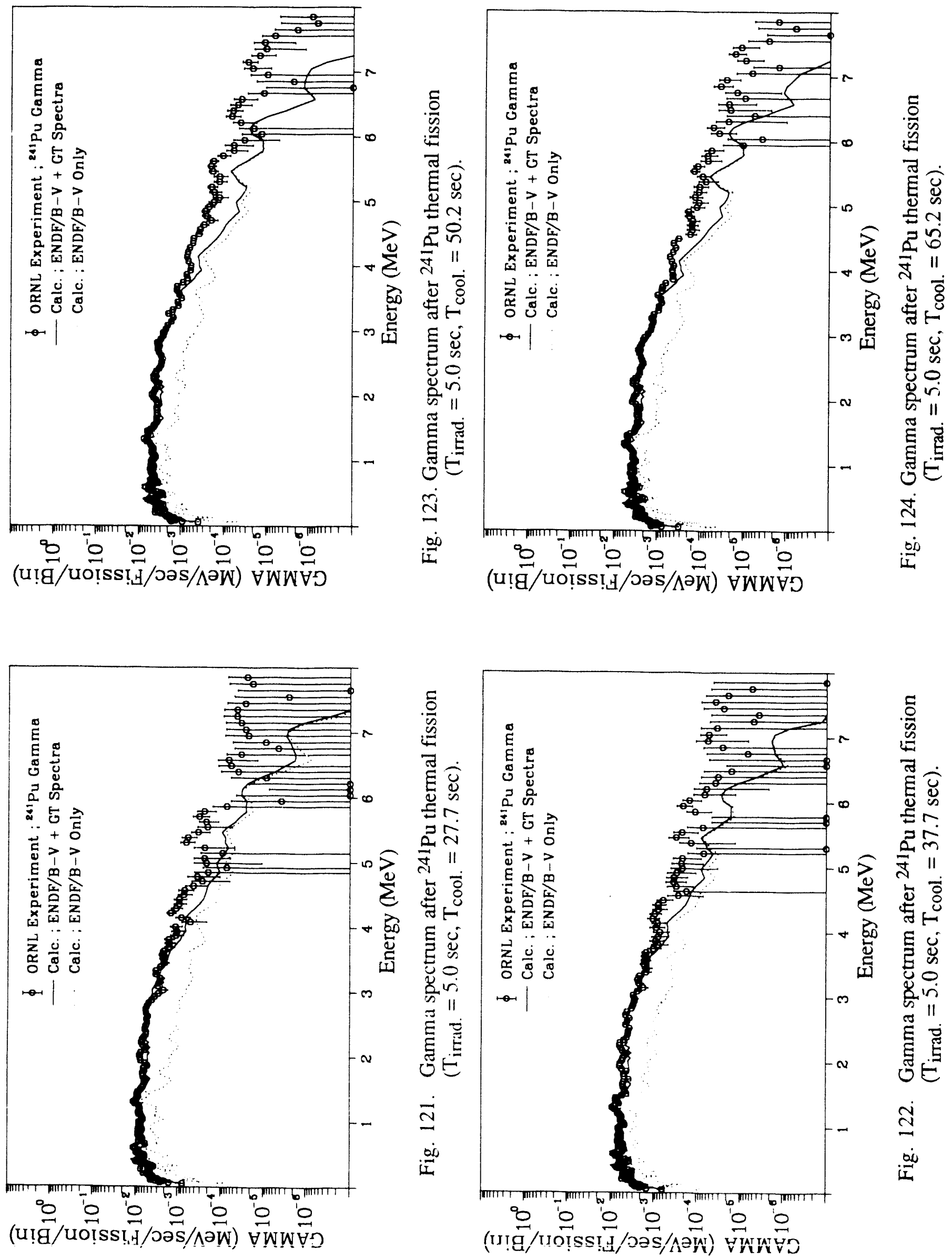


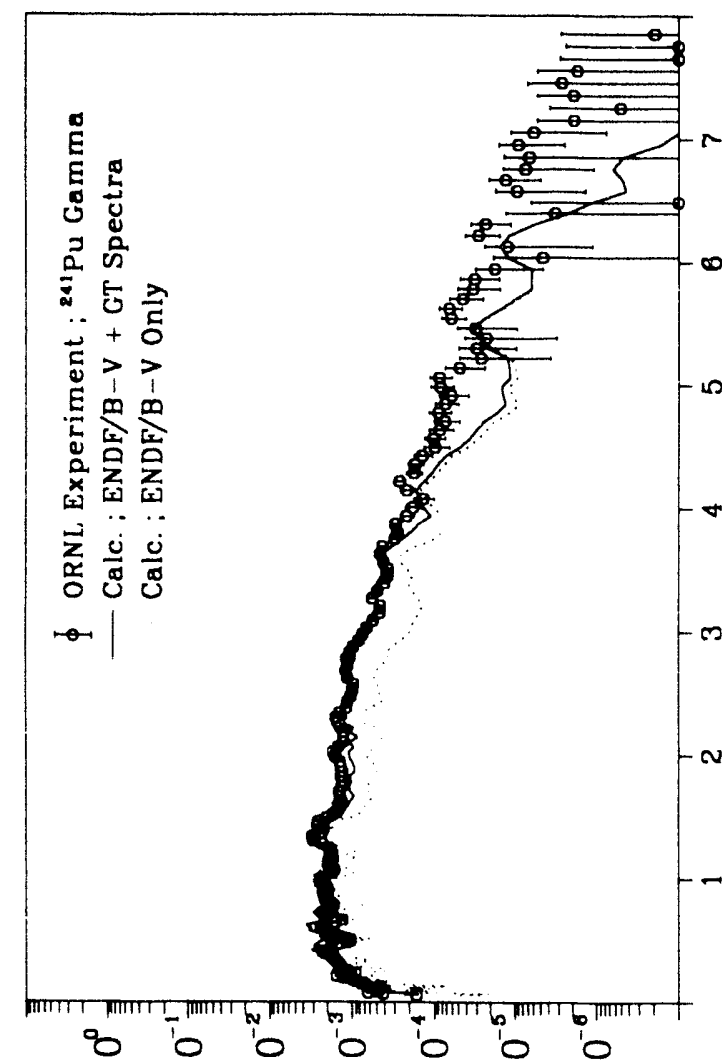

으 응으의 으응

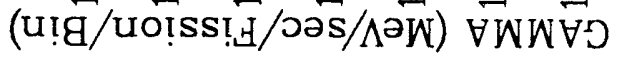
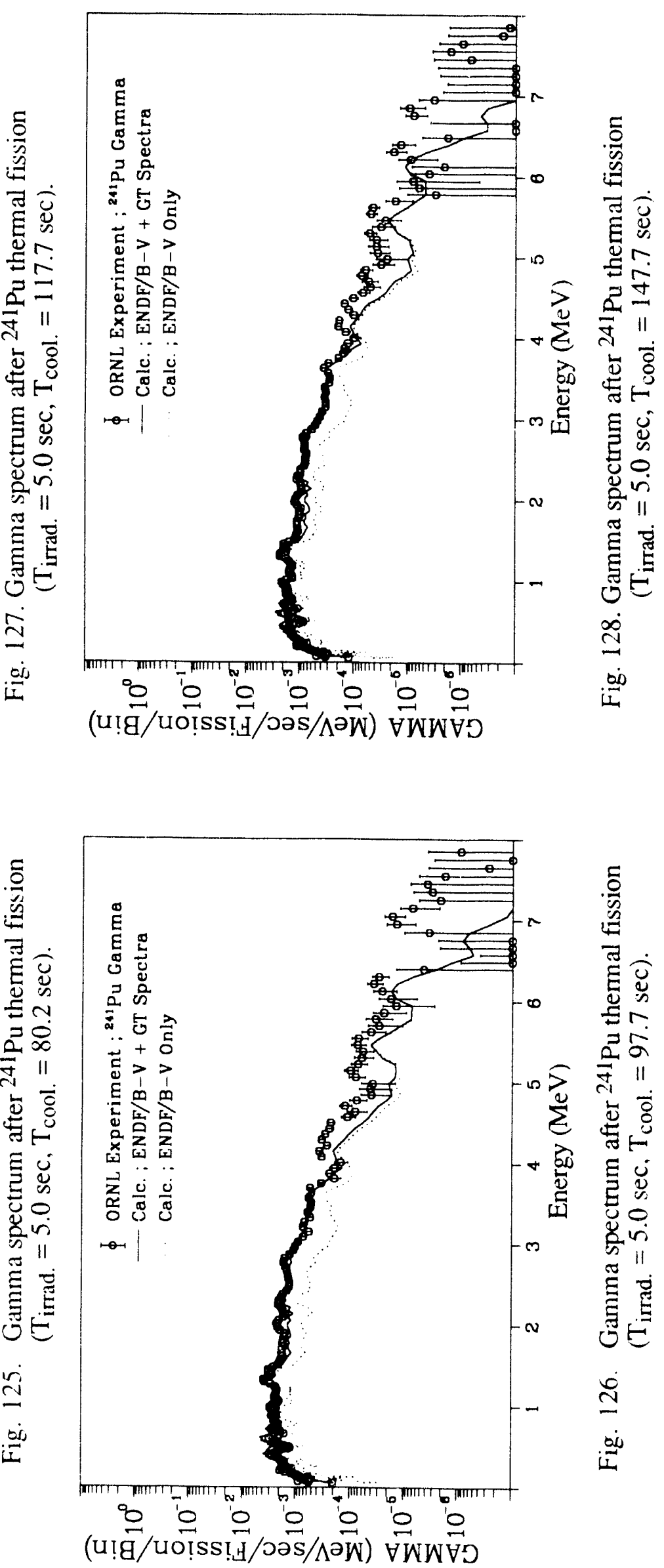

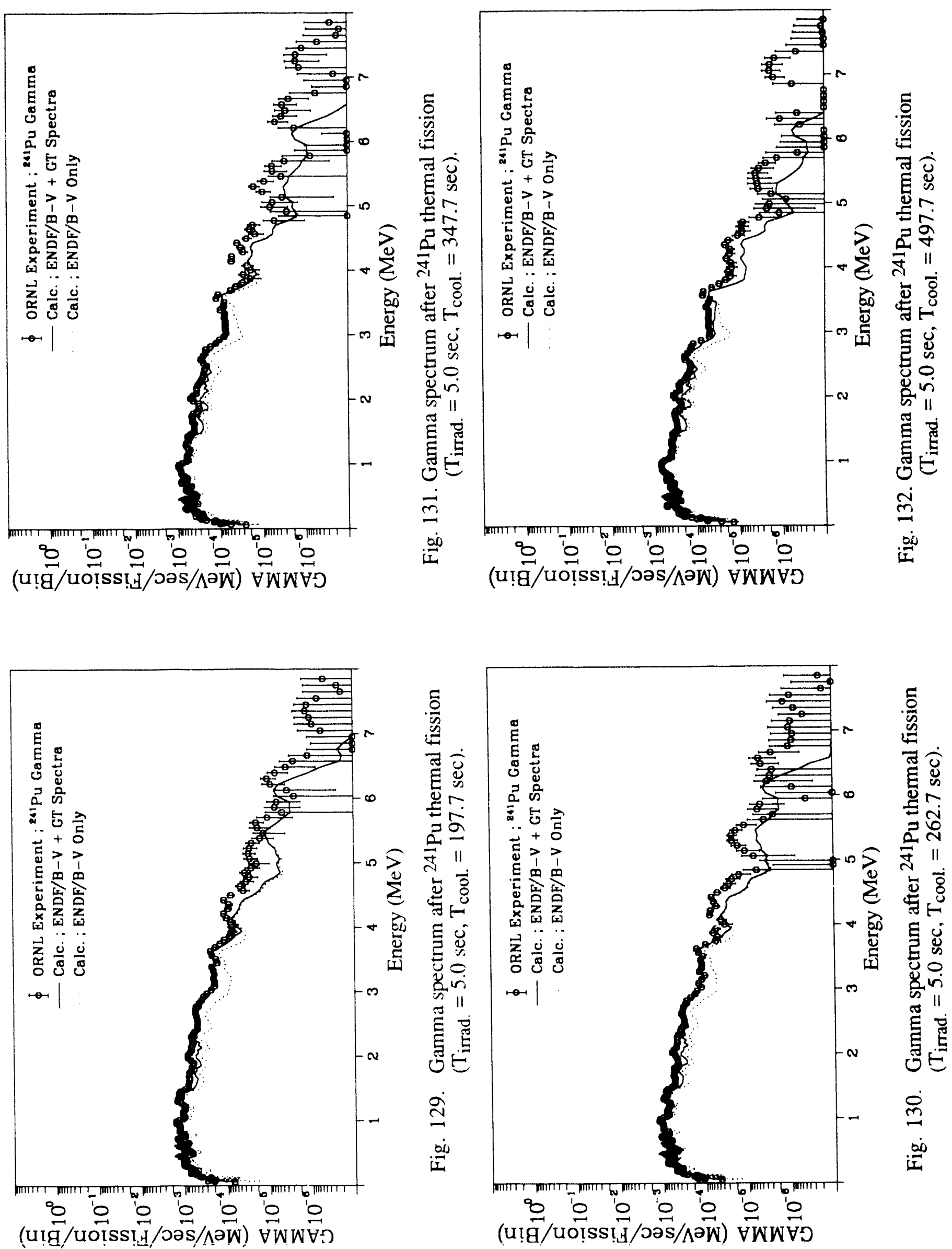


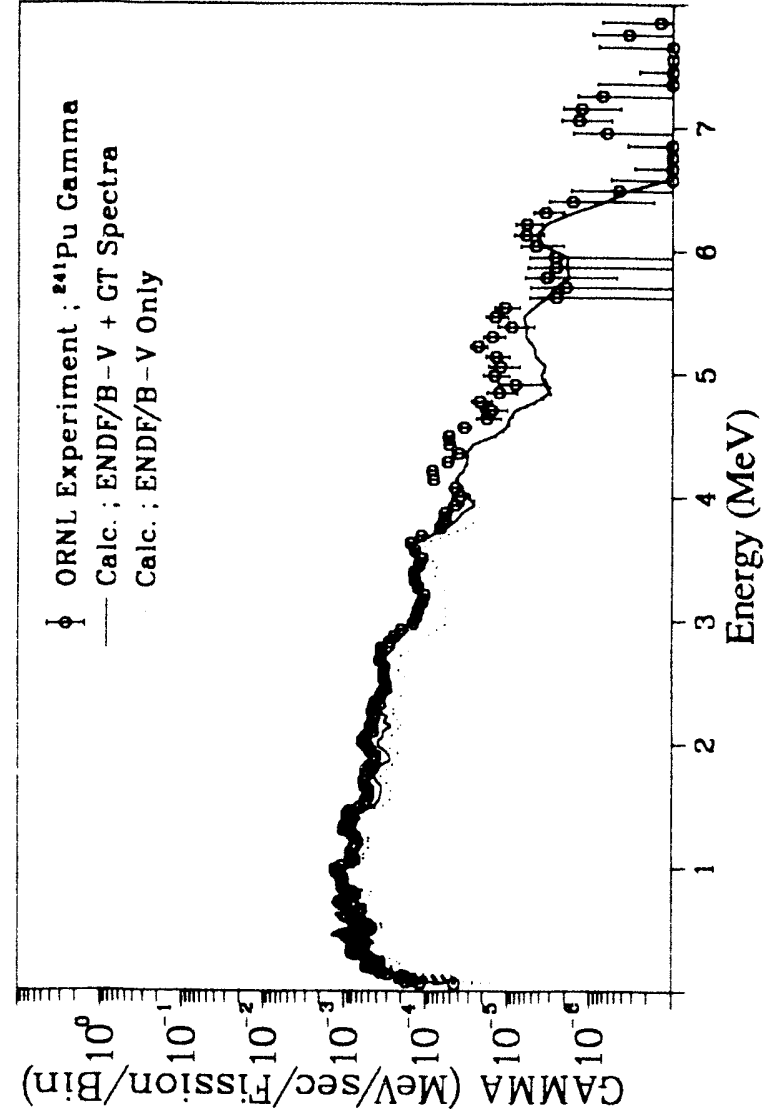

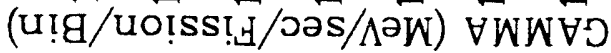

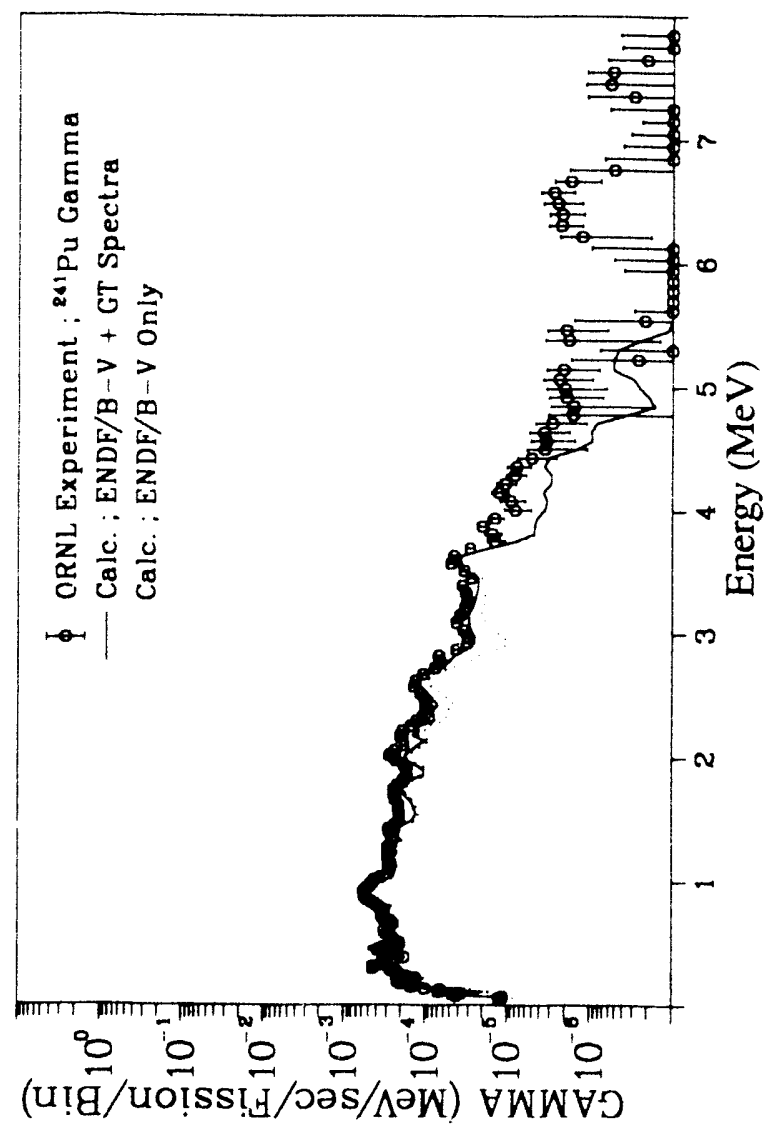

政
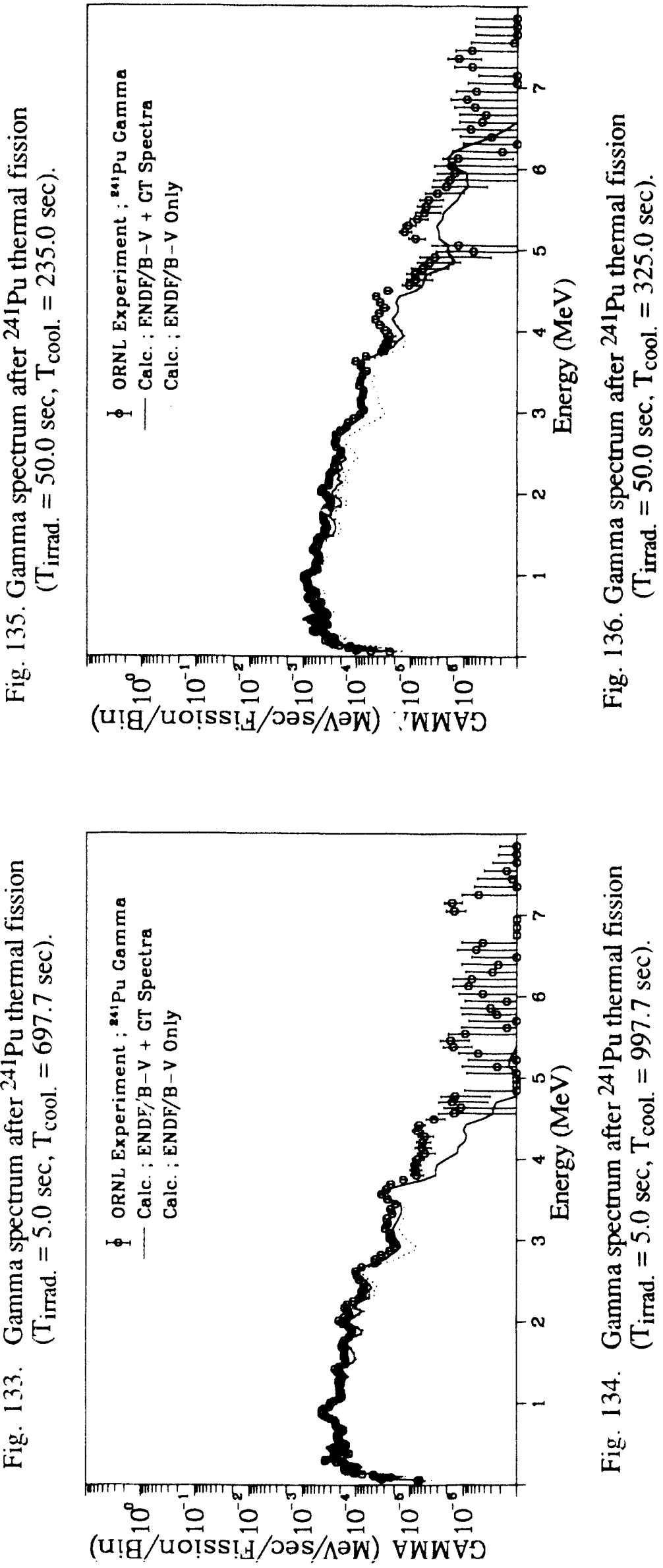


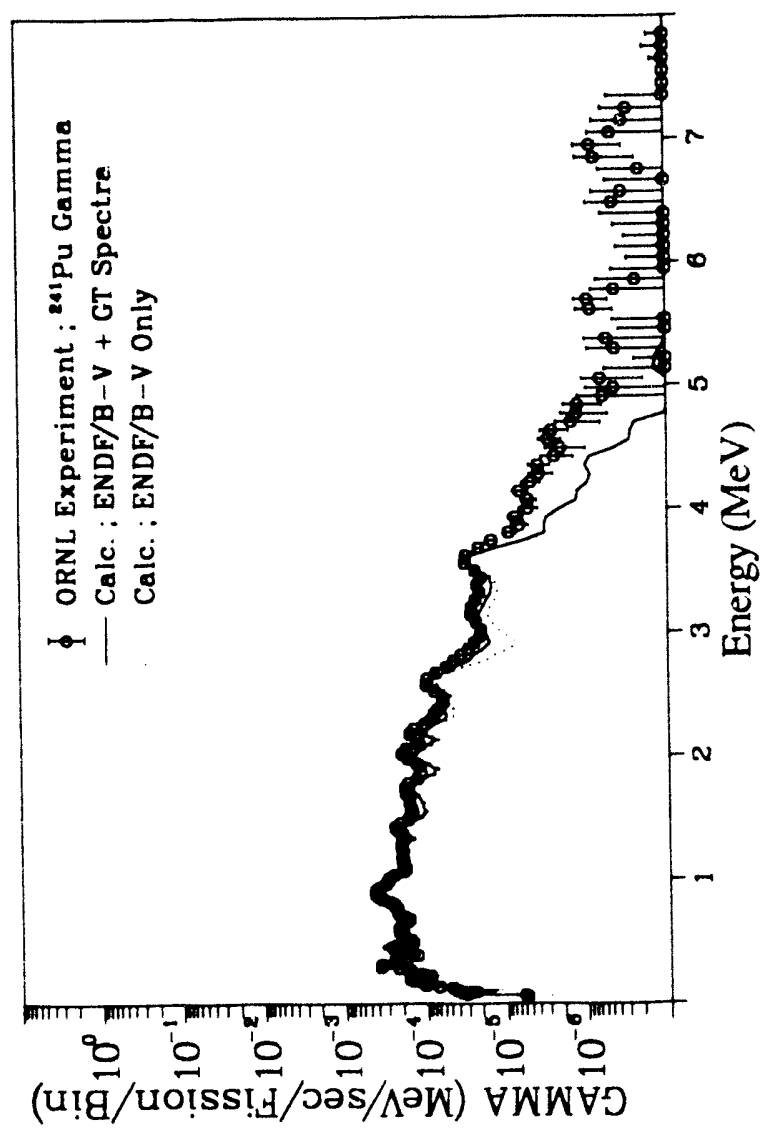

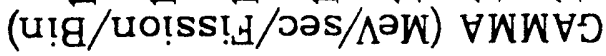
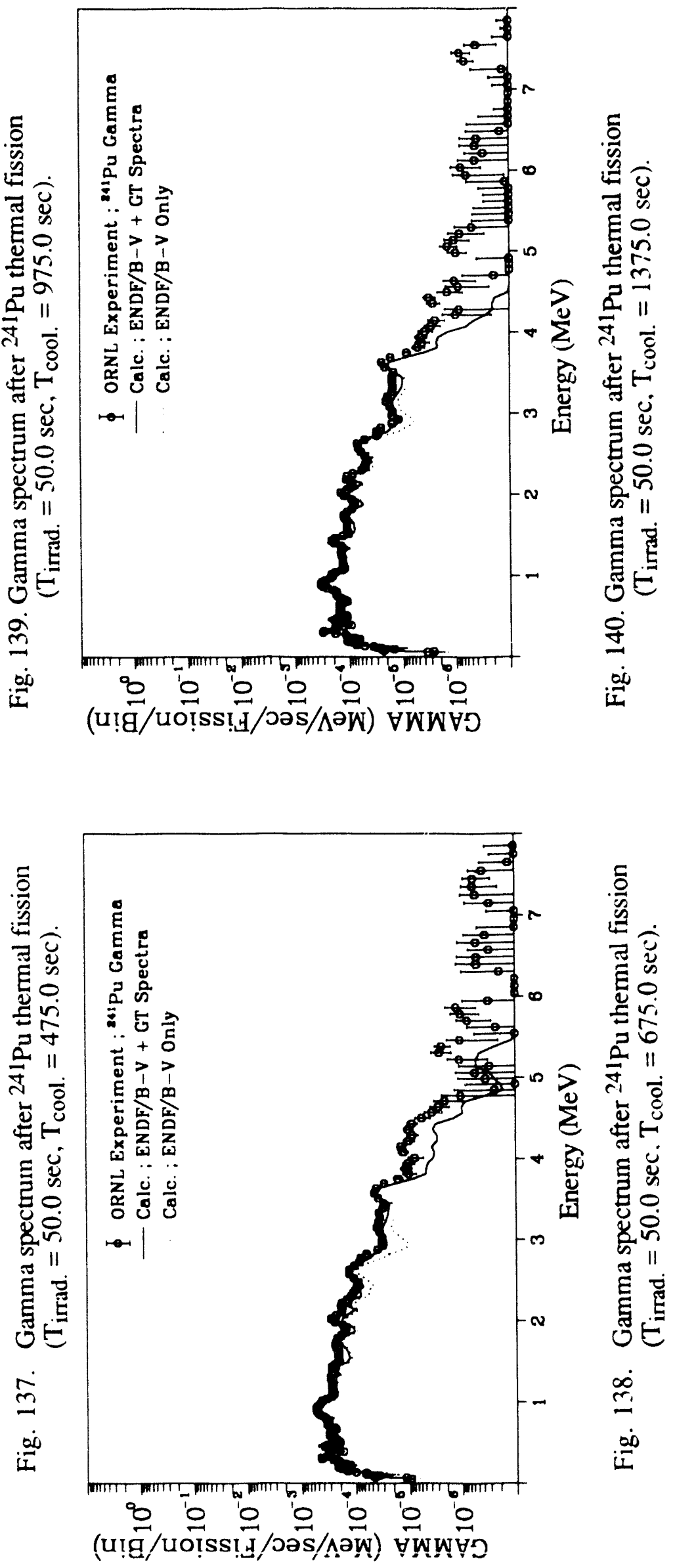

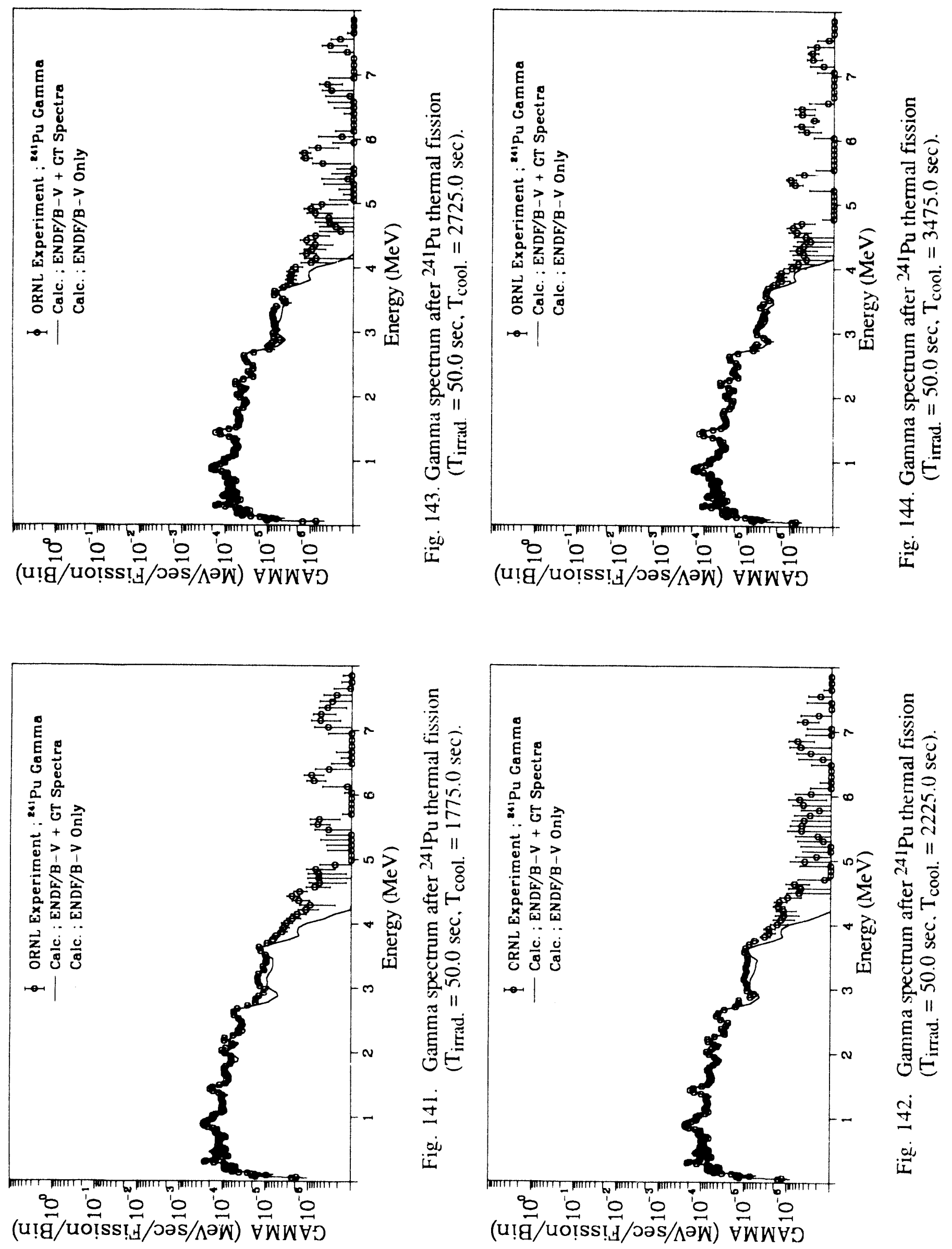






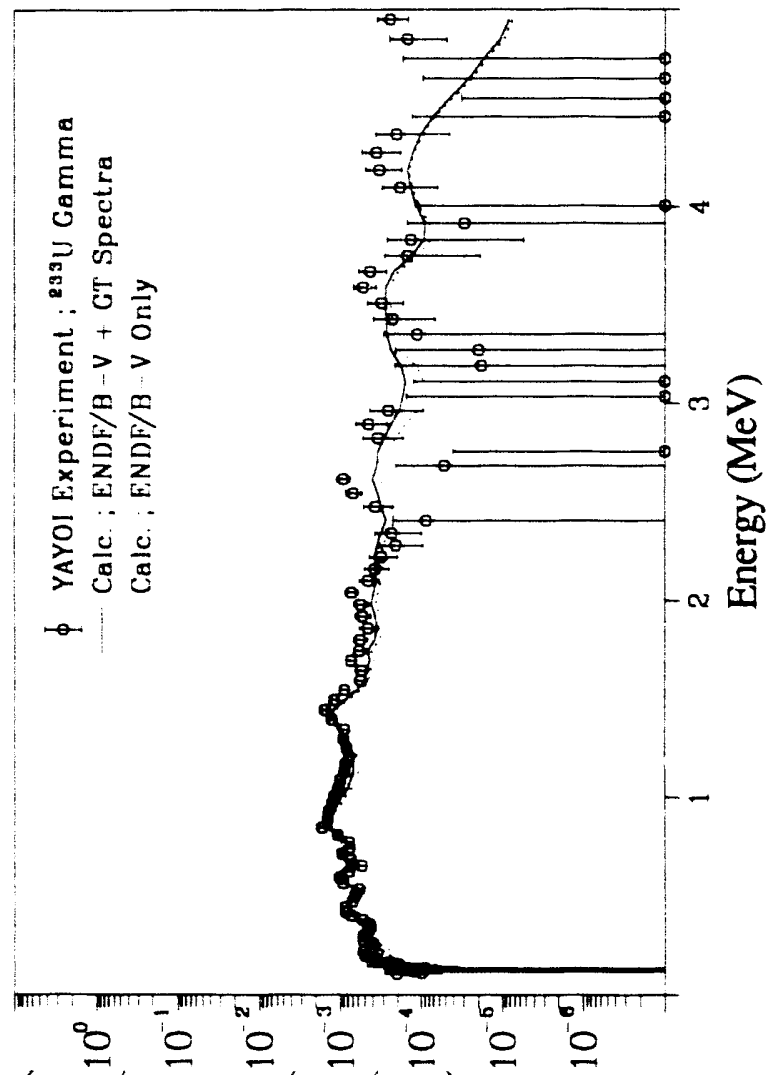

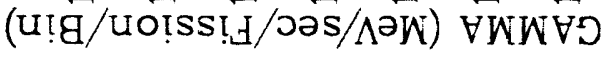

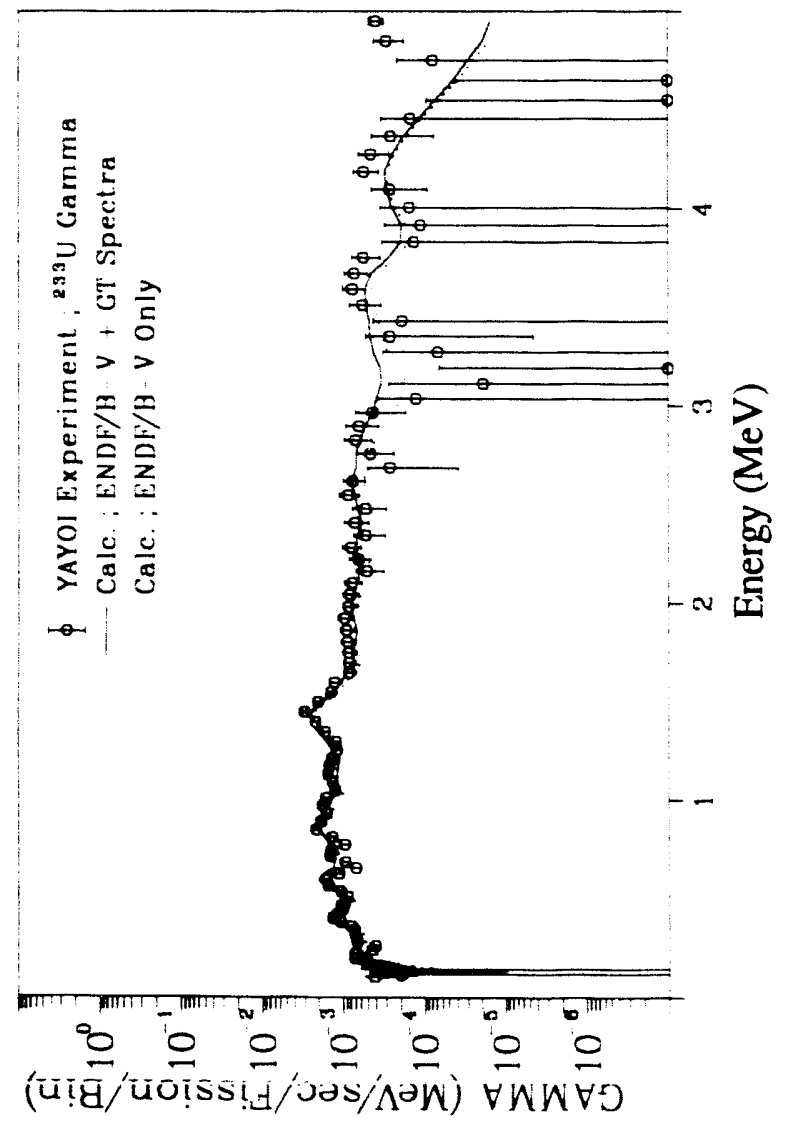

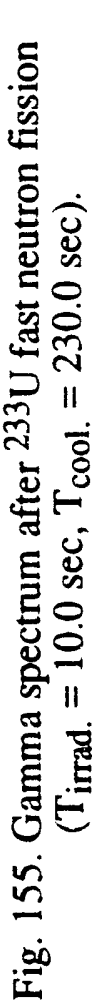

सागा। गागा।

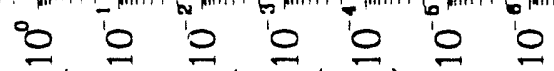

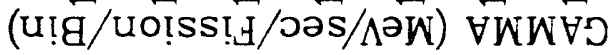

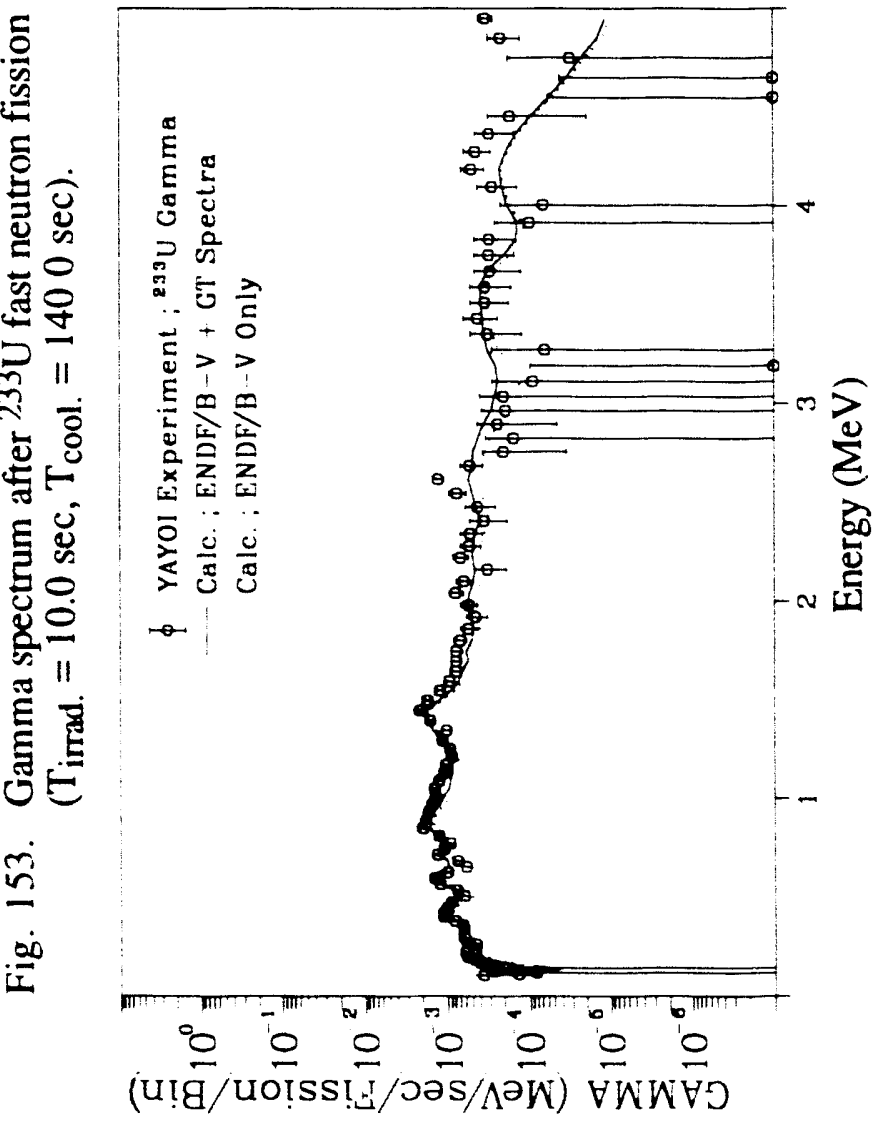




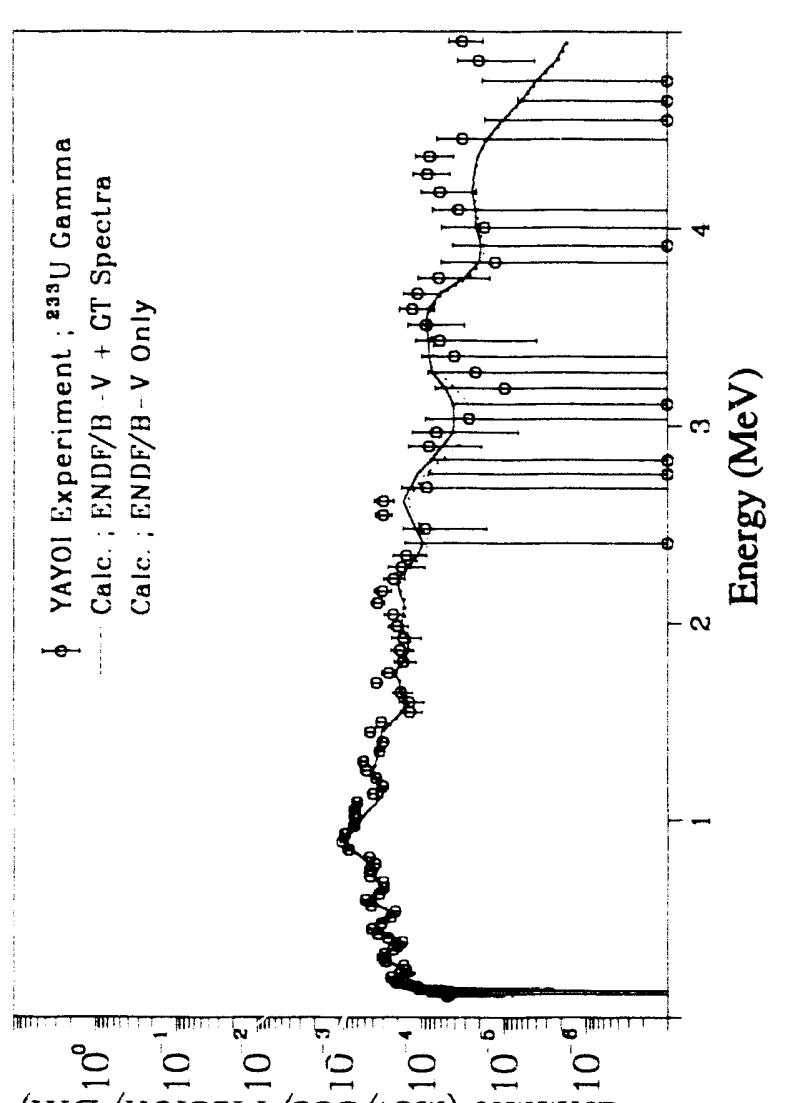

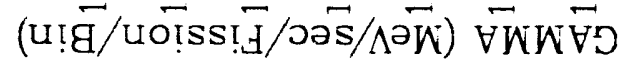
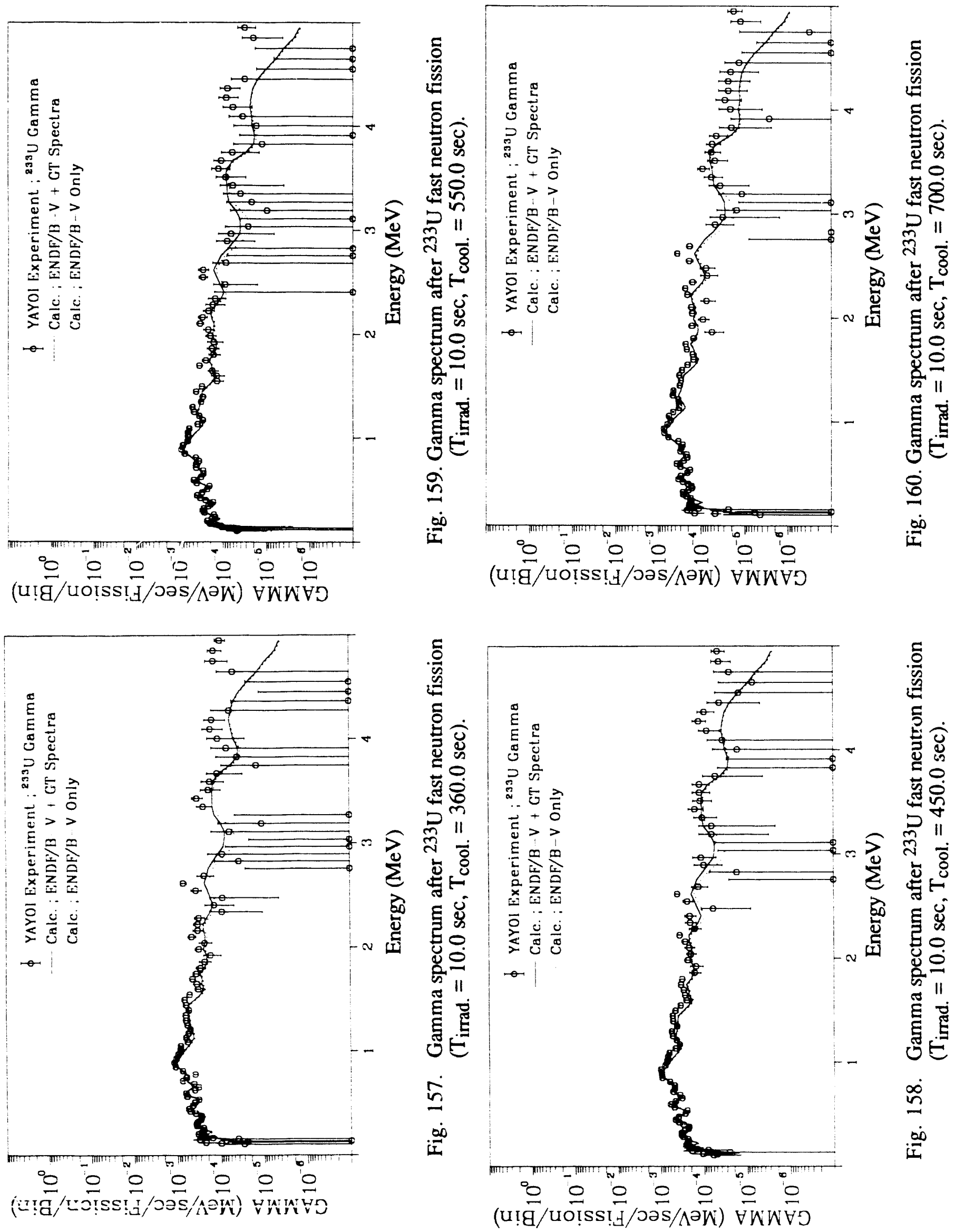

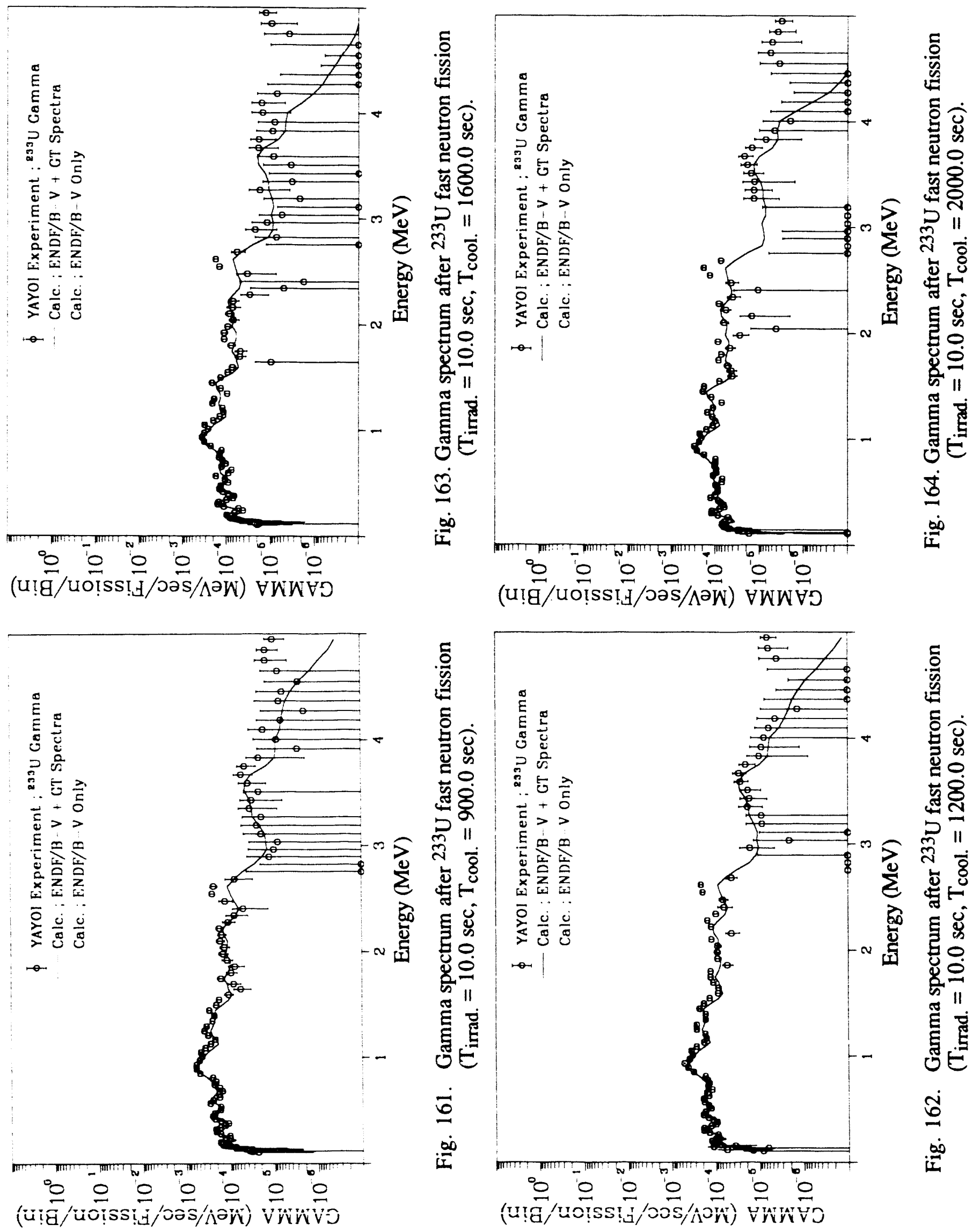




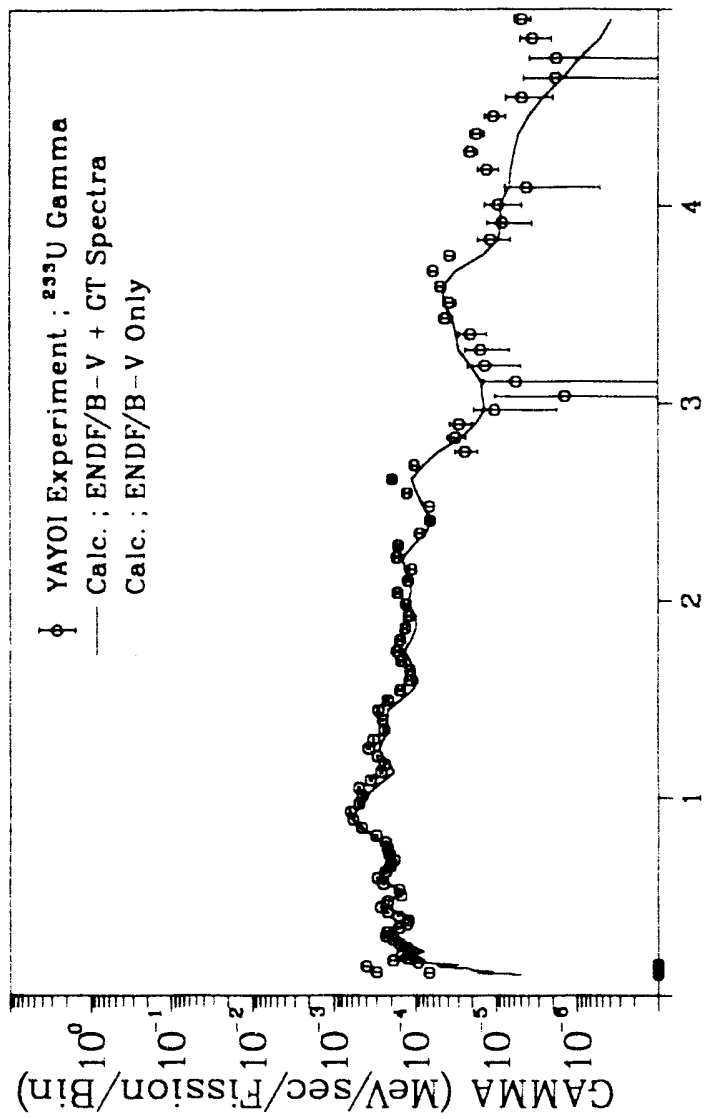

(u!g/uo!ss!]/Jas/ $\Lambda$ คW) $\forall W W \forall J$




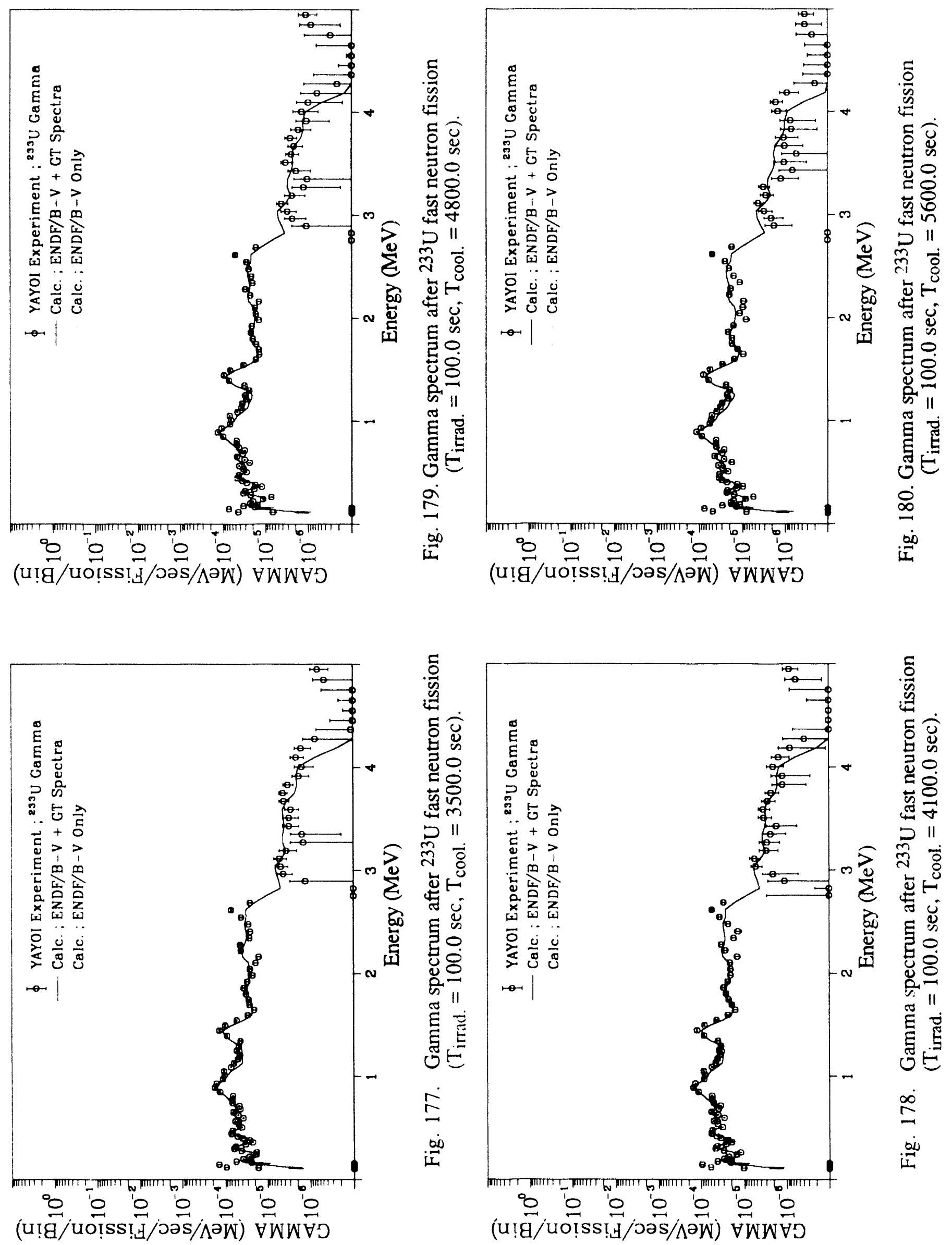

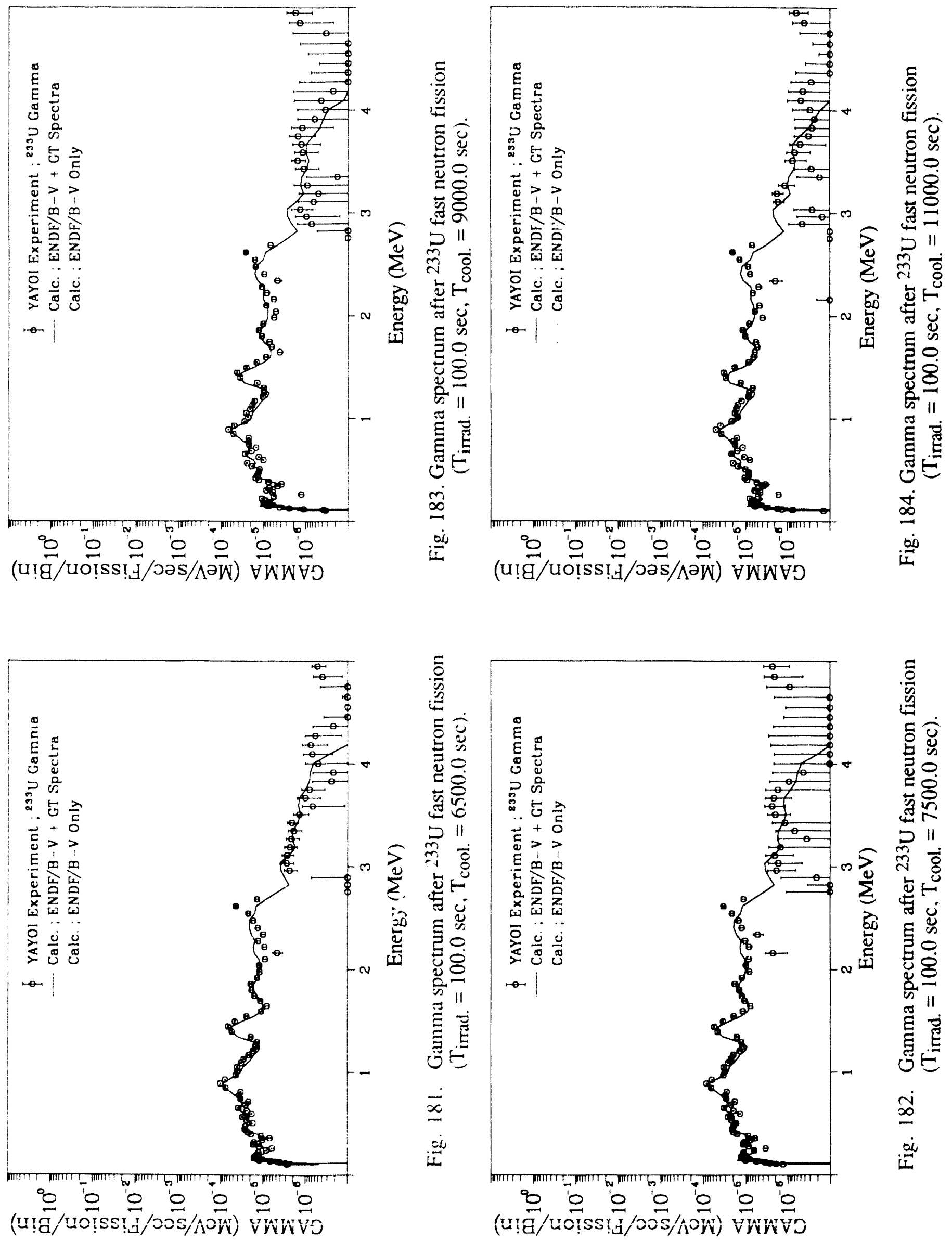


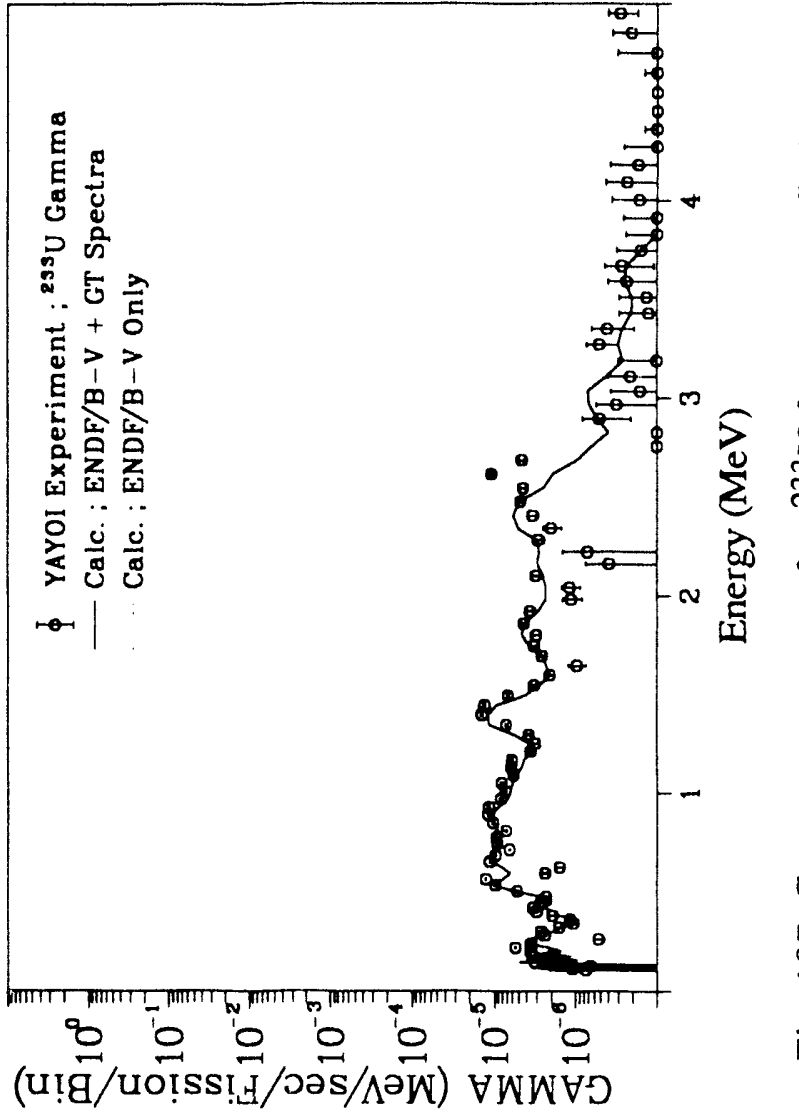







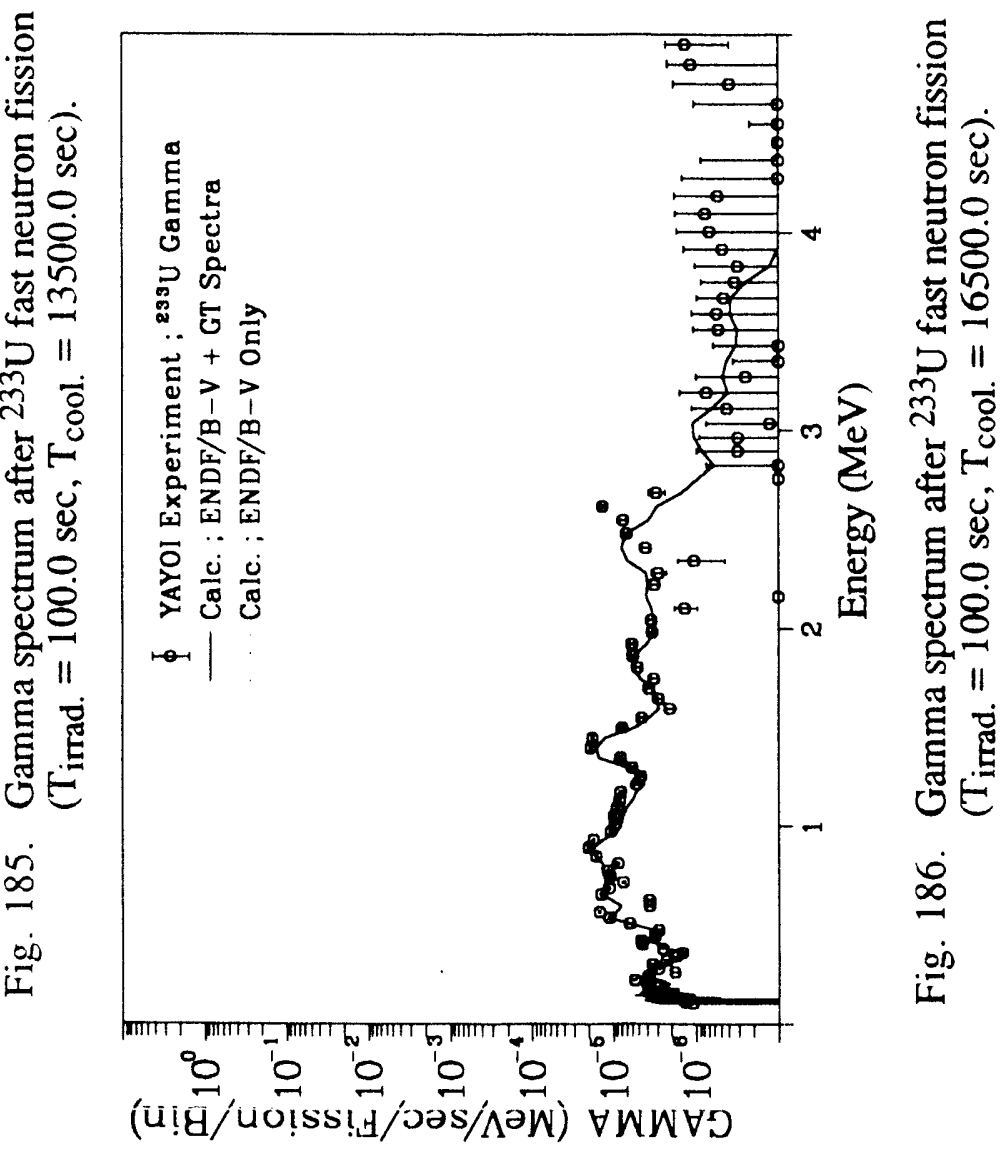











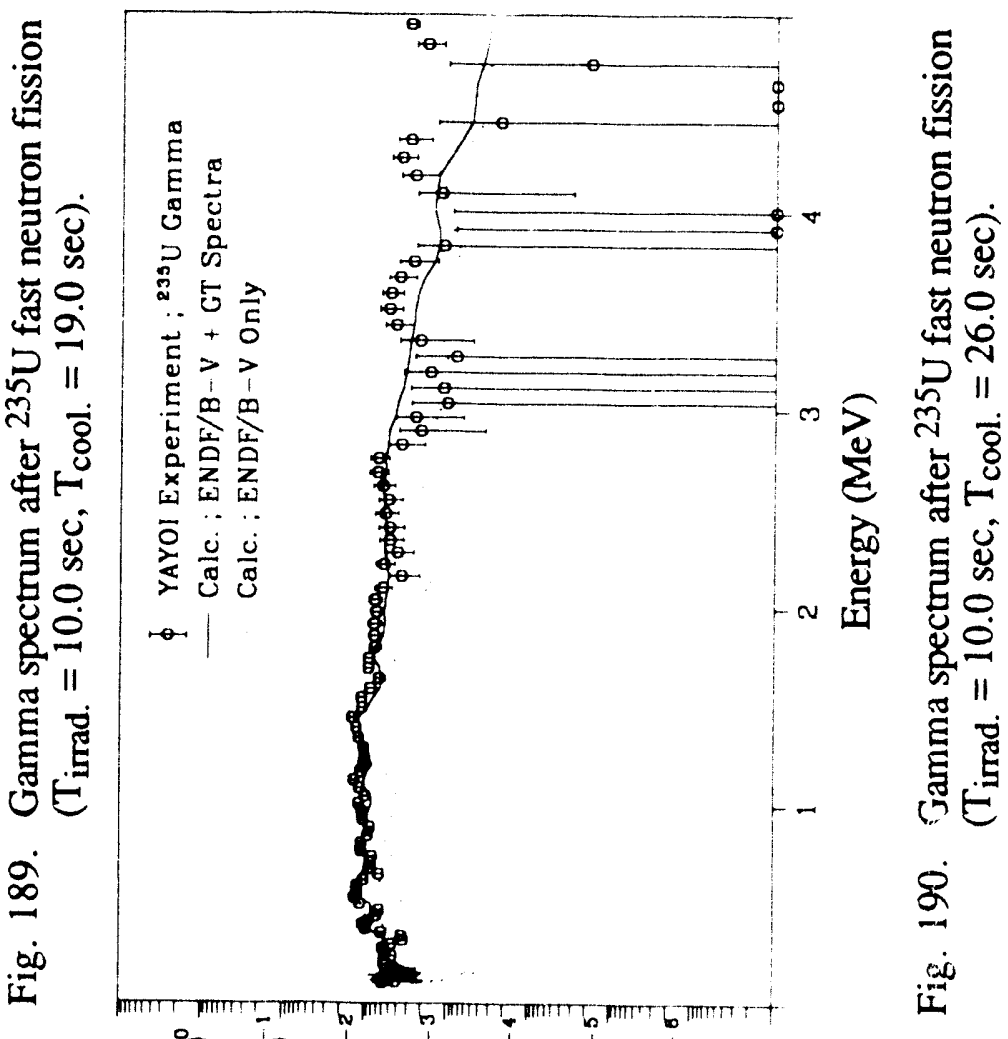







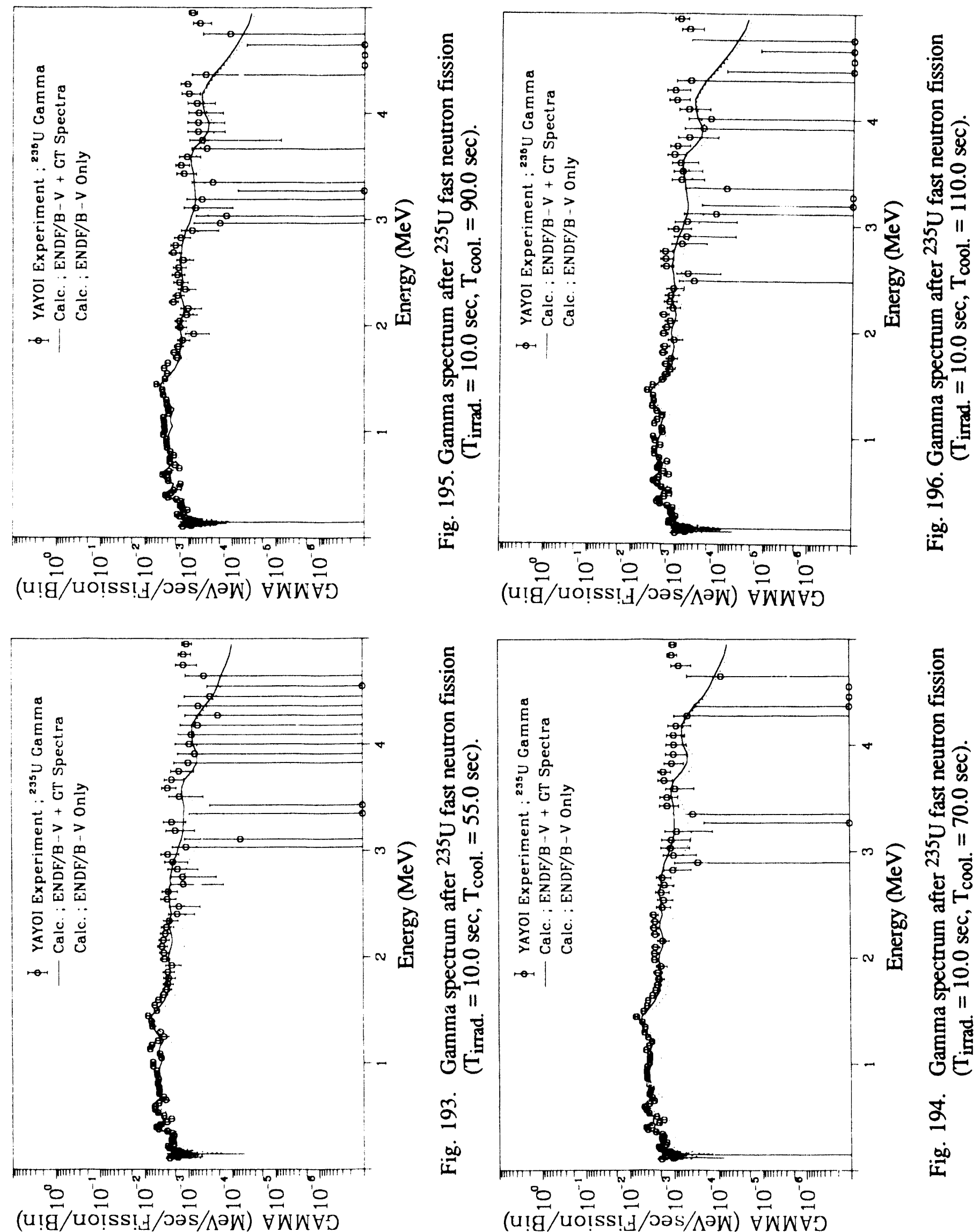

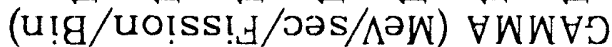



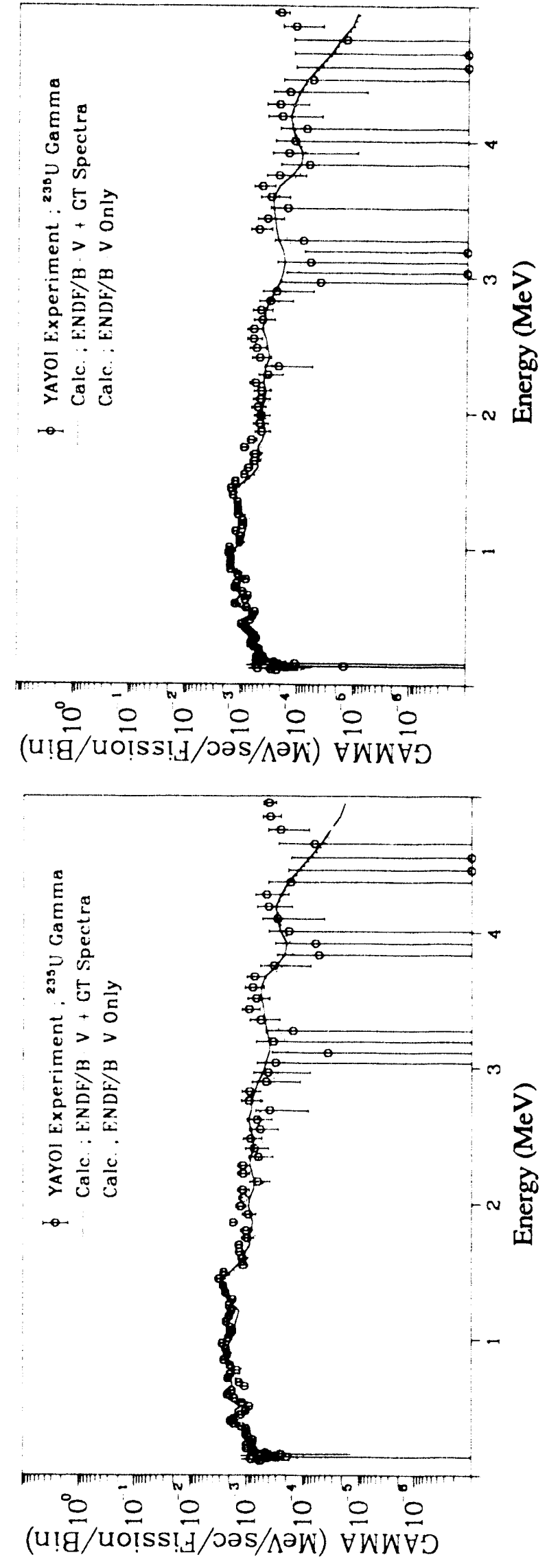

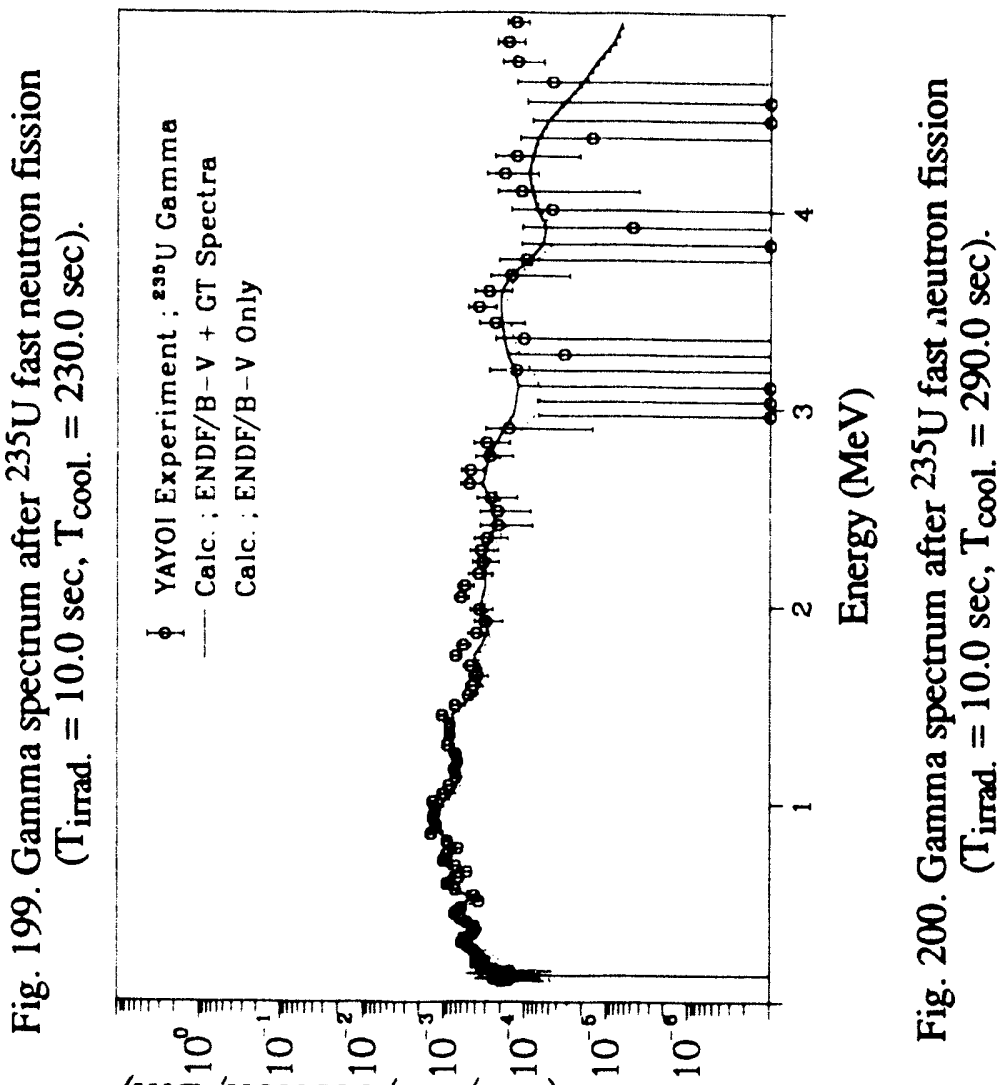

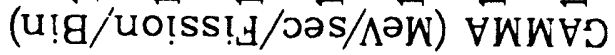

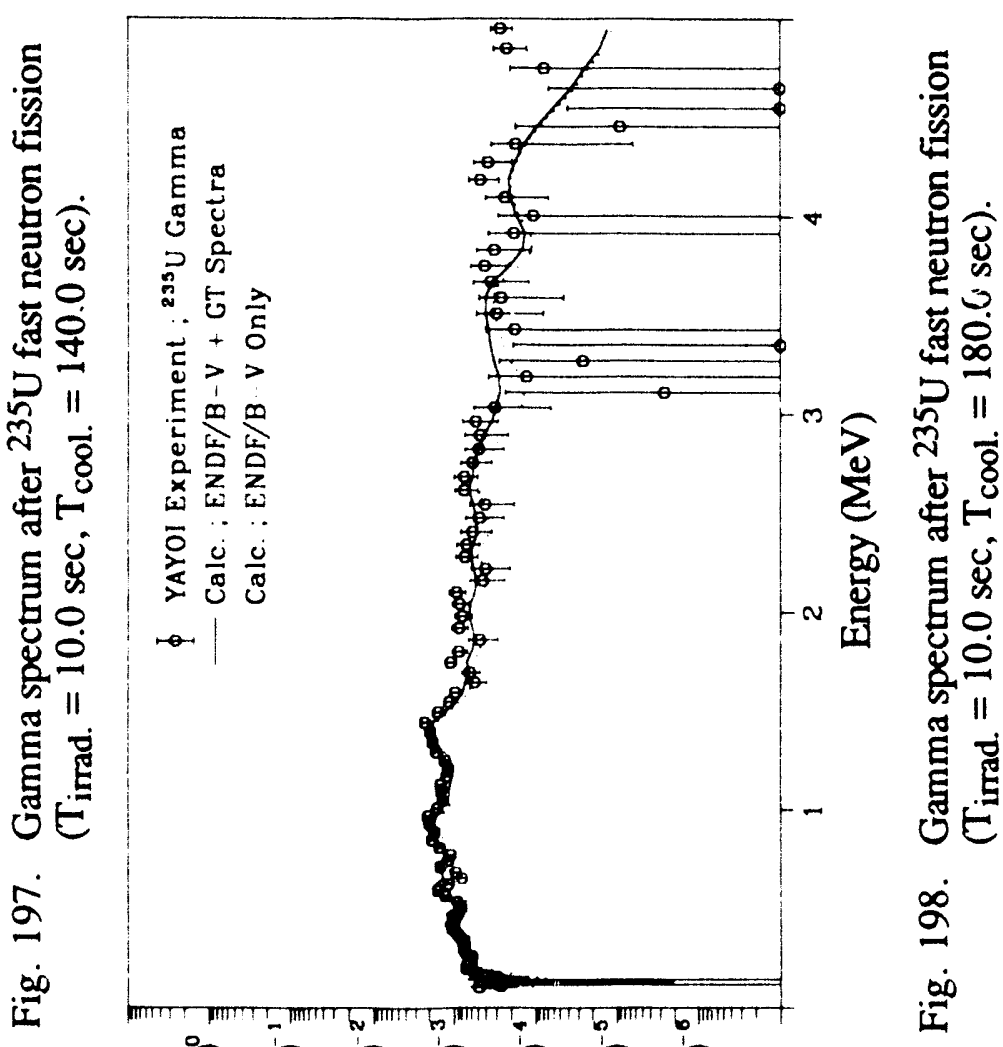

$\therefore$ 으의 0

(u!ต/uo!̣s! 

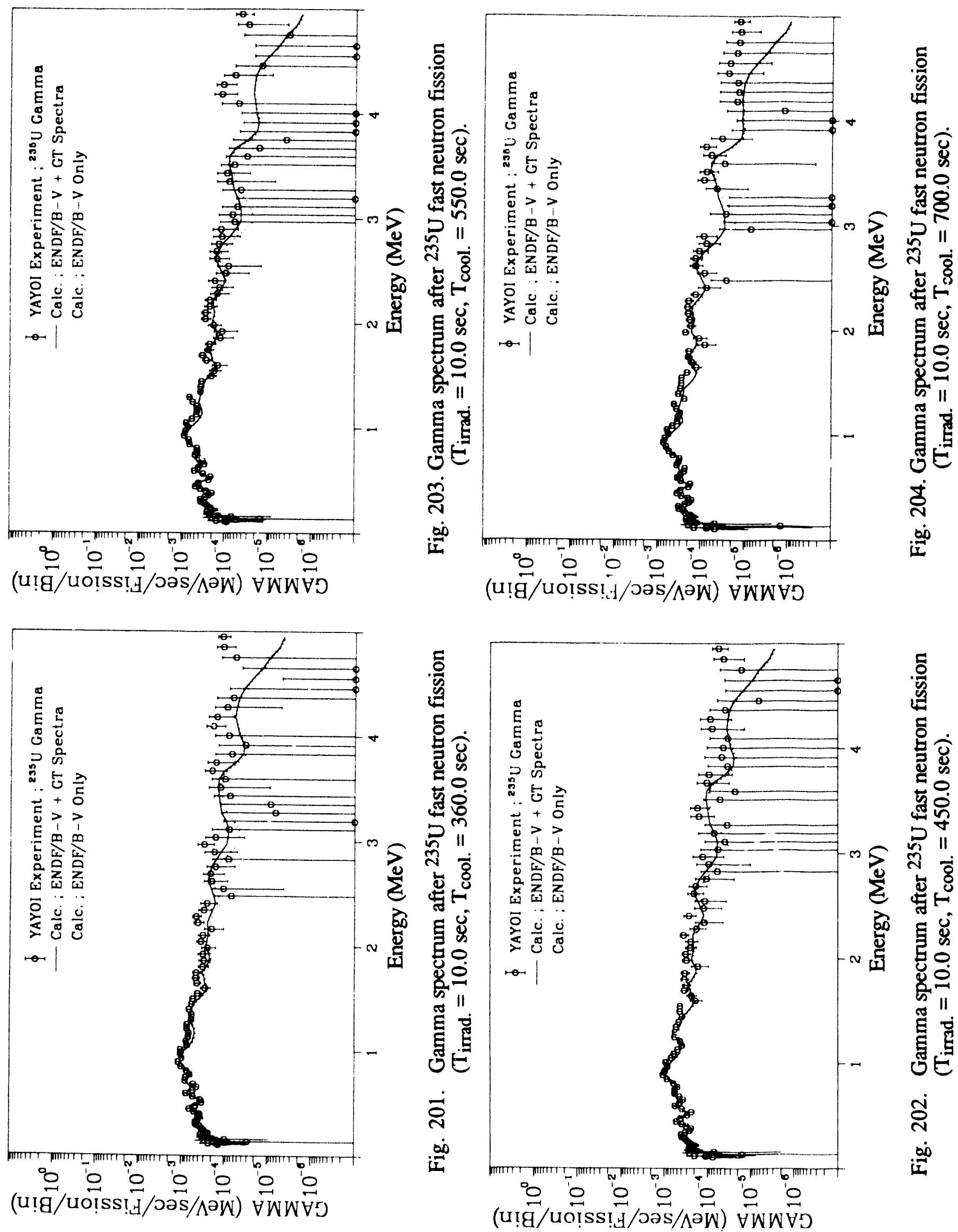

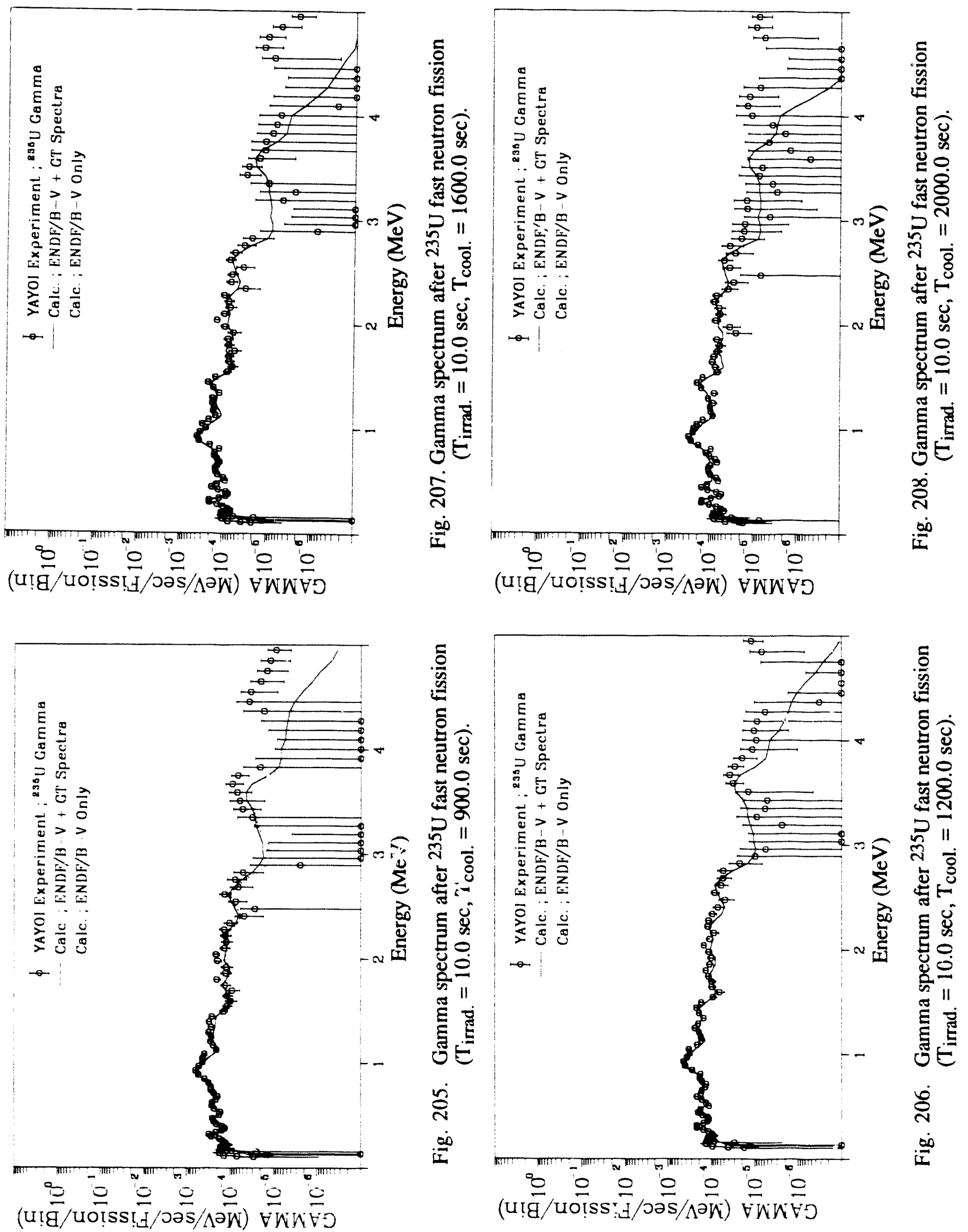


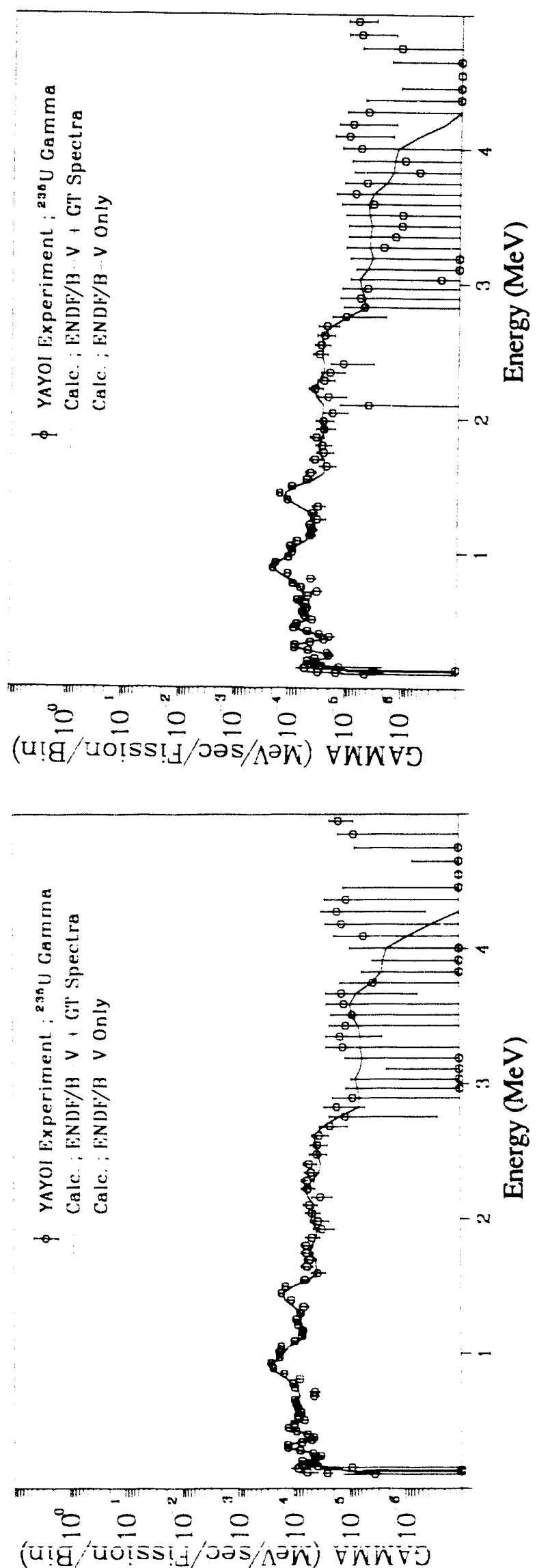

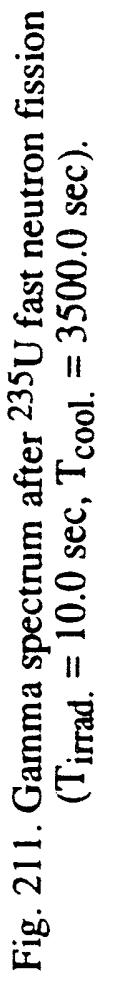
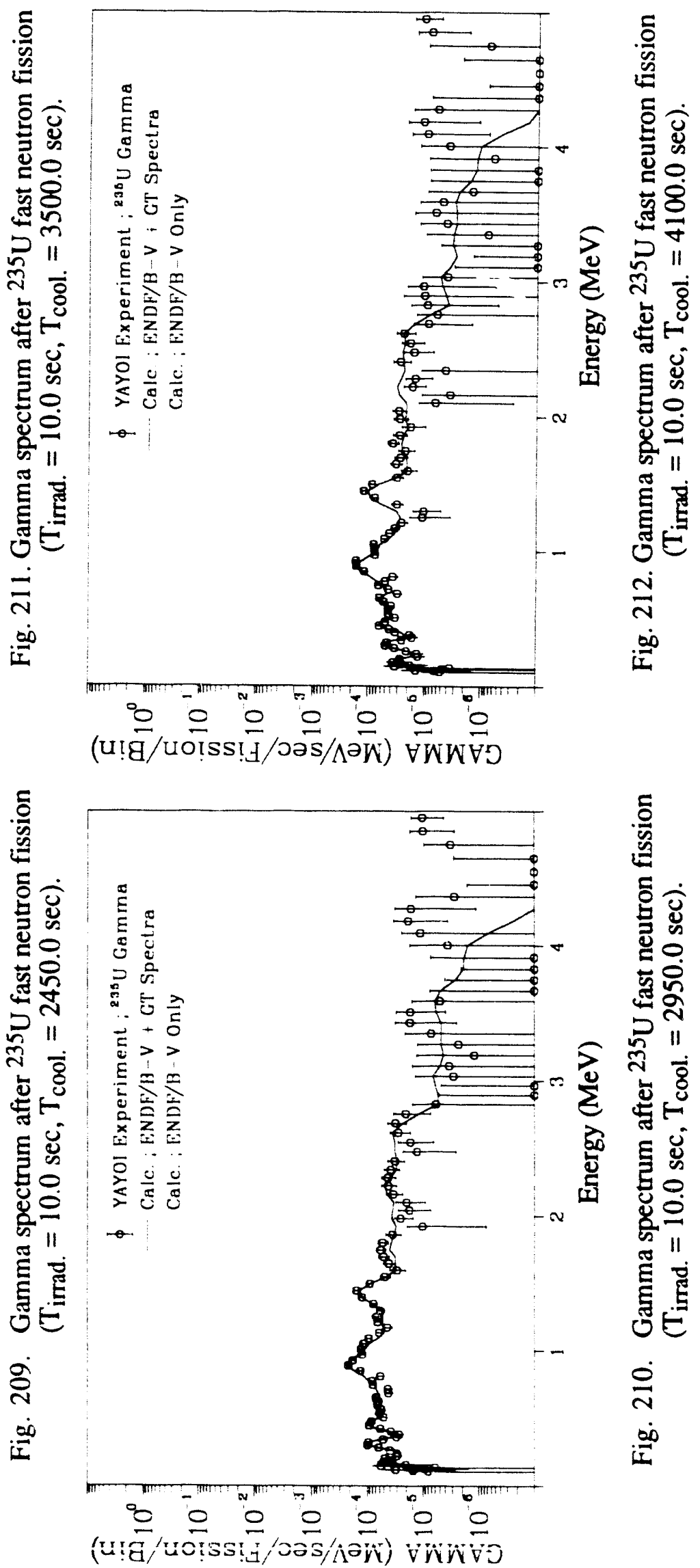


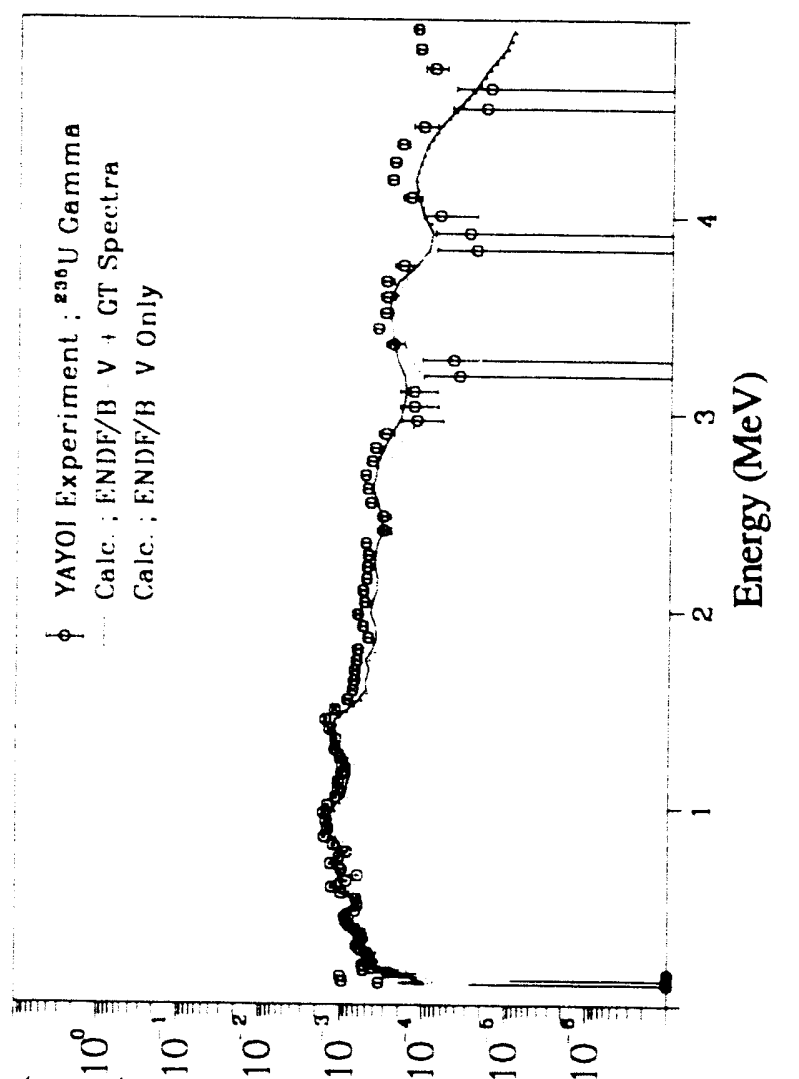

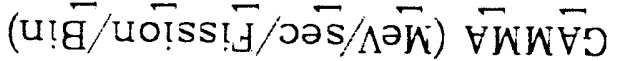

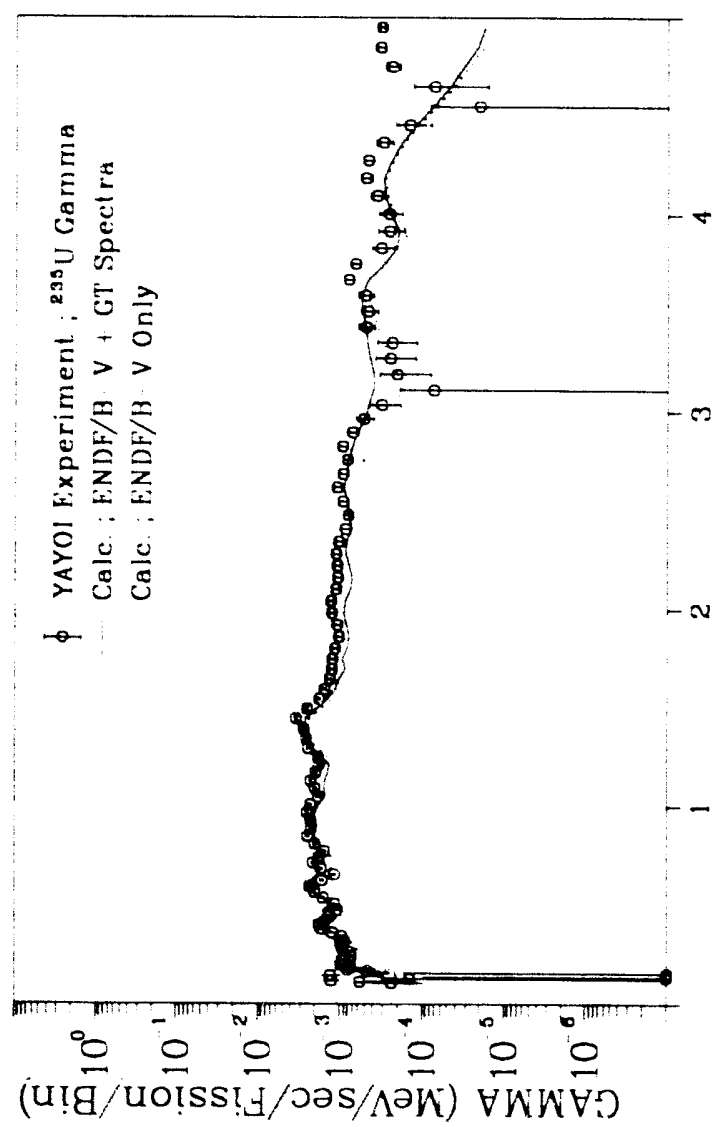

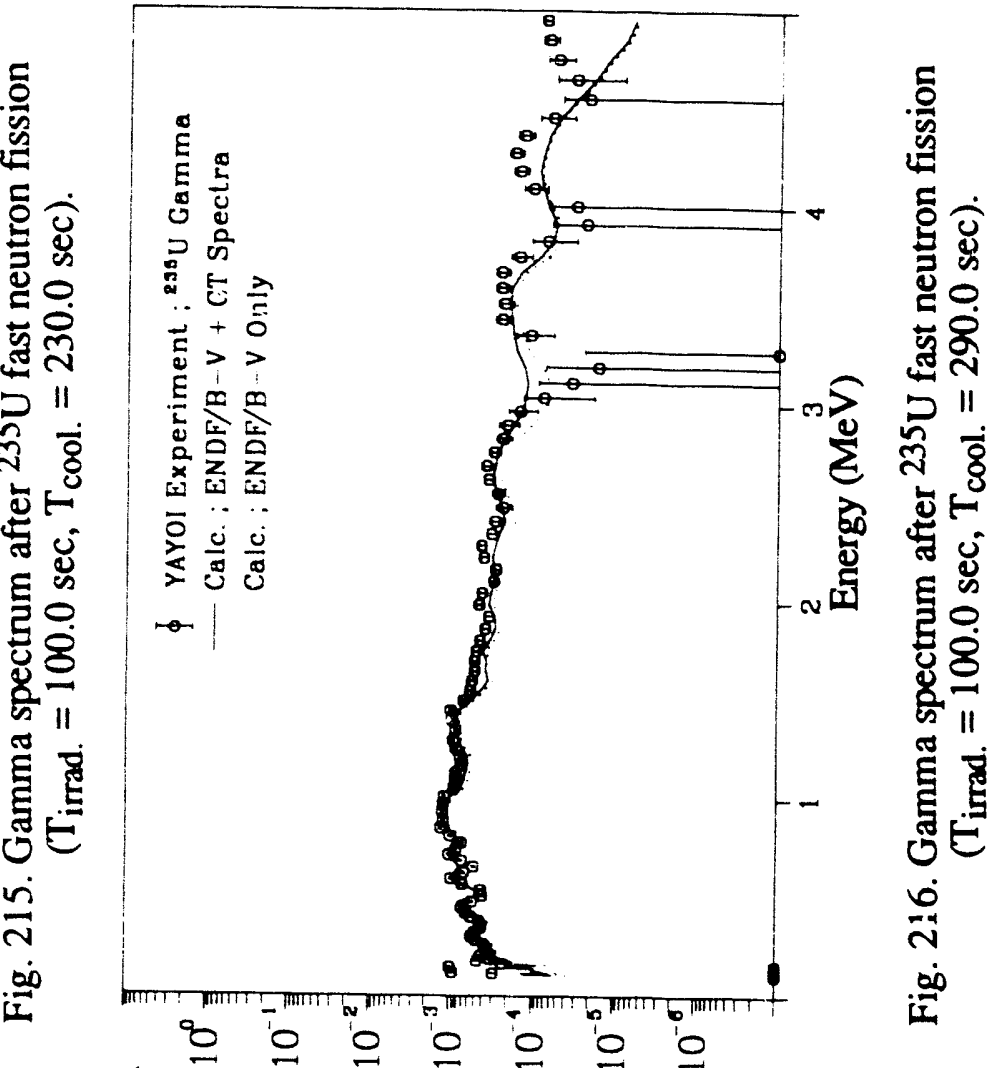



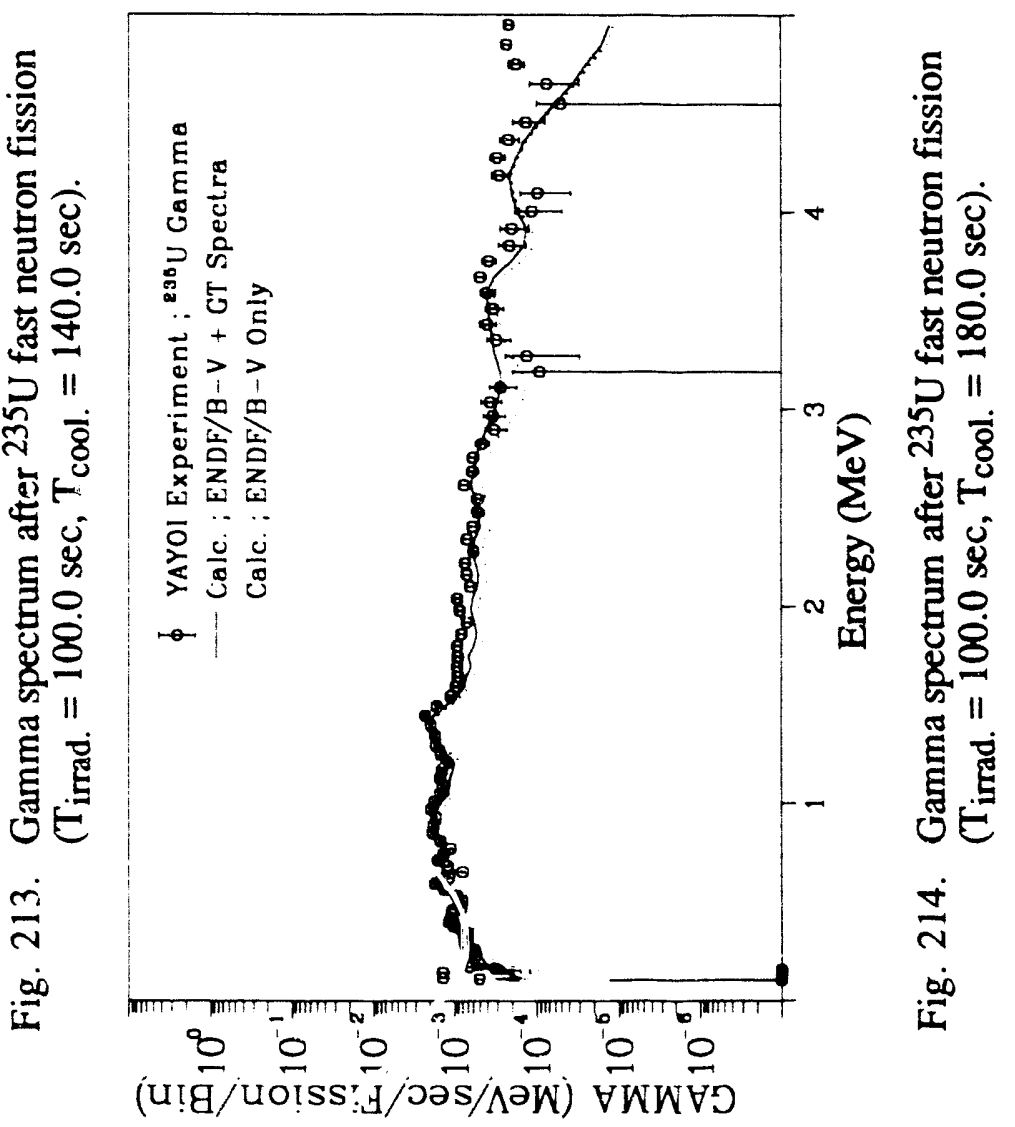




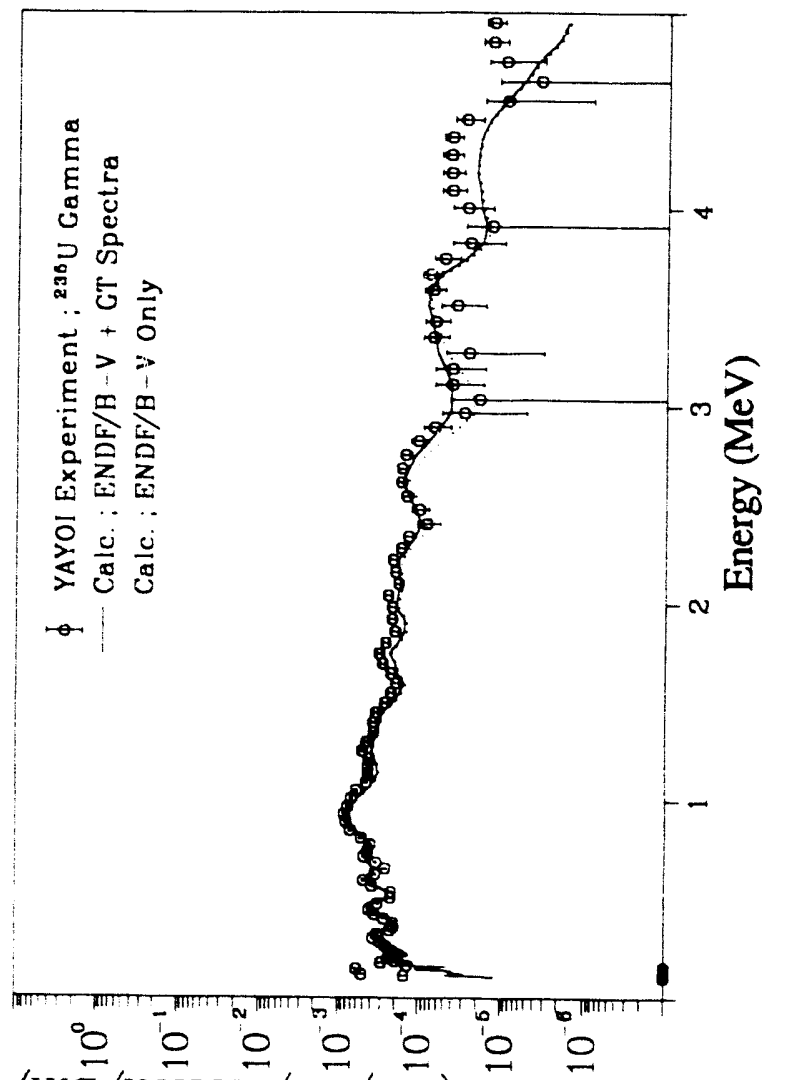

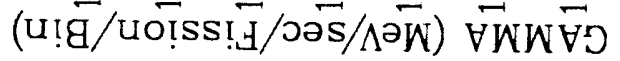

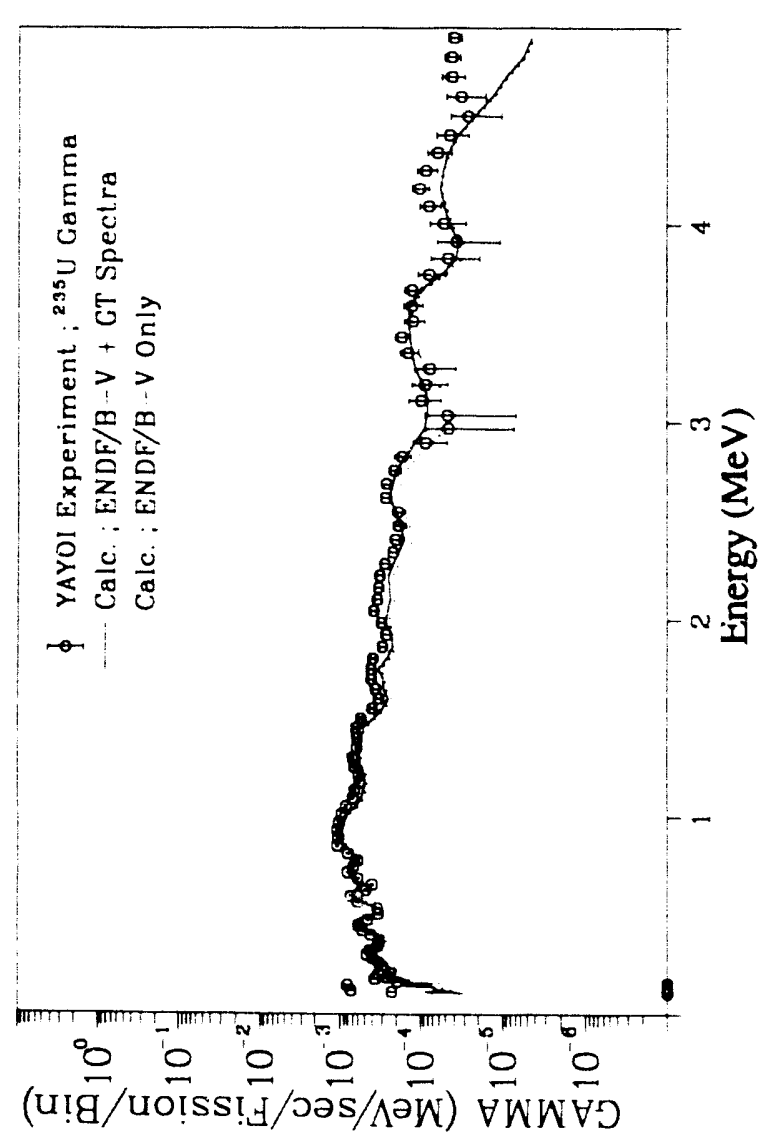

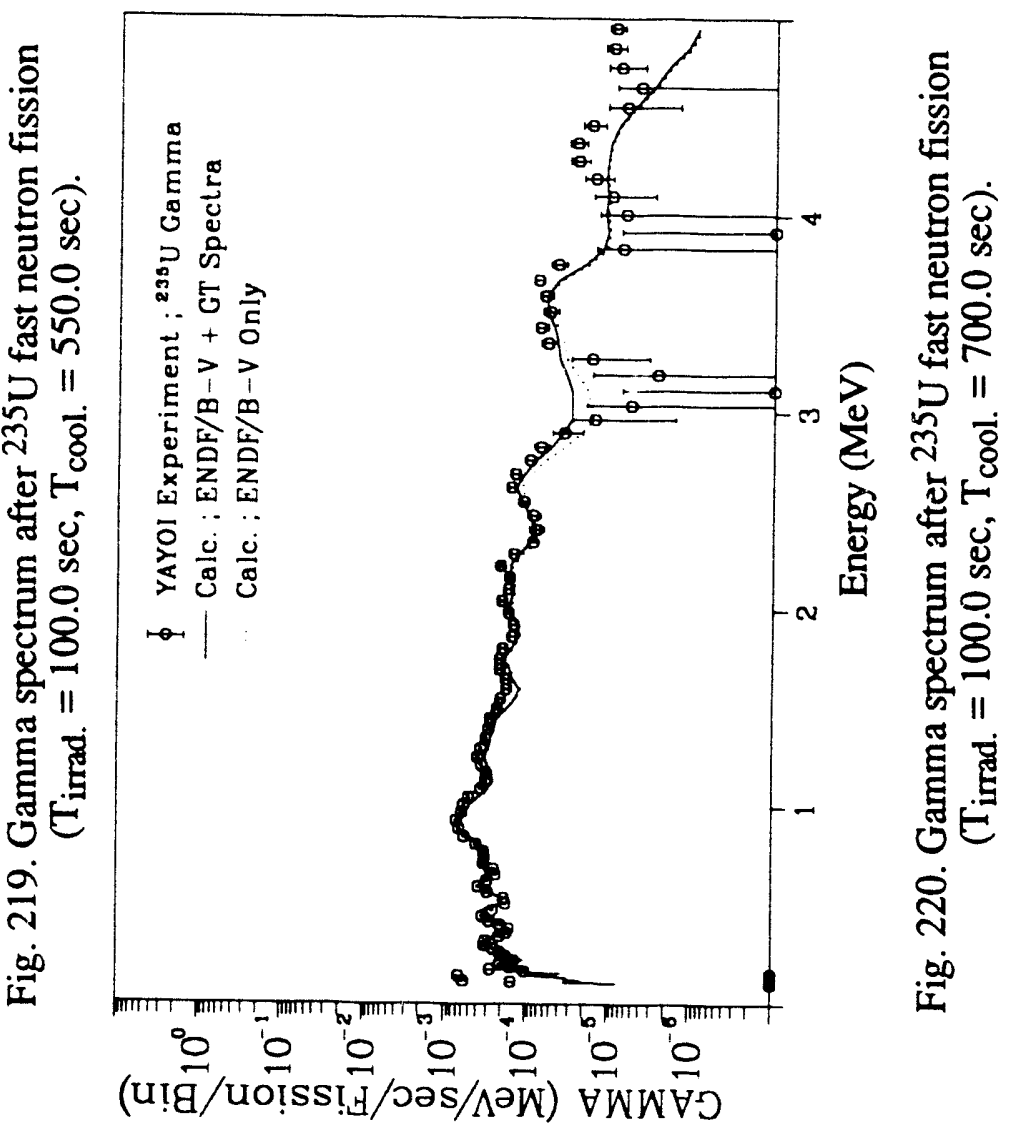

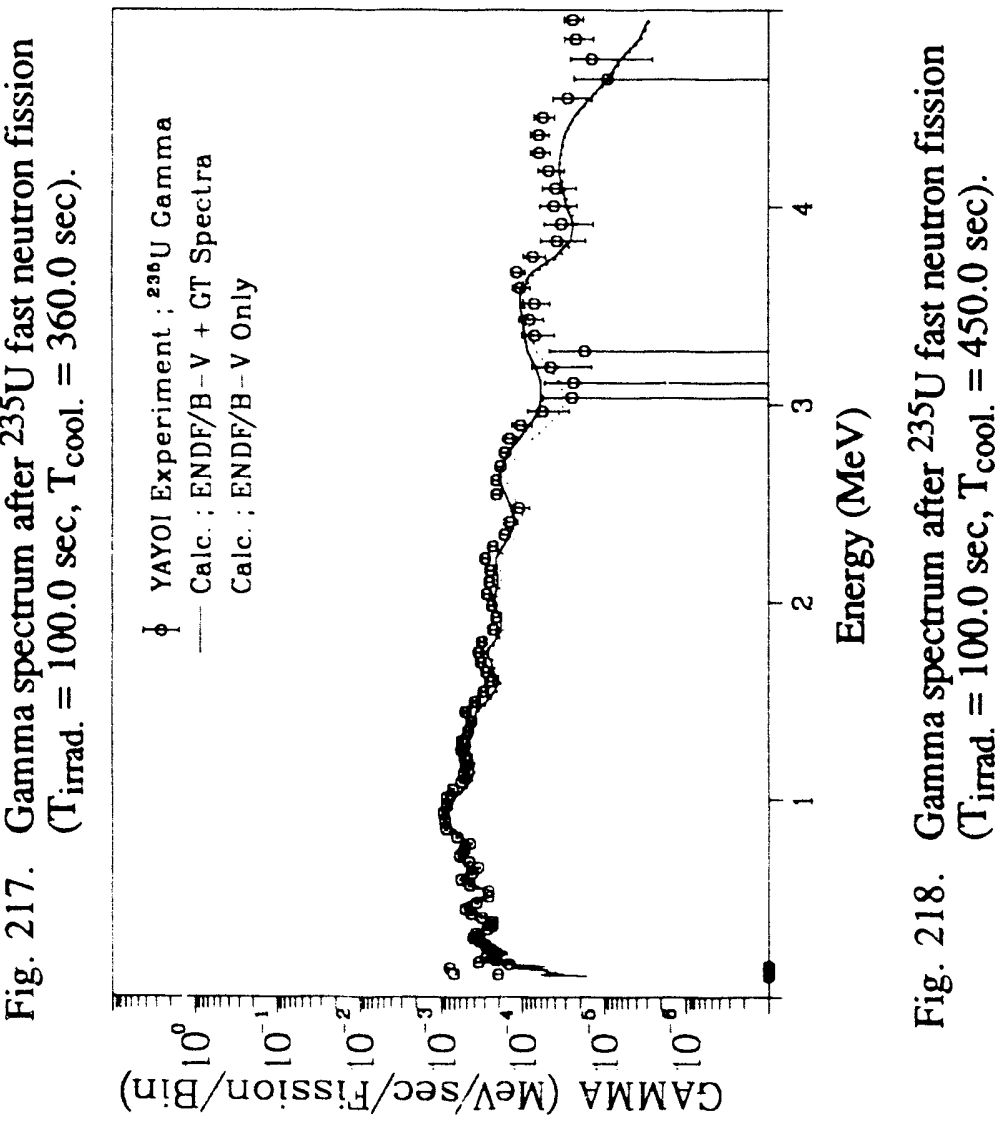



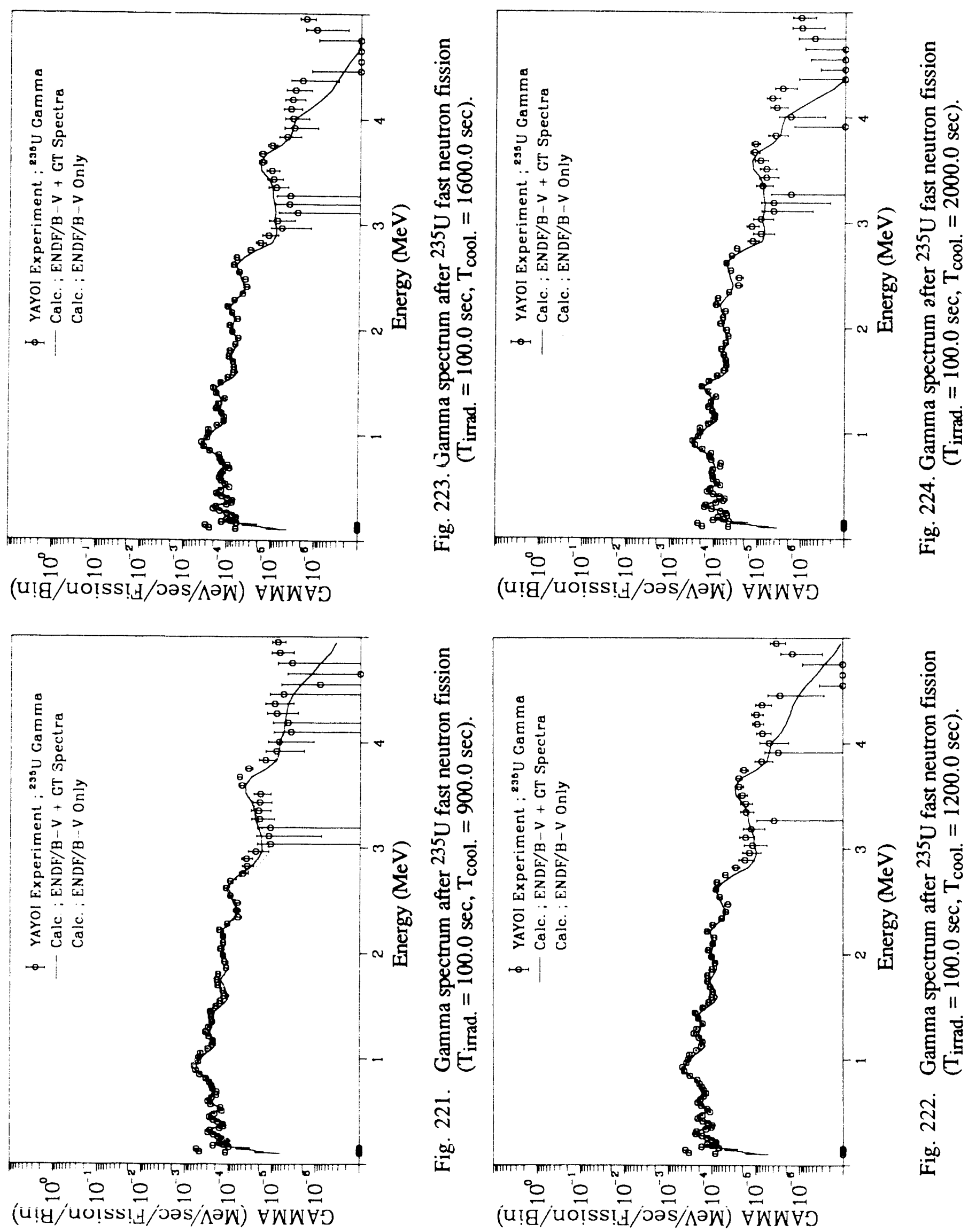


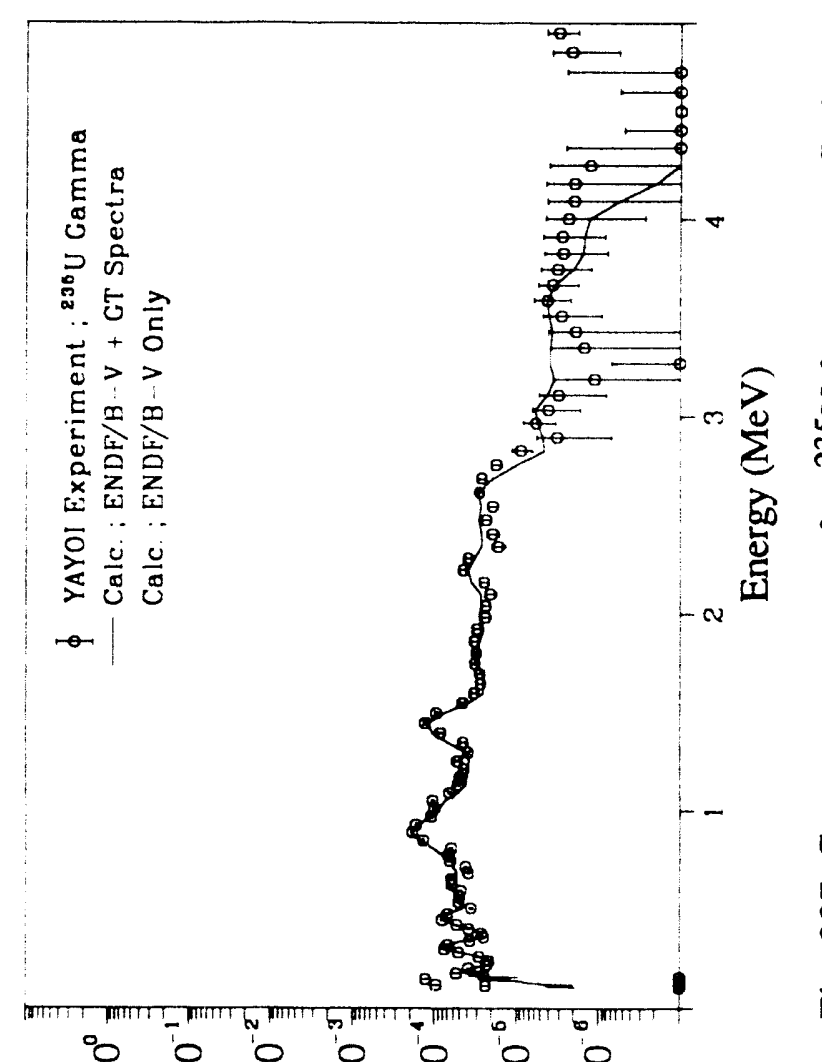

(u!̣/uo!̣ss!

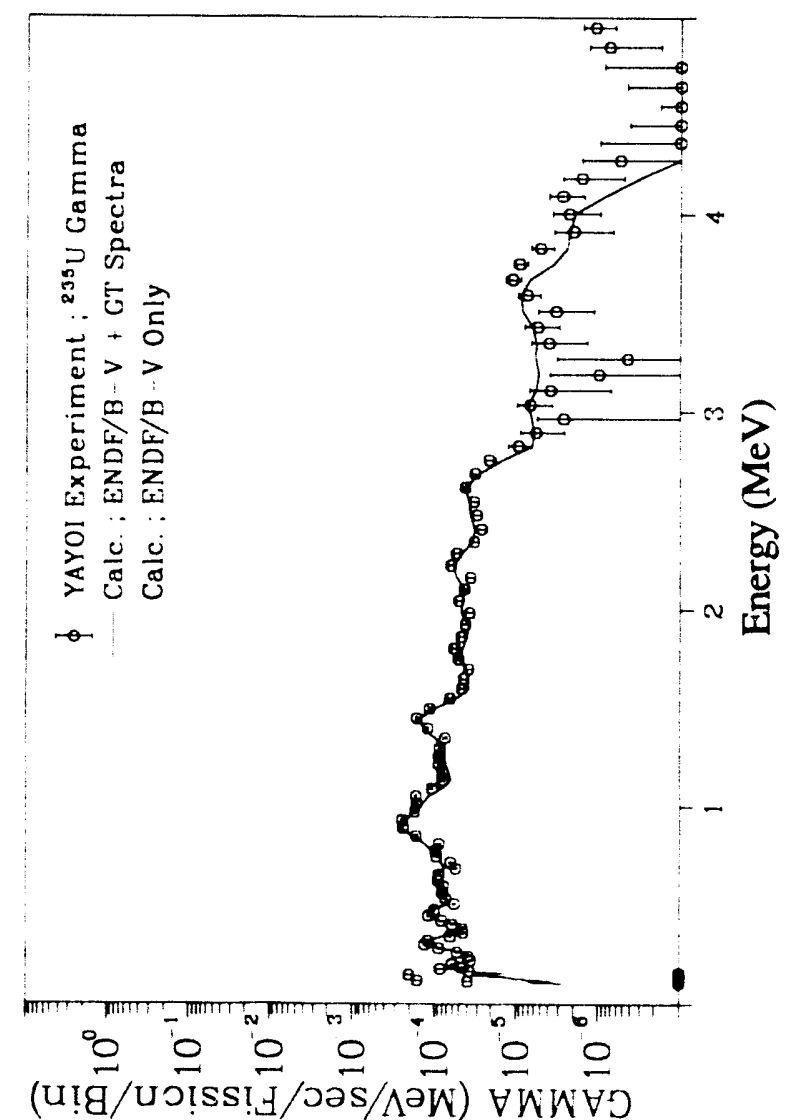

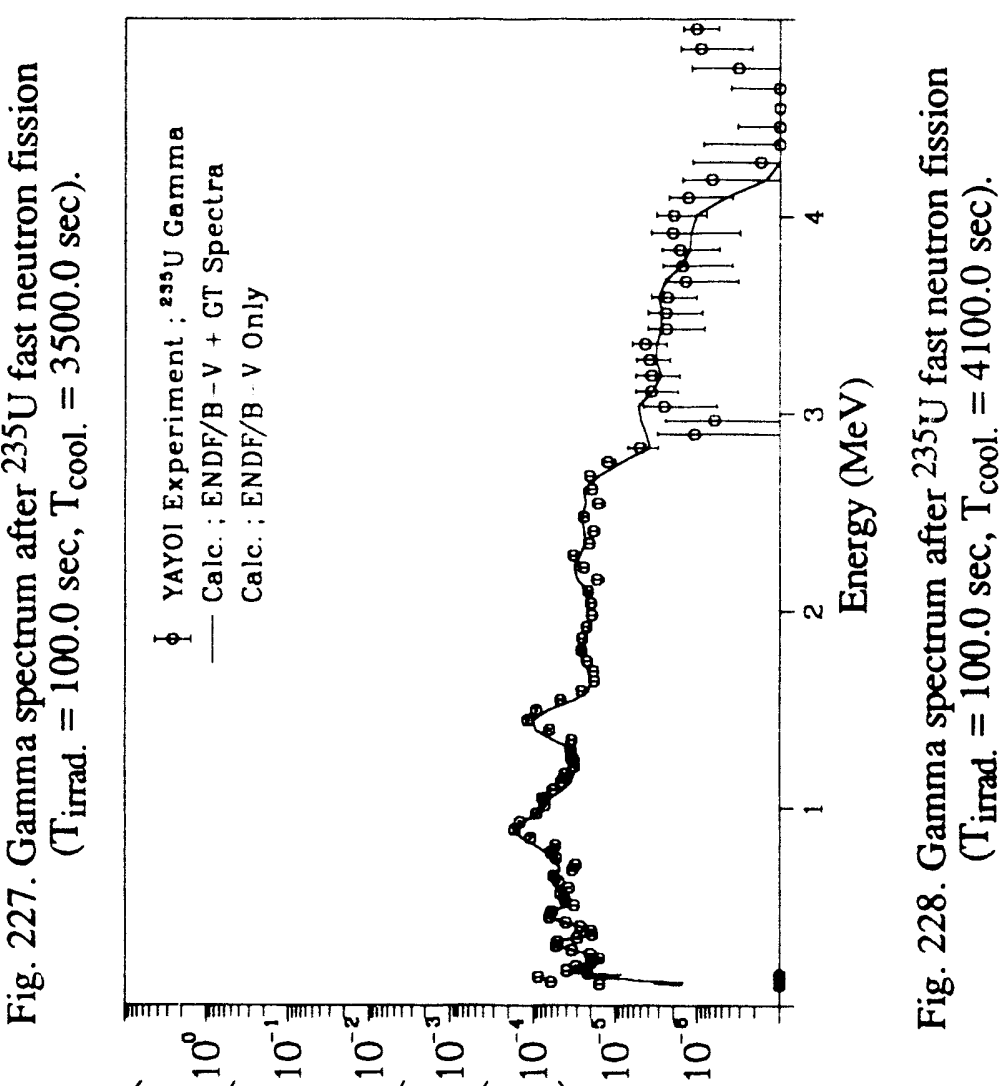

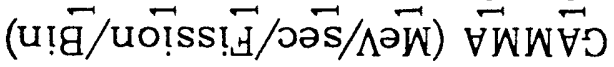

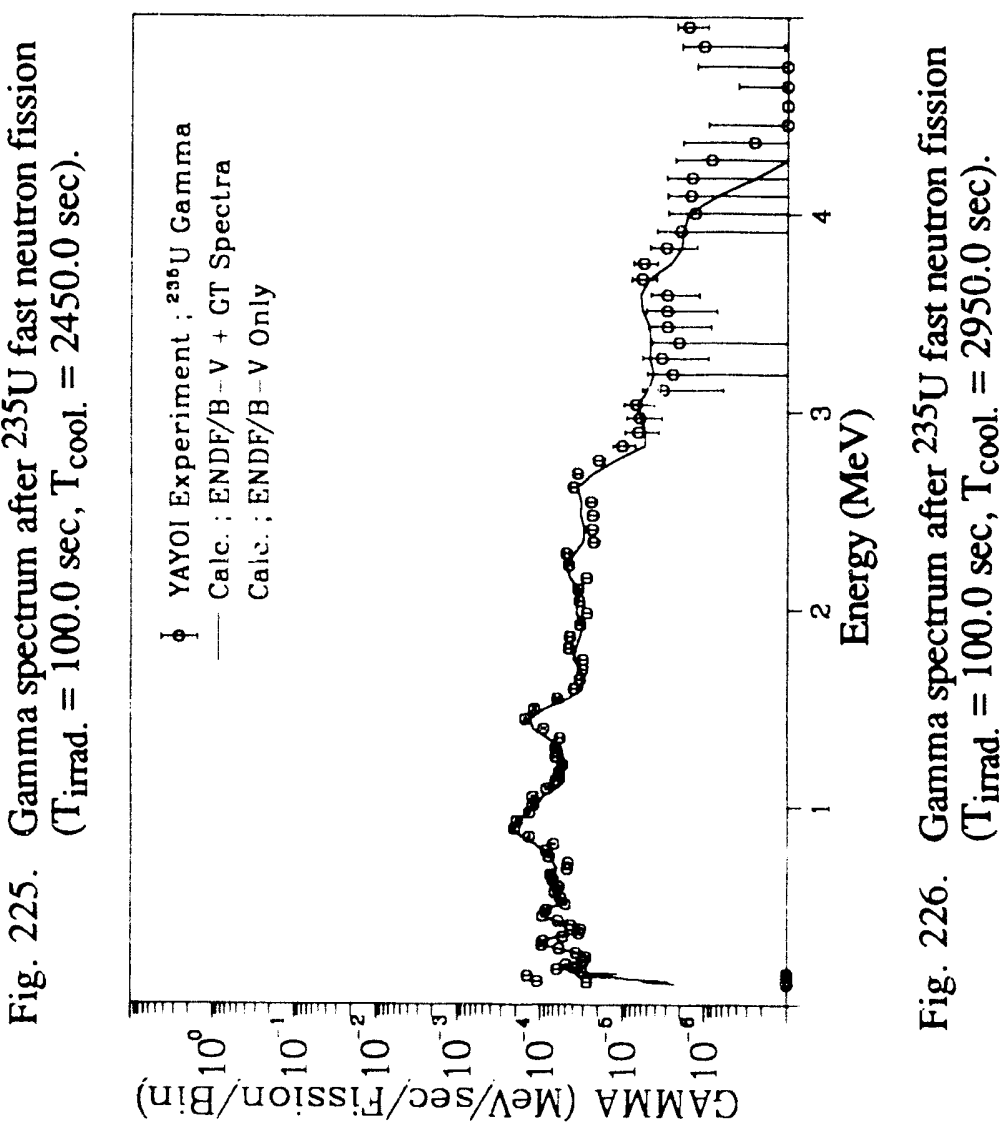



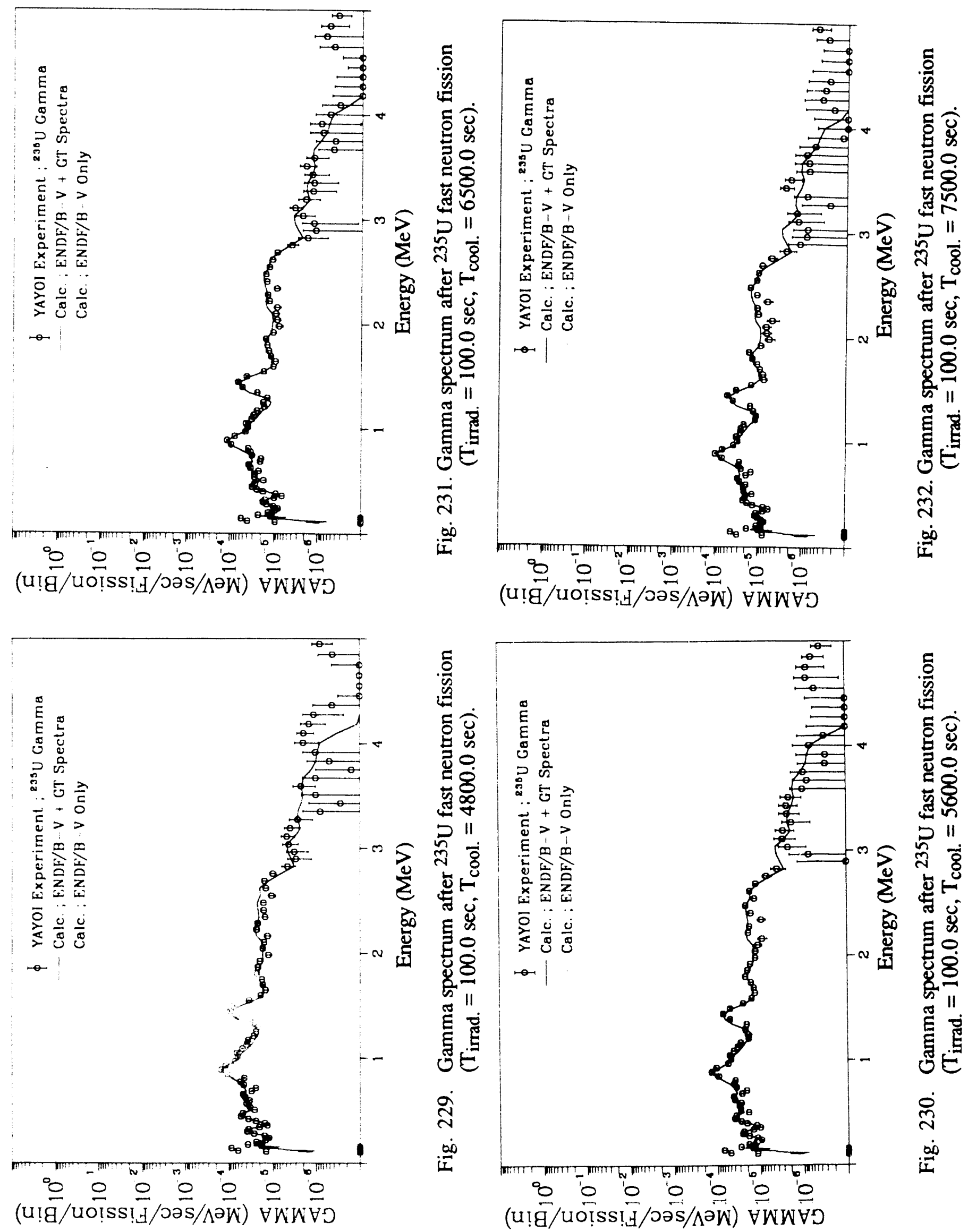

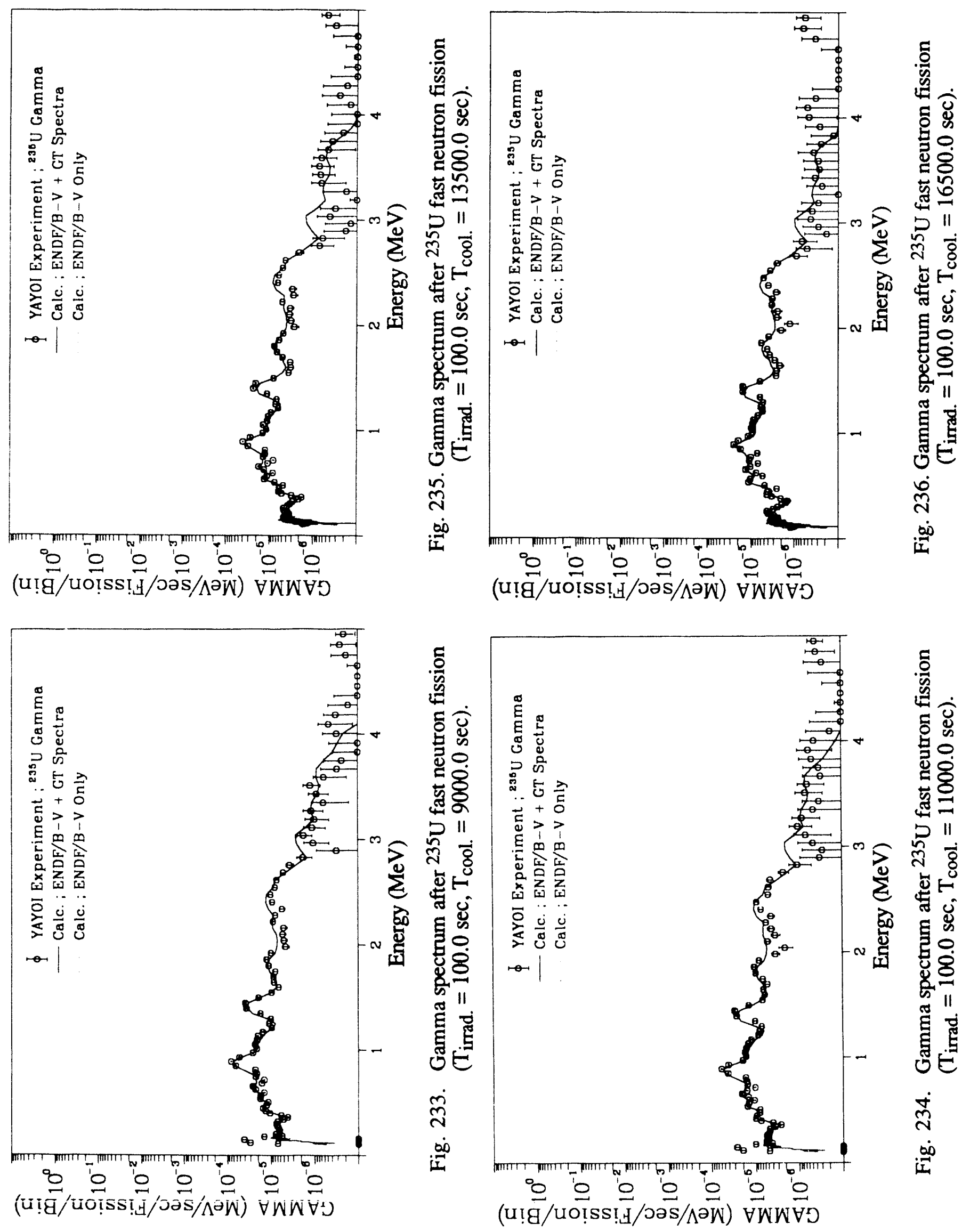


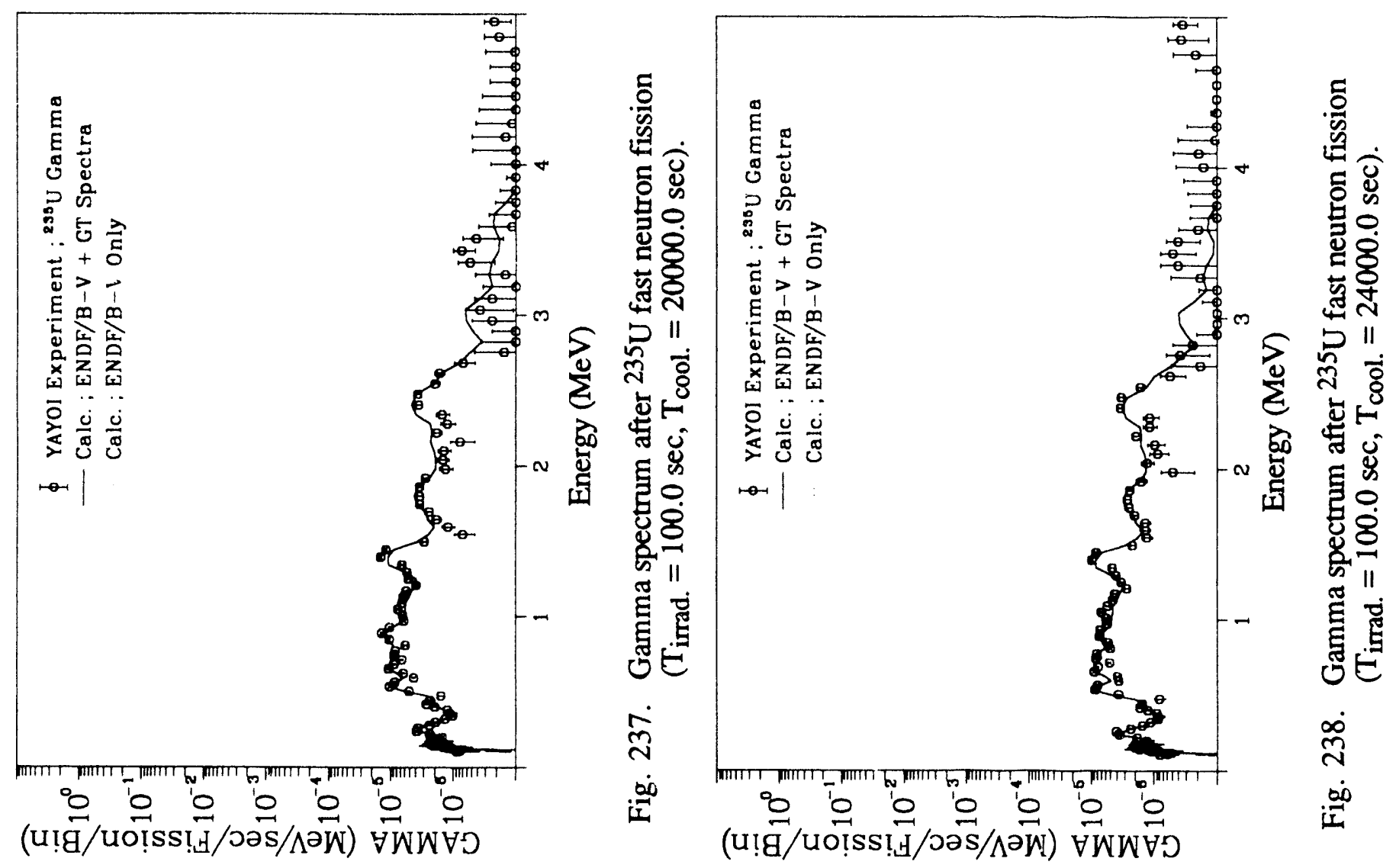




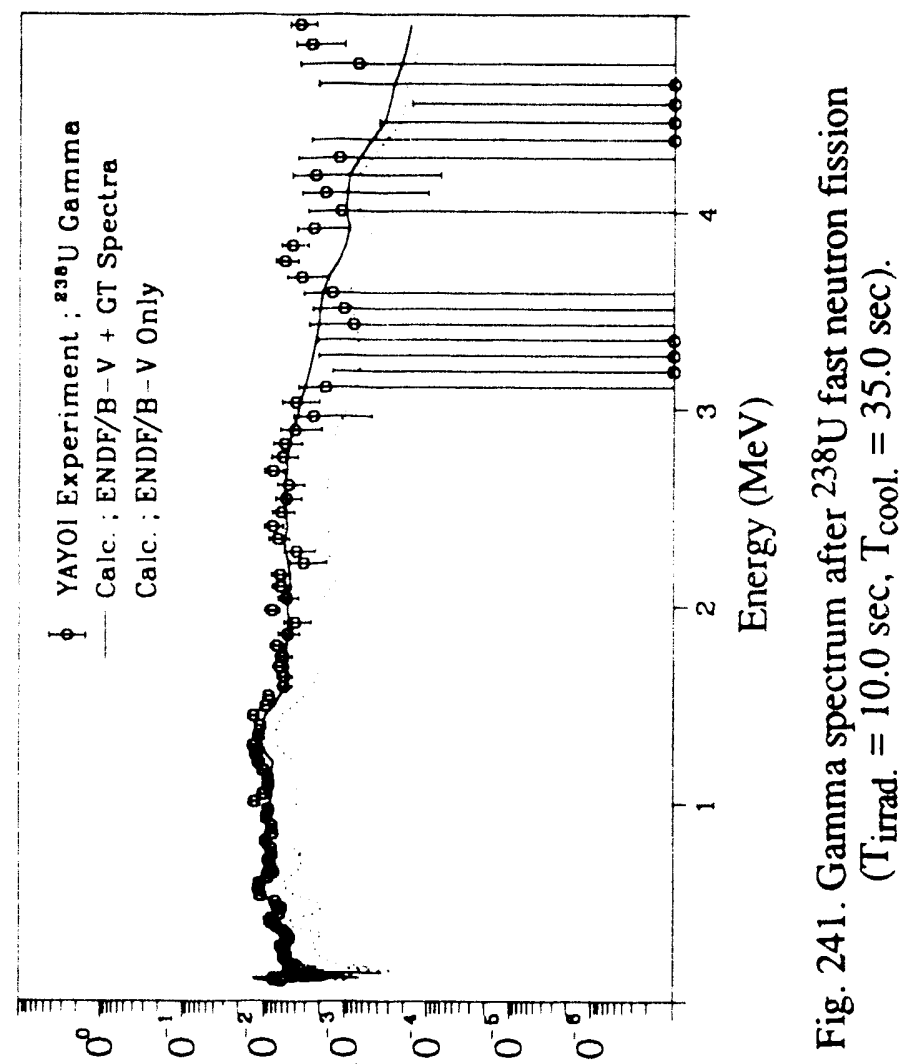

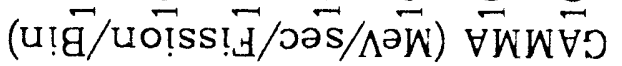
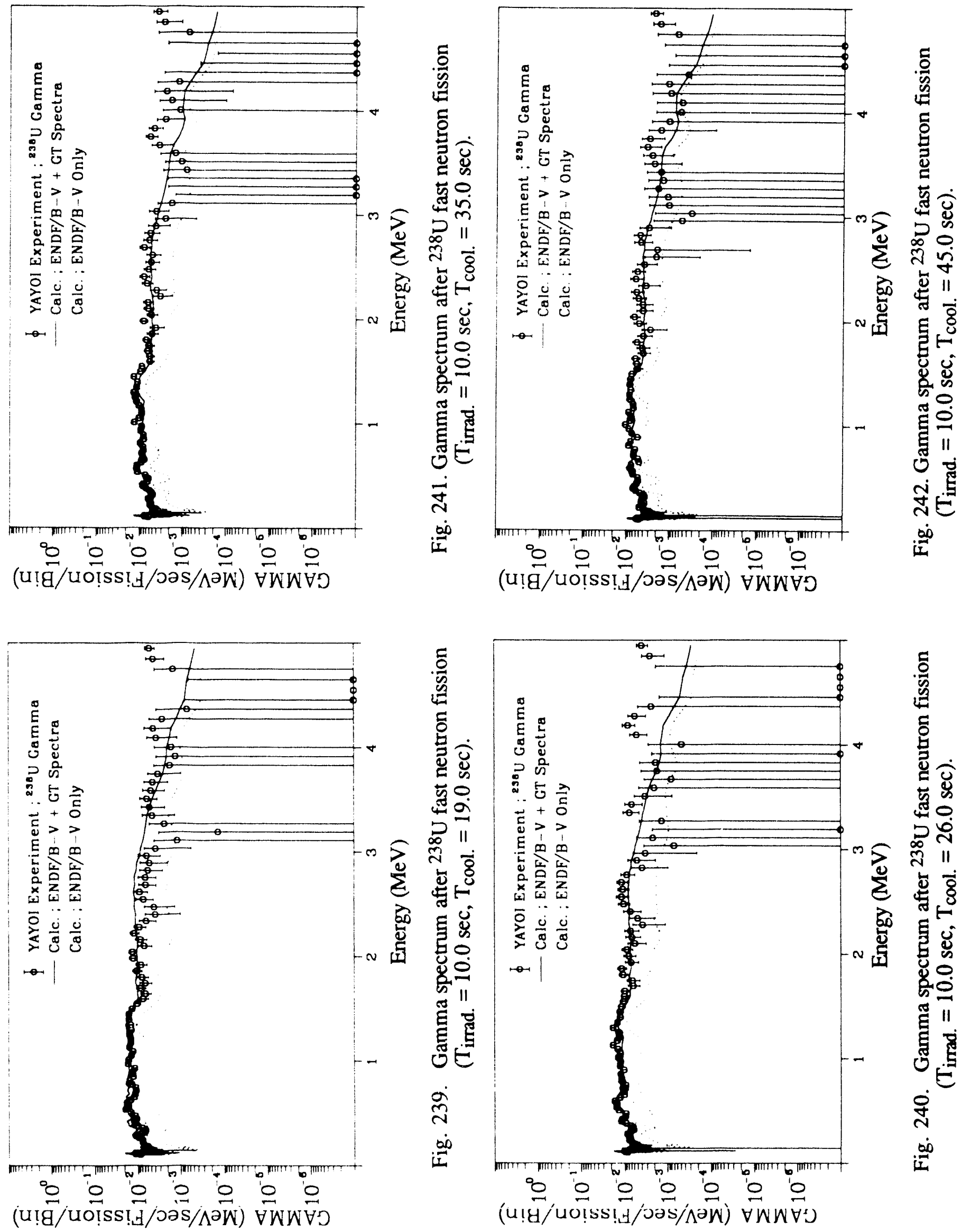

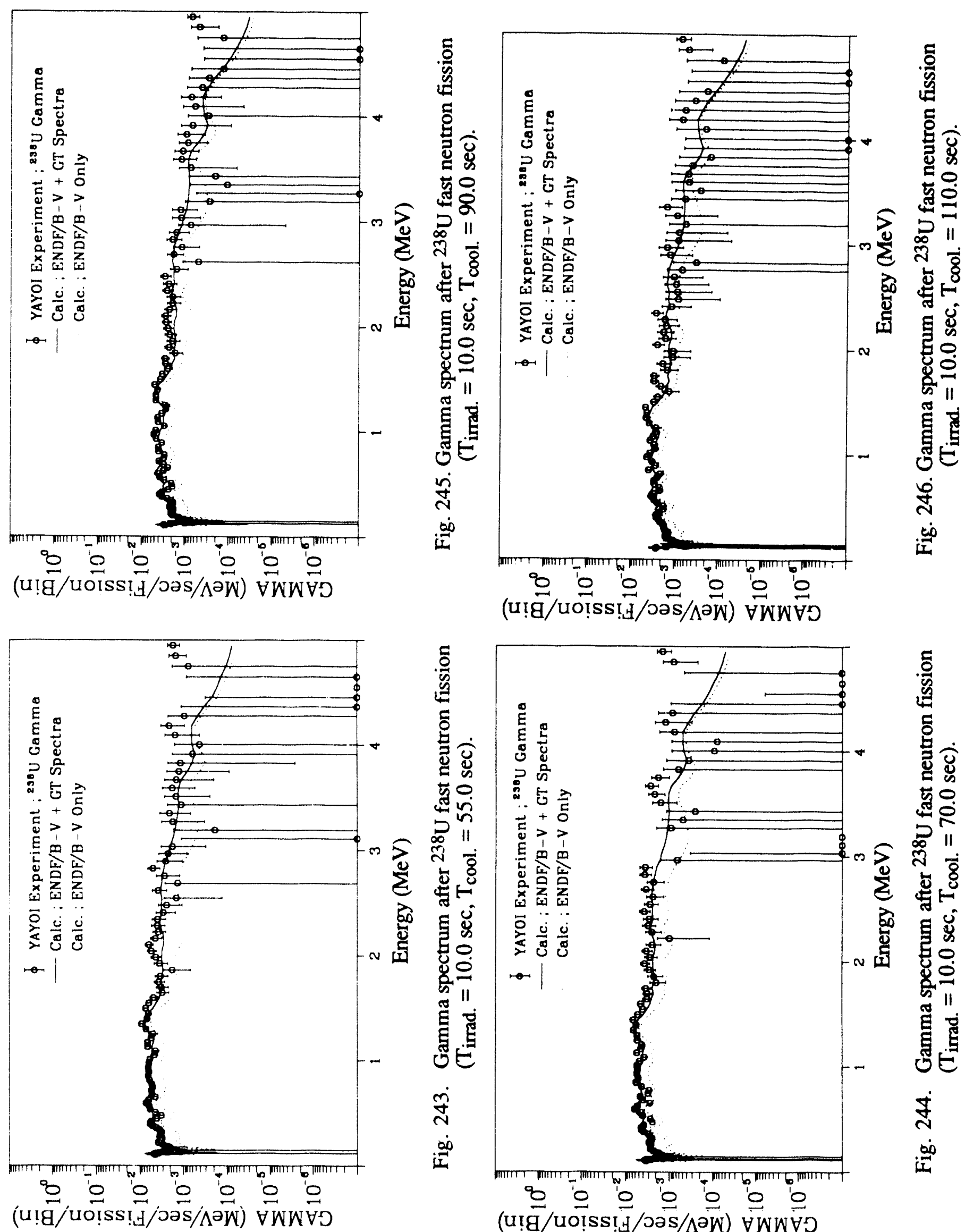

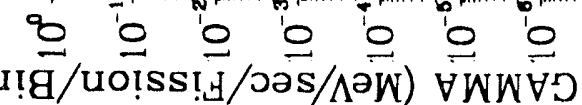




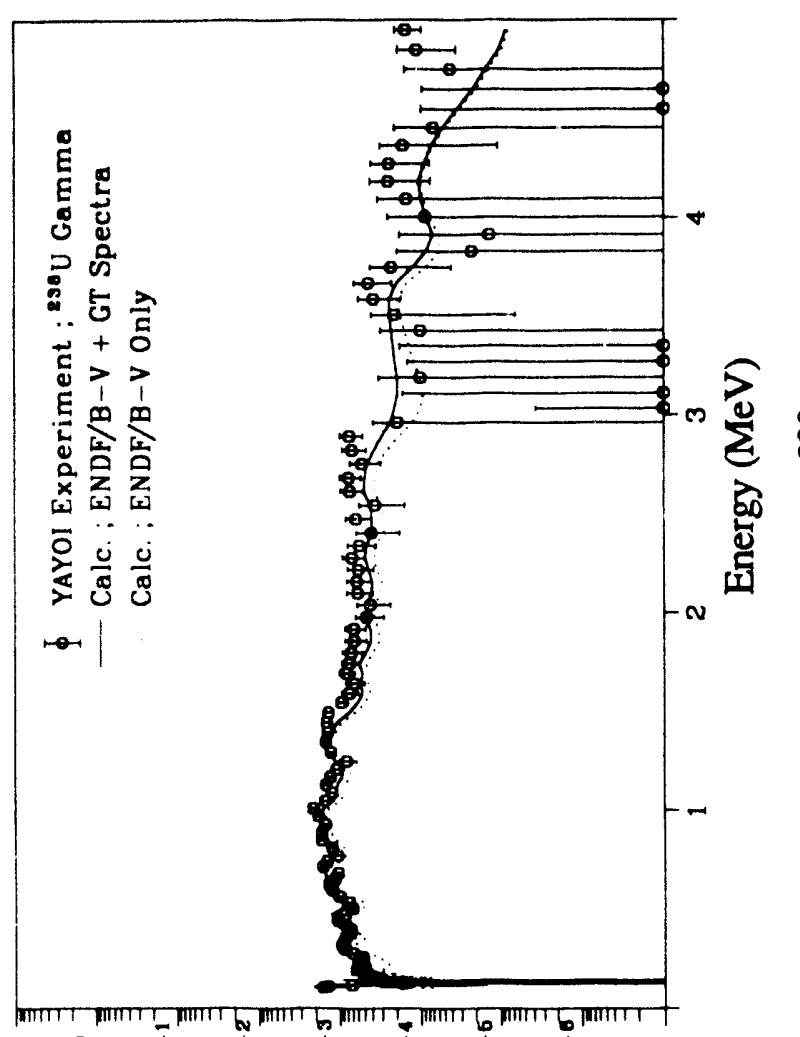

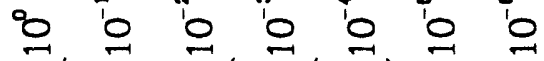

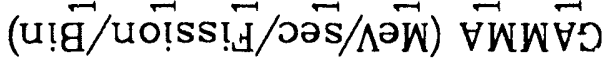

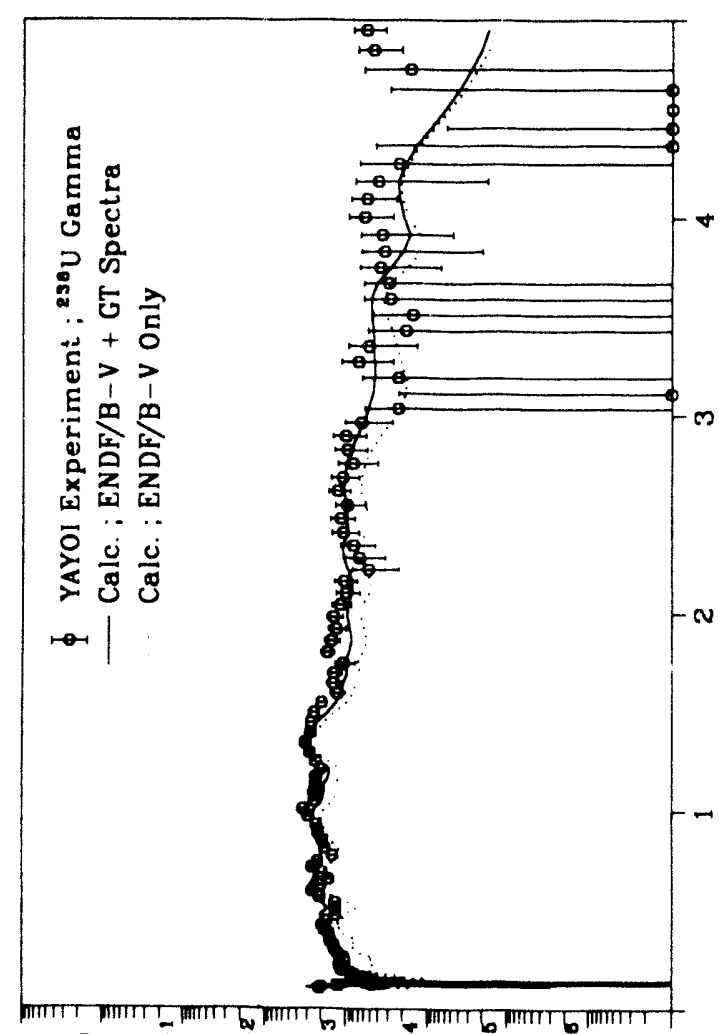

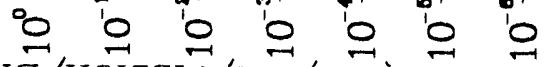

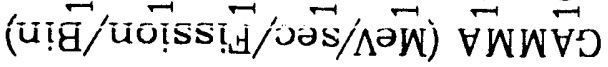

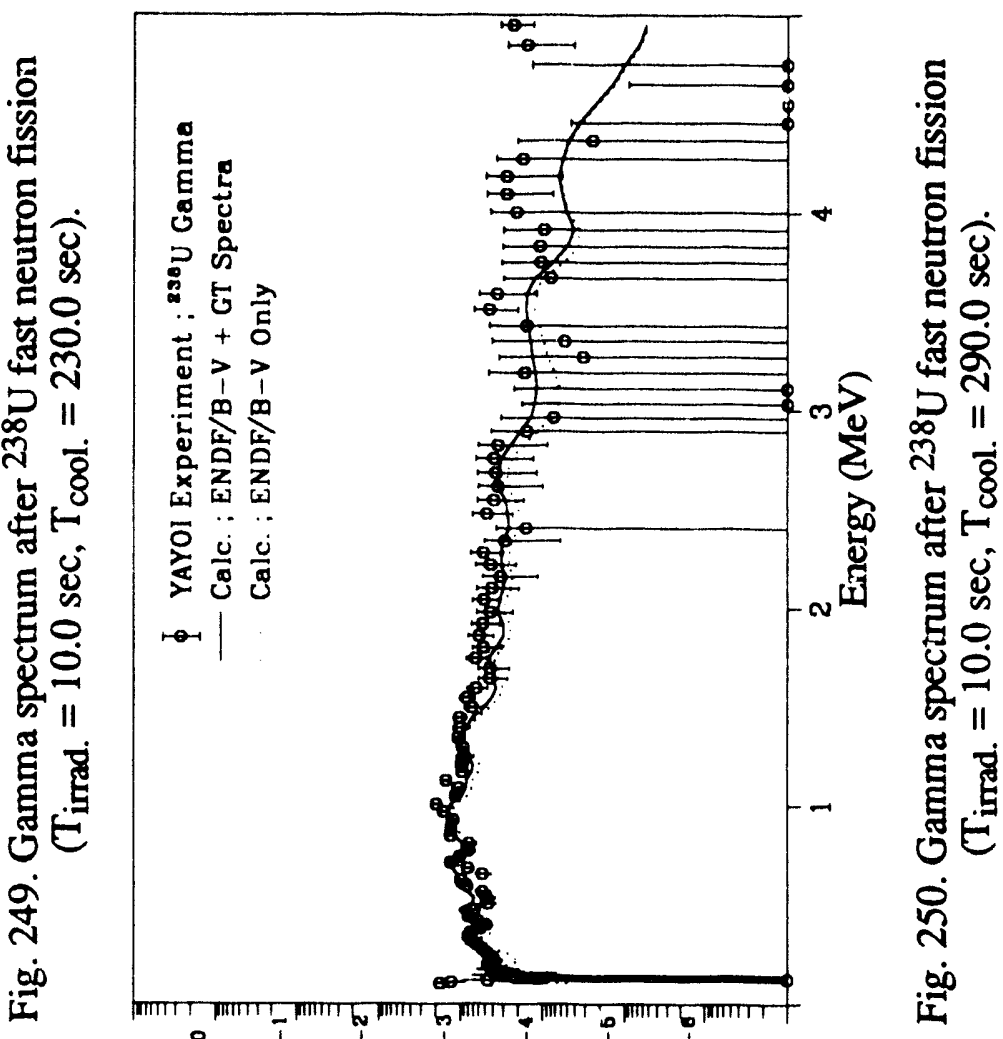

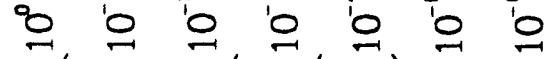

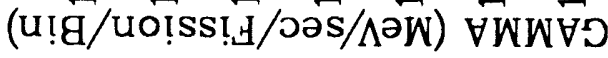
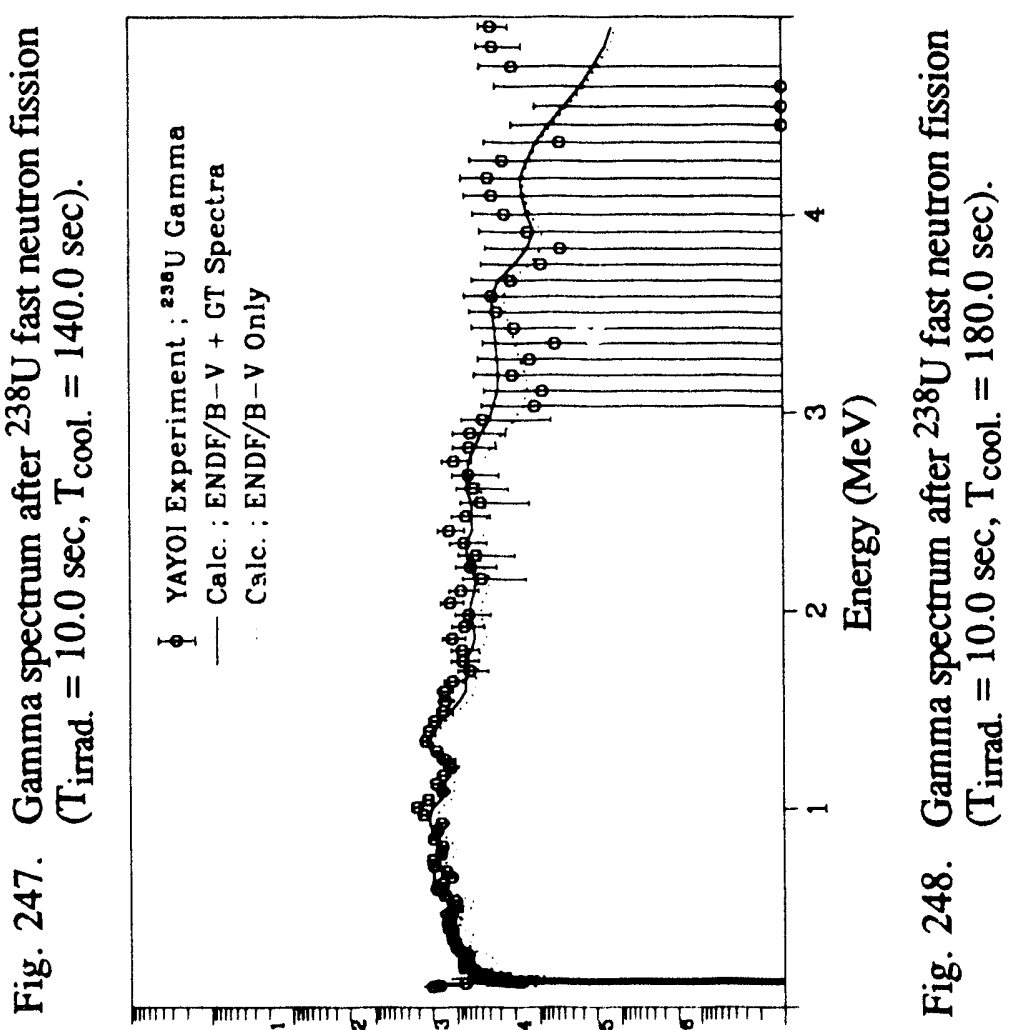

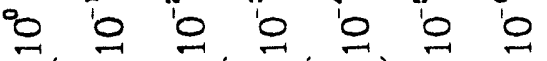

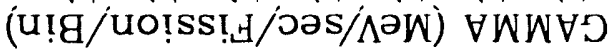



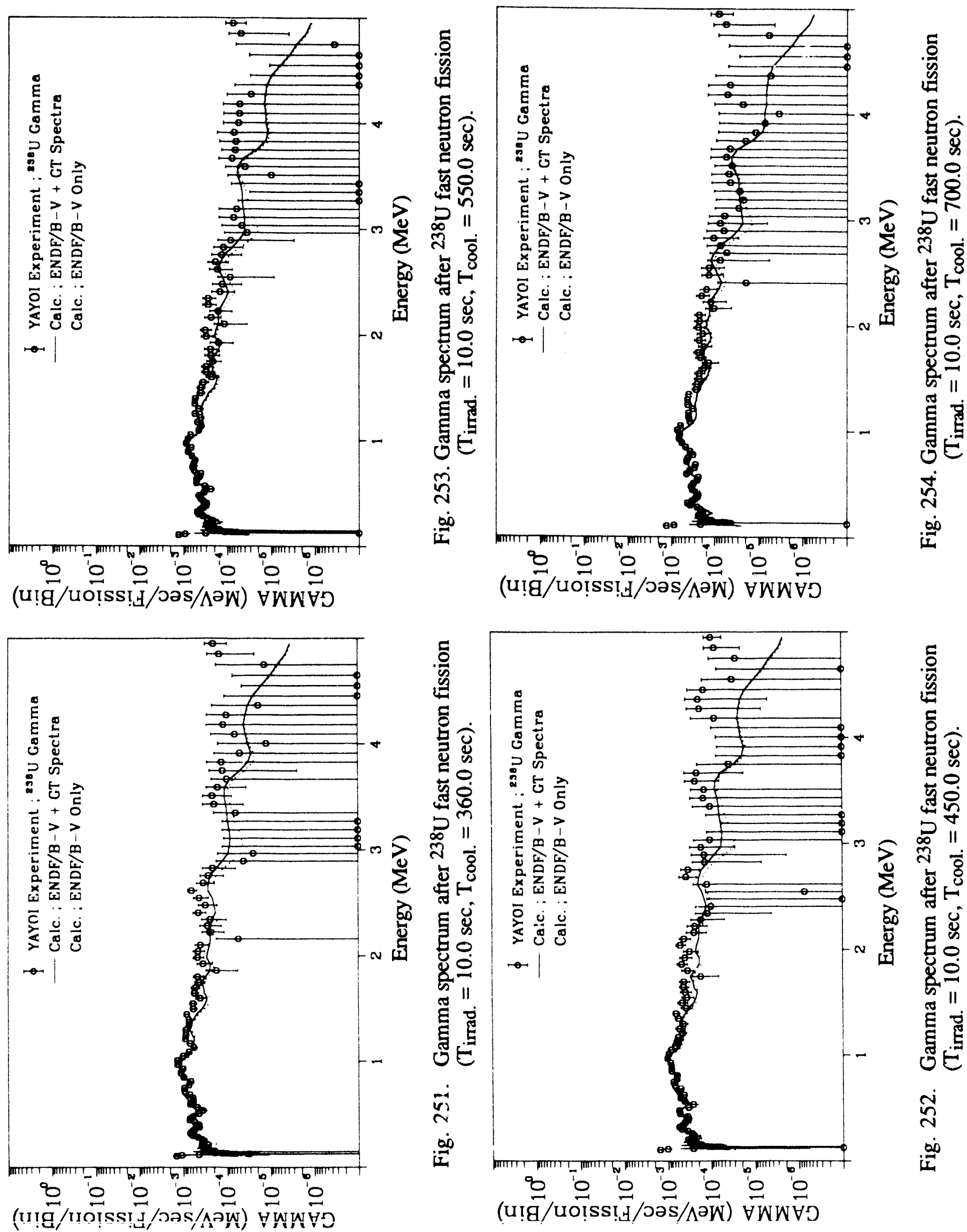

을 (u!g/uo!ss!ิ 


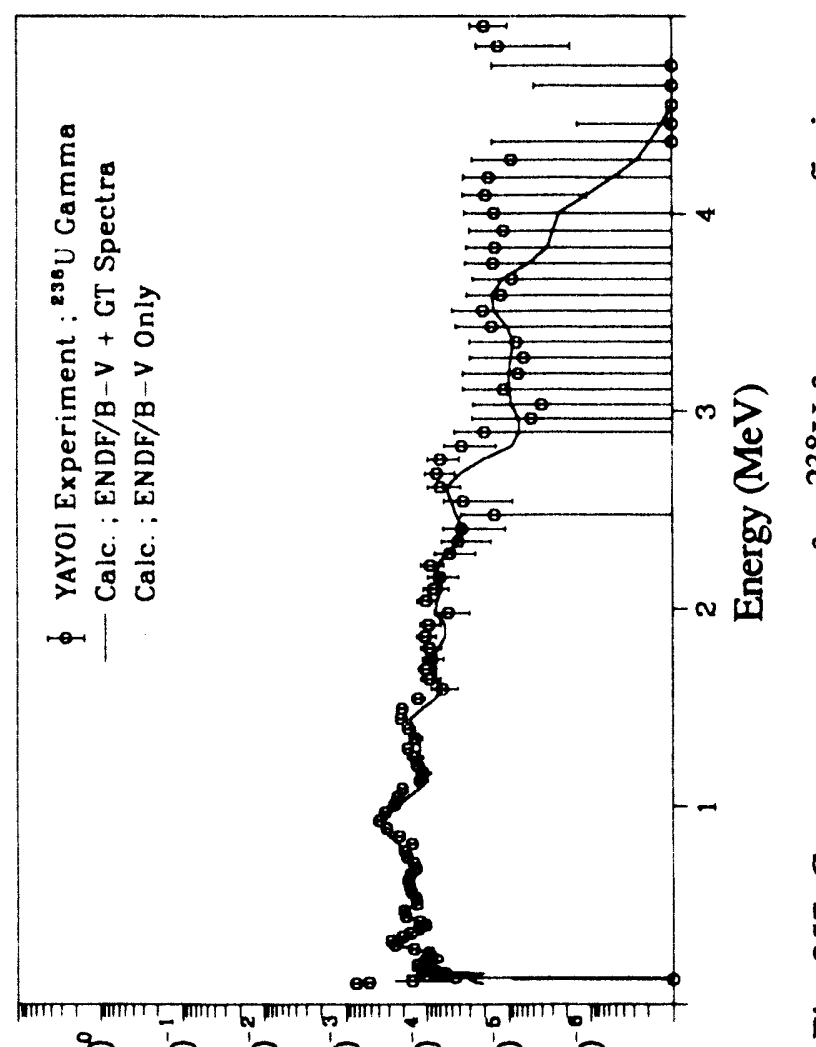

으 으 으 응으은 (u!̣/uoịss!

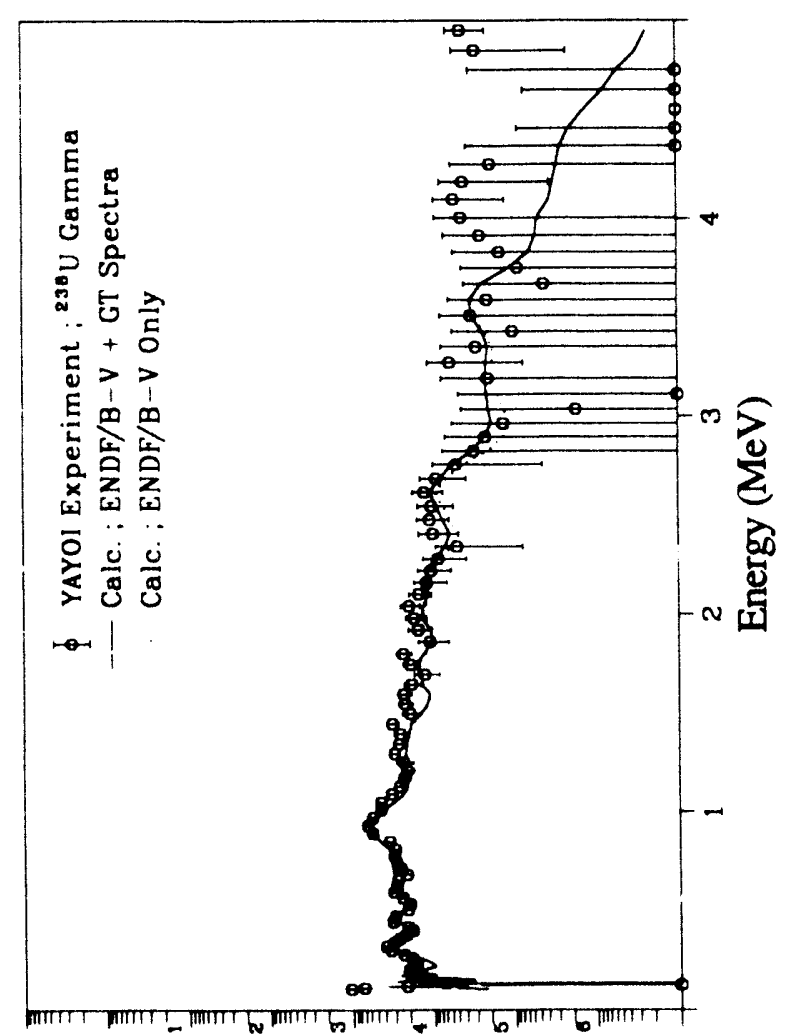

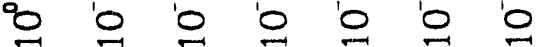

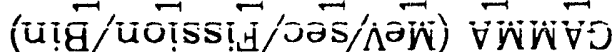

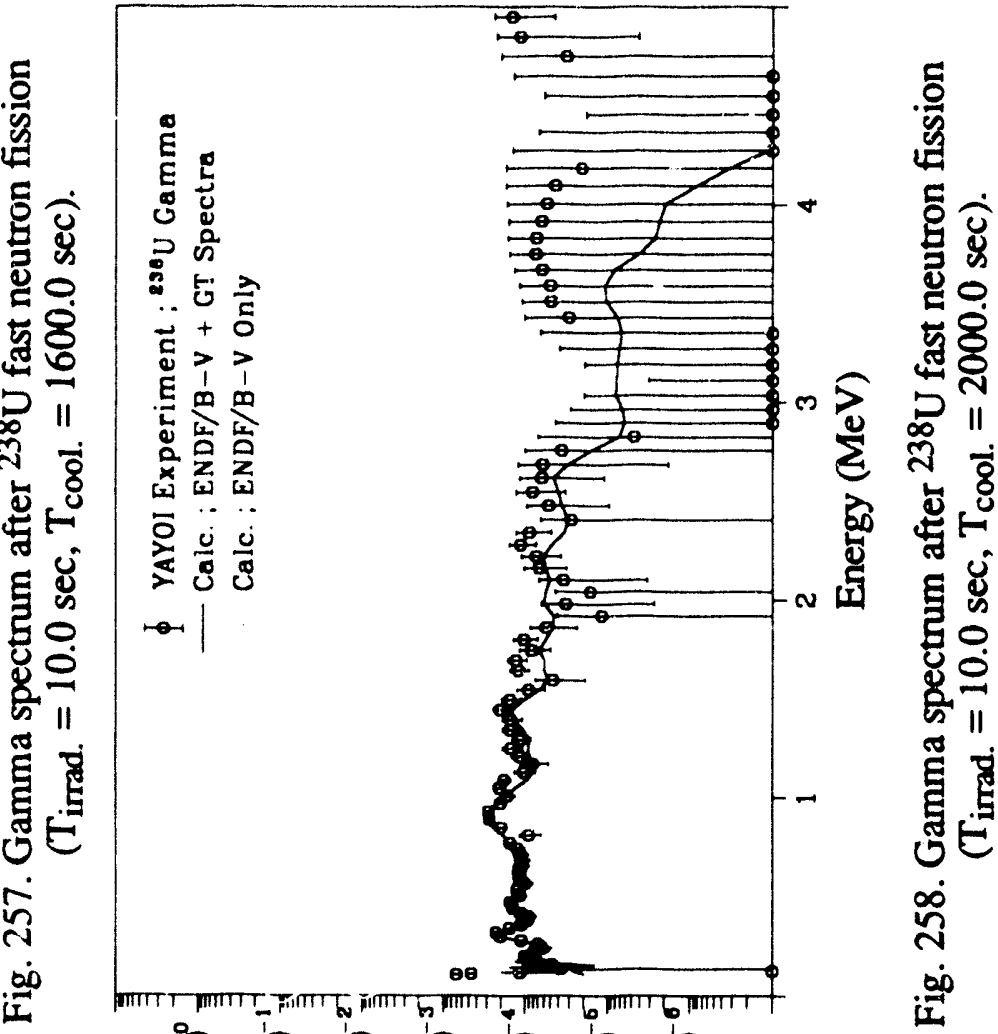

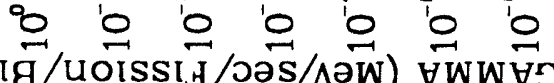
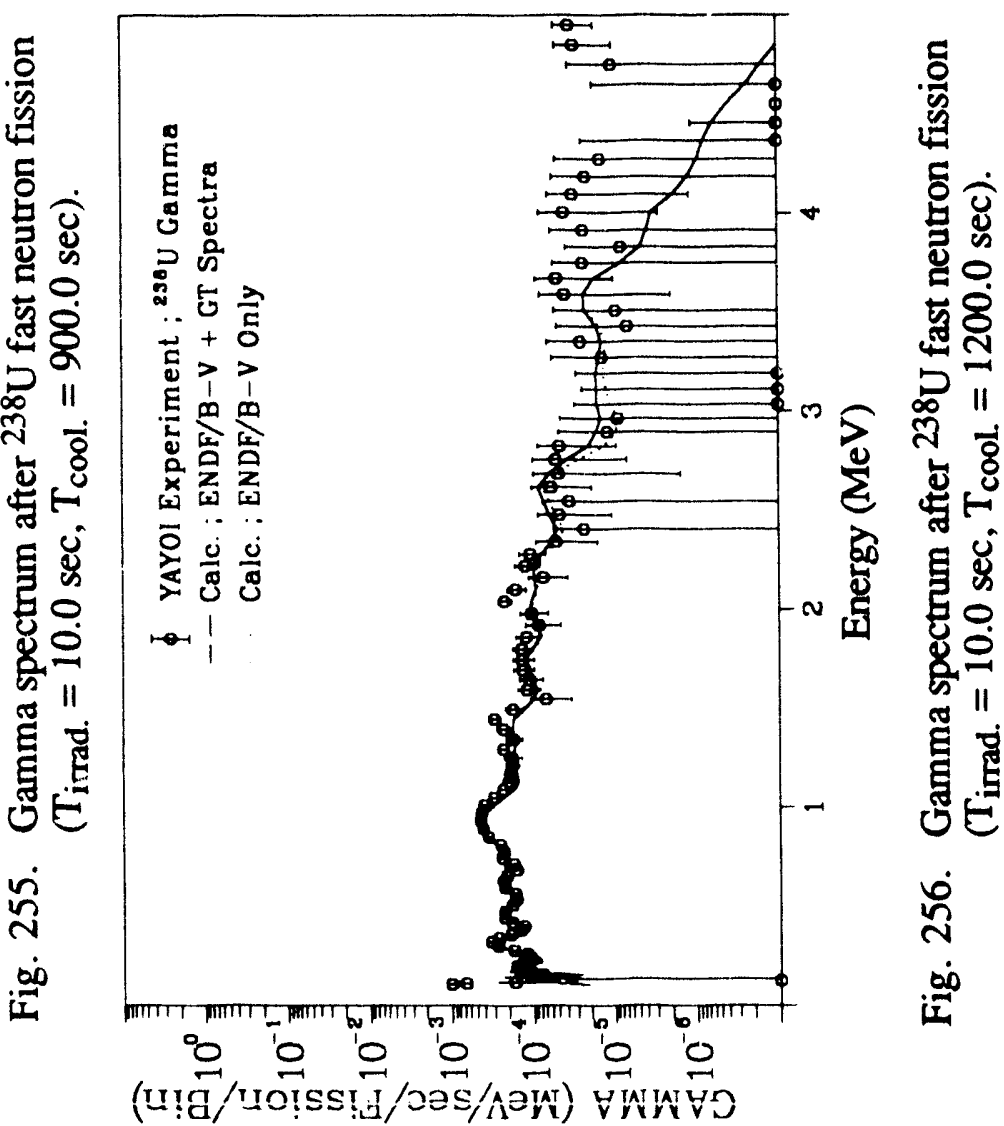


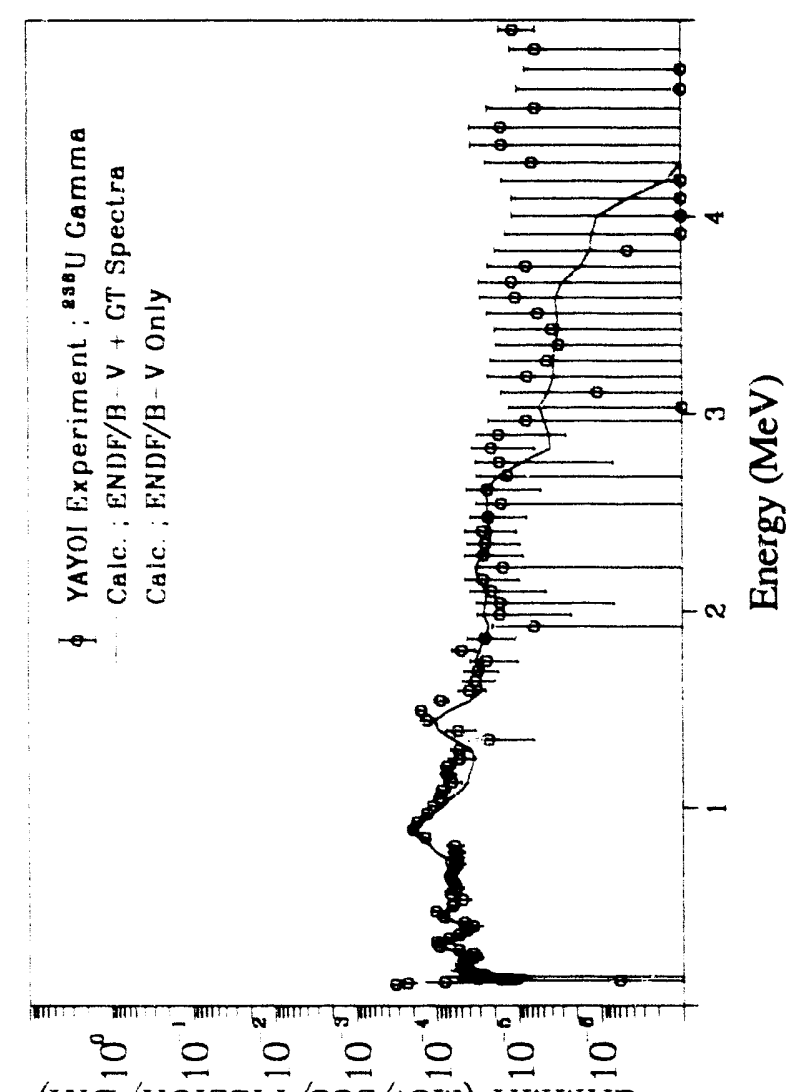

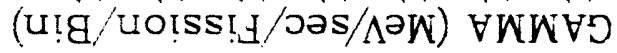

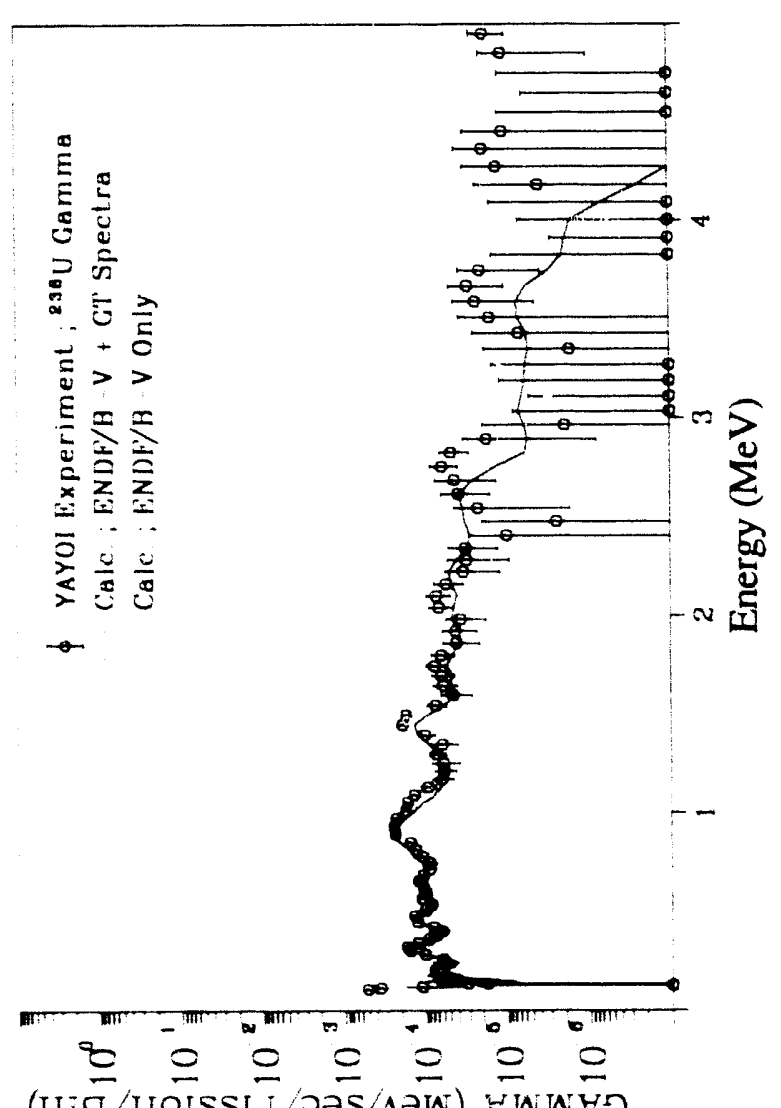

(uig/uotss!

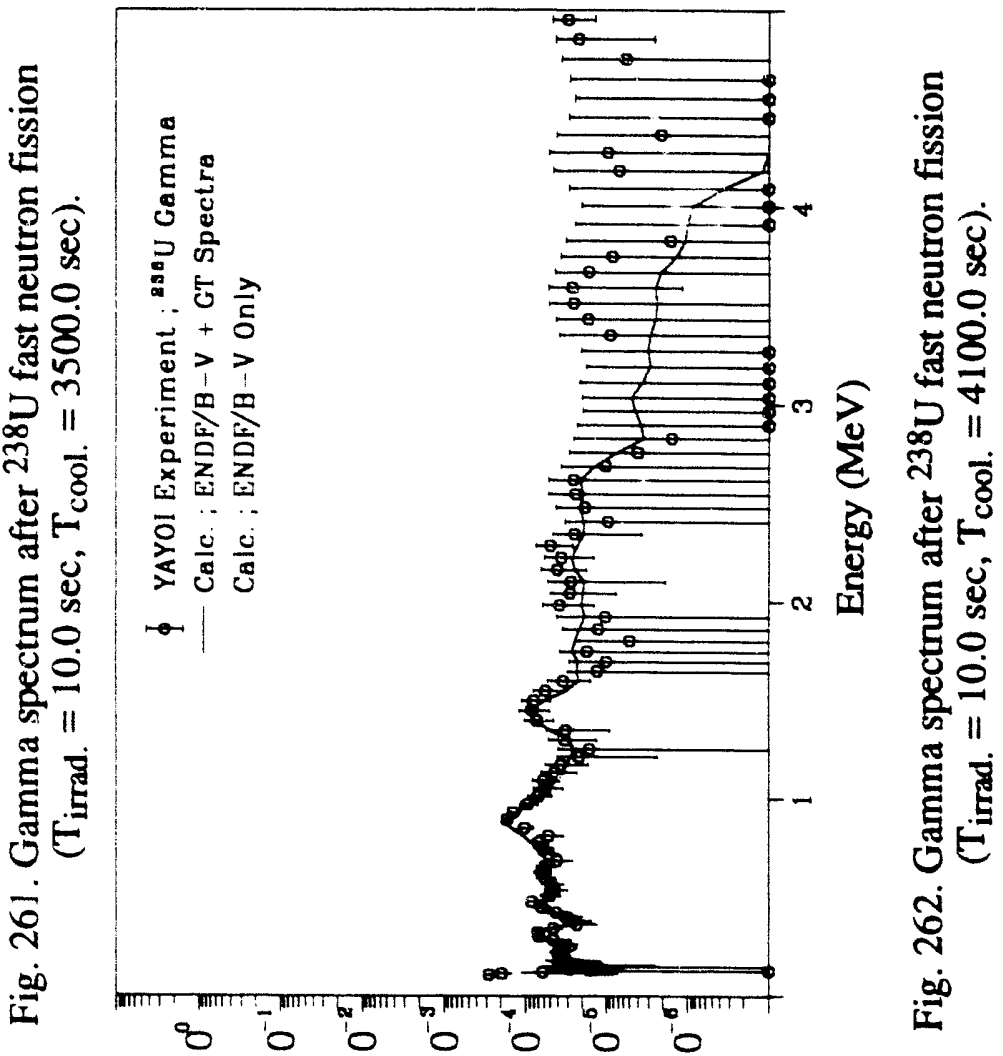

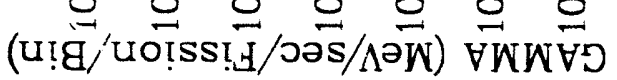

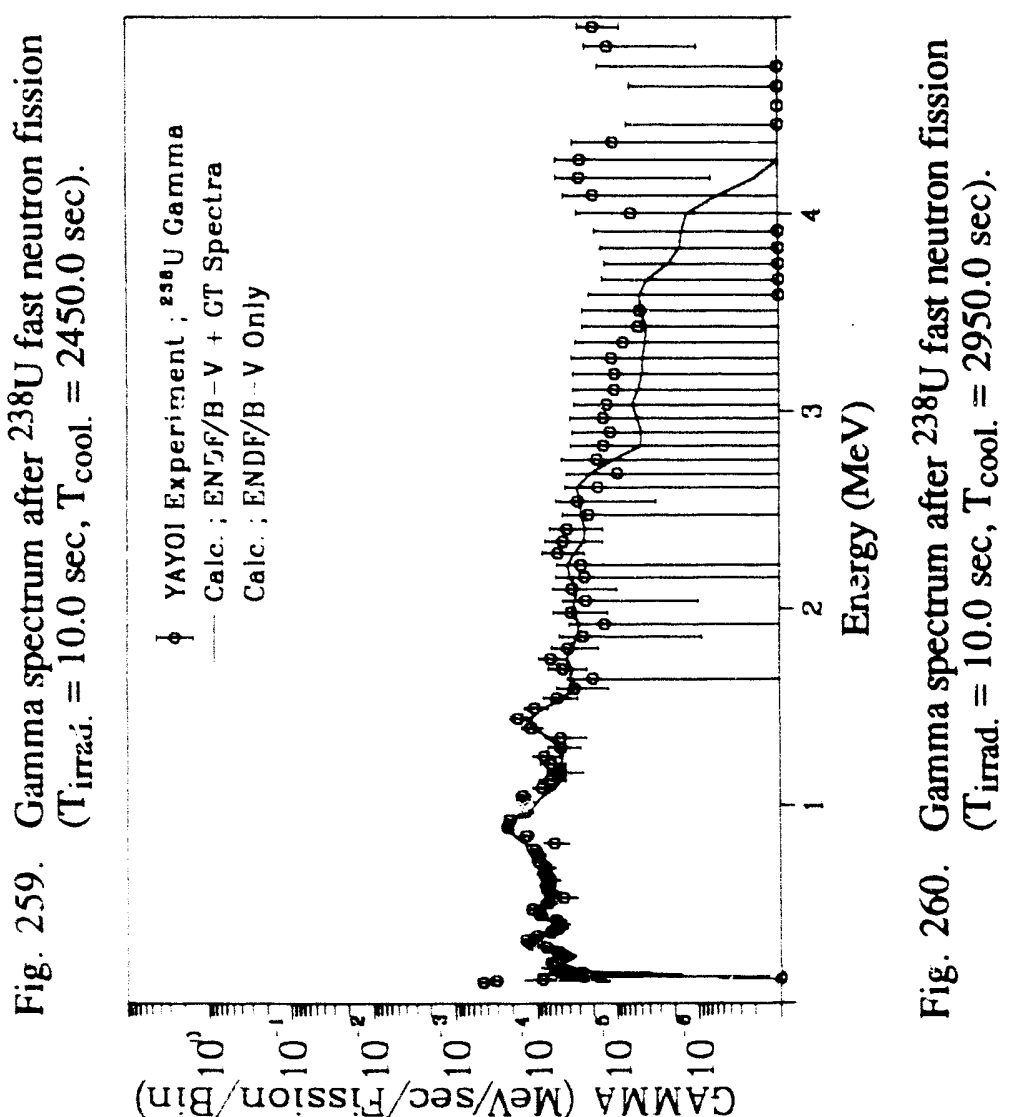



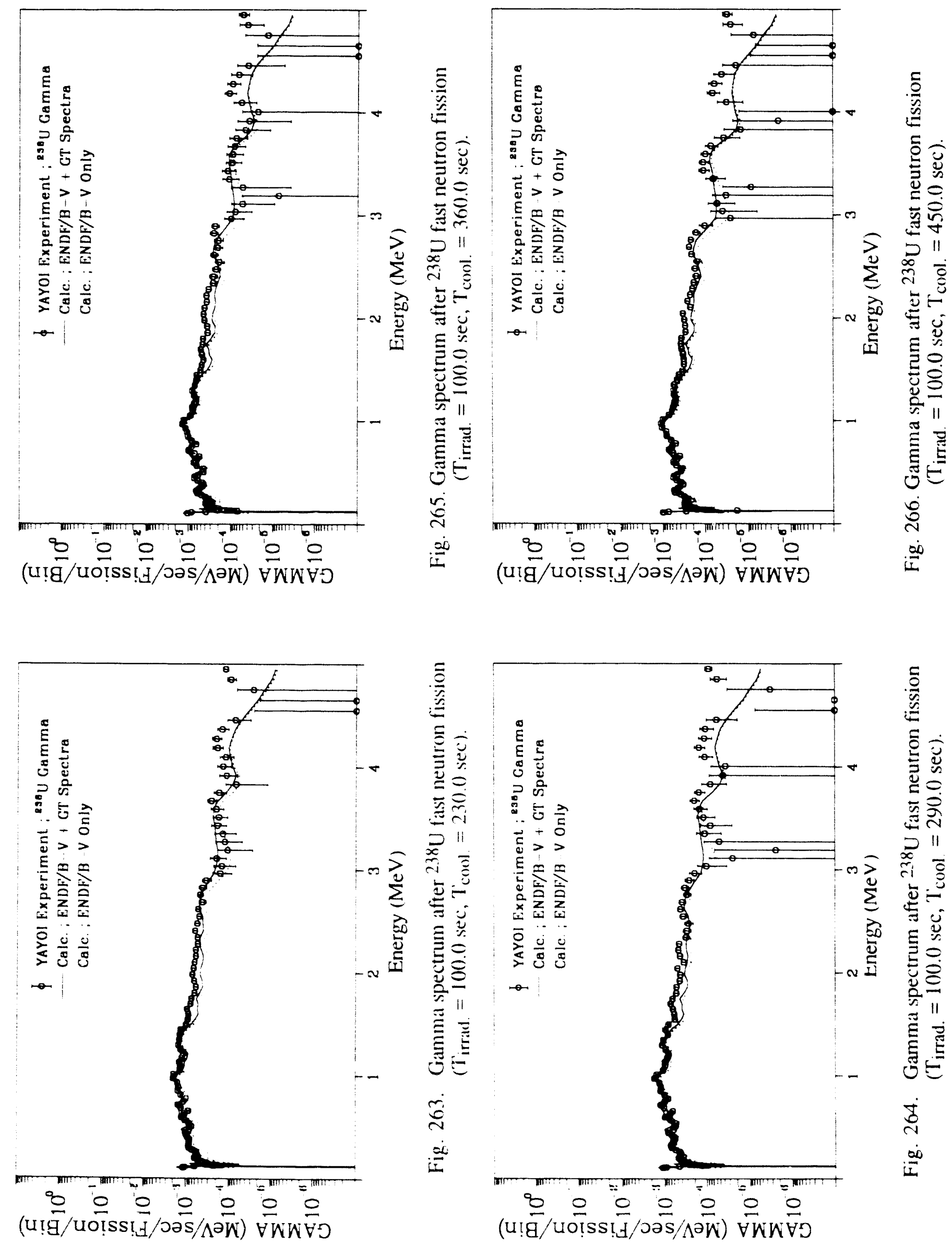

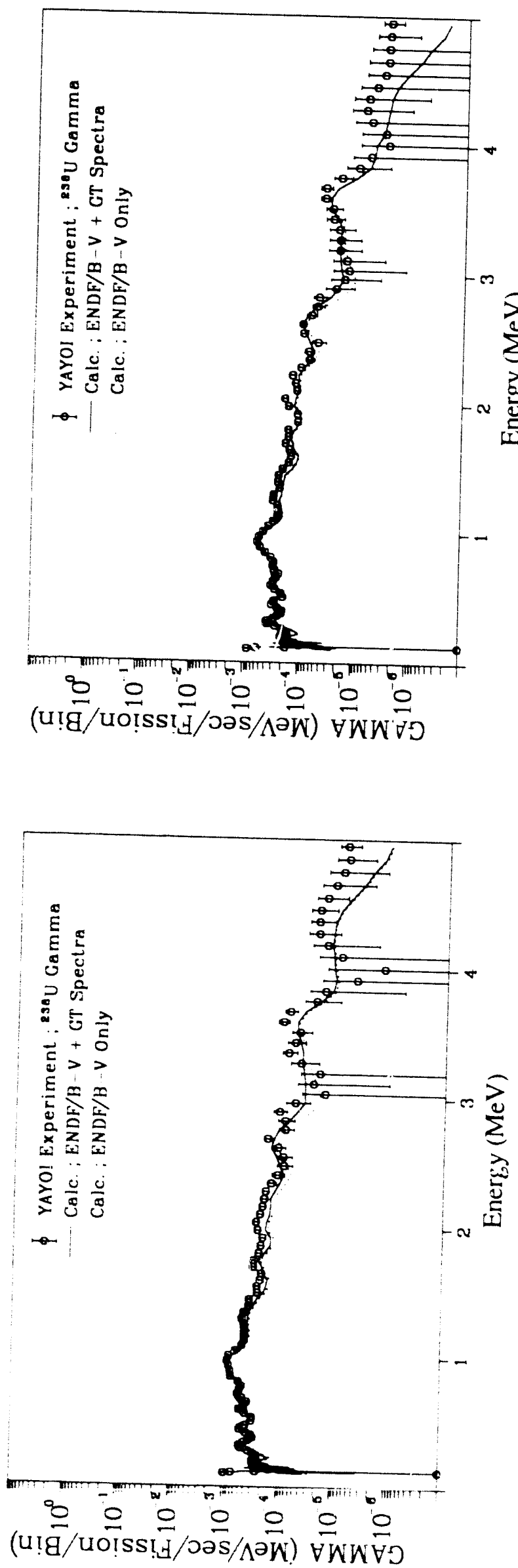
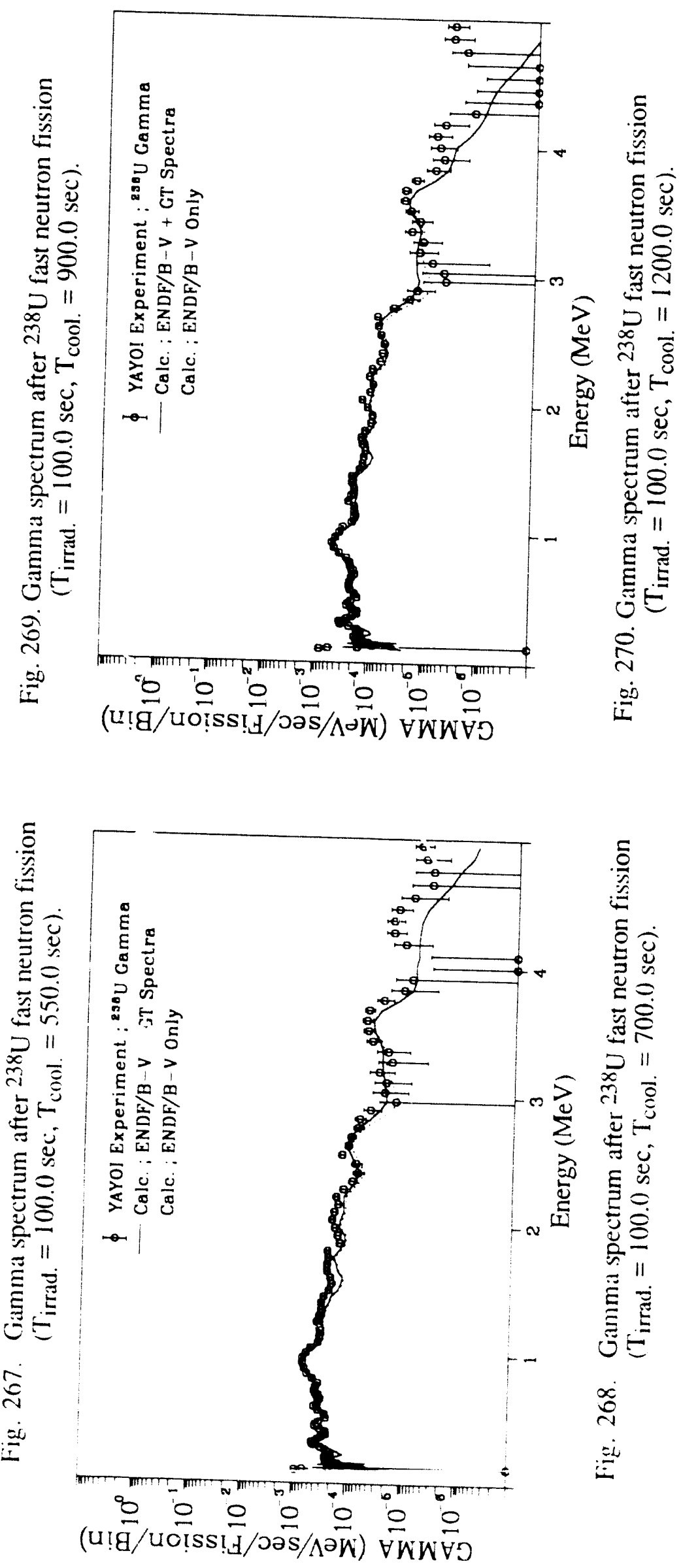


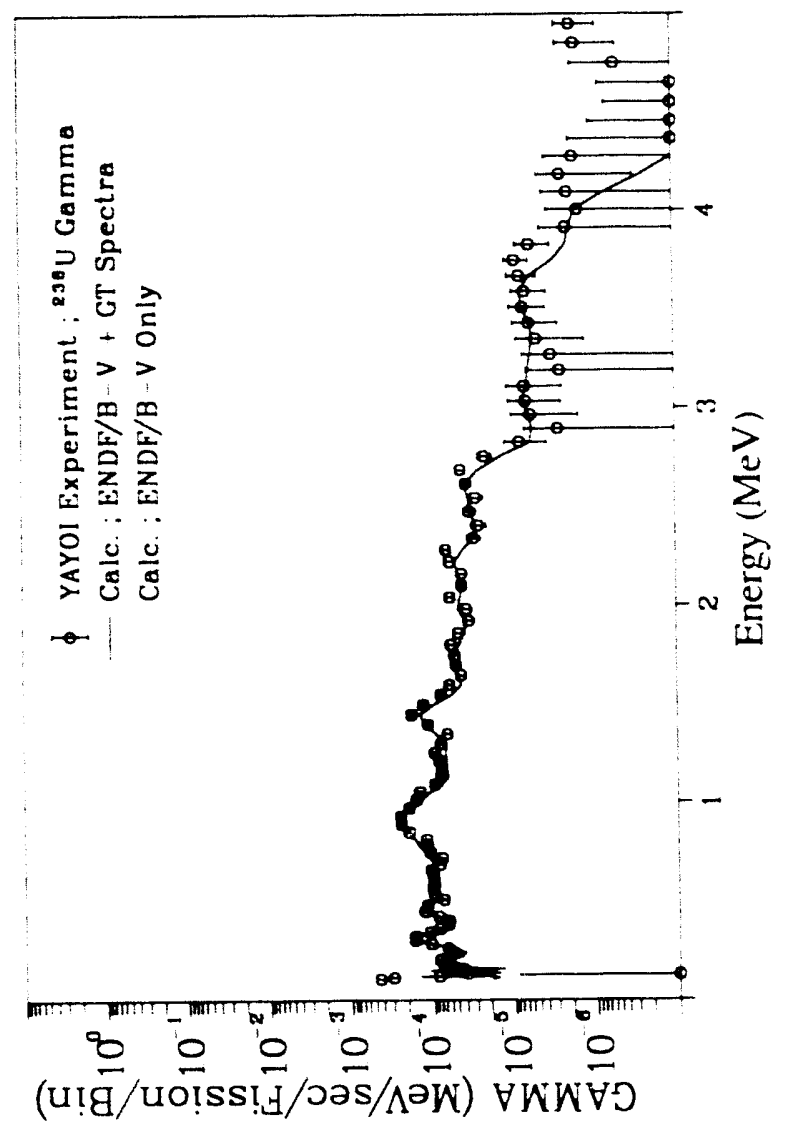

(u!g/uo!̣s!
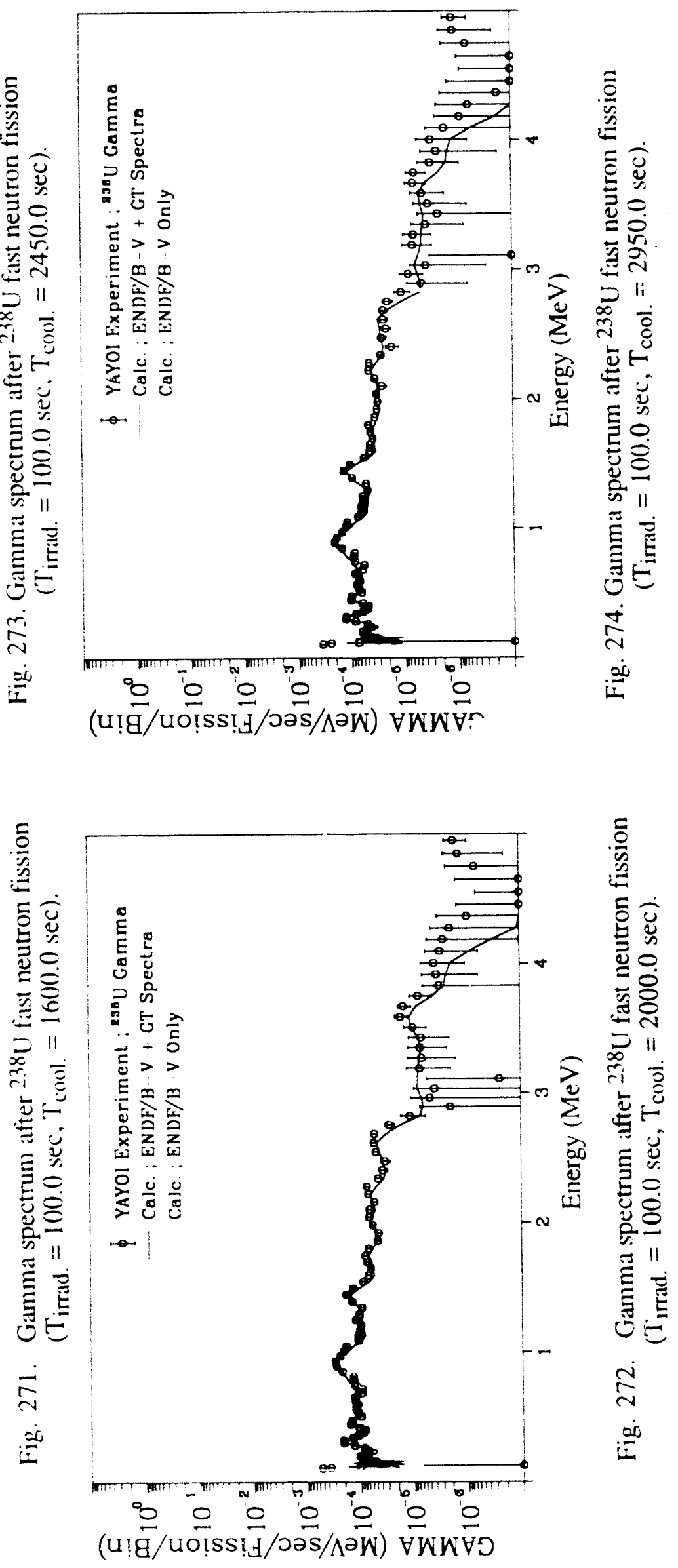

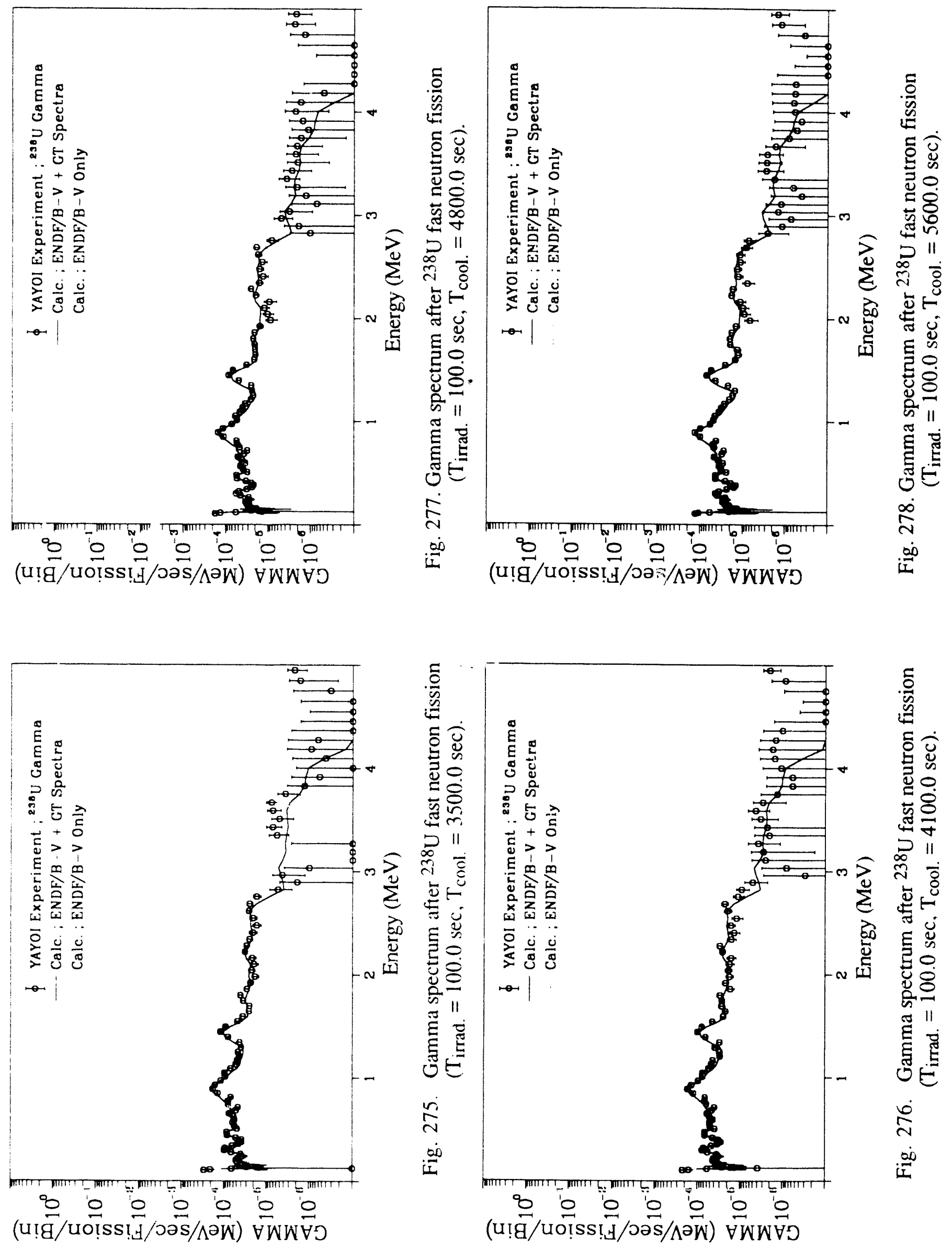

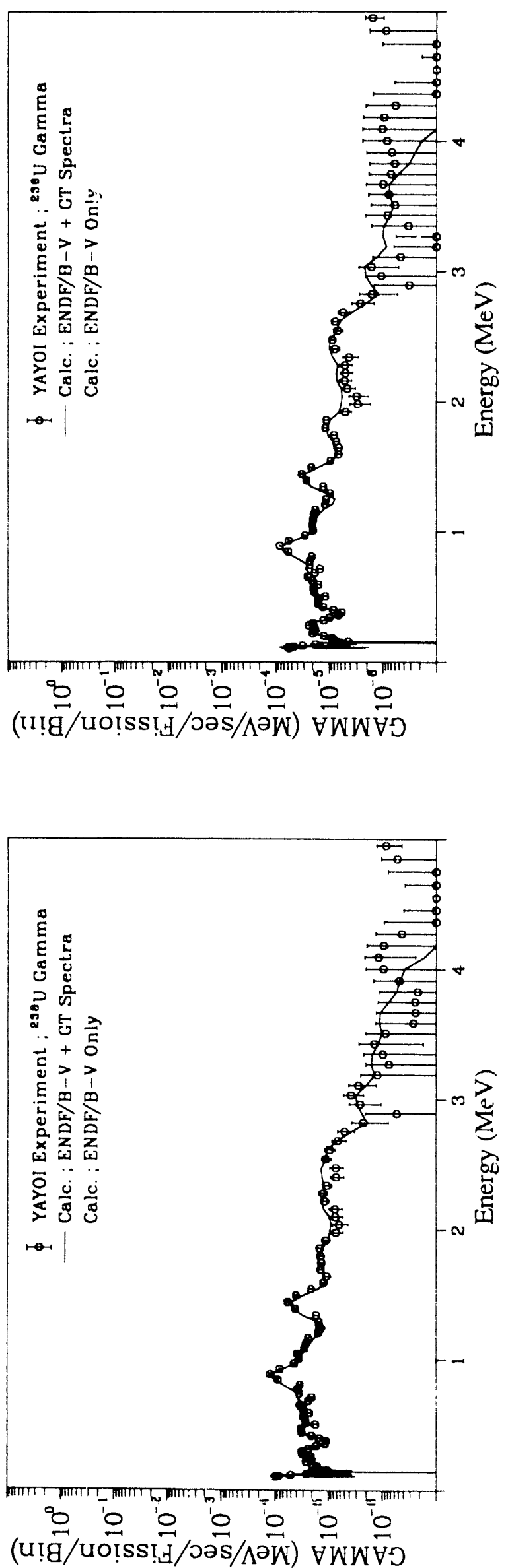



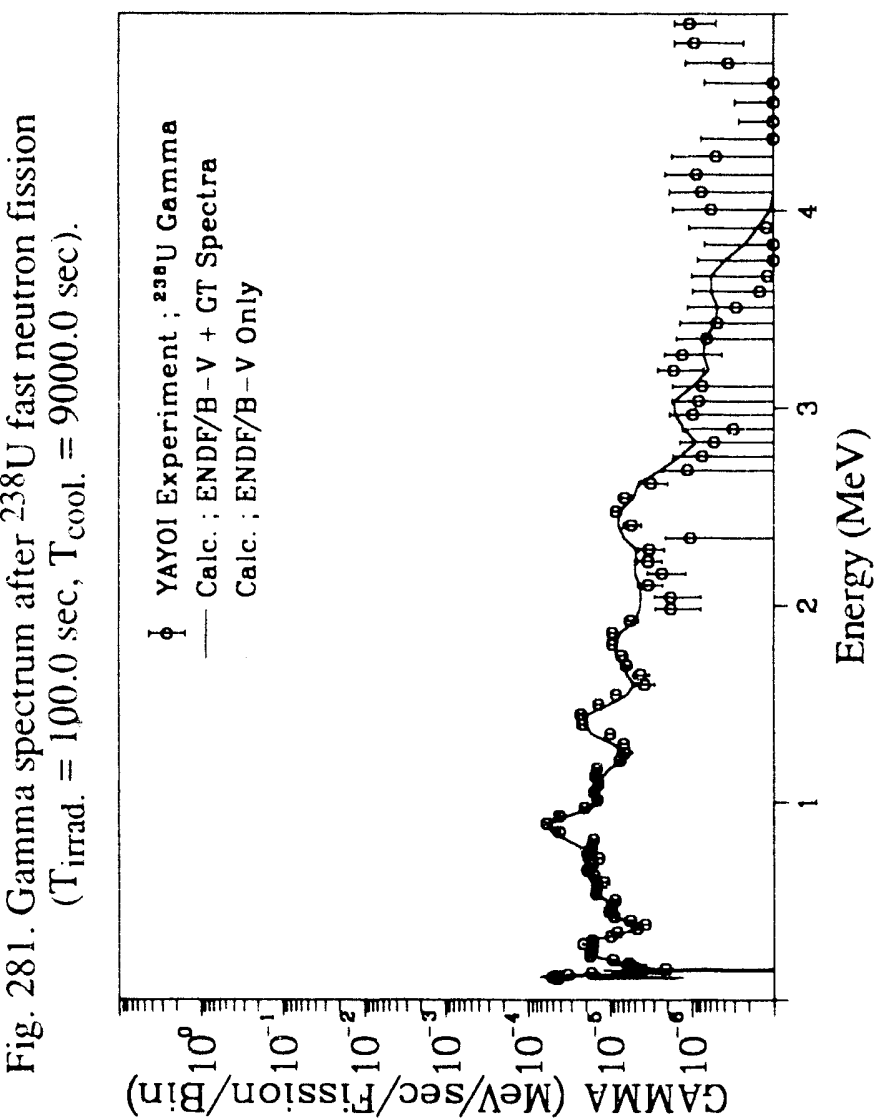

窇

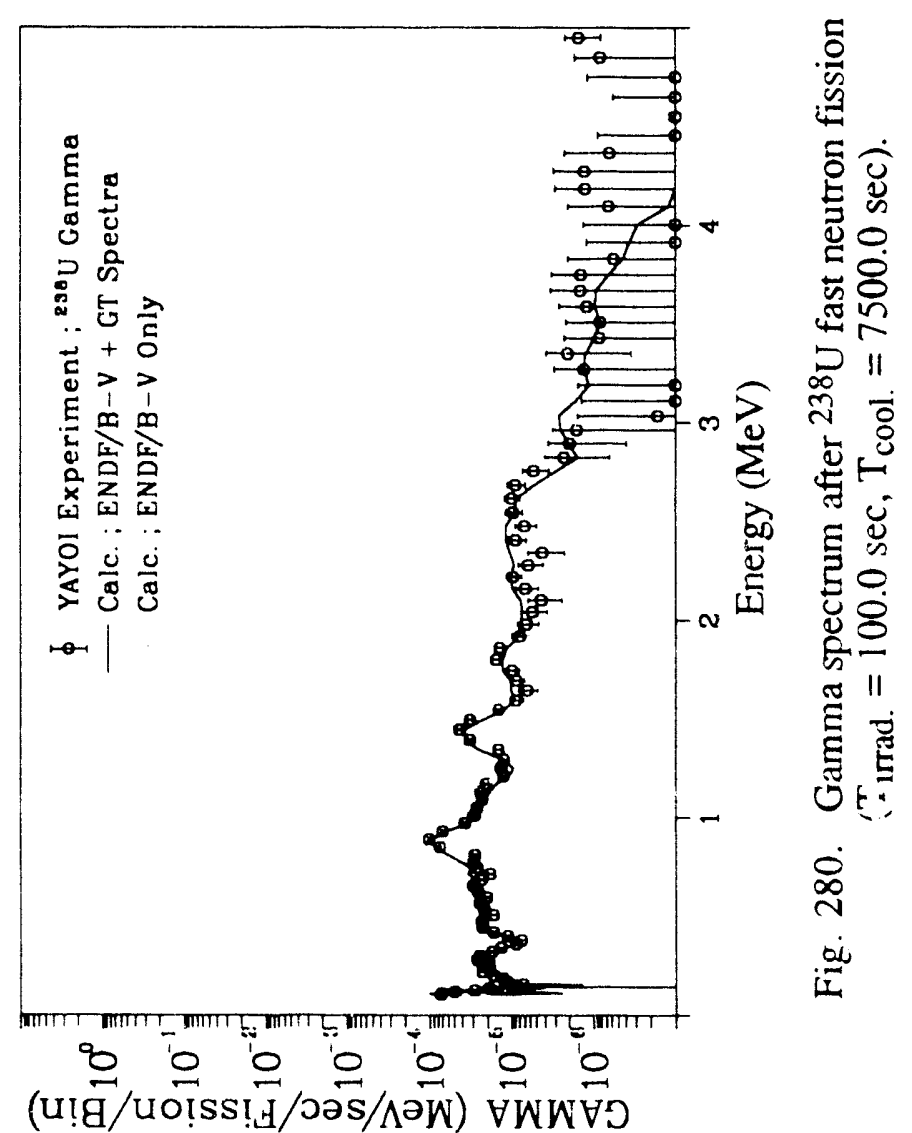



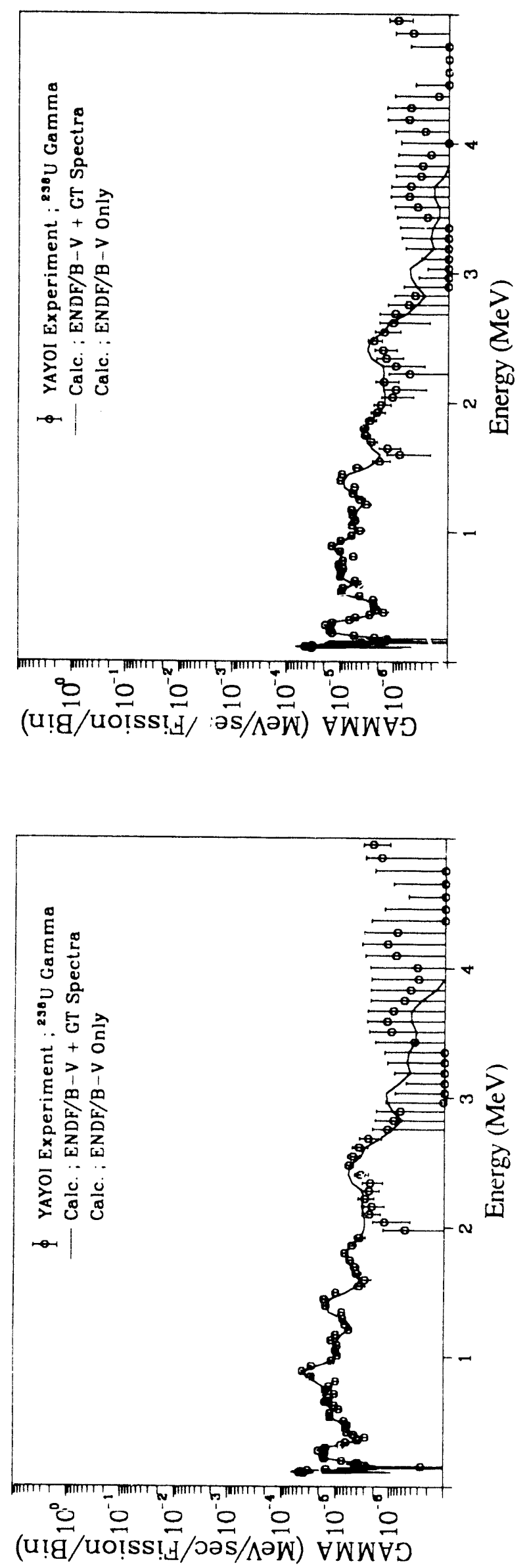
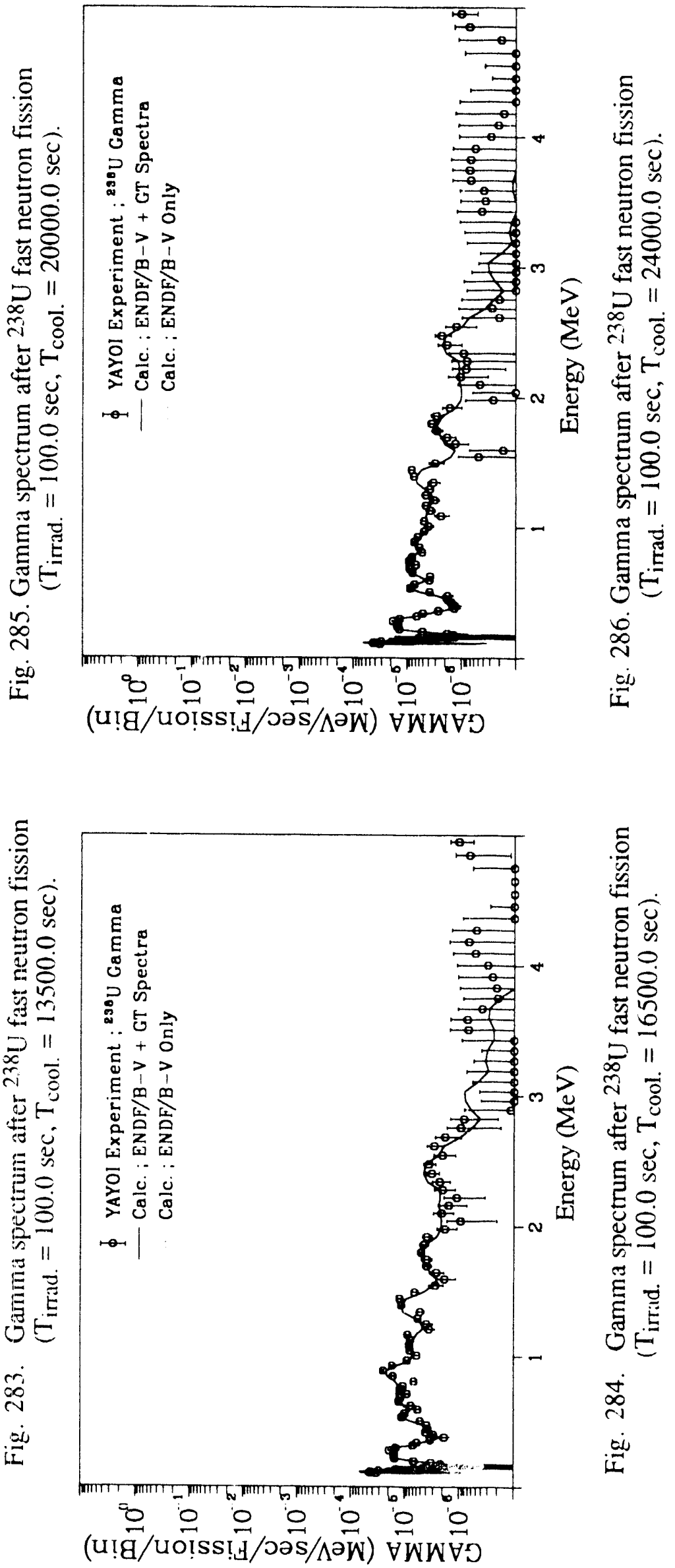

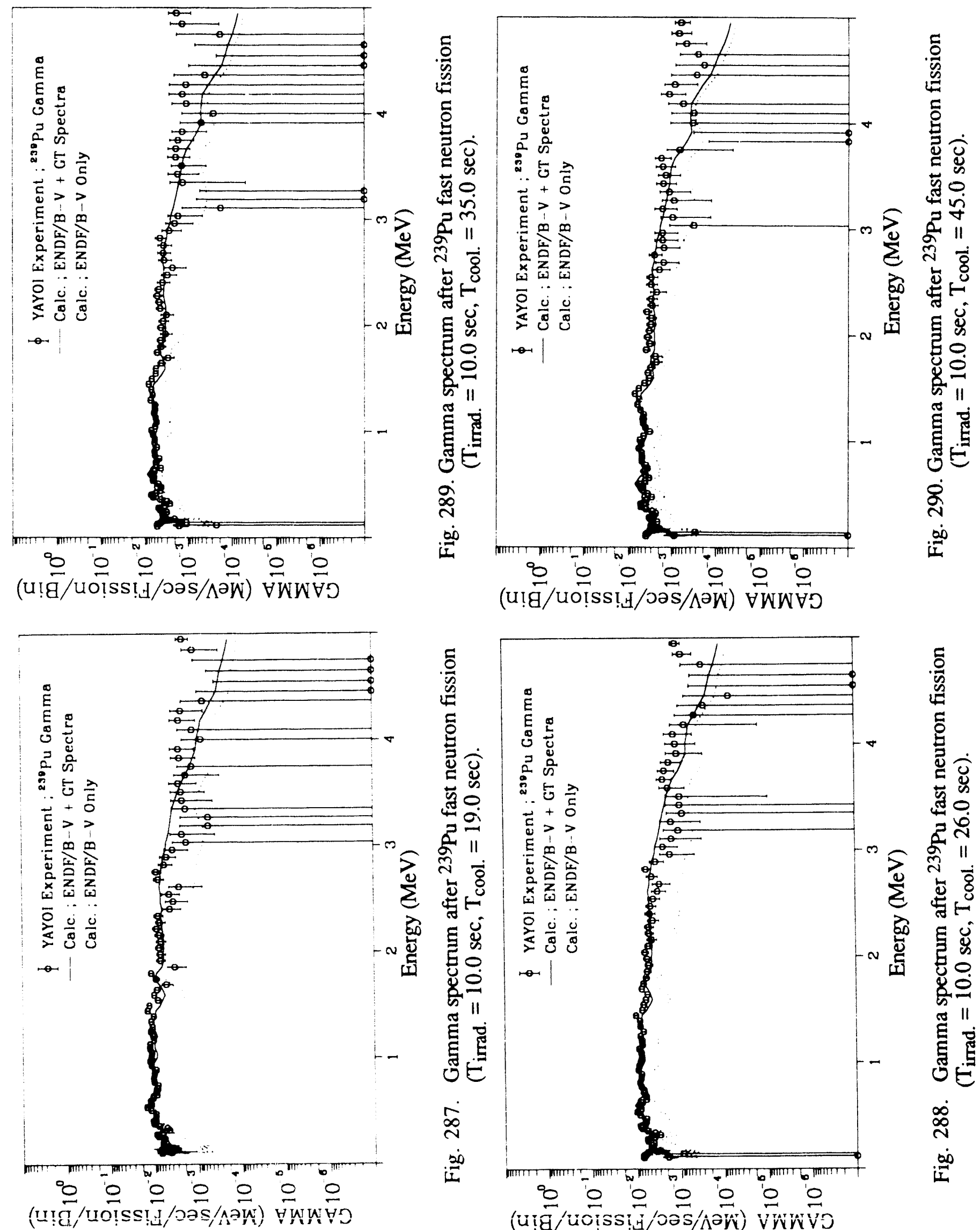


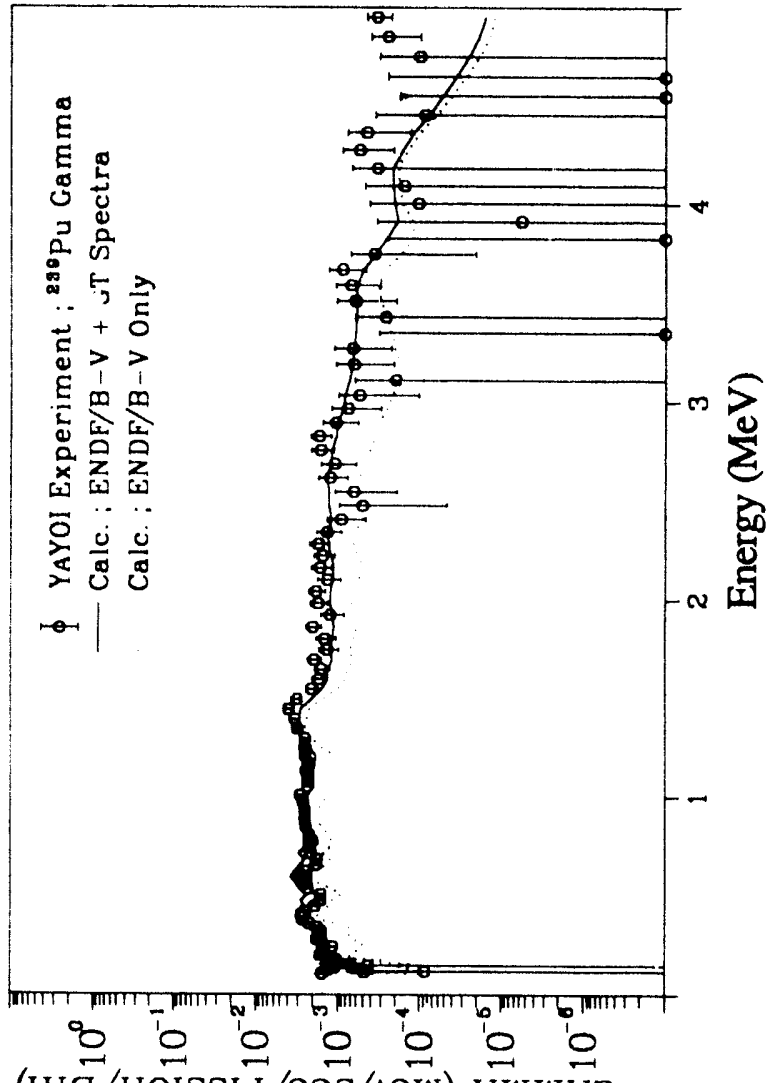

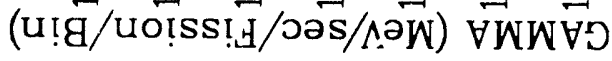

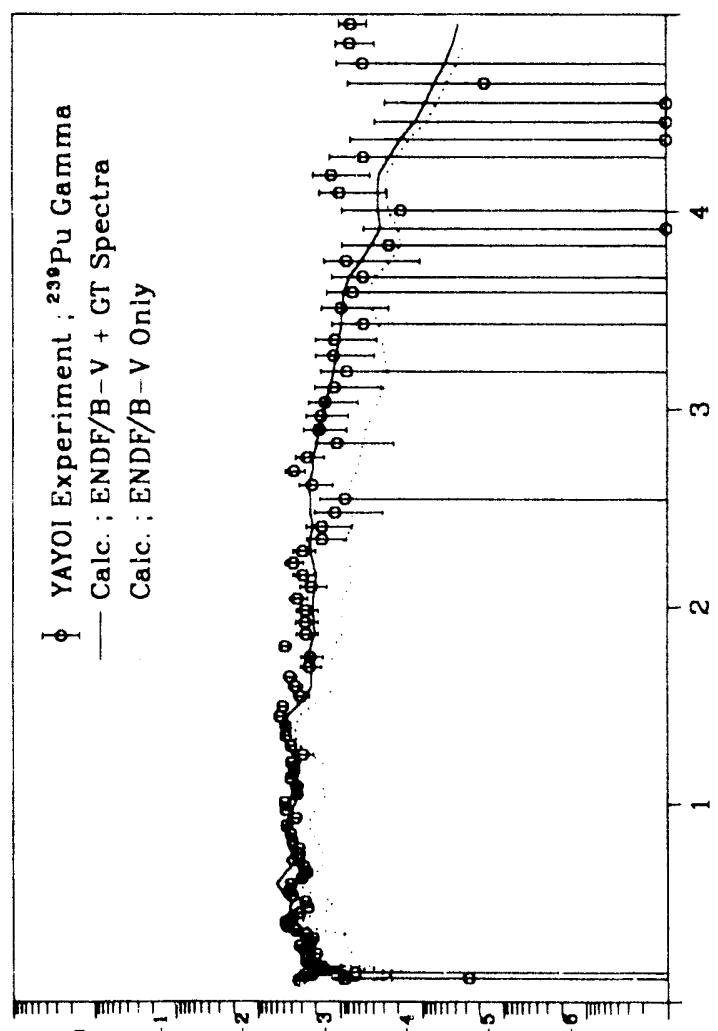

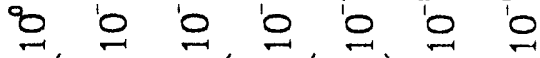

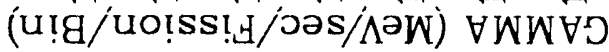

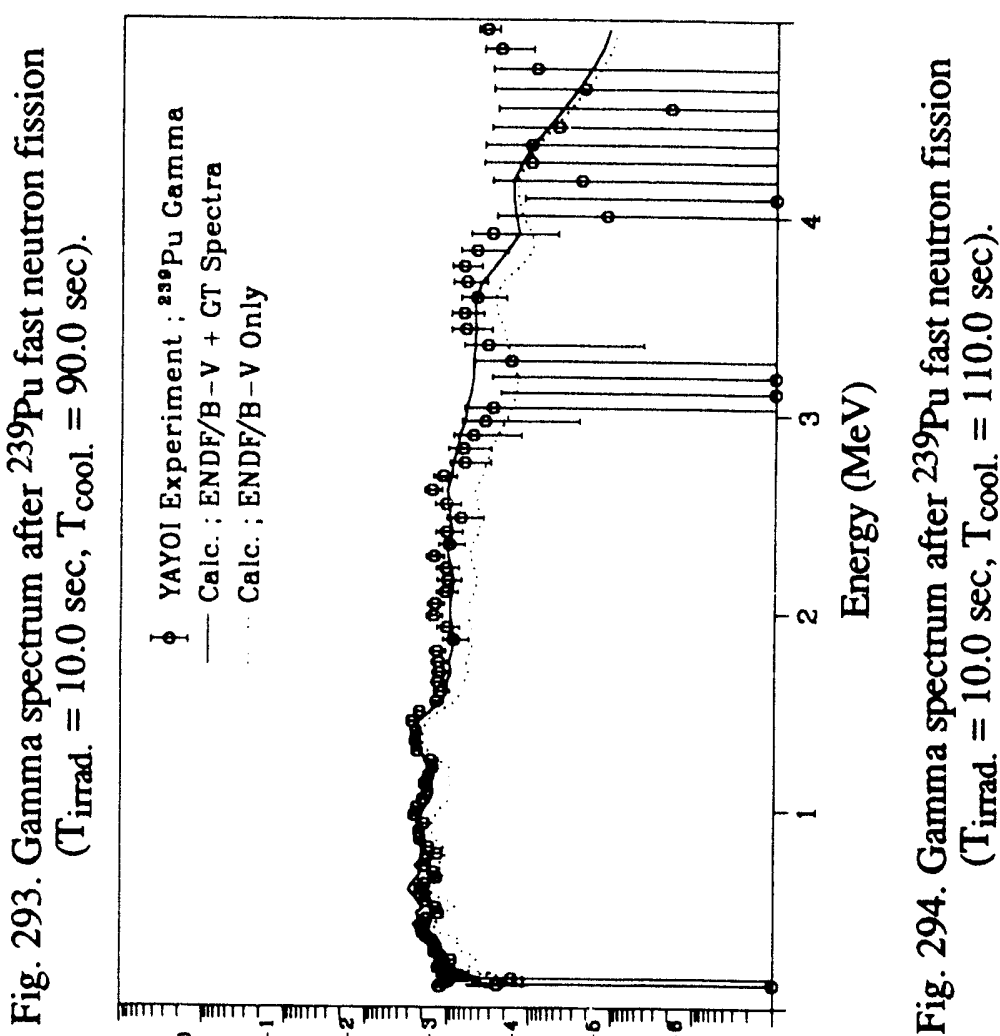

윽 으 으 io io io io

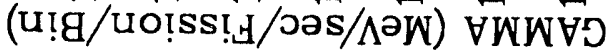

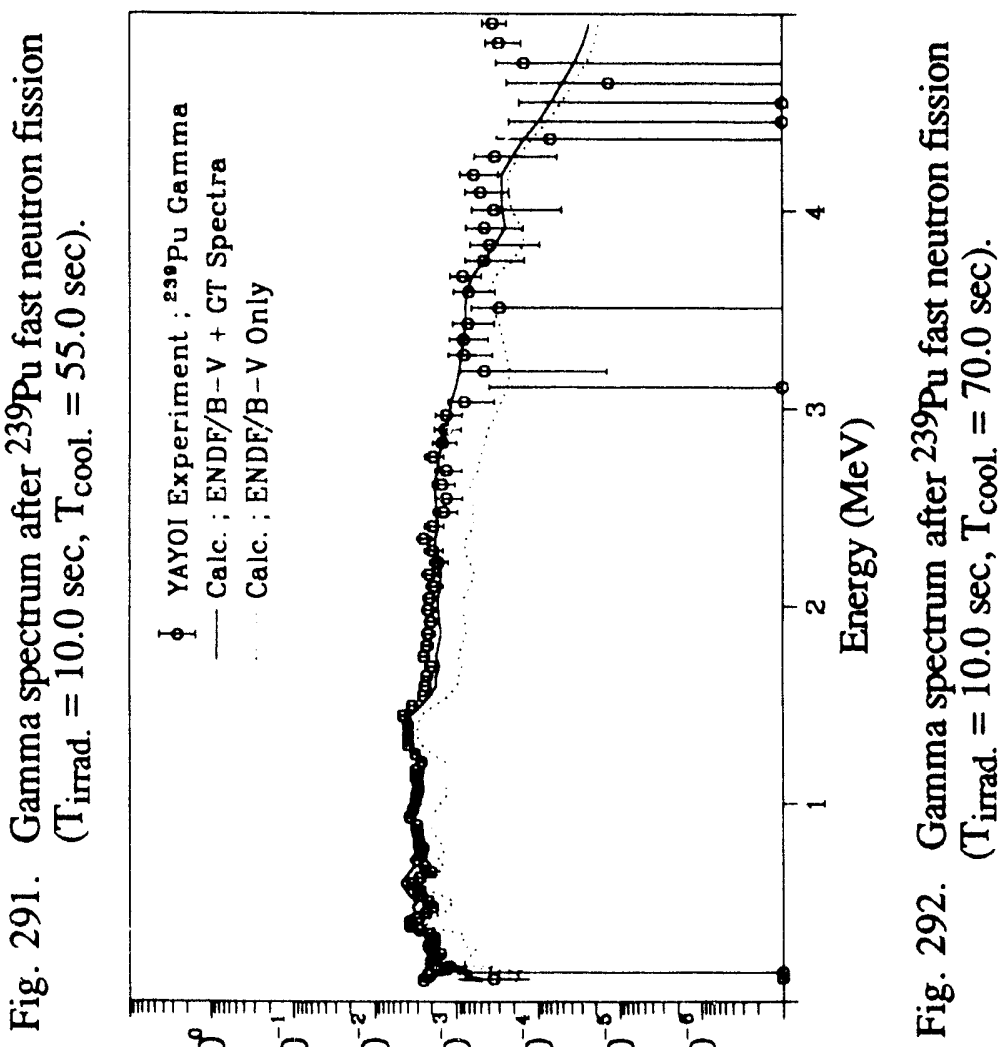

8 으 으 '응의 은

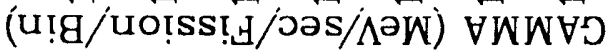



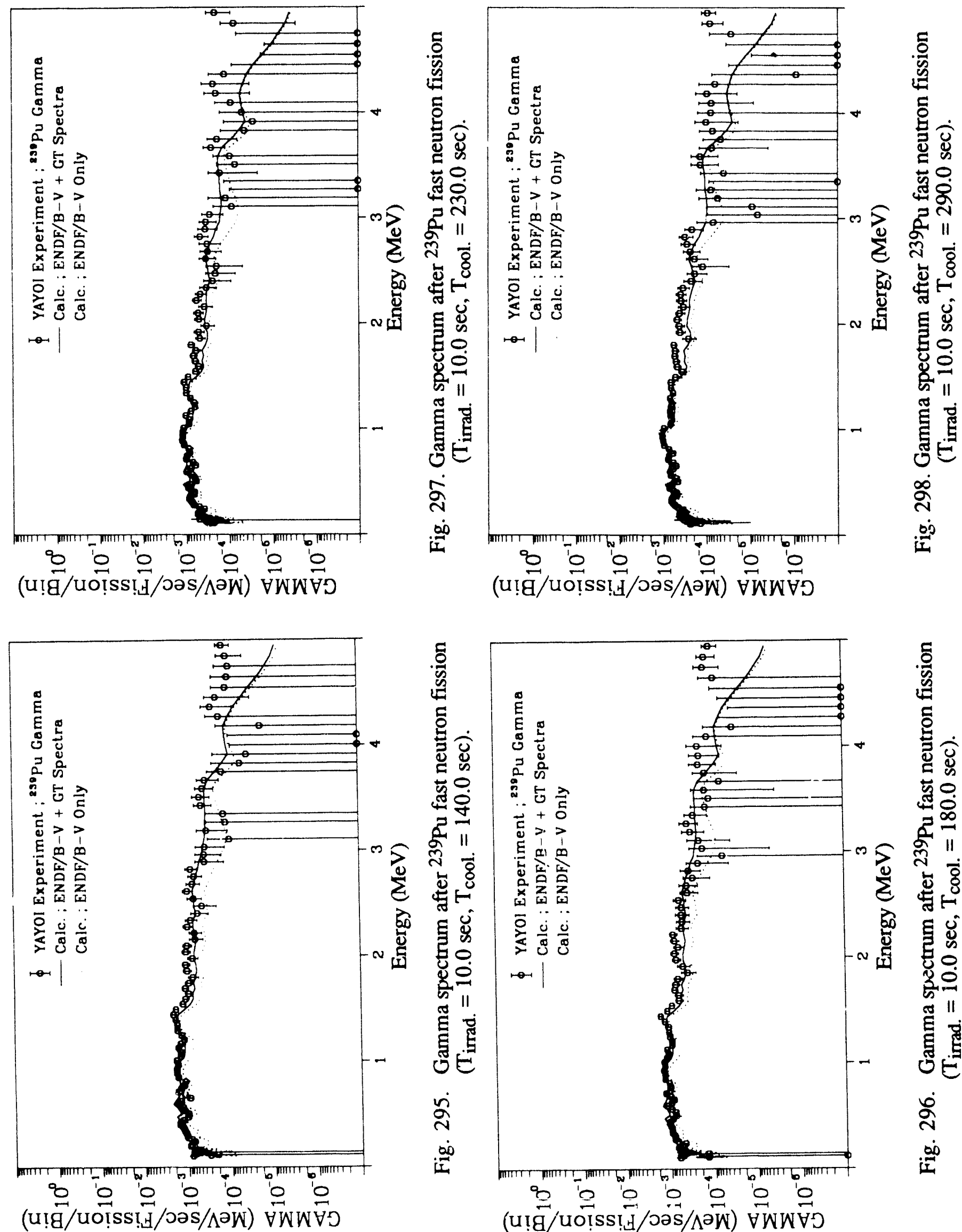

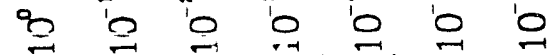

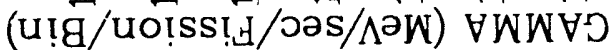



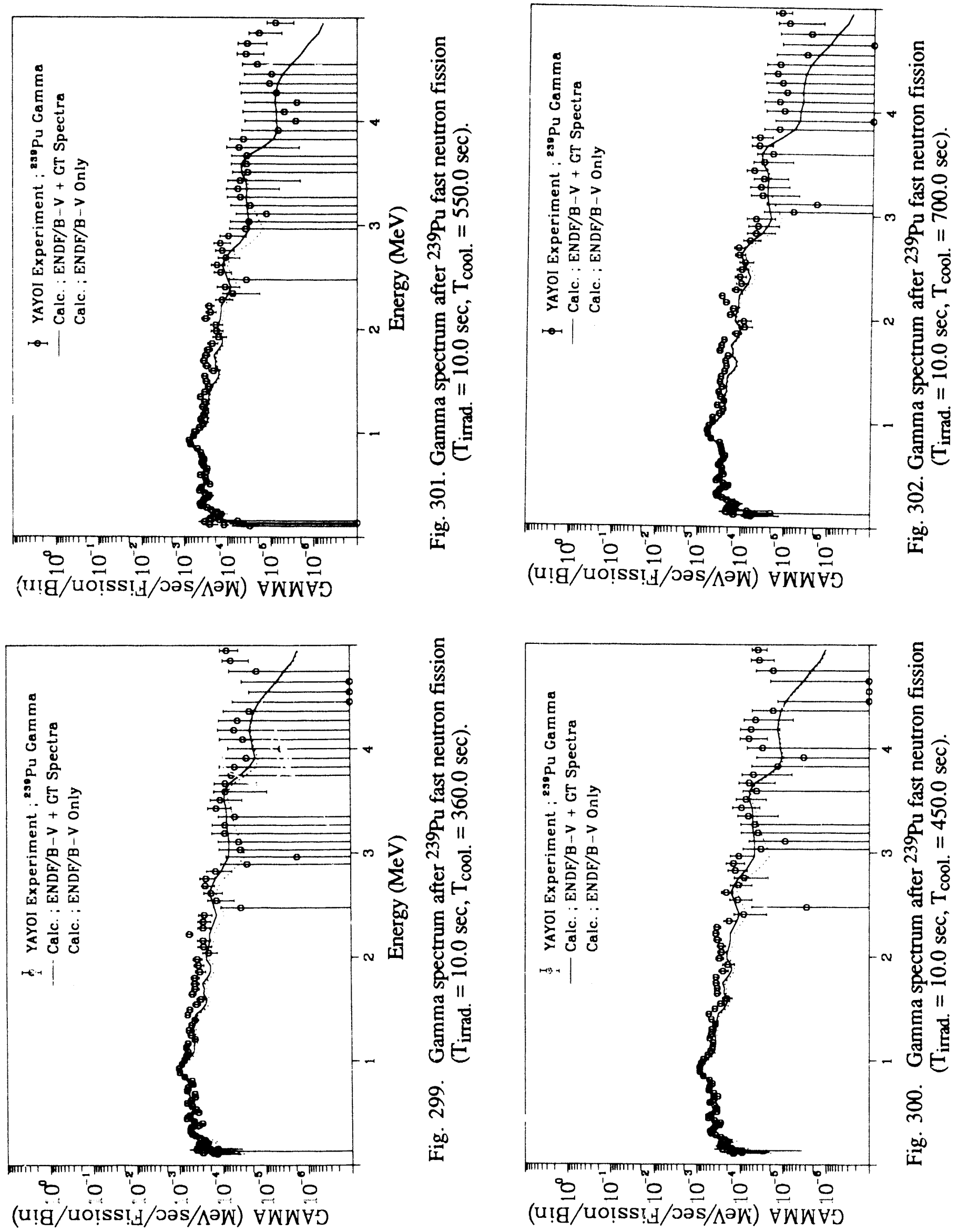


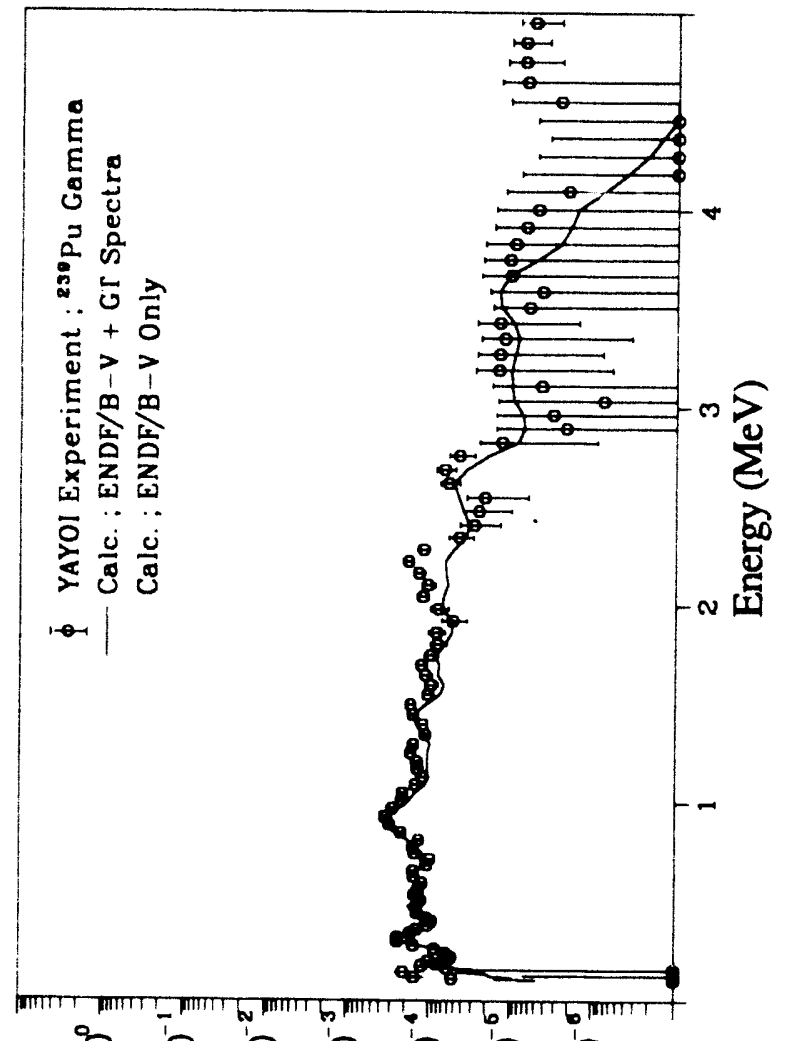

은응으의 응의

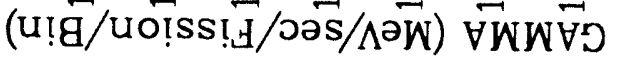

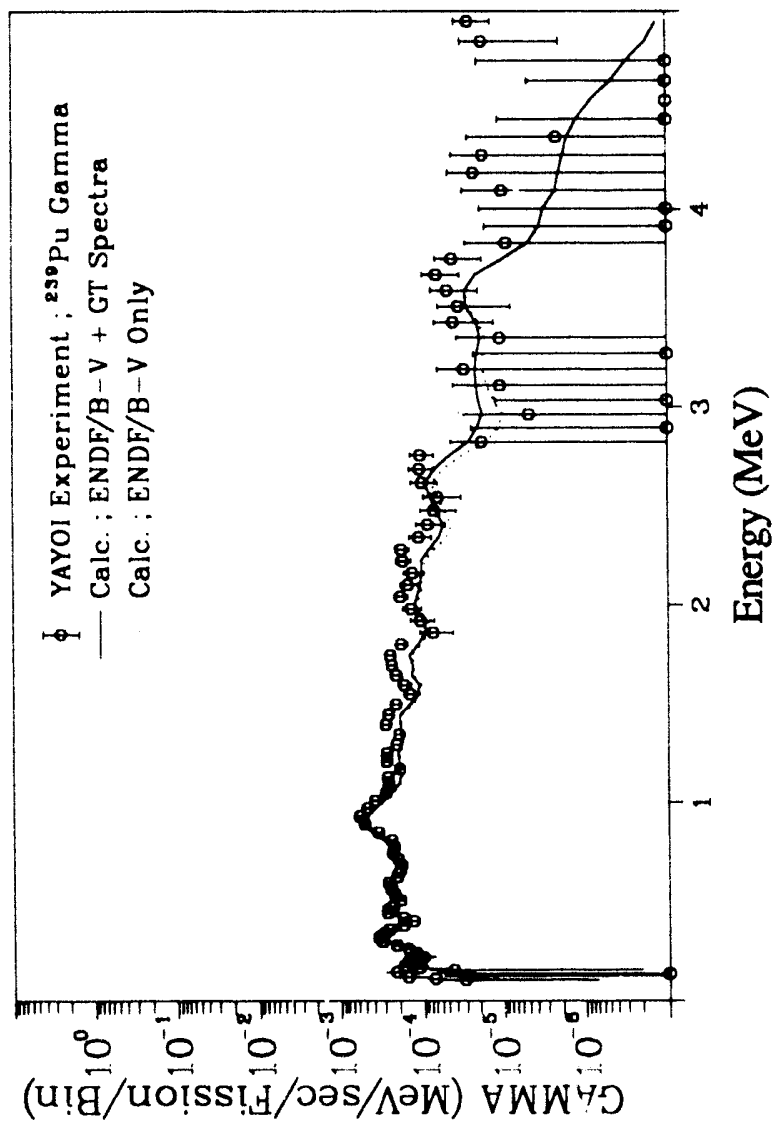

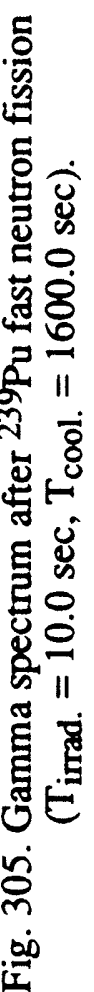

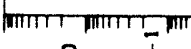

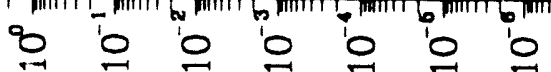

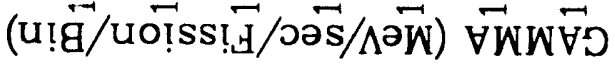

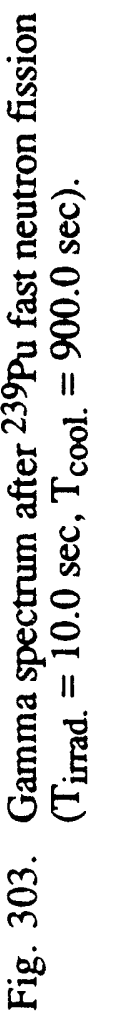



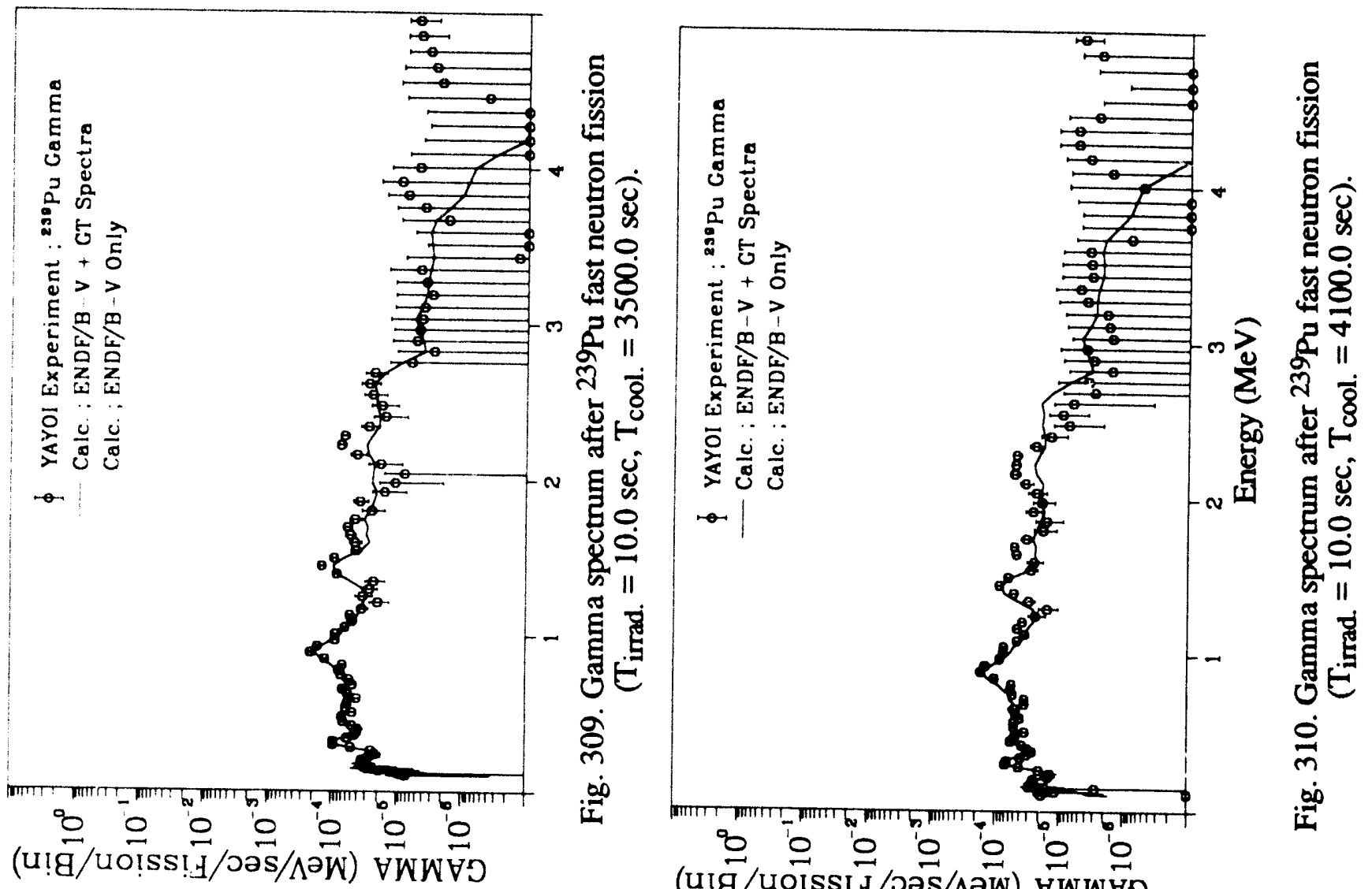

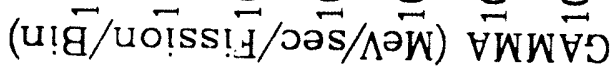

은 으 은 (u!̣/uO!
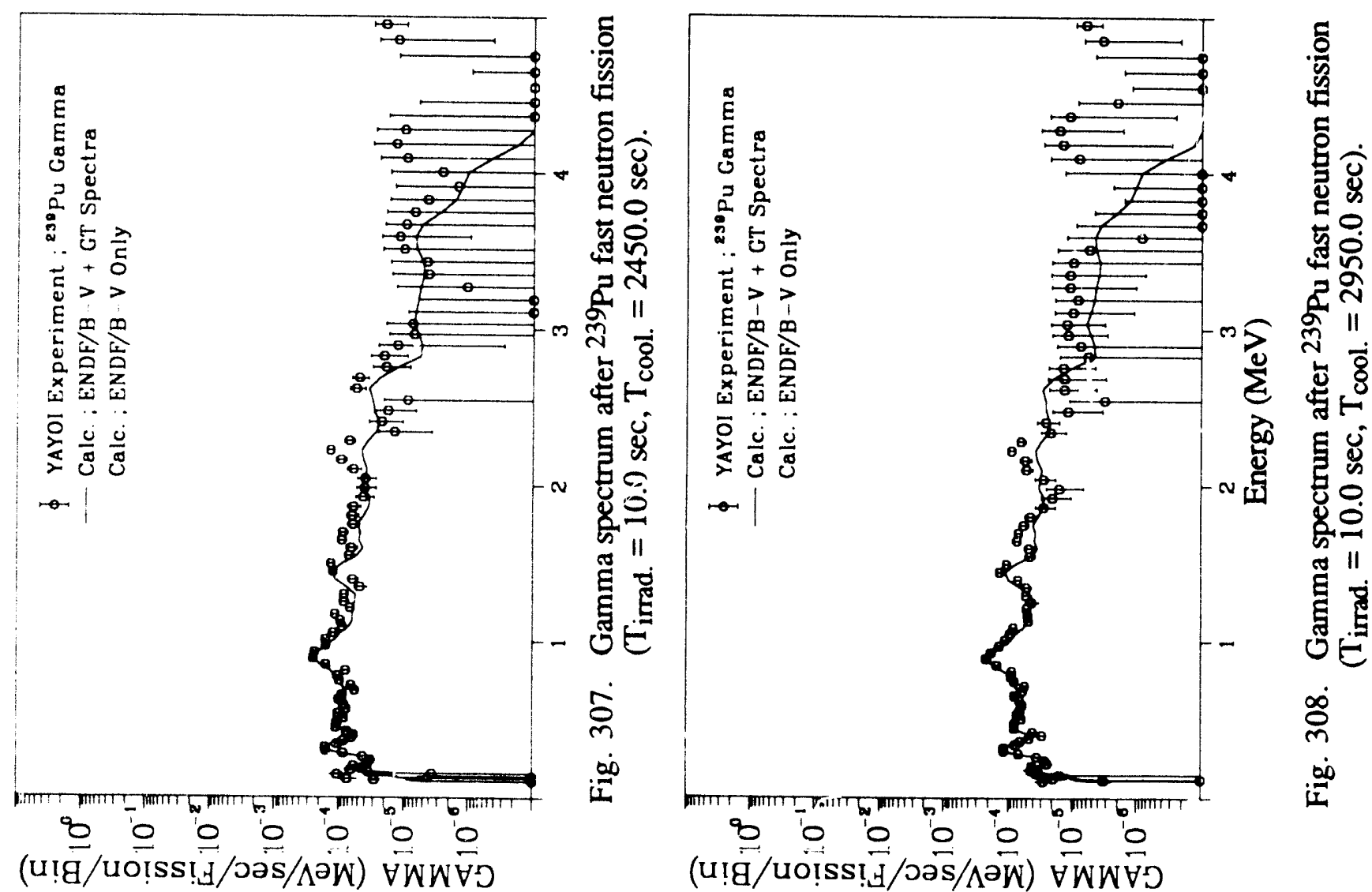

80 은 0 io

(u!g/uo!ss! 

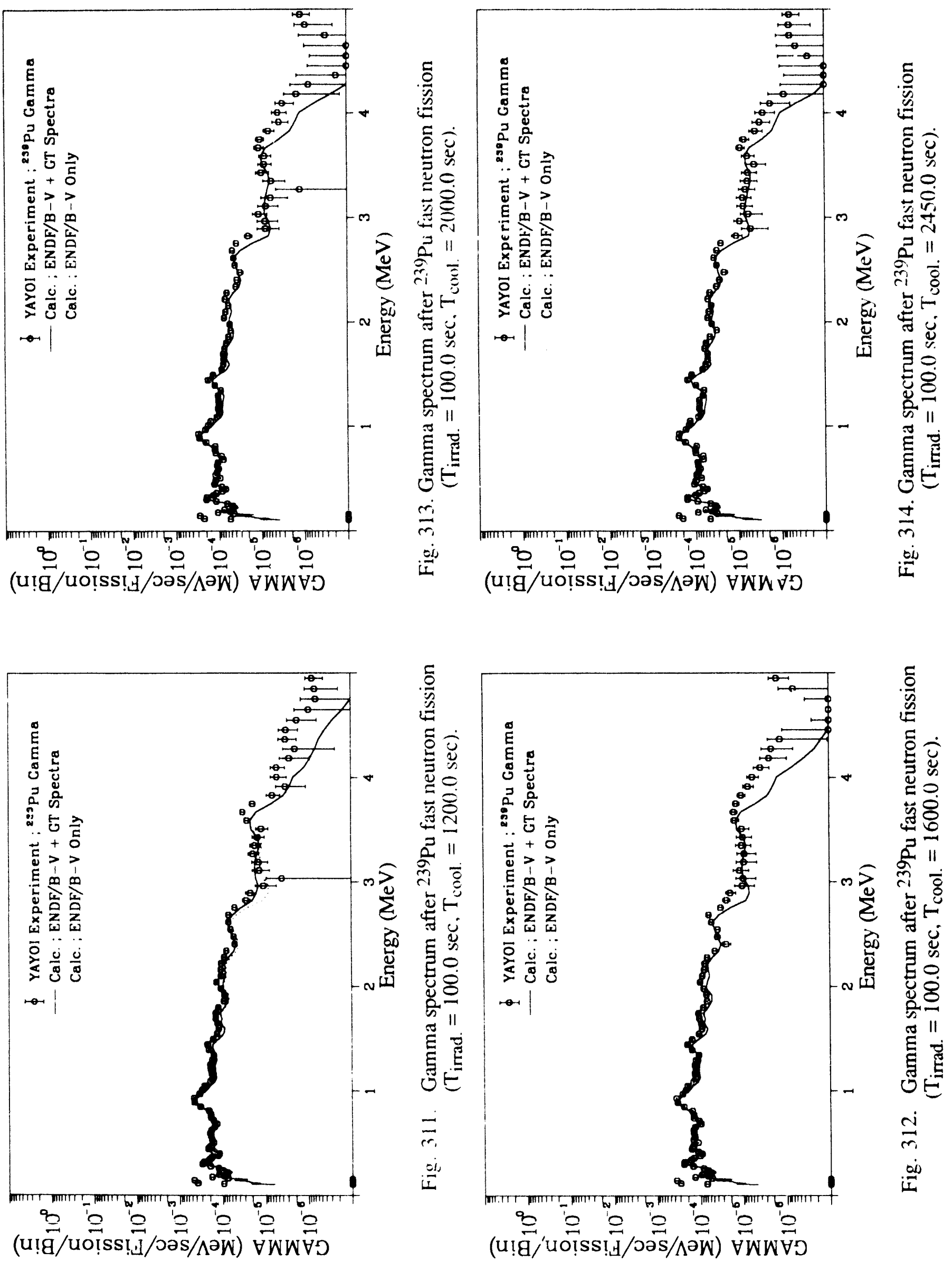


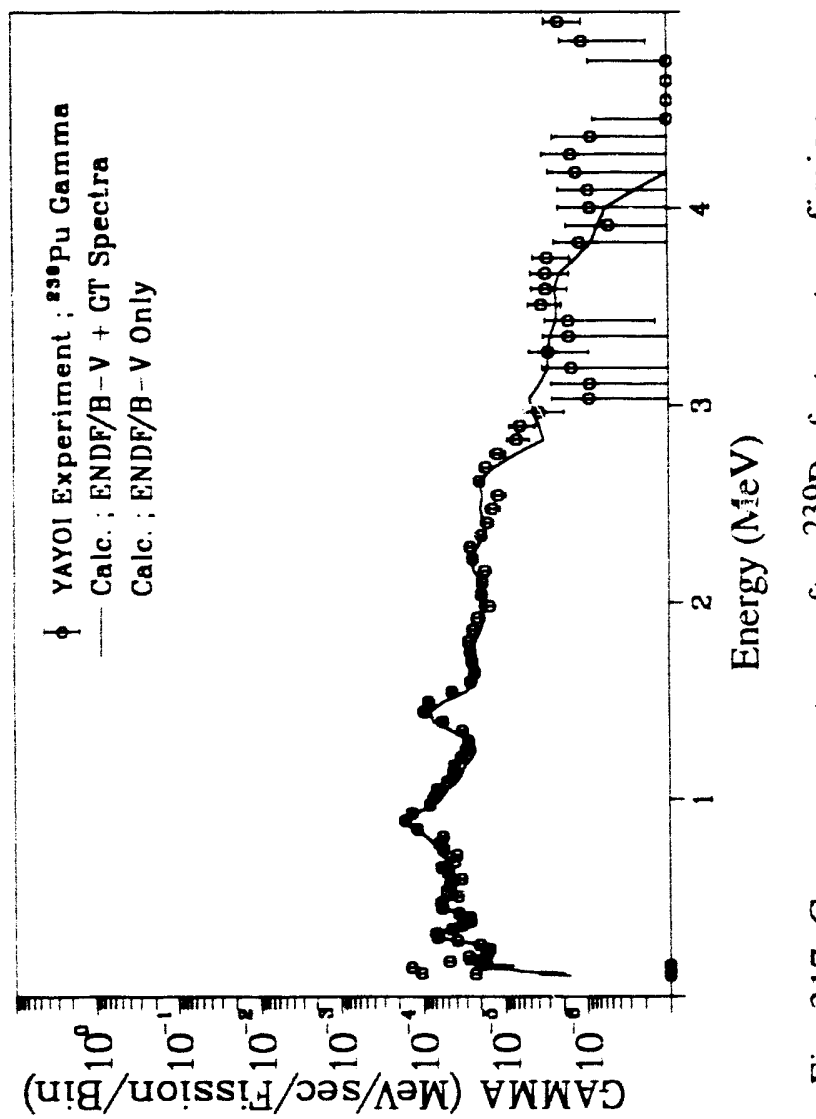

(uţ/นo!̣s

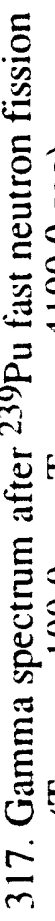

is
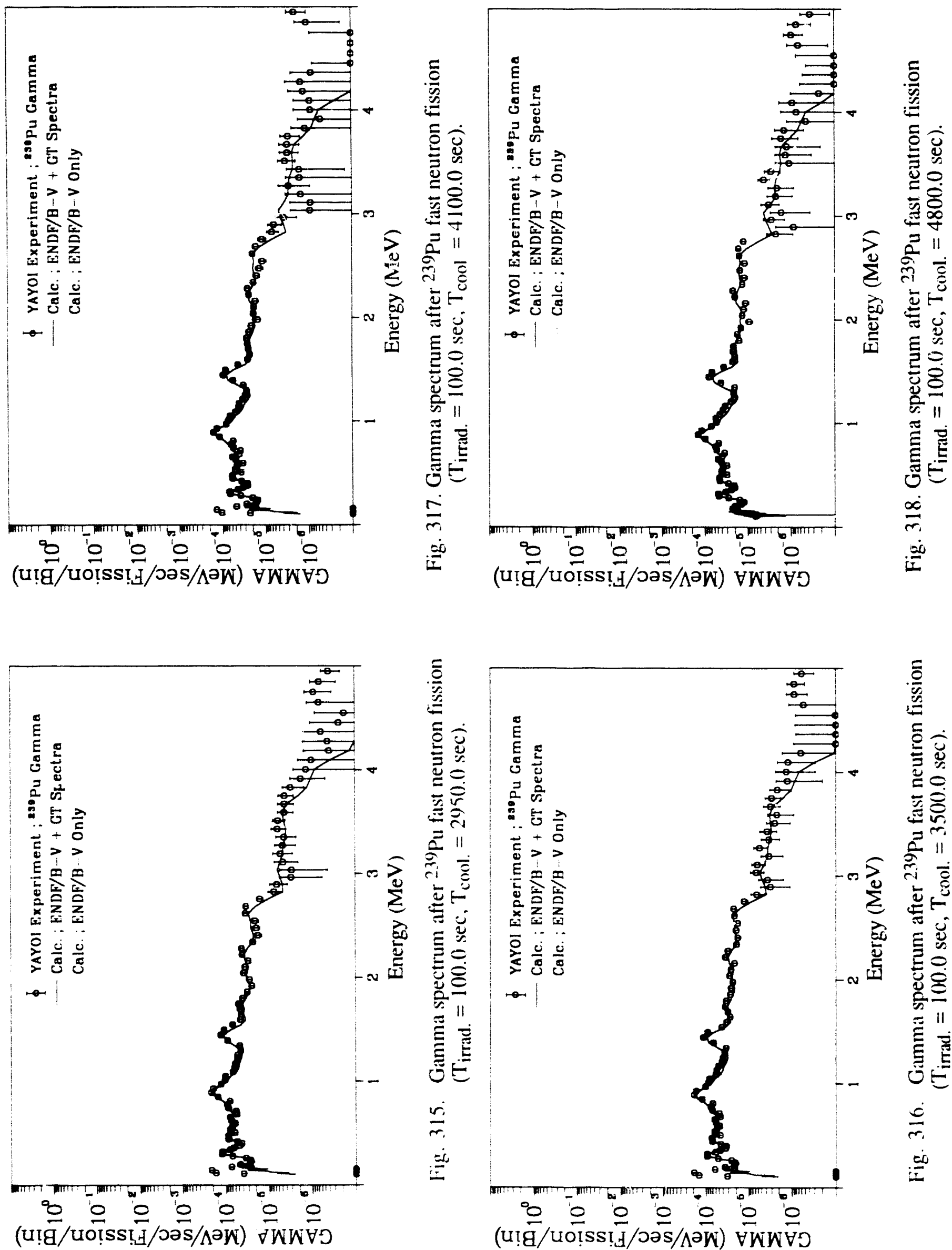

作

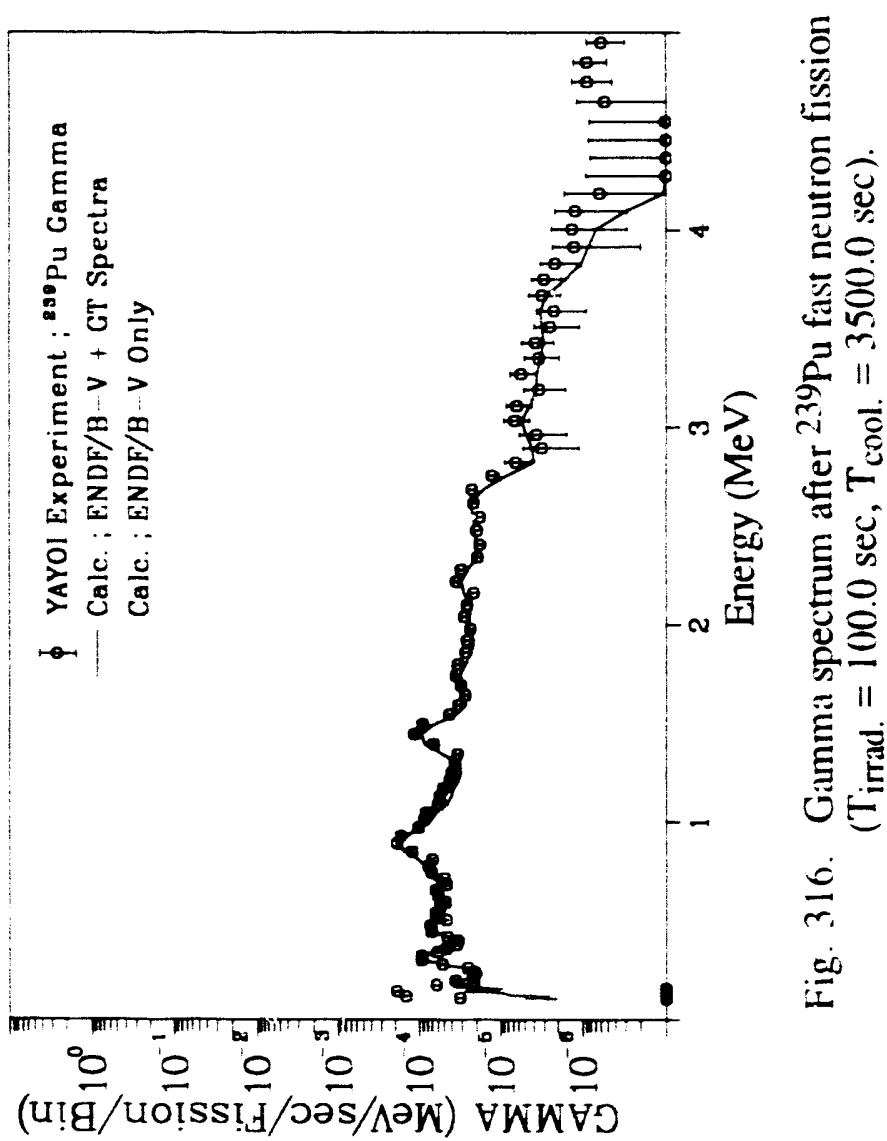



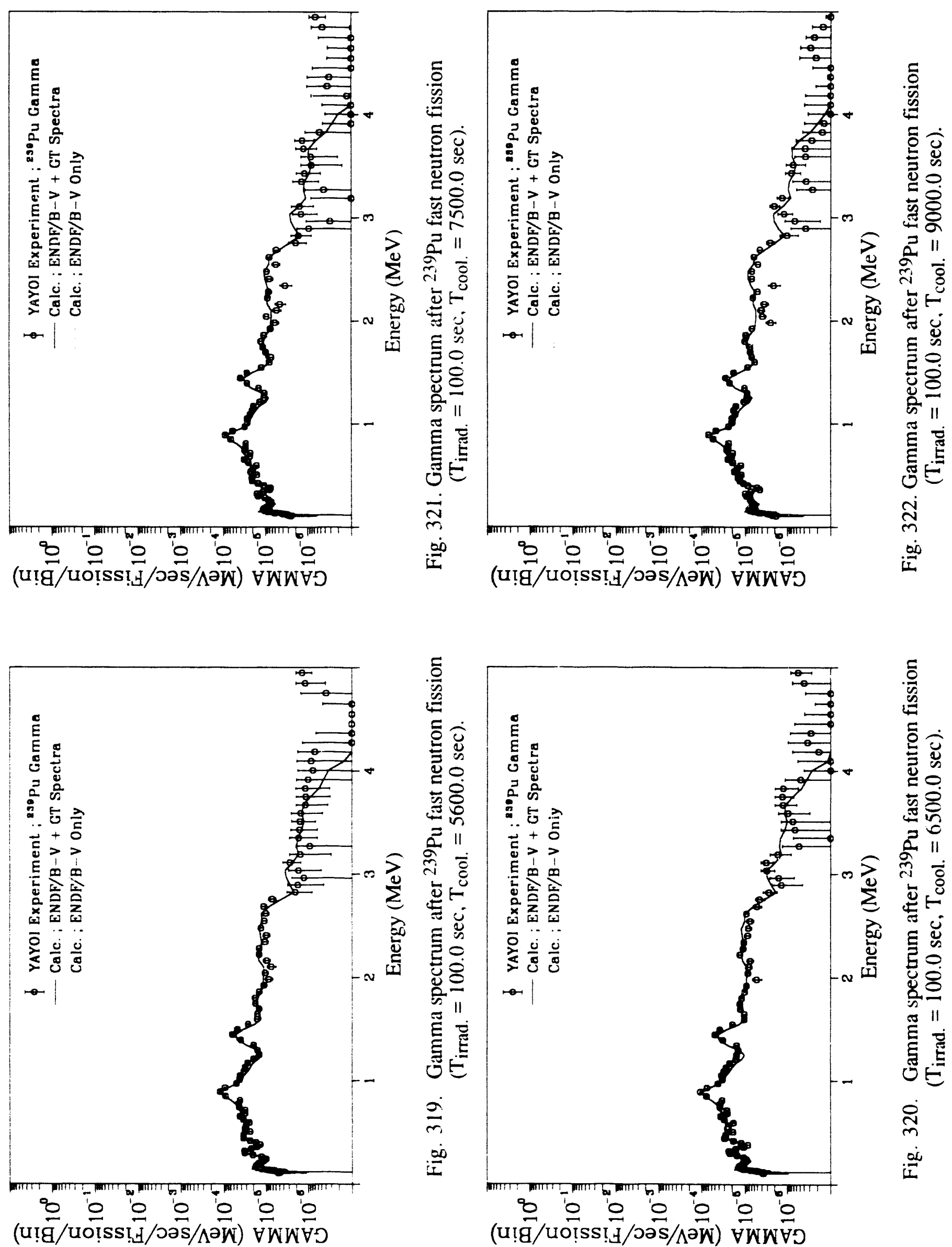

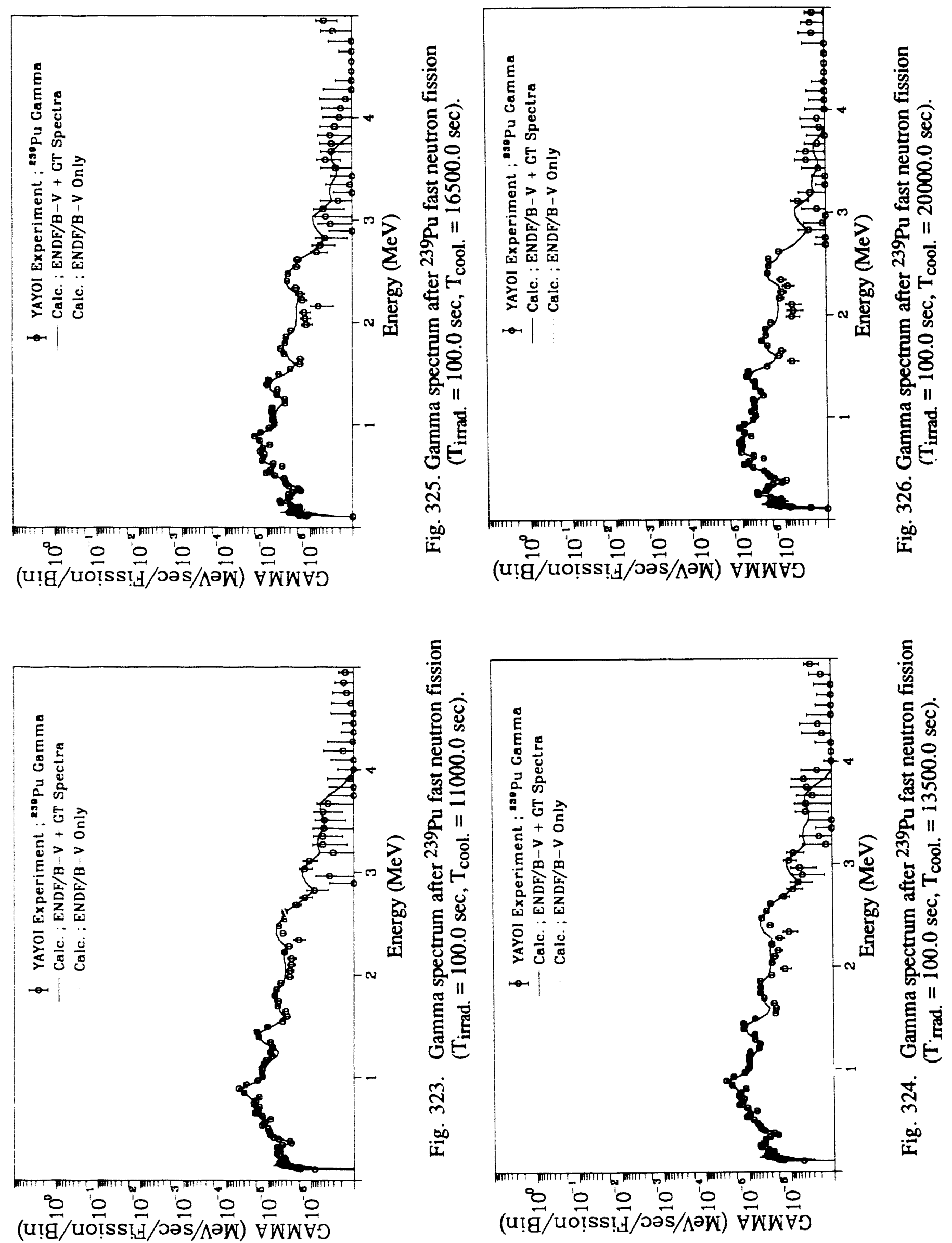


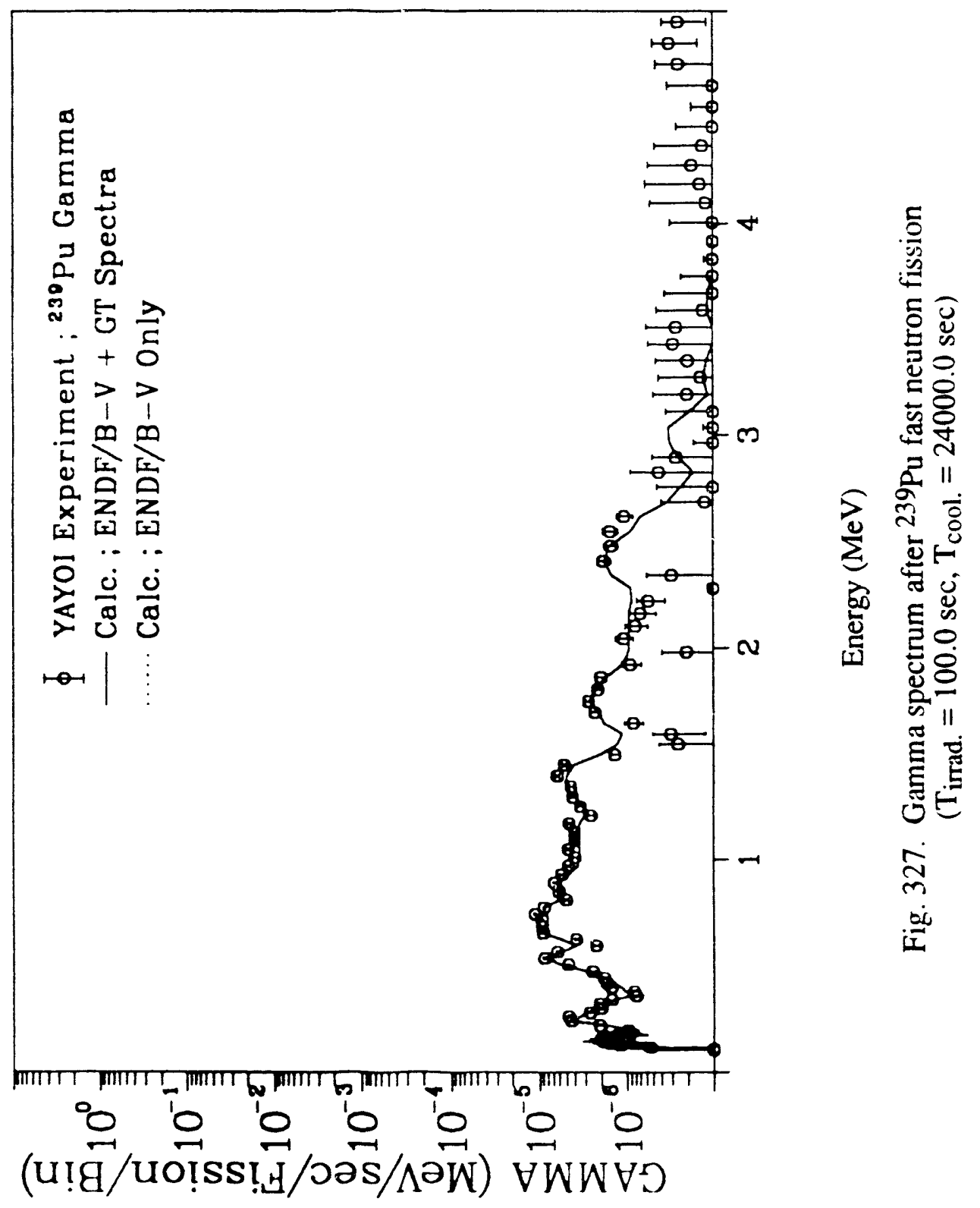



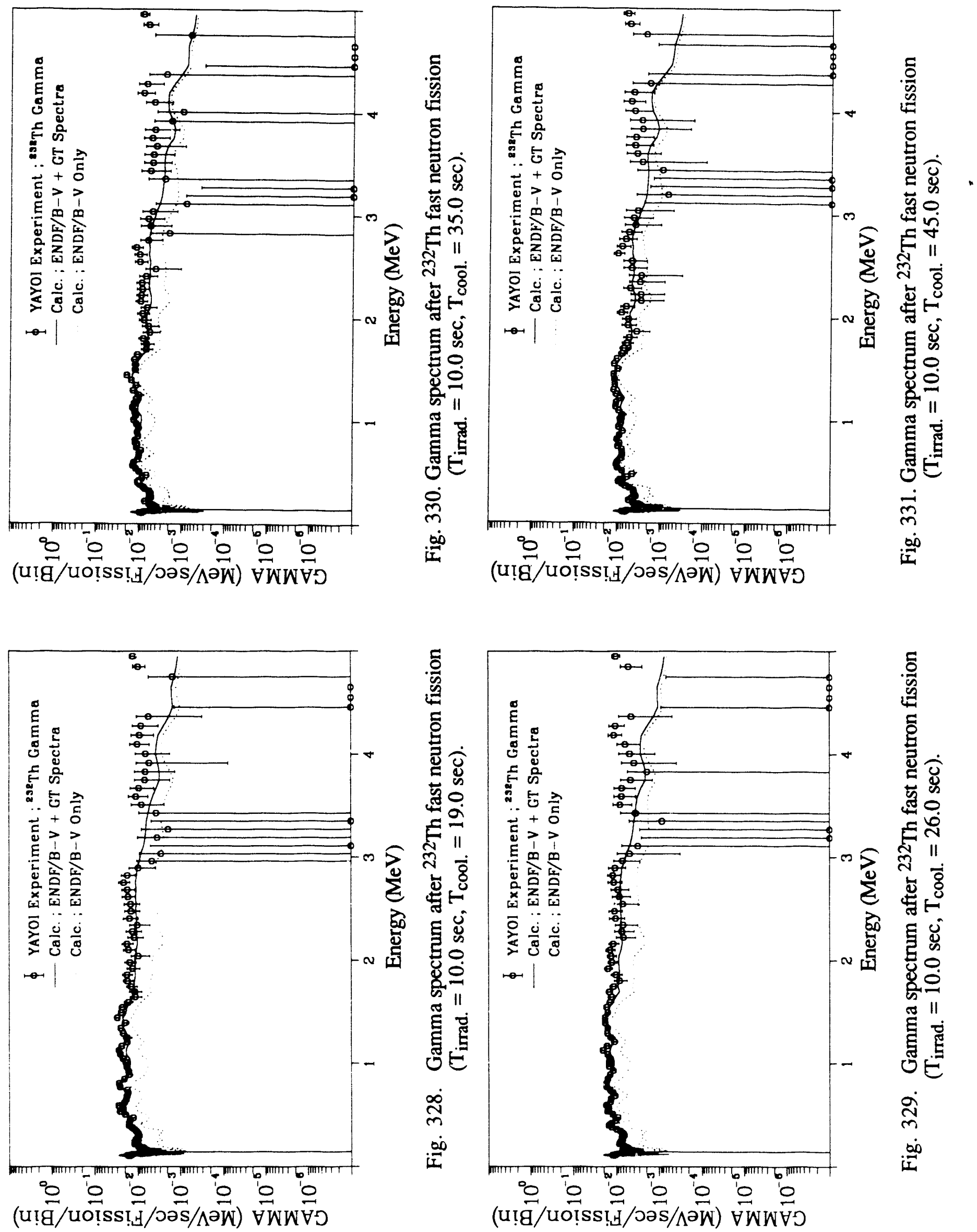

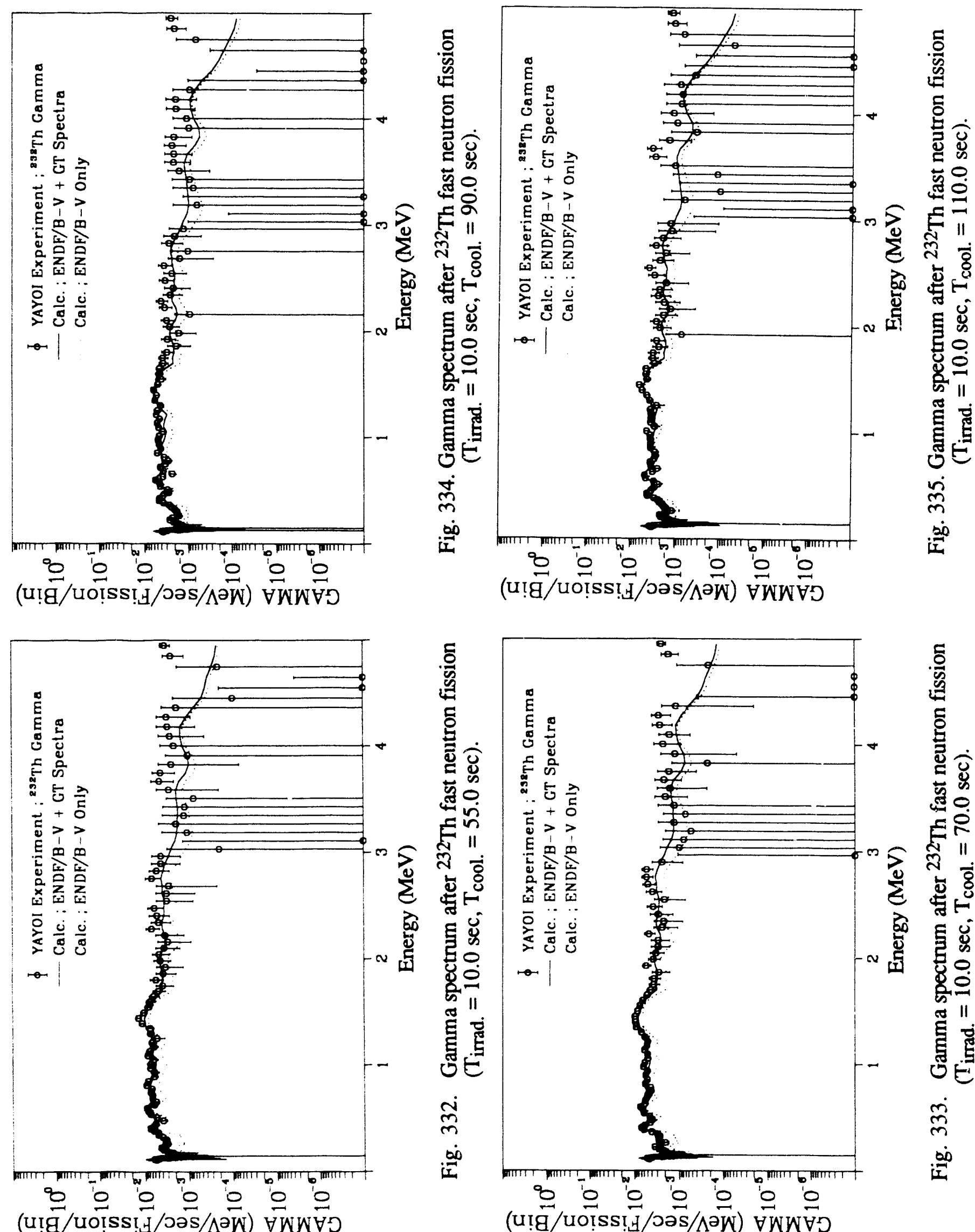

尊

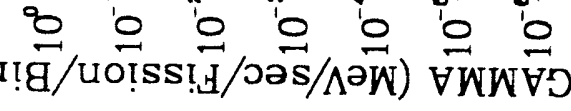

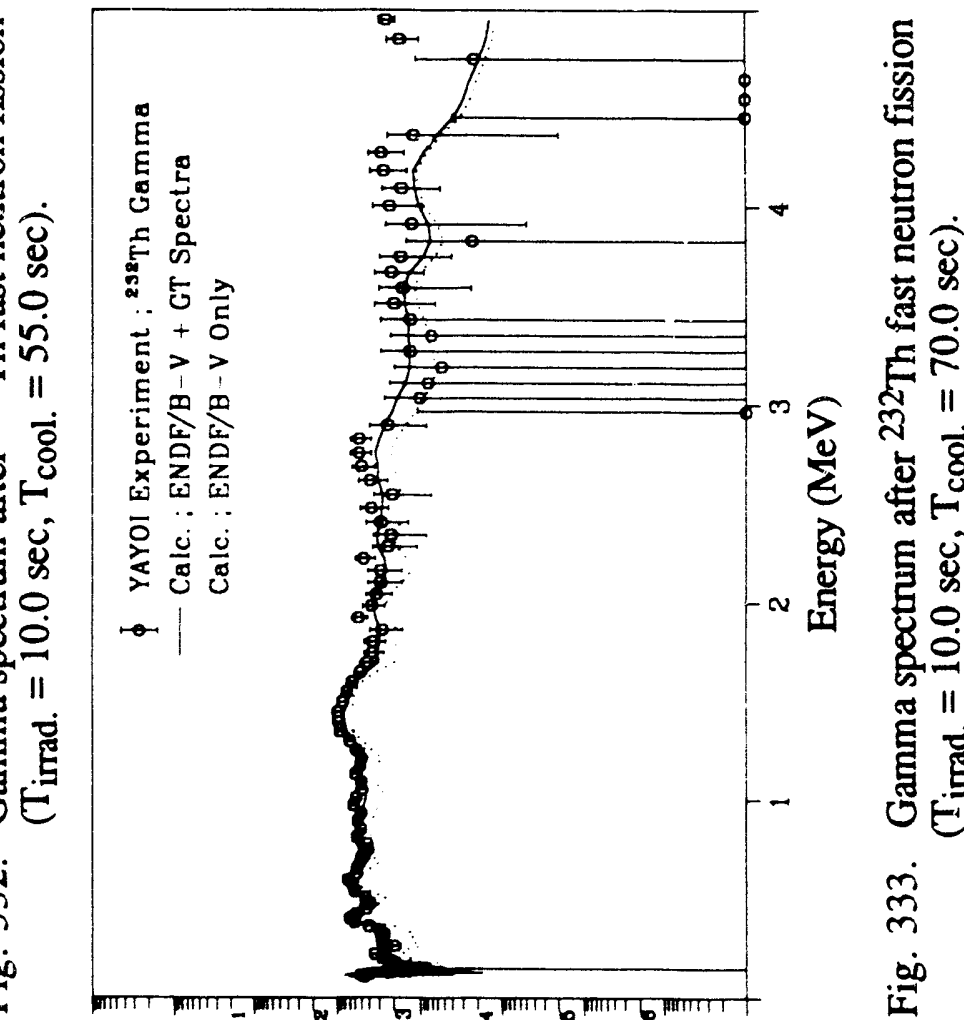

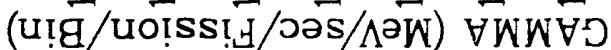

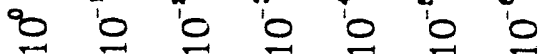

(u!g/uo!ss! 

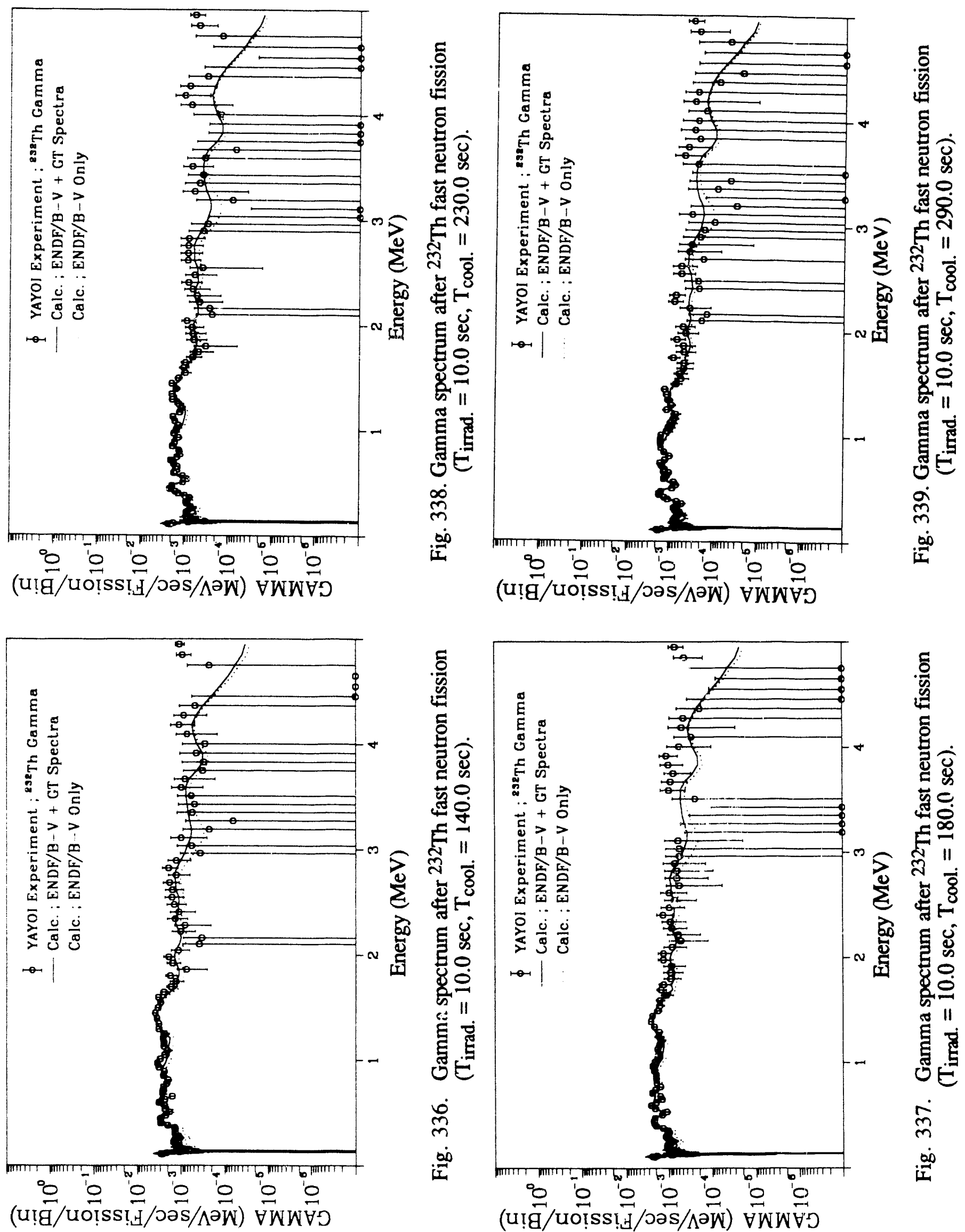

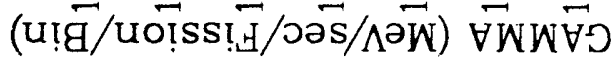

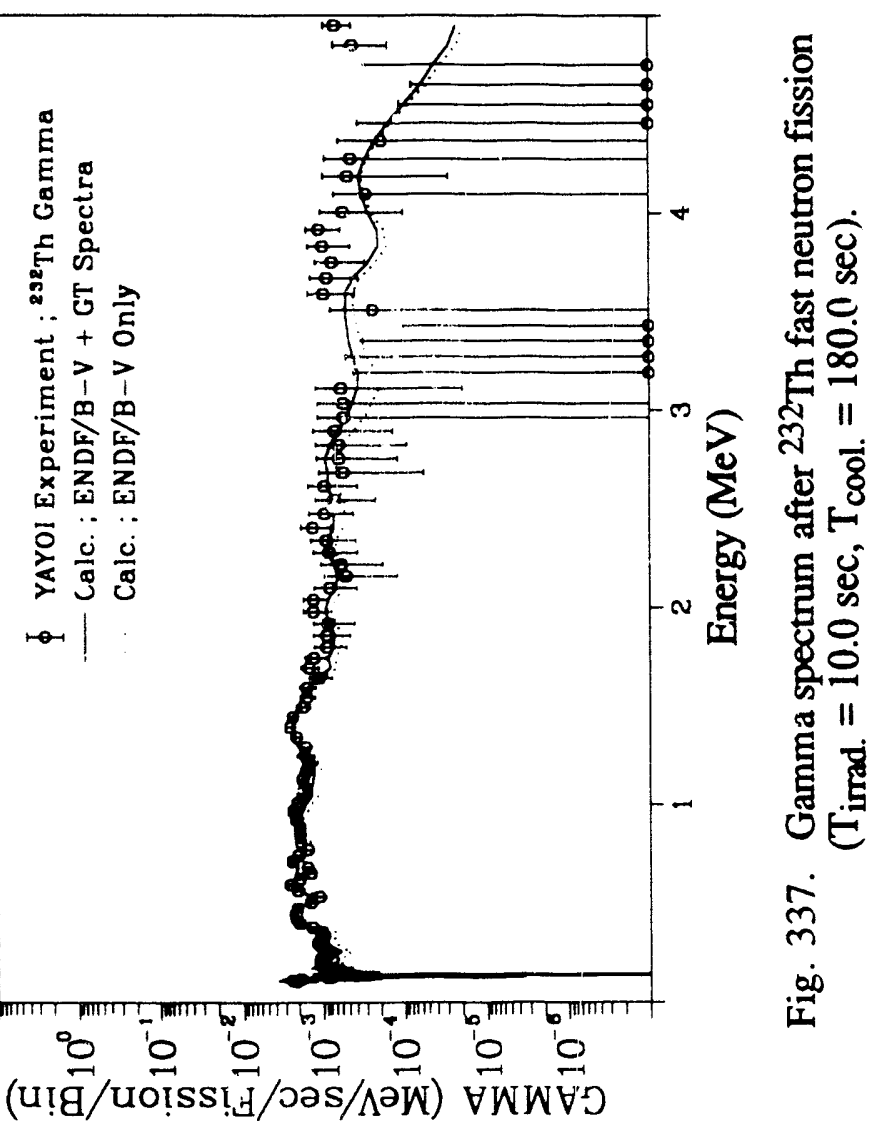




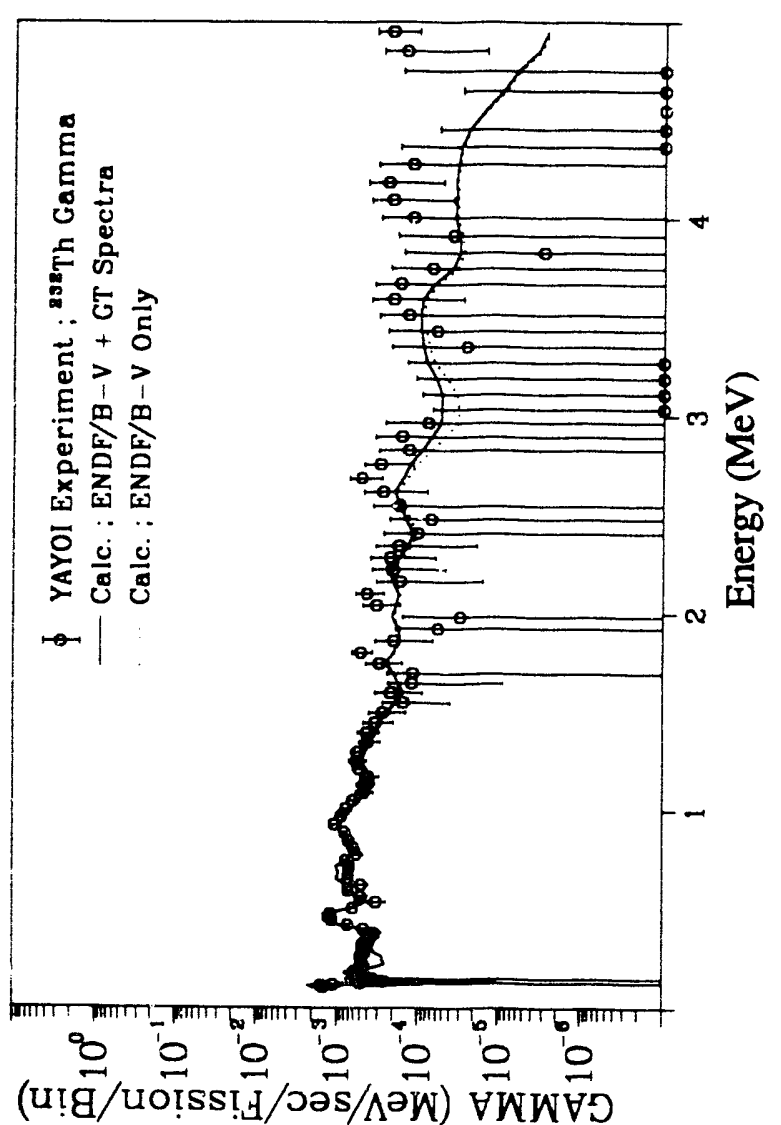

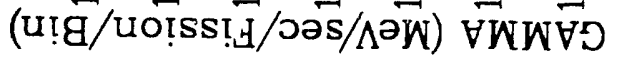

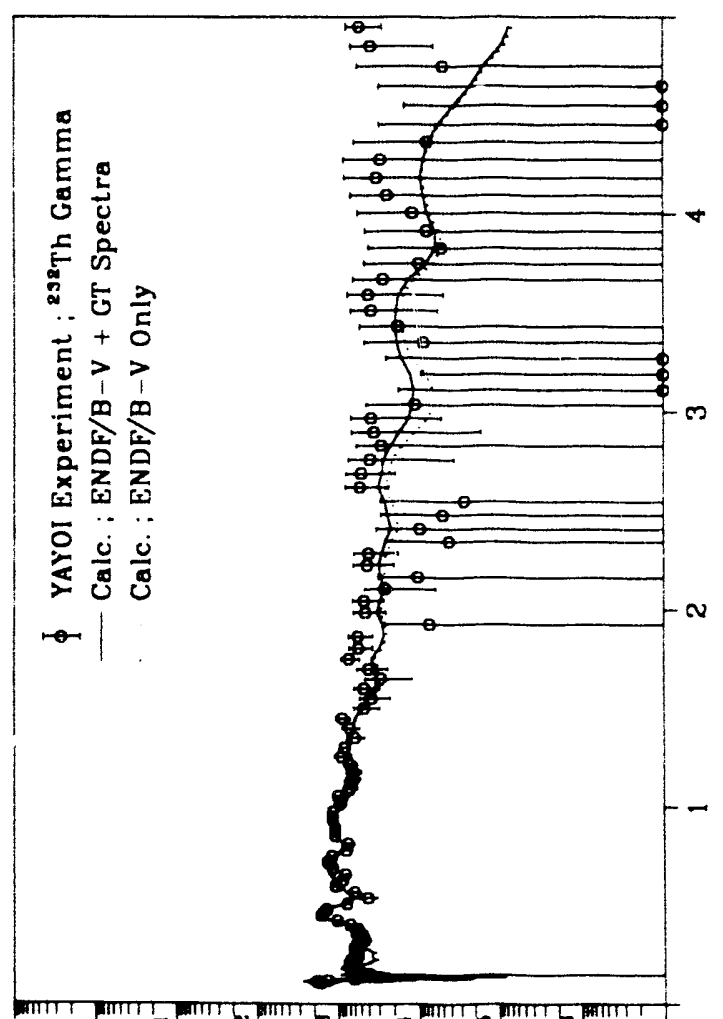

8 io 0 응으은

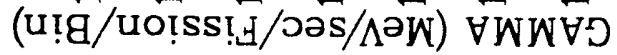

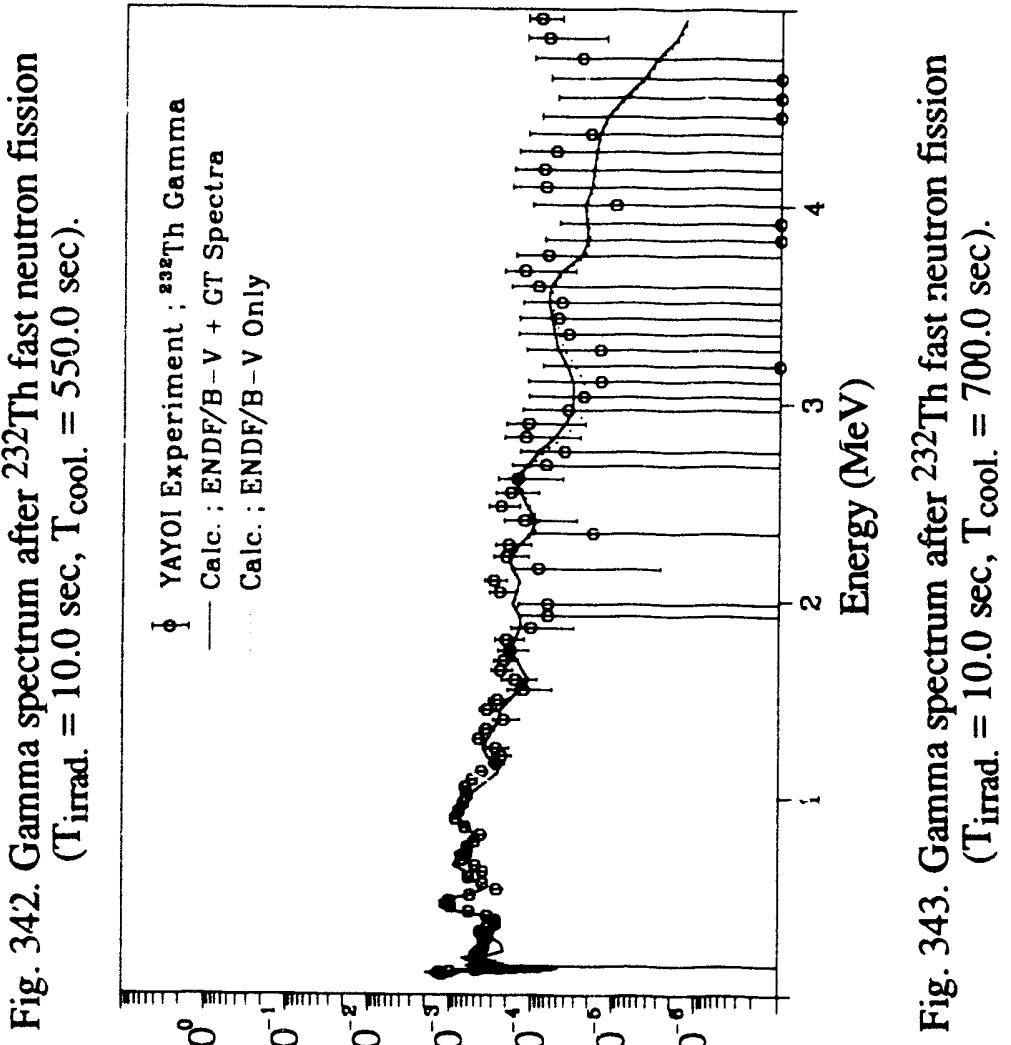

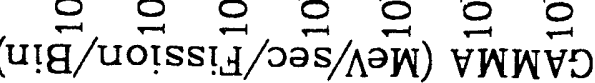

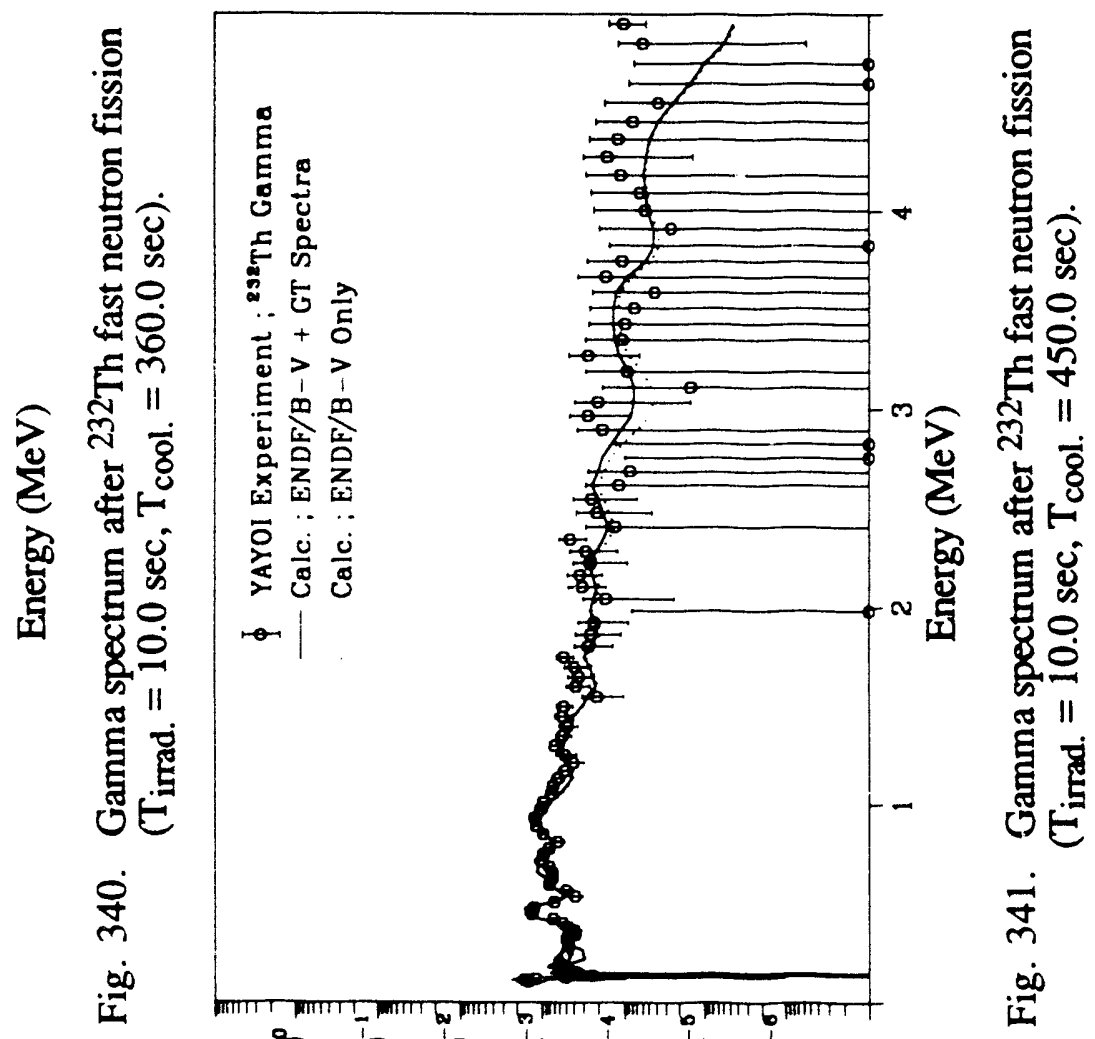

응으응의

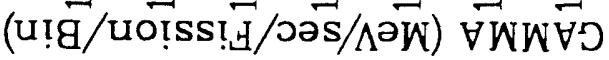



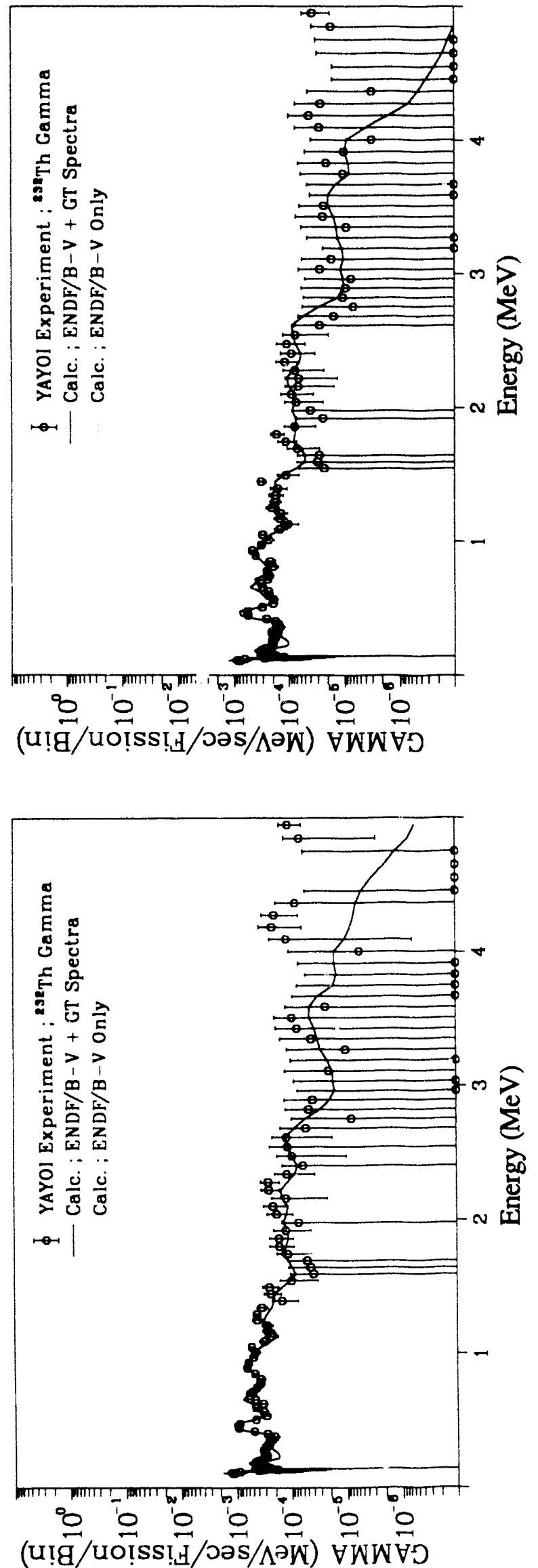

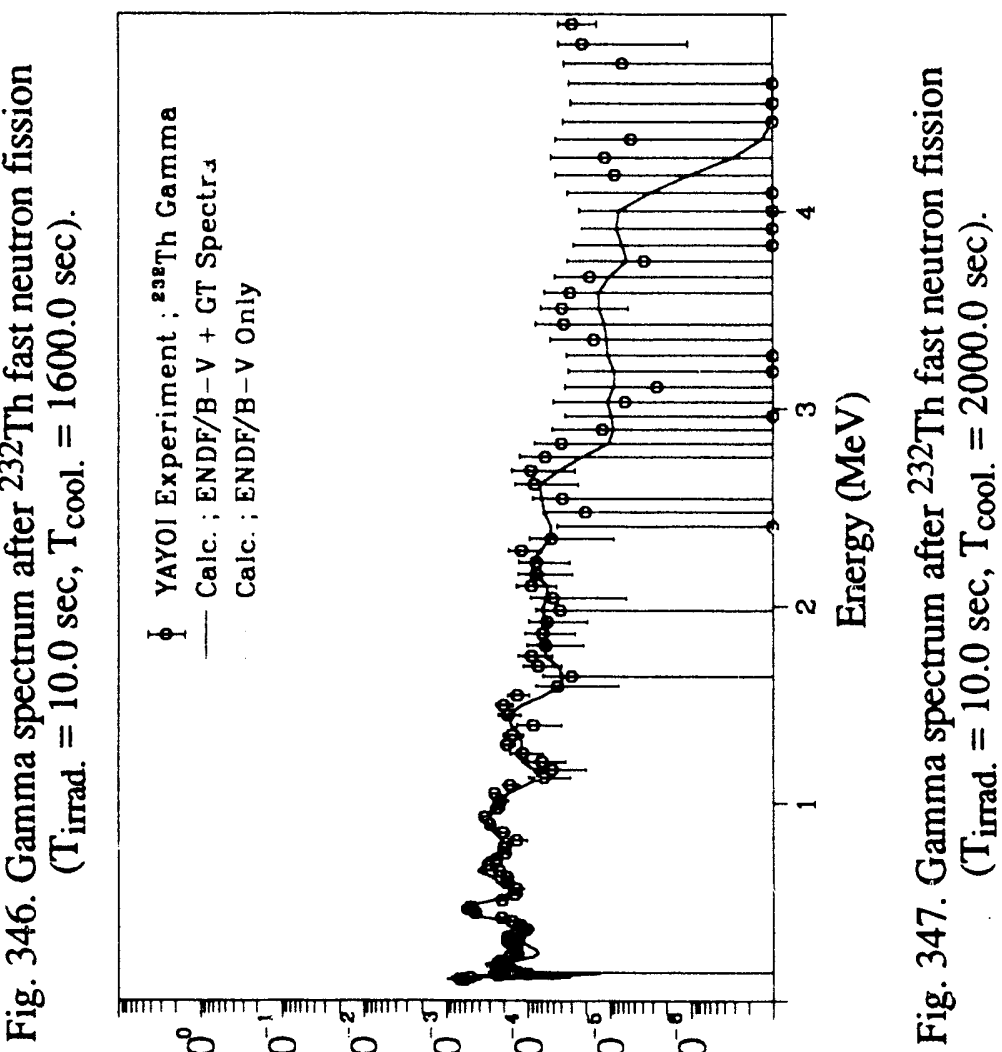

(uṭ/uo!̣ss!

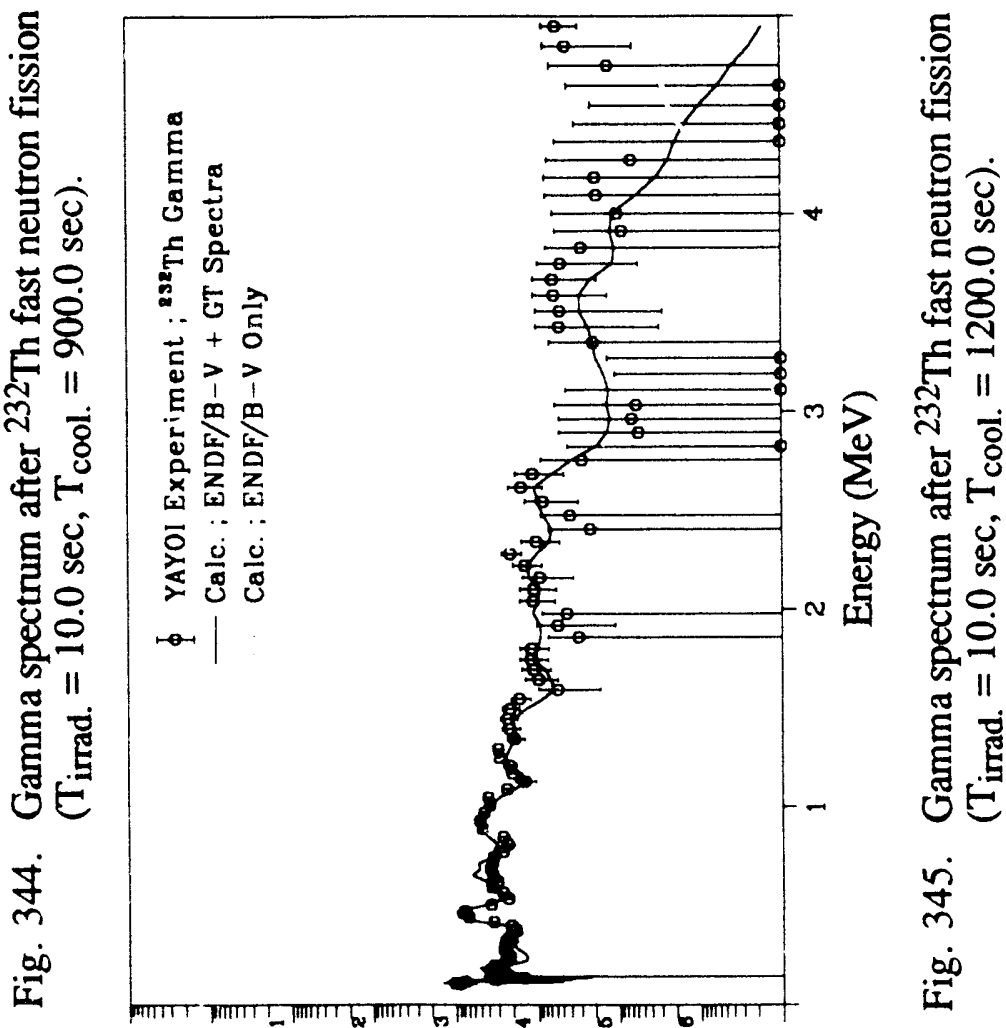

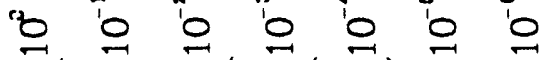
(u!g/uo!̣s! 

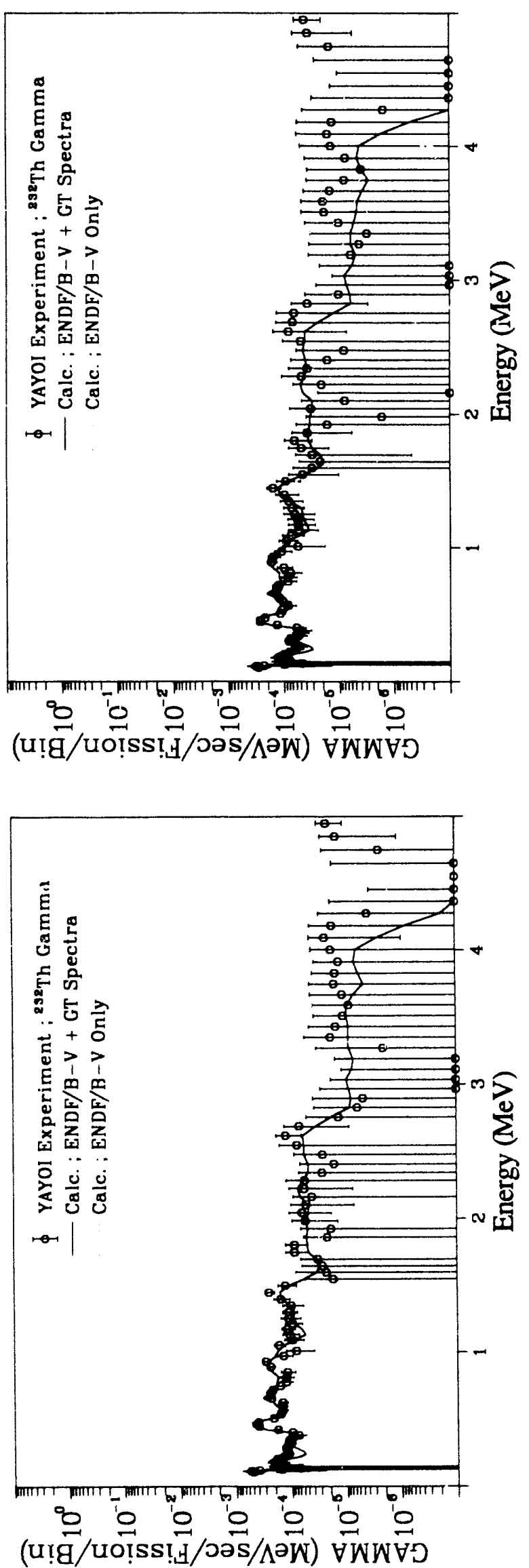
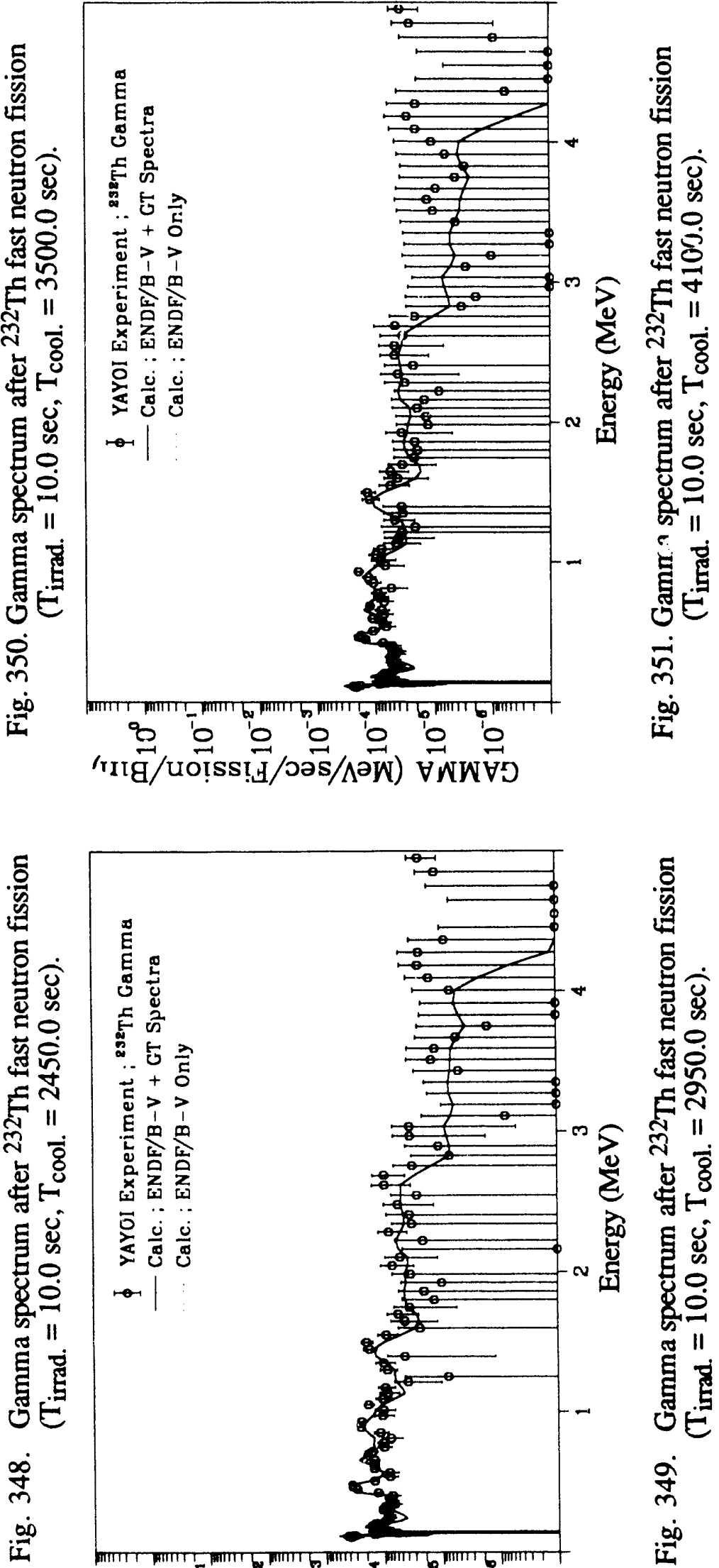

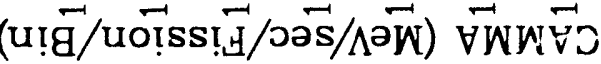




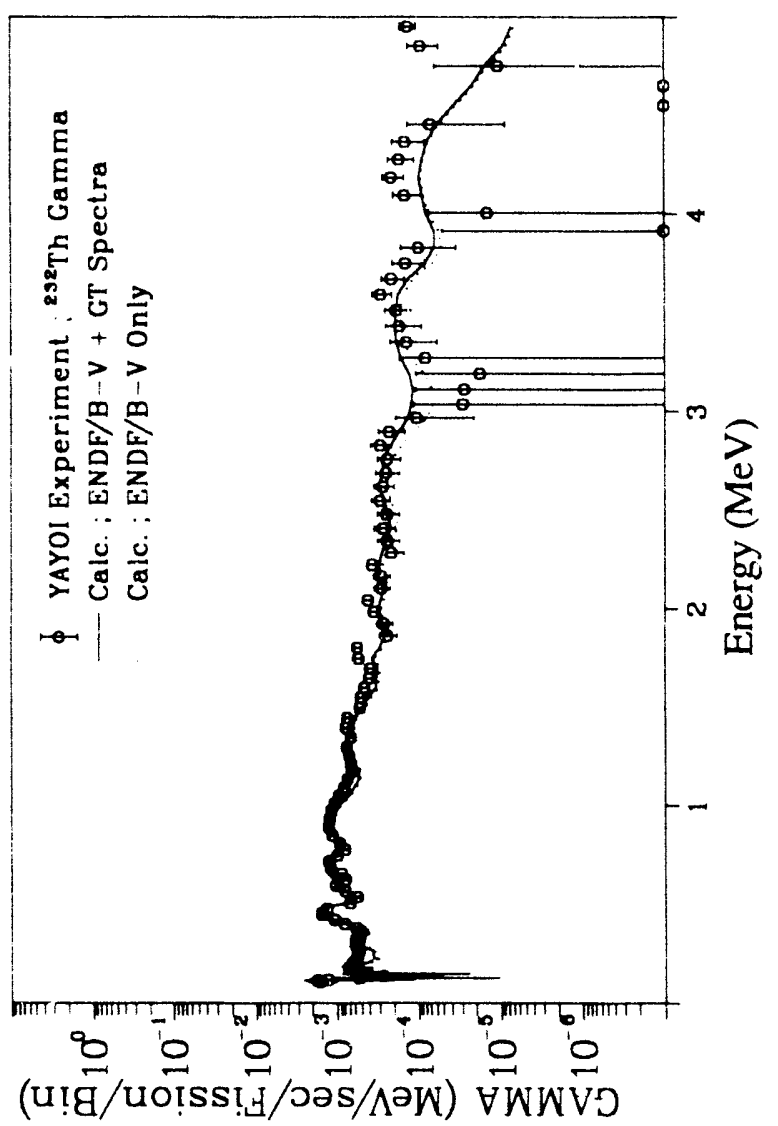

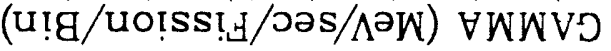
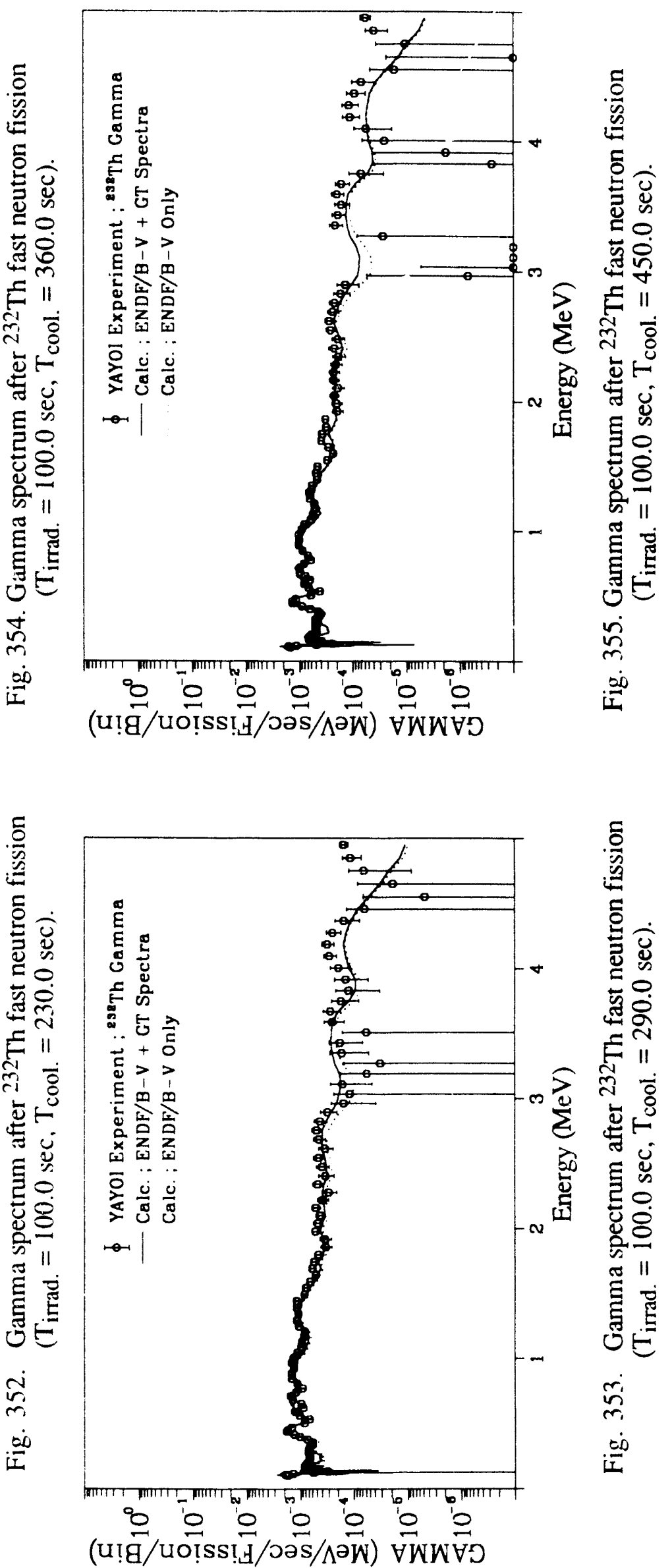

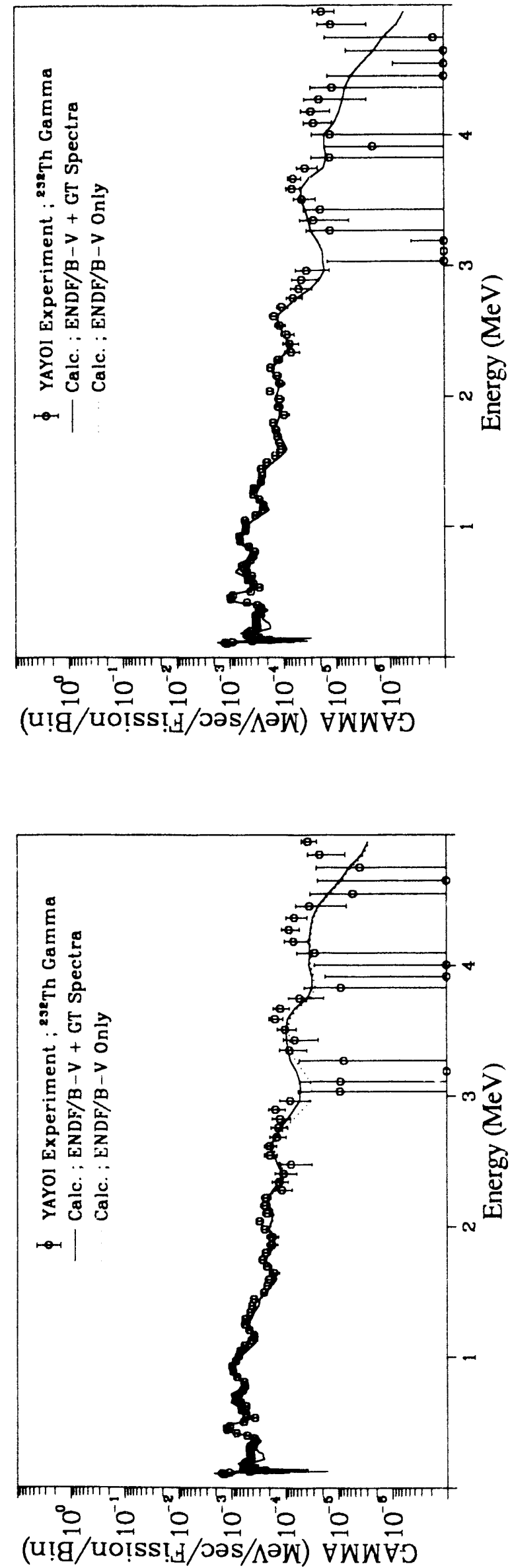

苍
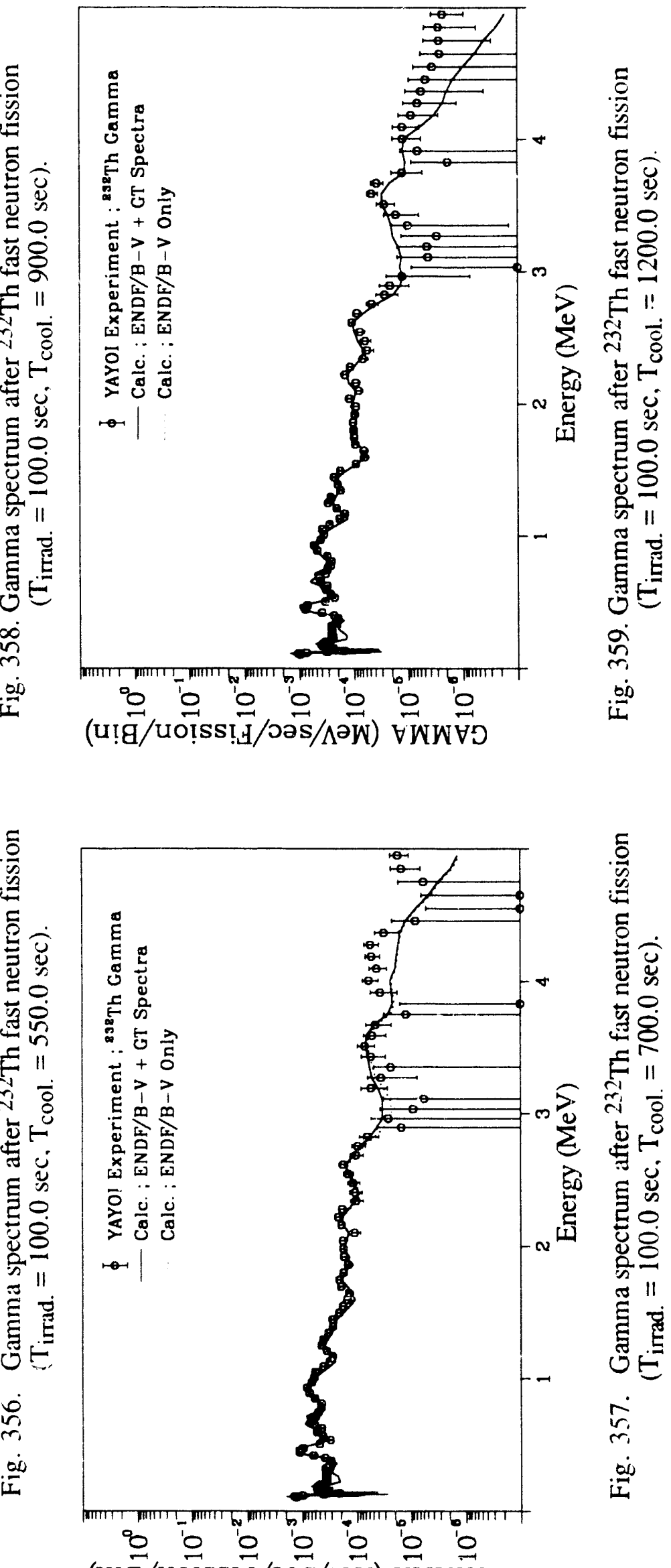


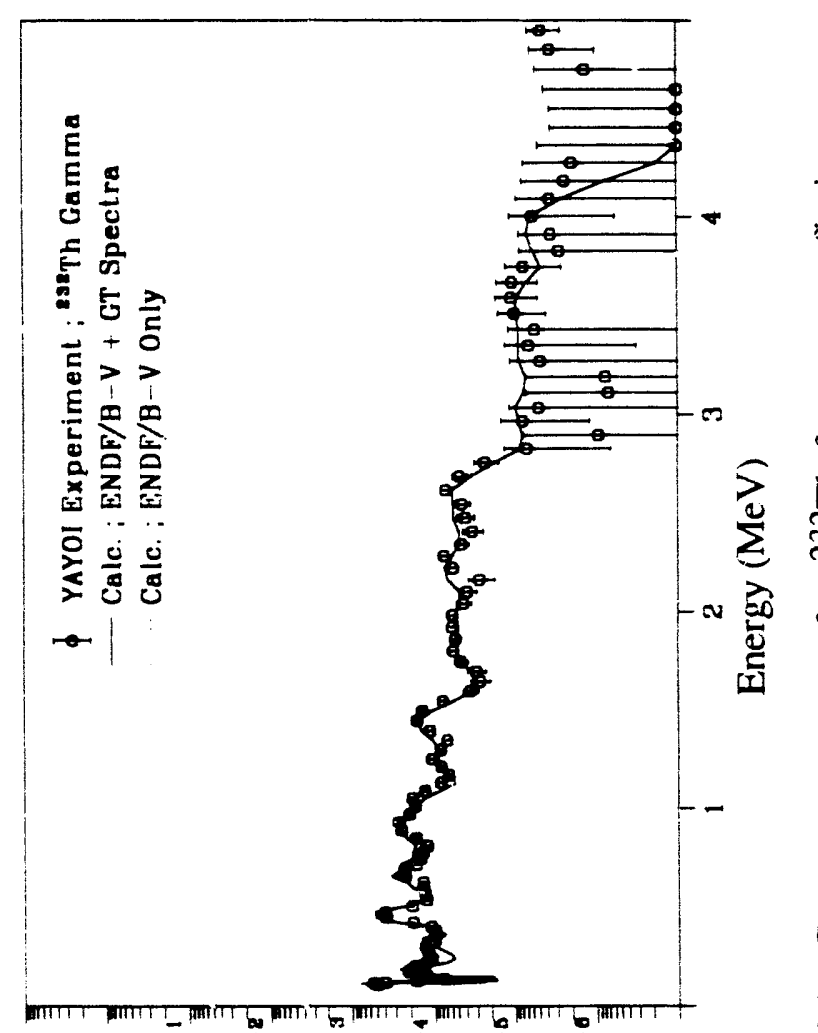

으 으으으으응 (utg/uo!ss!
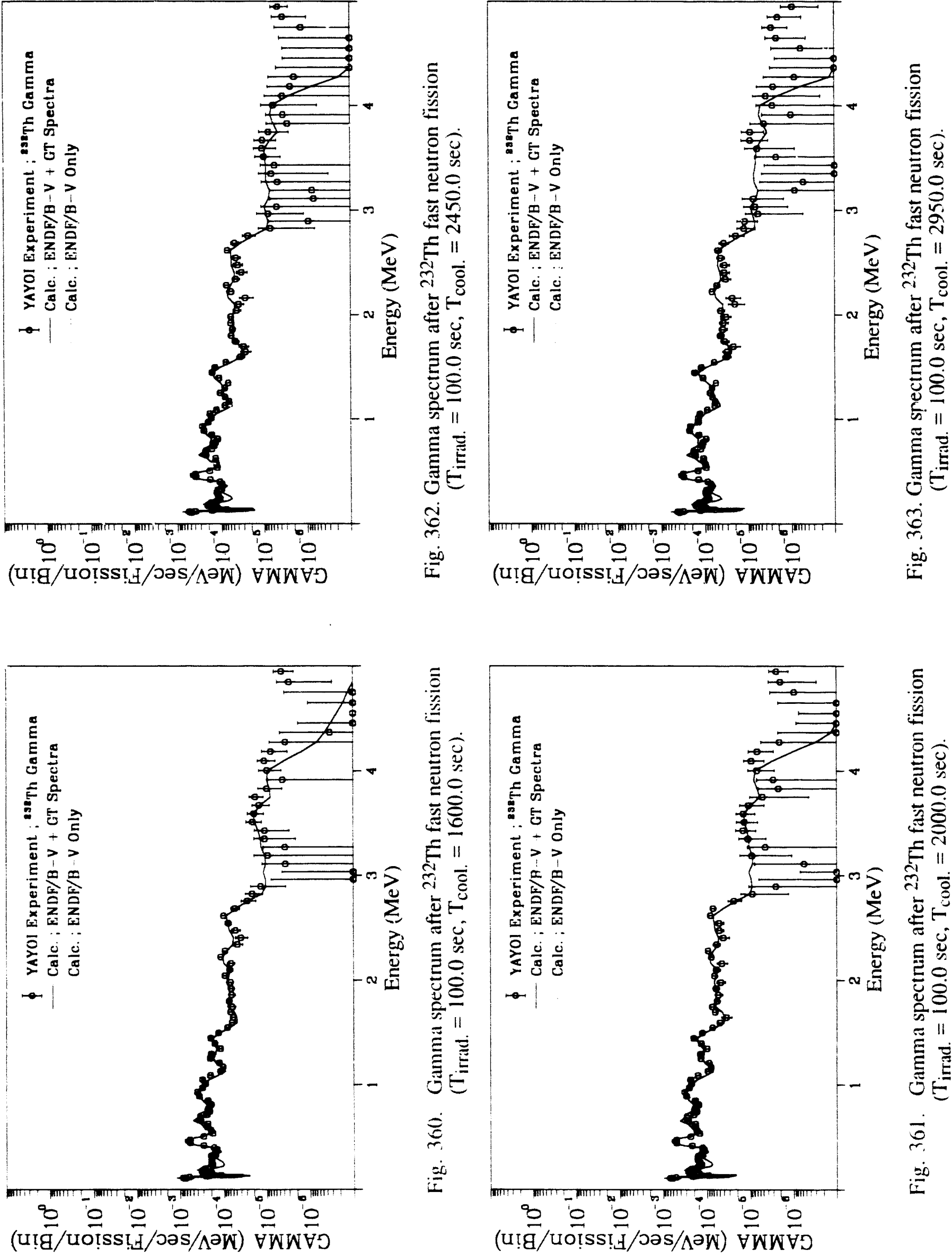

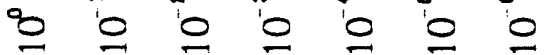

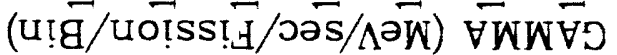



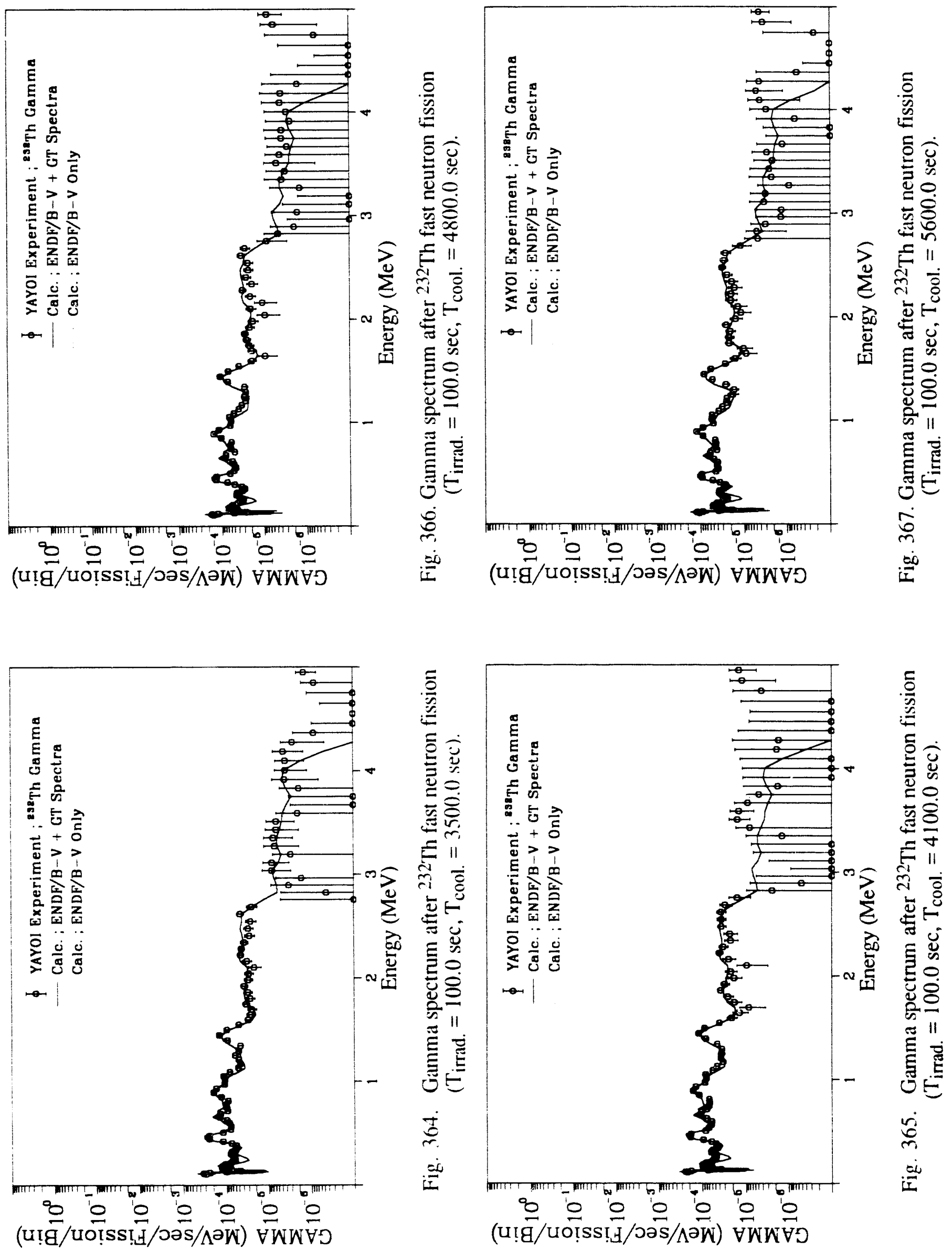

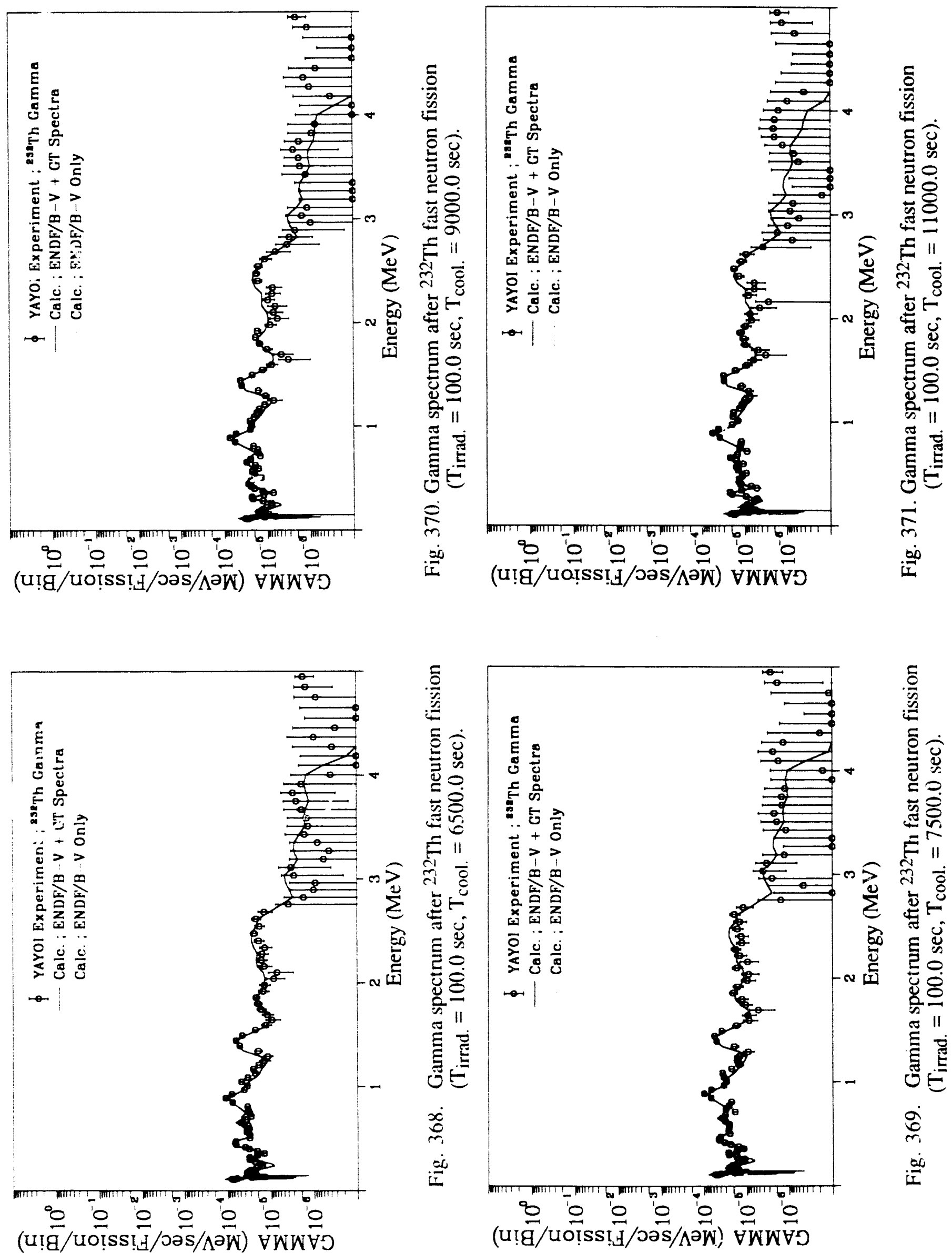


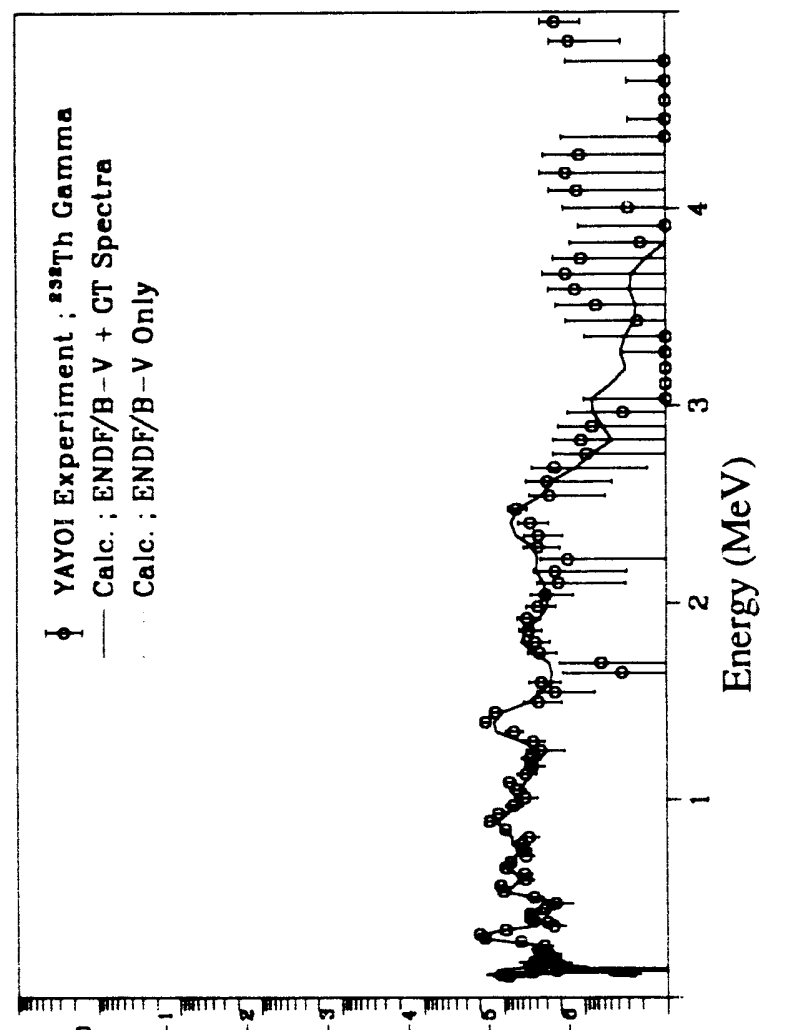

으 으 으 으 으 으

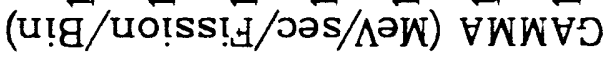

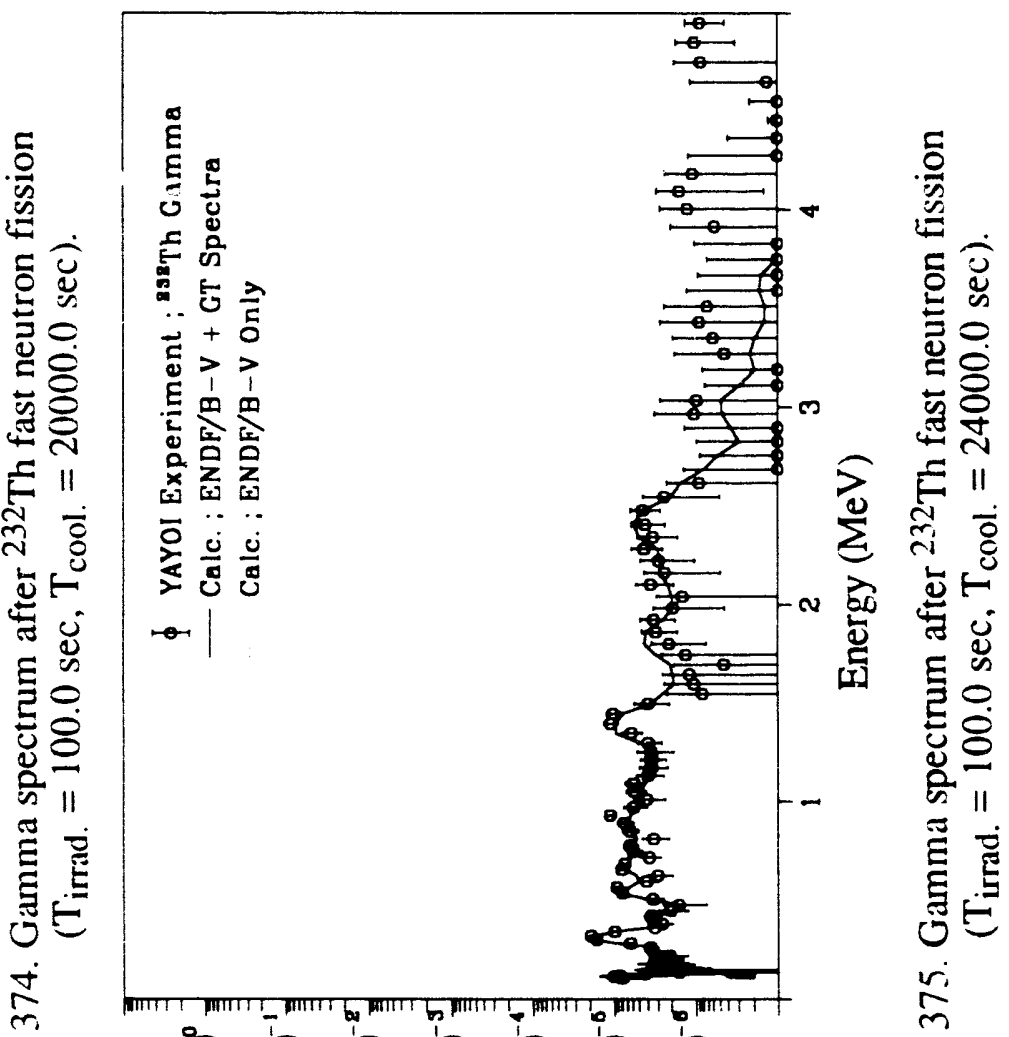

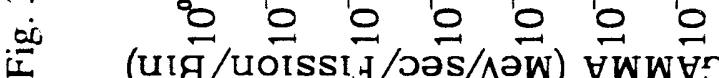

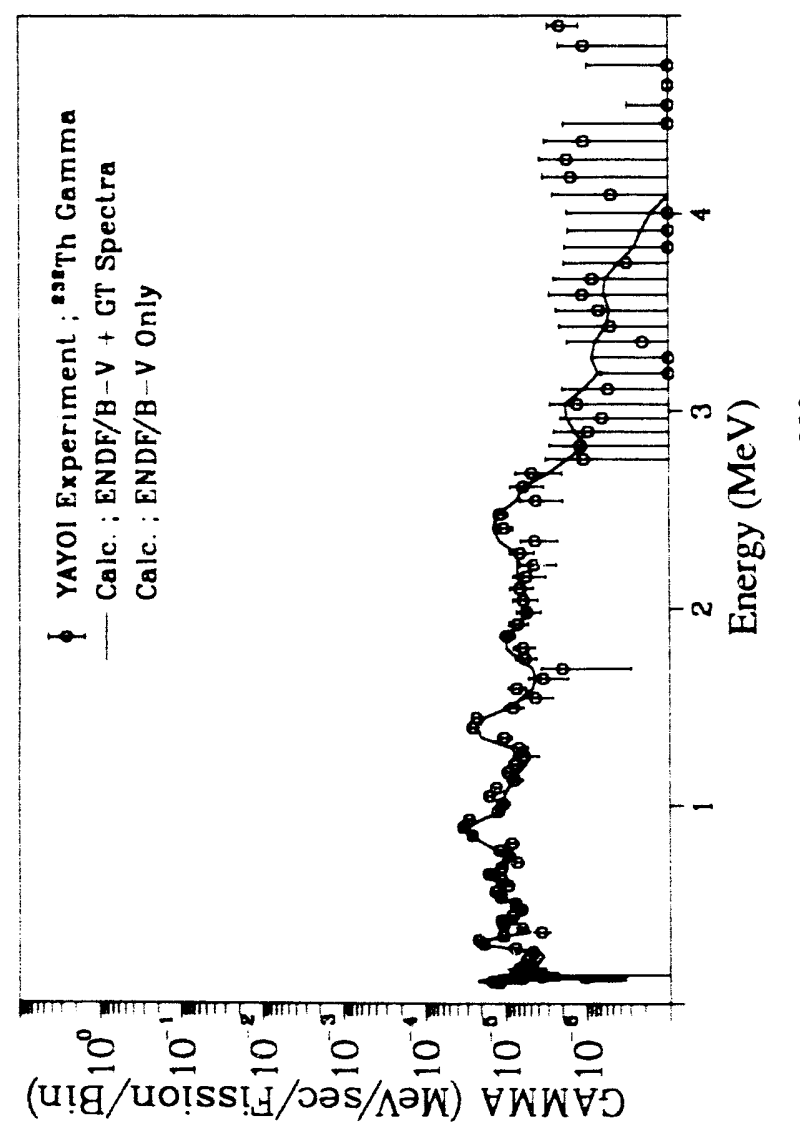

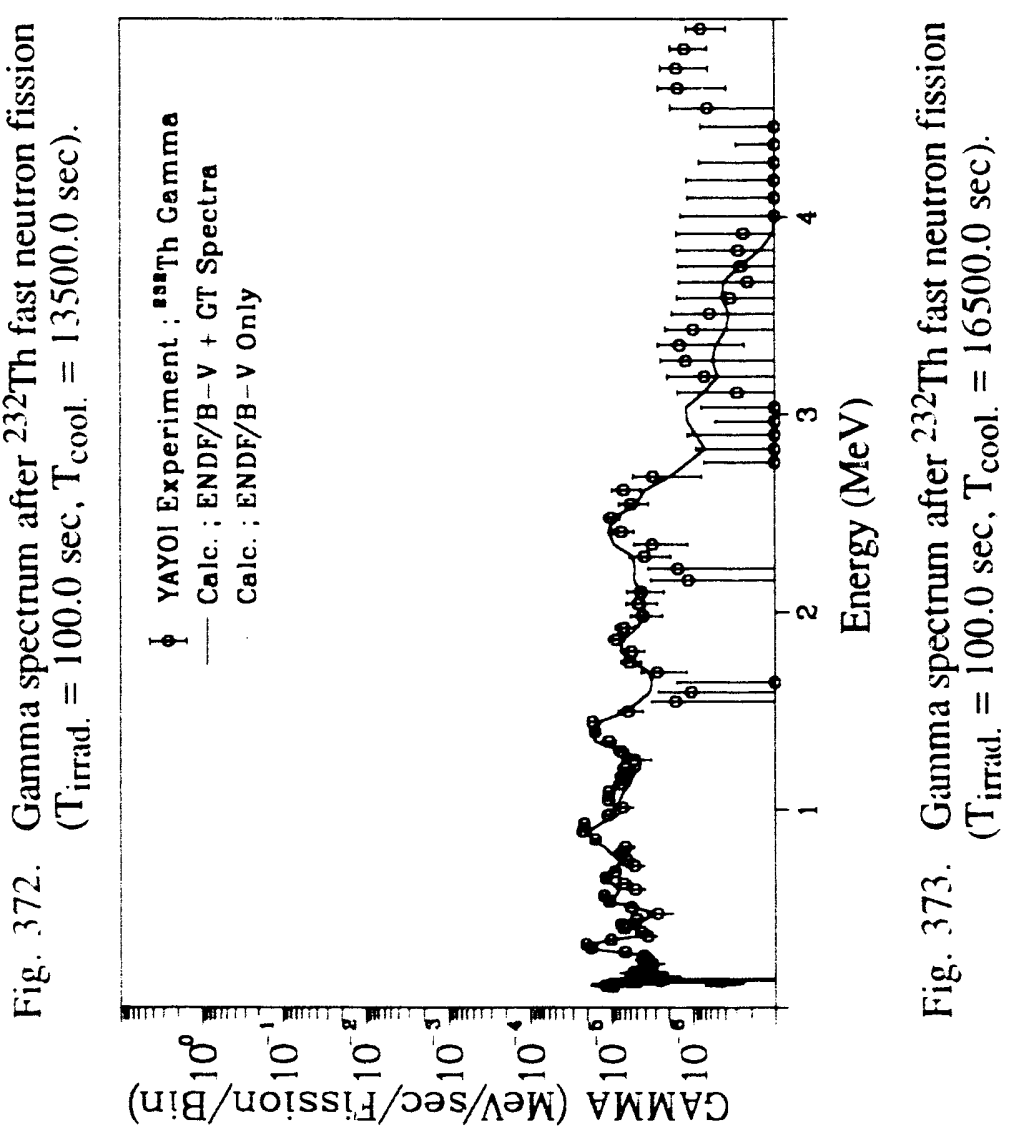



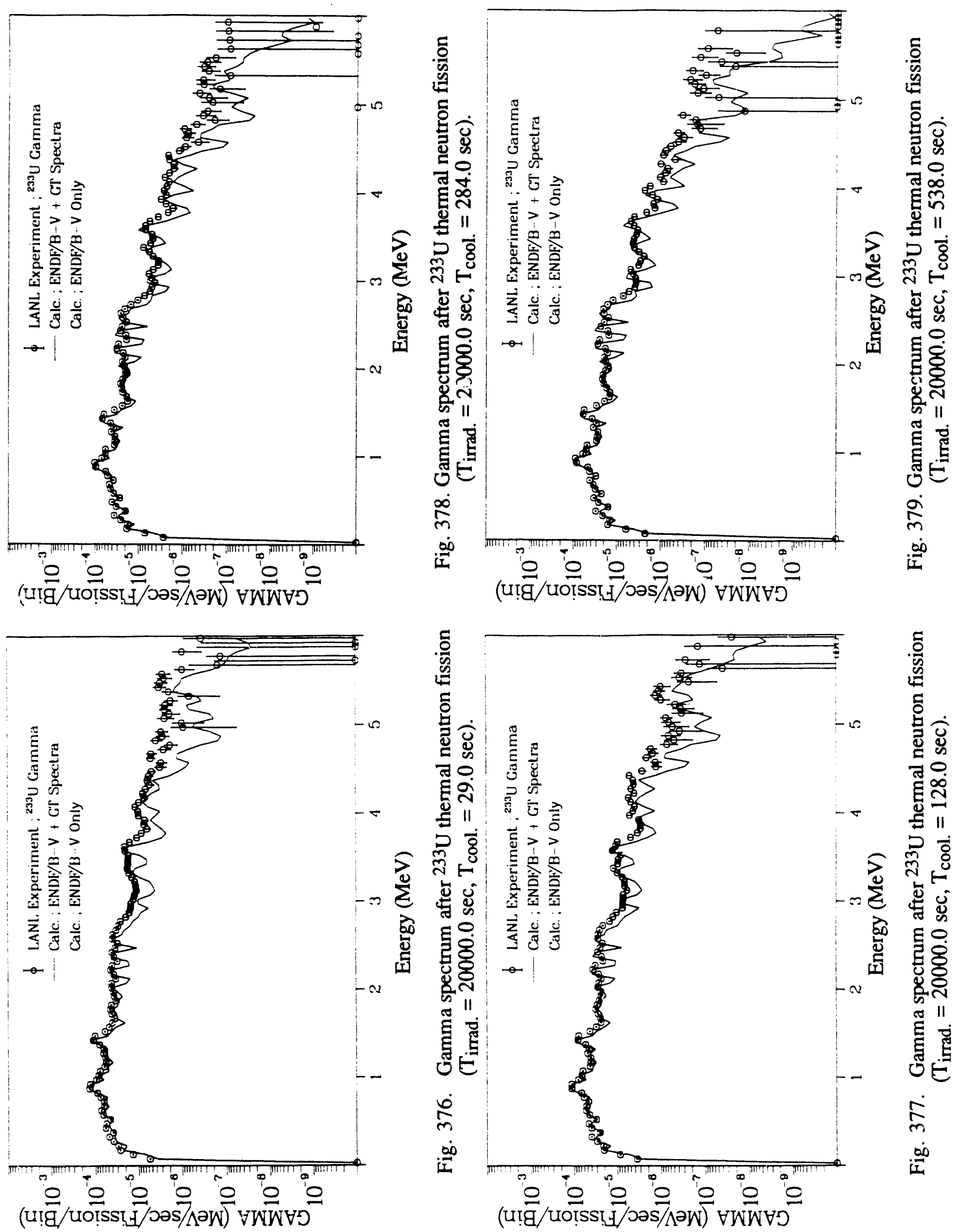


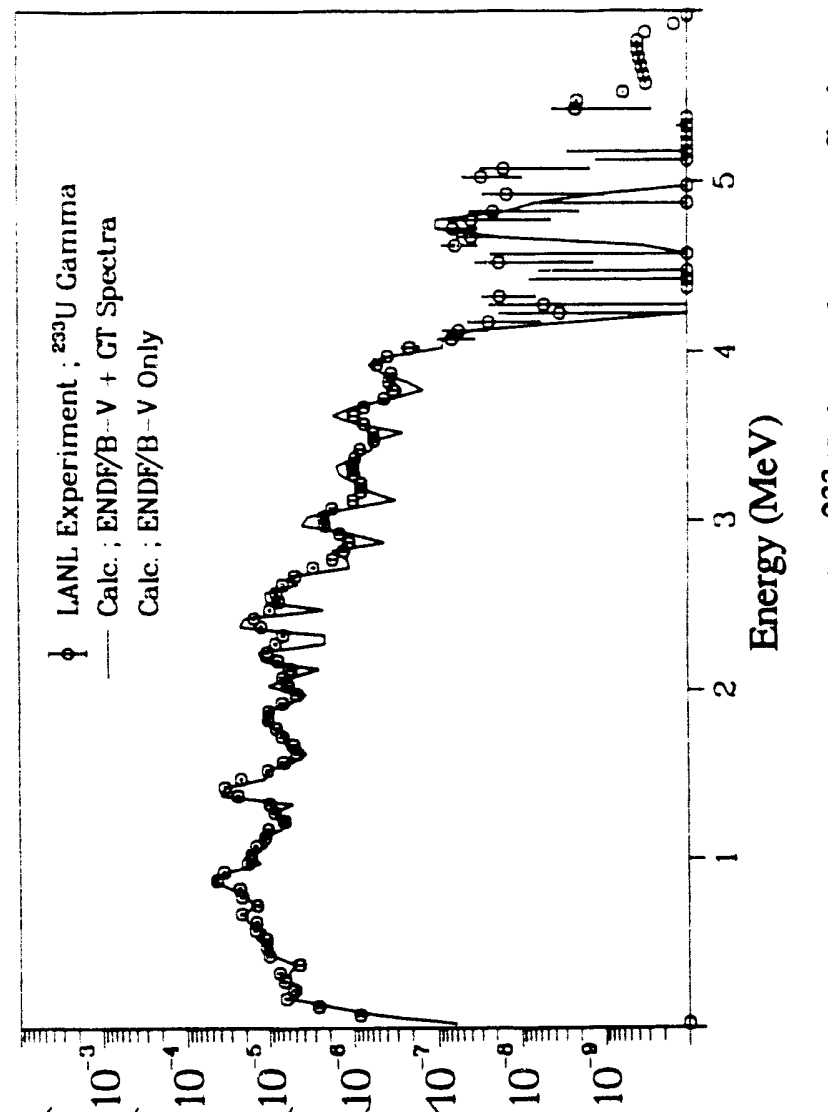

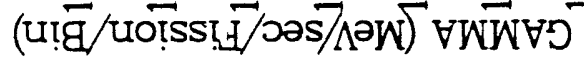

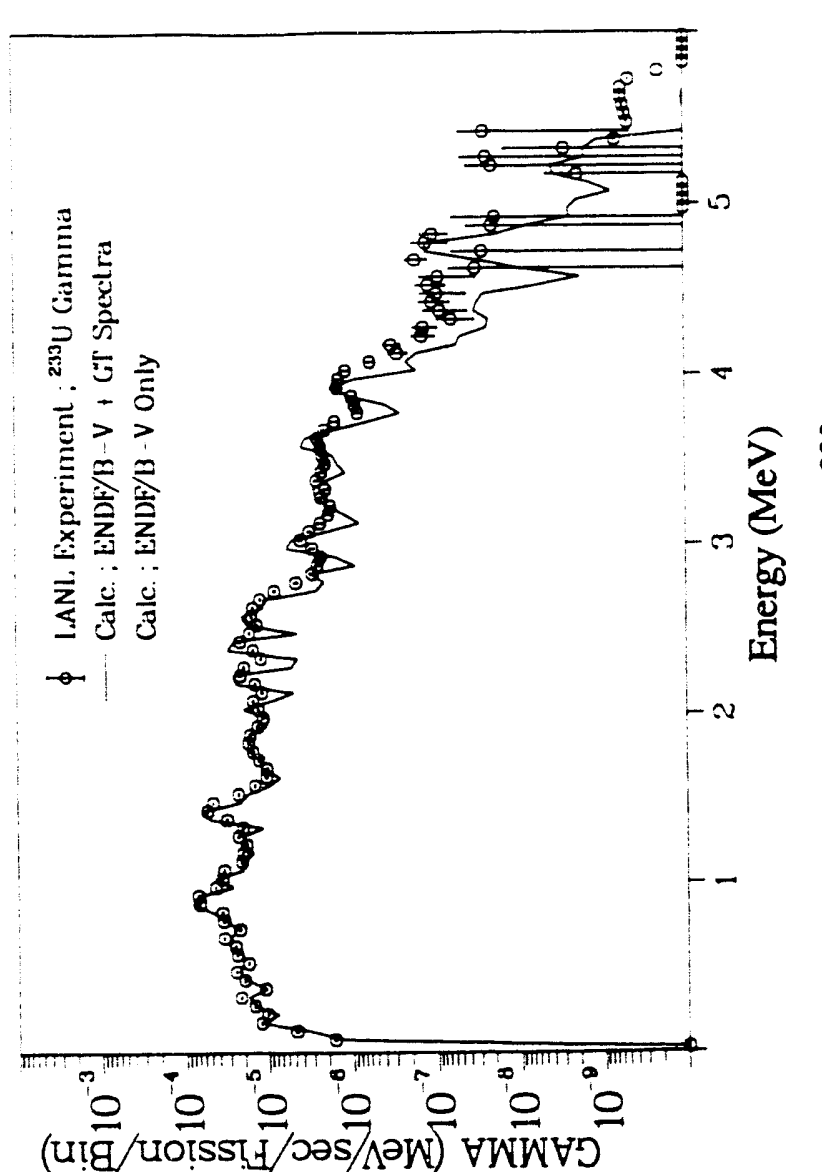

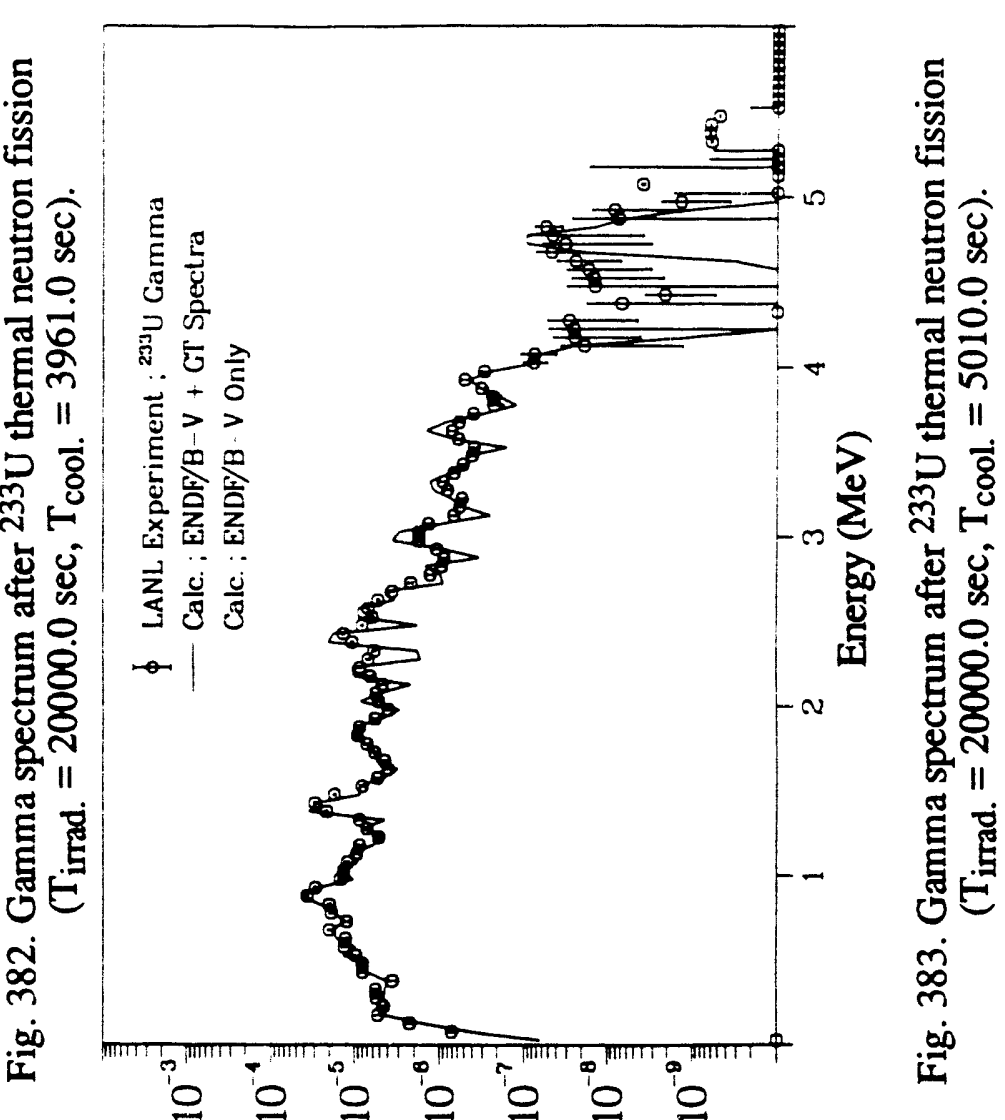

(u!̣/uotss!

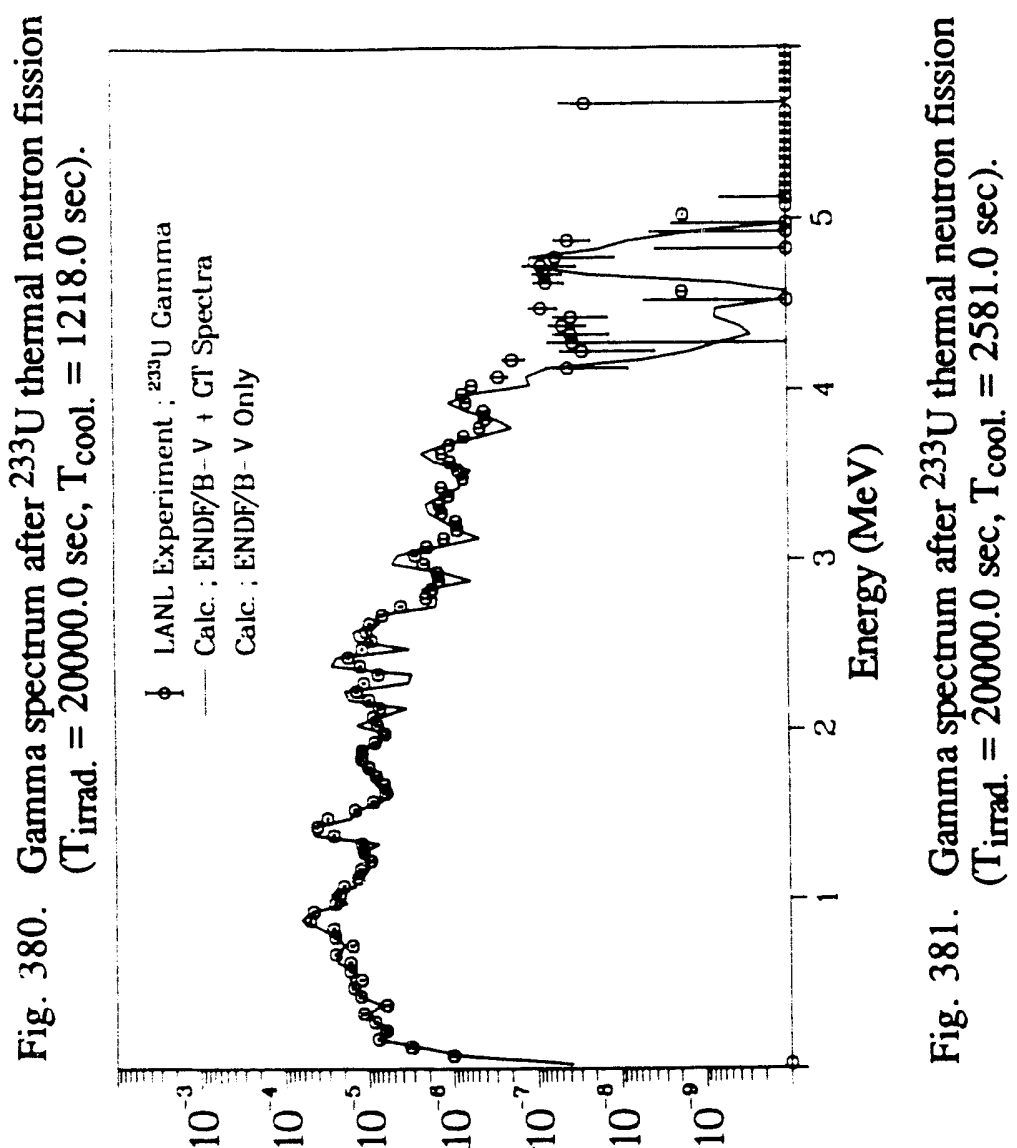

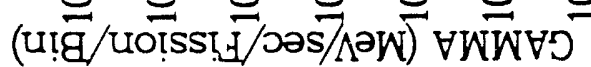




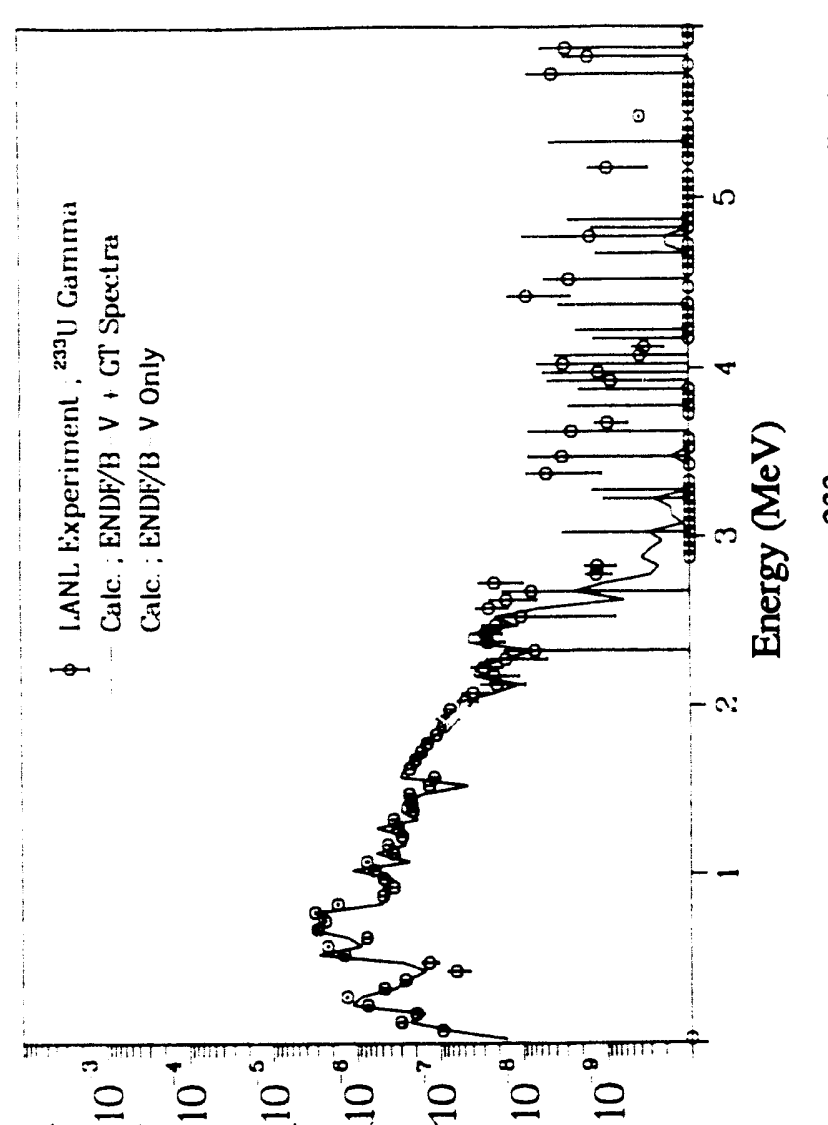

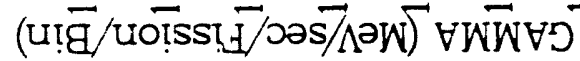

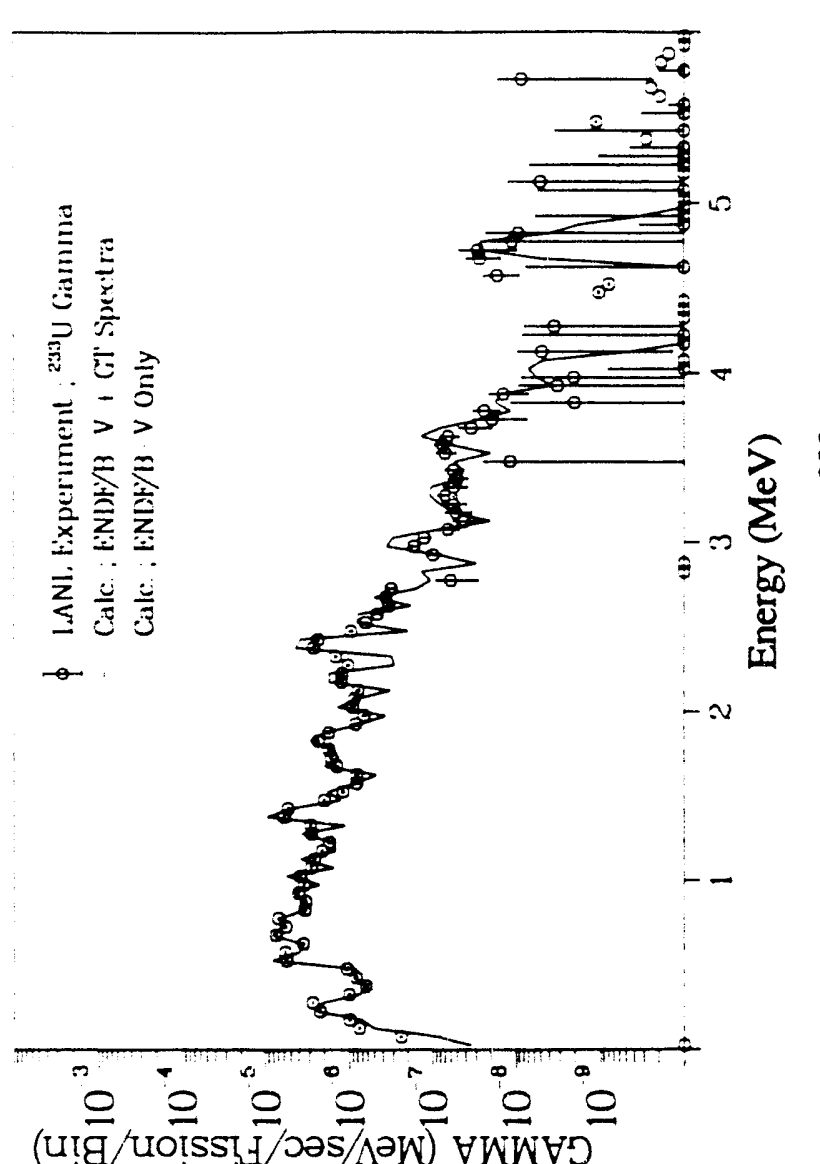

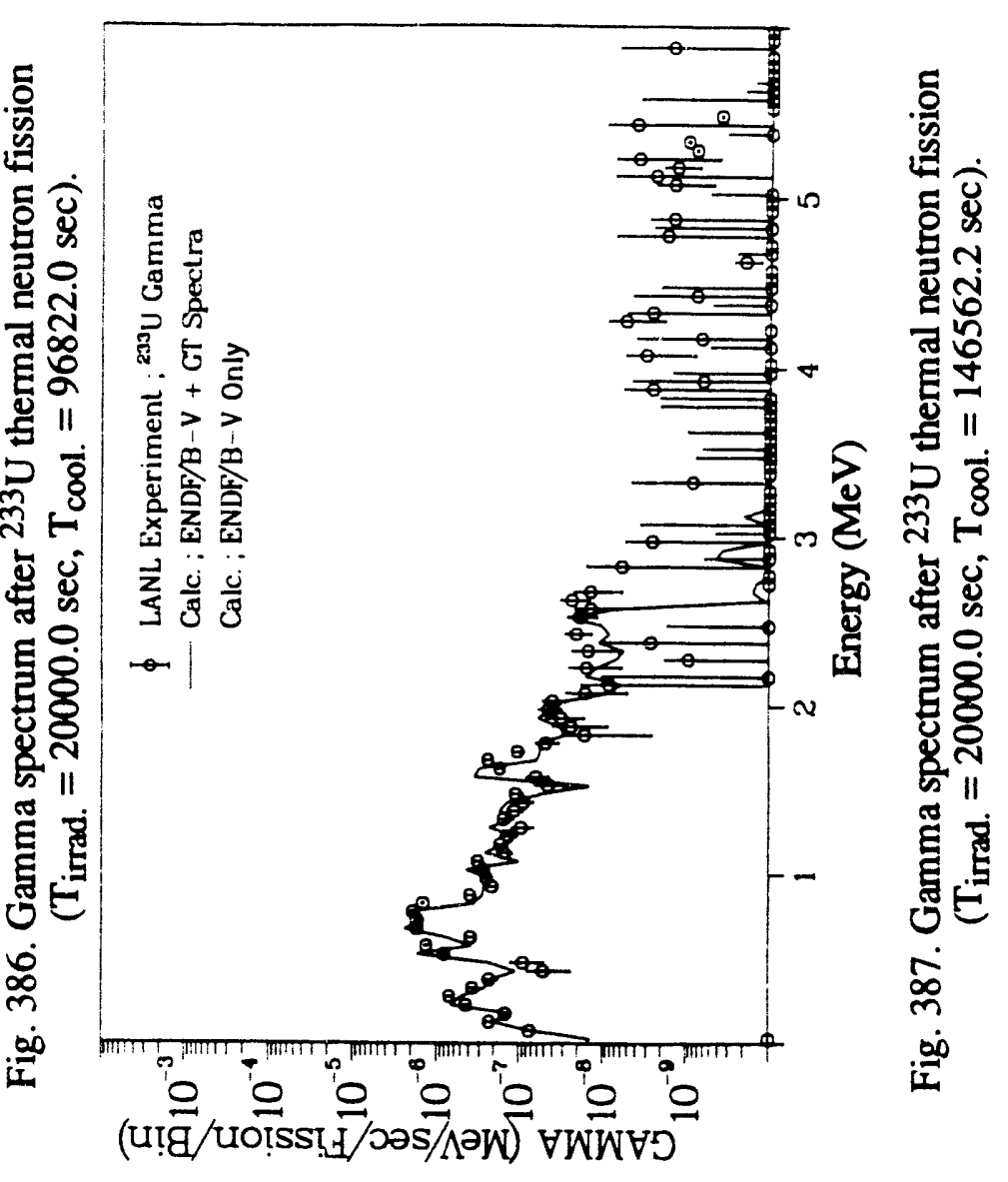

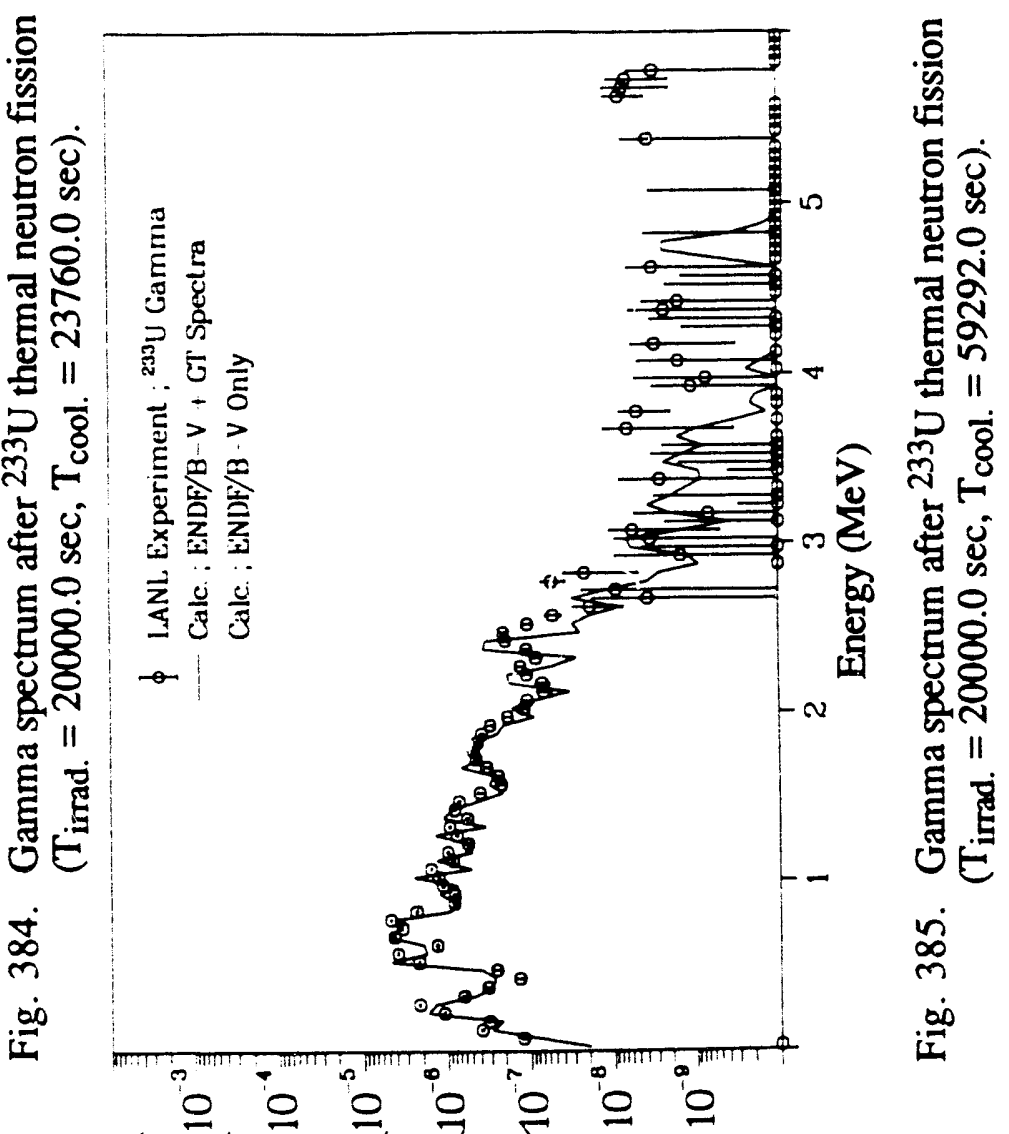

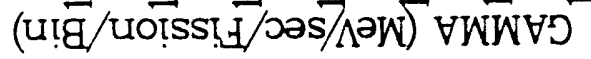




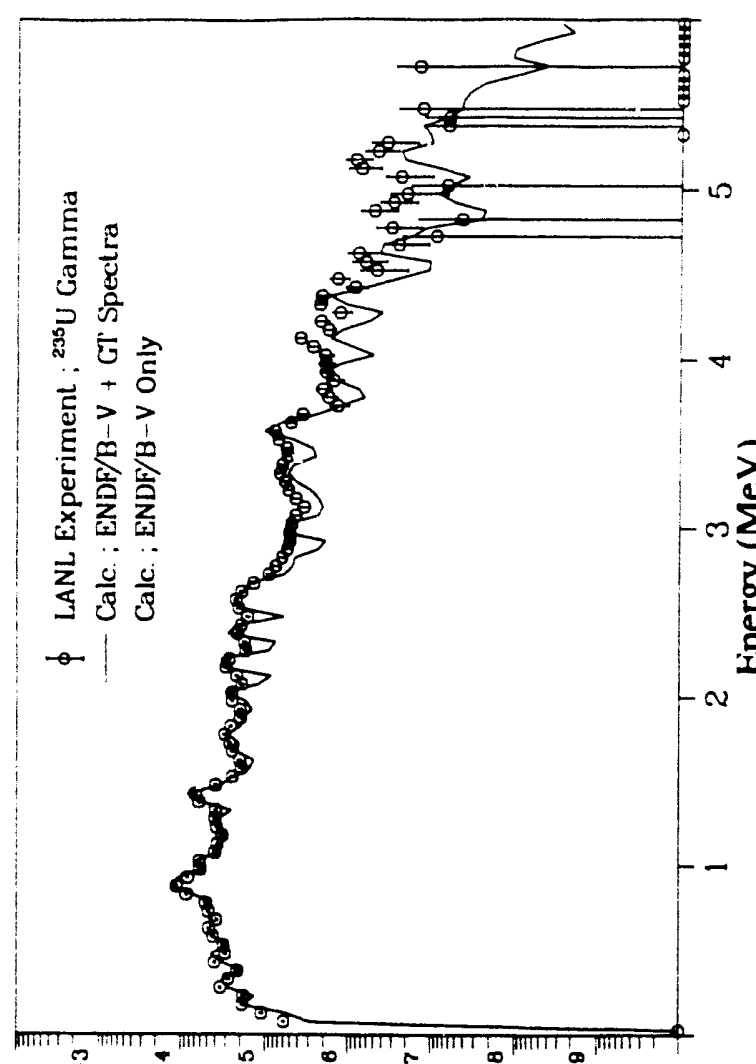

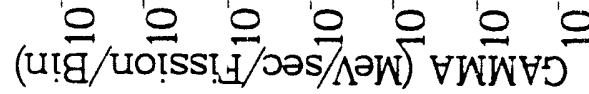

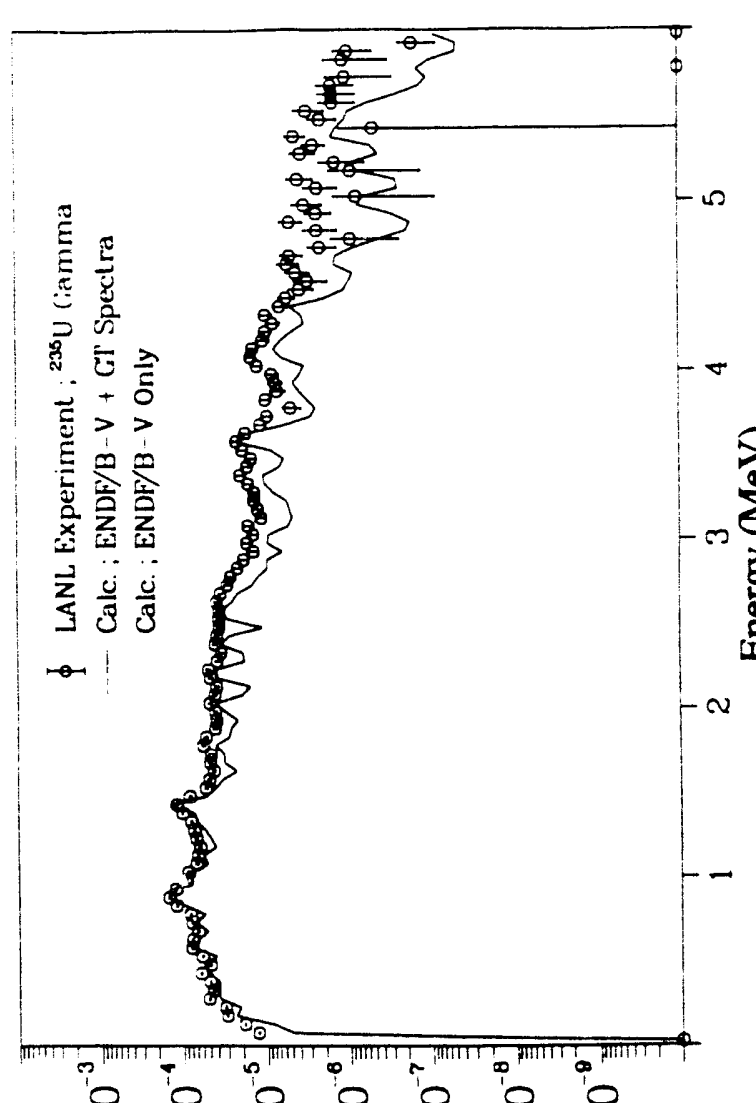

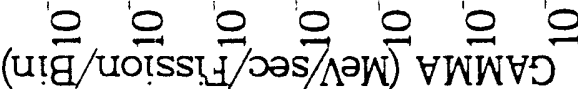

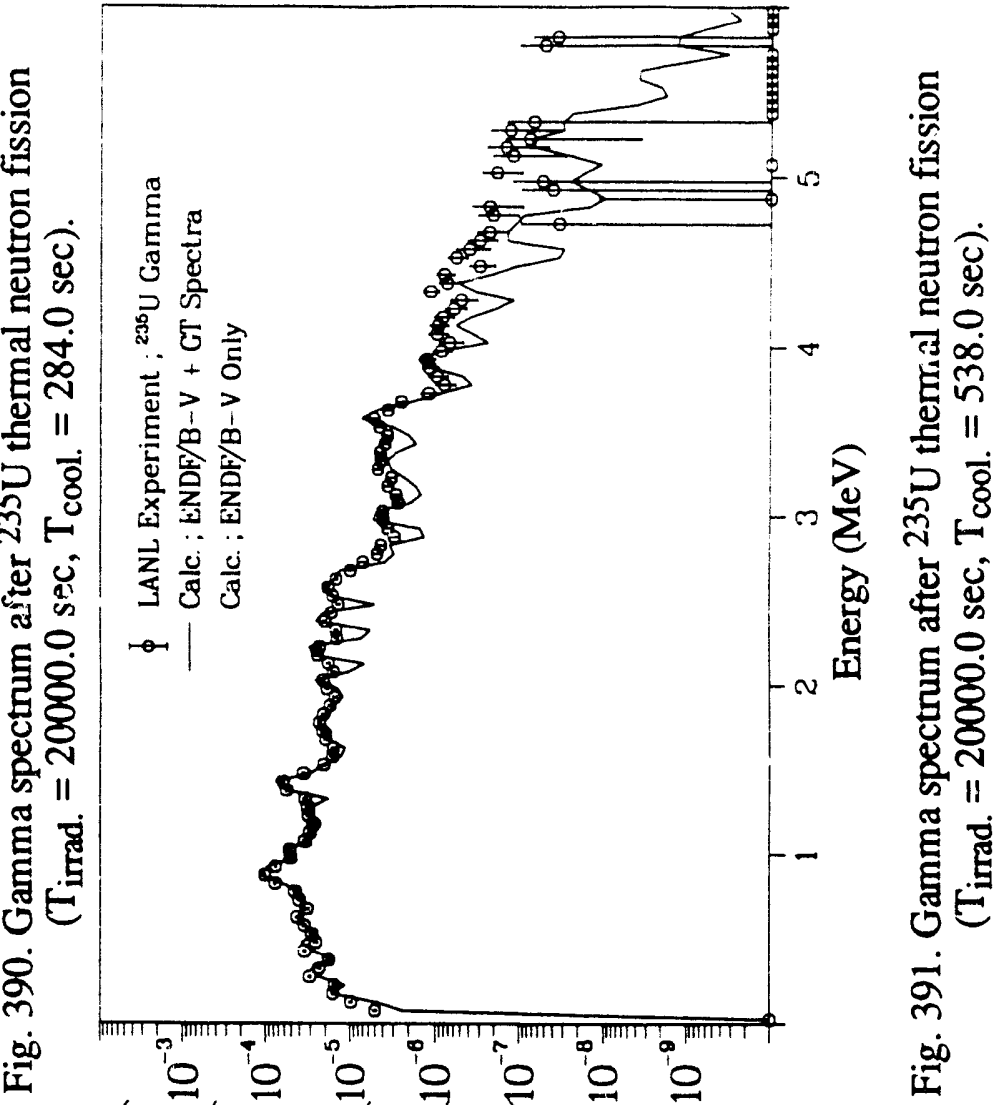

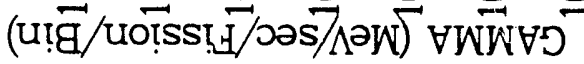
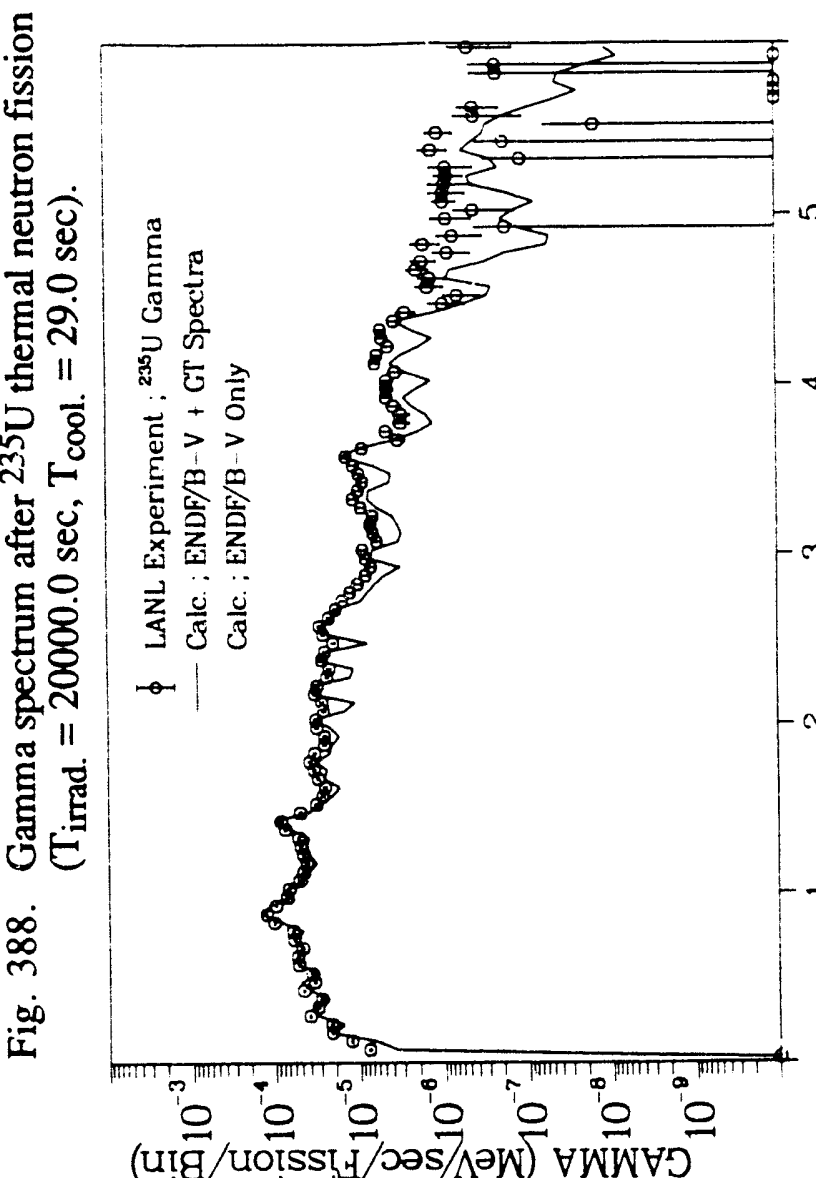

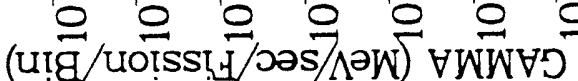



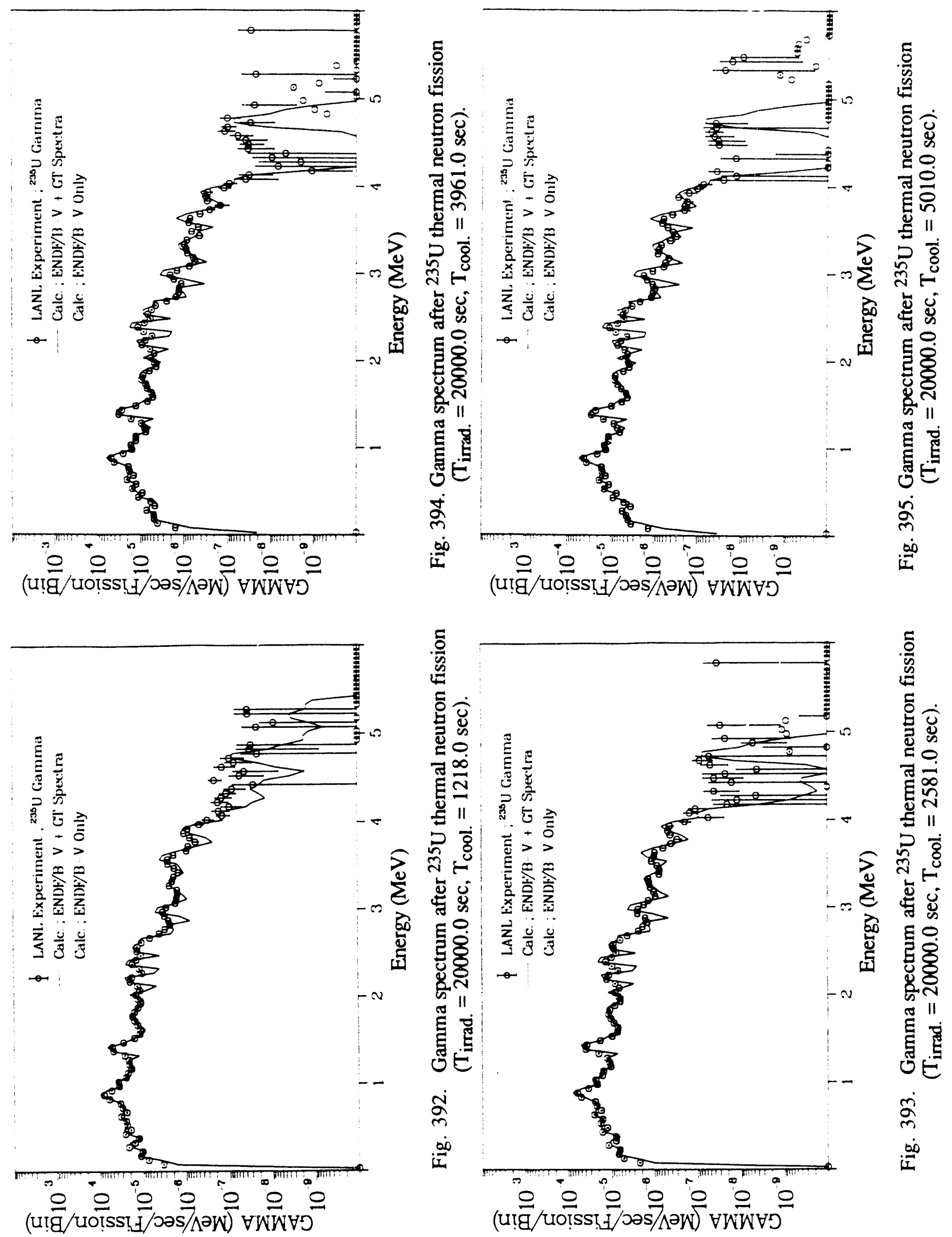


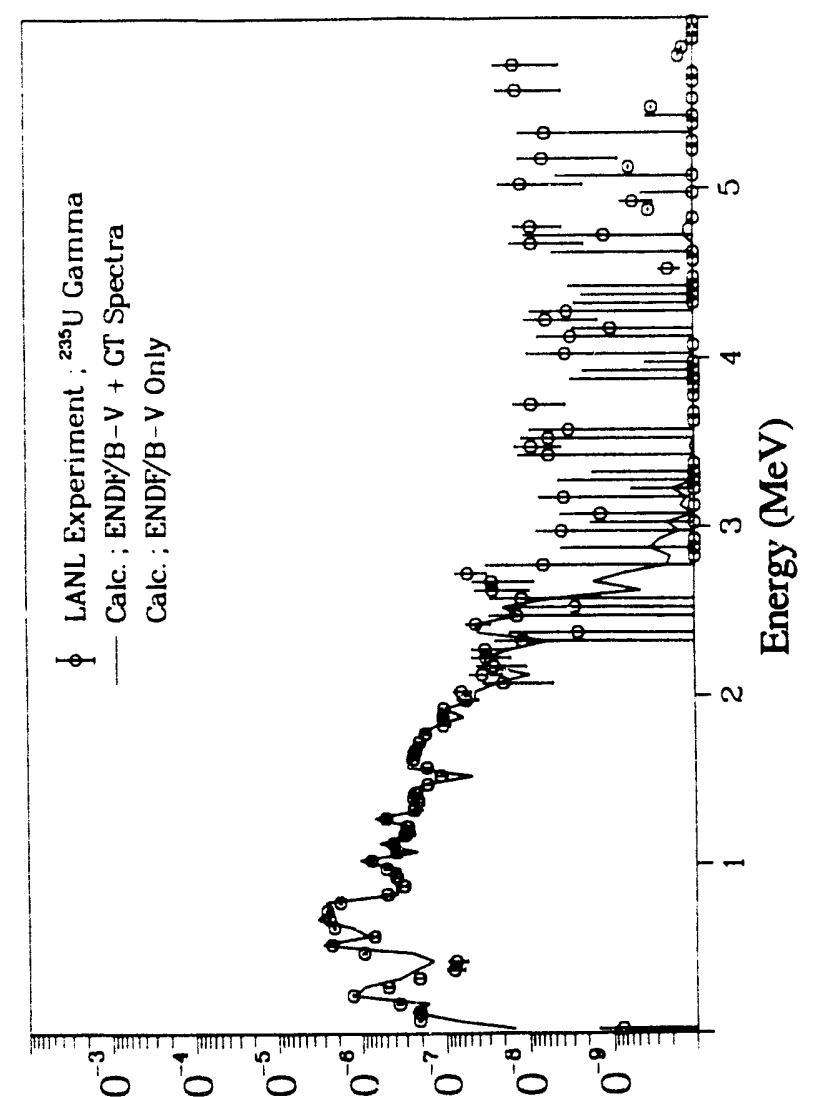

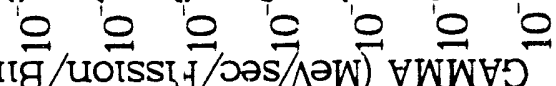

(utg.

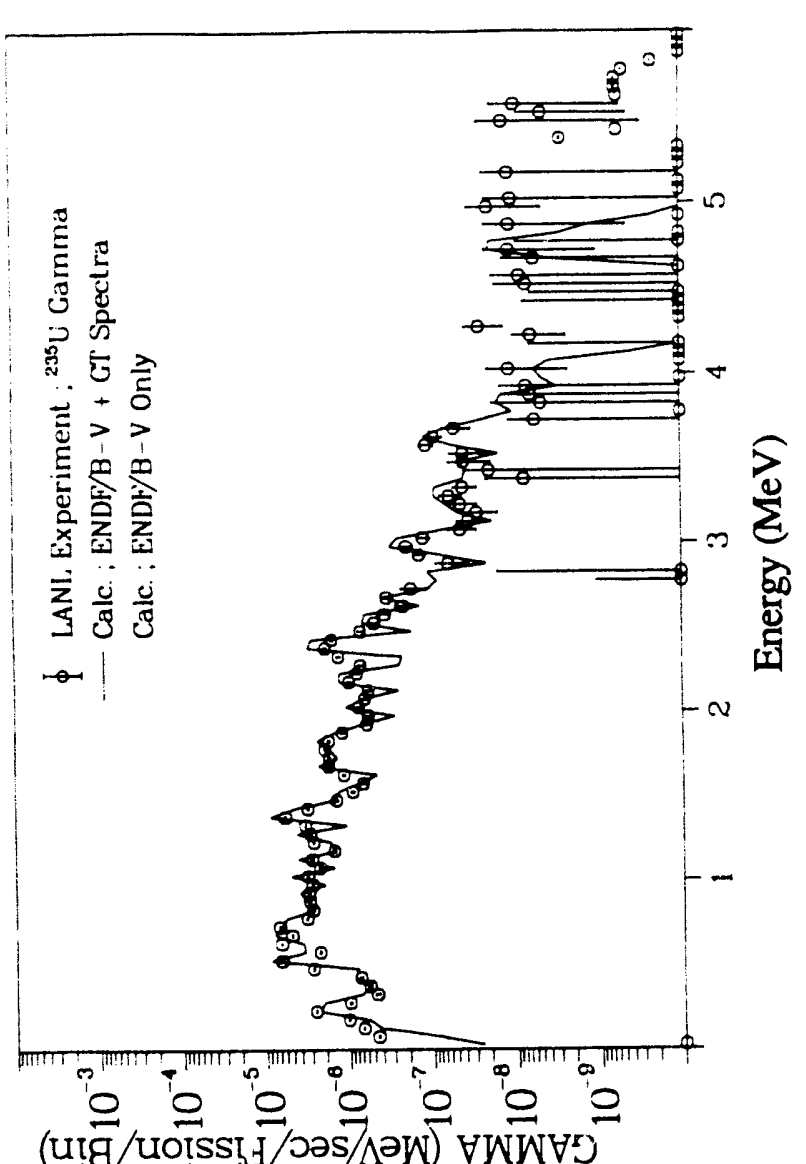

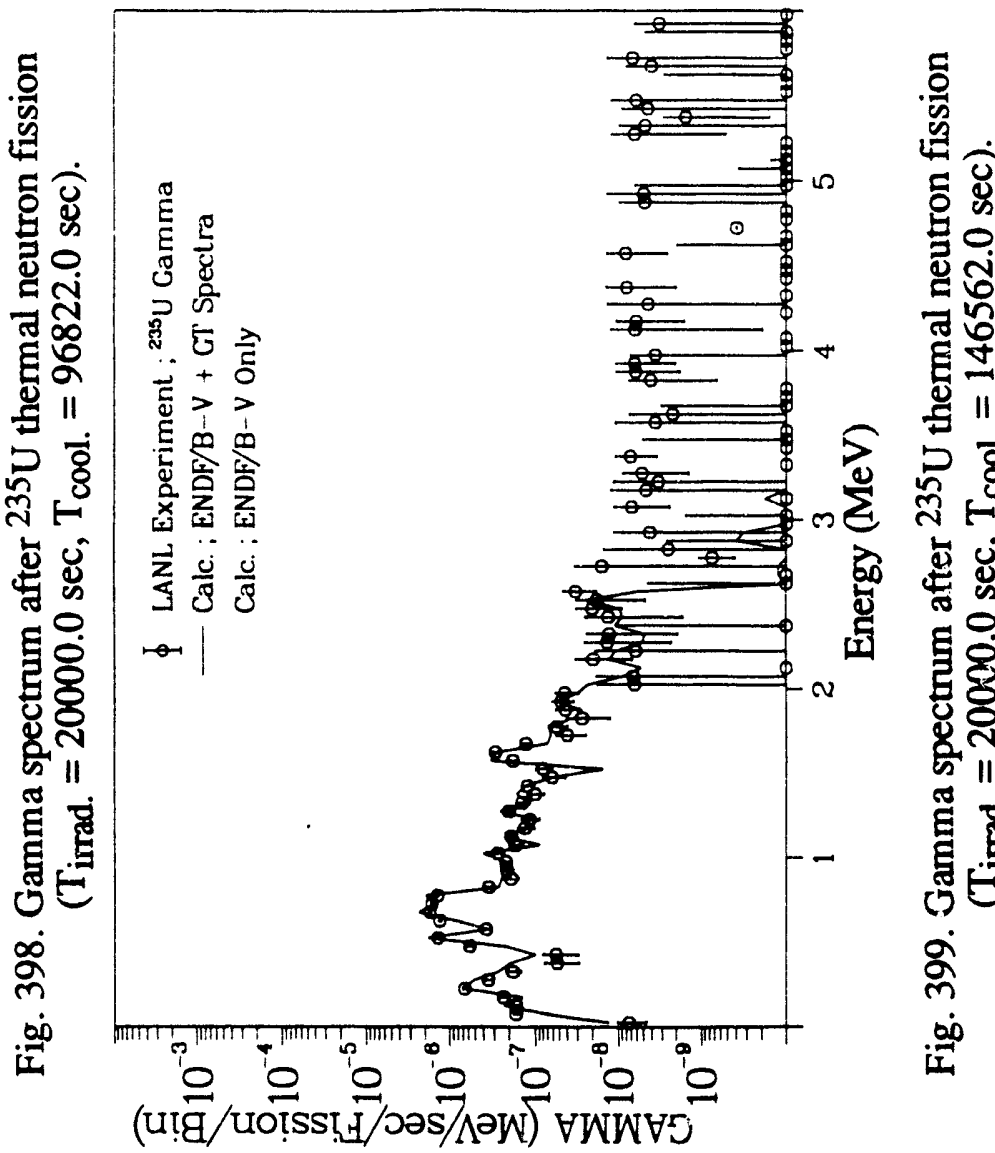

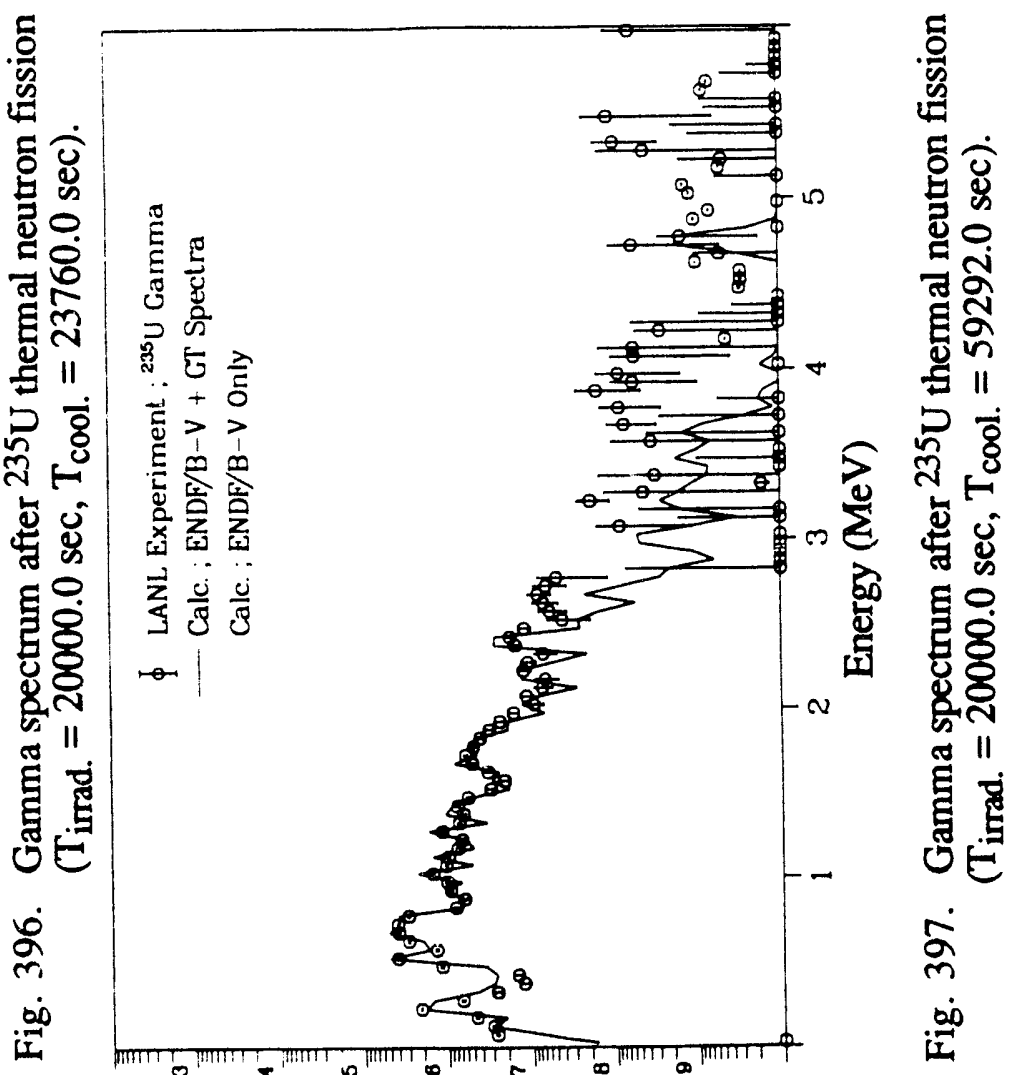

으으 으 '응으응

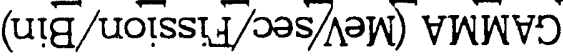



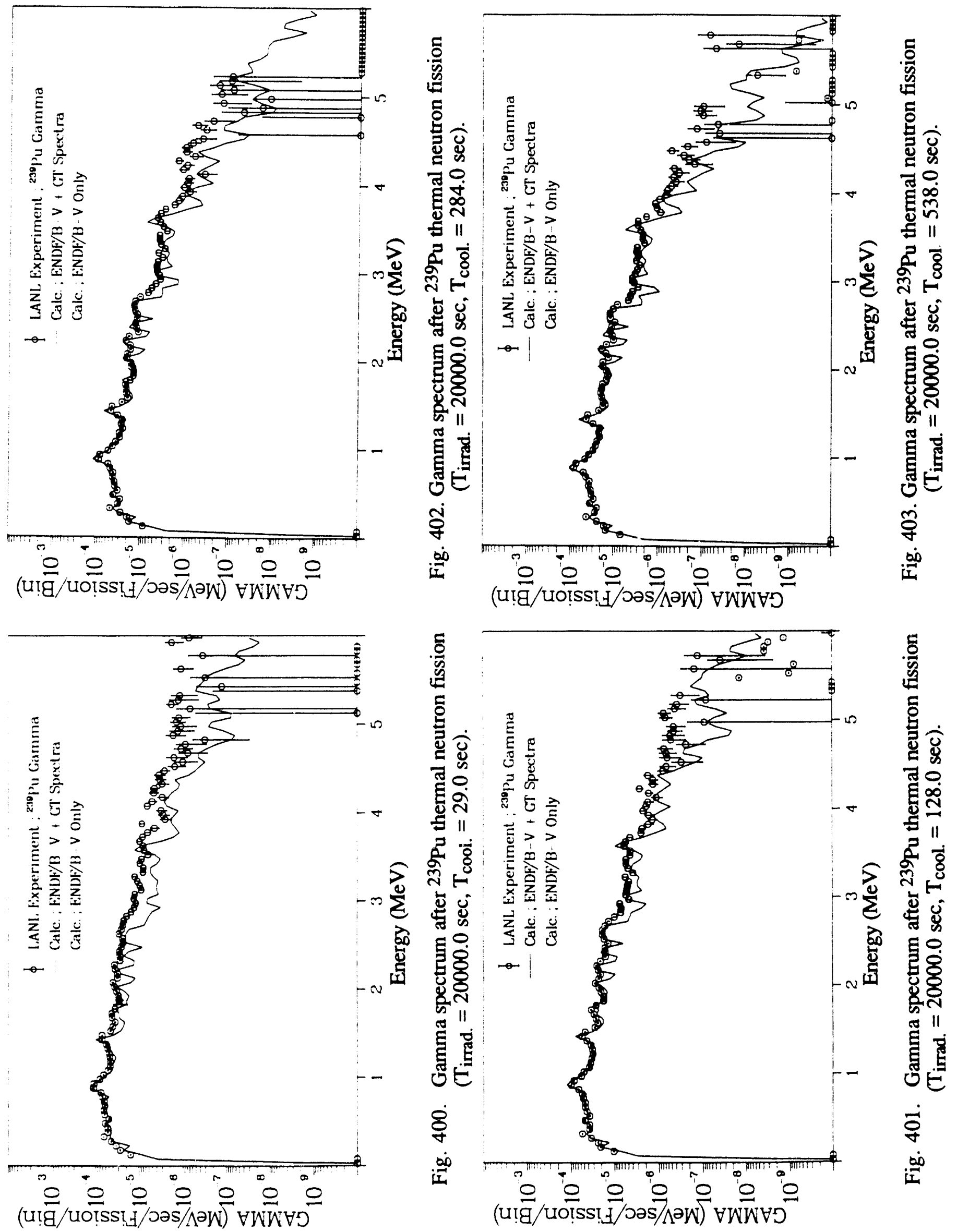


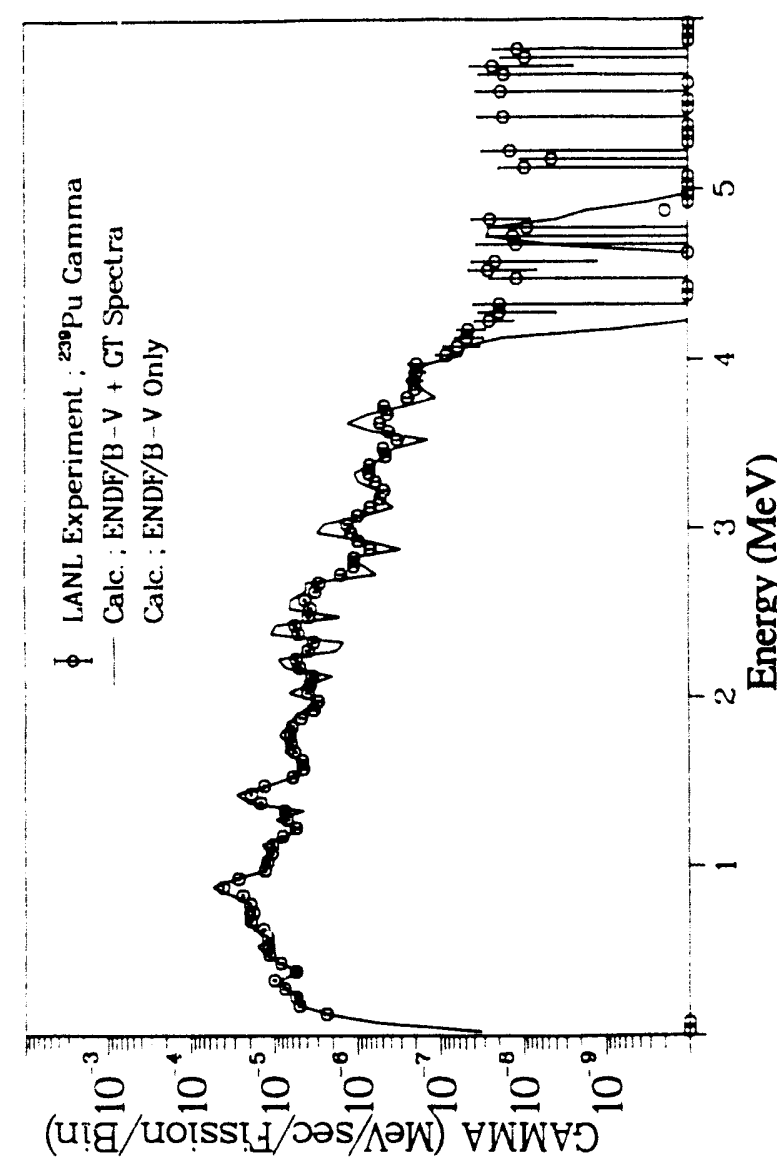


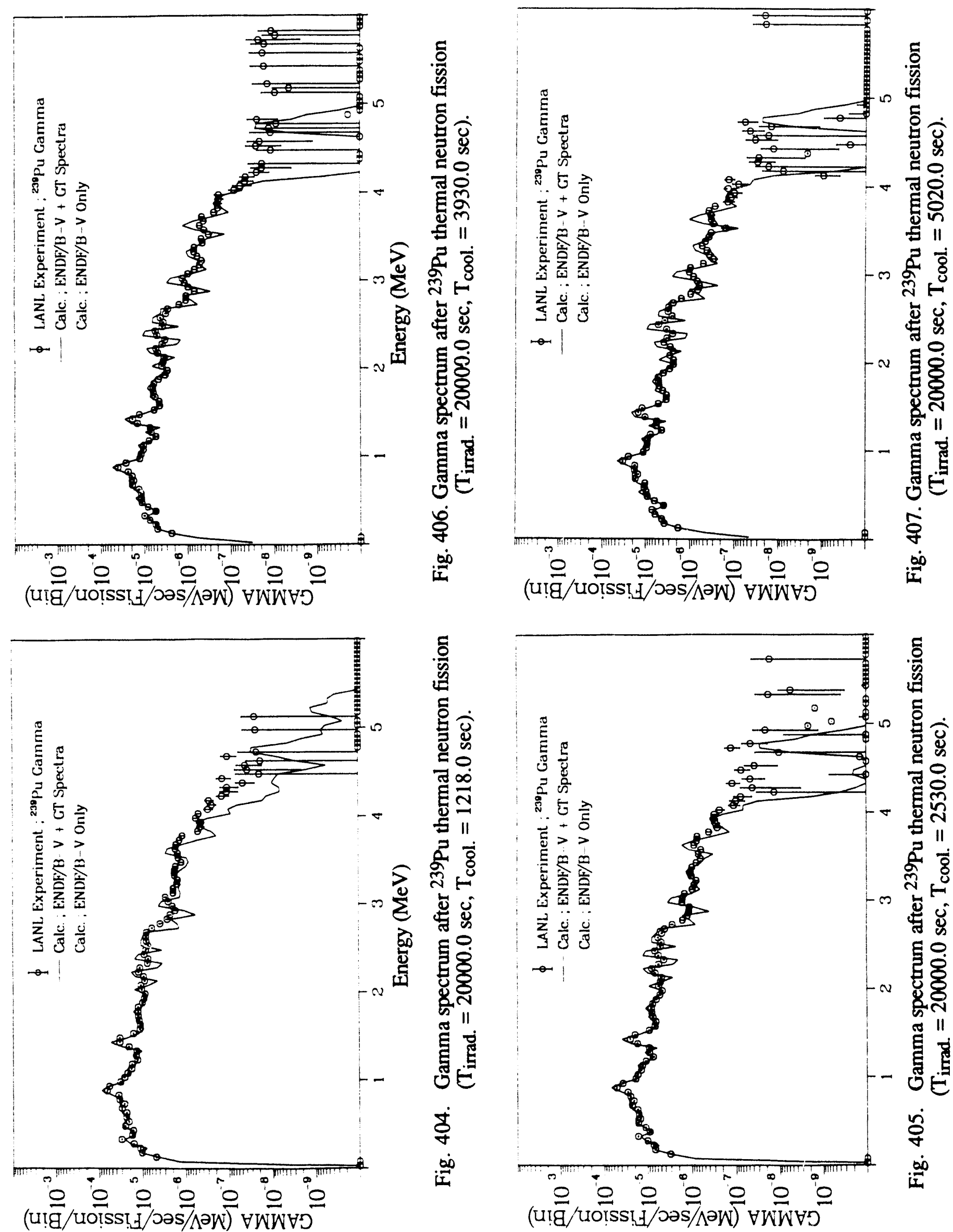


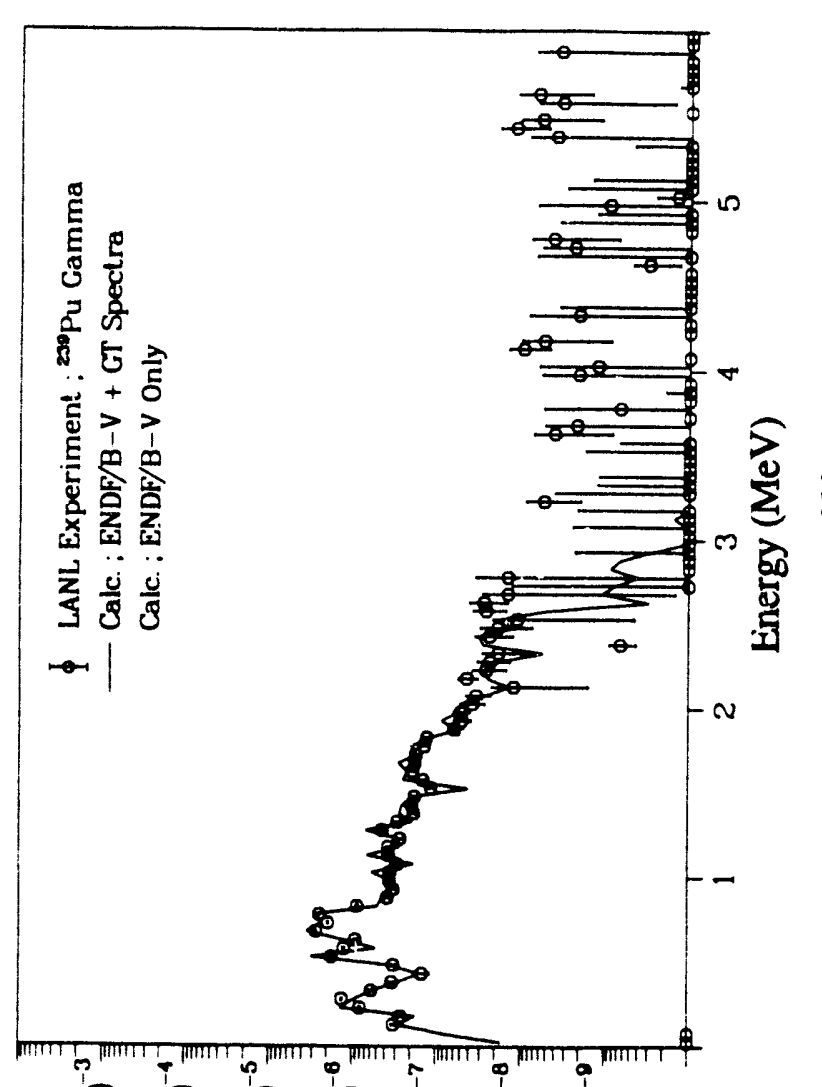

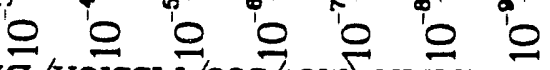

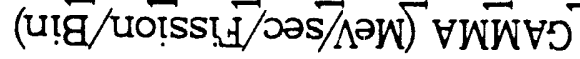

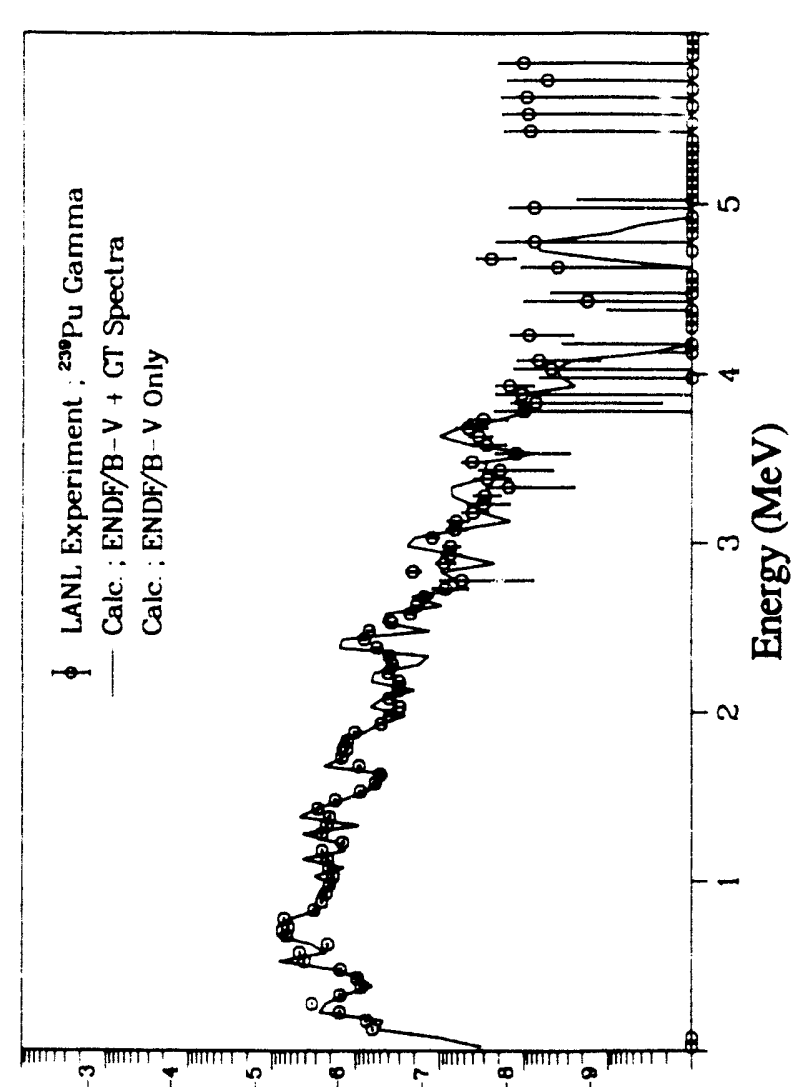

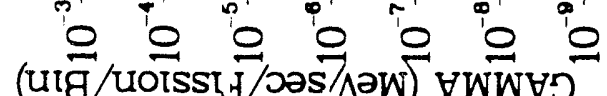

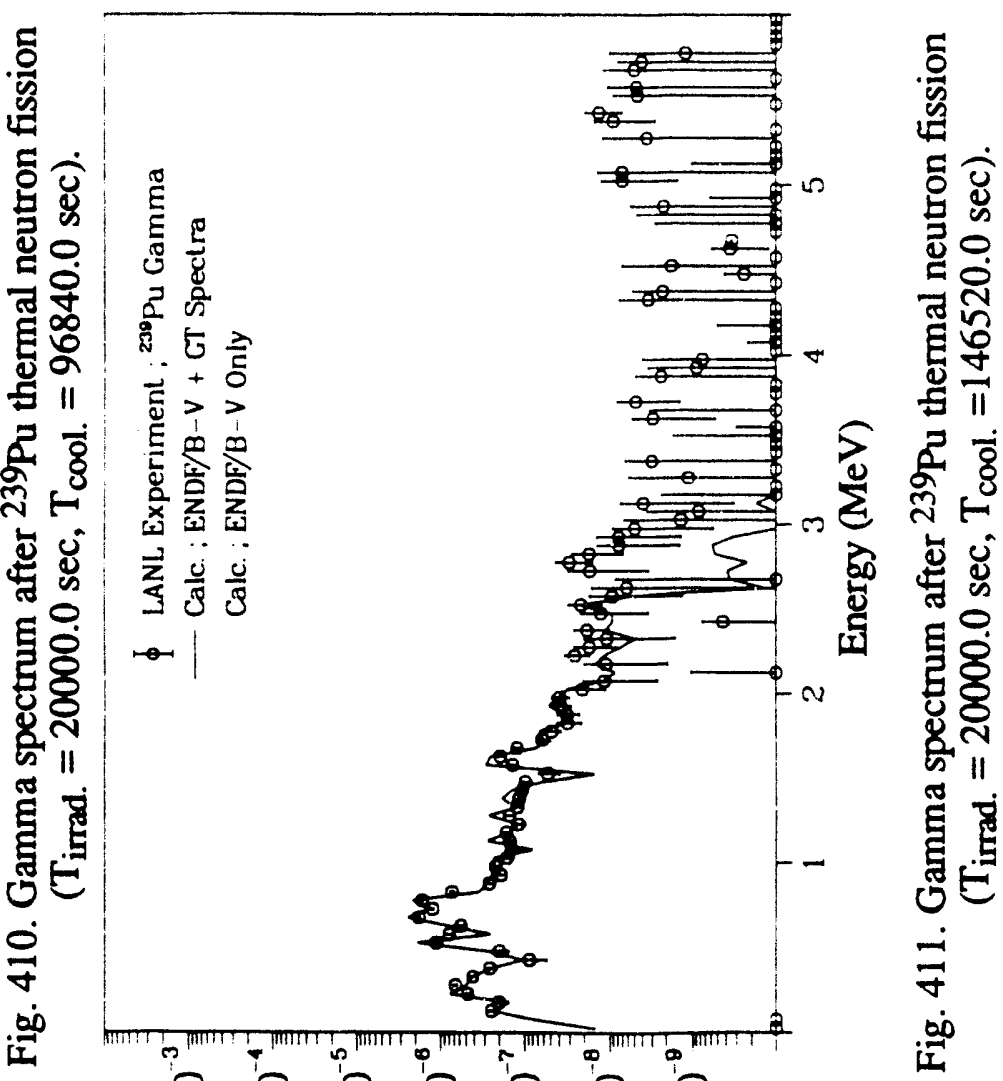

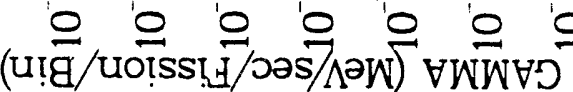

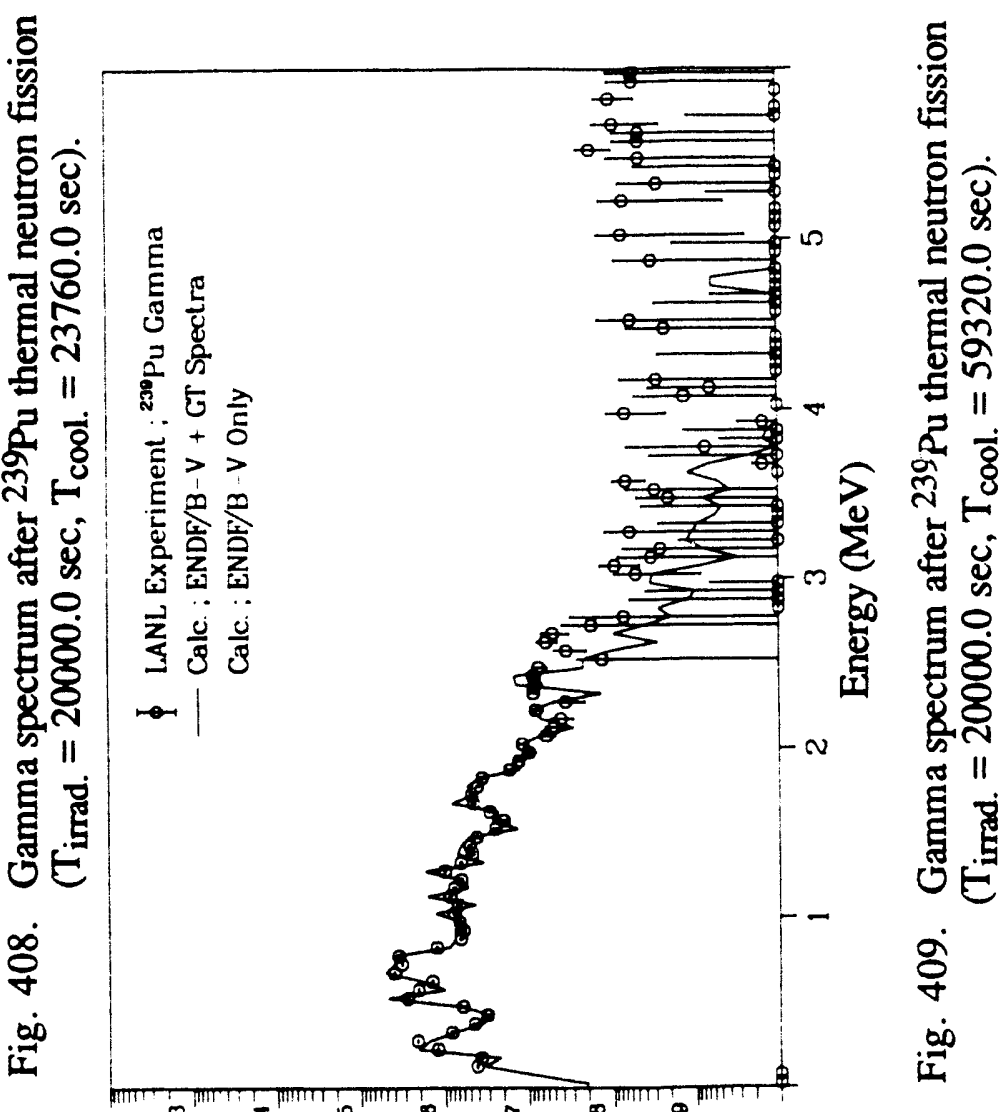

으응으응으

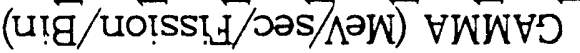




\section{REFERENCES:}

1. Proc. Nuclear Energy Agency Nuclear Data Committee (NEANDC) Specialists' Meeting on Yields and Decay Data of Fission Product Nuclides, Brookhaven National Laboratory, Oct. 24-27, 1983, Brookhaven report BNL 51778.

2. K. Tasaka, H. Ihara, M. Akiyama, T. Yoshida, Z. Matumot, and R. Nakasima, "JNDC Nuclear Data Library of Fission Products," Japan Atomic Energy Research Inst. report JAERI 1287 [NEANDC(J) 91/U, INDC(JAP0 78/L)] (1983).

3. T. Yoshida and J. Katakura, "Calculation of the Delayed Gamma-Ray Energy Spectra from Aggregate Fission Product Nuclides," Nucl. Sci. Eng. 23 (1986).

J. Katakura and T. Yoshida, "Gamma-Ray Spectrum Data Library of Fission Product Nuclides and its Assessment," Japan Atomic Energy Research Inst. report JAERI 1311 [NEANDC (J)-124/U, INDC(JPN-110/L)] (1988).

T. Yoshida and R. Nakasima, J. Nuc. Sci. Tech. 18, No. 6, 393-407 (June 1981).

T. Yoshida, "Theoretical Calculation of Decay Data of Short-Lived Nuclides for JNDC Fission Product Decay File, Japan Atomic Energy Research Inst. report JAERI-M-83-127 [NEANDC (J)-92/U, INDC(JAP0)-79/L] (August 1983).

4. T. R. England, W. B. Wilson, R. E. Schenter, and F. M. Mann, "Summary of ENDF/B-V Data for Fission Products and Actinides," Electric Power Research Inst. report EPRI NP3787 (1984).

5. J. K. Dickens, J. F. Emery, T. A. Love, J. W. McConnell, K. J. Northcutt, R. W. Peelle, and $\mathrm{H}$. Weaver, "Fission Product Energy Release for Times Following Thermal Neutron Fission of $235 \mathrm{U}$ Between 2 and 14,000 Seconds," Oak Ridge National Laboratory report ORNL/NUREG-14 (1977).

J. K. Dickens, J. F. Emery, T. A. Love, J. W. McConnell, K. J. Northcutt, R. W. Peelle, and H. Weaver, "Fission Product Energy Release for Times Following Thermal Neutron Fission of ${ }^{239}$ Pu Between 2 and 14,000 Seconds," Oak Ridge National Laboratory report NUREG/CR-0194, ORNL/NUREG-34 (1978).

J. K. Dickens, J. F. Emery, T. A. Love, J. W. McConnell, K. J. Northcutt, R. W. Peelle, and H. Weaver, "Fission Product Energy Release for Times Following Thermal Neutron Fission of 241 Pu Between 2 and 14,000 Seconds," Oak Ridge National Laboratory report NUREG/CR-0171, ORNL/NUREG-47 (1978).

J. K. Dickens, T. A. Love, J. W. McConnell, J. F. Emery, K. J. Northcutt, and R. W. Peelle, "Delayed Beta- and Gamma-Ray Production Due to Thermal-Neutron Fission of 235U, Spectral Distributions for Times after Fission Between 2 and 14,000 Seconds: Tabular and Graphical Data," Oak Ridge National Laboratory report NUREG/CR-0162, ORNL/NUREG-39 (1978).

J. K. Dickens, T. R. England, T. A. Love, J. W. McConnell, J. F. Emergy, K. J. Northcutt, and R. W. Peelle, "Delayed Beta- and Gamma-Ray Production Due to ThermalNeutron Fission of ${ }^{239} \mathrm{Pu}$ : Tabular and Graphical Spectral Distributions for Times After Fission Between 2 and 14,000 Seconds," Oak Ridge National Laboratory report NUREG/CR-1172, ORNL/NUREG-66 (1980). 
6. M. Akiyama, Dr. Eng. Thesis, University of Tokyo (1983).

M. Akiyama and J. Katakura, "Measured Data of Delayed Garmma-Ray Spectra from Fissions of ${ }^{232} \mathrm{Th},{ }^{233} \mathrm{U},{ }^{235} \mathrm{U},{ }^{238} \mathrm{U}$, and ${ }^{239} \mathrm{Pu}$ by Fast Neutrons: Tabular Data," Japan Atomic Energy Research Inst. report JAERI-M 88-252 (1988).

7. E. T. Jurney, P. J. Bendt, and T. R. England, "Fission Product Gamma Spectra," Los Alamos National Laboratory report LA-7620-MS (1979).

8. K. Takahashi, M. Yamada, and T. Kondoh, "Beta-Decay Half-Lives Calculated on the Gross Theory," Atom. Data and Nucl. Data Tables, 12, 101 (1973) (see also references therein).

9. D. M. Brink, Ph.D. Thesis, Oxford University (1955).

10. P. Axel, "Electric Dipole Ground-State Transition Width Strength Function and 7-MeV Photon Interactions," Phys. Rev. 126, 67 (1962).

11. A. Gilbert and A. G. W. Cameron, "A Composite Nuclear Level Density Formula with Shell Corrections," Can. T. Phys. 43, 1446 (1965).

12. J. Katakura and R. Nakasima, "Average Decay Energies in JNDC file Version 2," Proc. Specialists' Mtg. on Data for Decay Heat Prediction, Studsvik, Sweden, Sept. 7-10, 1987, NEACRP-302 'IJ', NEANDC-245 'U' (1987).

13. H. Thara, Ed., "Tabies and Figures from JNDC Nuclear Data Library of Fission Products, Version 2," Japan Atomic Energy Research Inst. report JAERI-M 90-204 (1989).

K. Tasaka, J. Katakura, H. Ihara, T. Yoshida, S. Iijima, R. Nakasima, T. Nakagawa, and H. Takano, "Second Version of JNDC Nuclear Data Library of Fission Products," to be published as a JAERI report.

14. T. R. England, R. Wilczynski, and N. L. Whittemore, "CINDER-7: An Interim Report for Users," Los Alamos Scientific Laboratory report LA-5885-MS (April 1975).

15. 'T. R. England and B. F. Rider, "Evaluation and Compilation of Fission Product Yields, 1989," (ENDF-349) draft form, to be published as a Los Alamos report as the primary documentation for ENDF/B-VI. 


\section{APPENDIX A \\ COMPARISONS USING ENDF/B-VI PRELIMINARY DATA}

The calculations using the ENDF/B-VI preliminary data (as of October 1989) were performed due to the fact that the data became available for testing during the time frame of this work effort. The results are shown in Figs. A-1 through A-6 for ${ }^{235} \mathrm{U},{ }^{239} \mathrm{Pu}$, and ${ }^{241} \mathrm{Pu}$ fission. Four kinds of calculations are demonstrated in each figure; i.e., the calculations using the ENDF/B-V line spectral data, the augmented ENDF/B-V data, the preliminary ENDF/B-VI line spectral data, and the augmented preliminary ENDF/B-VI data.

As evidenced in these figures, the preliminary ENDF/B-VI spectral data are greatly improved over the ENDF/B-V ones. However, the calculations using only the preliminary ENDF/B-VI spectral data seem to have a problem of missing gamma rays and they underestimate the measured aggregate spectral data. The augmented preliminary ENDF/B-VI data seem to give better agreement among them. These comparisons were done using the preliminary ENDF/B-VI file; some data in the file could be changed when the file is completed, but most of the improvement is expected by using the calculated spectra for the augmentation of the line spectral data. 


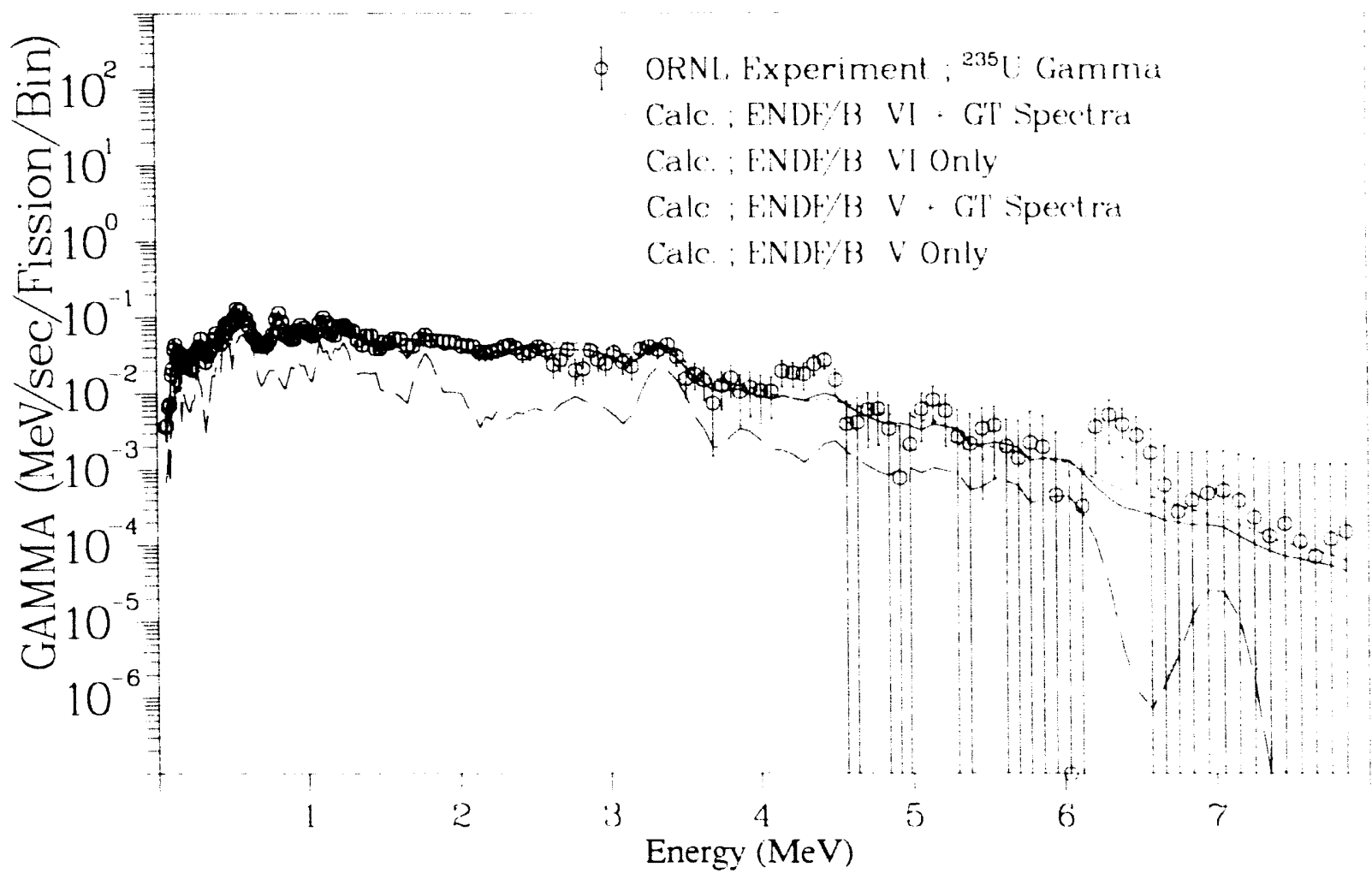

Fig. A-1. Gamma spectrum after $235 \mathrm{U}$ thermal neurron fission $\left(\mathrm{T}_{\mathrm{irrad}}=1.0 \mathrm{sec}, \mathrm{T}_{\text {cool. }}=2.2 \mathrm{sec}\right)$ (to $8 \mathrm{MeV}$ ).

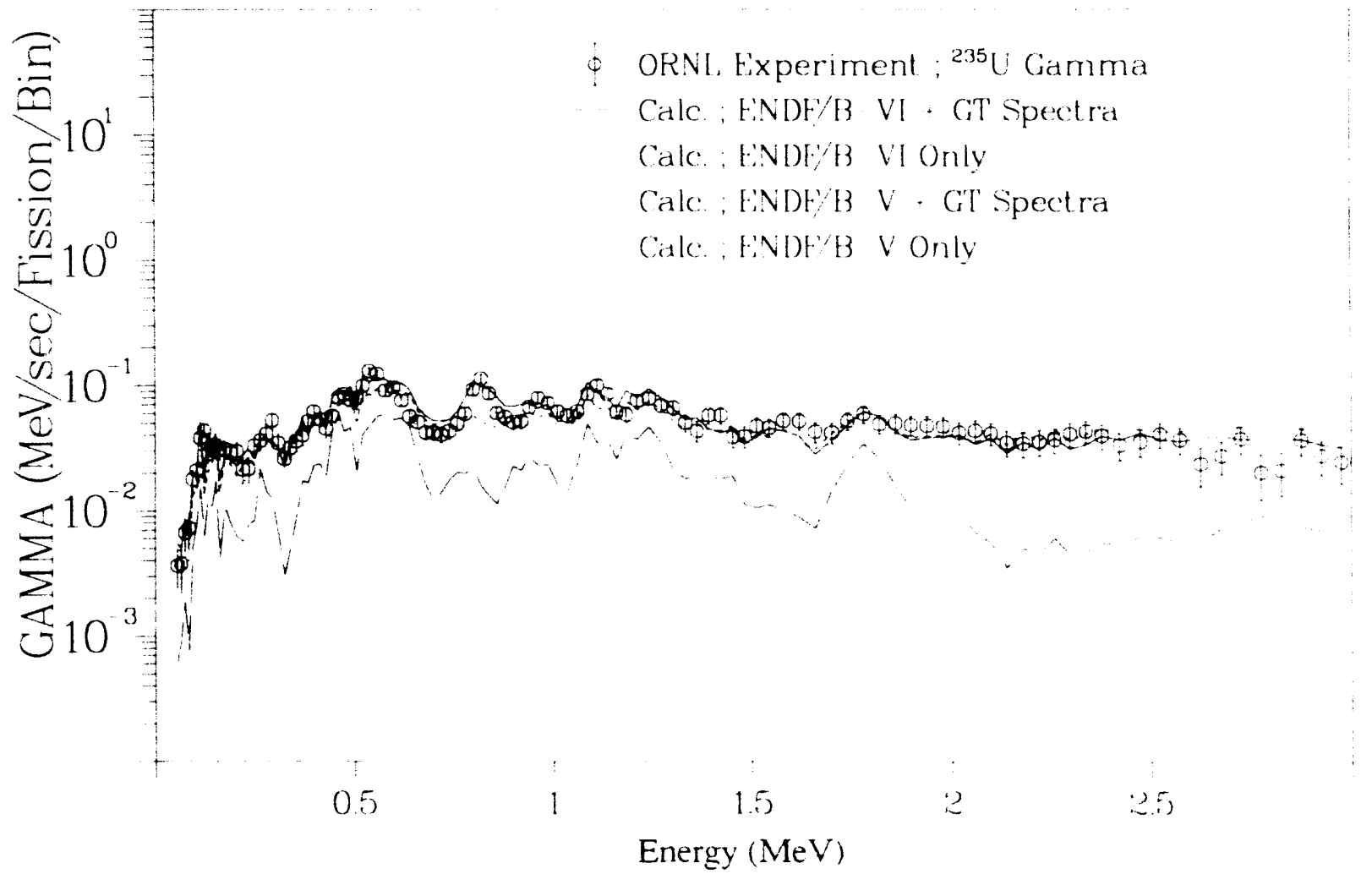

Fig. A-2. Gamma spectrum after $235 \mathrm{t}$ thermal neutron fission $\left(\mathrm{T}_{\mathrm{irrad}}=1.0 \mathrm{sec}, \mathrm{T}_{\text {cool }}=2.2 \mathrm{sec}\right)$ (to $3 \mathrm{MeV}$ ). 


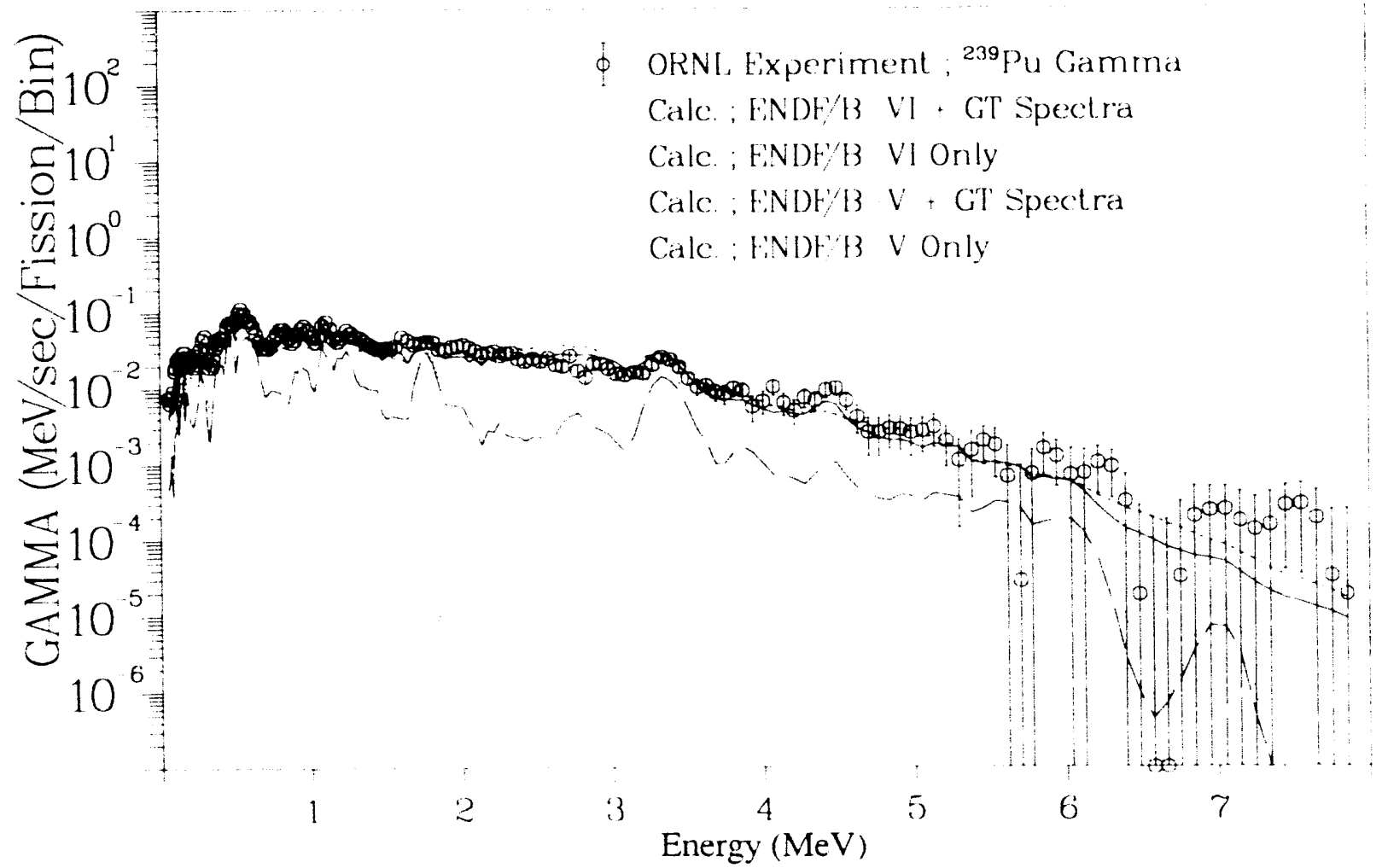

Fig. A-3. Gamma spectrum after ${ }^{239} \mathrm{Pu}$ therrmal neutron fission $\left(\mathrm{T}_{\text {irrad. }}=1.0 \mathrm{sec}, \mathrm{T}_{\text {cool. }}=2.2 \mathrm{sec}\right)$ (to $8 \mathrm{MeV}$ ).

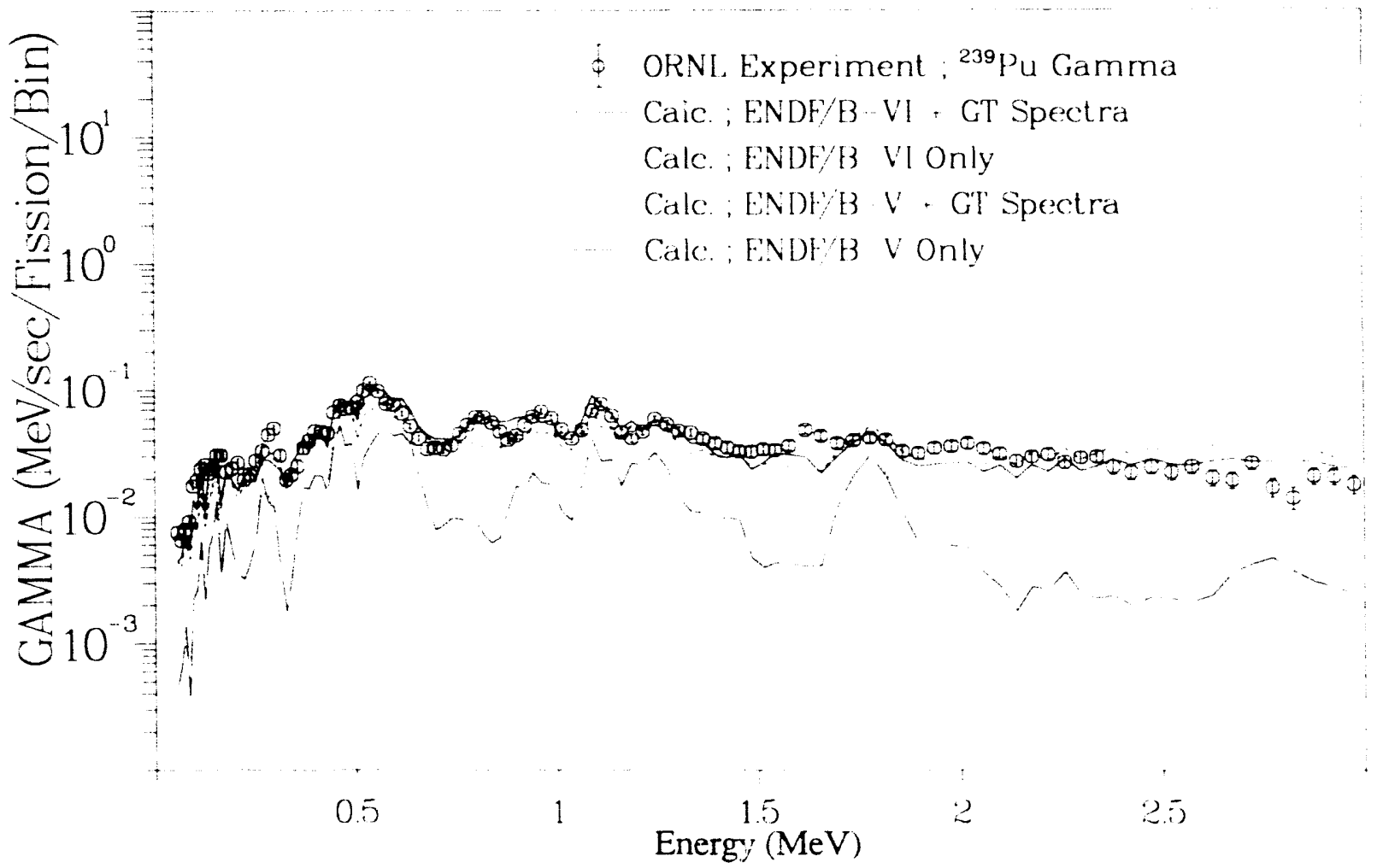

Fig. A-4. Gamma spectrum after ${ }^{239} \mathrm{Pu}$ thermal neutron fission $\left(\mathrm{T}_{\mathrm{irrad}}=1.0 \mathrm{sec}, \mathrm{T}_{\mathrm{cool}}=2.2 \mathrm{sec}\right)$ (to $3 \mathrm{MeV}$ ). 


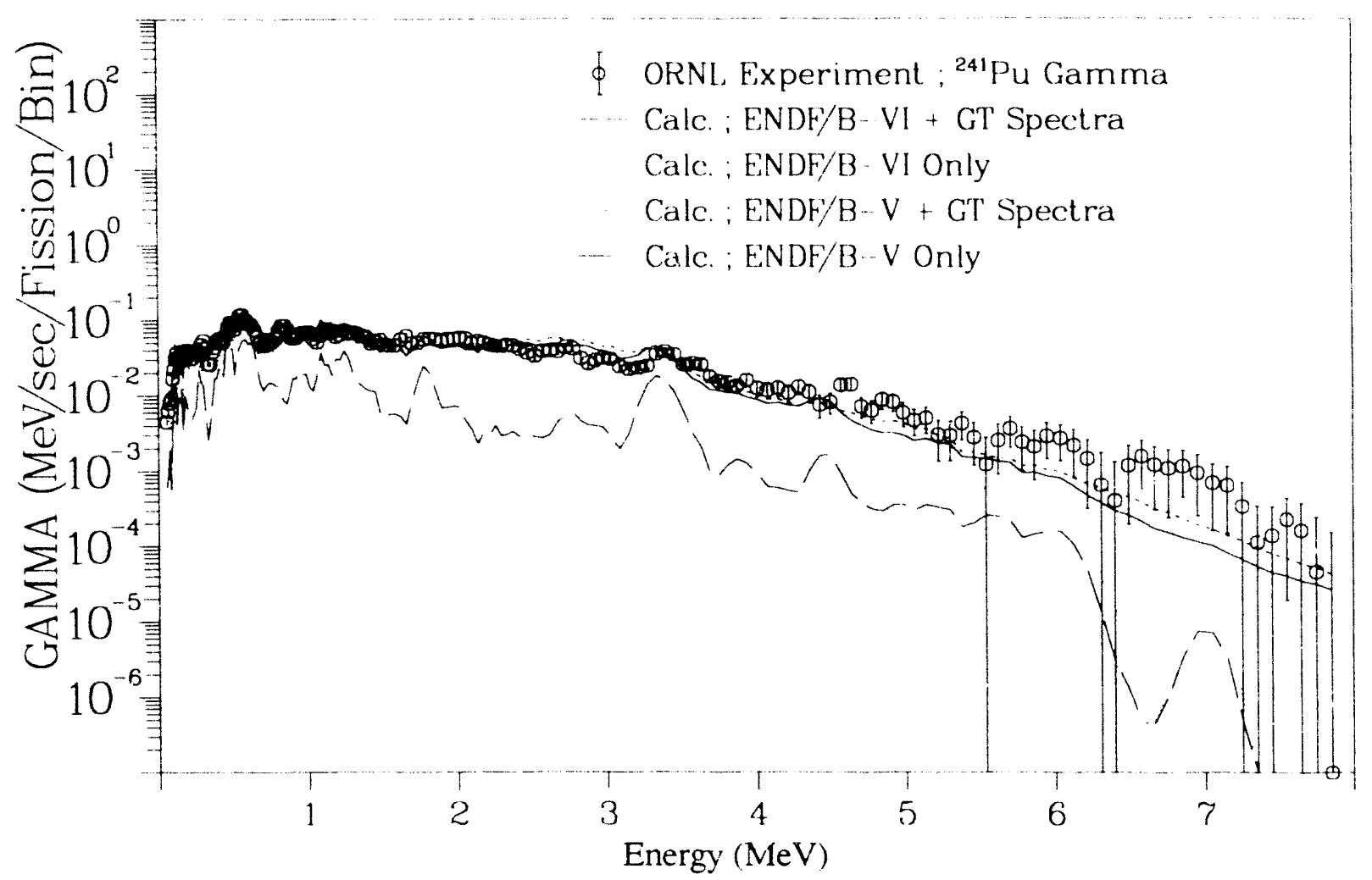

Fig. A-5. Gamma spectrum after ${ }^{241} \mathrm{Pu}$ thermal neutron fission $\left(\mathrm{T}_{\text {irrad. }}=1.0 \mathrm{sec}, \mathrm{T}_{\text {cool. }}=2.2 \mathrm{sec}\right.$ ) (to $8 \mathrm{MeV}$ ).

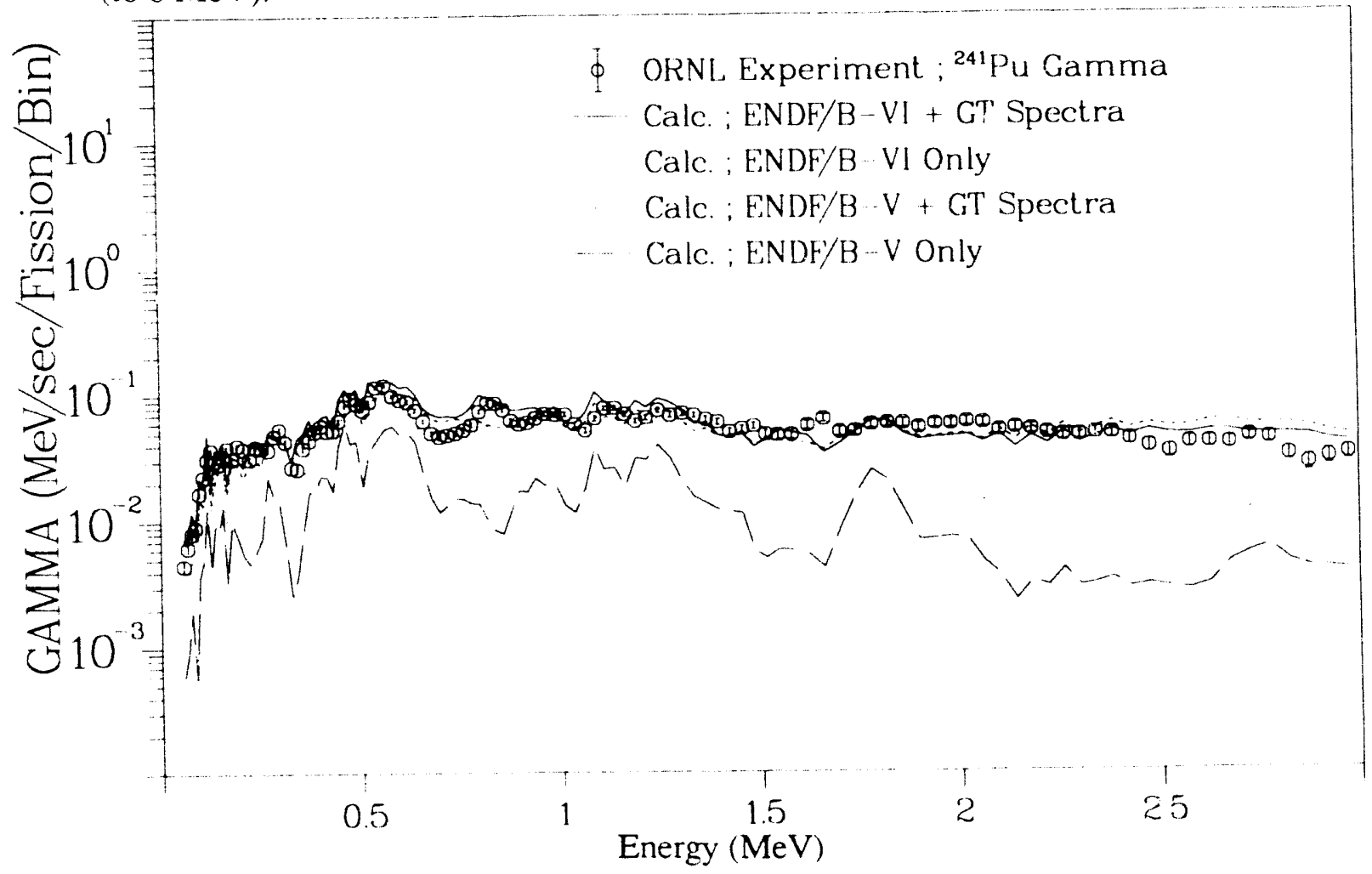

Fig. A-6. Gamma spectrum after ${ }^{241} \mathrm{Pu}$ thermal neutron fission $\left(\mathrm{T}_{\text {irrad. }}=1.0 \mathrm{sec}, \mathrm{T}_{\text {cool. }}=2.2 \mathrm{sec}\right.$ ) (to $3 \mathrm{MeV}$ ). 


\section{APPENDIX B}

\section{CALCULATION OF BETA-RAY SPECTRUM}

The calculation of gamma-ray spectra is discussed in the main text. The beta spectra calculation also involves a problem for some nuclides in that the measured spectrum enhances the high-energy part, because the beta transitions to higher energy levels of daughter nucleus may not be observed in measurements even if the transitions are to be allowed. The calculation of aggregate beta spectrum using such spectral data also enhances the high-energy part and depresses the lowenergy part. The situation is seen in the comparisons of the aggregate beta-ray spectra between the calculations and the measurements by Dickens et al. At very short cooling times after fission, when the nuclides with no measured data contribute to the spectra, the calculation falls below the measured values. At longer (though still short) cooling times in which the nuclides with incomplete spectral data are still important, the calculation shows an underestimation for the lowenergy part and an overestimation for the high-energy part. In order to improve the situation, we tried to calculate the beta spectra of each fission-product nuclide by the Gross Theory, which is used to complement the measured gamma spectra.

In the case of the beta-ray spectra., some different treatments or modifications from that of the gamma-ray spectra are needed. The average beta energy value that can force agreement with the measured decay heat value is smaller than the average energy from measured decay scheme data. Therefore, it is not suitable to simply add some fraction of the calculated spectrum to the measured one. We used the following procedure.

The calculation of beta rays by the Gross Theory was performed by assuming that there may have been some missing beta transitions to higher levels than the observed ones. Namely, the beta spectrum was calculated with the maximum energy equal to the difference between the $Q_{\beta}$ value and the maximum level energy. This calculated spectrum is normalized to be 1.0 when energy integration is carried out over the full energy range.

Let the average energy of the calculated spectrum be $E_{c}$ and the energy derived from the measured decay scheme be $E_{m}$. The average energy that is suitable to calculate the decay heat value is $E_{T}$; then $E_{T}$ is expressed as follows:

$$
E_{T}=\alpha \cdot E_{m}+\beta \cdot E_{c},
$$

whereas $\alpha+\beta=1.0, \alpha-$ and $\beta$-values can be calculated

$$
\begin{aligned}
& \alpha=\left(E_{\mathrm{T}}-E_{c}\right) /\left(E_{m}-E_{c}\right) \\
& \beta=\left(E_{m}-E_{T}\right) /\left(E_{m}-E_{c}\right) .
\end{aligned}
$$


As $E_{c}<E_{T}<E_{m}$ for most of the nuclides having the problem, $\alpha$ and $\beta$ values become positive. In the case of $E_{\mathrm{T}}>E_{\mathrm{m}}$, the calculated spectrum is simply added to augment the difference between $E_{T}$ and $E_{m} ; \alpha=1, \beta=\left(E_{T}-E_{m}\right) / E_{c}$ in this case. The spectrum, then, becomes

$$
S\left(E_{\beta}\right)=\alpha \cdot S_{m}\left(E_{\beta}\right)+\beta \cdot S_{c}\left(E_{\beta}\right)
$$

By this method, the beta-ray spectra were calculated for nuclides whose average beta energy value has inconsistency between that from the decay scheme and that used for decay heat calculations.

An example of the spectrum is shown in Fig. B-1. This is a case of ${ }^{97} \mathrm{Sr}$ decay. The average energy value derived from the measured decay scheme is 2.646 and its spectrum is illustrated by the dotted line. The calculated spectrum, assuming that beta transitions occur only to higher levels than observed ones, is shown by a dashed line, and its average energy is $1.923 \mathrm{MeV}$. The modified or augmented spectrum is seen as a solid line. This modified spe`trum gives the average energy of $2.282 \mathrm{MeV}$, which is nearly equal to the value of the energy used for the decay heat calculation in the JNDCV2 library.

The modified spectra of each fission product nuclide were used to calculate the aggregate beta-ray spectra after fission. One result is shown in Fig. B-2 together with the measured spectrum. The calculation using only the ENDF/B-V spectra data shows an underestimation for the low-energy part. The calculation using the modified spectra (ENDF/B-V + GT spectra in the figure) shows rather good agreement with the measurement. 


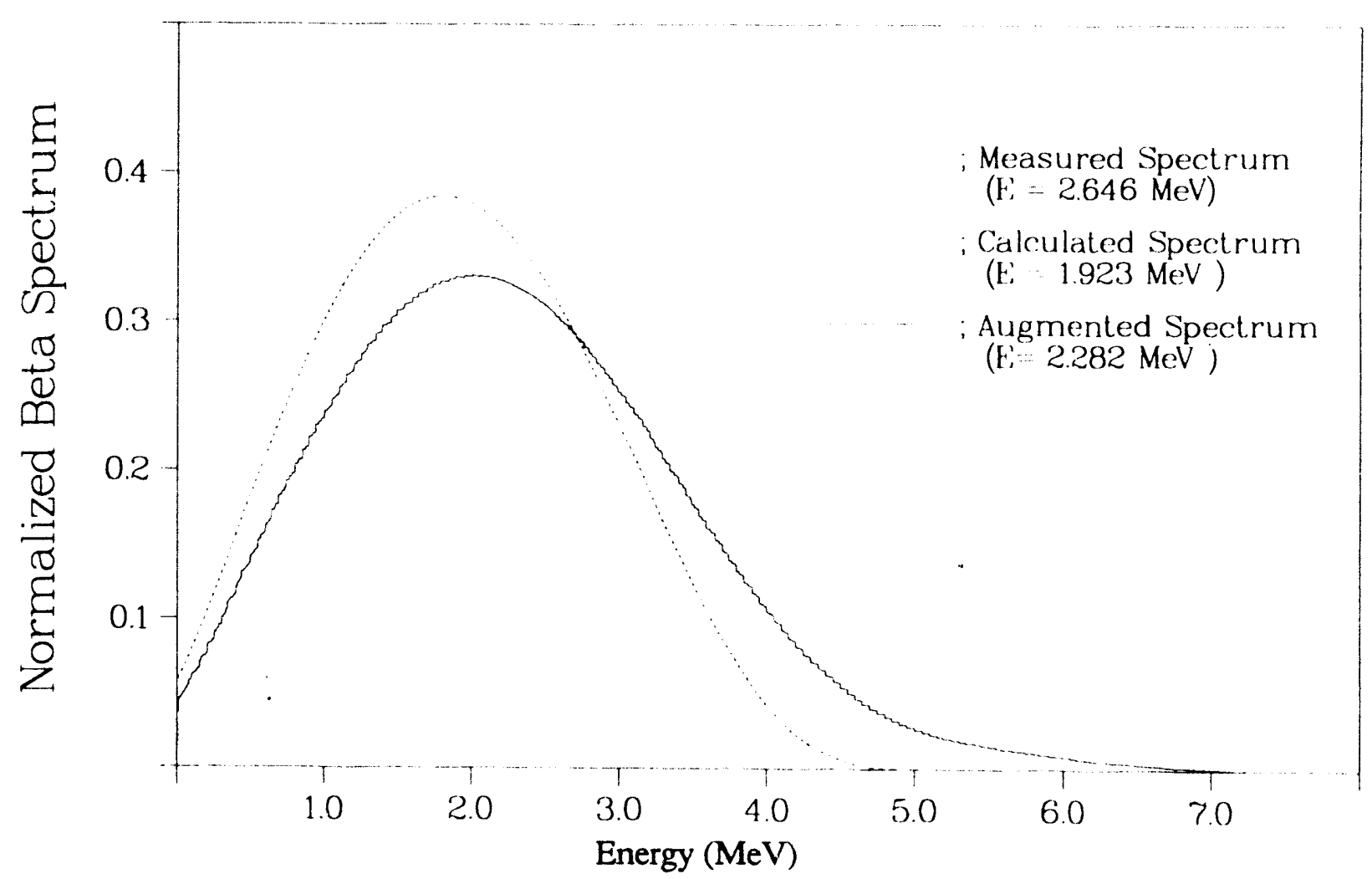

Fig. B-1. Beta-ray spectrum of ${ }^{97} \mathrm{Sr}$ decay $(\mathrm{Q}=7.4 \mathrm{MeV})$.

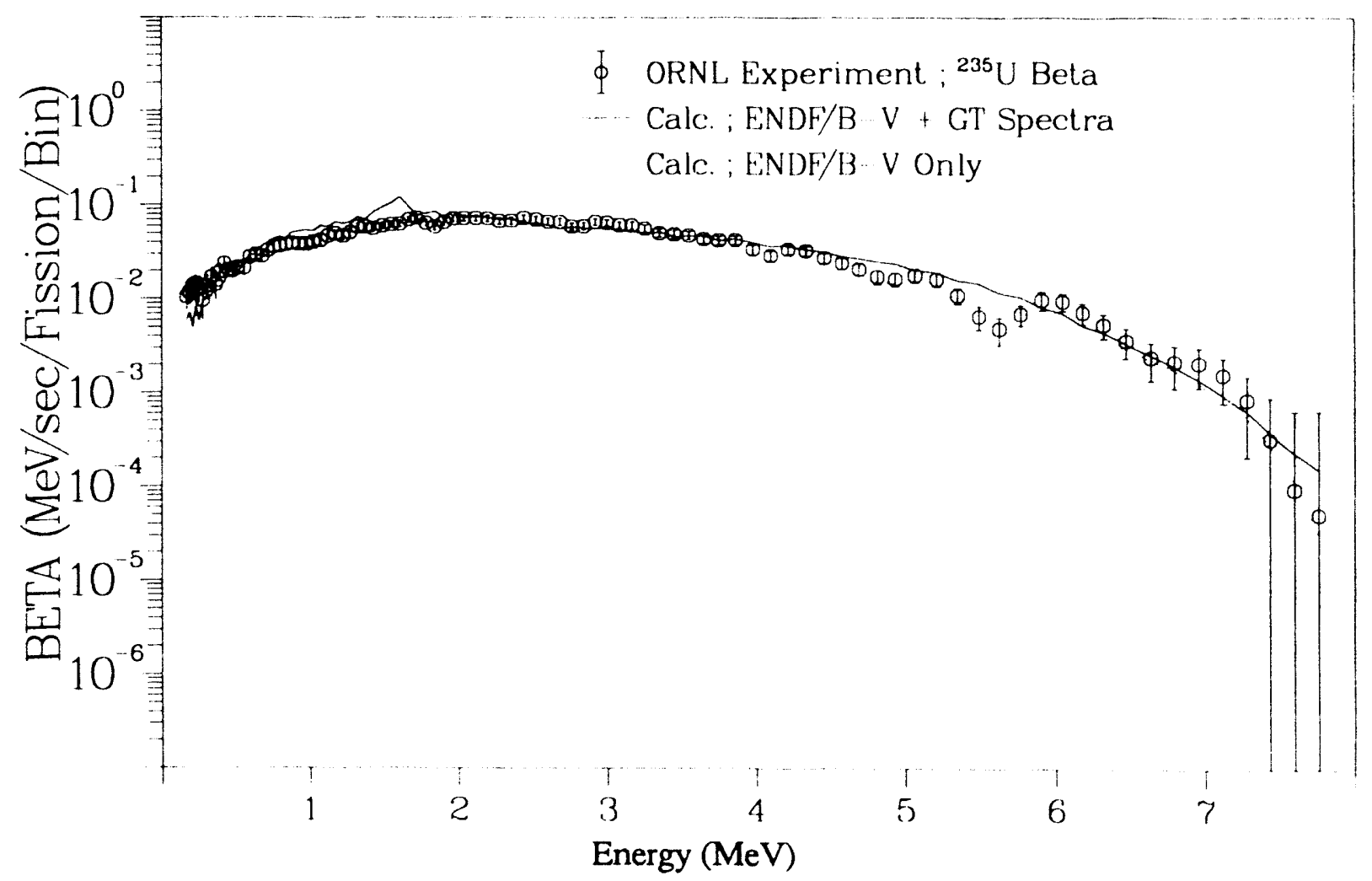

Fig. B-2. Beta-ray spectrum after ${ }^{235} \mathrm{U}$ thermal neutron fission $\left(\mathrm{T}_{\text {irrad. }}=1.0 \mathrm{sec}, \mathrm{T}_{\text {cool. }}=2.2 \mathrm{sec}\right.$ ) (to $8 \mathrm{MeV}$ ). 


\section{APPENDIX C \\ FISSION PRODUCTS: \\ PRELIMINARY DECAY ENERGIES, HALF-LIVES, AND BRANCHINGS FOR ENDF/B-VI}

In Table C-1 we have listed total beta, gamma, and alpha energies from the most recent data file used in this report. Branchings by decay are also listed, as are half-lives. All energies are in $\mathrm{eV}$, half-lives in seconds, and branchings are fractions per decay. Spectral data are too extensive for inclusion in this report. The table identifies 127 products as stable; these are needed in calculations involving a neutron flux. Their cross sections will be found in ENDF/B-VI files. The meaning of the columns in Table C-1 are:

\section{Col. Heading}

Symbol

ZZAAAS

Half-life

E-beta, E-gamma, E-alpha

RTYP

RFS

Q

Branching

NDK

NSP

MAT

\section{Quantity}

chemical symbol preceded by the $Z$ value and followed by the atomic number. Nuclides that are isomeric states have $m, n, \ldots$ following the atomic number meaning $1 \mathrm{st}, 2 \mathrm{nd}, \ldots$ isomeric states (the files generally include isomeric states having half-lives $\geqq 0.1 \mathrm{~s}$ ).

is a numeric identifier consisting of the quantity $\mathrm{Z} \times 10000+\mathrm{A} \times 10+\mathrm{S}$, where $S$ is the isomeric state number $(0=$ ground, $1=1$ st isomeric state, etc.).

the total decay half-life in seconds.

generally are average beta, gamma, and alpha decay energies, but have more precise definitions. Thus, E-beta is the total electron-related radiation, such as $\beta^{-}, \beta^{+}$, conversion electron, Auger, etc. E-gamma is the average energy of all "electromagnetic" radiation, such as gamma rays, $\mathrm{x}$ rays, and annihilation radiation. E-alpha is the average energy of all heavy charged particles and delayed neutrons. The alpha decay energy includes the recoil energy. The sum of the three average energies is the recoverable energy per decay (neutrino energies are excluded). All values are given in units of eV. (Delayed neutron energy is not tabulated but will be included in the final ENDF/B-VI files and summed into E-alpha.)

identifies the initial or primary decay mode for the listed line of data (see below).

identifies the daughter state following the decay $(0 .=$ ground, $1 .=1 \mathrm{st}$ isomeric state, etc.).

is the total Q-value for the decay mode.

is the fraction of decays from type RTYP to state RFS

is the number of decay modes.

is the number of spectral types included in the ENDF/B files.

is the material identification number assignment in the ENDF/B files. 
The decay mode identification, RTYP, has the following definitions.

$\underline{\text { RTYP }}$

Decay Mode

\begin{tabular}{|c|c|c|}
\hline 1. & $\beta-$ & Beta decay \\
\hline 2. & e.c., $\left(\beta^{+}\right)$ & Electron capture and/or positron emission \\
\hline 3. & $\mathrm{TT}$ & Isomeric transition \\
\hline 4. & $\alpha$ & Alpha decay \\
\hline 5. & $\mathrm{n}$ & $\begin{array}{l}\text { Neutron emission but not delayed neutron } \\
\text { decay (see below) }\end{array}$ \\
\hline 6. & $\mathrm{SF}$ & \begin{tabular}{l} 
Spontaneous fission \\
\hline 7.
\end{tabular} \\
\hline 10. & $\mathrm{p}$ & Proton emission \\
\hline
\end{tabular}

Multiple particle emission is also defined by combining the RTYP indicators as decimal digits in the sequence in which particles are emitted. Thus, a beta decay followed by a delayed neutron is RTYP $=1.5$, and a positron followed by alpha decay is RTYP $=2.4$, etc. Such compound RTYP values therefore indicate intermediate states having lifetimes that are too short for explicit inclusion in the files. The Q-value for such cases is the energy difference based on masses of the initial and final states.

Spectral files contain a particle indicator, STYP, defined similarly to RTYP, but with the added values of $0 .=$ gamma rays, 8. = discrete electrons, and 9. $=x$ rays. In this report we do not include the average energies for each type of spectra. As can be seen from the NSP column, most nuclides do have spectra in the files.

These data are preliminary because they have not been officially accepted by the Cross Section Evaluation Working Group (CSEWG). We anticipate that the decay energies will be accepted as mod 0 . Figures $\mathrm{C}-1$ through $\mathrm{C}-3$ show a comparison of aggregate decay energies (beta, gamma, and total) with Tokyo measurements following a ${ }^{235} \mathrm{U}$ fast fission pulse. The "Pandemonium" problem is no longer evident. Additional comparisons will be included in ENDF/B-VI documentation following a phase-1 review of the decay and yield files. 


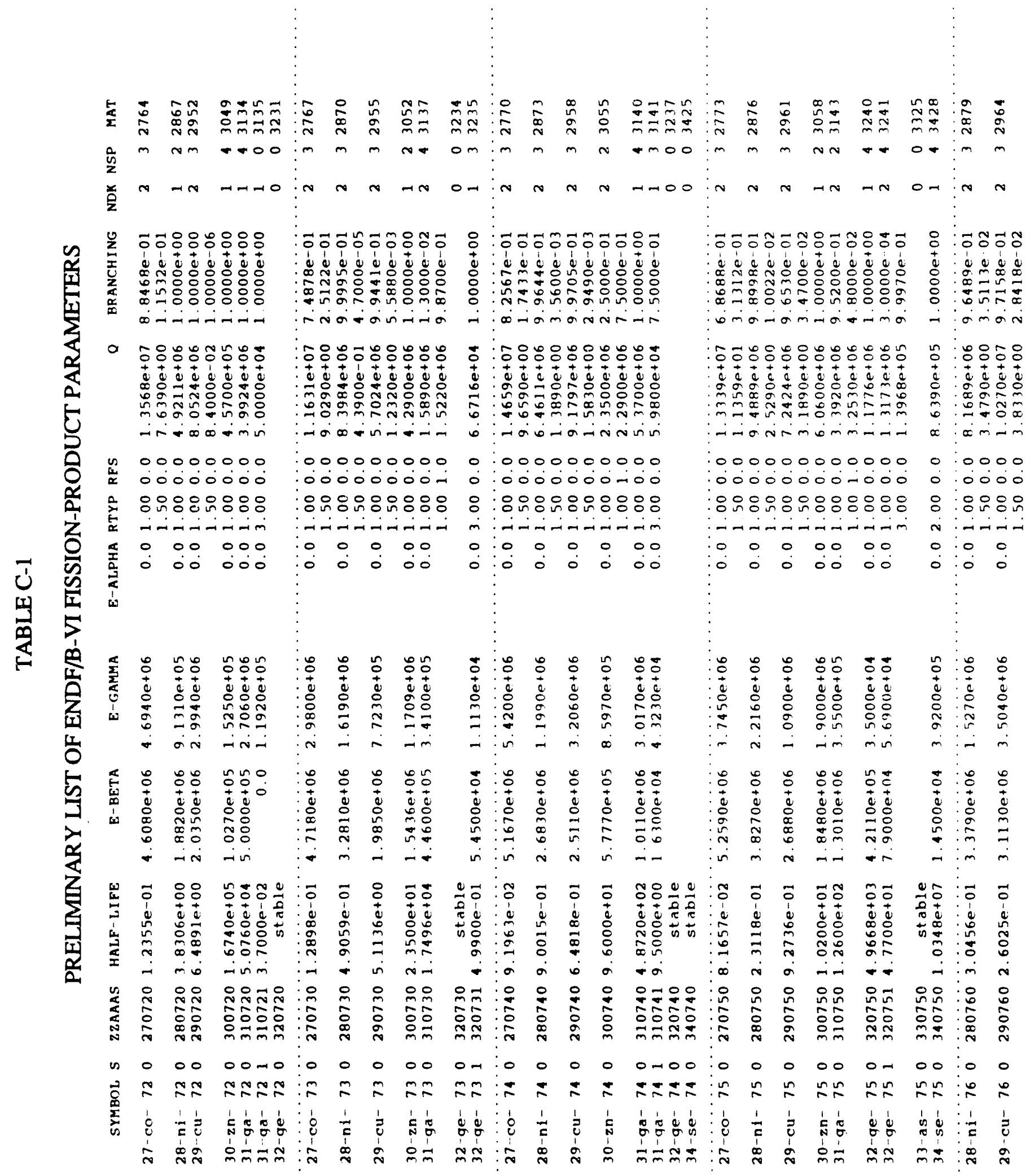




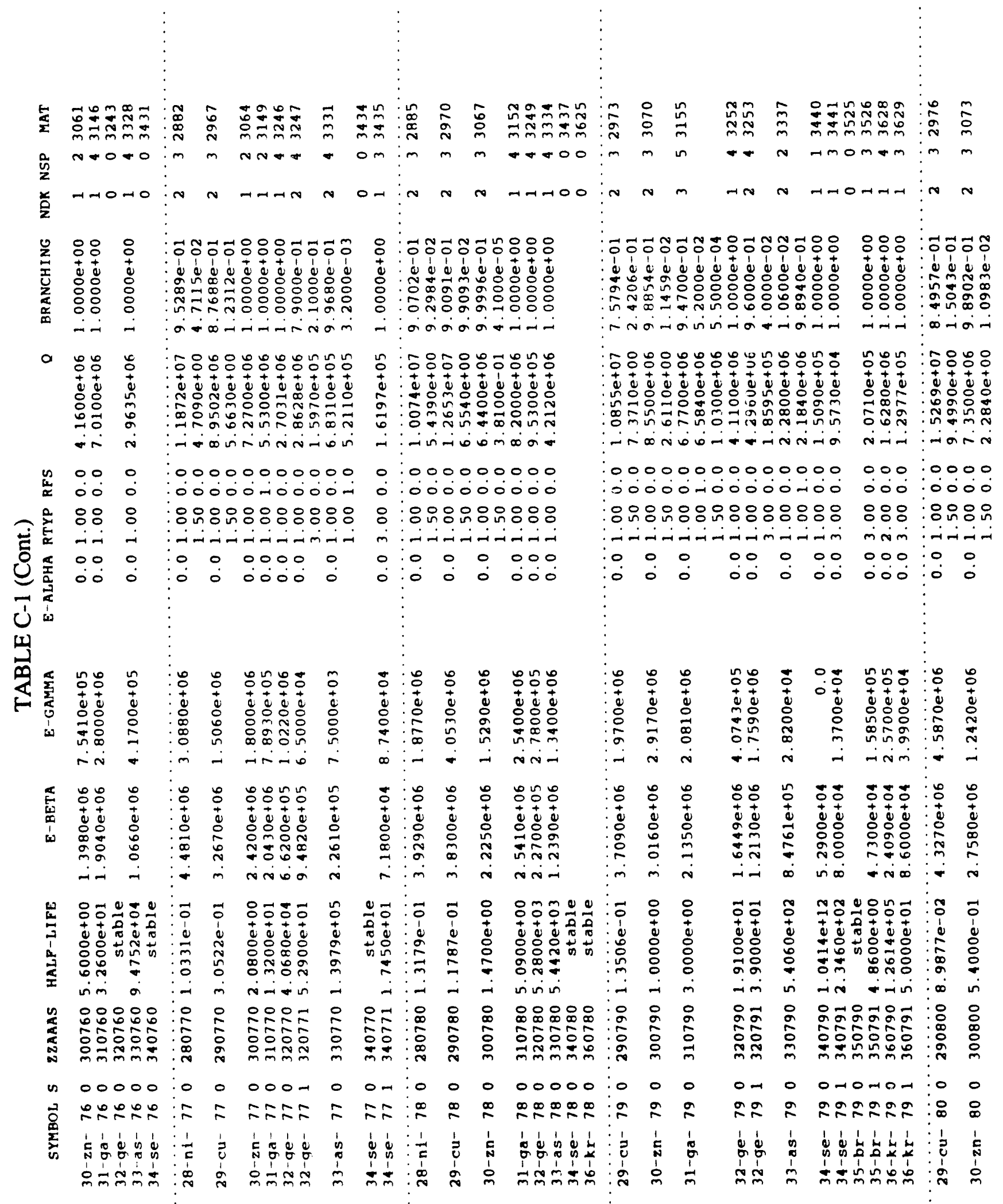




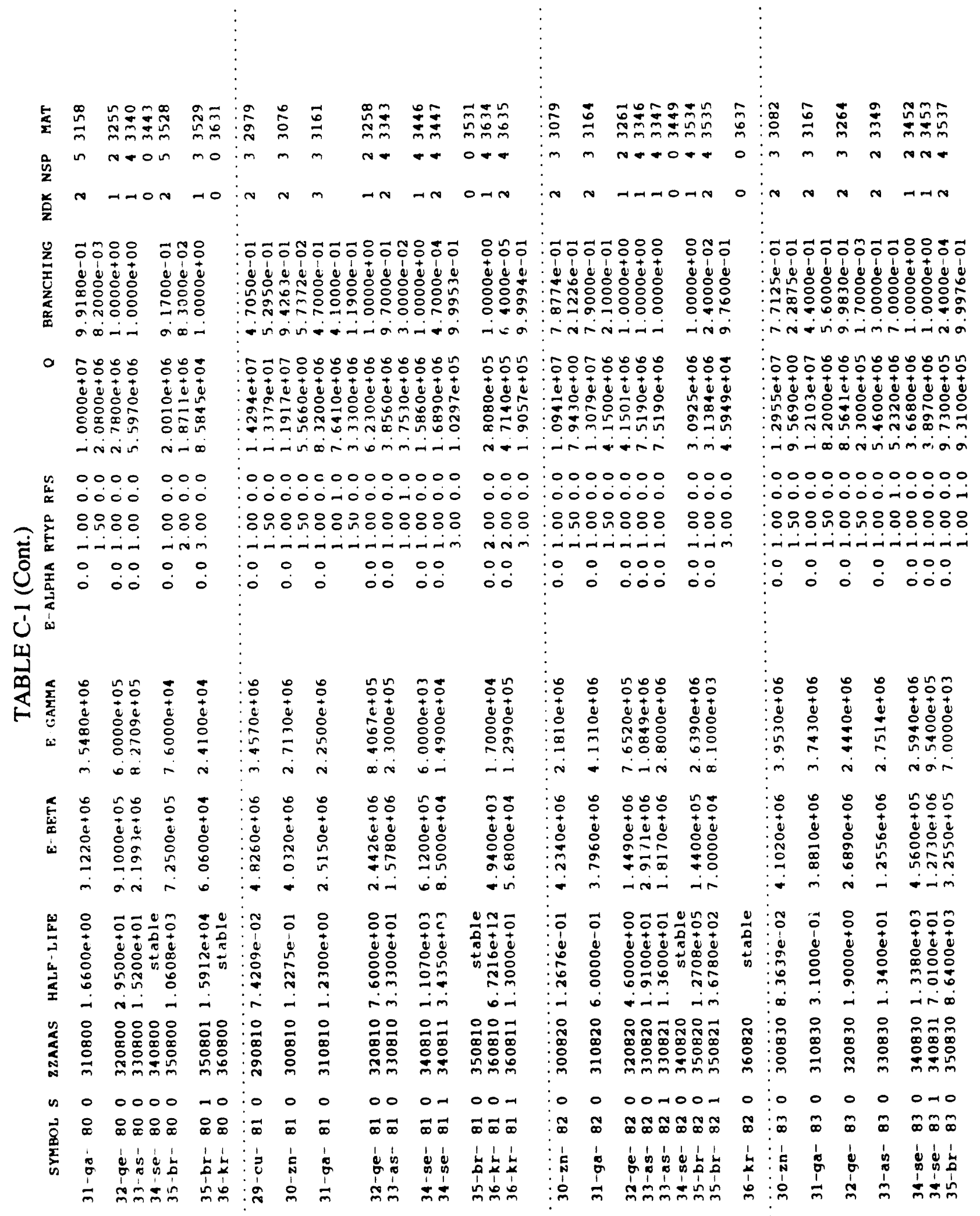




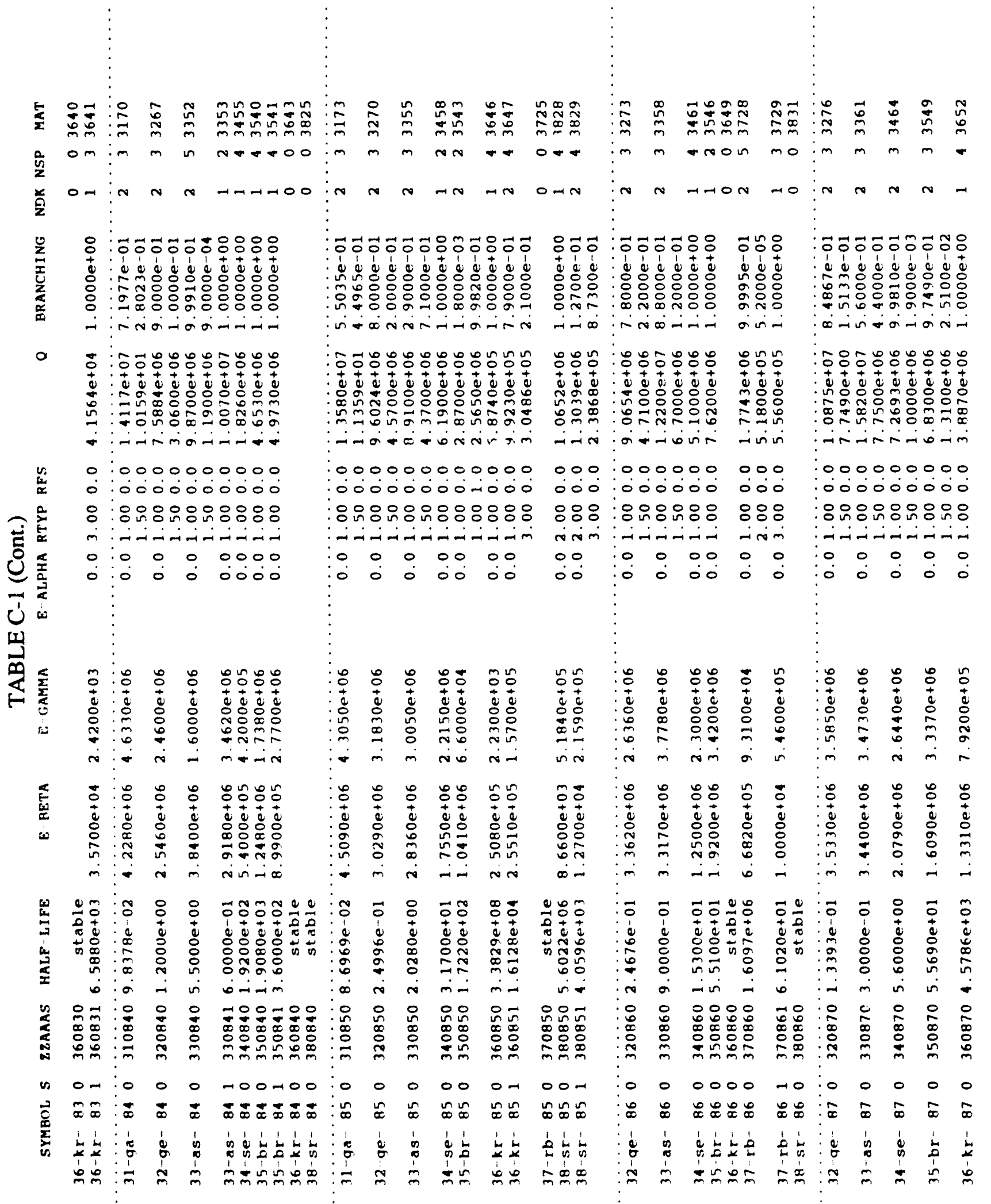




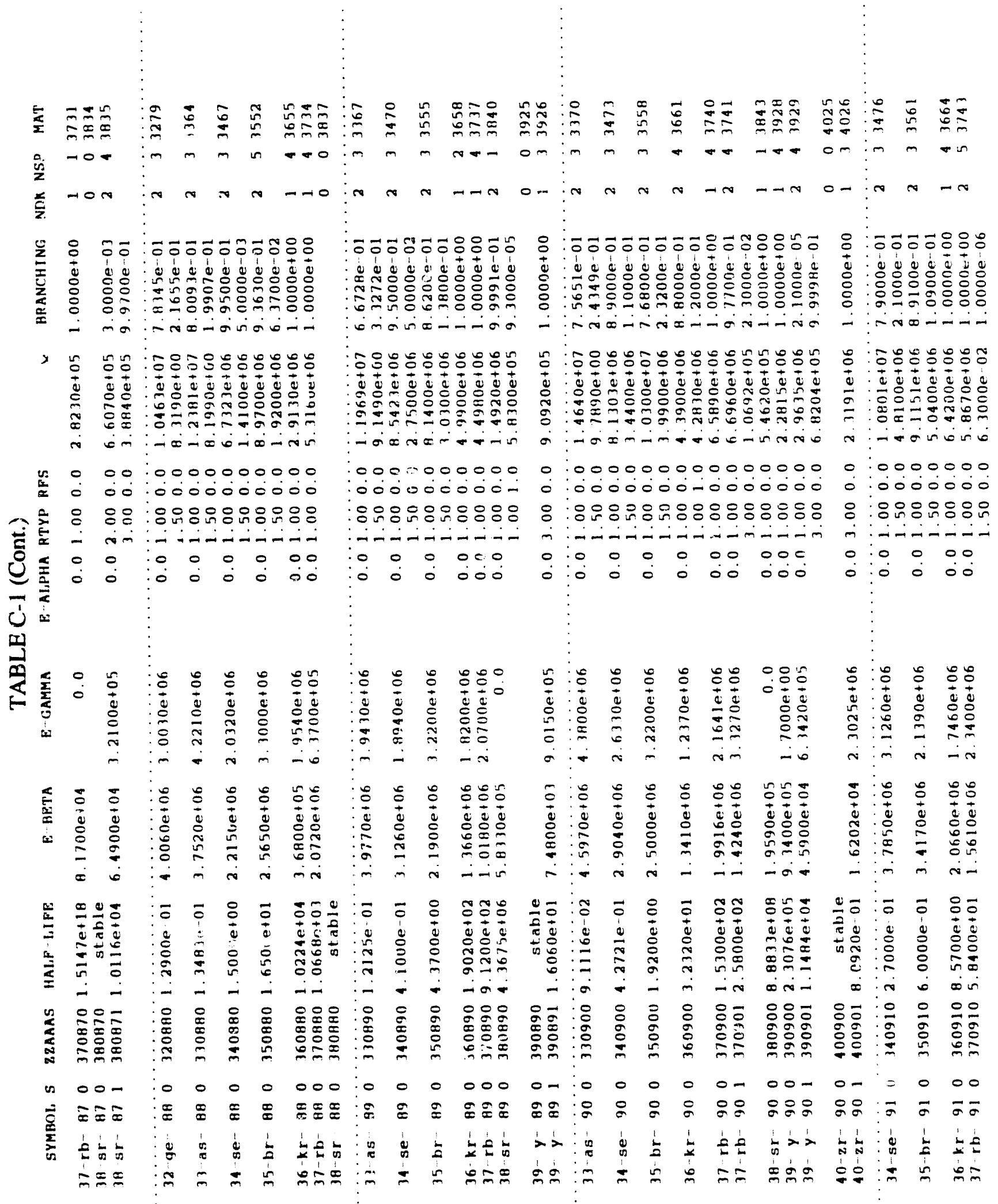




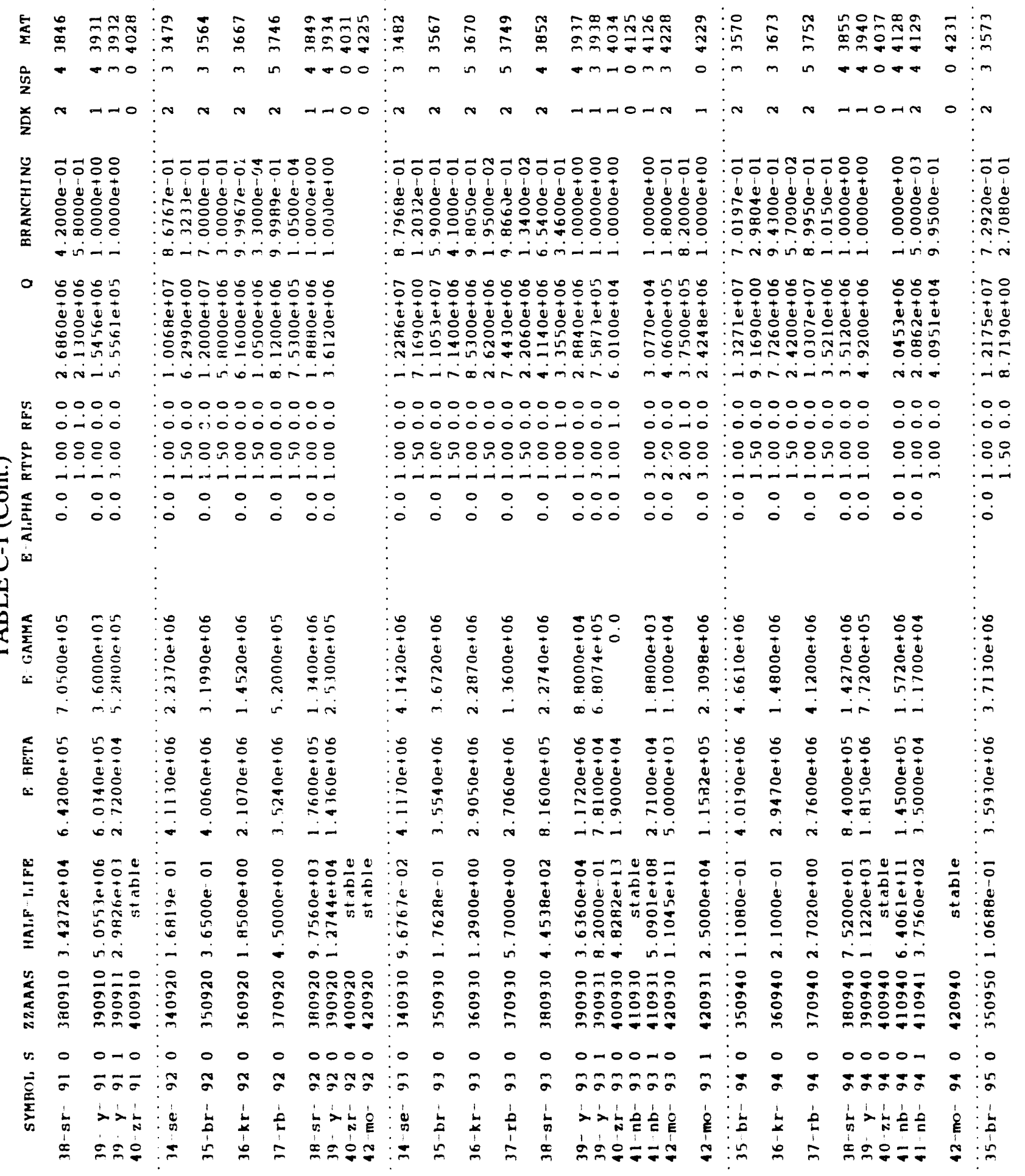




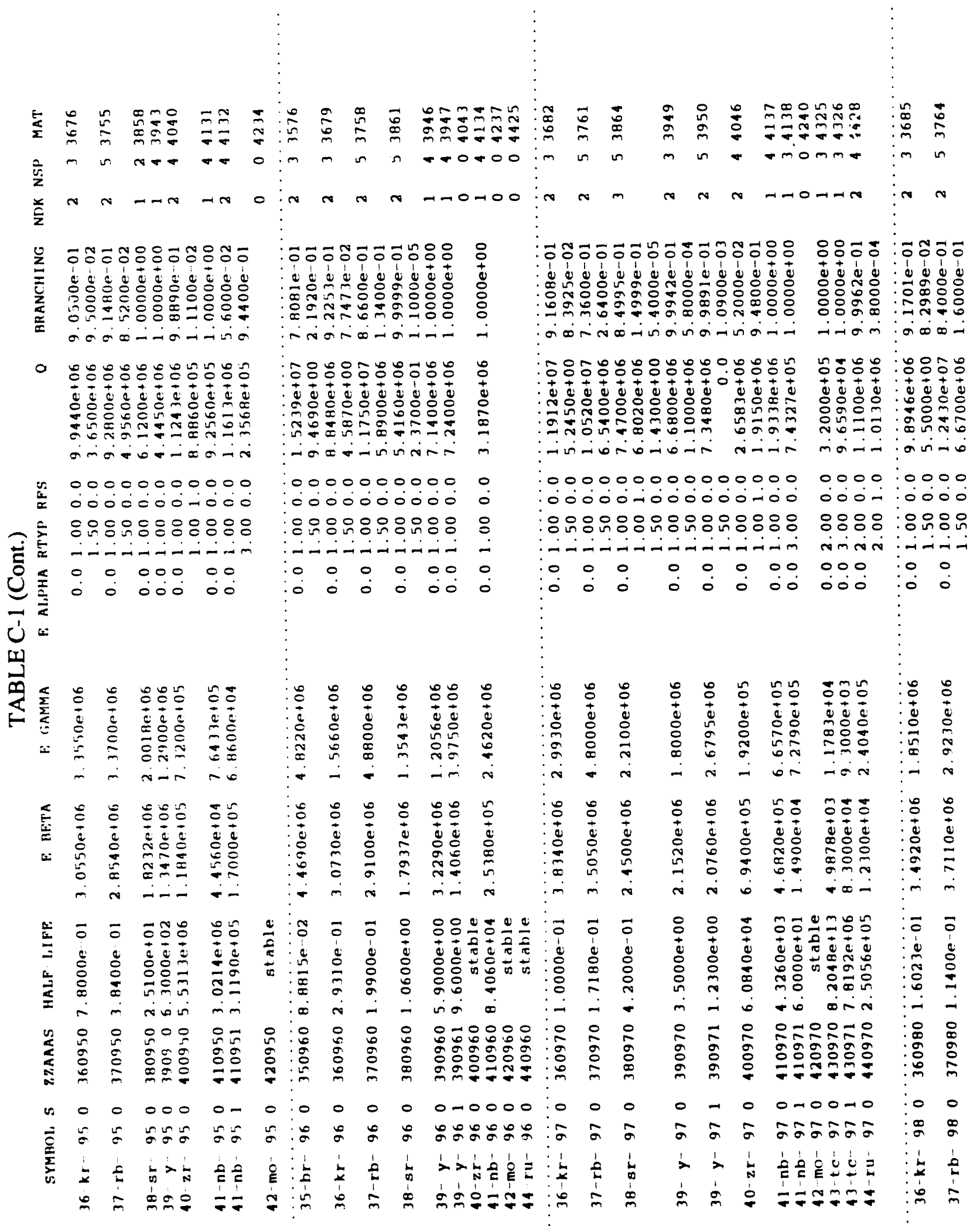




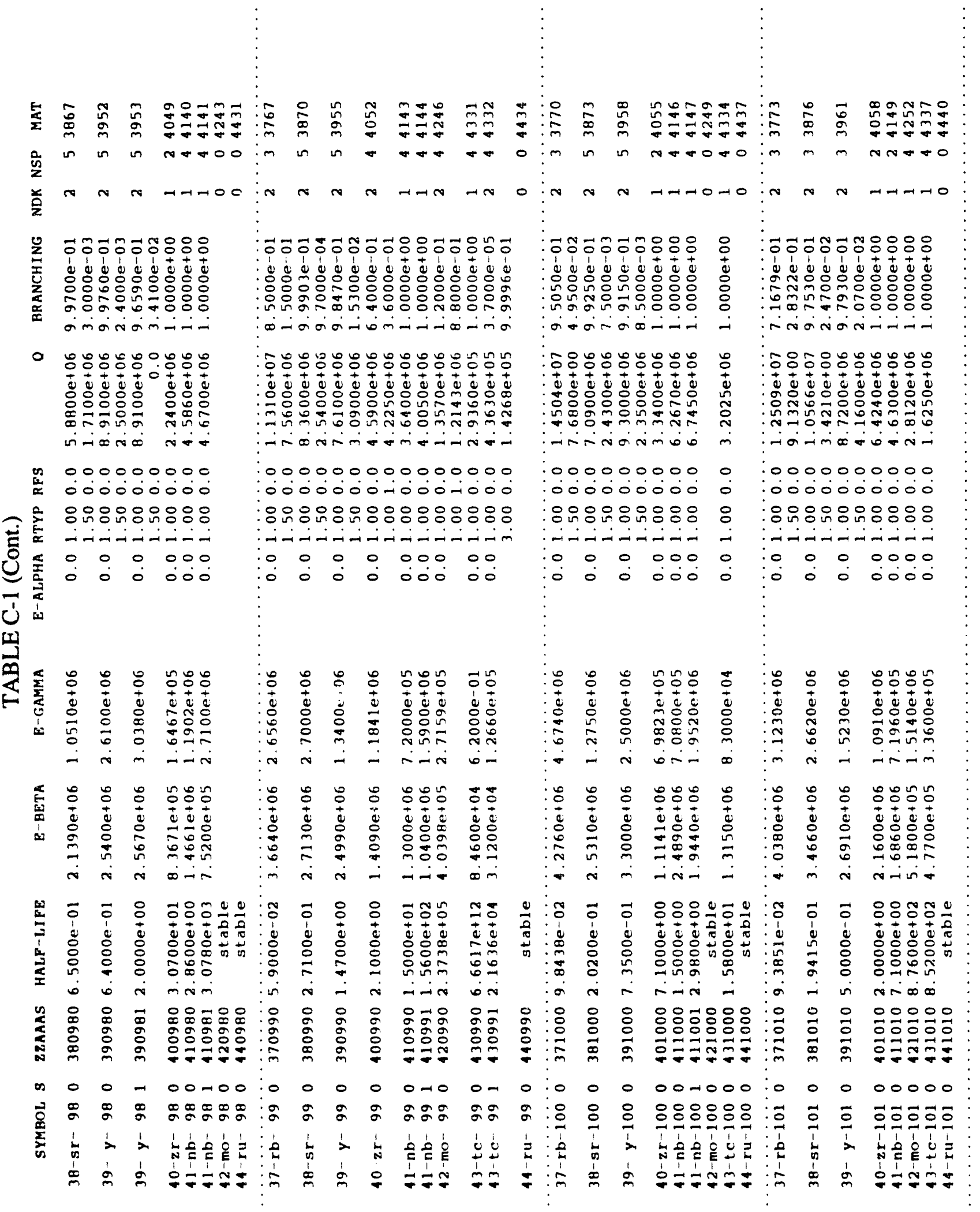




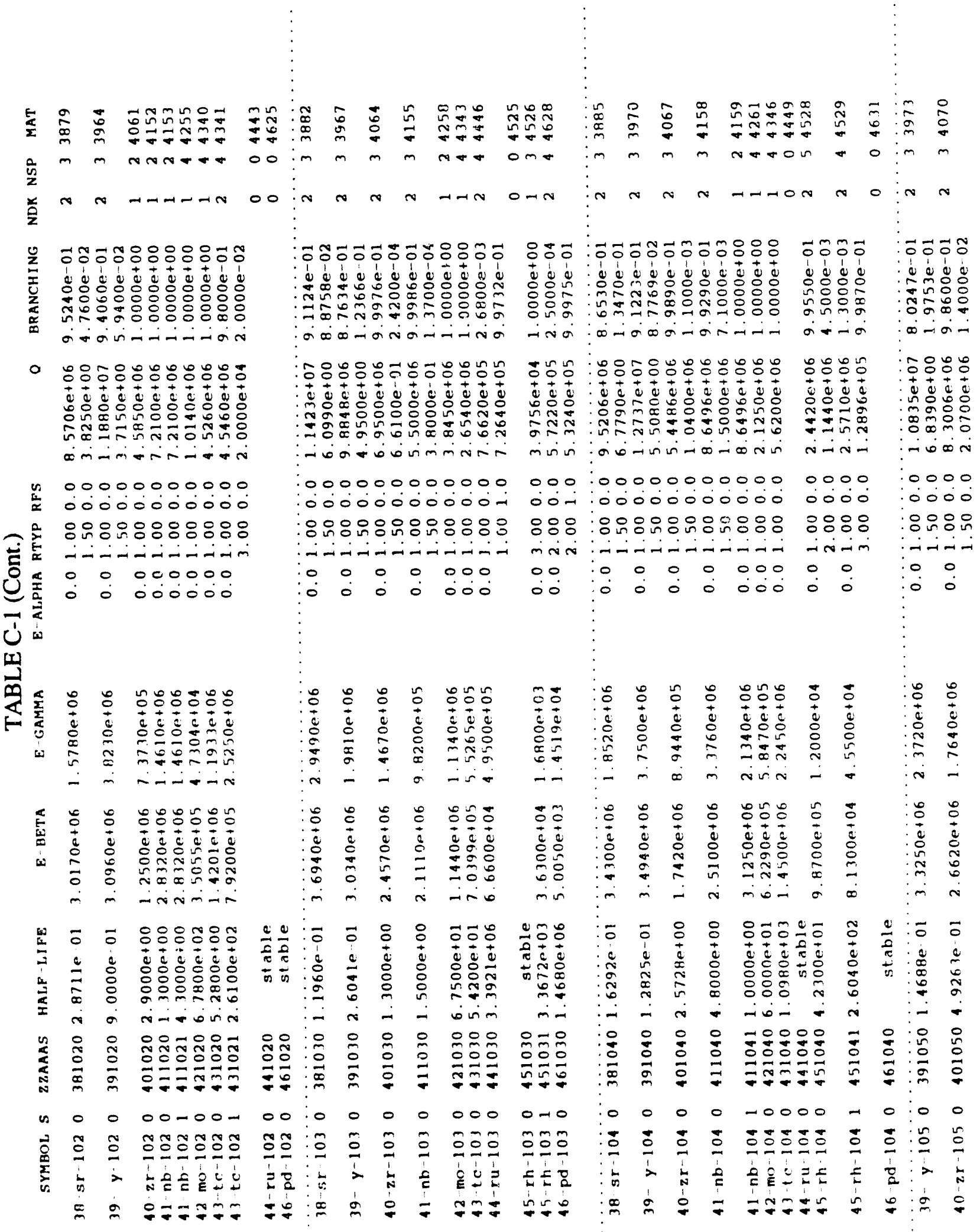




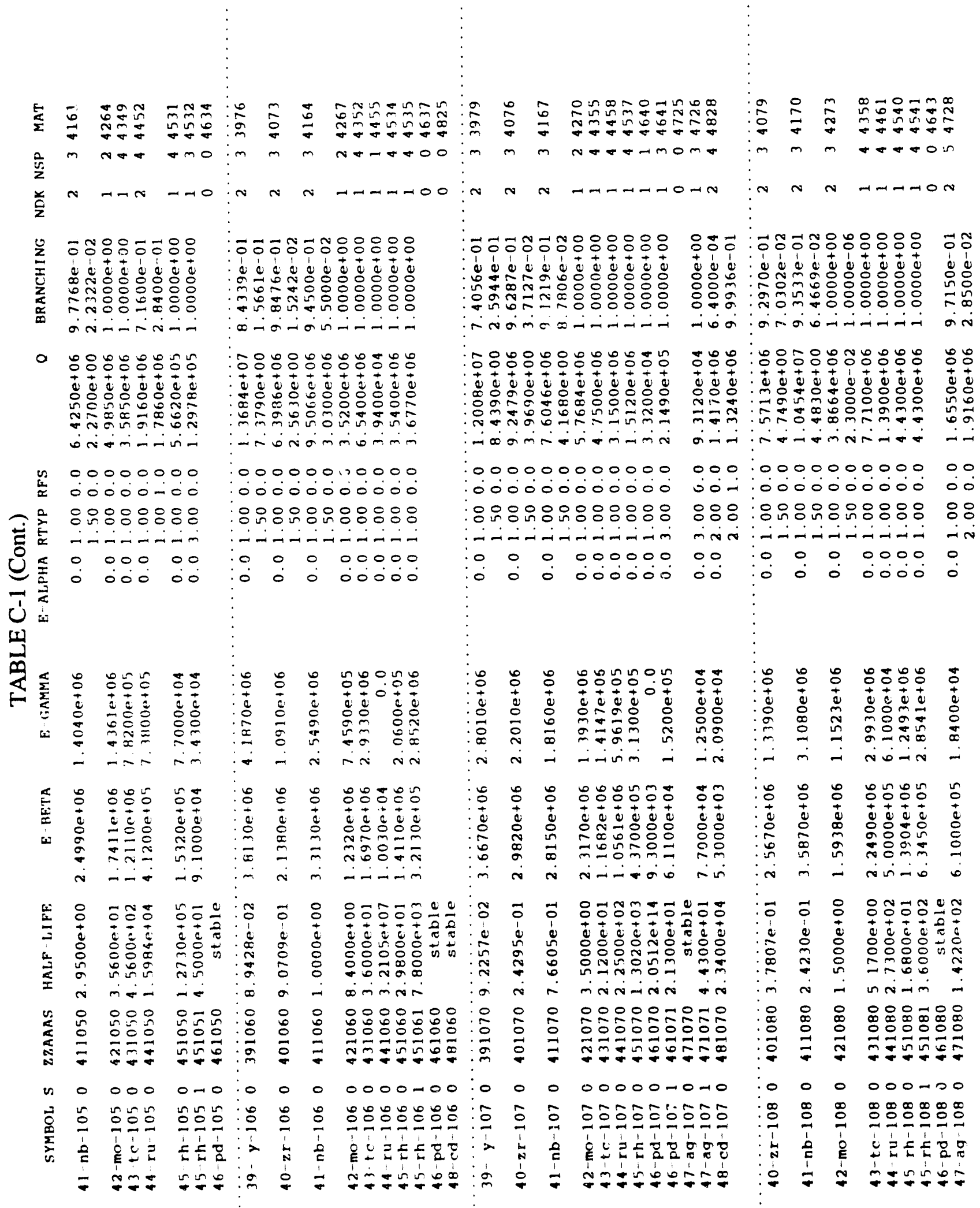




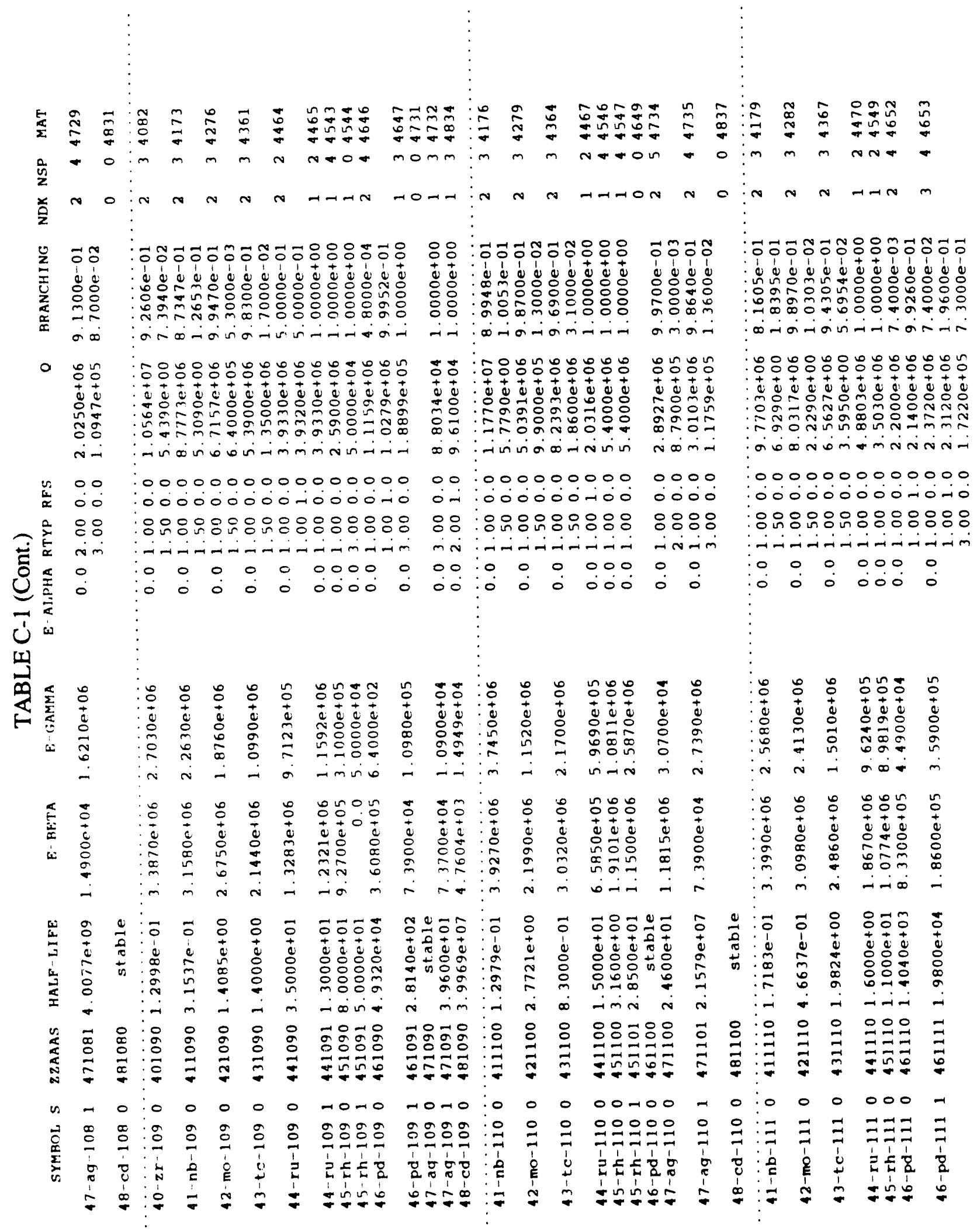




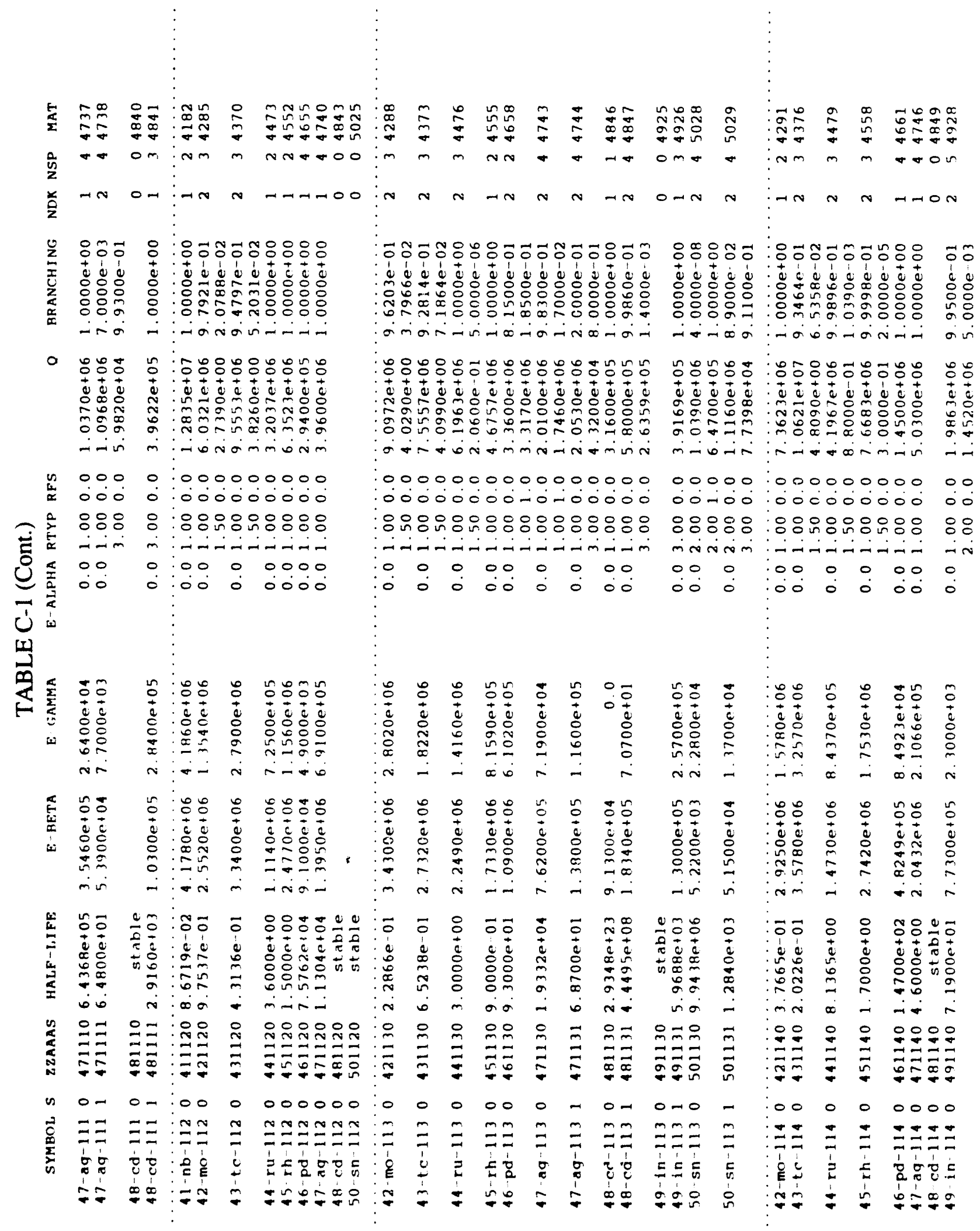




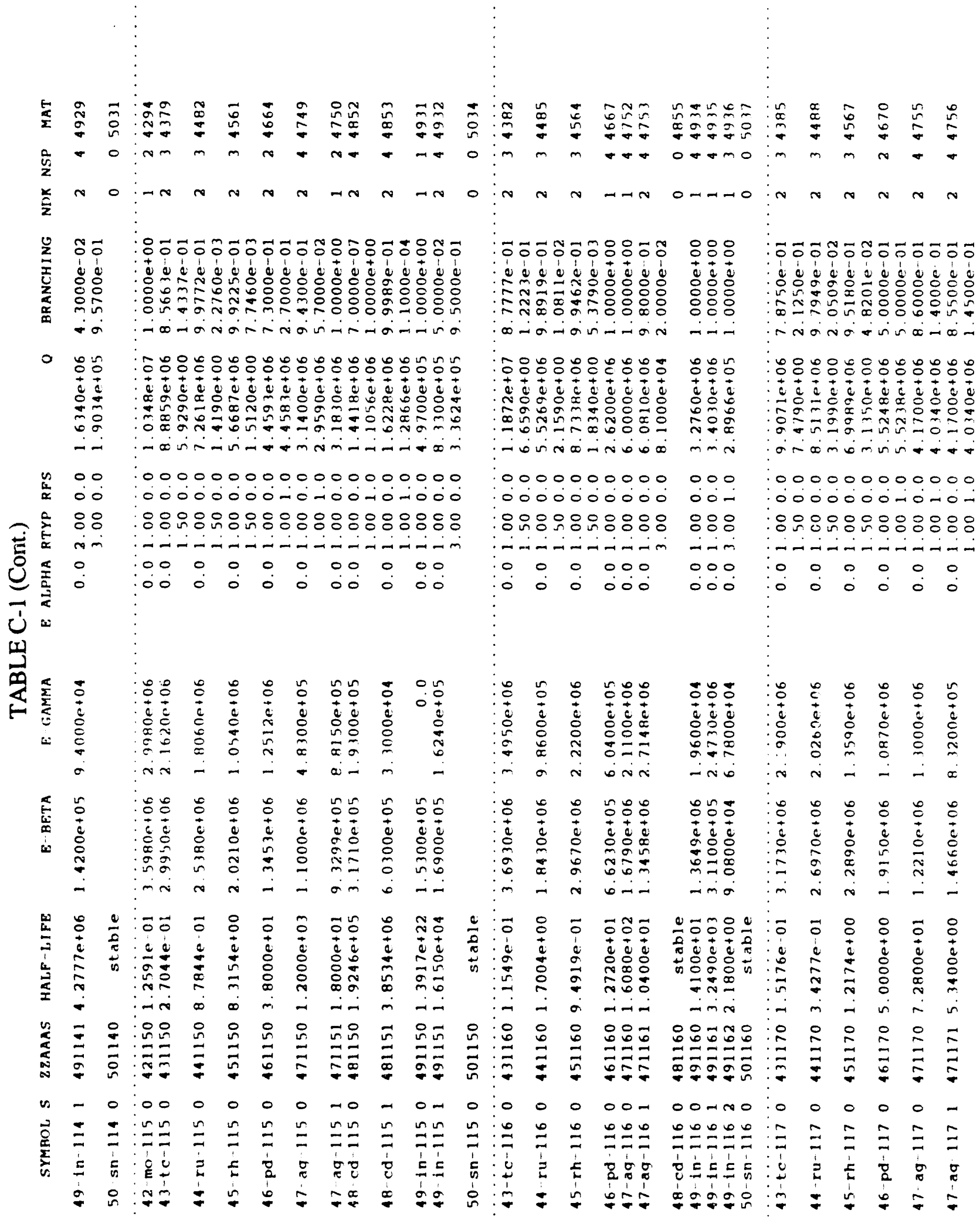




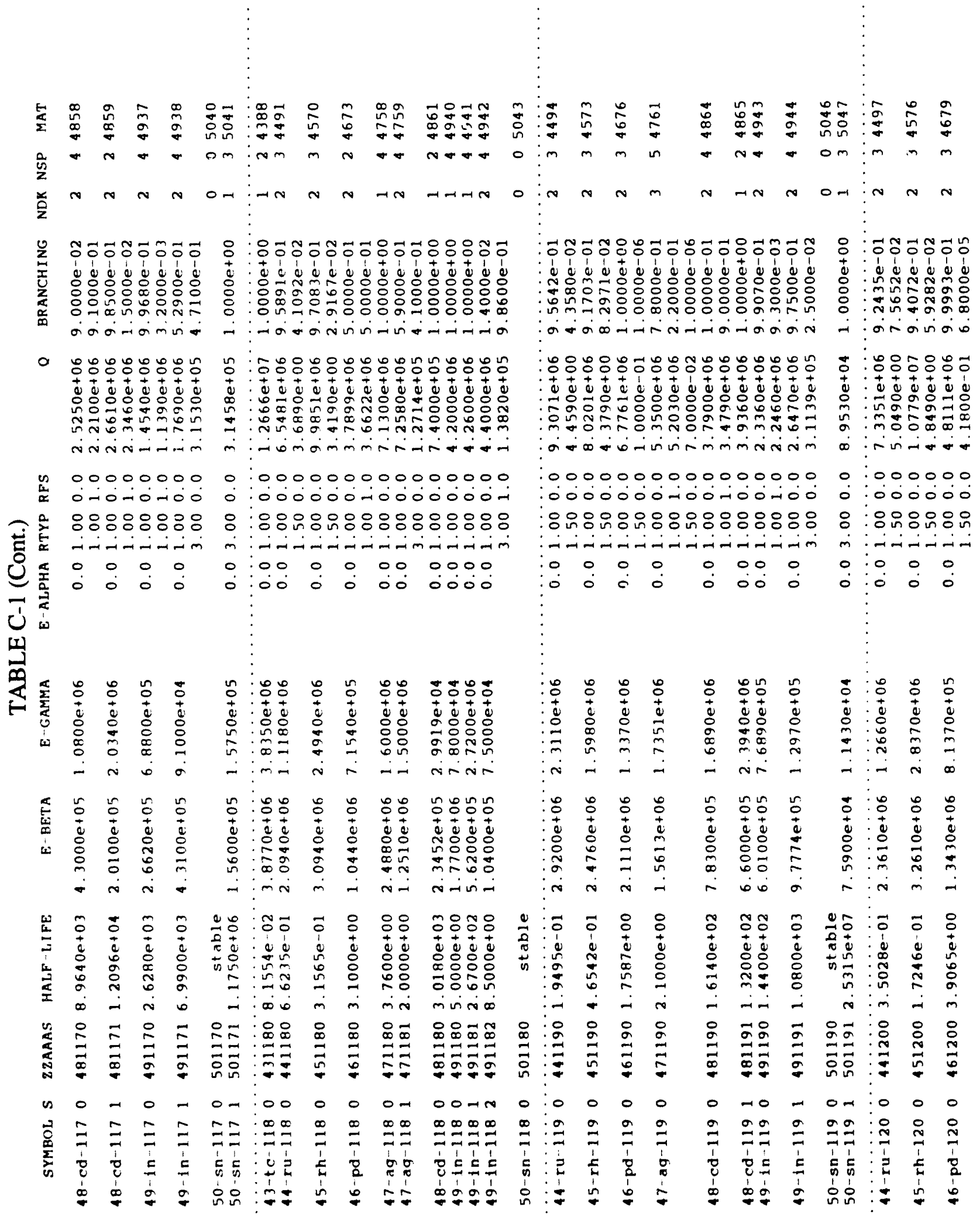




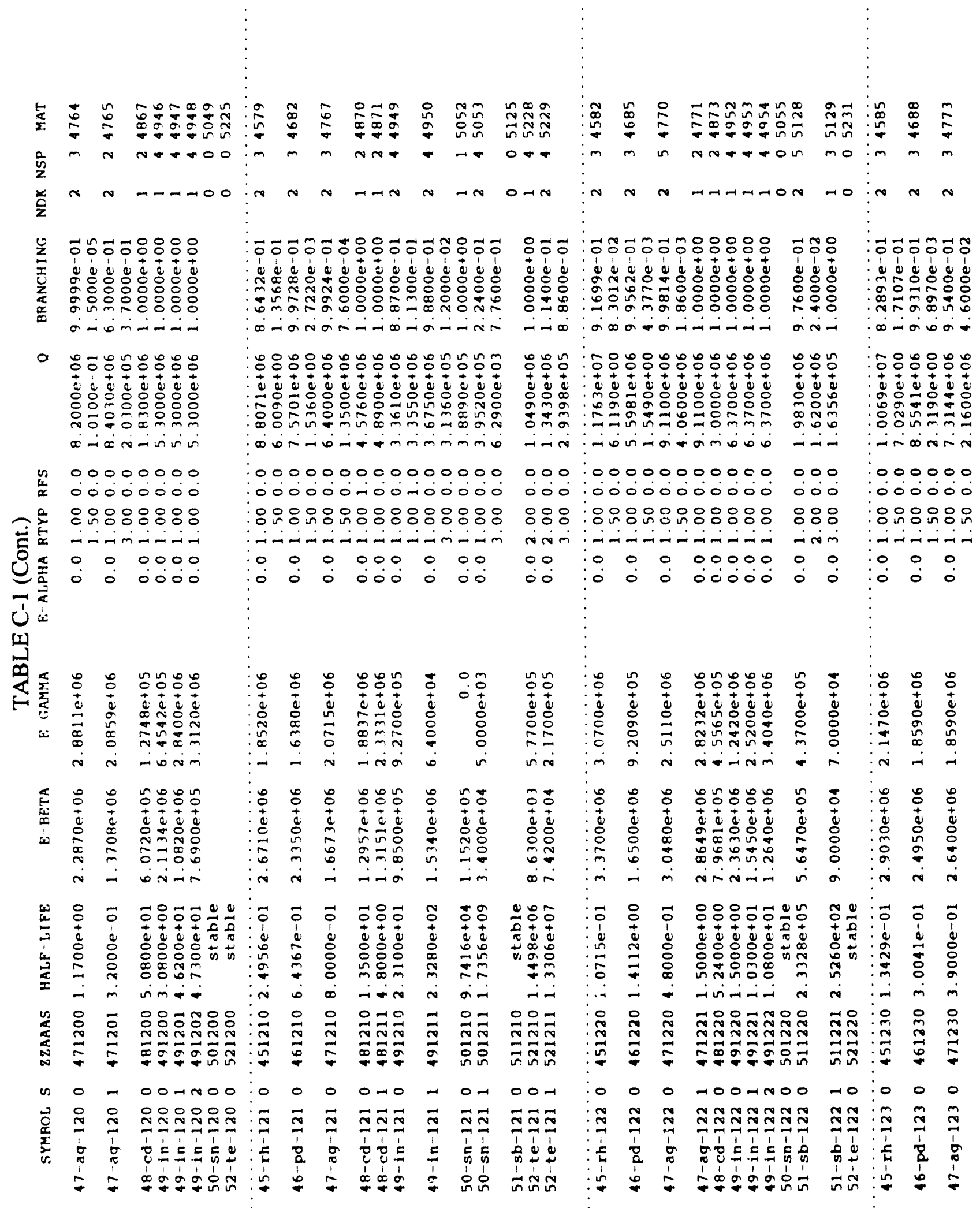




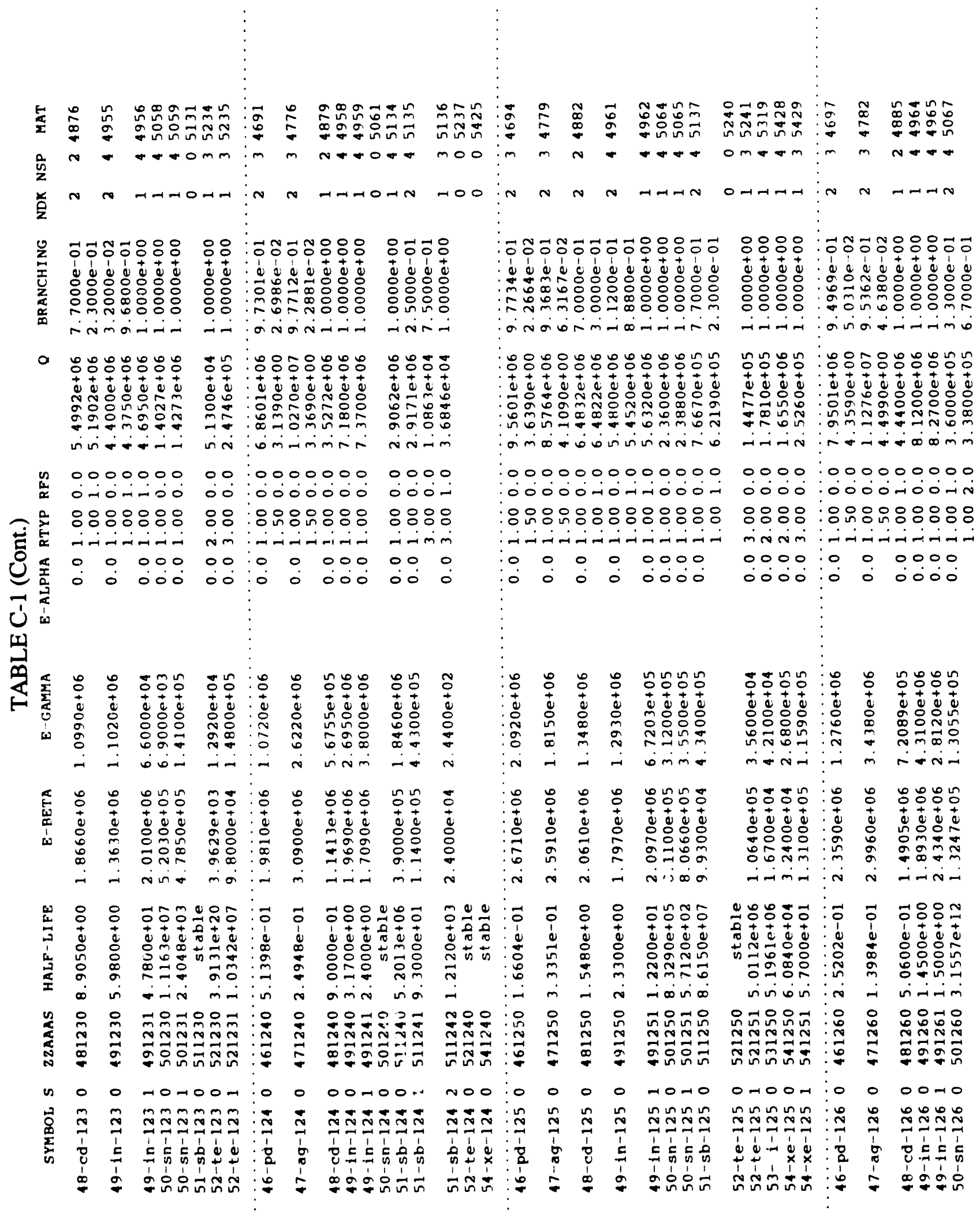




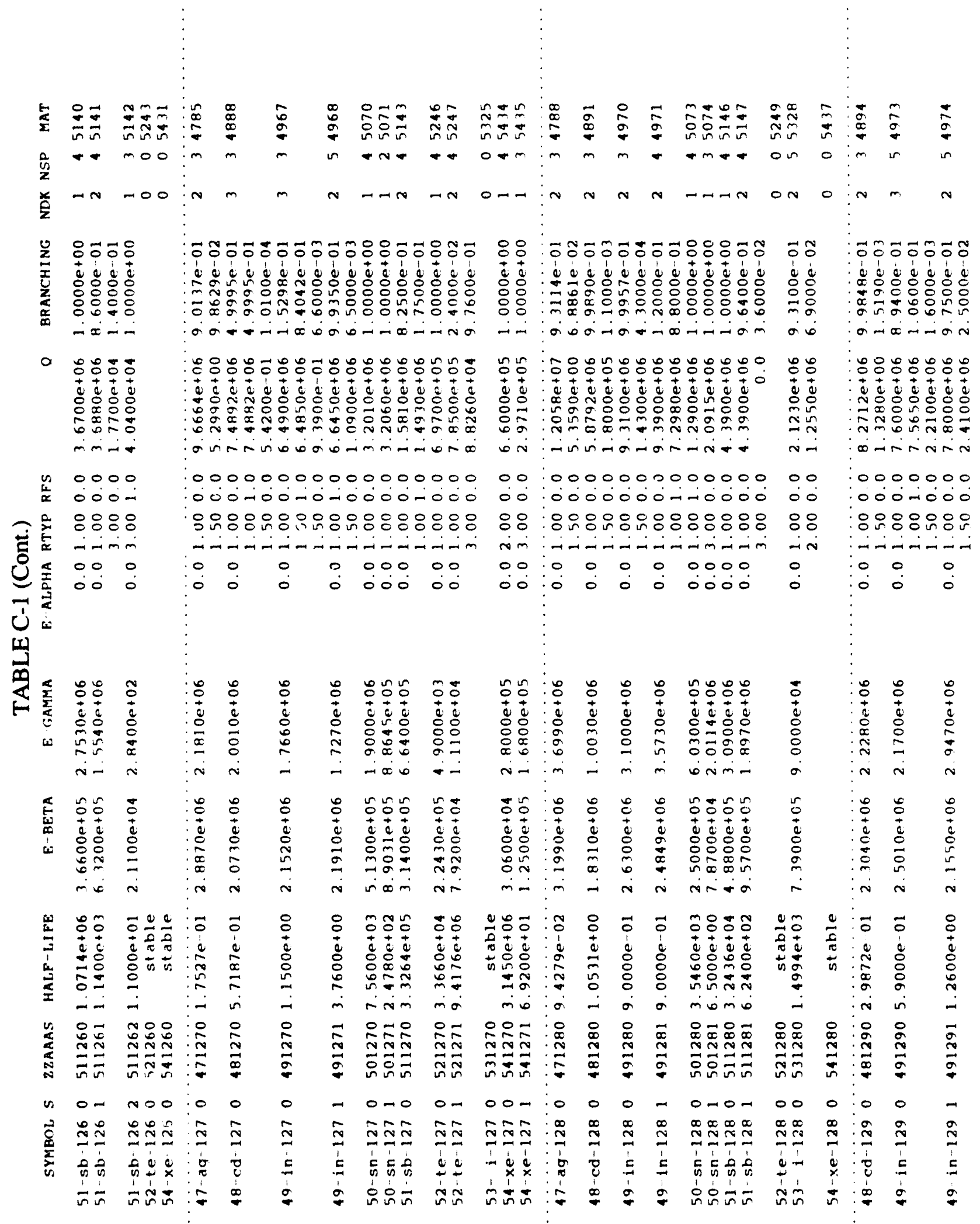




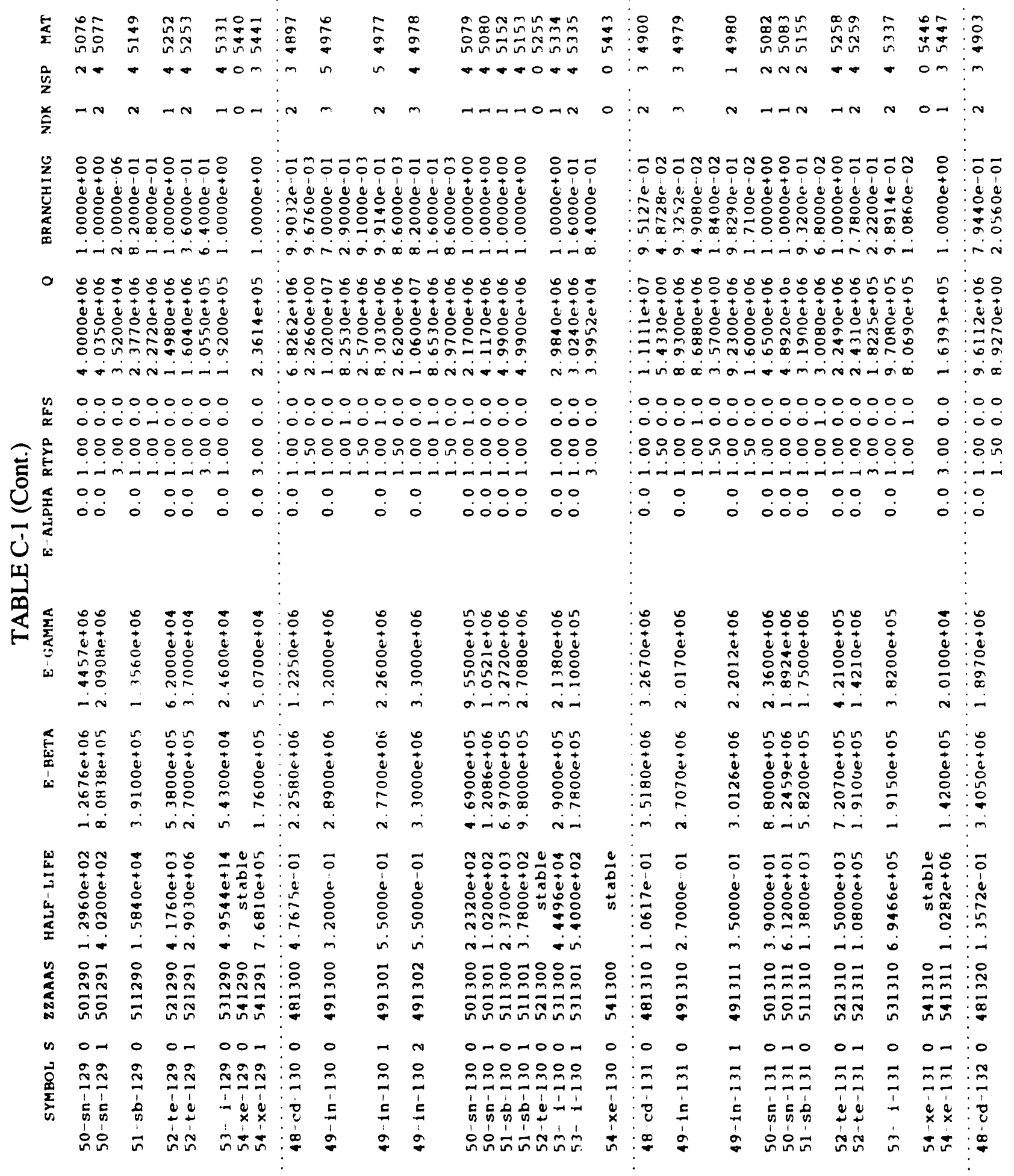




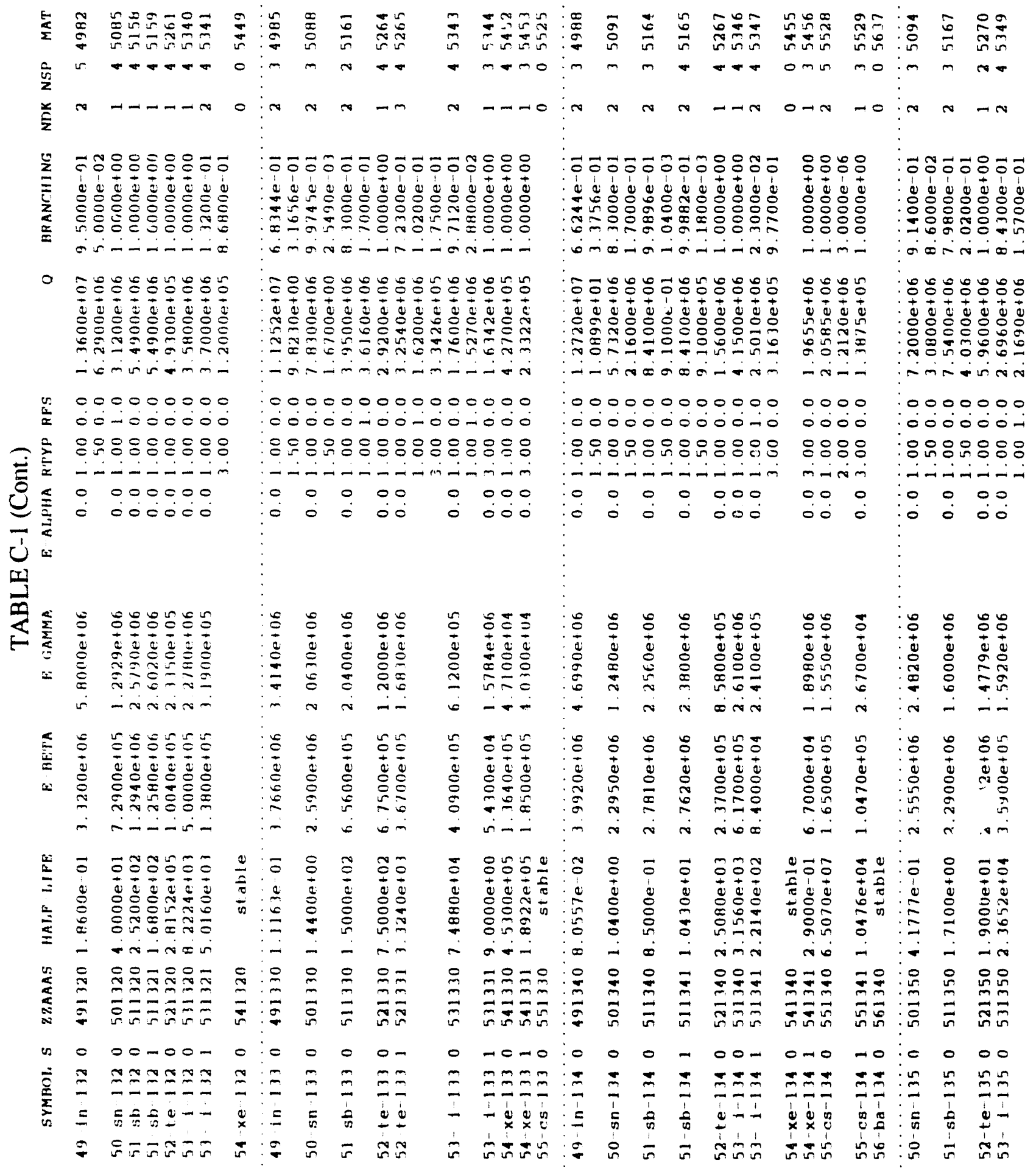




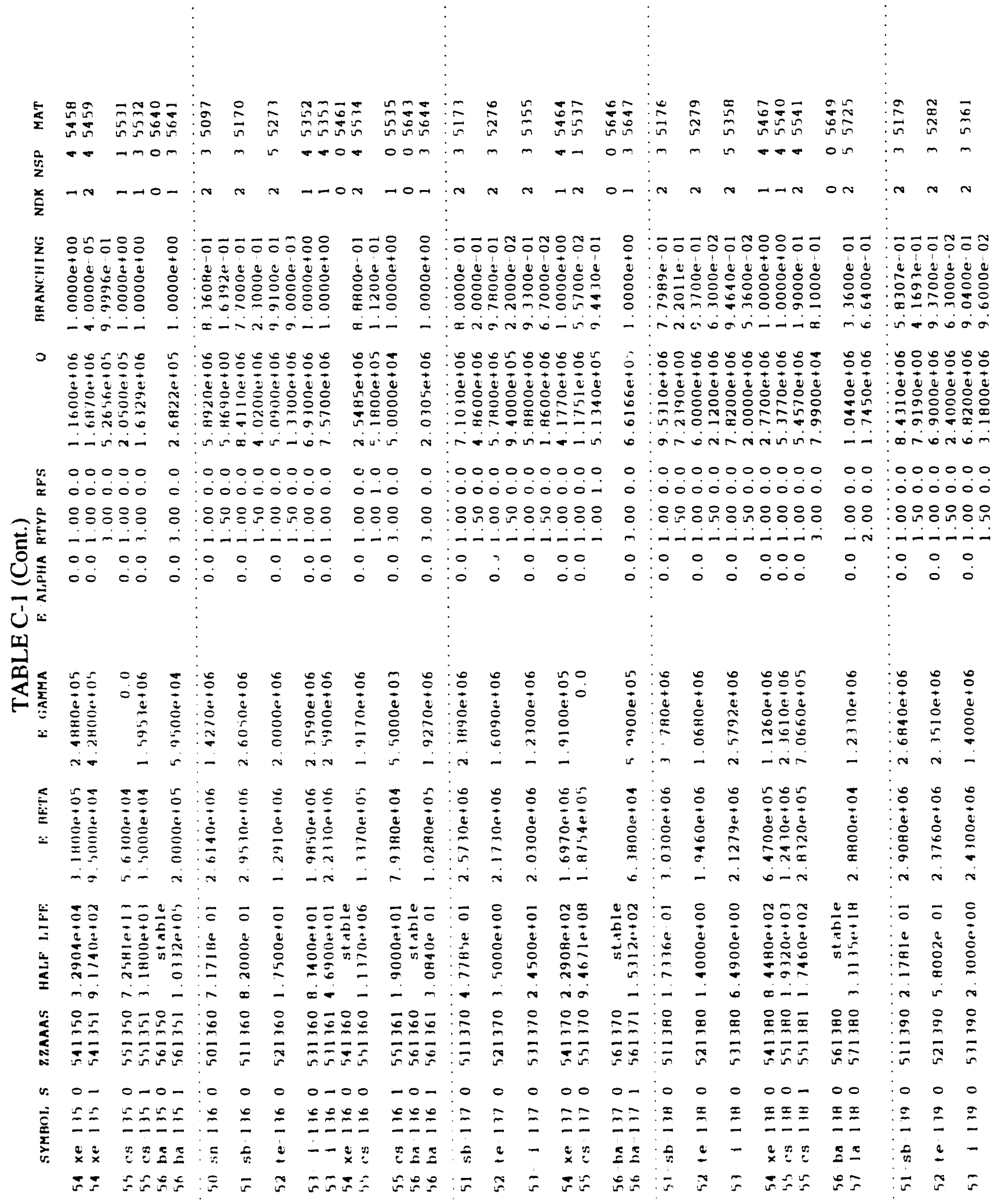




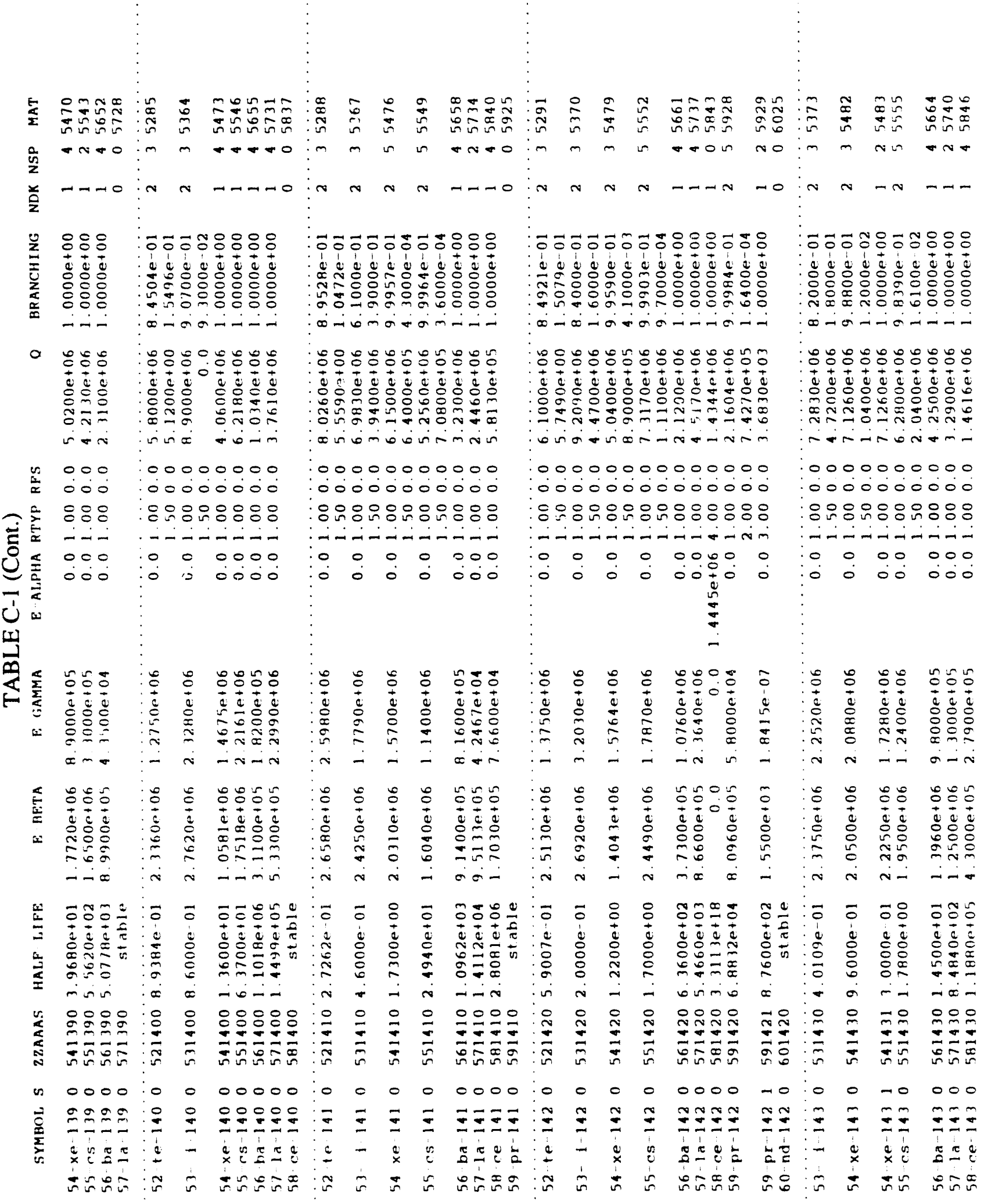




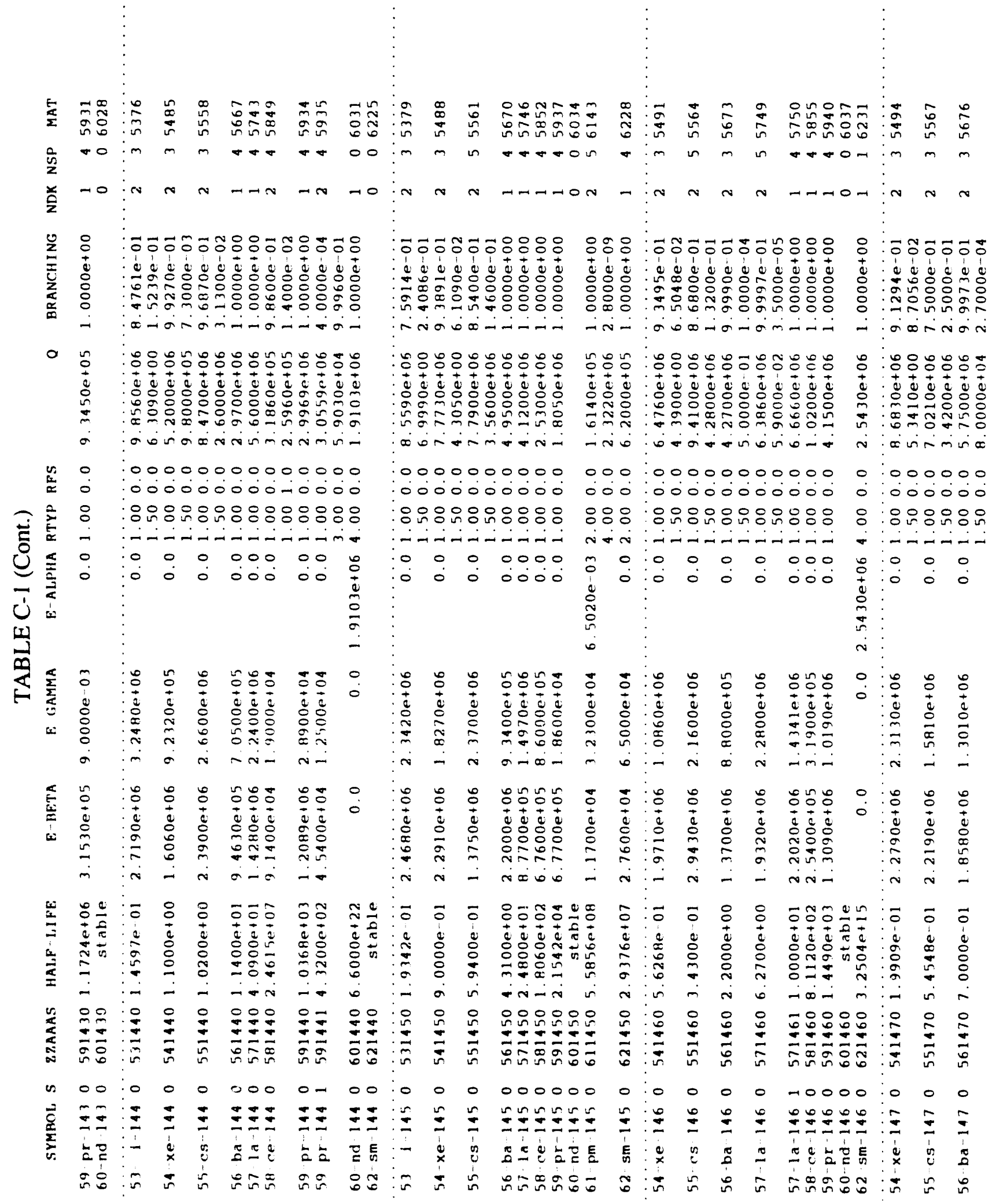




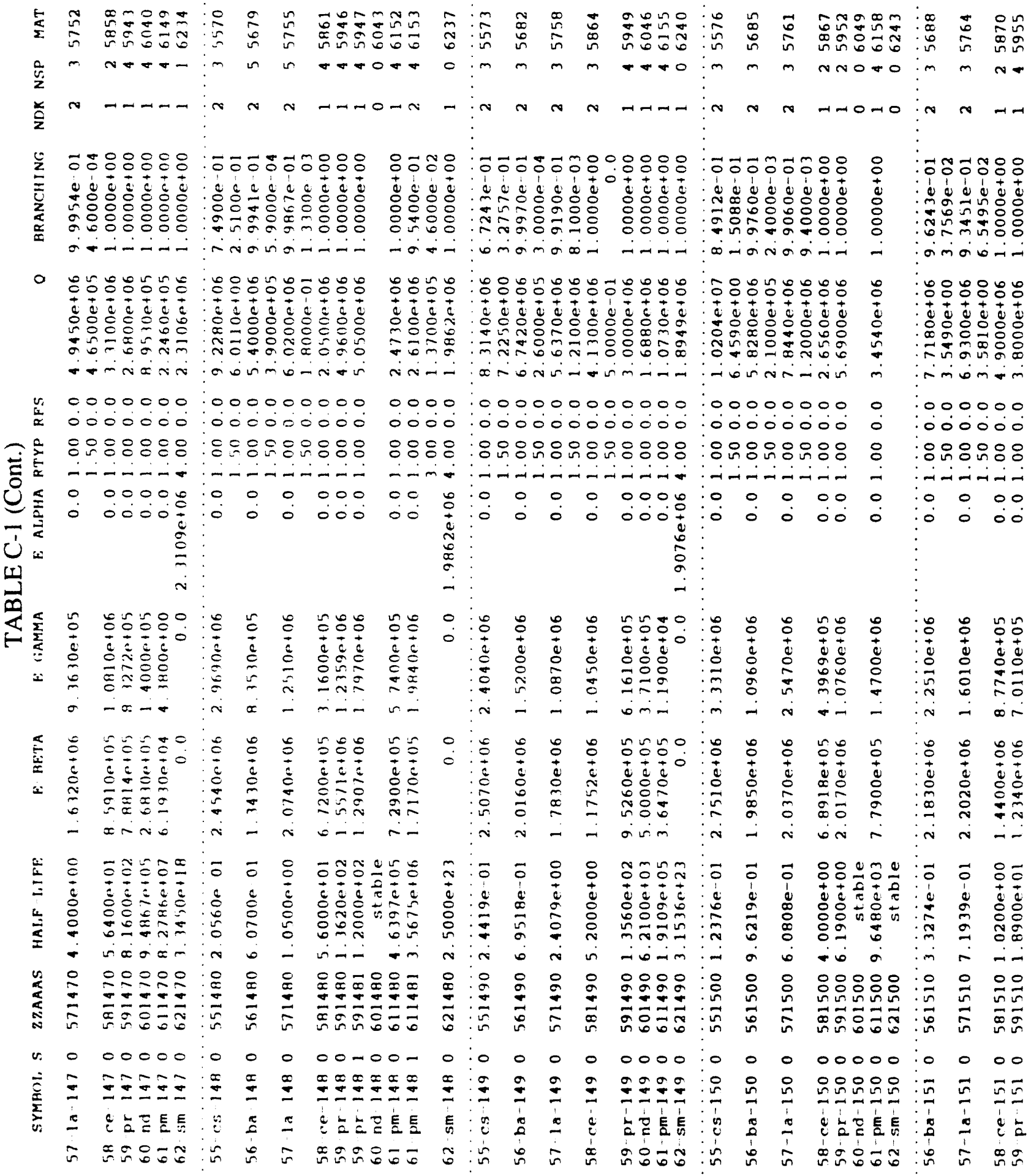




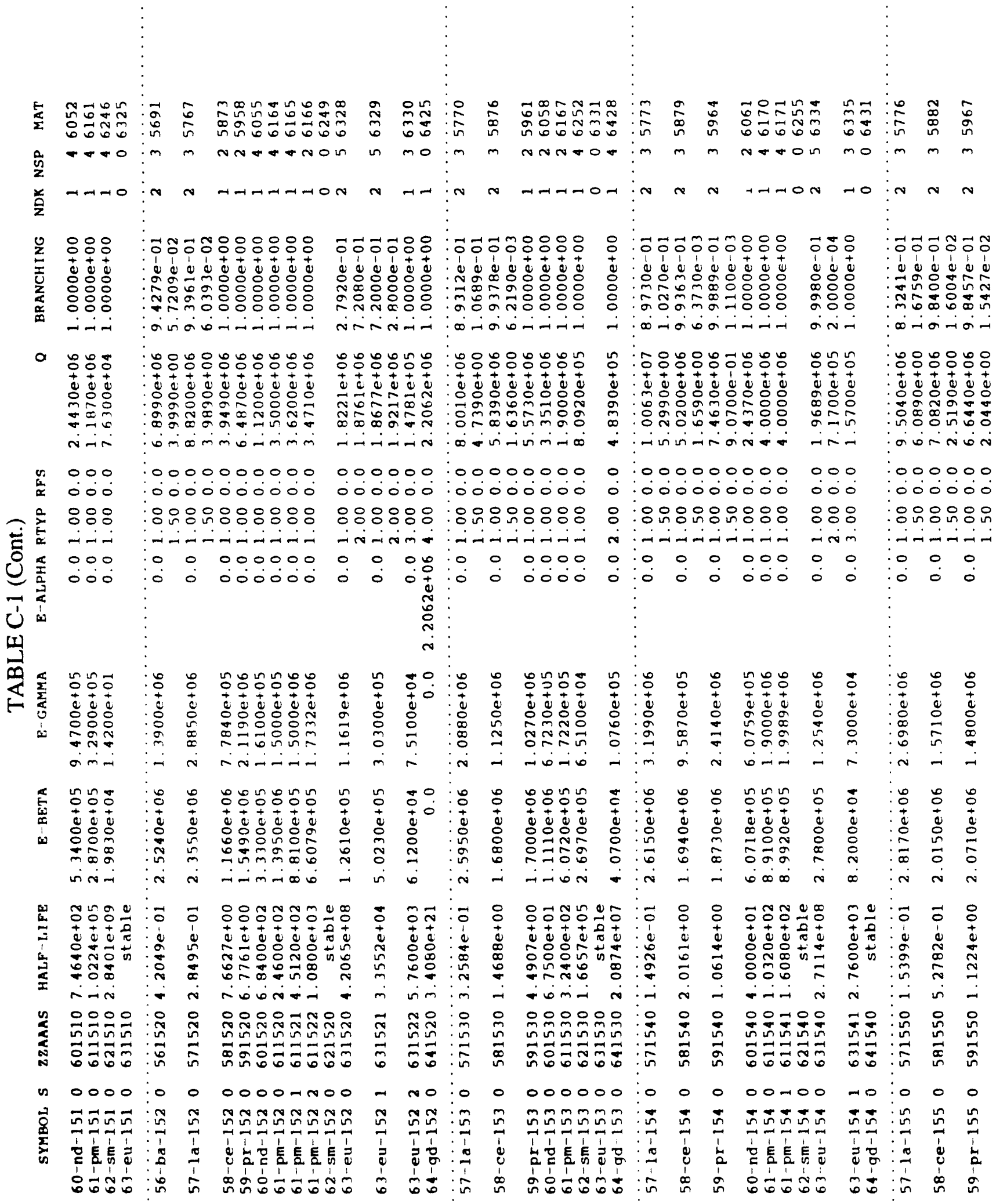




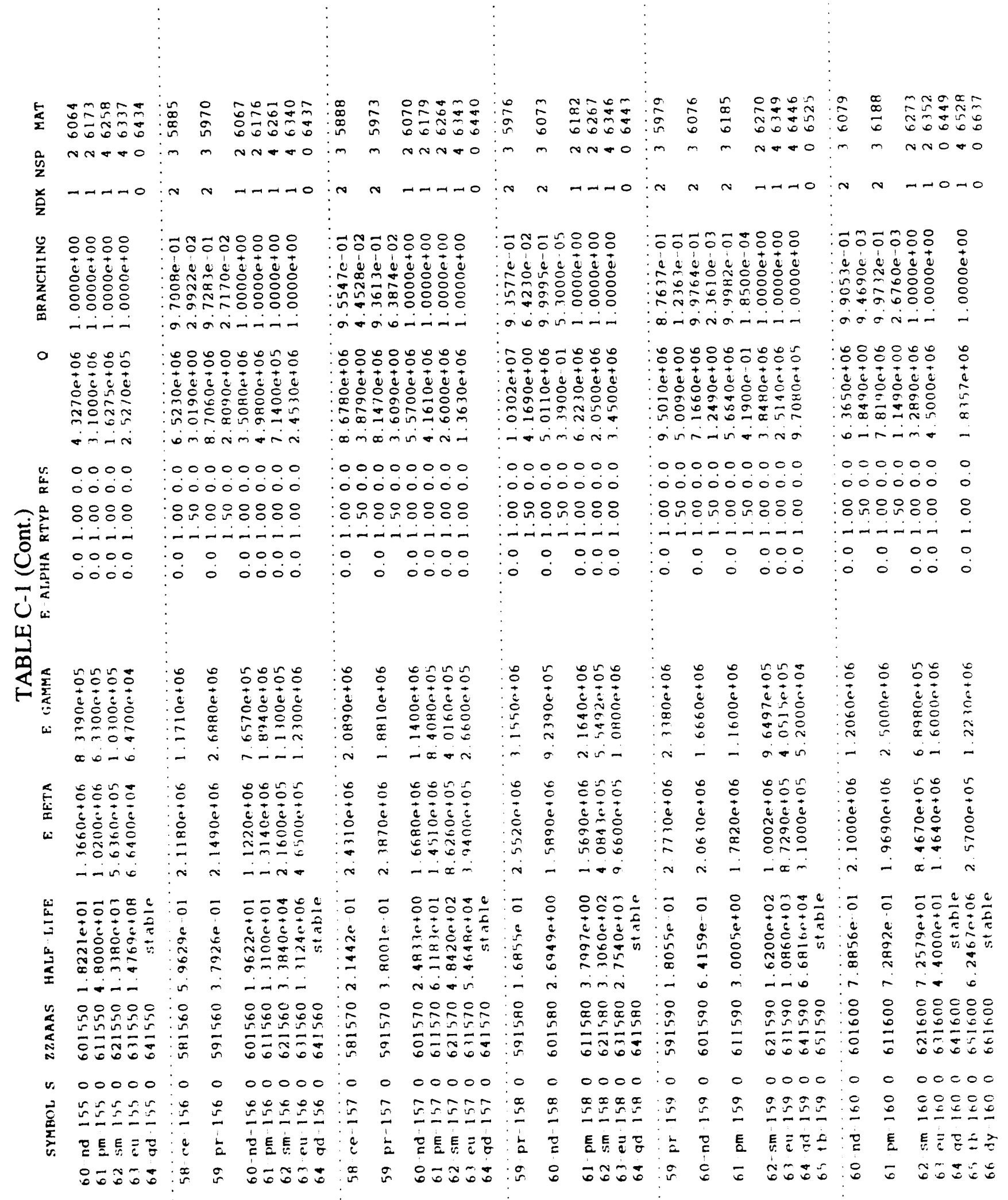




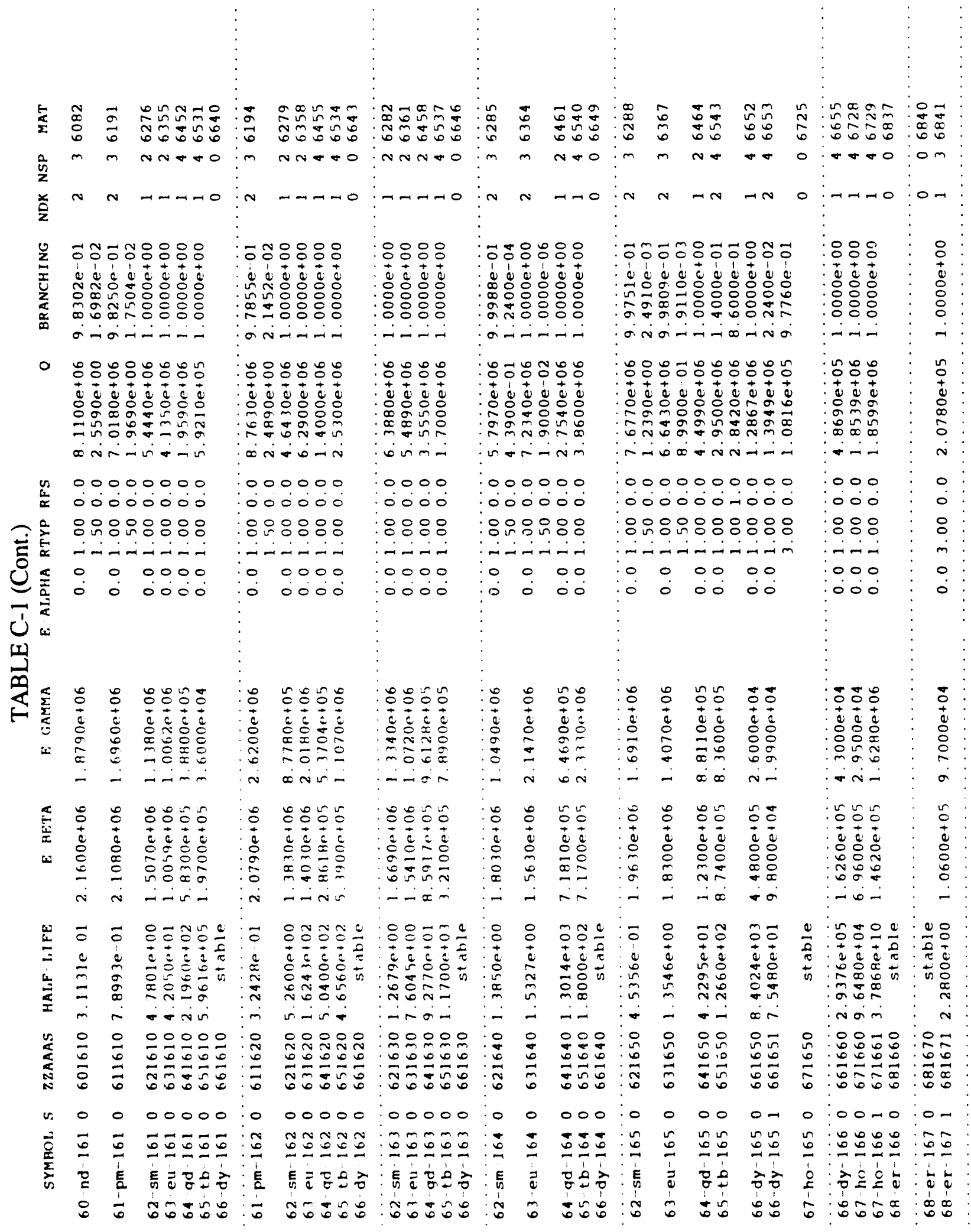


TABLE C-1 (Cont.)

Data Count

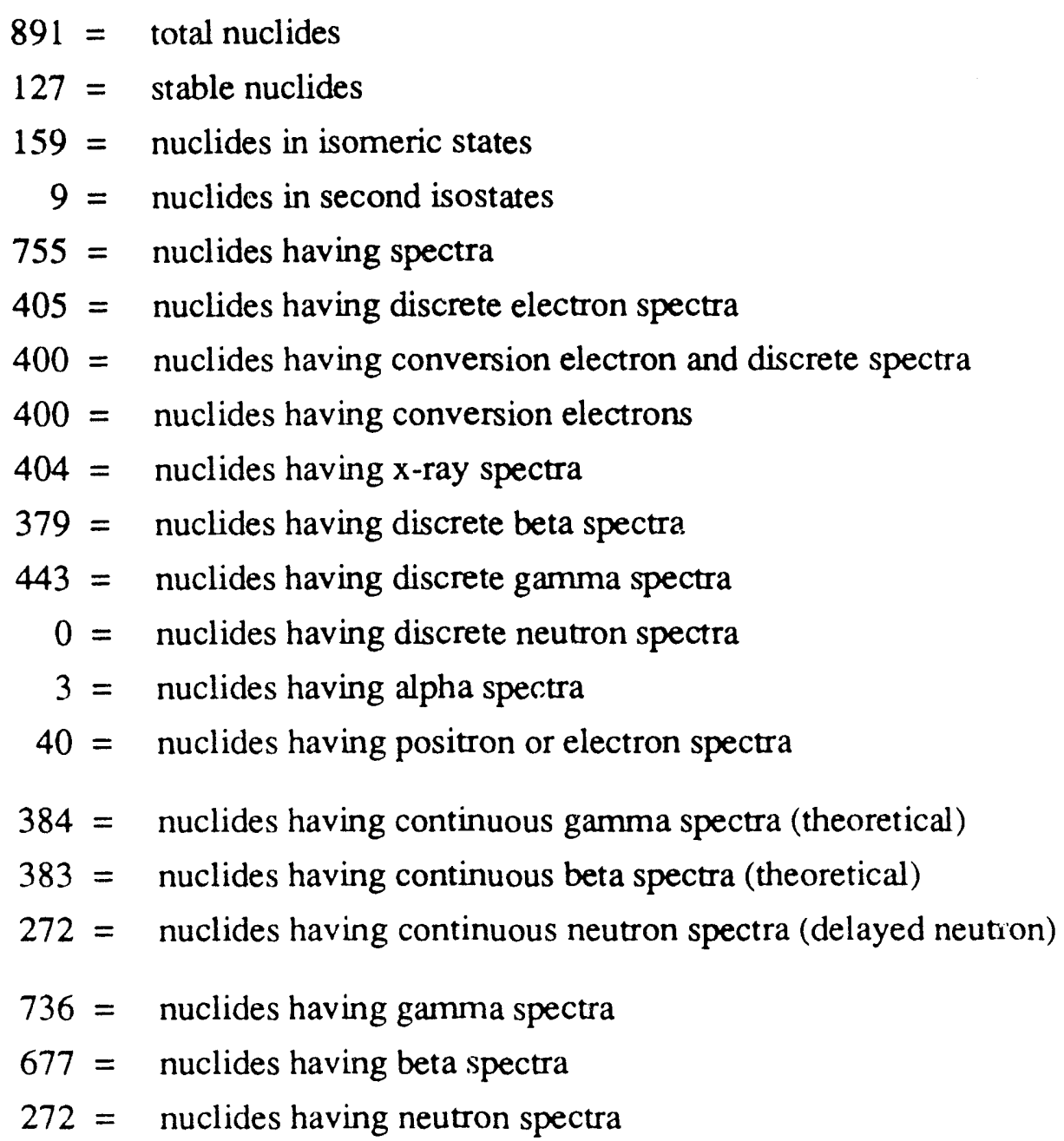

\section{ENDF/B-VI Decay File: Summary of Modifications, Supplements, and General Sources.}

The fission-product decay data will be combined with the activation and actinide decay files to produce a single decay file of $\sim 970$ nuciides. Fifty nuclides are common in these files. The fission product and actinide files take prece lence over the earlier evaluated activation files. All files are now at Brookhaven National Laboratoly [the activation and actinide files having been sent earlier by Fred Mann, HEDL (Hanford Engineering Development Laboratory)].

Differing from previous versions of ENDF/B, in the preliminary Version VI, the fission products were formed as a sequence of libraries, testing each with an extensive range of integral measurements. Unlike the activation and actinide portions of the ENDF/B-VI decay file, many fission products have no spectral measurements and some lack even half-life measurements. Other products have incomplete measurements of spectra. 
Recognizing that measured data were extensive but inadequate for calculations of decay heat and spectra, it was decided to:

1. Use measured data where they existed in 1989;

2. Otherwise, use theory.

3. Supplement measurements believed to be incomplete with theoretical values.

4. Complete a library having spectra that would closely match the average beta and gamma energies.

5. Add in the delayed neutron spectra.

In the process of supplementation we did not want to lose any evaluated measurements, and for spectra, that is the case. Average energies for $\sim 73$ nuclides were known to be in error and they were replaced by theoretical values. Approximately 45 others were believed to be in error and were replaced by theory. Many others $(\sim 244)$ had no measurements, average energies, or spectra, and were necessarily replaced by theory. None had delayed neutron spectra.

In toto, 420 of 764 nuclides now have theoretical data and/or delayed neutron data. We believe the supplements are essentially correct; however, the user should be aware that some are certainly questionable and the need for some specific supplements were necessarily subjective. A few ( 116) have since been measured, but they will have to be used in a subsequent "mod." The process of forcing agreement of average energies derived from spectra with file averages complicates the simple additions of new spectral measurements. Perhaps more importantly, this first file has already been compared with essentially all measured aggregate decay heat, beta, and gamma spectra. As was the original motivation for the product data, it is an excellent file that will serve as an essential complete fiducial data set. All but 9 of 764 unstable nuclides now have a decay spectra (compared with 264 in ENDF/B-V). All theoretical values are inserted as continuous spectra. Evaluations of measured data have not been changed except in a few cases where the beta normalization was adjusted by changing FD (the discrete spectrum normalization factor for beta decay apDearing in the File 8 representation of decay spectra in ENDF/B-VI), to avoid negative spectra.

Most of the evaluated measurements were supplied by Charles Reich of INEL (Idaho National Engineering Lab.), with a close adherence to ENSDF (Evaluated Nuclear Structure Data File) and converted to ENDF/B format at HEDL by F. Mann. All of the supplements to these data and those for unmeasured parameters were made at Los Alamos by the authors. The additions expanded the file from $\sim 600000$ to $>300000$ lines, or approximately 25000000 bytes.

Table C-2 provides a list of information that may be of great interest to present and future evaluators. This information, as well as more detailed additional material, can now be recovered from File 1. The meaning of the various numbers is given at the end of the table. The table relates only to beta, gamma, and delayed neutron energies; there are other spectra in the files. 


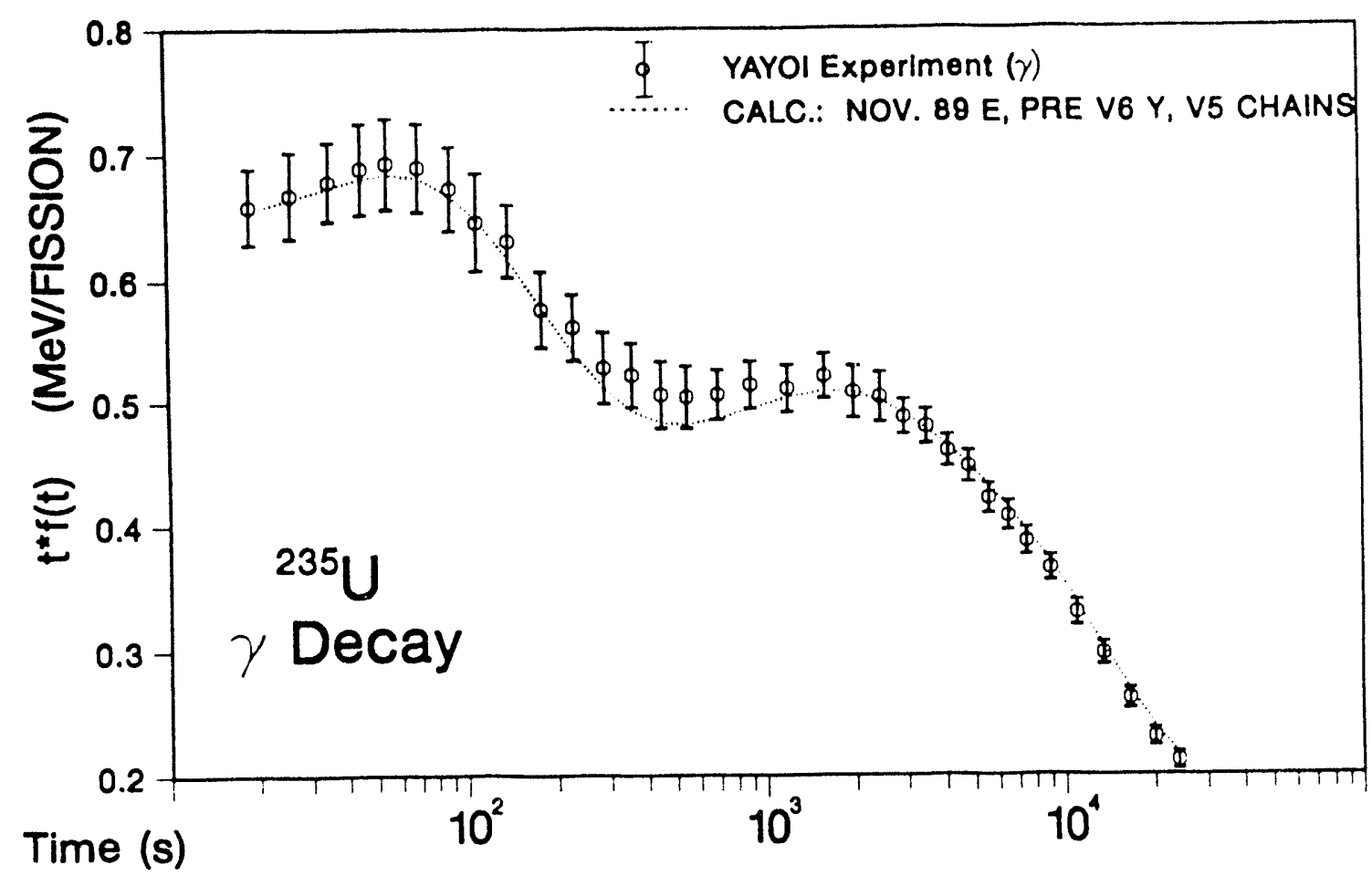

Fig. C-1. Gamma decay energy after $235 \mathrm{U}$ fast fission (pulse).

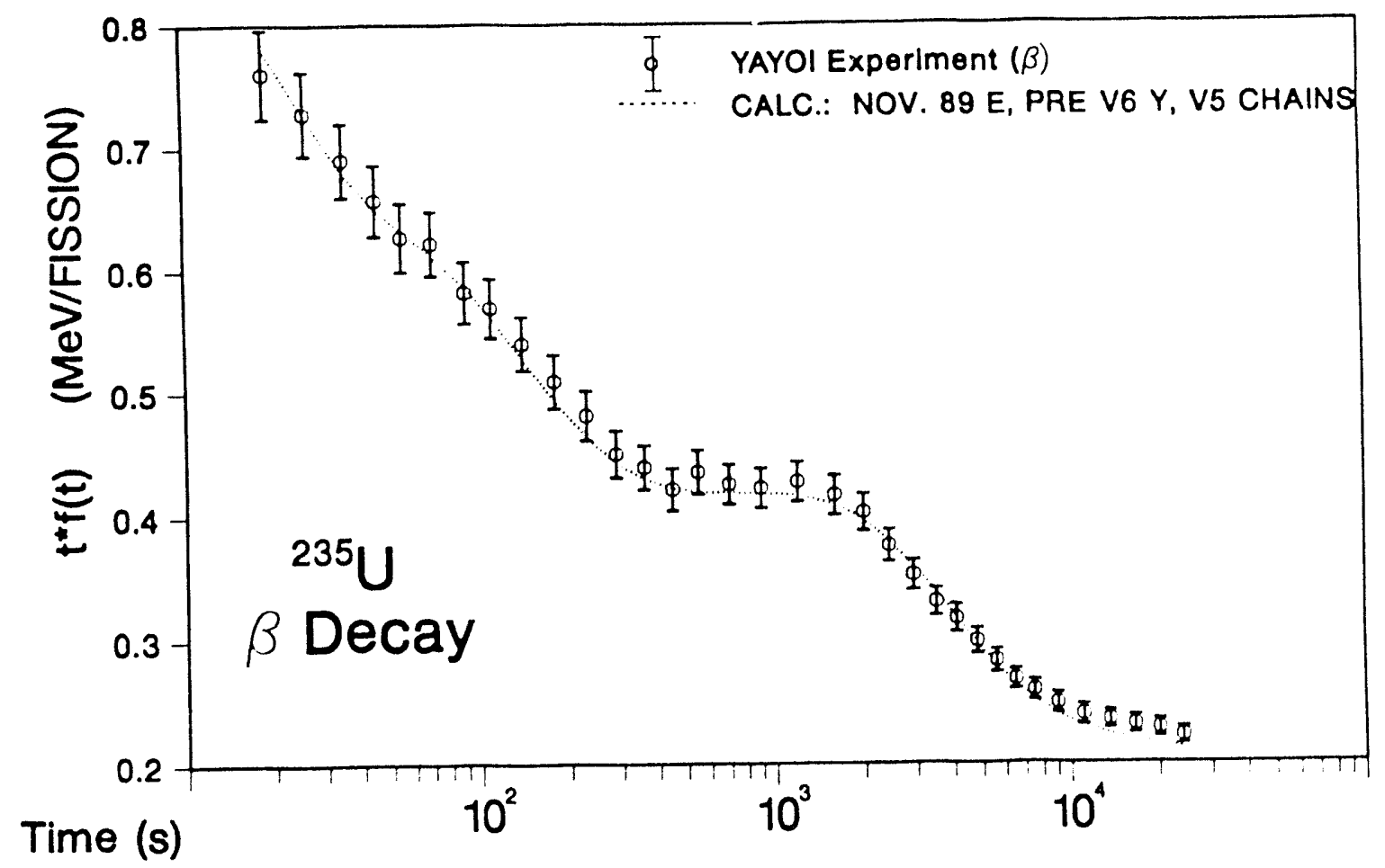

Fig. C-2. Beta decay energy after ${ }^{235} \mathrm{U}$ fast fission (pulse). 


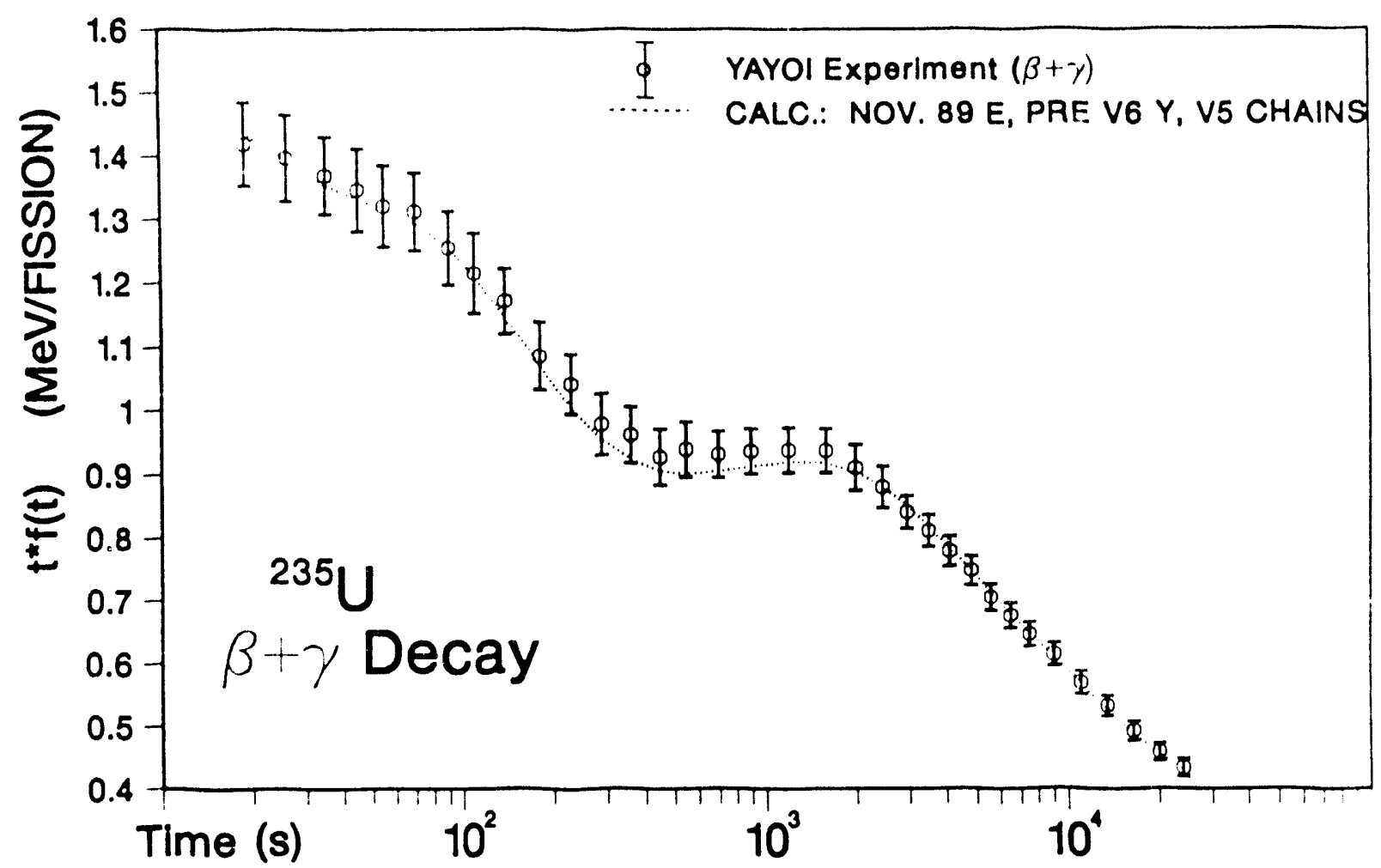

Fig. C-3. Total decay energy after ${ }^{235} U$ fast fission (pulse). 
TABLE C-2

\section{FISSION-PRODUCT DECAY FILE: SUPPLEMENTS, ADDITIONS, AND SOURCES ${ }^{a}$}

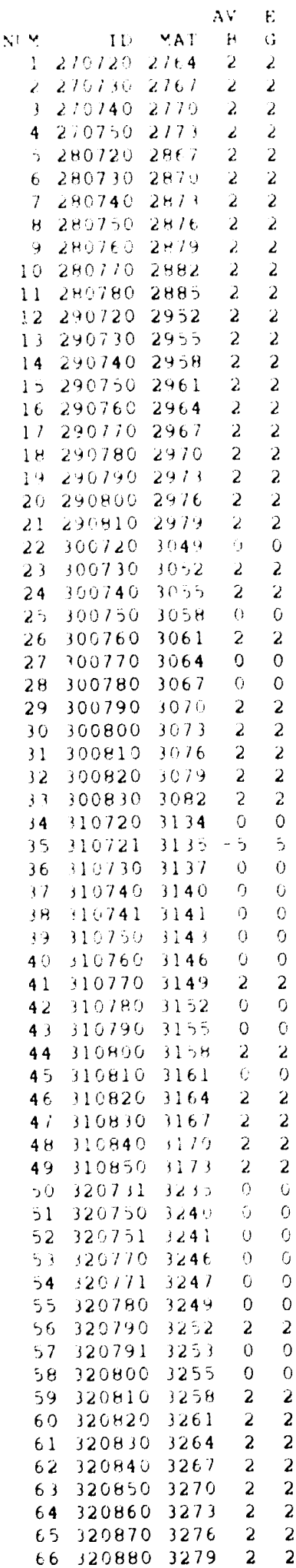

\begin{tabular}{|c|c|c|c|c|c|c|c|}
\hline$\Delta p$ & TYP & & ionT & E COMPONE & $\therefore \mathrm{T}$ & & \\
\hline $\mathrm{H}$ & G & $\therefore$ & HETA & IANMA & NEII & NEW & $\mathrm{HI}$ \\
\hline & 1 & 1 & $46081+06$ & $4.6940+06$ & $\Rightarrow 2009+04$ & & . \\
\hline 1 & 1 & 1 & $4.1180+06$ & 2. $9800+06$ & $2.1642+05$ & & $\cdots$ \\
\hline 1 & 1 & 1 & $3.1669+06=$ & $5.4200+06$ & 1. $5428+05$ & & $\cdots$ \\
\hline$?$ & 1 & 1 & $3.2591+06$ & $31449+06$ & $2.9849+05$ & & $\cdots-\cdots$ \\
\hline$!$ & 1 & -1 & 1. $8820+96$ & $9.1313+05$ & $\ldots$ & & $\cdots$ \\
\hline 1 & 1 & 1 & $3.2809+06$ & $1.6189+06$ & $7.2241+00$ & & $\cdots$ \\
\hline 1 & 1 & 1 & $2.6831+06$ & $1.1990+06$ & $11609+03$ & & $-\cdots$ \\
\hline 1 & $i$ & 1 & $3.8269+06$ & $2.2159+06$ & $4.4904+03$ & & $-\cdots$ \\
\hline 1 & 1 & 1 & $3.3790+06$ & $1.5270+06$ & 1. $8386+04$ & & $\cdots$ \\
\hline - & 1 & 1 & $4.4910+06$ & $3.0841+06$ & $2.8536+04$ & & $\cdots-$ \\
\hline 1 & 1 & 1 & $39289+06$ & $18769+06$ & $6.0141+04$ & & $\cdots$ \\
\hline , & 1 & 1 & $2.0350+06$ & $2.9940+06$ & $4.7074-02$ & & $\cdots \cdots$ \\
\hline 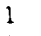 & 1 & 1 & 1. $9849+06$ & $7.7230+05$ & $1.7105+03$ & & $\cdots-$ \\
\hline 1 & 1 & 1 & 2. $5109+06$ & $3.2060+06$ & $1.0334+03$ & & $\cdots$ \\
\hline 1 & 1 & 1 & 2. $6880+06$ & $1.0900+06$ & $1.7,002+04$ & & $\cdots-$ \\
\hline 1 & 1 & 1 & $3.1131+06$ & $3.5040+6$ & 1. $5625+04$ & & $\cdots$ \\
\hline 1 & 1 & 1 & $32671+96$ & $15060+10$ & $81783+04$ & & $\cdots$ \\
\hline 1 & 1 & 1 & $3.8302+06$ & $40511+06$ & $7.0359+04$ & & $\cdots$ \\
\hline 1 & 1 & 1 & $3.7089+06$ & $19700+00$ & $1.8111+00$ & & $-\cdots$ \\
\hline 1 & 1 & 1 & 4. $3263+06$ & $45871+06$ & $1.2097+05$ & & $\cdots$ \\
\hline 1 & 1 & 1 & $4.8260+06$ & $3.4570+06$ & $3.2712+05$ & & $\cdots$ \\
\hline 1 & -1 & -1 & $\cdots$ & $\ldots$ & $\cdots$ & & $\cdots-$ \\
\hline 2 & 2 & -1 & $3.8465+05$ & $10519+06$ & -- & $6.26+3$ & -03 \\
\hline 2 & 2 & -1 & $1.8033+0 j$ & $5.8172+05$ & $\cdots$ & 4.95 & -03 \\
\hline 1 & -1 & -1 & $\cdots$ & $\ldots \cdot-$ & $\cdots$ & & $\cdots$ \\
\hline 2 & 2 & -1 & 1. $7295+05$ & $2.0810+05$ & $-\cdots$ & 7.7388 & -03 \\
\hline 2 & -1 & -1 & $2.8500+25$ & $-\cdots$ & $-\cdots-$ & & $\cdots-$ \\
\hline-1 & -1 & 1 & $\cdots$ & -- & $5.6341+00$ & & -- \\
\hline 1 & 1 & 1 & $3.0160+06$ & $2.9170+06$ & $5.0842+03$ & & $\cdots$ \\
\hline 1 & 1 & 1 & $2.7581+06$ & 1. $2420+06$ & 4. $3256+03$ & & $-\cdots$ \\
\hline 1 & 1 & 1 & $4.0320+06$ & $2.7131+06$ & $3.6837+04$ & & $\cdots$ \\
\hline 1 & 1 & 1 & $4.2339+06$ & $2.1810+06$ & $1.6182+05$ & & $\cdots$ \\
\hline 1 & 1 & 1 & $4.1021+06$ & $3.9531+06$ & 1. $9025+05$ & & $\cdots$ \\
\hline 1 & -1 & -1 & $-\cdots$ & $\cdots$ & $\ldots$ & & $\cdots$ \\
\hline 1 & 1 & -1 & $\cdots$ & -- & $\ldots$ & & --- \\
\hline 1 & -1 & -1 & $\cdots$ & $\cdots$ & $\cdots$ & & $\cdots$ \\
\hline 1 & 1 & -1 & $\cdots$ & $-\cdots$ & $\cdots$ & & $\ldots-\cdots$ \\
\hline$\cdots 1$ & -1 & -1 & $\cdots$ & $\cdots$ & $\ldots$ & & $\cdots$ \\
\hline-1 & -1 & -1 & $\cdots$ & $\cdots$ & $\cdots$ & & $\cdots$ \\
\hline-1 & 1 & $\cdots 1$ & $\cdots$ & $-\cdots$ & $\cdots$ & & $\cdots \cdots$ \\
\hline 1 & 1 & 1 & $2.04: 0+06$ & $7.8930+05$ & --- & & $\cdots$ \\
\hline 1 & -1 & -1 & $\cdots \cdots$ & $\ldots$ & $\cdots$ & & $-\cdots$ \\
\hline 1 & -1 & 1 & $\cdots$ & $\cdots$ & $1.6635+02$ & & $\cdots$ \\
\hline 2 & 2 & 1 & $4.4322+05$ & $7.7800+05$ & $2.7848+03$ & & $\cdots$ \\
\hline 2 & -1 & 1 & 3.0899 .05 & & 4,$4 ; 81+04$ & & $\cdots$ \\
\hline 1 & 1 & 1 & $3.7361+06$ & 4.1311+06 & $1.3732+05$ & & $\cdots$ \\
\hline 1 & 1 & 1 & 3. $8810+06$ & $3.7429+06$ & $4.4794+05$ & & $\cdots$ \\
\hline 1 & 1 & 1 & 4. $22.80+06$ & $4.6330+06$ & 2. $3871+05$ & & $-\cdots$ \\
\hline 1 & 1 & 1 & 4. $5090+06$ & 4. $3050+06$ & $4.0263+05$ & & $\cdots-$ \\
\hline-1 & -1 & -1 & $\cdots$ & $-\cdots$ & $\cdots-$ & & $\cdots-$ \\
\hline-1 & -1 & -1 & -- & $\cdots$ & --- & & --- \\
\hline-1 & 1 & 1 & --- & $-\cdots$ & $\cdots$ & & $\cdots$ \\
\hline-1 & -1 & -1 & $-\cdots$ & $\cdots$ & $\ldots-$ & & $-\cdots$ \\
\hline-1 & -1 & -1 & $\cdots$ & $\ldots .$. & $\cdots$ & & $\ldots$ \\
\hline-1 & -1 & -1 & $-\cdots$ & --- & $\ldots$ & & --- \\
\hline-1 & 2 & -1 & $-\cdots$ & $9.9269+04$ & $\cdots$ & & -- \\
\hline-1 & -1 & -1 & $\cdots$ & -- & --- & & --- \\
\hline 2 & -1 & -1 & $6.4631+04$ & -- & --- & 8.0511. & -03 \\
\hline 1 & 1 & -1 & $2.4426+06$ & 8. $4067+05$ & -- & & -- \\
\hline 1 & 1 & -1 & 1. $4490+06$ & $7.6519+05$ & --- & & --- \\
\hline 1 & 1 & 1 & $2.6891+06$ & $2.4440+06$ & $3.6673+02$ & & -- \\
\hline 1 & 1 & 1 & $2.5461+06$ & $2.4600+06$ & $5.6600+04$ & & $-\cdots$ \\
\hline 1 & 1 & 1 & $3.0291+06$ & $3.1829+06$ & $1.3881+05$ & & $-\cdots$ \\
\hline 1 & 1 & 1 & 3. $3620+06$ & $2.6360+06$ & $1.4859+05$ & & --+ \\
\hline 1 & 1 & 1 & $3.5330+06$ & $3.5850+06$ & $1.1063+05$ & & -- \\
\hline 1 & 1 & 1 & $4.0061+06$ & $3.0030+06$ & $1.6309+05$ & & -- \\
\hline
\end{tabular}


Table C-2 (Cont.)

\begin{tabular}{|c|c|c|c|c|c|c|c|c|c|c|c|c|c|c|}
\hline & & & $A V$ & $\mathrm{E}$ & $\$ P$ & IY & & $(\cdot) \mathrm{NI}$ & $\mathrm{E}$ & $\because(\because x p) N$ & $\because \vee$ & & & \\
\hline NIYY & II & $\because A \Gamma$ & $\mathrm{H}$ & $G$ & $B$ & $(;$ & $N$ & HE:TA & & $G A \times M A$ & LE:L & VE!'! & $\therefore$ Sh & F' \\
\hline 67 & 330760 & $332 \mathrm{H}$ & (i) & 5 & -1 & 1 & $\cdots$ & & & & & & & \\
\hline 68 & 330710 & 3311 & 0 & 0 & 1 & 1 & 1 & & & & & & & \\
\hline 69 & 330780 & $1: 44$ & 0 & 0 & 1 & 1 & 1 & $\cdots$ & & & & - & & \\
\hline 70 & 330790 & 33:i & 6 & 6 & -1 & 2 & $\cdot 1$ & & 8 & $9001+03$ & & & & \\
\hline 71 & 330800 & 3340 & 2 & 2 & 1 & 2 & 1 & $\cdots$ & 1. & $900 H+05$ & & & & \\
\hline 12 & 330810 & 1343 & 0 & 0 & 1 & 1 & -1 & $-\cdot$ & & & & & & \\
\hline 13 & 330820 & 3146 & 2 & 2 & 2 & 2 & 1 & $4.9313+04$ & 7. & $.490+0$ & & & 4.1004 & -03 \\
\hline 14 & 330821 & $334 \%$ & 0 & 0 & -1 & 1 & -1 & & & $\cdots$ & & & & \\
\hline 75 & 330830 & $3: 49$ & 6 & 6 & 1 & 1 & -1 & $1.2556+06$ & 2 & $1514+06$ & & & & \\
\hline 76 & 330840 & 33,2 & $n$ & 0 & -1 & 1 & 1 & $\cdots$ & & $\cdots \cdots$ & $2=$ & $440+02$ & & \\
\hline 77 & 330841 & 3333 & 2 & 2 & 1 & 1 & 1 & $2917 y+06$ & 3 & $4620+06$ & & & & \\
\hline 78 & 330850 & 3353 & 2 & 2 & 1 & 1 & 1 & $2.8360+06$ & 3 & $00 \div 0+06$ & $5.0 \%$ & $0294+6$ & & $\cdots$ \\
\hline 79 & 330860 & $335 \mathrm{H}$ & 2 & 2 & 1 & 1 & 1 & 3.3170 .06 & 3 & $7779+06$ & H4 & $4909+1) 4$ & & $\cdots \cdots$ \\
\hline 80 & 330870 & 3361 & 2 & 2 & 1 & 1 & 1 & $3.4400+06$ & 3 & $4731+06$ & 3.3 & $370 b+09$ & & $\cdots$ \\
\hline 81 & 330480 & 3364 & 2 & 2 & 1 & 1 & 1 & $3.7521+06$ & 4. & $2211+06$ & 14 & $4884+05$ & & $\cdots$ \\
\hline 82 & 330890 & 3367 & 2 & 2 & 1 & 1 & 1 & $3.9768+06$ & 3. & $9429+06$ & 2.6 & $6130+05$ & & $\cdots$ \\
\hline 83 & 330960 & 3310 & 2 & 2 & 1 & 1 & 1 & 4. $5968+06$ & 4 & $3800+06$ & 1.9 & $9676+03$ & & $\cdots \cdot$ \\
\hline 84 & 340750 & 3424 & 0 & 0 & -1 & -1 & -1 & $\cdots$ & & $\cdots \cdots$ & & $\cdots$ & & $\cdots$ \\
\hline 85 & 340771 & 3435 & 0 & 0 & -1 & -1 & -1 & $-\quad-$ & & $\cdots$ & & $\cdots$ & & $\cdots$ \\
\hline 86 & 340790 & 3440 & 0 & 0 & -1 & -1 & -1 & $\cdots-$ & & $\cdots$ & & $\cdots$ & & $\cdots$ \\
\hline 87 & 340791 & 3441 & 0 & 0 & -1 & -1 & -1 & $\cdots$ & & $\cdots \cdots$ & & $\cdots$ & & $\cdots$ \\
\hline 88 & 340810 & 3446 & 0 & 0 & -1 & 1 & $\cdot 1$ & $\cdots$ & & & & $-\cdots$ & & $\ldots$ \\
\hline 89 & 340811 & 3447 & 0 & 0 & $\cdots 1$ & -1 & 1 & $\cdots$ & & $\cdots \cdots$ & & $\cdots$ & & \\
\hline 90 & 340830 & $34 \supset 2$ & 0 & 0 & -1 & -1 & $\sim 1$ & $\cdots$ & & & & $\cdots$ & & $\cdots \cdots$ \\
\hline 91 & 340831 & 34,3 & 0 & 0 & -1 & -1 & -1 & $\cdots$ & & $-\cdots$ & & $\cdots$ & & $\cdots$ \\
\hline 92 & 340840 & 3455 & 0 & 0 & $\cdot 1$ & -1 & -1 & $\cdots$ & & $\cdots-$ & & 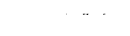 & & $\cdots$ \\
\hline 93 & $340850 j$ & 3438 & 0 & 0 & -1 & -1 & -1 & $\cdots$ & & $\cdots$ & & $\cdots-$ & & $\cdots$ \\
\hline 94 & 340860 & 3461 & 0 & 0 & -1 & -1 & -1 & $\cdots$ & & $-\cdots$ & & $\cdots$ & & $\cdots$ \\
\hline 95 & 340870 & 3464 & 2 & 2 & 1 & 1 & 1 & $2.0791+06$ & 2 & 6440.06 & 4. 3 & $3577+02$ & & $\cdots-$ \\
\hline 96 & 340880 & 3467 & 2 & 2 & 1 & 1 & 1 & $2.21: 1+06$ & 2 & $0320+06$ & 2.4 & $4953+03$ & & $\cdots$ \\
\hline 97 & 340890 & 3470 & 2 & 2 & 1 & 1 & 1 & $3.1261+06$ & 1. & $8940+06$ & 3.1 & $1279+04$ & & $\cdots$ \\
\hline 98 & 340900 & 3473 & 2 & 2 & 1 & 1 & 1 & $2.5040+06$ & 2. & $6330+06$ & 70 & $0072+04$ & & $\cdots$ \\
\hline 99 & 340910 & 3476 & 2 & 2 & 1 & 1 & 1 & $3.7830+06$ & 3 & $1260+06$ & 1.5 & $5110+05$ & & $\cdots$ \\
\hline 100 & 340920 & 3479 & 2 & 2 & 1 & 1 & 1 & $4.1130+06$ & 2 & $2370+06$ & 9.4 & $44:-3+04$ & & $\cdots$ \\
\hline 101 & 340930 & 3482 & 2 & 2 & 1 & 1 & 1 & $4.1169+06$ & 4. & $14 ! 9+06$ & $H$. & $\therefore H+04$ & & $\cdots$ \\
\hline 102 & 350791 & 3526 & 0 & 0 & -1 & -1 & -1 & $\cdots$ & & $\cdots$ & & $\cdots$ & & $\cdots-$ \\
\hline 103 & 350800 & 3528 & 0 & 0 & -1 & 1 & -1 & $\cdots$ & & & & $\cdots$ & & $\cdots \cdots$ \\
\hline 104 & 350801 & 3329 & 0 & 0 & $\cdots 1$ & $\cdots 1$ & -1 & & & $\cdots$ & & $\cdots$ & & $\ldots$. \\
\hline 105 & 350820 & 3534 & 0 & 0 & -1 & -1 & -1 & $-\cdots \cdot$ & & $\cdots$ & & $\cdots-$ & & $\cdots$ \\
\hline 106 & 350821 & 3535 & 0 & 0 & -1 & -1 & $\cdots 1$ & $\cdots$ & & $\cdots$ & & $\cdots$ & & $\cdots$ \\
\hline 101 & 350830 & 3531 & 0 & 0 & -1 & -1 & -1 & $\cdots$ & & $\cdots \cdots$ & & $\cdots$ & & $\cdots$ \\
\hline 108 & 350840 & 3540 & 0 & 0 & -1 & -1 & -1 & $\cdots$ & & $\cdots \cdots$ & & $\cdots$ & & $\ldots-\cdots$ \\
\hline 109 & 350841 & 3541 & 0 & 0 & -1 & -1 & -1 & $\cdots$ & & $\cdots-$ & & -- & & $\cdots \cdots$ \\
\hline 110 & 350850 & 3543 & 0 & 0 & 1 & 1 & $\cdot 1$ & $\cdots \cdots$ & & $\cdots \cdot$ & & $\cdots$ & & $\cdots$ \\
\hline 111 & 350860 & 3546 & 1 & 1 & -1 & -1 & -1 & $\cdots$ & & & & & & $\cdots$ \\
\hline 112 & 350870 & 3549 & 0 & 0 & -1 & -1 & 1 & $\cdots$ & & & 54 & $4221+03$ & & $\cdots$ \\
\hline 113 & 350880 & 3552 & 0 & 0 & -1 & -1 & 1 & $\cdots$ & & & 1. 6 & $637+04$ & & $\cdots$ \\
\hline 114 & 350890 & 3555 & 1 & 1 & 2 & 2 & 1 & $17132+06$ & 1. & $5500+06$ & 7.1 & $1476+04$ & 1.8 & -03 \\
\hline 115 & 350900 & 3558 & 1 & 1 & 2 & 2 & 1 & $2.0580+06$ & 1. & $6800+06$ & 1.5 & $5125+05$ & & ;-0? \\
\hline 116 & 350910 & 3561 & 2 & 2 & 1 & 1 & 1 & $34171+06$ & 2. & $1390+06$ & 71 & $1622+04$ & & $\cdots \cdots$ \\
\hline 117 & 350920 & 3564 & 2 & 2 & 1 & 1 & 1 & 4. $0059+06$ & 3. & $1990+06$ & 4.7 & $1618+05$ & & $-\cdots \cdot$ \\
\hline 118 & 350930 & 3,67 & 2 & 2 & 2 & 1 & 1 & $3.5540+06$ & 3. & $6719+06$ & 3.0 & $0795+05$ & & $\cdots$ \\
\hline 119 & 350940 & 3570 & 2 & 2 & 1 & 1 & 1 & $40190+06$ & 4. & $6610+06$ & 2.2 & $2901+05$ & & $\cdots$ \\
\hline 120 & 350950 & 3573 & 2 & $?$ & 1 & 1 & 1 & $3.5929+06$ & 3 & $7130+06$ & 2.0 & $0035+05$ & & $-\cdots$ \\
\hline 121 & 350960 & 3576 & 2 & 2 & 1 & 1 & 1 & $4.4689+06$ & 4 & $8219+06$ & 1.6 & $6863+05$ & & $\cdots$ \\
\hline 122 & 360790 & 3628 & 0 & 0 & -1 & -1 & -1 & $\cdots$ & & $\cdots-$ & & $\cdots$ & & $\cdots$ \\
\hline 123 & 360791 & 3629 & 0 & 0 & -1 & -1 & -1 & $\cdots$ & & $\cdots$ & & $\cdots$ & & -- \\
\hline 124 & 360810 & 3634 & 0 & 0 & -1 & -1 & -1 & $\cdots$ & & $\cdots$ & & $-\cdots-$ & & $\ldots-$ \\
\hline 125 & 360811 & 3635 & 0 & 0 & -1 & -1 & -1 & $\ldots-$ & & $\cdots$ & & $\cdots$ & & $\ldots$ \\
\hline 126 & 360831 & 3641 & 0 & 0 & -1 & -1 & -1 & $\cdots$ & & $\cdots$ & & $\cdots$ & & $\ldots-$ \\
\hline 127 & 360850 & 3646 & 0 & 0 & -1 & -1 & -1 & --- & & $\cdots \cdots$ & & $\cdots$ & & $\cdots$ \\
\hline 128 & 260851 & 3647 & 0 & 0 & -1 & -1 & -1 & $\cdots$ & & $\cdots$ & & $\cdots$ & & $\cdots$ \\
\hline 129 & 360870 & 3652 & 0 & 0 & -1 & -1 & -1 & $\cdots$ & & $\cdots$ & & $\cdots$ & & $\cdots$ \\
\hline 130 & 360880 & 3655 & 0 & 0 & -1 & -1 & -1 & $\cdots$ & & $\cdots$ & & --- & & $\cdots$ \\
\hline 131 & 360890 & 3658 & 0 & 0 & -1 & -1 & -1 & $\cdots-$ & & $\cdots-$ & & --- & & --- \\
\hline 132 & 360900 & 3661 & 0 & 0 & -1 & -1 & -1 & -- & & $\cdots$ & & $\cdots$ & & $\cdots$ \\
\hline 133 & 360910 & 3664 & 0 & 0 & -1 & -1 & -1 & -- & & --- & & $\cdots$ & & $\cdots$ \\
\hline 134 & 360920 & 3667 & 0 & 0 & -1 & -1 & 1 & $\cdots$ & & $-\cdots$ & 8,3 & $3248+01$ & & $\cdots$ \\
\hline
\end{tabular}


Table C-2 (Cont.)

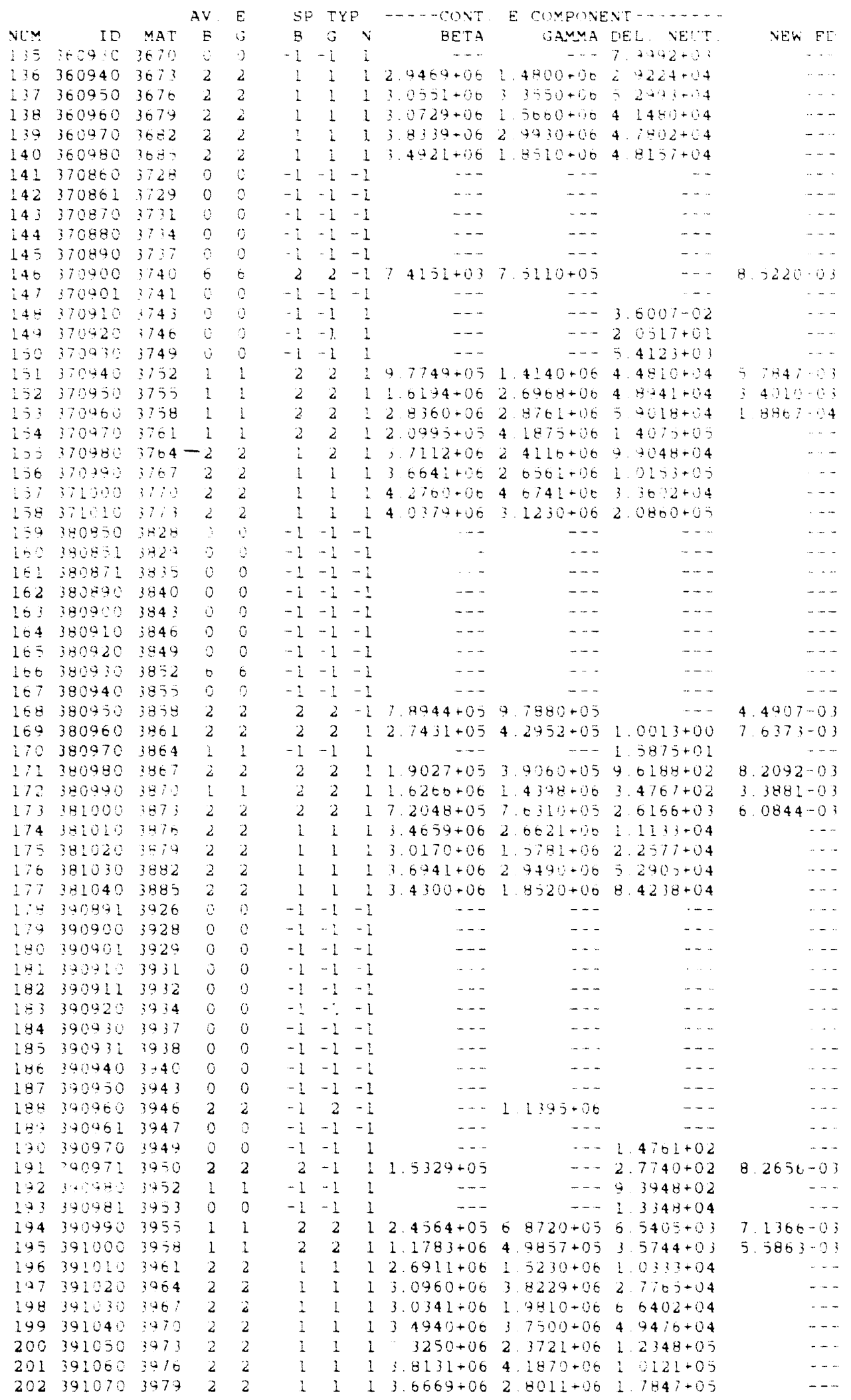


Table C-2 (Cont.)

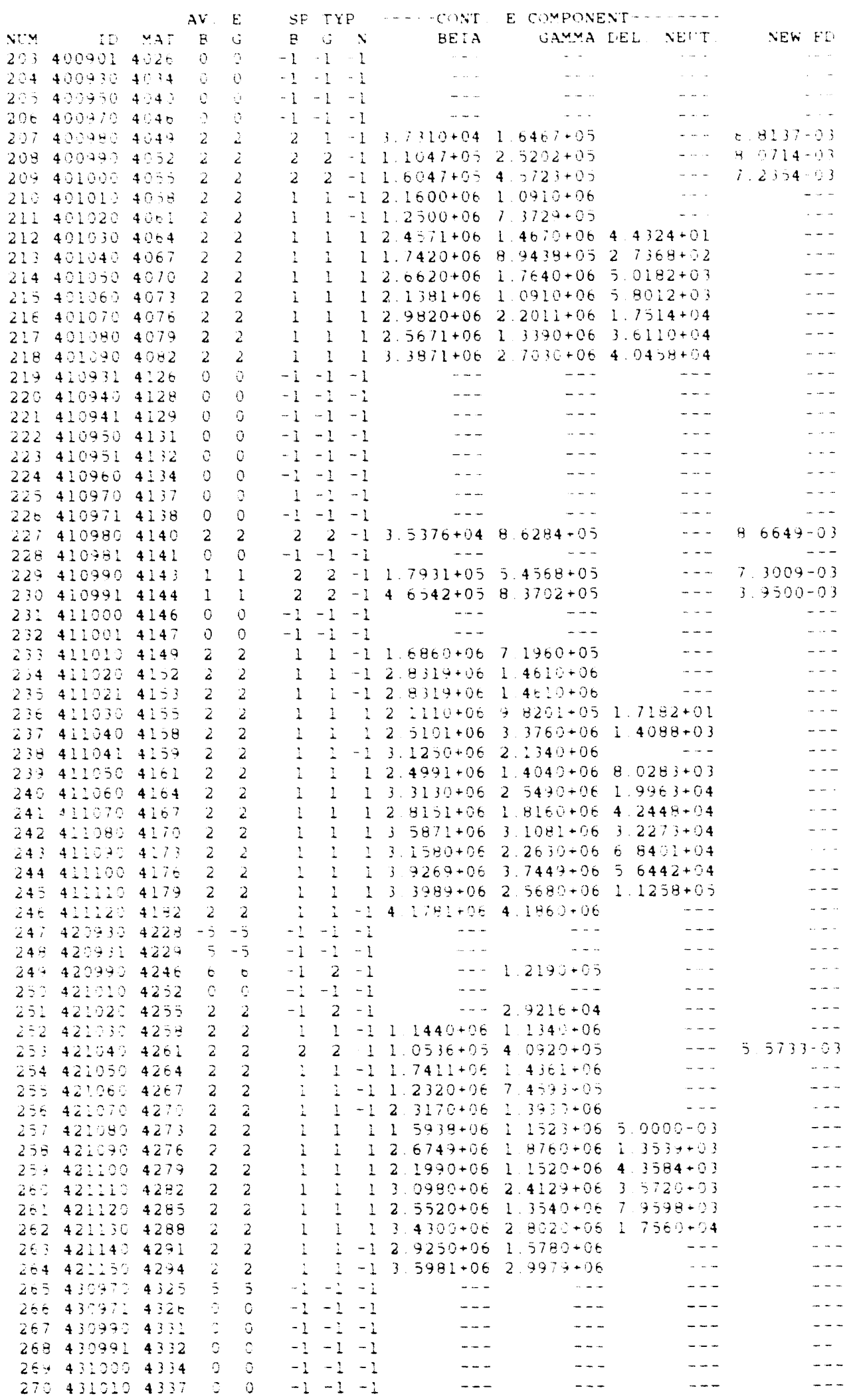


Table C-2 (Cont.)

\begin{tabular}{|c|c|c|c|c|}
\hline & & & $A V$ & \\
\hline NLM & ID & $M A T$ & B & \\
\hline 279 & $1: 1020$ & $474 . j$ & 2 & \\
\hline 272 & 431021 & $434 !$ & 3 & \\
\hline 273 & 431030 & $4: 43$ & 2 & \\
\hline 274 & $4: 104 \%$ & 4346 & $\mathrm{c}$ & \\
\hline 275 & $4: 10 \div$ & 4347 & 0 & \\
\hline 276 & 43150 & 4352 & 2 & \\
\hline 277 & $4: 1: i$ & 4355 & 2 & \\
\hline 278 & $4 i 1080$ & 4358 & $\angle$ & \\
\hline 279 & 432040 & $436 !$ & 2 & \\
\hline 290 & 431100 & 4304 & 2 & \\
\hline $24 !$ & 431210 & $4 i 0 i$ & 2 & \\
\hline 282 & $4: 1120$ & 4370 & 2 & \\
\hline 293 & $4: 1: 30$ & $4 ? 73$ & 2 & \\
\hline 284 & 431140 & 4370 & 2 & \\
\hline 285 & 431150 & 4379 & 2 & \\
\hline 200 & $43: 56$ & $43 \times 2$ & 2 & \\
\hline 287 & $4: 1170$ & $4: 85$ & 2 & \\
\hline 288 & 4.1180 & 4384 & 2 & \\
\hline 289 & 440970 & 4428 & 8 & \\
\hline 290 & 441033 & 4440 & 3 & \\
\hline 291 & 441251 & $4+22$ & 0 & \\
\hline $2 \div 2$ & 441000 & 4455 & $\mathrm{a}$ & \\
\hline 293 & 441070 & 4458 & 2 & \\
\hline 294 & 441080 & $44+1$ & 0 & \\
\hline 275 & 441090 & 4464 & 2 & \\
\hline 236 & $44: 99:$ & 4465 & 2 & \\
\hline 297 & $441: 00$ & $440 \%$ & 2 & \\
\hline 298 & 441210 & 4470 & 2 & \\
\hline 299 & $44: 220$ & 4473 & 2 & \\
\hline 300 & 441230 & 4476 & 2 & \\
\hline 301 & $44: 140$ & 4479 & 2 & \\
\hline 302 & $441: 50$ & 4432 & 2 & \\
\hline 303 & 441160 & 4485 & 2 & \\
\hline 304 & 441170 & 4488 & 2 & \\
\hline 305 & 441190 & 4491 & 2 & \\
\hline 306 & 441290 & 444 & 2 & \\
\hline 307 & 441220 & 4497 & 2 & \\
\hline 308 & 451031 & 4520 & 3 & \\
\hline 309 & 451040 & $4>28$ & 0 & \\
\hline 310 & $4: 1041$ & 4524 & 0 & \\
\hline 311 & 451650 & 4331 & 0 & \\
\hline$\$ 12$ & 451051 & 4532 & ن & \\
\hline 313 & $4 \lessgtr 10 \in 0$ & 4534 & 0 & \\
\hline 314 & 451001 & 4535 & 0 & \\
\hline 315 & $4 \equiv: 070$ & 4537 & 2 & \\
\hline 316 & 451090 & 4549 & 2 & \\
\hline 317 & 451081 & 4,41 & 2 & \\
\hline 319 & 451090 & 4543 & 0 & \\
\hline 319 & $4>1091$ & $4 \supset 44$ & -5 & \\
\hline 320 & 451100 & 4546 & 2 & \\
\hline 321 & 451101 & 4547 & ? & \\
\hline 322 & $451: 10$ & 4549 & 2 & \\
\hline$\because 3$ & 451120 & 4552 & 2 & \\
\hline$\therefore 4$ & 451136 & 4555 & 2 & \\
\hline 325 & $451: 40$ & 4538 & 2 & \\
\hline 26 & 451.50 & $456 ́ 1$ & 2 & \\
\hline 327 & $45110 ?$ & $4 \div E 4$ & 2 & \\
\hline 328 & 431179 & 4507 & 2 & \\
\hline 329 & 451180 & 4570 & 2 & \\
\hline 330 & 451190 & 4573 & 2 & \\
\hline 331 & $45: 200$ & 4576 & 2 & \\
\hline 332 & 453210 & 4577 & 2 & \\
\hline 333 & 421220 & 4582 & 2 & \\
\hline 334 & 451230 & 4585 & 2 & \\
\hline 335 & 461030 & 4628 & $\therefore$ & \\
\hline 336 & 461070 & 4640 & 0 & \\
\hline 337 & 461071 & 4641 & 0 & \\
\hline 338 & 461090 & 4646 & 0 & \\
\hline
\end{tabular}

\begin{tabular}{|c|c|c|c|c|c|c|c|c|c|}
\hline \multicolumn{3}{|c|}{ TYP } & -...cont & \multicolumn{3}{|c|}{ E COMPONENI $\ldots \ldots$} & \multirow{2}{*}{\multicolumn{3}{|c|}{$\therefore E N$ FD }} \\
\hline 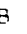 & $\dot{G}$ & 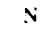 & BEIA & GAYMA & LEE L & $V E: 5$ & & & \\
\hline & 2 & -1 & $1.4054+05$ & $1.1123+00$ & & $\cdots$ & $\epsilon$ & $3741-$ & -03 \\
\hline & : & $-i$ & $\cdots$ & $\cdots$ & & $\cdots$ & & & -- \\
\hline & 2 & -1 & $1.4617+05$ & $2.9824+05$ & & $\cdots-$ & j & $6713-$ & -03 \\
\hline & -1 & -1 & 3. $8492+04$ & $\cdots$ & & $-\cdots$ & 7. & $7937-$ & -03 \\
\hline & -1 & -1 & $-\cdots$ & --- & & --- & & & $\cdots$ \\
\hline & 2 & -1 & $5.4848+05$ & $7.3271+05$ & & -- & 6 & 0945 & -03 \\
\hline & 2 & -1 & $6.4955+05$ & $8.9960+0 j$ & & $\cdots$ & 2. & 69 & -03 \\
\hline & 2 & -1 & $9.5352+05$ & $2.1064+06$ & & -- & 4 & 14 & -03 \\
\hline & 1 & $i$ & $2.1440+06$ & 1. $0990+06$ & 3.21 & $42+03$ & & & $\cdots$ \\
\hline & 1 & 1 & $3.0319+06$ & $2.1700+00$ & 1.01 & $5+04$ & & & $-\cdots$ \\
\hline & $i$ & 1 & $2.4861+00$ & $1.5010+06$ & $2.5:$ & $105+94$ & & & $\cdots$ \\
\hline & 1 & $i$ & $33401+00$ & $2.7999+06$ & 2.35 & $55+04$ & & & $\cdots-$ \\
\hline & 1 & 1 & $27320+06$ & $1.8220+06$ & 3.35 & $25+04$ & & & --- \\
\hline & 1 & 1 & $3.5779+00$ & $3.2569+06$ & 3.25 & $1+04$ & & & --- \\
\hline & 1 & $i$ & $29950+06$ & $2.1620+06$ & 797 & $40+04$ & & & -- \\
\hline & 1 & 1 & $3.6 \div 30+06$ & $3.4949+06$ & 7.27 & $35+04$ & & & -- \\
\hline & 1 & 1 & $3.1731+06$ & $2.3900+00$ & 1. 3 & $60+05$ & & & --- \\
\hline & 2 & -1 & $3.8771+00$ & $3.8349+06$ & & $\cdots-$ & & & $\cdots$ \\
\hline & -1 & -1 & $\cdots$ & --- & & $\ldots-$ & & & -- \\
\hline & -1 & -1 & --- & $\cdots$ & & $\cdots-$ & & & --- \\
\hline & -1 & -1 & $\cdots$ & $\cdots$ & & $\cdots$ & & & -- \\
\hline & -1 & -1 & $\cdots$ & $\cdots$ & & $\cdots$ & & & --- \\
\hline 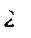 & 2 & -1 & $39426+04$ & $\therefore 4: 2+95$ & & $\cdots$ & $\theta$ & $i-$ & -03 \\
\hline & -1 & -1 & $\cdots$ & $\cdots$ & & $\ldots$ & & & -- \\
\hline & 1 & -1 & $13283+00$ & $9.7126+05$ & & $\cdots$ & & & $\cdots$ \\
\hline & $i$ & -1 & 1. $232 i+60$ & $1.15+2+06$ & & -- & & & -- \\
\hline & 1 & -1 & $6.5849+05$ & $59589+05$ & & $\cdots-$ & & & -- \\
\hline & 1 & -1 & $1.8670+06$ & $9.6244+05$ & & -- & & & --- \\
\hline & 1 & -1 & $11140+06$ & $72501+05$ & & $-\cdots$ & & & --- \\
\hline & 1 & i & $2.2491+00$ & $1.4160+06$ & 3.94 & $3-01$ & & & --- \\
\hline & 1 & 1 & $1.4730+06$ & $8.4371+05$ & 2.1 & $116+02$ & & & --- \\
\hline 1 & 1 & 1 & $2.5380+00$ & $1.8060+06$ & 6.1 & $199+02$ & & & $-\cdots$ \\
\hline & 1 & 1 & $1.8430+06$ & $9.8000+05$ & 360 & $6+03$ & & & --- \\
\hline & 1 & 1 & $2.6970+06$ & $2.0261+06$ & 8.30 & $8+0 ;$ & & & $-\cdots$ \\
\hline & 1 & 1 & $2.0941+00$ & $11180+06$ & 1.7 & $796+04$ & & & $-\cdots$ \\
\hline & 1 & 1 & $2.9201+06$ & $2.3109+06$ & 2.06 & $563+04$ & & & $-\cdots$ \\
\hline : & 1 & $!$ & $2.3609+06$ & 1. $2660+00$ & 3.8 & $011+04$ & & & -- \\
\hline & 1 & -1 & $-\cdots \cdots$ & $\cdots$ & & $\cdots$ & & & --- \\
\hline & -1 & -1 & $\cdots$ & $-\cdots$ & & $\cdots$ & & & $\ldots$ \\
\hline & -1 & $-:$ & $\cdots$ & $\ldots$ & & $\cdots$ & & & $-\cdots$ \\
\hline & -1 & -1 & $\cdots$ & --- & & --- & & & $\cdots$ \\
\hline 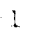 & -1 & $-:$ & --- & $--\infty$ & & $\cdots-$ & & & --- \\
\hline & -1 & -1 & -- & $\cdots$ & & $\cdots$ & & & --- \\
\hline & $-i$ & $\cdots 1$ & $\cdots-$ & $\cdots-$ & & $-\cdots$ & & & $\cdots$ \\
\hline$i$ & -1 & -1 & --- & $\cdots$ & & $\cdots$ & & & $-\cdots$ \\
\hline 2 & 2 & -1 & $11928+05$ & $93023+03$ & & $\cdots$ & 7. & & -03 \\
\hline 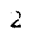 & 2 & -1 & e.2145+05 & $5.9113+05$ & & $-\cdots$ & 9. & 58 & -03 \\
\hline 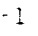 & -1 & -1 & -- & $\cdots$ & & $\ldots$ & & & -- \\
\hline 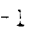 & -1 & -1 & $\cdots$ & $\cdots$ & & $-\infty$ & & & $\cdots$ \\
\hline 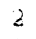 & 2 & -1 & $7.3251+04$ & $8.5998+05$ & & +- & 7. & 9950 & -03 \\
\hline$\vdots$ & $\because 1$ & -1 & $-\cdots$ & $\cdots$ & & $\cdots$ & & & -- \\
\hline$\vdots$ & 1 & -1 & $1.0774+06$ & $8.9817+05$ & & $\ldots-$ & & & $\ldots$ \\
\hline 1 & 1 & -1 & $2.4770+06$ & 1. $1560+00$ & & --- & & & $\cdots$ \\
\hline 1 & 1 & -1 & $1.7330+06$ & $8.159 j+05$ & & $\cdots$ & & & $\ldots$ \\
\hline$\vdots$ & 1 & 1 & $2.7421+06$ & $1.7531+06$ & 2.0 & $0+00$ & & & -- \\
\hline i & 1 & 1 & $2.0210+00$ & $1.0540+106$ & 2.1 & $1+03$ & & & --- \\
\hline 1 & 1 & 1 & 2. $9670+06$ & $2.2200+06$ & 1.6 & $8+03$ & & & --- \\
\hline & 1 & 1 & $2.2890+06$ & $13590+06$ & 1.9 & $324+04$ & & & $\cdots$ \\
\hline 1 & 1 & 1 & $3.0940+06$ & $2.4940+06$ & 1.2 & $160+04$ & & & $\cdots$ \\
\hline i & 1 & 1 & $2.4760+06$ & 1. $5981+06$ & 3.8 & $986+04$ & & & -- \\
\hline 1 & 1 & 1 & $3.2609+06$ & $2.8370+06$ & 2.9 & $1: 10+04$ & & & $\cdots$ \\
\hline & 1 & 1 & $2.6710+06$ & $1.8520+06$ & 7.4 & $063+04$ & & & $\cdots$ \\
\hline & 1 & 1 & 3. $3701+06$ & $3.0699+06$ & 4.5 & $538+74$ & & & $\cdots$ \\
\hline & 1 & 1 & $2.9030+06$ & $2.1470+06$ & 1. 0 & $017+65$ & & & -- \\
\hline & -1 & -1 & $\cdots$ & --- & & $\cdots-$ & & & $\cdots$ \\
\hline & -1 & -1 & $\cdots$ & $\cdots$ & & $\cdots$ & & & $\ldots$ \\
\hline & -1 & -1 & -- & --- & & $-\cdots$ & & & $\ldots$ \\
\hline & 1 & -1 & -- & -- & & -- & & & 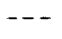 \\
\hline
\end{tabular}


Table C-2 (Cont.)

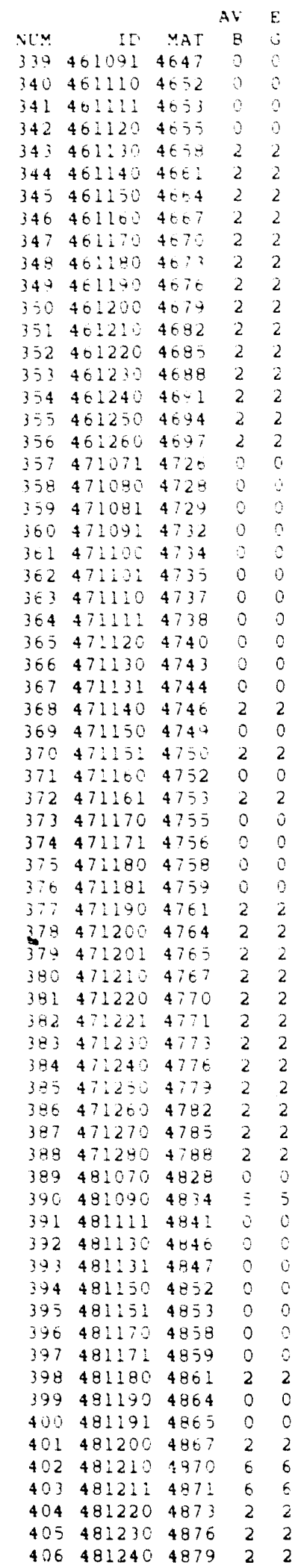

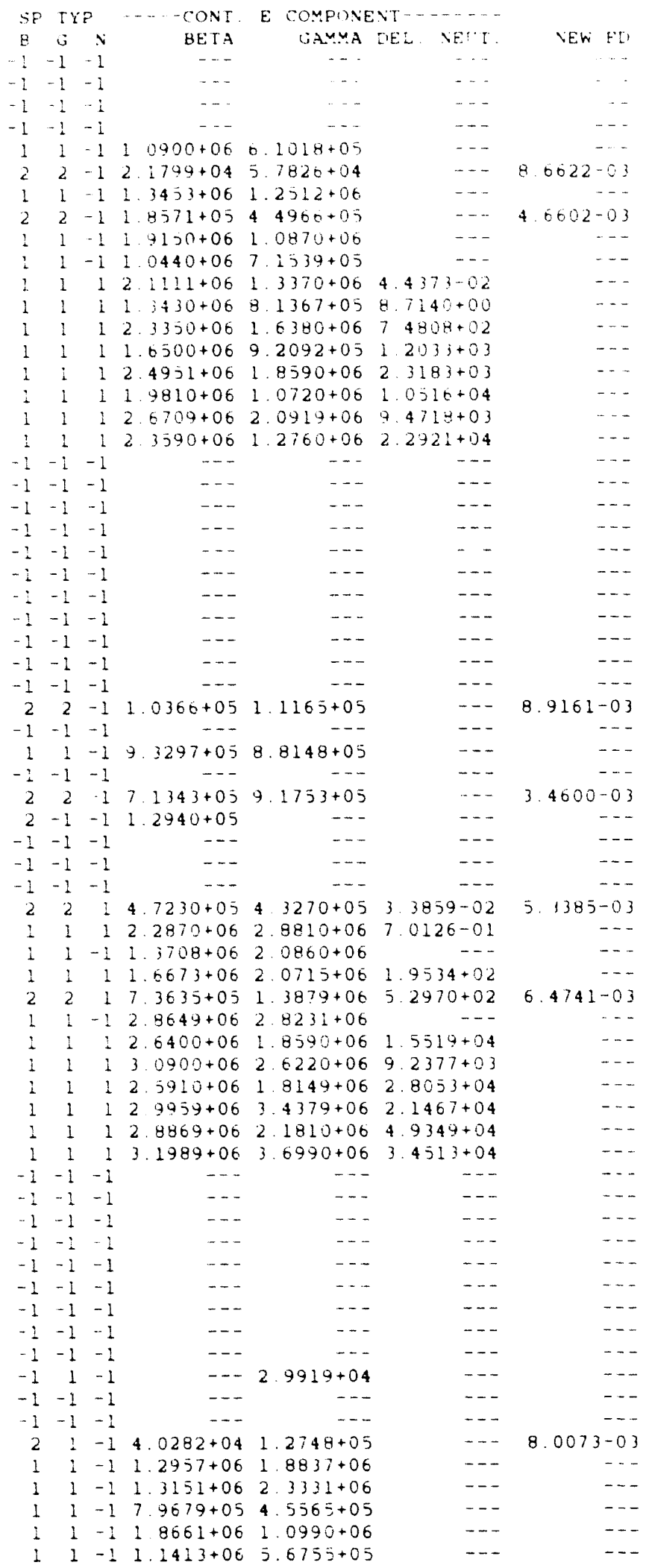


Table C-2 (Cont.)

\begin{tabular}{|c|c|c|c|c|c|c|c|c|c|c|c|c|}
\hline & & & $A v$ & $\mathrm{E}$ & $S E$ & $T Y$ & & $\cdots-\operatorname{coNT}$ & ONE & $\mathrm{NI}$ & & \\
\hline NLY & ID & MAI & $\mathrm{B}$ & G & $\mathrm{B}$ & $\bar{G}$ & $\therefore$ & BETA & GAMAA & NEII & VEW & $E L$ \\
\hline 407 & 481250 & 4882 & 2 & 2 & 1 & 1 & -1 & $2.0010+00$ & 1. $3480+00$ & $\cdots$ & & $\therefore$ \\
\hline 408 & 481260 & 4885 & 2 & 2 & 1 & $i$ & -1 & 1. $4905+00$ & $i 2089+05$ & $\cdots$ & & $\cdots$ \\
\hline 09 & 481270 & 4888 & 2 & 2 & 1 & $!$ & 1 & $2.0730+06$ & $2.0011+06$ & $1.5105+01$ & & $\ldots$ \\
\hline 410 & 481280 & 4891 & 2 & 2 & 1 & 1 & 1 & 1. $8310+06$ & $1.0030+00$ & 2. $3804+02$ & & $\cdots$ \\
\hline 411 & 481290 & 4894 & 2 & 2 & 1 & 1 & 1 & $2.3040+00$ & $2.2280+06$ & $3.7534+02$ & & $\ldots \ldots$ \\
\hline 12 & 481300 & 4897 & 2 & 2 & 1 & 1 & 1 & $2.2580+06$ & 1. $2250+06$ & $3.127 i+03$ & & $\cdots$ \\
\hline 413 & 481310 & 4900 & 2 & 2 & 1 & 1 & 1 & 3. $5181+06$ & $32671+06$ & $2.4307+04$ & & 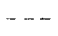 \\
\hline 414 & 481320 & 4903 & 2 & 2 & 1 & 1 & 1 & $3.4049+06$ & $1.8971+06$ & 1. $304 i+05$ & & $-\cdot-$ \\
\hline 15 & 491131 & 4926 & 0 & $c$ & -1 & -1 & -1 & $\cdots$ & $\cdots$ & $\cdots$ & & $\cdots$ \\
\hline 16 & 491140 & 4928 & 0 & 0 & -1 & -1 & -1 & $\cdots$ & $\ldots \ldots$ & --- & & $-\cdots-$ \\
\hline 17 & 491141 & 4929 & 0 & 0 & -1 & -1 & -1 & $\ldots$ & $\ldots$ & $\cdots$ & & $\ldots$ \\
\hline 418 & 491150 & 4931 & 0 & 0 & -1 & -1 & -1 & $\cdots$ & $\ldots$ & -- & & $\ldots$ \\
\hline 419 & 491151 & 4932 & 0 & 0 & -1 & -1 & -1 & $\ldots$ & $\cdots \cdots$ & $\cdots$ & & $\ldots$ \\
\hline 420 & 491160 & 4934 & 0 & 0 & -1 & -1 & -1 & $\cdots$ & $\ldots$ & $\ldots$ & & $\ldots$ \\
\hline 421 & 491161 & 4935 & 0 & 0 & -1 & -1 & -1 & -- &.-- & $\cdots$ & & $\cdots$ \\
\hline 422 & 491162 & 4936 & 0 & 0 & -1 & -1 & -1 & $\cdots$ & $\cdots$ & $-\cdots-$ & & $\cdots$ \\
\hline 423 & 421170 & 4937 & 0 & 0 & -1 & -1 & -1 & -- & $\cdots$ & $\cdots$ & & $\ldots$. \\
\hline 424 & 491171 & 4938 & 0 & 0 & -1 & -1 & -1 & $-\cdots$ & $\ldots$ & $\cdots$ & & $\cdots$ \\
\hline 425 & $491: 80$ & 4440 & 0 & 0 & -1 & -1 & -1 & $\ldots$ & $\ldots$ & $\ldots$ & & $\ldots$ \\
\hline 420 & 491181 & 4941 & 0 & 0 & -1 & -1 & -1 & -- & $\cdots$ & $\cdots-$ & & $\ldots$ \\
\hline 427 & 401182 & 4942 & 0 & 0 & -1 & -1 & -1 & $-\cdots$ & $\cdots$ & $\ldots$ & & $\cdots$ \\
\hline 428 & 401190 & 4943 & 0 & 0 & -1 & -1 & -1 & $\cdots$ & $\cdots$ & $\cdots$ & & $\ldots$ \\
\hline 429 & 441191 & 4444 & 2 & 2 & 2 & 2 & -1 & $3.9001+04$ & 1. $1846+05$ & $\ldots$ & A. 7996 & -03 \\
\hline 430 & 491200 & 4946 & 2 & 2 & -1 & 2 & -1 & $\ldots$ & 3. $1442+05$ & $-\cdots$ & & $\ldots$ \\
\hline 431 & 491201 & 4947 & 0 & 0 & -1 & -1 & -1 & $\cdots$ & $\ldots$ & $-\cdots$ & & $\cdots-$ \\
\hline 432 & 491202 & 4948 & 0 & 0 & -1 & -1 & -1 & $\ldots$ & $\ldots$ & -- & & $\ldots$ \\
\hline 433 & 491210 & 4949 & 0 & 0 & -1 & -1 & -1 & $\cdots$ & $\ldots$ & -- & & --- \\
\hline 434 & 491211 & 4950 & 0 & . & -1 & -1 & -1 & --- & $\cdots$ & $\ldots$ & & -- \\
\hline 435 & 491220 & 4952 & 2 & 2 & 2 & 2 & -1 & $1.8134+05$ & $6.0199+03$ & -- & 8.60 & -03 \\
\hline 436 & 491221 & 4953 & 0 & 0 & 2 & -1 & -1 & $2.2341+05$ & $\ldots$ & -- & & $\ldots$ \\
\hline 437 & 491222 & 4954 & 0 & 0 & -1 & -1 & -1 & $\ldots$ & $\cdots$ & -- & & $\cdots$ \\
\hline 438 & 491230 & 4955 & 0 & 0 & -1 & -1 & -1 & $\cdots$ & $\cdots$ & -- & & --- \\
\hline 439 & 491231 & 4956 & 0 & 0 & -1 & -1 & -1 & $-\cdots$ & $\ldots$ & -- & & $\cdots$ \\
\hline 440 & 491240 & 4958 & 0 & 0 & -1 & -1 & -1 & $\cdots$ & --- & $\cdots$ & & $-\cdots$ \\
\hline $44 i$ & 491241 & 4959 & 0 & 0 & -1 & -1 & -1 & $\ldots$ & -- & -- & & $\ldots$ \\
\hline 442 & 491250 & 4961 & 0 & 0 & -1 & -1 & -1 & $\ldots$ & $\ldots$ & $\cdots$ & & $\ldots$ \\
\hline 443 & 491251 & 4962 & 2 & 2 & 2 & 2 & -1 & $2.7575+05$ & $5.0533+05$ & --- & 7.5687 & -03 \\
\hline 444 & 491260 & 4964 & 0 & 0 & 2 & -1 & -1 & 1. $3752+06$ & $\ldots$ & $\cdots$ & 2.132 & -03 \\
\hline 445 & 491261 & 4965 & 0 & 0 & -1 & -1 & -1 & $--\cdots$ & $\cdots$ & $\ldots$. & & $\cdots$ \\
\hline 446 & 491270 & 4967 & 0 & 0 & -1 & -1 & 1 & $-\cdots$ & $\cdots$ & $1.3635+03$ & & $\cdots$ \\
\hline 447 & 491271 & 4968 & 2 & 2 & 2 & 2 & 1 & 6. $1617+05$ & $1.2263+00$ & 1. $3429+03$ & 3.7159 & -03 \\
\hline 448 & 491280 & 4970 & 1 & 1 & -1 & -1 & 1 & $\cdots$ & $\cdots$ & $1.1085+02$ & & $\cdots$ \\
\hline 449 & 491281 & 4971 & 2 & 2 & -1 & 2 & -1 & $-\cdots$ & $1.7523+06$ & $\ldots$ & & $\cdots$ \\
\hline 450 & 491290 & 4973 & 0 & 0 & -1 & -1 & 1 & $\cdots$ & -- & $8.7615+02$ & & $\cdots$ \\
\hline $4 j 1$ & 491291 & 4974 & 2 & 2 & 2 & 2 & 1 & $2+00$ & 2. $5857+06$ & 1. $3690+04$ & 2.45 & $5-03$ \\
\hline 452 & 491300 & 4976 & 0 & 0 & -1 & -1 & 1 & $\ldots$ & --- & $4.8671+03$ & & $\cdots$ \\
\hline 453 & 491301 & 4977 & 0 & 0 & -1 & $\cdots 1$ & 1 & $\cdots$ & $\cdots$ & 4. $5996+03$ & & $\cdots$ \\
\hline 454 & 491302 & 4978 & 0 & 0 & -1 & -1 & -1 & -- & $-\cdots$ & $-\cdots$ & & $\cdots$ \\
\hline 453 & 491310 & 4979 & 2 & 2 & 1 & 1 & 1 & $2.7071+06$ & $2.0170+06$ & $7.4402+03$ & & $\ldots$ \\
\hline 456 & 491311 & 4980 & 2 & 2 & -1 & -1 & 1 & $\ldots$ & $\ldots$ & $6.914 j+03$ & & $\ldots$ \\
\hline 457 & 491320 & 4982 & 0 & 0 & -1 & -1 & 1 & $\cdots$ & --- & 2. $5952+04$ & & $\ldots-\ldots$ \\
\hline 458 & 491330 & 4983 & 2 & 2 & 1 & 1 & 1 & $3.7661+06$ & $3.4140+06$ & $2.1073+03$ & & --- \\
\hline 459 & 491340 & 4988 & 2 & 2 & 1 & 1 & 1 & 3. $9920+06$ & $4.6901+06$ & $2.3581+05$ & & $\ldots$ \\
\hline 460 & 501130 & 5028 & 0 & 0 & -1 & -1 & -1 & $\ldots$ & $\cdots$ & $\cdots$ & & $\ldots$ \\
\hline 461 & 501131 & 5029 & 0 & 0 & -1 & -1 & -1 & $\cdots$ & $\cdots$ & $\cdots$ & & $\ldots$ \\
\hline $4 \in 2$ & 501171 & 5041 & 0 & 0 & -1 & -1 & -1 & $\cdots$ & $\cdots$ & $\cdots \cdots$ & & $\ldots$ \\
\hline 463 & 501191 & 5047 & 0 & 0 & -1 & -1 & -1 & -- & $\cdots$ & --- & & $\ldots$ \\
\hline 464 & 501210 & 5052 & 0 & 0 & -1 & -1 & -1 & -- & $\cdots$ & $-\cdots-$ & & $\ldots$ \\
\hline 465 & 501211 & 5053 & 0 & 0 & -1 & -1 & -1 & $\ldots$ & $\ldots$ & $\ldots$ & & $\cdots$ \\
\hline 466 & 501230 & 3058 & 0 & 0 & -1 & -1 & -1 & $-\cdots$ & $\ldots$ & -- & & -- \\
\hline 467 & 501231 & 5059 & 0 & 0 & -1 & -1 & -1 & --- & $\ldots$ & $\ldots$ & & $\ldots$ \\
\hline 468 & 501250 & 5064 & 0 & 0 & -1 & -1 & -1 & $\ldots$ & $\cdots$ & $\cdots$ & & $\ldots$ \\
\hline 469 & 501251 & 5065 & 0 & 0 & -1 & -1 & -1 & $\ldots$ & -- & $\cdots$ & & $\ldots$ \\
\hline 470 & 501260 & 5067 & 0 & 0 & 2 & 2 & -1 & $2.6171+04$ & $7.4452+04$ & $\ldots$ & & $\ldots$ \\
\hline 471 & 501270 & 5070 & 0 & 0 & -1 & -1 & -1 & $\ldots$ & $\ldots$ & --- & & $\ldots$ \\
\hline 472 & 501271 & 5071 & 2 & 2 & 2 & 2 & -1 & $8.9376+04$ & $3.1845+05$ & $\cdots$ & 72156 & $5-03$ \\
\hline 473 & 501280 & 5073 & 0 & 0 & -1 & -1 & -1 & --- & $\cdots$ & -- & & -- \\
\hline 474 & 501281 & 5074 & 0 & 0 & -1 & -1 & -1 & $-\cdots$ & $\cdots$ & --- & & -- \\
\hline
\end{tabular}


Table C-2 (Cont.)

\begin{tabular}{|c|c|c|c|c|c|c|c|c|c|c|c|c|}
\hline & & & $A V$ & $\mathrm{E}$ & $S P$ & $T Y$ & & CONT & OMPONE & ENT $\ldots . .$. & & \\
\hline$N 1 x$ & II) & $\therefore A I$ & $\mathrm{H}$ & $i j$ & E & $\dot{~ ن ~}$ & $\mathrm{~N}$ & BETA & GAMMA & IELL NELT & NEW & $\mathrm{FD}$ \\
\hline 475 & 501200 & $20 \% 0$ & i & $i$ & 2 & 2 & $\cdot 1$ & 1. $4808+05$ & $8.0011+05$ & $\cdots$ & $7.94,5-$ & -03 \\
\hline 476 & 501291 & 5078 & i & $i$ & 2 & 2 & -1 & $4.8707+07$ & $4.7850+05$ & $\cdots$ & $3.3296 \%$ & 03 \\
\hline 477 & 501300 & 3019 & 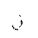 & 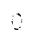 & $i$ & -1 & -1 & $\ldots \ldots$ & $-\cdots$ & $\cdots$ & & $\cdots$ \\
\hline 478 & 501301 & 3040 & 2 & 2 & 2 & 2 & -1 & $8.1946+04$ & $3.4720+05$ & $\cdots$ & $6.7135-$ & -03 \\
\hline 479 & 501310 & 3082 & 1 & 1 & 1 & 1 & -1 & 8. $19004+05$ & 2. $3600+06$ & --- & & $\ldots$ \\
\hline 480 & 501311 & 5083 & 2 & 2 & $i$ & 1 & -1 & 1. $2459+06$ & 1. $8924+06$ & $\ldots$ & & $\cdots$ \\
\hline 481 & 501320 & 3085 & 0 & 0 & -1 & $\cdots 1$ & -1 & $\cdots$ & --- & $\ldots$ & & $\cdots$ \\
\hline 482 & 501330 & 5ORH & 1 & 1 & 2 & 2 & 1 & $7.2485+05$ & $1.7900+06$ & $6.9831+02$ & $5.4954-$ & -03 \\
\hline 483 & 501340 & 3041 & 2 & 2 & 1 & 1 & 1 & $2.2950+06$ & 1. $2480+06$ & $9.0706+04$ & & $\ldots$ \\
\hline 484 & 501350 & 5094 & 2 & 2 & 1 & 1 & 1 & $2.5550+06$ & 2. $4820+06$ & 4. $0836+04$ & & $\cdots$ \\
\hline 485 & 501360 & 5097 & 2 & 2 & 1 & 1 & 1 & $2.6141+00$ & $14270+06$ & 8. $3410+04$ & & $\cdots$ \\
\hline 486 & 511220 & 5128 & 0 & 0 & -1 & -1 & -1 & $\cdots$ & -- & -- & & -- \\
\hline 487 & 511221 & 5129 & 0 & 0 & -1 & -1 & -1 & $-\cdots$ & $\cdots$ & $\cdots$ & & -- \\
\hline 488 & 311240 & 5134 & 0 & 0 & -1 & -1 & -1 & $-\cdots$ & -- & $\cdots$ & & $-\cdots$ \\
\hline 489 & 511241 & 5135 & 0 & 0 & -1 & -1 & -1 & -- & $\cdots$ & $\ldots$ & & $\ldots$ \\
\hline 490 & 511242 & 5136 & 0 & 0 & -1 & -1 & -1 & $\ldots$ & $\cdots$ & $\ldots$ & & $\ldots$ \\
\hline 491 & 511250 & 5137 & 0 & 0 & -1 & -1 & -1 & $-\cdots$ & $\cdots$ & $\ldots-$ & & $\ldots$ \\
\hline 492 & 511260 & 5140 & 0 & 0 & -1 & -1 & -1 & $\cdots$ & -- & $\cdots$ & & $\ldots$ \\
\hline 493 & 511261 & 5141 & 0 & 0 & -1 & -1 & -1 & $\cdots$ & $\cdots$ & $\ldots$ & & $\ldots$ \\
\hline 494 & 511262 & 5142 & 0 & 0 & -1 & -1 & $\cdot 1$ & $\cdots$ & $\cdots$ & $\cdots$ & & $\ldots$ \\
\hline $49 j$ & 511270 & 5143 & 0 & 0 & -1 & -1 & -1 & $-\cdots$ & $\ldots$ & $\ldots$ & & $\cdots$ \\
\hline 496 & 511280 & 5146 & 0 & 0 & -1 & -1 & -1 & $\cdots$ & $\ldots$ & $\ldots$ & & $\cdots$ \\
\hline $4 \unlhd 7$ & 511281 & 5147 & 0 & 0 & -1 & -1 & -1 & $\ldots$ & $\ldots$ & $\ldots$ & & $\cdots$ \\
\hline 498 & 511290 & 5149 & 0 & 0 & -1 & -1 & -1 & $\ldots$ & $\cdots$ & $\ldots$ & & $\cdots-$ \\
\hline 499 & 511300 & 5152 & 0 & 0 & -1 & -1 & -1 & -- & $\cdots-$ & $\ldots$ & & $-\cdots$ \\
\hline 500 & 511301 & 5153 & 0 & 0 & -1 & -1 & -1 & -- & $\cdots$ & -. & & -- \\
\hline 501 & 511310 & 5155 & 0 & 0 & -1 & -1 & -1 & $\ldots$ & $\ldots$ & $-\cdots$ & & -- \\
\hline 502 & 511320 & 5158 & 0 & 0 & -1 & -1 & -1 & $\ldots$ & $-\cdots$ & --- & & $\ldots$ \\
\hline 503 & 511321 & 5159 & 0 & 0 & -1 & -1 & -1 & -- & -- & -- & & $\cdots$ \\
\hline 504 & 511330 & 5161 & 0 & 0 & -1 & -1 & -1 & --- & --- & --- & & $\cdots$ \\
\hline 505 & 511340 & 5164 & 2 & 2 & 1 & 1 & 1 & $2.7810+06$ & $2.2560+06$ & $37+02$ & & --- \\
\hline 306 & 511341 & 5165 & 1 & 1 & -1 & 2 & -1 & $-\cdots$ & $3.4390+05$ & --- & & -- \\
\hline 507 & 511350 & 5167 & 1 & 1 & 1 & 1 & 1 & $2.2900+06$ & $1.6000+06$ & $1.6475+05$ & & -- \\
\hline 508 & 511360 & 5170 & 2 & 2 & 1 & 1 & 1 & $2.9529+06$ & $2.6051+06$ & $1.0769+05$ & & $\cdots$ \\
\hline 509 & 511370 & 5173 & 2 & 2 & 1 & 1 & 1 & $2.5730+06$ & 2. $3891+06$ & $1.0036+05$ & & $\cdots$ \\
\hline 510 & 511380 & 5176 & 2 & 2 & 1 & 1 & 1 & $3.0300+06$ & 3. $5780+06$ & 1. $2349+05$ & & $\cdots$ \\
\hline 311 & 511390 & 5179 & 2 & 2 & 1 & 1 & 1 & $2.9080+06$ & $2.6840+06$ & $2.4376+05$ & & -- \\
\hline 512 & 521210 & 5228 & 0 & 0 & -1 & -1 & -1 & $-\cdots$ & -- & $\ldots$ & & $\ldots$ \\
\hline 513 & 521211 & 5229 & 0 & 0 & -1 & -1 & -2 & $\cdots$ & $\cdots$ & $\cdots$ & & $-\cdots$ \\
\hline 514 & 521230 & 5234 & 5 & 5 & -1 & -1 & -1 & $\ldots$ & $\ldots$ & -- & & $\cdots$ \\
\hline 513 & $32: 231$ & 5235 & 0 & 0 & -1 & $-i$ & -1 & $\cdots$ & +- & $\cdots$ & & $-\cdots$ \\
\hline 516 & 521251 & 5241 & 0 & 0 & -1 & -1 & -1 & $\ldots$ & $-\cdots$ & -- & & $\ldots$ \\
\hline 517 & 521270 & 5246 & 0 & 0 & -1 & -1 & -1 & $-\cdots$ & $\cdots-$ & $\cdots$ & & $-\cdots$ \\
\hline 518 & 521271 & 5247 & 0 & 0 & -1 & 2 & -1 & $\cdots$ & 4. $.0001+01$ & --- & & --- \\
\hline 519 & $52: 290$ & 5252 & 0 & 0 & -1 & -1 & -1 & -- & $\cdots$ & --- & & $\ldots$ \\
\hline 520 & 521291 & 5253 & 0 & 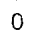 & -1 & -1 & -1 & $\cdots$ & $\ldots$ & --- & & $\ldots$ \\
\hline 521 & 521310 & 5258 & 0 & 0 & -1 & -1 & -1 & --- & $\cdots-$ & $\ldots$ & & --- \\
\hline 522 & 521311 & 5259 & 0 & 0 & -1 & -1 & -1 & --- & --- & $-\cdots$ & & $\cdots$ \\
\hline 523 & 521320 & 5261 & 0 & 0 & -1 & -1 & -1 & -- & --- & -- & & $-\cdots$ \\
\hline 524 & 521330 & 5264 & 0 & 0 & -1 & -1 & -1 & -- & -- & $\cdots$ & & $\ldots$ \\
\hline 525 & 521331 & 5265 & 0 & 0 & -1 & -1 & -1 & --- & --- & $\ldots$ & & $\ldots$ \\
\hline 526 & 521340 & 5267 & 0 & 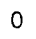 & -1 & -1 & -1 & --- & $-\cdots$ & $\ldots$ & & $-\cdots$ \\
\hline 527 & 521350 & 5270 & 2 & 2 & 2 & 2 & -1 & $3.7034+05$ & $9.6790+0 j$ & $-\cdots$ & 7.1800 & .03 \\
\hline 528 & 521360 & 5273 & 0 & 0 & -1 & -1 & 1 & -- & -- & $2.6734+03$ & & $-\cdots$ \\
\hline 529 & 521370 & 5276 & 2 & 2 & 1 & 1 & 1 & $0+06$ & $1.6090+05$ & $41+03$ & & $-\cdots$ \\
\hline 530 & 521380 & 5279 & 2 & 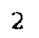 & 1 & 1 & 1 & $1.9460+06$ & $1.0680+06$ & $2.0845+04$ & & -- \\
\hline 531 & 521390 & 5282 & 2 & 2 & 1 & 1 & 1 & $2.3760+06$ & $2.3510+06$ & $2.8410+04$ & & $\cdots$ \\
\hline 532 & 521400 & 5285 & 2 & 2 & 1 & 1 & 1 & $2.3360+06$ & 1. $2750+06$ & $7.2588+04$ & & $\ldots$ \\
\hline 533 & 521410 & 5288 & 2 & 2 & 1 & 1 & 1 & $2.658 i+06$ & $2.5980+06$ & $5.0932+04$ & & $\cdots$ \\
\hline 534 & 521420 & 5291 & 2 & 2 & 1 & 1 & 1 & $2.5130+06$ & 1. $3750+06$ & $7.4319+04$ & & --- \\
\hline 535 & 531250 & 5319 & 0 & 0 & -1 & -1 & -1 & $\ldots$ & $\ldots$ & $-\cdots$ & & $-\cdots$ \\
\hline 536 & 531280 & 5328 & 0 & 0 & -1 & -1 & -1 & $\cdots$ & $-\cdots$ & $-m$ & & $\cdots$ \\
\hline 537 & 531290 & 5331 & 0 & 0 & -1 & -1 & -1 & $\ldots$ & $\rightarrow--$ & $-\cdots$ & & --- \\
\hline 538 & 531300 & 5334 & 0 & 0 & -1 & -1 & -1 & -- & $\cdots$ & -- & & $\ldots$ \\
\hline 539 & 531301 & 5335 & 0 & 0 & -1 & -1 & -1 & $\cdots$ & $\cdots-$ & $\ldots$ & & --- \\
\hline 540 & 531310 & 5337 & 0 & 0 & -1 & -1 & -1 & $\cdots$ & $-\infty$ & $-\cdots$ & & --- \\
\hline 541 & 531320 & 5340 & 0 & 0 & -1 & -1 & -1 & -- & --- & --- & & -- \\
\hline 542 & 531321 & 5341 & 0 & 0 & 2 & -1 & -1 & $7.0197+03$ & --- & -- & 9.8884 & -03 \\
\hline
\end{tabular}


Table C-2 (Cont.)

\begin{tabular}{|c|c|c|c|c|c|c|c|c|c|c|c|c|}
\hline \multirow[b]{2}{*}{ Ne: } & \multicolumn{3}{|c|}{$A V$} & \multirow{2}{*}{$\begin{array}{l}E \\
G\end{array}$} & \multirow{2}{*}{$\begin{array}{l}S P \\
B\end{array}$} & \multicolumn{2}{|c|}{ TYP } & \multirow{2}{*}{$\begin{array}{r}\text { CONT } \\
\text { BETA }\end{array}$} & \multirow[t]{2}{*}{$E$} & \multicolumn{2}{|c|}{ COMPONENT $\cdots$} & \\
\hline & I $\mathrm{O}$ & $M A I$ & in & & & G & $N$ & & & GAMMA & NELT & NEW FD \\
\hline $54 ?$ & 531330 & 5343 & 0 & $n$ & -1 & -1 & -1 & --- & & $-\cdots$ & $\cdots-$ & $\cdots$ \\
\hline 544 & 531331 & 5344 & $c$ & 0 & -1 & $\cdots 1$ & -1 & $-\cdots$ & & $\cdots-$ & $\cdots$ & $\cdots$ \\
\hline 545 & 331340 & 3346 & 0 & 0 & -1 & -1 & -1 & --- & & -- & $\cdots$ & $\cdots$ \\
\hline 546 & 531341 & 5347 & 0 & 0 & -1 & -1 & -1 & --- & & $-\cdots$ & $\cdots$ & -- \\
\hline 347 & 531350 & 5340 & 0 & 0 & -1 & -1 & -1 & --- & & --- & $\cdots$ & $\cdots-$ \\
\hline 548 & 531360 & 5352 & 0 & 0 & -1 & -1 & -1 & $\cdots-$ & & $--\cdots$ & $\cdots$ & -- \\
\hline 549 & 531361 & 5333 & 1 & 1 & 2 & 2 & -1 & $.4454+04$ & 4. & $8650+05$ & --- & $8.2505-0$ \\
\hline 550 & 531370 & 5355 & 1 & 1 & -1 & -1 & 1. & --- & & $-\cdots$ & $4.2194+04$ & -- \\
\hline 351 & 531380 & 5358 & 2 & 2 & 2 & 2 & 1 & $6.4218+05$ & 1 . & $1191+06$ & $2.0145+04$ & $.3909-0$ \\
\hline 552 & 531390 & 5361 & 1 & 1 & 1 & 1 & 1 & $2.4299+06$ & 1. & $4000+06$ & $3.9890+04$ & -- \\
\hline 553 & 531400 & 5364 & 2 & 2 & 1 & 1 & 1 & $2.7621+06$ & 2 . & $3280+06$ & $4.0215+04$ & -- \\
\hline 354 & 331410 & 3367 & 2 & 2 & 1 & 1 & 1 & $2.4251+06$ & 1. & $7790+06$ & $1.3514+05$ & -- \\
\hline 555 & 531420 & 3310 & 2 & 2 & 1 & 1 & 1 & $2.6920+06$ & & $2030+06$ & $8.2623+04$ & -- \\
\hline 556 & 531430 & 3373 & 2 & 2 & 1 & 1 & 1 & $2.3751+06$ & 2 . & $2520+06$ & $9.8113+04$ & -- \\
\hline 557 & 531440 & 5376 & 2 & 2 & 1 & 1 & 1 & $2.7189+06$ & 3. & $2481+06$ & $7.8132+04$ & -- \\
\hline $55 B$ & 531450 & 5379 & 2 & 2 & 1 & 1 & 1 & $2.4681+06$ & 2. & $3421+06$ & 1. $2962+05$ & -- \\
\hline & 541250 & 5428 & 0 & 0 & -1 & -1 & -1 & --- & & -- & -- & -- \\
\hline 560 & 541251 & 5429 & 0 & 0 & -1 & -1 & -1 & --- & & $-\cdots$ & $\ldots$ & - \\
\hline 561 & 341270 & 3434 & 0 & 0 & -1 & -1 & -1 & --- & & $\cdots$ & --- & - \\
\hline 562 & 541271 & 3435 & 0 & 0 & -1 & -1 & -1 & --- & & $\cdots-$ & $\cdots-$ & -- \\
\hline 563 & 541291 & 3441 & -0 & 0 & -1 & -1 & -1 & --- & & $\cdots \cdots$ & $\cdots$ & -- \\
\hline 264 & 541311 & 5447 & 0 & 0 & -1 & -1 & -1 & $-\cdots$ & & $-\cdots$ & $\cdots$ & -- \\
\hline 50 & 341330 & 5452 & 0 & 0 & -1 & -1 & -1 & $\cdots-$ & & $-\cdots$ & $-\cdots$ & -- \\
\hline 566 & 541331 & 5453 & 0 & 0 & -1 & -1 & -1 & $\cdots$ & & --- & --- & --- \\
\hline 567 & 341 & 5456 & 0 & 0 & -1 & -1 & -1 & $-\cdots$ & & -- & --- & -- \\
\hline 568 & 341350 & 5458 & 0 & 0 & -1 & -1 & -1 & $\cdots$ & & --- & --- & $-\infty$ \\
\hline 569 & 541351 & 5459 & 0 & 0 & 1 & 1 & --1 & $-\cdots$ & & --- & $-\cdots$ & -- \\
\hline 570 & 541370 & 5464 & 0 & 0 & -1 & -1 & -1 & --- & & -- & $-\cdots$ & - \\
\hline 71 & 41380 & 3467 & 0 & 0 & -1 & -1 & -1 & --- & & --- & $\cdots-$ & -- \\
\hline 572 & 90 & 5470 & 0 & 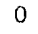 & -1 & -1 & -1 & $\cdots$ & & -- & --- & -- \\
\hline 573 & 400 & 5473 & 2 & 2 & -1 & 2 & -1 & $-\cdots$ & 3. & $6970+05$ & --- & -- \\
\hline 574 & 41410 & 3416 & 1 & 1 & 2 & 2 & 1 & 3. $3528+04$ & 1. & $0267+06$ & $6.8564+01$ & $2-03$ \\
\hline 375 & 541420 & 5479 & 2 & 2 & 1 & 1 & 1 & 1. $4043+06$ & 1 . & $5764+06$ & $55+02$ & --- \\
\hline 576 & 541430 & 5432 & 2 & 2 & 1 & 1 & 1 & $2.0500+06$ & 2 . & $0880+06$ & $4.4114+03$ & --- \\
\hline 577 & 31 & 5483 & 2 & 2 & 1 & 1 & -1 & $2.2251+06$ & 1 . & $7280+06$ & --- & -- \\
\hline 578 & $54 i 440$ & 5485 & 2 & 2 & 1 & 1 & 1 & $1.6060+06$ & 9. & $2320+05$ & $2.8032+03$ & -- \\
\hline 579 & 541450 & 5488 & 2 & 2 & 1 & 1 & 1 & $2.2910+06$ & 1. & $8270+06$ & 2. $5784+04$ & -- \\
\hline 280 & 541460 & 5491 & 2 & 2 & 1 & 1 & 1 & $1.9711+06$ & 1 . & +06 & $2.7629+04$ & --- \\
\hline 581 & 541470 & 5494 & 2 & 2 & 1 & 1 & 1 & $2.2789+06$ & 2. & $3131+06$ & $47+04$ & --- \\
\hline 382 & 40 & 5728 & 0 & 0 & -1 & -1 & -1 & --- & & $\cdots-$ & --- & -- \\
\hline 583 & 551341 & 5529 & 0 & 0 & -1 & -1 & -1 & $-\cdots$ & & $-\cdots$ & $-\cdots$ & -- \\
\hline 584 & 551350 & 5531 & 0 & 0 & -1 & -1 & -1 & --- & & $--\cdots$ & $\cdots$ & - \\
\hline 385 & 551351 & 5532 & 0 & 0 & -1 & -1 & -1 & $-\cdots$ & & --- & --- & $\ldots$ \\
\hline 506 & 531360 & 5334 & 0 & $?$ & -1 & -1 & -1 & $\cdots$ & & $-\cdots$ & $-\cdots$ & -- \\
\hline 547 & 361 & 535 & 6 & 6 & -1 & 1 & -1 & $\cdots$ & & $\cdots$ & $\cdots$ & - \\
\hline $5 H Q$ & 251370 & 5331 & 0 & 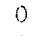 & -1 & -1 & -1 & --- & & $--\infty$ & $\cdots$ & $\cdots$ \\
\hline 589 & 531380 & 5340 & 0 & 0 & -1 & -1 & -1 & $-\cdots$ & & $-\cdots$ & -- & $\cdots$ \\
\hline 590 & 531381 & 5541 & 2 & 2 & 2 & 2 & $-1-$ & $0+04$ & 2. & $9029+05$ & $\cdots-$ & -- \\
\hline 591 & 351390 & 5543 & 0 & () & -1 & -1 & -1 & --- & & --- & $\cdots$ & - \\
\hline, 92 & 100 & 5546 & 6 & $t$ & 2 & 2 & -1 & $2+03$ & 6. & $1+05$ & $-\cdots$ & $8-0$ \\
\hline 593 & 551410 & 5549 & 1 & 1 & 2 & 2 & 1 & $1.5176+04$ & 3. & $6100+05$ & $7.9177+01$ & 7.80 \\
\hline 394 & $351+20$ & 3052 & 2 & 2 & 1 & 2 & 1 & $2.4490+06$ & 7. & $6682+05$ & $2.4996+02$ & -- \\
\hline 593 & 551430 & $55 j 5$ & 1 & 1 & 2 & 2 & 1 & $5.0195+05$ & 8. & +05 & $4.1149+03$ & $5.6760-0$ \\
\hline 596 & 551440 & 5558 & 1 & 1 & 1 & 1 & 1 & $2.3900+06$ & 2. & $6601+06$ & $9.8195+03$ & -- \\
\hline 597 & 551450 & 3561 & 1 & 1 & 2 & 2 & 1 & $1.0891+06$ & 1. & $7101+06$ & $6.2998+04$ & 8.26 \\
\hline 596 & 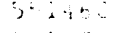 & 5564 & 1 & 1 & 2 & 2 & 1 & $5.3204+05$ & 1. & $3427+06$ & $8.1035+04$ & 6.87 \\
\hline 54 & $914 i$ & $\therefore 56$ & 2 & 2 & 1 & 1 & 1 & $2.2190+06$ & 1. & $5810+06$ & $1.4630+05$ & -- \\
\hline 010 & .1440 & $\because 1$ & 2 & 2 & 1 & 1 & 1 & $2.4540+06$ & 2. & $9690+06$ & $1.2391+05$ & \\
\hline $60 !$ & $214+$ & $7 i$ & 2 & 2 & 1 & 1 & 1 & $2.5069+06$ & 2. & $4041+06$ & $1.7669+05$ & -- \\
\hline 69 & $\vdots$ & $\cdot$ & 2 & 2 & 1 & 1 & 1 & $2.7510+06$ & 3. & $3311+06$ & $7.6691+04$ & - \\
\hline$\therefore 03$ & , e . , i & $24 i$ & 0 & 0 & -1 & -1 & -1 & -- & & --- & --- & -- \\
\hline$n 64$ & $26: 36 i$ & $\therefore 4$ & 0 & 0 & -1 & -1 & -1 & $\cdots$ & & --- & --- & -- \\
\hline $6: 5$ & $+1+i$ & 1641 & 0 & 0 & -1 & -1 & -1 & $\cdots$ & & -- & --- & -- \\
\hline 606 & 261390 & 3652 & 0 & 0 & -1 & -1 & -1 & $-\cdots$ & & --- & --- & -- \\
\hline 607 & 561400 & 5655 & 0 & 0 & -1 & -1 & -1 & --- & & $\cdots$ & -- & -- \\
\hline 608 & 561410 & 5658 & 0 & 0 & 2 & -1 & -1 & $4.6161+03$ & & --- & -- & $8.9573-03$ \\
\hline 609 & 561420 & 5661 & 0 & 0 & -1 & -1 & -1 & $\cdots$ & & $-\cdots$ & --- & -- \\
\hline 610 & 561430 & 5664 & 0 & 0 & -1 & -1 & -1 & --- & & --- & $-\cdots$ & - \\
\hline
\end{tabular}


Table C-2 (Cont.)

\begin{tabular}{|c|c|c|c|c|}
\hline & & & $A V$ & \\
\hline $\mathrm{NLM}$ & ID & $\therefore A T$ & $B$ & $G$ \\
\hline 611 & 561440 & 3607 & 2 & 2 \\
\hline 612 & 561450 & 5670 & 1 & 1 \\
\hline 613 & 561460 & 5673 & 1 & 1 \\
\hline 614 & 561470 & 5676 & 2 & 2 \\
\hline 615 & 561480 & 5679 & 2 & 2 \\
\hline 616 & 561490 & 5682 & 2 & 2 \\
\hline 617 & 561500 & 5685 & 2 & 2 \\
\hline 618 & 561510 & 5688 & 2 & 2 \\
\hline 619 & 561520 & 3691 & 2 & 2 \\
\hline 620 & 571380 & 5725 & 0 & 0 \\
\hline 621 & 571400 & 5731 & 0 & 0 \\
\hline 622 & 571410 & 5734 & 6 & 6 \\
\hline 623 & 571420 & 5737 & 0 & 0 \\
\hline 624 & 571430 & 5740 & 1 & 1 \\
\hline 625 & 571440 & 5743 & 1 & 1 \\
\hline 626 & 571450 & 5746 & 1 & 1 \\
\hline 627 & 571460 & 5749 & 1 & 1 \\
\hline 628 & 571461 & 5750 & 0 & 0 \\
\hline 629 & 571470 & 5752 & 2 & 2 \\
\hline 630 & 571480 & 5755 & 0 & 0 \\
\hline 631 & 571490 & 5758 & 2 & 2 \\
\hline 632 & 571500 & 5761 & 2 & 2 \\
\hline 633 & 571510 & 3764 & 2 & 2 \\
\hline 634 & 571520 & 5767 & 2 & 2 \\
\hline 635 & 571530 & 5770 & 2 & 2 \\
\hline 636 & 571540 & 5773 & 2 & 2 \\
\hline 637 & 571550 & 5776 & 2 & 2 \\
\hline 638 & 581410 & 5840 & 0 & 0 \\
\hline 639 & 581420 & 5843 & -5 & -5 \\
\hline 640 & 581430 & 5846 & 0 & 0 \\
\hline 641 & 581440 & 5849 & 0 & 0 \\
\hline 642 & 581450 & 5852 & 0 & 0 \\
\hline 643 & 581460 & 5855 & 0 & 0 \\
\hline 644 & 581470 & 5858 & 2 & 2 \\
\hline 645 & 581480 & 5861 & 0 & 0 \\
\hline 646 & 581490 & 5864 & 2 & 2 \\
\hline 647 & 581500 & 5867 & 2 & 2 \\
\hline 648 & 581510 & 5870 & 2 & 2 \\
\hline 649 & 581520 & 5873 & 2 & 2 \\
\hline 650 & 581530 & 5876 & 2 & 2 \\
\hline 651 & 581540 & 5879 & 2 & 2 \\
\hline 652 & 581550 & 5882 & 2 & 2 \\
\hline 653 & 581560 & 5885 & 2 & 2 \\
\hline 654 & 581570 & 5888 & 2 & 2 \\
\hline 655 & 591420 & 5928 & 0 & 0 \\
\hline 656 & 591421 & 5929 & 0 & 0 \\
\hline 657 & 591430 & 5931 & 0 & 0 \\
\hline 658 & 591440 & 5934 & 0 & 0 \\
\hline 659 & 591441 & 5935 & 0 & 0 \\
\hline 660 & 591450 & 5937 & 0 & 0 \\
\hline 661 & 591460 & 5940 & 0 & 0 \\
\hline 662 & 591470 & 5943 & 6 & 6 \\
\hline 663 & 591480 & 5946 & 2 & 2 \\
\hline 664 & 591481 & 5947 & 2 & 2 \\
\hline 665 & 591490 & 5949 & 2 & 2 \\
\hline 666 & 591500 & 5952 & 2 & 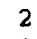 \\
\hline 667 & 591510 & 5955 & 2 & 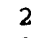 \\
\hline 668 & 591520 & 5958 & 2 & 2 \\
\hline 669 & 591530 & 5961 & 2 & 2 \\
\hline 670 & 591540 & 5964 & 2 & 2 \\
\hline 671 & 591550 & 5967 & 2 & 2 \\
\hline 672 & 591560 & 5970 & 2 & 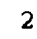 \\
\hline 673 & 591570 & 5973 & 2 & 2 \\
\hline 674 & 591580 & 5976 & 2 & 2 \\
\hline 675 & 591590 & 5979 & 2 & 2 \\
\hline 676 & 601440 & 6031 & -5 & -5 \\
\hline 677 & 601470 & 6040 & 0 & 0 \\
\hline 678 & 601490 & 6046 & 0 & 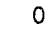 \\
\hline
\end{tabular}

\begin{tabular}{|c|c|c|c|c|c|c|c|}
\hline & Y & & cont & & $r-$ & & \\
\hline$B$ & i & 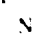 & BEIA & (FAYMA & NELC'T & $\therefore E W$ & $\mathrm{FD}$ \\
\hline 1 & 2 & 1 & $9.4629+05$ & $1.014(1+0)$ & $\ldots$ & & $\ldots$ \\
\hline 2 & 2 & -1 & $3.3070+05$ & $3.4740+0=$ & & & $\cdots-\infty$ \\
\hline 1 & -1 & 1 & 1. $3700+00$ & $\cdots$ & $1.3+10+01$ & & $\ldots \ldots$ \\
\hline 1 & 1 & 1 & $1+1580+06$ & $13010+06$ & 6. $3630+00$ & & $\ldots$. \\
\hline & 2 & 1 & $2.169+05$ & $62300+05$ & $6.7759+01$ & 7.3510 & -03 \\
\hline & 1 & 1 & $2.0160+06$ & $1.5200+06$ & $9.4217+01$ & & $\cdots-$ \\
\hline 1 & 1 & 1 & $1.9850+06$ & $1.0960+06$ & $9.8791+02$ & & $\ldots$ \\
\hline 1 & 1 & 1 & $2.1831+06$ & $2.2>10+06$ & 1. $4108+04$ & & $\ldots$. \\
\hline 1 & 1 & 1 & 2. $5239+06$ & 1. $3900+06$ & $2.2730+04$ & & $\ldots$ \\
\hline & -1 & -1 & $\cdots$ & $-\ldots$ & $\ldots$ & & $\ldots$ \\
\hline & -1 & -1 & --- & $\ldots$ & $\ldots$ & & $\ldots$ \\
\hline & 2 & -1 & $\cdots$ & $1.5667+04$ & $-\cdots$ & & -- \\
\hline & -1 & -1 & $-\cdots$ & $\ldots .$. & --- & & $\cdots$ \\
\hline & -1 & -1 & $\ldots$ & -- & $\cdots$ & & $\ldots$ \\
\hline 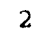 & -1 & -1 & $3.3460+04$ & -- & $\ldots$ & 8.5307 & -03 \\
\hline 2 & 2 & -1 & $6.1160+05$ & $8.4008+05$ & $\ldots$ & $16:$ & -03 \\
\hline 2 & 2 & 1 & $2.4979+05$ & $1.0540+06$ & $9.9295-01$ & 7.89 & -03 \\
\hline 3 & -1 & -1 & $\ldots$ & $\ldots$ & --- & & $\ldots \ldots$ \\
\hline 1 & 2 & 1 & $1.6320+06$ & $7.5630+05$ & $7.6282+01$ & & $-\ldots$ \\
\hline-1 & -1 & 1 & $\ldots$ & $\ldots$ & $8.9599+01$ & & --- \\
\hline 1 & 1 & 1 & $1.7830+06$ & $1.0870+06$ & $35+03$ & & $-\cdots$ \\
\hline 1 & 1 & 1 & $2.0370+06$ & $2.5470+06$ & $2+03$ & & $-\cdots$ \\
\hline 1 & 1 & 1 & $2.2020+06$ & 1.601 & $6+04$ & & $\cdots$ \\
\hline 1 & 1 & 1 & $2.3550+06$ & 2.884 & $5+04$ & & $-\cdots$ \\
\hline 1. & 1 & 1 & 2. $5950+06$ & 2.088 & $0+04$ & & -- \\
\hline 1 & 1 & 1 & $2.6149+06$ & 3.198 & $4+04$ & & --- \\
\hline 1 & 1 & 1 & $2.8169+06$ & 2.698 & $4+04$ & & -- \\
\hline 1 & -1 & -1 & $\ldots$ & $\cdots$ & -- & & -- \\
\hline 1 & -1 & -1 & $\ldots$ & $\ldots$ & -- & & --- \\
\hline 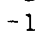 & -1 & -1 & -- & $\ldots$ & -- & & --- \\
\hline- & -1 & -1 & $\ldots$ & $\ldots$ & $-\cdots$ & & --- \\
\hline 1 & -1 & -1 & $\ldots$ & $\ldots$ & $\ldots-$ & & $\ldots$ \\
\hline 1 & -1 & -1 & --- & $-\ldots$ & $\ldots-$ & & $\ldots$ \\
\hline 1 & 2 & -1 & $8+05$ & $9.8303+05$ & $\cdots$ & & $\cdots$ \\
\hline 1 & -1 & -1 & $\ldots$ & $\ldots$ & -- & & $\ldots$ \\
\hline 1 & 1 & 1 & 1. $1752+06$ & 1.04 & --- & & --- \\
\hline 1 & 1 & -1 & $6.8918+05$ & 4.396 & -- & & $\ldots$ \\
\hline 1 & 1 & -1 & $1.4400+06$ & 8.774 & --- & & $-\cdots$ \\
\hline 1 & 1 & -1 & $1.1660+06$ & 7.784 & -- & & $-\cdots$ \\
\hline 1 & 1 & 1 & $1.6800+06$ & 1.125 & $4+03$ & & --- \\
\hline 1 & 1 & 1 & $1.6940+06$ & $9.5867+05$ & 1.6 & & $-\infty$ \\
\hline 1 & 1 & 1 & $2.0150+06$ & 1.571 & 4.9 & & $\ldots$ \\
\hline 1 & 1 & 1 & $2.1180+06$ & 1.17 & +04 & & $-\cdots$ \\
\hline 1 & 1 & 1 & $2.4309+06$ & 2.08 & +04 & & -- \\
\hline 1 & -1 & $\cdots 1$ & $\ldots$ & $\ldots$ & $\ldots$ & & --1 \\
\hline-1 & $-i$ & -1 & $\ldots$ & -- & $\cdots-$ & & $\ldots$ \\
\hline 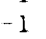 & -1 & -1 & $\ldots$ & --- & $\ldots$ & & $\ldots$ \\
\hline & -1 & -1 & -- & -- & $-\cdots$ & & $\ldots$ \\
\hline 1 & -1 & -1 & --- & $\ldots$ & - & & $\ldots$ \\
\hline 1 & -1 & -1 & --- & $\ldots-$ & $\ldots$ & & $-\cdots$ \\
\hline 1 & -1 & -1 & $-\cdots$ & $\ldots$ & --- & & $\ldots$ \\
\hline 2 & 2 & -1 & $5+03$ & 4. 24 & --- & 8. & -0 \\
\hline 2 & 2 & -1 & $2.9362+04$ & 3.63 & $-\cdots$ & & -07 \\
\hline 2 & 2 & -1 & $6.0288+04$ & 8. 58 & $\ldots$ & 7. & -0 \\
\hline 2 & 2 & -1 & $5.3163+04$ & 2.46 & $\cdots$ & 8 & -03 \\
\hline 1 & 1 & -1 & $2.0170+06$ & 1.076 & --- & & $\ldots$ \\
\hline 2 & 2 & -1 & $1.9456+05$ & $2.4890+05$ & $\ldots$ & 7.4 & -03 \\
\hline 1 & 1 & -1 & 1. $5490+06$ & $2.1190+06$ & --- & & $\cdots-$ \\
\hline 1 & 1 & -1 & $1.7000+06$ & $1.0270+06$ & --- & & $-\cdots$ \\
\hline 1 & 1 & 1 & $1.8730+06$ & $2.4140+06$ & 2.0 & & $-\cdots$ \\
\hline 1 & 1 & 1 & $2.0710+06$ & 1. $4 \varepsilon^{2} 0+06$ & 4. 33 & & $\ldots$ \\
\hline 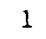 & 1 & 1 & $2.1490+06$ & $2.6879+06$ & $8.9302+03$ & & - \\
\hline 1 & 1 & 1 & $2.3870+06$ & $1.8809+06$ & $2.3721+04$ & & $\ldots \ldots$ \\
\hline 1 & $i$ & 1 & $2.5519+06$ & $3.1550+06$ & 2. $5556+04$ & & $\ldots$ \\
\hline 1 & 1 & 1 & $2.7730+06$ & 2. $3380+06$ & $5.3750+04$ & & $\ldots$ \\
\hline 1 & -1 & -1 & $\ldots$ & $\ldots$ & - - & & -- \\
\hline-1 & -1 & -1 & $\ldots$ & $-\ldots$ & $-\cdots$ & & \\
\hline-1 & -1 & -1 & $-\cdots$ & $\ldots$ & --- & & - \\
\hline
\end{tabular}


Table C-2 (Cont.)

\begin{tabular}{|c|c|c|c|c|c|c|c|c|c|c|c|}
\hline & & & Av. & $\mathrm{E}$ & $S P$ & TYP & & $\operatorname{coNT}$ & E COMPONE & ENT - - & \\
\hline$N L M$ & I [ & MAT & $\mathrm{B}$ & c; & $\mathrm{B}$ & i & $v$ & BE'TA & GAMMA & NEUT. & NEW FD \\
\hline 679 & 601510 & 6052 & 0 & 0 & -1 & -1 & -1 & $\cdots$ & $\ldots$ & $\ldots$ & $\cdots$ \\
\hline 080 & 601520 & 0057 & 0 & 0 & 1 & -1 & -1 & $\ldots$ & $\ldots$ & $\cdots$ & $\cdots-$ \\
\hline 681 & 601530 & 0,058 & 2 & 2 & $i$ & 1 & -1 & $1.1110+06$ & $6.7228+05$ & $-\cdots$ & $\cdots+\cdots$ \\
\hline 682 & 601540 & 6061 & 2 & 2 & 1 & 1 & -1 & $6.0718+05$ & $6.0760+05$ & -- & $-\cdots$ \\
\hline 683 & 601550 & 0064 & 2 & 2 & 1 & 1 & -1 & 1. $3660+06$ & 8. $3390+05$ & $\ldots$ & $\cdots$ \\
\hline 684 & 601560 & 0067 & 2 & 2 & 1 & 1 & -1 & 1. $1220+06$ & $7.6567+05$ & $\cdots$ & $\cdots$ \\
\hline 685 & 601570 & 6070 & 2 & 2 & 1 & 1 & -1 & $1.6680+06$ & $1.1400+06$ & $\cdots \cdots$ & $-\cdots-$ \\
\hline 686 & 601580 & 6073 & 2 & 2 & 1 & 1 & 1 & 1. $5890+06$ & $9.2387+05$ & 5. $3826+00$ & $-\cdots$ \\
\hline 687 & 601590 & 6076 & 2 & 2 & 1 & 1 & 1 & $2.0629+06$ & 1. $6660+06$ & $5.1090+02$ & $\cdots$ \\
\hline 688 & 601600 & 6079 & 2 & 2 & 1 & 1 & 1 & $2.0999+06$ & 1. $2060+06$ & $2.4922+03$ & $-\cdots$ \\
\hline 689 & 601610 & 6082 & 2 & 2 & 1 & 1 & 1 & $2.1600+06$ & $1.8790+06$ & $5.2440+03$ & $\cdots$ \\
\hline 690 & 611450 & 0143 & 0 & 0 & -1 & -1 & -1 & --- & -- & $-\cdots$ & -- \\
\hline 691 & 611470 & 6149 & 0 & 0 & -1 & -1 & -1 & -- & $\ldots$ & $\ldots-$ & --- \\
\hline 692 & 611480 & 6152 & 0 & 0 & -1 & -1 & -1 & $\ldots$ & -- & $\ldots-$ & $\cdots$ \\
\hline 693 & 611481 & 6153 & 0 & 0 & -1 & -1 & -1 & $\cdots$ & $\ldots$ & $\ldots$ & --- \\
\hline 694 & 611490 & 6155 & 0 & 0 & -1 & -1 & -1 & $\ldots$ & --- & $\ldots \ldots$ & $\ldots$ \\
\hline 695 & 611300 & 6158 & 0 & 0 & -1 & -1 & -1 & $\ldots$ & -- & $\ldots$ & $\cdots$ \\
\hline 696 & 611510 & 6161 & 0 & 0 & -1 & -1 & -1 & $\ldots$ & $\cdots$ & $\cdots-$ & $-\cdots$ \\
\hline 697 & 611520 & 6164 & 0 & 0 & -1 & -1 & -1 & $\ldots$ & --- & $\cdots$ & $\cdots$ \\
\hline 698 & 611521 & 6165 & 0 & 0 & -1 & -1 & -1 & $\cdots-$ & -- & $--\cdots$ & $\cdots$ \\
\hline 699 & 611522 & 6166 & 2 & 2 & 1 & 1 & -1 & $6.6079+05$ & $1.7332+06$ & --- & --- \\
\hline 100 & 611530 & 6167 & 2 & 2 & 1 & 1 & -1 & $6.0721+05$ & $1.7220+05$ & --- & $\cdots$ \\
\hline 701 & 611540 & 6170 & 0 & 0 & -1 & -1 & -1 & $\ldots-$ & $-\cdots$ & $\cdots$ & $\cdots$ \\
\hline 702 & 611541 & 6171 & 6 & 6 & 2 & 2 & -1 & 3. $4463+04$ & $6.8460+05$ & --- & $7.4952-03$ \\
\hline 703 & 611550 & 6173 & 2 & 2 & 1 & 1 & -1 & $1.0200+06$ & $6.3301+05$ & $-\cdots$ & $\cdots$ \\
\hline 704 & 611560 & 6176 & 2 & 2 & 1 & 1 & -1 & 1. $3140+06$ & $1.8940+06$ & $\cdots$ & $-\cdots$ \\
\hline 705 & 611570 & 6179 & 2 & 2 & 1 & 1 & -1 & $1.4510+06$ & 8. $4079+05$ & $\cdots$ & $\ldots$ \\
\hline 706 & 611580 & 6182 & 2 & 2 & 1 & 1 & -1 & 1. $5690+06$ & $2.1640+06$ & $-\cdots$ & -- \\
\hline 707 & 611590 & 6185 & 2 & 2 & 1 & 1 & 1 & $1.7819+06$ & 1. $1599+06$ & $2.1557+01$ & --- \\
\hline 708 & 611600 & 6188 & 2 & 2 & 1 & 1 & 1 & $1.9690+06$ & $2.4999+06$ & $5.5300+02$ & --- \\
\hline 709 & 611610 & 6191 & 2 & 2 & 1 & 1 & 1 & $2.1081+06$ & $1.6961+06$ & $4.7400+03$ & $\cdots$ \\
\hline 710 & 611620 & 6194 & 2 & 2 & 1 & 1 & 1 & $2.0790+06$ & $2.6200+06$ & $6.5128+03$ & -- \\
\hline 711 & 621450 & 6228 & 0 & 0 & -1 & -1 & -1 & $-\cdots-$ & . & $-\cdots$ & --- \\
\hline 712 & 621460 & 6231 & -5 & -5 & -1 & -1 & -1 & $\cdots$ & $\cdots$ & --- & --- \\
\hline 713 & 621470 & 6234 & -5 & -5 & -1 & -1 & -1 & $\cdots$ & --- & --- & --- \\
\hline 714 & 621480 & 6237 & -5 & -5 & -1 & -1 & -1 & -- & $-\cdots-$ & -- & --- \\
\hline 715 & 621490 & 6240 & -5 & -5 & -1 & -1 & -1 & --- & --- & -- & --- \\
\hline 716 & 621510 & 6246 & 0 & 0 & -1 & -1 & -1 & $-\cdots$ & $--\cdots$ & $-\cdots$ & $-\cdots$ \\
\hline 717 & 621530 & 6252 & 0 & 0 & -1 & -1 & -1 & -- & --- & $-\cdots$ & --- \\
\hline 718 & 621550 & 6258 & 0 & 0 & $-i$ & -1 & -1 & $\cdots$ & --- & $\ldots$ & $-\cdots$ \\
\hline 719 & 621560 & 6261 & 0 & 0 & -1 & -1 & -1 & $\cdots$ & $\cdots$ & $\cdots$ & $\cdots$ \\
\hline 720 & 621570 & 6264 & 2 & 2 & 1 & 1 & -1 & $8.6261+05$ & $4.0160+05$ & --- & $\ldots$ \\
\hline 721 & 621580 & 6267 & 2 & 2 & 1 & 2 & -1 & $4.0843+05$ & $2.1592+05$ & --- & $\rightarrow$ \\
\hline 722 & 621590 & 6270 & 2 & 2 & 1 & 1 & -1 & $1.0002+06$ & $9.6499+05$ & --- & $\ldots$ \\
\hline 723 & 621600 & 0273 & 2 & 2 & 1 & 1 & -1 & $8.4669+05$ & $6.8978+05$ & --- & $-\infty$ \\
\hline 724 & 621610 & 6276 & 2 & 2 & 1 & 1 & -1 & $1.5070+06$ & 1. $1380+06$ & --- & $\ldots$ \\
\hline 725 & 621620 & 6279 & 2 & 2 & 1 & 1 & -1 & 1. $3830+06$ & $8.7783+05$ & $-\cdots$ & $-\cdots-$ \\
\hline 726 & 621630 & 6282 & 2 & 2 & 1 & 1 & -1 & $1.6690+06$ & 1. $3340+06$ & --- & -- \\
\hline 727 & 621640 & 6285 & 2 & 2 & 1 & 1 & 1 & $1.8029+06$ & $1.0490+06$ & $1.4716+01$ & -- \\
\hline 728 & 621650 & 6288 & 2 & 2 & 1 & 1 & 1 & $1.9630+06$ & 1. $6910+06$ & $5.2722+02$ & -- \\
\hline 729 & 631520 & 6328 & 0 & 0 & -1 & $-i$ & -1 & -- & --- & $\cdots$ & -- \\
\hline 730 & 631521 & 6329 & 0 & 0 & -1 & -1 & -1 & -- & $-\cdots$ & $\cdots$ & -- \\
\hline 731 & 631522 & 6330 & 0 & 0 & -1 & -1 & -1 & $\ldots$ & --- & $\ldots$ & 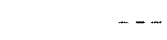 \\
\hline 732 & 631540 & 6334 & 0 & 0 & -1 & -1 & -1 & $-\cdots$ & $\cdots$ & $\cdots-$ & - \\
\hline 733 & 631541 & 6335 & 0 & 0 & -1 & -1 & -1 & $-\cdots$ & - & $\ldots$ & -- \\
\hline 734 & 631550 & 6337 & 0 & 0 & -1 & $-i$ & -1 & $-\cdots$ & -- & --- & -- \\
\hline 735 & 631360 & 6340 & 0 & 0 & -1 & -1 & -1 & $\ldots$ & $\ldots$ & $\ldots$ & \\
\hline 736 & 631570 & 6343 & 0 & 0 & -1 & -1 & -1 & $\cdots$ & $-\cdots-$ & $\cdots$ & -- \\
\hline 737 & 631580 & 6346 & 0 & 0 & -1 & -1 & -1 & $-\cdots$ & --- & $-\ldots$ & -- \\
\hline 738 & 631590 & 6349 & 2 & 2 & 1 & 2 & -1 & $8.7290+05$ & $2.2555+05$ & $\ldots$ & - \\
\hline 739 & 631600 & 6352 & 0 & 0 & -1 & -1 & -1 & $\ldots$ & - & $-\cdots$ & $-\cdot$ \\
\hline 740 & 631610 & 6355 & 2 & 2 & 1 & 1 & -1 & 2. .0059+06 & $1.0062+06$ & -- & $-\cdots$ \\
\hline 741 & 631620 & 6358 & 2 & 2 & 1 & 1 & -1 & $1.4030+06$ & $2.0180+06$ & $\cdots$ & - \\
\hline 742 & 631630 & 6361 & 2 & 2 & 1 & 1 & -1 & $1.5410+06$ & 1. $0720+06$ & $-\cdots$ & $-\cdots$ \\
\hline 743 & 631640 & 6364 & 2 & 2 & 1 & 1 & 1 & $1.5629+06$ & $2.1469+06$ & $5.0000 \cdots 03$ & - \\
\hline 744 & 631650 & 6367 & 2 & 2 & 1 & 1 & 1 & $1.8301+06$ & 1. $4070+06$ & 3. $4214+02$ & $-\cdots$ \\
\hline 745 & 641520 & 6425 & -5 & -5 & -1 & -1 & -1 & $-\cdots$ & -- & -- & $\cdots$ \\
\hline 746 & 641530 & 6428 & 0 & 0 & -1 & -1 & -1 & $\cdots$ & --- & --- & - \\
\hline
\end{tabular}


Table C-2 (Cont.)

\begin{tabular}{|c|c|c|c|c|c|c|c|c|c|c|c|c|}
\hline & & & Av. & $\mathrm{E}$ & $S P$ & TTY & & CONT. & E COMPONENT $\cdots$ & $\ldots \ldots$ & & \\
\hline עי N' & II & MAT & $B$ & G & B & (i) & $N$ & BETA & GAMMA DEL. & NEUT & NEW & $\mathrm{FD}$ \\
\hline 747 & 641590 & 6446 & 0 & 0 & -1 & -1 & $\cdots 1$ & --- & $\cdots$ & $\cdots$ & & $\cdots$ \\
\hline 148 & 041610 & $64 \div 2$ & 0 & 0 & -1 & -1 & -1 & $\cdots$ & $\cdots$ & - & & $\cdots$ \\
\hline 149 & $64: 620$ & 645 & 2 & 2 & 2 & 2 & -1 & $3.7416+04$ & $1.1273+05$ & --- & 7.3435 & -03 \\
\hline 750 & 641630 & $645 k$ & 2 & 2 & 1 & 1 & -1 & $8.5918+05$ & $9.6129+05$ & --- & & $\ldots-\cdots$ \\
\hline 751 & 641640 & 0401 & 2 & 2 & 1 & 1 & -1 & $7.1809+05$ & $6.4691+05$ & -- & & $\cdots$ \\
\hline 752 & 041650 & 6464 & 2 & 2 & 1 & 1 & -1 & $1.2300+06$ & $8.8110+05$ & --- & & $\cdots$ \\
\hline 753 & 651600 & 6528 & 0 & 0 & -1 & -1 & -1 & --- & $-\cdots$ & --- & & $\cdots-$ \\
\hline 154 & 651610 & 6331 & 0 & 0 & -1 & -1 & -1 & --- & $\cdots$ & --- & & $\ldots$ \\
\hline 755 & 651620 & 6534 & 0 & 0 & -1 & $\because 1$ & -1 & --- & $\cdots$ & $-\cdots$ & & $\cdots$ \\
\hline 756 & 651630 & 6537 & 0 & 0 & -1 & -1 & -1 & --- & $\rightarrow-$ & -- & & $-\cdots$ \\
\hline 757 & 651640 & 6540 & 0 & 0 & -1 & -1 & -1 & $\cdots$ & $\cdots-$ & $-\cdots$ & & $\cdots$ \\
\hline 758 & 651650 & 6543 & 0 & 0 & -1 & -1 & -1 & $\cdots$ & $\cdots$ & --- & & --- \\
\hline 759 & 661650 & 6652 & 0 & 0 & -1 & -1 & -1 & --- & -- & $\cdots$ & & $-\cdots$ \\
\hline 760 & 661651 & 6653 & 0 & 0 & -1 & -1 & -1 & -- & --- & $-\cdots$ & & --- \\
\hline 761 & 661660 & 6655 & 0 & 0 & -1 & -1 & -1 & --- & --- & $\cdots$ & & $-\cdots$ \\
\hline 762 & 671660 & 6728 & 0 & 0 & -1 & -1 & -1 & $-\cdots$ & $\cdots$ & $\cdots-$ & & $\cdots$ \\
\hline 763 & 671661 & 6729 & 0 & 0 & -1 & -1 & -1 & --- & $\cdots$ & -- & & $\cdots$ \\
\hline 764 & 681671 & 6841 & 0 & 0 & -1 & -1 & -1 & --- & --- & $\cdots$ & & $\cdots \cdots$ \\
\hline
\end{tabular}

a"NUM" corresponds to the order in the fission-product decay file before combining all decay data into a single ENDF/B-VI file.

"ID" $=Z * 10000+A * 10+S$ is a numerical identification for the nuclide.

"MAT" = ENDF MAT number

Under "AV. E," the B (beta) and G (gamma) numbers mean:

0 no change in INEL spectroscopic data (Fall 1989)

1 direct measurement by G. Rudstam from INEL data

2 theory, LANL (using slightly modified Gross Theory code of T. Toshida)

5 same as ENDF/B-V

-5 no average energy as in ENDF/B-V

6-7 JNDC 1989; probably based on evaluated measurement for gamma and/or beta

Note: these two columns are primarily used to indicate with "2" where Gross Theory has been used as the source of average energy. Many such values will probably agree with JENDL2.

Under "SP TYP" for B, G, and N, the numbers mean:

$-1 \quad$ no change in spectroscopic spectra made

1 corresponding spectra entirely free from Gross Theory, except delayed neutrons are based on LANL evaluation of measured and theory (referenced in File 1).

2 Gross Theory spectra supplements spectroscopic

Under "CONT.E COMPONENT" are the beta, gamma, and delayed neutron energies derived from the continuous energy files.

Under "NEW FD," the discrete normalization factor for beta is listed if it has changed from the spectroscopic value (usually 1.0e-02). 

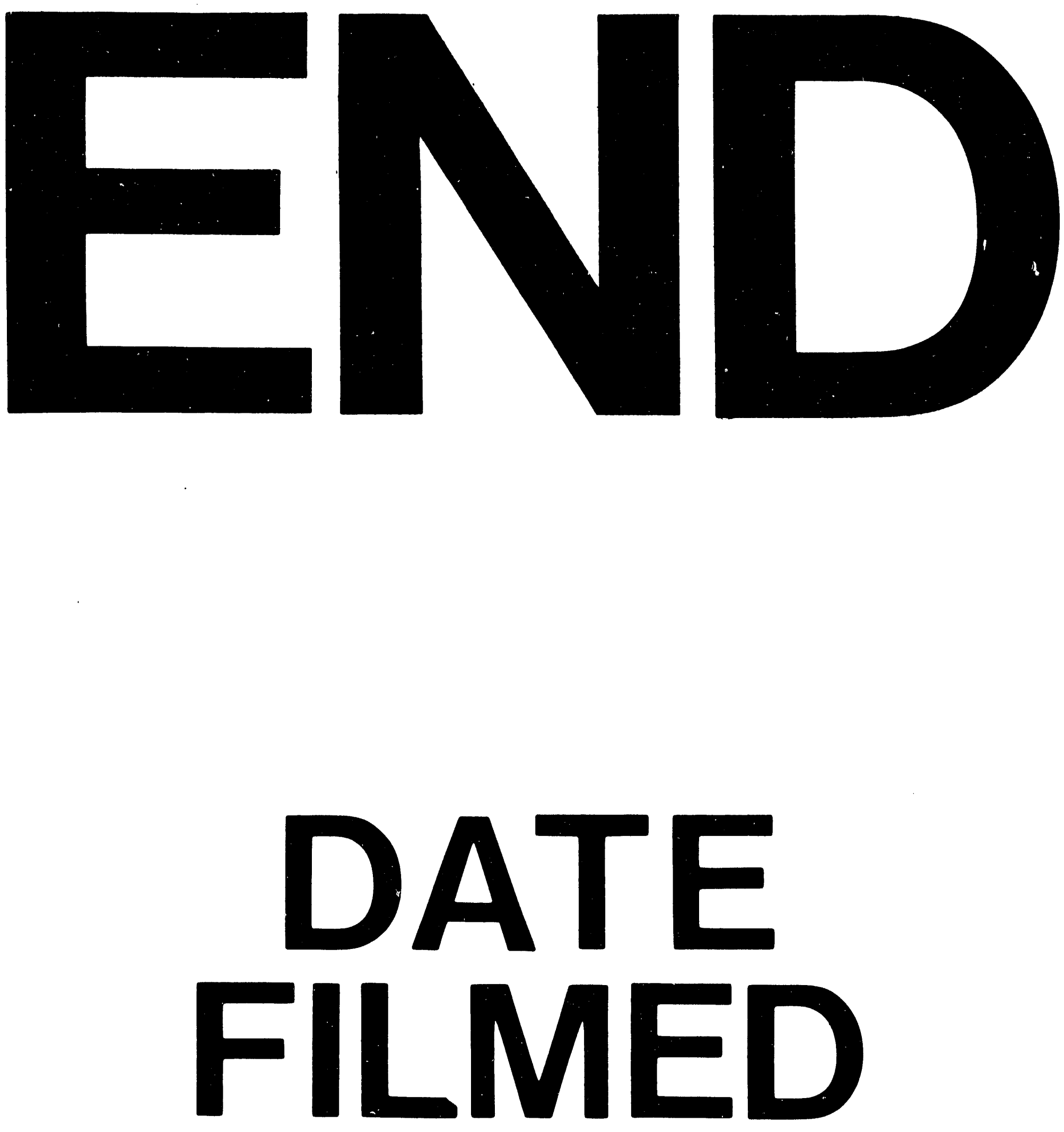

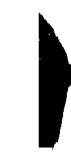

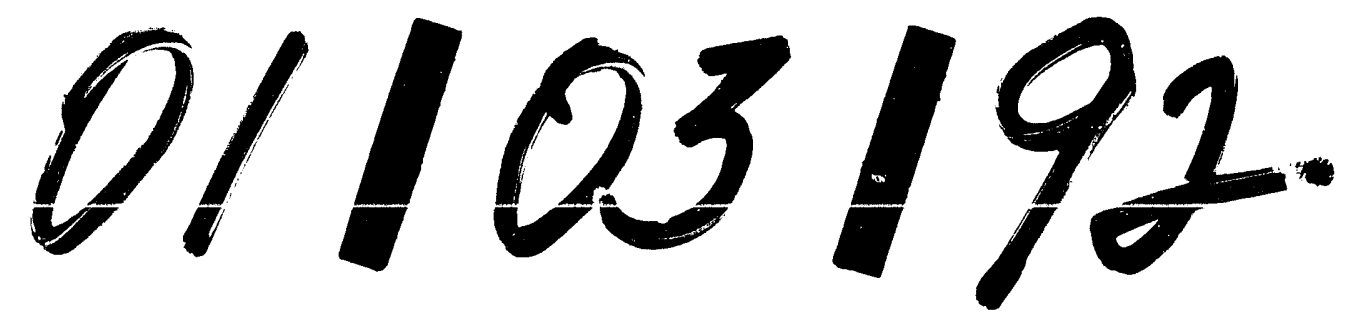


